\title{
Skin and Bones
}

Citation for published version (APA):

de Vos, I. J. H. M. (2018). Skin and Bones: Studying the effects of MMP14 mutations. [Doctoral Thesis, Maastricht University]. Datawyse / Universitaire Pers Maastricht. https://doi.org/10.26481/dis.20181219iv

Document status and date:

Published: 01/01/2018

DOI:

10.26481/dis.20181219iv

Document Version:

Publisher's PDF, also known as Version of record

\section{Please check the document version of this publication:}

- A submitted manuscript is the version of the article upon submission and before peer-review. There can be important differences between the submitted version and the official published version of record.

People interested in the research are advised to contact the author for the final version of the publication, or visit the DOI to the publisher's website.

- The final author version and the galley proof are versions of the publication after peer review.

- The final published version features the final layout of the paper including the volume, issue and page numbers.

Link to publication

\footnotetext{
General rights rights.

- You may freely distribute the URL identifying the publication in the public portal. please follow below link for the End User Agreement:

www.umlib.nl/taverne-license

Take down policy

If you believe that this document breaches copyright please contact us at:

repository@maastrichtuniversity.nl

providing details and we will investigate your claim.
}

Copyright and moral rights for the publications made accessible in the public portal are retained by the authors and/or other copyright owners and it is a condition of accessing publications that users recognise and abide by the legal requirements associated with these

- Users may download and print one copy of any publication from the public portal for the purpose of private study or research.

- You may not further distribute the material or use it for any profit-making activity or commercial gain

If the publication is distributed under the terms of Article $25 \mathrm{fa}$ of the Dutch Copyright Act, indicated by the "Taverne" license above, 
Skin and Bones: Studying the effects of MMP14 mutations Ivo J.H.M. de Vos

ISBN 978-94-6380-082-2

Cover design and layout by Ivo J.H.M. de Vos

Printed by Datawyse | Universitaire Pers Maastricht

Copyright (C) 2018 I.J.H.M. de Vos, Maastricht, the Netherlands

All rights reserved. No part of this thesis may be reproduced, stored or transmitted in any form or by any means without prior written permission of the author.

Cover illustration: transmitted light extended depth-of-focus microscopic image of an adult zebrafish anal fin, showing individual melanocytes (black), xanthophores (yellow) and segmented, branching bony fin rays. 


\section{Skin and Bones}

\section{Studying the effects of MMP14 mutations}

\section{DISSERTATION}

to obtain the degree of Doctor at Maastricht University, on the authority of the Rector Magnificus, Prof. Dr. Rianne M. Letschert in accordance with the decision of the Board of Deans, to be defended in public on Wednesday $19^{\text {th }}$ of December 2018, at 16:00 hours

$$
\text { by }
$$

Ivo Johannes Hendrikus Marie de Vos born on Thursday $18^{\text {th }}$ of July 1985 in Maastricht 


\section{Supervisors}

Prof. Dr. M.A.M. van Steensel (Nanyang Technological University, and Skin Research Institute of Singapore, Agency for Science, Technology, and Research, Singapore) Prof. Dr. P.M. Steijlen

\section{Co-supervisor}

Dr. B.J. Coull (University of Dundee, United Kingdom)

\section{Assessment Committee}

Prof. Dr. H.J.M. Smeets (chairman)

Prof. Dr. N.V.A.M. Knoers (University Medical Center Utrecht)

Prof. Dr. C.T.R.M. Stumpel

Prof. Dr. M.H. Vermeer (Leiden University Medical Center)

This work was financially supported by the Agency for Science, Technology and Research (A*STAR), the Skin Research Institute of Singapore (SRIS), the Biomedical Research Council Singapore (BMRC), the Wellcome Trust, Tenovus Scotland, the Victorian Government, the Australian Government, the Dutch Arthritis Society, and Stichting de Weijerhorst. 


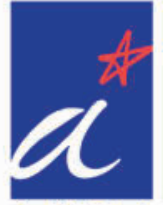

$A \star S T A R$

Institute of

Medical Biology

Skin Research

Institute of Singapore
$A$ * STAR
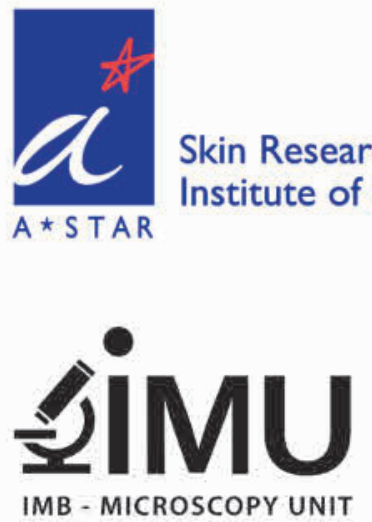

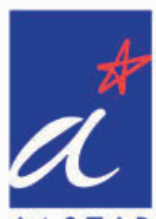

Institute of

Molecular and

Cell Biology

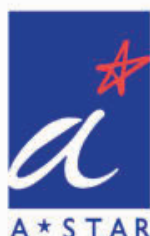

Singapore

Bioimaging

Consortium 

To my parents 



\section{Contents}

CHAPTER $1 \quad$ General introduction

CHAPTER 2 A novel homozygous missense mutation in MMP14

causes a mitigated form of Winchester syndrome

CHAPTER 3

The role of MMP14 in de novo lumen formation and

branching morphogenesis in acne pathogenesis

CHAPTER 4 A zebrafish model for Winchester syndrome

CHAPTER 5

General discussion

201

CHAPTER 6

Addendum 1

Addendum 2

Addendum 3

CHAPTER 7

Valorisation

CHAPTER 8

Summary 273

Samenvatting 279

Acknowledgements / Dankwoord 289

Curriculum vitae 294 



\section{CHAPTER 1}

\section{General introduction}

Adapted from:

Ivo J.H.M. de Vos, Evelyn Yaqiong Tao, Sheena Li Ming Ong, Julian L. Goggi, Thomas Scerri, Gabrielle R. Wilson, Chernis Guai Mun Low, Arnette Shi Wei Wong, Dominic Grussu, Alexander P.A. Stegmann, Michel van Geel, Renske Janssen, David J. Amor, Melanie Bahlo, Norris R. Dunn, Thomas J. Carney, Paul J. Lockhart, Barry J. Coull and Maurice A.M. van Steensel. Functional analysis of a hypomorphic allele shows that MMP14 catalytic activity is the prime determinant of the Winchester syndrome phenotype. Human Molecular Genetics 2018; 27(16):2775-2788

Ivo J.H.M. de Vos, Arnette Shi Wei Wong, Tim J.M. Welting, Barry J. Coull and Maurice A.M. van Steensel. Multicentric osteolytic syndromes represent a phenotypic spectrum defined by defective collagen remodelling. In revision for American Journal of Medical Genetics Part A 


\section{CHAP'TER 2}

\section{A novel homozygous missense mutation in MMP14 causes a mitigated form of Winchester syndrome}

Adapted from:

Ivo J.H.M. de Vos, Evelyn Yaqiong Tao, Sheena Li Ming Ong, Julian L. Goggi, Thomas Scerri, Gabrielle R. Wilson, Chernis Guai Mun Low, Arnette Shi Wei Wong, Dominic Grussu, Alexander P.A. Stegmann, Michel van Geel, Renske Janssen, David J. Amor, Melanie Bahlo, Norris R. Dunn, Thomas J. Carney, Paul J. Lockhart, Barry J. Coull and Maurice A.M. van Steensel. Functional analysis of a hypomorphic allele shows that MMP14 catalytic activity is the prime determinant of the Winchester syndrome phenotype. Human Molecular Genetics 2018; 27(16):2775-2788 


\section{Abstract}

In 2007, we reported two Dutch brothers with an apparently recessive syndrome consisting of dysmorphic facial features, progressive mitral valve insufficiency, severe nodulocystic acne, thoracic kyphosis and reduced bone mineral density. Recently, we identified a novel homozygous p.R111H mutation in MMP14 (MIM 600754), encoding the membrane-bound matrix metalloproteinase 14. Accordingly, we revised their diagnosis to Winchester syndrome (MIM 277950), awaiting confirmation of the mutation's pathogenicity. To assess the effect of the p.R111H mutation on the protein level, and compare it to the previously reported human and murine MMP14 mutations, we established a novel in vitro $2 \mathrm{D}$ cell culture model. We provide evidence that a mild manifestation of Winchester syndrome is caused by a novel hypomorphic MMP14 p.R111H allele. This mutation, in contrast to previously reported human and murine MMP14 mutations, does not affect MMP14's transport to the cell membrane. Instead, it partially impairs MMP14's proteolytic activity. This residual activity likely accounts for the mitigated phenotype observed in our patients. Our observations suggest that loss of MMP14's catalytic activity might be a prime determinant of disease severity. In addition, our model provided additional insights into normal MMP14 posttranslational processing. We present the first evidence of MMP14 signal peptide removal upon ER insertion. Our data furthermore suggest that the $\mathrm{R}^{89}-\mathrm{R}-\mathrm{P}-\mathrm{R}-\downarrow-\mathrm{C}^{93}$ proprotein convertase recognition site plays a more important role in pro-MMP14 activation than previously thought. 


\section{Introduction}

In 2007, we reported two Dutch brothers with an apparently recessive syndrome consisting of skeletal anomalies, dysmorphic facial features, progressive mitral valve insufficiency and severe nodulocystic acne [1]. We diagnosed them with Borrone dermatocardio-skeletal syndrome, as their phenotype was similar to that of two patients described by Borone et al., albeit milder [2]. Recently, a homozygous deletion of SH3PXD2B at 5q25.1 was identified in the latter patients [3]. In our patients however, mutation or copy number variation of $S H 3 P X D 2 B$ was absent [3]. Instead, we identified a novel homozygous c.332G > A mutation in MMP14 at 14q11.2 (MIM 600754), encoding the membrane-bound matrix metalloproteinase 14 [4]. This missense mutation results in the substitution p.Arg111His (R111H), which was predicted to be possibly damaging by multiple algorithms. Additional lines of evidence suggest that this mutation is pathogenic. Firstly, the protein products of SH3PXD2B and MMP14 cooperate during the formation of podosomes, which are specialized membrane structures involved in extracellular matrix (ECM) degradation and invasive cell motility [5]. SH3PXD2B recruits MMP14 from intracellular stores to the podosome membrane, making them functionally active [5]. Secondly, a homozygous MMP14 loss-of-function mutation (n.284C $>$ G, p.T17R) has previously been identified in a patient with Winchester syndrome (WS, MIM 277950) [7]. Although the phenotype of this individual was more severe than that of our patients, they share a short stature, premature osteoporosis and have similar facial dysmorphology $[1,7]$. Finally, two additional missense mutations, p.R92C (Sabe) and p.S466P (Cartoon), result in similar features in mice when present homozygously [8, 9]. Therefore, we revised our patients' diagnosis to WS, pending confirmation of the mutation's pathogenicity.

MMP14 is a transmembrane proteinase that exerts its main catalytic function in the pericellular space [10]. Apart from transcriptional control, multiple processes posttranslationally regulate the activity of MMP14. These affect the activation of the latent pro-enzyme (see below), exposure of the active enzyme on the cell surface, or its lysosomal degradation [11-17].

MMP14 is synthesized as a latent pre-pro-enzyme [4, 18]. Prior to its appearance on the cell surface in its active form, the N-terminal part of MMP14 undergoes a series of stepwise inter- and intradomain cleavages (Fig. 2.1B) [12]. Interestingly, the human and murine mutations introduced above are all located at cleavage sites or in domains that play a role in activation and/or trafficking of MMP14 to the cell surface.

MMP14 is a class I transmembrane protein, that has a 24 amino acid stretch at its Cterminus which is inserted in the plasma membrane $[4,18]$. To reach the cell surface, MMP14 needs to access the exocytotic pathway [10]. For this, full-length pro-MMP14 is synthesised with an $\mathrm{N}$-terminal signal peptide (SP) that regulates incorporation into the ER membrane (Fig. 2.1A) [4, 18]. It is generally assumed that the SP present in both secreted and membrane-bound MMPs is removed upon ER insertion, however 

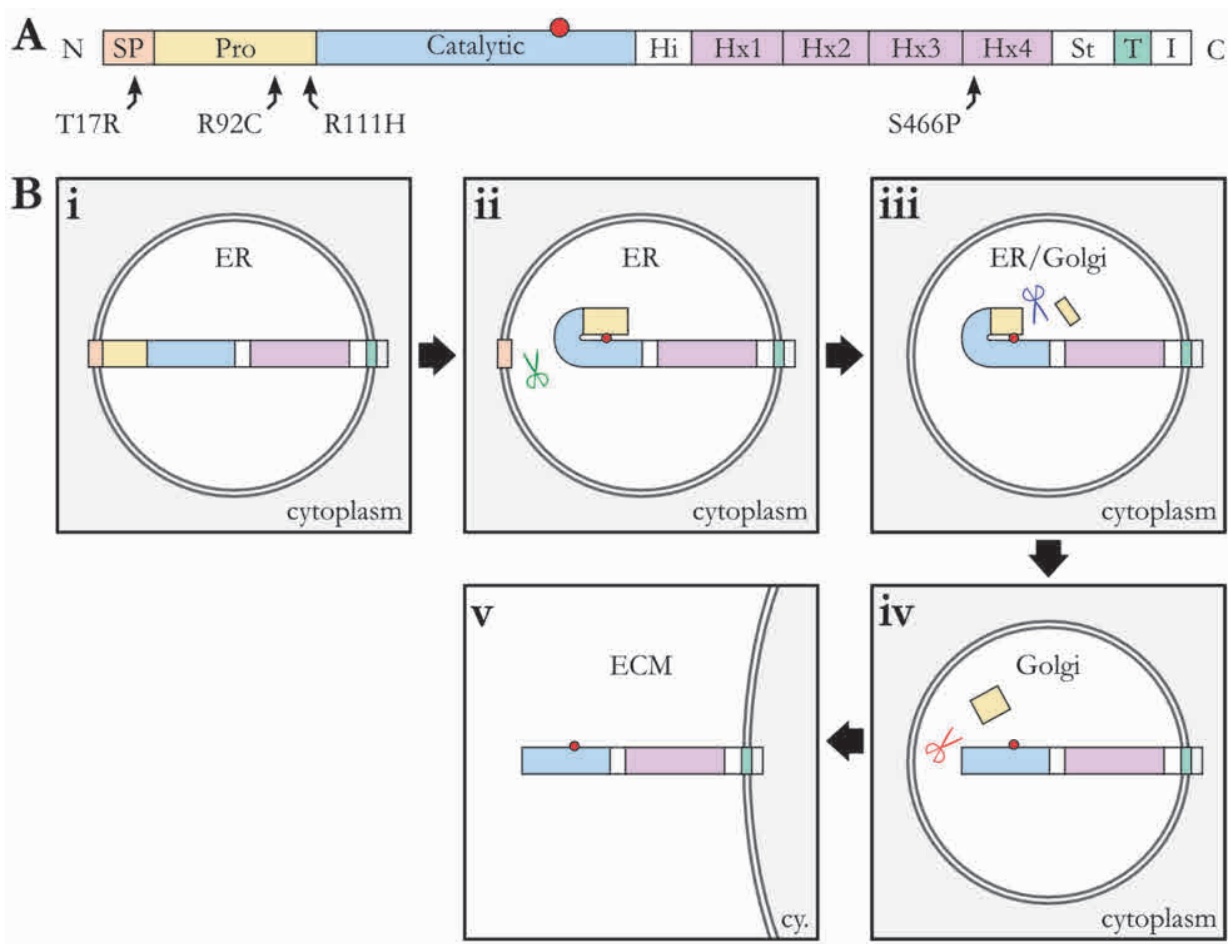

C N-region

H-region

C-region

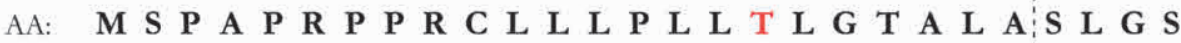
pos. $\quad \begin{array}{lllllllllllllllllllllllllll} & 2 & 3 & 4 & 5 & 6 & 7 & 8 & 9 & 10 & 11 & 12 & 13 & 14 & 15 & 16 & 17 & 18 & 19 & 20 & 21 & 22 & 23 & 24 & 25 & 26 & 27\end{array}$

D $\begin{array}{lcccccc:cccc}\text { AA: } & \mathbf{Y} & \mathbf{L} & \mathbf{P} & \mathbf{P} & \mathbf{G} & \mathbf{D} & \mathbf{L} & \mathbf{R} & \mathbf{T} & \mathbf{H} \\ \text { pos. } & 44 & 45 & 46 & 47 & 48 & 49 & 50 & 51 & 52 & 53\end{array}$

$\mathbf{E}$ \begin{tabular}{llllllll:llll} 
& & & \multicolumn{1}{c}{ PC cleavage site 1} & & & \\
\cline { 5 - 9 } AA: & $\mathbf{K}$ & $\mathbf{A}$ & $\mathbf{M}$ & $\mathbf{R}$ & $\mathbf{R}$ & $\mathbf{P}$ & $\mathbf{R}$ & $\mathbf{C}$ & $\mathbf{G}$ & $\mathbf{V}$ & $\mathbf{P}$ \\
pos. & 86 & 87 & 88 & 89 & 90 & 91 & 92 & 93 & 94 & 95 & 96
\end{tabular}

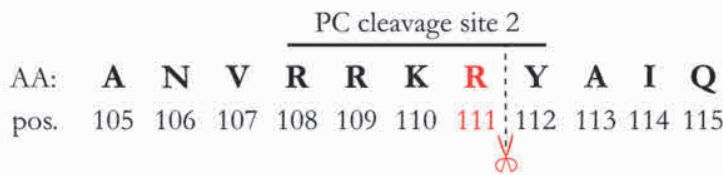

Figure 2.1 - Overview of MMP14 processing. A, schematic overview of MMP14 protein domains including the localisation of the human Winchester syndrome mutations p.T17R and p.R111H and the two 
Figure 2.1 (continued) - murine mutations p.R92C and p.S466P. MMP14 is synthesised as a pre-proprotein, with an N-terminal signal peptide (SP), a prodomain (Pro) adjacent to the catalytic domain, and a C-terminal transmembrane domain (T) with a short cytoplasmic tail (I). The red dot represents the catalytic zinc ion. B, schematic representation of the sequential processing of pro-MMP14. MMP14 is inserted into the ER membrane through a translocon by its $\mathrm{N}$-terminal signal peptide (i). Note that at one point, proMMP14 is inserted into the ER membrane with both the signal peptide and the transmembrane domain. For clarity, MMP14 is depicted as a straight bar. The signal peptide is removed in the ER by a signal peptidase (green scissors), and the pro-domain folds back to shield off the adjacent catalytic site, keeping proMMP14 inactive (ii). In the ER or Golgi apparatus, the prodomain of pro-MMP14 is cleaved by active MMPs (blue scissors), generating the activation intermediate (iii). Subsequently, the prodomain is cleaved by proprotein convertases (PC, red scissors) in the Golgi apparatus / trans-Golgi network, thereby releasing the pro-domain from the catalytic domain and activating MMP14 (iv), prior to insertion of active MMP14 into the cell membrane (v). C, MMP14 signal peptide with predicted signal peptidase cleavage site. $\mathbf{D}$, the region of the MMP14 prodomain containing the MMP cleavage site. E, regions of the MMP14 prodomain containing the proprotein convertase cleavage sites 1 and 2 . The bonds that are cleaved are highlighted in C-E by the dashed lines. AA, amino acid; C, carboxyl-terminus; cy, cytoplasm; ECM, extracellular matrix; Hi, hinge region; Hx, hemopexin-like domain; I, intracellular tail region; N, amino-terminus; pos, position; St, stalk region.

there is no direct evidence supporting this statement for MMP14 [11, 19-21]. MMP14 has an N-terminal amino acid sequence typical of SPs of class I single-pass transmembrane proteins (Supp. Fig. 1.1A) which is predicted to span the ER membrane (Supp. Fig. 1.1B) [6, 22-24]. Additional in silico analysis by SignalP 4.0 software predicted that MMP14's SP is likely to be cleaved, namely between amino acids 23 and 24 (Fig. 2.1C and Supp. Fig. 1.1C) [25]. This site is in perfect agreement with the consensus for SP cleavage (Supp. Fig. 1.1A) [22, 23, 26]. Similar to other class I transmembrane proteins, it is therefore likely that MMP14's SP is cleaved upon insertion into the ER membrane (Fig. 2.1B, panel ii) $[23,27]$. The p.T17R mutation is thought to disturb normal ER insertion and subsequent processing and trafficking of MMP14, in agreement with lower levels of mutant MMP14 T17R at the cell surface, especially its active form [6].

MMP14 is a zinc-dependent endopeptidase, with a conserved motif in its catalytic domain in which three histidine residues interact with the catalytic $\mathrm{Zn}^{2+}$ ion (Fig. $2.1 \mathrm{~A}$, red dot). The cysteine residue at position 93 in the adjacent prodomain serves as the fourth ligand of this $\mathrm{Zn}^{2+}$ ion, thereby keeping the protein in its latent zymogen state. Activation of pro-MMP14, therefore, requires displacement of the $\mathrm{C}^{93}$ residue form the catalytic $\mathrm{Zn}^{2+}$, thereby allowing the latter to bind a water molecule necessary for hydrolysis of MMP14's substrates; a process called the "cysteine switch" $[4,10,11,18$, 19, 28-32]. After SP removal, the cysteine switch is executed by serial proteolytic removal of the MMP14 prodomain in the secretory pathway [10]. It has been demonstrated that prodomain removal starts with its proteolysis by activated MMPs, including MMP14, at the P47-G-D- $\downarrow-L^{50}$ cleavage motif in the so-called "bait region". This cleavage generates an activation intermediate, which starts with the $\mathrm{N}$-terminal $\mathrm{L}^{50}$ (Fig. 2.1B, panel iii, and D) [33-35]. Cleavage of the bait region initiates subsequent prodomain proteolysis by proprotein convertases (PCs) [33, 34]. The PCs were first 
identified as mammalian homologues of the $S$. cerevisiae endopeptidase Kex2, which cleave proteins $\mathrm{C}$-terminally of a $\mathrm{R}^{-4}-\mathrm{X}-\mathrm{K} / \mathrm{R}-\mathrm{R}^{-1}$ motif (with $\mathrm{X}$ being non-basic residues) in the secretory pathway [36]. A similar R-X-R-X-K-R motif was subsequently identified in MMP11, and demonstrated to be necessary for intracellular activation of the pro-enzyme [37]. The MMP14 prodomain contains two conserved PC recognition sites, R ${ }^{89-R-P-R-\downarrow-C 93}$ and R ${ }^{108}-\mathrm{R}-\mathrm{Y}-\mathrm{R}-\downarrow_{-} \mathrm{T}^{112}$ (Fig. 2.1E) [12, 38, 39]. It has been shown that furin, a membrane-bound PC that resides mainly in the Golgi/trans-Golgi network, is the PC that most effectively cleaves these domains in vitro [12, 37, 41]. Mature, furin-cleaved, active MMP14 starts N-terminally with $\mathrm{T}^{112}$ (Fig. 2.1B, panel iv) [41]. Moreover, cleavage of this motif has been demonstrated to be crucial for MMP14's proteolytic activity $[12,38,42]$. As our newly identified p.R111H mutation is situated adjacent to this cleavage site, we hypothesised it impairs prodomain cleavage, thereby possibly disturbing both maturation and cell surface localisation of MMP14 [12]. Similar to the p.R111H mutation, the p.R92C Sabe mutation is present adjacent to the cleavage site in the more $\mathrm{N}$-terminally located $\mathrm{PC}$ recognition motif [8]. This site is part of the P91-R-C-G-V-P-D ${ }^{97}$ cysteine switch motif [18, 19, 33]. Although furin is capable of cleaving MMP14 at this motif in vitro, in silico modelling of the prodomain suggests it is inaccessible to furin in the native pro-enzyme $[12,33,40]$. It is therefore currently unclear how the p.R92C affects MMP14 to cause the resulting Sabe phenotype.

Situated between MMP14's catalytic domain and transmembrane domain, there are four repeated regions that share homology with the glycoprotein hemopexin $[4,18,43$, 44]. This hemopexin-like $(\mathrm{Hx})$ domain has been demonstrated to mediate proteinprotein interactions that are essential for multiple aspects of MMP14 function. These include heterodimerisation with CD44 needed for cell surface localisation, homodimerisation involved in pro-MMP2 cleavage, and binding to substrates such as type I collagen fibrils prior to their cleavage [10, 11, 32, 45-51]. In addition, the presence of the Hx domain has been shown to be crucial for normal activating cleavage of the prodomain [52]. Crystal structure studies demonstrated that the Hx domain forms a four-bladed $\beta$-propeller, the fourth blade of which is essential for MMP14 homodimerisation [19, 46, 53]. As the p.S466P Cartoon mutation is present in this highly conserved fourth blade, we hypothesized it disrupts the activation, trafficking and substrate proteolysis of MMP14.

Although multiple lines of evidence support a possible pathogenic role of the novel MMP14 p.R111H mutation, further confirmation is required. Similarly, although previous studies have clearly implied the p.T17R, p.R92C and p.S466P mutations disrupt normal MMP14 function, the exact mechanism of how these changes elicit a phenotypic response is yet to be elucidated. To assess the effect of the p.R111H mutation on the protein level, and compare it to the previously reported mutations, we utilised a novel in vitro model. By expressing either wild type or mutant MMP14 with two differ- 
ent tags at the $\mathrm{N}$ - and $\mathrm{C}$-terminus, respectively, we were able to study the effect of the mutations on posttranslational processing and subcellular localisation of MMP14. We found that the p.R111H mutation, in contrast to the other mutations, does not seem to affect prodomain cleavage or the subcellular localisation of MMP14; rather, we observed that the p.R111H mutation partially impairs MMP14's function to activate pro-MMP2 and cleave gelatin in vitro. Compared to the p.T17R mutations, the R111H mutant still maintained residual catalytic activity, which might explain our patients' milder phenotype. Although the phenotype of the Sabe and Cartoon mice are almost identical, the p.S466P mutation impaired pro-MMP2 activation to a lesser extent than p.R92C. In addition, gelatin degradation of MMP14 S466P remained unaltered. This might be explained by altered substrate specificity caused by this $\mathrm{Hx}$ domain mutation. In contrast to previous studies, the $\mathrm{R}^{22}$ residue turned out to be crucial for normal prodomain cleavage, cell surface localisation, and subsequent MMP14-dependent proMMP2 activation and gelatin cleavage in vitro. In addition, we presented the first evidence of MMP14 SP removal upon ER insertion. Taken together, these studies confirm pathogenicity of the novel p.R111H mutation. In addition, they shed new light on the origins of the clinical phenotypes and normal MMP14 posttranslational processing.

\section{Results}

\section{Designing a double-tagged MMP14 fusion protein to study MMP14 subcellular localisation and posttranslational processing}

To test the hypothesis that the MMP14 p.R111H mutation impairs MMP14 activating cleavage and plasma membrane localisation, it was necessary to be able to distinguish between intact pro-MMP14 and cleaved MMP14, and visualise both in 2D cell culture. As there were no commercially available antibodies raised against the pro-domain of MMP14, this distinction could not be made with antibody staining alone. Therefore, expression vectors encoding either wild type (WT) or mutant human pro-MMP14 with an N-terminal triple hemagglutinin (3HA) tag and a C-terminal EGFP were created, resulting in the fusion protein 3HA-MMP14-EGFP (Fig. 2.2). Utilising site-directed mutagenesis, the p.T17R, p.R92C, p.R111H, and p.S466P mutations, and various combinations thereof, were generated using the WT vector as template, resulting in eight mutant vectors in total (see Supp. Fig. 1.2). As the N-terminal region of proMMP14 is reported to be cleaved at different sites, the smaller 3HA-tag was attached $\mathrm{N}$-terminally of the SP sequence to prevent disruption of pro-MMP14 processing. The C-terminus has been tagged previously, indicating it can be safely tagged with EGFP without disrupting MMP14 localisation or functional activity $[38,53]$. Both the $3 \mathrm{HA}$ tag and the EGFP were fused to pro-MMP14 with flexible linkers (Fig. 2.2B). Given correct processing of 3HA-MMP14-EGFP, the 3HA tag should not be detectable in a 
A

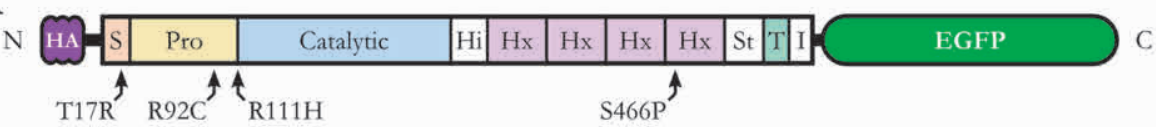

B

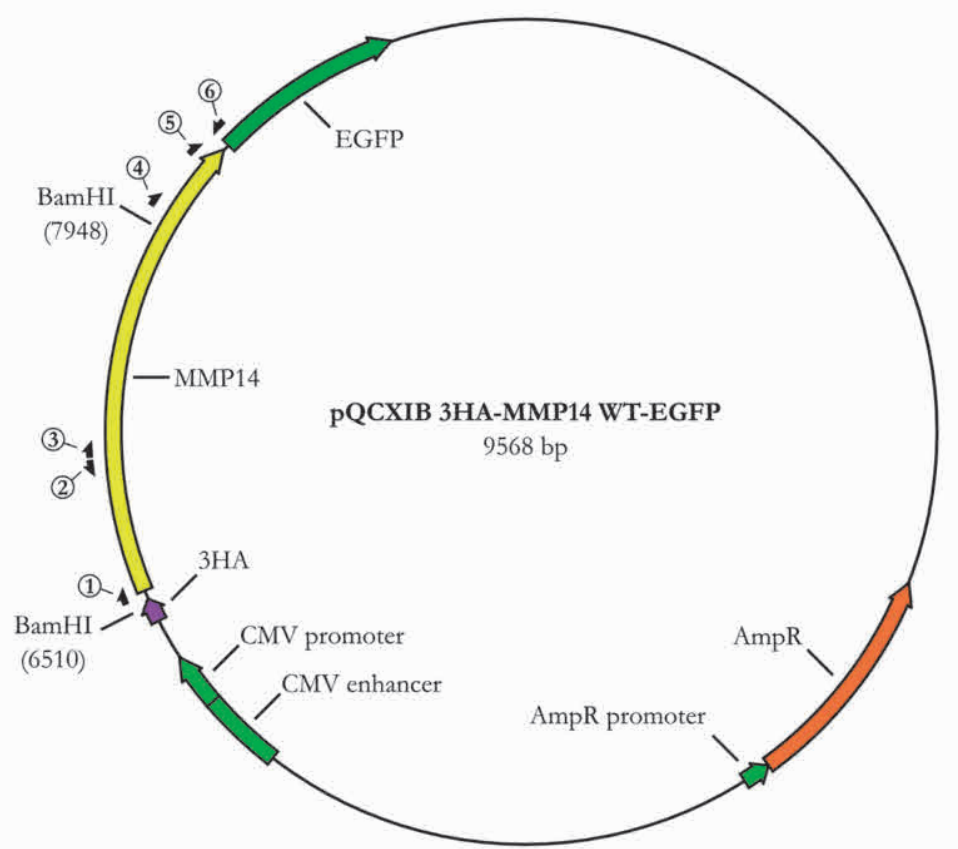

Figure 2.2 - Design of the 3HA-MMP14-EGFP fusion protein and encoding vector. A, schematic representation of the MMP14 fusion protein with N-terminal triple (3) HA tag and C-terminal EGFP. Both tags are linked to MMP14 with flexible linkers (indicated by the black lines). The domains of MMP14 are indicated, as are the position of the two human (p.T17R and p.R111H) and two murine (p.R92C and p.S466P) mutations. B, simplified vector map of the pQCXIB 3HA-MMP14 WT-EGFP expression vector, encoding the wild type fusion protein under cytomegalovirus (CMV) promoter. The binding sites for primers that were used for sequencing of the insert are indicated (numbers 1-6). Variants of the vector encoding the mutant fusion proteins were created by site-directed mutagenesis to generate the missense mutations indicated in A. AmpR, ampicillin resistance cassette; C, carboxyl-terminus; EGFP, enhanced green fluorescent peptide; HA, hemagglutinin; Hi, hinge domain; Hx, hemopexin-like domain; I, cytoplasmic tail; N, amino-terminus; Pro, prodomain; $\mathrm{S}$, signal peptide; St, stalk region; $\mathrm{T}$, transmembrane domain.

similar subcellular location to EGFP. Conversely, the EGFP signal should be visible at various cellular compartments including the Golgi/trans-Golgi network, the cell membrane and along the route by which the protein traffics. Aberrant processing and subsequent abnormal trafficking ought to be reflected in an altered subcellular distribution of both tags. To control for any putative influence of the tags on the subcellular localisation of the MMP14 fusion proteins, a control vector was designed expressing the 3HA tag and EGFP fused to each other by the two linkers (Fig. 2.3A). To generate 
A

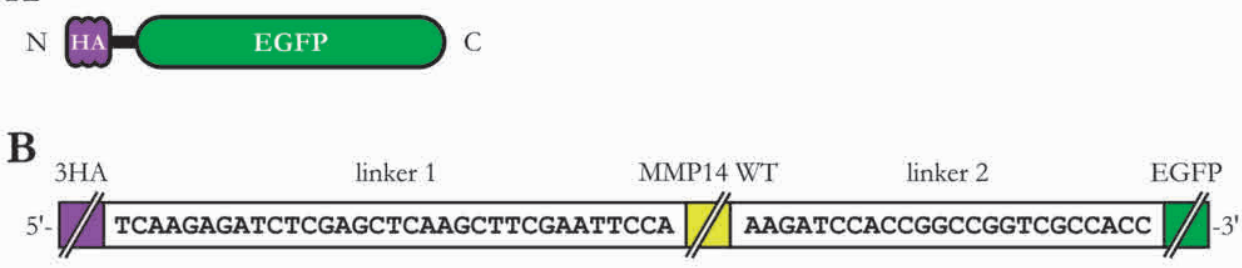

C

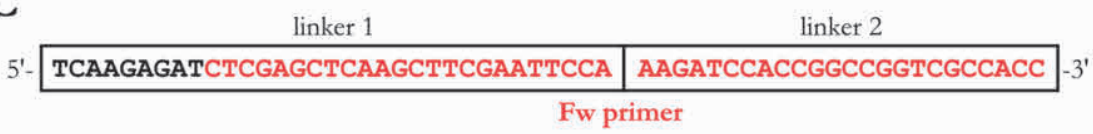

D

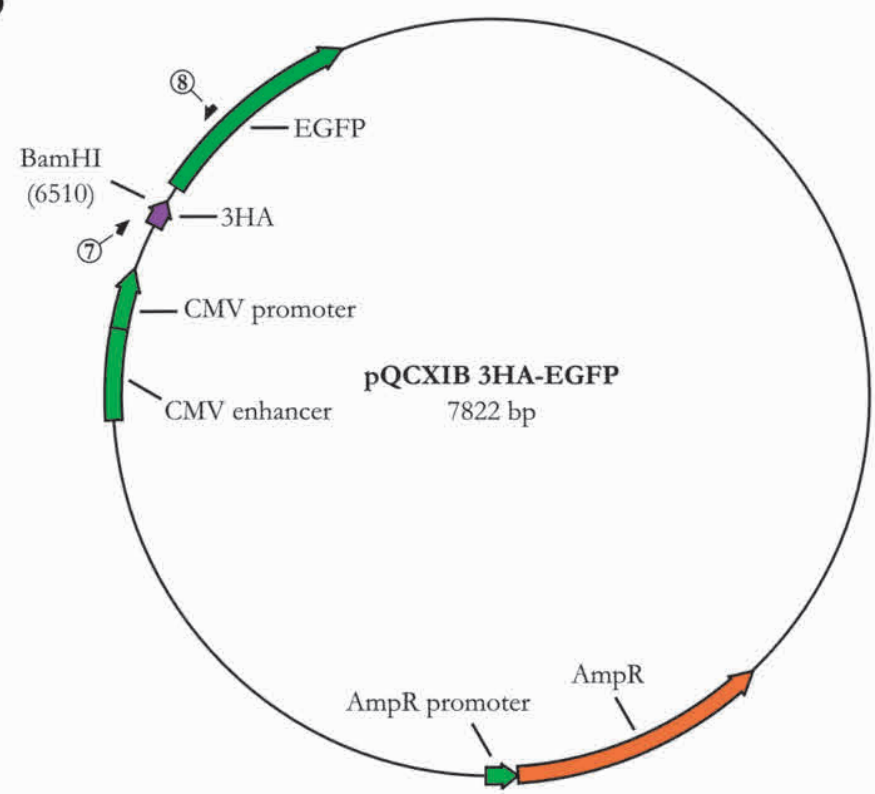

Figure 2.3 - Design of the HA-EGFP fusion protein and encoding vector. A, schematic representation of the 3HA-EGFP fusion protein. The two tags are fused by the two flexible linkers (linker 1 and linker 2, respectively) encoded by the PQCXIB 3HA-MMP14 WT-EGFP vector, connecting the two tags to MMP14 in the encoded fusion protein, as shown in B. C, in order to remove the MMP14 coding sequence by inverse-PCR, primers were designed to bind parts of both linker 1 and linker 2 coding sequence. Forward (Fw) primer is identical to the sequence highlighted in red; reverse primer is its reverse complement. D, resulting vector after removal of the MMP14 coding sequence. The binding sites for primers used for sequencing of the vector are indicated (numbers 7 and 8). Abbreviations as in Fig. 2.2.

this vector, primers binding to parts of the two linker sequences in PQCXIB 3HAMMP14 WT-EGFP were used to effectively remove the pro-MMP14 coding sequence by inverse-PCR (Fig. 2.3B-D and Supp. Fig. 1.3). After verifying all ten vectors by 
diagnostic restriction digestion and Sanger sequencing (Supp. Fig. 1.2 and 1.3), the fusion proteins could subsequently be expressed in a relevant cell type.

\section{MRC-5V1 cells express very low levels of endogenous MMP14}

MMP14 is expressed by a variety of cell types [4, 54]. MMP14 can form a homodimer at the cell surface through interaction of the Hx domains, which is necessary for cleavage of type I collagen fibrils and pro-MMP2 [39, 48-50]. To minimize confounding of enzymatic activity or even subcellular localisation of 3HA-MMP14-EGFP fusion proteins due to homodimerisation with endogenous MMP14, expressing the fusion proteins in a cell type with low endogenous MMP14 levels would be preferential. MRC$5 \mathrm{~V} 1$ is a well-documented and widely used human lung fibroblast cell line, isolated from a male foetus in 1970 and subsequently SV40 immortalised in 1983 [55, 56]. This cell type is relevant to the disease phenotype, which includes connective tissue and lung abnormalities in respectively patients and mouse models $[57,58]$. Moreover, the MRC-5V1 cells are relatively easy to culture and transfect, which is essential to the experiments outlined above. Expression levels of endogenous MMP14 were assessed in MRC-5V1 cells by immunoblot against MMP14 on whole cell protein extract (Fig. 2.4A). Protein extract of HT-1080 human fibrosarcoma cells, a cell type with a known high expression of MMP14, served as control [59]. In HT-1080 lysate, a strong band between 46 and $58 \mathrm{kDa}$ could be observed, corresponding to the cleaved, active form of MMP14 (54-57 kDa in literature) [13, 35, 60]. The two fainter bands directly above it could be accounted for by sequential cleavage of pro-MMP14 (63-66 kDa in lit.) [12, 13, 35, 60-62]. In MRC-5V1 lysate, bands at corresponding heights are hardly visible, suggesting that expression levels of endogenous MMP14 in MRC-5V1 cells are very low compared to those in HT-1080 cells. The localisation of exogenously expressed MMP14 is therefore unlikely to be affected by endogenous MMP14 in MRC-5V1 cells, making these cells suitable for the aforementioned experiments. Before analysing the MMP14 fusion proteins, the subcellular localisation of endogenous MMP14 was assessed in both cell types.

\section{Endogenous MMP14 localises to the cell surface and perinuclear aggregates in MRC-5V1 cells}

To assess the subcellular localisation of endogenous MMP14, HT-1080 cells were stained with two commercially available anti-MMP14 antibodies (Fig. 2.4B and Table 2.4). Both antibody stainings showed that endogenous MMP14 localises to perinuclear aggregates and to the cell surface, as reported before by others [4]. Staining MRC-5V1 cells with one of these antibodies revealed a highly similar subcellular localisation of endogenous MMP14, although the signal intensity was much lower (Fig. 2.4C) consistent with our Western blot results (Fig. 2.4A). Cells were co-stained with fluorescently labelled phalloidin, a toxin binding filamentous (F)-actin, which localises (among other locations) just below the plasma membrane [63]. Signal intensity plots through 
optical sections of immunofluorescently (IF) labelled MRC-5V1 cells (Fig. 2.4D-E) demonstrate colocalisation of MMP14 with F-actin at the cell surface, supporting plasma membrane localisation of MMP14.

\section{MRC-5V1 cells retained their fibroblast identity after immortalisation}

As becomes clear in Figure 2.4D, MRC-5V1 cells do not have the typical spindleshaped morphology of fibroblasts. As published before, primary MRC-5 cells are uniform in size and have an elongated spindle-shaped morphology. In contrast, the immortalised MRC-5V1 cell line is known to be less uniform in cell size and shape. These cells reportedly have an epithelial to fibroblast-like morphology, which becomes more epithelial as the culture becomes confluent, as is true for the cells in our lab (Supp. Fig. 1.4A) [56]. Given the morphology of MRC-5V1 differs significantly from primary MRC-5 cells, our MRC-5V1 cells were stained with two commercially available antibodies against vimentin, a widely used fibroblast marker. As shown in Supplemental Figure 1.4B, both anti-vimentin antibodies revealed a filamentous vimentin pattern, characteristic for fibroblasts [64]. This supported their fibroblast identity and justified the use of this cell type in subsequent experiments.

\section{The subcellular localisation of $3 H A-M M P 14$ WT-EGFP is indistinguishable from that of endogenous MMP14}

Having established the subcellular distribution of endogenous MMP14 in MRC-5V1 cells, it was subsequently compared to the localisation of the exogenously expressed 3HA-MMP14 WT-EGFP fusion protein. Expression of the 3HA-EGFP fusion protein served as a transfection control. Staining 3HA-EGFP-expressing cells with three different anti-MMP14 antibodies all revealed an identical subcellular localisation for endogenous MMP14, at the cell surface and in a perinuclear aggregate (Fig. 2.5A). This distribution was furthermore similar to that observed in untransfected cells (Fig. 2.4), suggesting it is unaffected by the transfection and expression of 3HA-EGFP. MRC5V1 cells expressing the 3HA-MMP14 WT-EGFP fusion protein showed a strong EGFP signal that localised similarly to endogenous MMP14 (Fig. 2.5B), suggesting the exogenous fusion protein localises normally. This distribution was moreover indistinguishable from the anti-MMP14 staining pattern, indicating the EGFP signal accurately reflects the subcellular localisation of the exogenous MMP14 fusion protein.

\section{Visualising the $H A$ tag of the fusion proteins by indirect IF microscopy}

To assess posttranslational cleavage of full-length WT and mutant MMP14 fusion proteins, it was necessary to visualise both the EGFP and the 3HA tag. For the latter, a commercially available anti-HA antibody was obtained and tested for indirect IF microscopy. The HA signal in 3HA-EGFP-expressing MRC-5V1 cells was found to be 

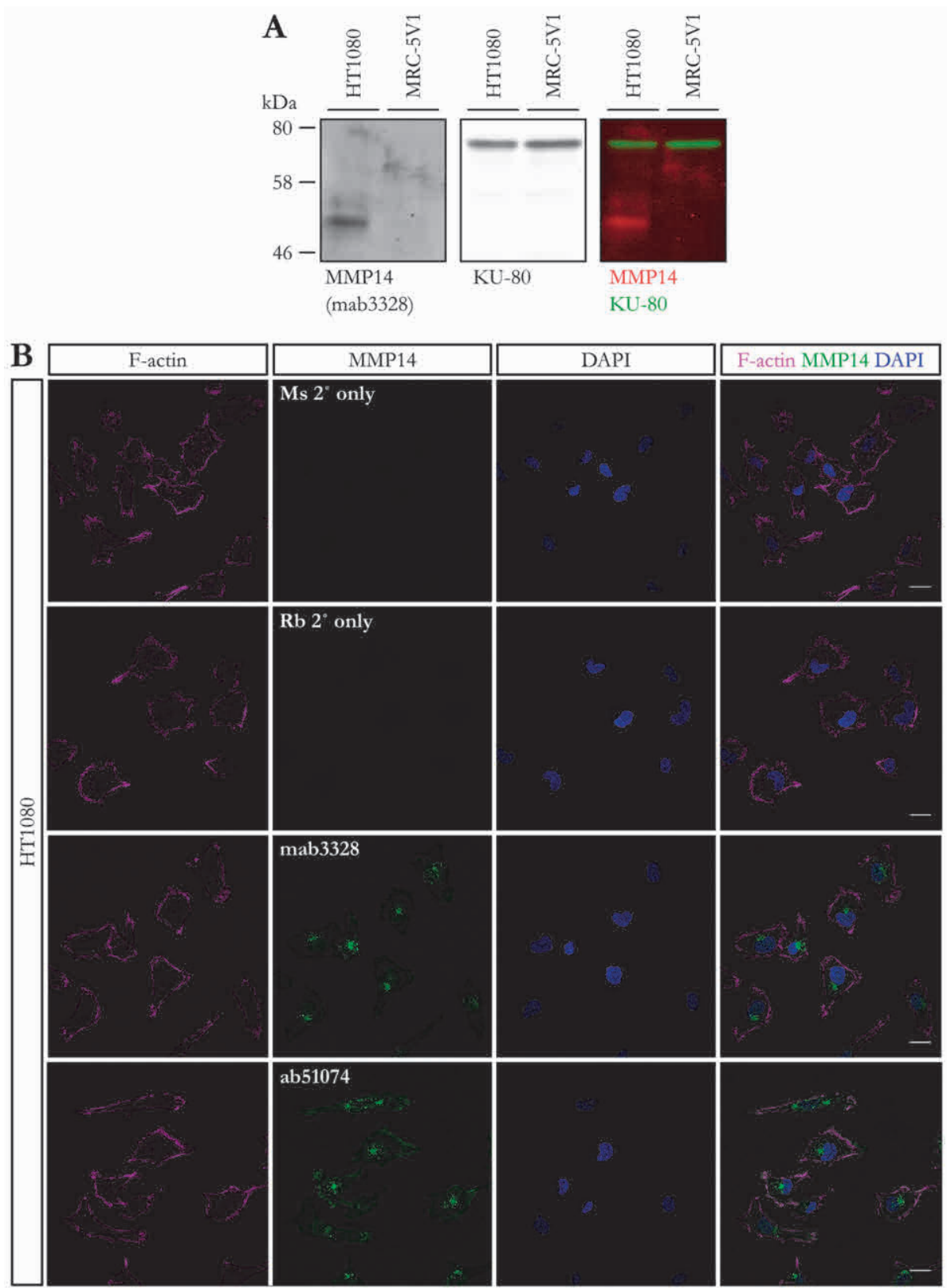

Figure 2.4 - Endogenous MMP14 expression in MRC-5V1 is low compared to HT-1080 cells, but localises similarly. A, expression levels of endogenous MMP14 in MRC-5V1 fibroblasts are very low compared to those in HT-1080 fibrosarcoma cells, as shown by immunoblot on whole cell protein extracts with anti-MMP14 antibody (mab3328) and anti-KU-80 antibody as loading control. Multiple bands can be detected in both cell types, although the intensity is much higher in HT-1080 lysate. B-E, IF microscopy images of untransfected MRC-5V1 cells (seeded at 50,000 cells per mL medium) and HT-1080 cells (seeded at 30,000 cells per mL medium) stained with anti-MMP14 primary antibody 

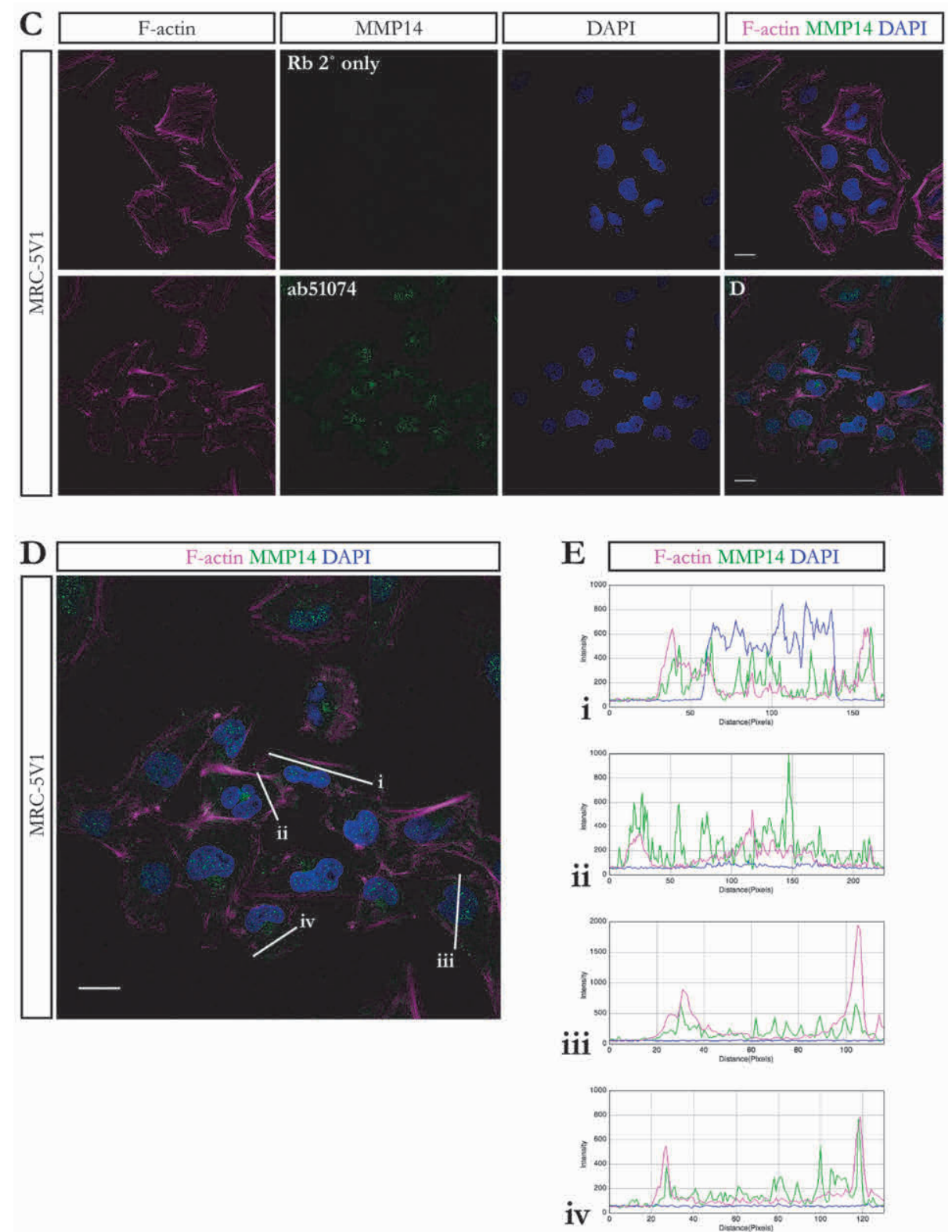

Figure 2.4 (continued) - (mab3328 or ab51074) and/or Alexa 488-conjugated goat anti-mouse or anti-rabbit secondary antibody. In both HT-1080 cells (B) and MRC-5V1 cells (C), MMP14 localises almost identically to the cell surface and to a perinuclear aggregate. Line plots (E) through MRC-5V1 cells (C, enlarged in D), showing signal intensity along for the different channels along the lines drawn, clearly demonstrate colocalisation of MMP14 and F-actin in the cell periphery, supporting plasma membrane localisation of MMP14. Scale bar equals $20 \mu \mathrm{m}$. 
distributed throughout the cell, with its signal intensity being highest in the peripheral cytoplasm and lowest in the nucleus (Supp. Fig. 1.5). Although the EGFP signal was present in both compartments as well, the signal intensity was distributed differently and notably highest in the nucleus. One possible explanation for the discrepancy in nuclear signal intensity for the two tags is suboptimal antibody penetrance due to insufficient permeabilisation. However, repeating the staining in cells permeabilised with increasing concentrations of Triton X-100 did not increase the nuclear HA signal intensity (Supp. Fig. 1.6). Alternatively, the 3HA-EGFP fusion protein might undergo posttranslational cleavage, separating the two tags. Western blotting data supports this hypothesis (see Fig. 2.7). Nevertheless, the HA signal in 3HA-MMP14 WT-EGFPexpressing cells was distributed differently; its intensity was found highest near the perinuclear EGFP aggregate and decreased towards the cell periphery (discussed in detail below). The contrasting distribution of the two tags in cells expressing the MMP14 fusion protein and cells expressing tags only suggests that the tags do not interfere with the localisation of the MMP14 fusion protein. In conclusion, the 3HAMMP14 WT-EGFP and 3HA-EGFP fusion proteins could, therefore, serve as proper positive and negative controls, respectively, for the subcellular localisation of the mutant MMP14 fusion proteins discussed hereafter.

\section{The p.T17R, p.R92C and p.S466P mutations disturb the subcellular localisation of MMP14}

To assess the effect of the aforementioned missense mutations on the subcellular localisation and posttranslational processing of MMP14, the WT and mutant MMP14 fusion proteins were transiently expressed in MRC-5V1 cells and their tags visualised by IF microscopy. As described above, in cells expressing 3HA-MMP14 WT-EGFP, the EGFP signal localised to the cell surface and a perinuclear aggregate, whereas the HA signal formed a decreasing gradient towards the cell periphery (Fig. 2.6A). In line with previously published work, the cell surface-localized MMP14 WT contained the EGFP but not the 3HA tag. The lack of HA and EGFP co-localisation is consistent with published data demonstrating MMP14 pro-peptide removal precedes plasma membrane insertion [12].

Surprisingly, the subcellular localisation of both tags of 3HA-MMP14 R111H-EGFP was identical to those of the MMP14 WT fusion protein, indicating trafficking of this mutant protein is normal. The lack of any HA signal at the cell surface suggests that the R111H mutation does not markedly impact SP removal. The HA signal of 3HAMMP14 R92C-EGFP-expressing cells formed a gradient similar to cells expressing the MMP14 WT fusion protein. However, unlike MMP14 R111H, MMP14 R92C-EGFP was absent from the cell membrane. Instead, it accumulated in perinuclear aggregates, suggesting the p.R92C mutation impairs trafficking to the plasma membrane after removal of the N-terminal SP and HA tag. In cells expressing the MMP14 T17R fusion protein, the HA and EGFP tags were distributed diffusely throughout the cyto- 
plasm. It is noteworthy that the subcellular distribution of both tags (partially) colocalised, indicating inefficient removal of the SP and HA tag. These findings suggest that the p.T17R mutation impairs incorporation into the ER membrane, subsequently affecting both the processing and trafficking of the fusion protein to the plasma membrane. Accordingly, neither tag was detected at the cell surface. In cells expressing 3HA-MMP14 S466P-EGFP, there was extensive perinuclear co-localisation of the HA tag and EGFP. Membrane localisation of this mutant protein was markedly reduced compared to WT, with only a minority of cells $(<5 \%)$ demonstrating distinct membrane localisation of the EGFP signal (not shown here). This observation suggests that this single amino acid substitution in the Hx domain compromises MMP14 processing and subsequent trafficking.

As shown in Figure 2.6B, cell surface localisation of all double and triple mutant MMP14 fusion proteins was severely impaired, with EGFP being absent from the cell surface. Only for the R111H S466P fusion protein, EGFP could be detected in a minority $(<5 \%)$ of cells at the cell surface; this was not detected upon repeating the experiment. The HA signal for all of these double and triple mutants invariably formed a gradient similar to the MMP14 W'T fusion protein. There was significant colocalisation of the HA tag and EGFP for the MMP14 R92C S466P double and MMP14 R92C R111H S466P triple mutant fusion protein, but not for the MMP14 R92C R111H and MMP14 R111H S466P double mutants. Taken together, these data suggest that the p.T17R, p.R92C, and p.S466P mutations disturb normal processing and trafficking of pro-MMP14, in contrast to the p.R111H mutation.

\section{The p.T17R, p.R92C and p.S466P mutations disturb MMP14 processing}

To further examine the posttranslational processing of WT and mutant MMP14, protein extract of MRC-5V1 cells expressing the fusion proteins shown in Fig. 2.6 was analysed by Western blotting with commercial antibodies against the two tags. As shown in Figure 2.7, immunoblot against the HA tag (top panel) showed an intense band between 80 and $100 \mathrm{kDa}$ for cells expressing every MMP14 fusion protein. Immunoblot for GFP (middle panel) showed a band that overlapped with this HA band (merged in the bottom panel), suggesting it corresponds to the full-length proprotein (expected size of $96 \mathrm{kDa}$ ). The anti-HA antibody detected an additional faint band between 58 and $80 \mathrm{kDa}$, which was also present in protein extract of nontransfected cells (not shown in Fig. 2.7) and therefore deemed nonspecific. For cells expressing the MMP14 WT fusion protein, there were three additional smaller GFP bands. The band around $80 \mathrm{kDa}$ likely corresponds to active, cleaved MMP14EGFP (expected size of $82 \mathrm{kDa}$ ), whereas the two bands around $58 \mathrm{kDa}$ likely correspond to inactive MMP14 degradation products with intact C-terminus (thus containing EGFP) [62]. This supports normal processing of the WT fusion protein and fits with the IF microscopy data shown in Fig. 2.6A. For cells expressing the 

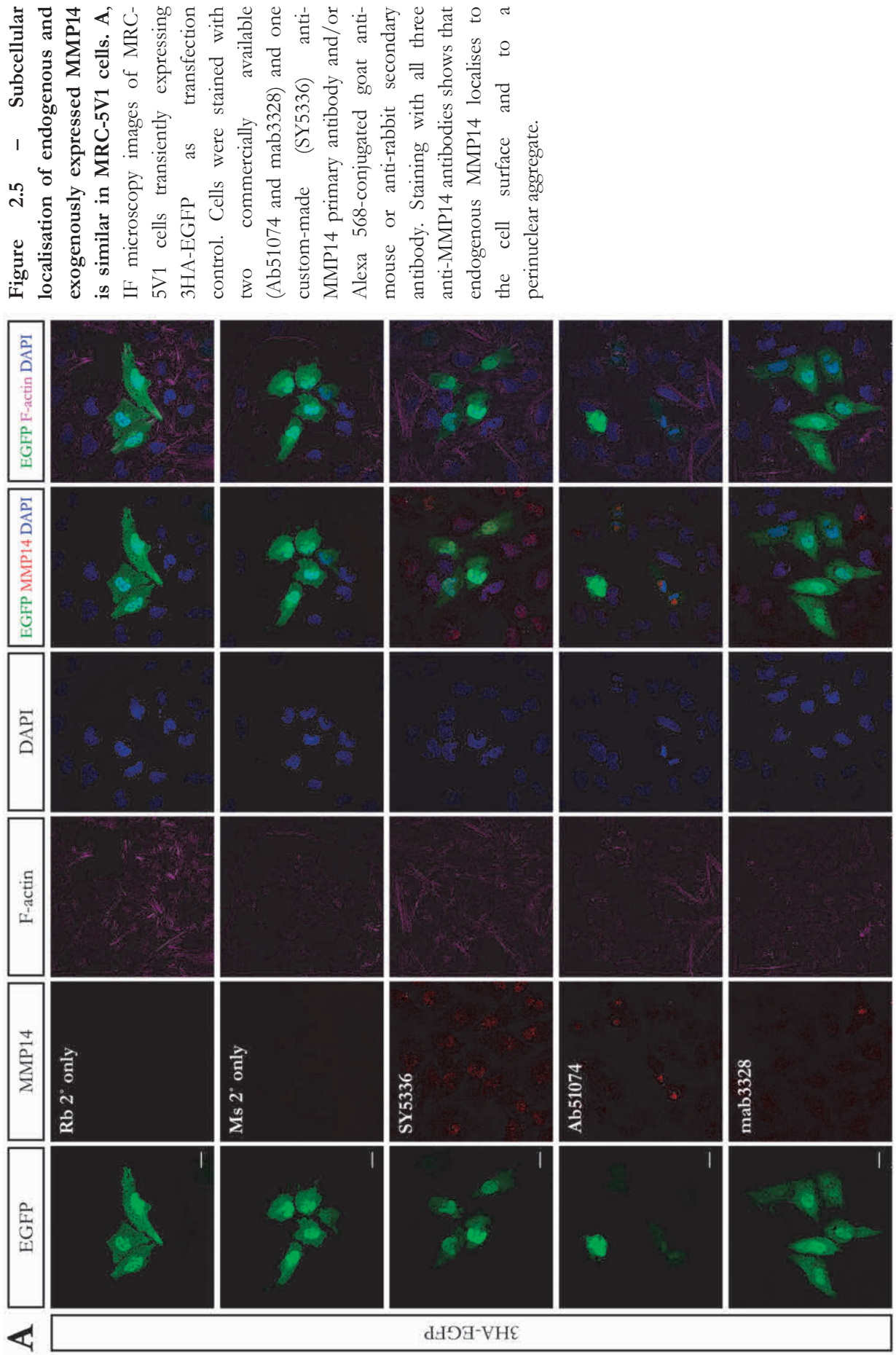

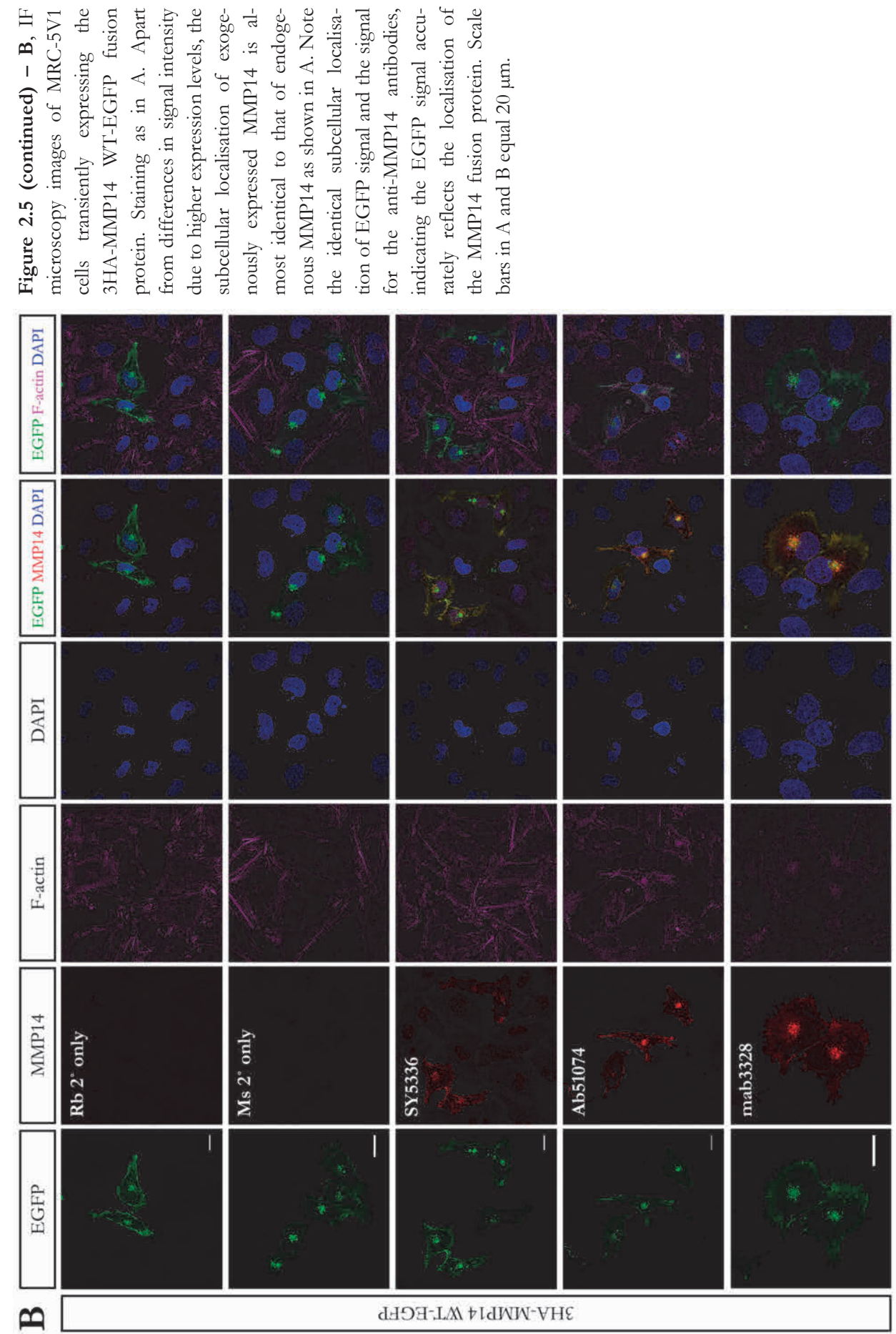
MMP14 R111H mutant fusion protein, bands detected with both antibodies were identical to MMP14 WT-expressing cells, suggesting the R111H mutation does not affect posttranslational processing of MMP14. In protein extract of MMP14 T17Rexpressing cells, both antibodies detected a single overlapping band between 80 and $100 \mathrm{kDa}$. This suggests that 3HA-MMP14 T17R-EGFP does not undergo any posttranslational cleavage, which supports the IF microscopy results that show colocalisation of both tags (Fig. 2.6A). In protein extract of cells expressing any other single, double or triple mutant fusion protein, both the $96 \mathrm{kDa}$ and the $82 \mathrm{kDa}$ GFP band could be detected, suggesting that the prodomain of these mutant proteins gets removed. However, further processing seems abnormal, given the absence of bands around $58 \mathrm{kDa}$. As smaller degradation products of comparable size have been detected on the cell surface in multimers with active MMP14, it is possible that cell surface localisation and the generation of these smaller forms are linked [62]. The fact that these smaller forms of MMP14-EGFP are only detected in cells expressing MMP14 fusion proteins that are present at the cell surface would support this hypothesis. In protein extract of cells expressing 3HA-EGFP, both antibodies detected an overlapping band around $30 \mathrm{kDa}$, corresponding to the intact 3HA-EGFP fusion protein. The anti-GFP antibody detected two additional bands around 25 and $20 \mathrm{kDa}$, suggesting that the 3HA-EGFP fusion protein is cleaved. This apparent cleavage could explain the discrepancy in subcellular localisation of the two tags observed by IF microscopy (see Fig. 2.6 and Supp. Fig. 1.5 and 1.6 in Addendum 1). As both antibodies only detected a single band for 3HA-MMP14 T17R-EGFP, another fusion protein with primarily cytoplasmic distribution and thus exposed to the same enzymes, the cleavage of one of the tags and/or linkers seems to be specific to 3HA-EGFP.

To rule out that the subcellular localisation and posttranslational processing of MMP14 fusion proteins depend on the cell type in which they are expressed, the experiment shown in Figure 2.6 was partially repeated with mIMCD-3 murine immortalised renal epithelial cells (see Chapter 3 for details on this cell line) [65]. As shown in supplemental Figure 1.7, the subcellular distribution of the two tags of both WT and mutant 3HA-MMP14-EGFP was similar to that observed in MRC-5V1 cells, demonstrating the subcellular localisation (and posttranslational cleavage) of the expressed fusion proteins is not cell-type dependent.

\section{Perinuclear MMP14 aggregates partially colocalise with the Golgi apparatus}

Having established the posttranslational processing of MMP14 WT both by IF microscopy and Western blotting, it was subsequently assessed in which subcellular compartment the sequential steps of N-terminal MMP14 cleavage take place. As essential pro-MMP14 cleavages are expected to take place in the ER and Golgi/trans-Golgi network (Fig. 2.1B), the subcellular localisation of the HA-gradient and the perinuclear 
MMP14 aggregates were compared to that of known markers for these compartments. As shown in Addendum 1, antibodies against Golgin97 (Supp. Fig. 1.8) respectively sarcoplasmic/endoplasmic reticulum calcium ATPase 2a (SERCA2A, Supp. Fig. 1.9) were optimised for indirect IF microscopy. Subsequently, MRC-5V1 cells were costained for endogenous MMP14 and these markers. As shown in Figure 2.8, the perinuclear MMP14 aggregates colocalised minimally with the ER (see panel A, enlarged in panel B, and line plots in panel C). In contrast, MMP14 aggregates colocalised to a greater extent, but not completely, with the Golgi apparatus (Fig. 2.8A, D E). The position of MMP14 aggregates relative to the ER and Golgi apparatus became even clearer in cells co-stained for endogenous MMP14 and both markers (Fig 2.8F-H), where the MMP14 signal intensity more closely followed the distribution of the Golgin97 signal as opposed to the SERCA2A signal (Fig. 2.8I). This apparent accumulation of MMP14 in the Golgi apparatus fits with previously published data indicating that pro-MMP14 is being cleaved by furin, which mainly resides in the Golgi apparatus and the trans-Golgi network $[12,37,41]$.

\section{The MMP14 signal peptide gets removed in the ER}

From optimisation of the anti-SERCA2A antibody shown in Supplemental Figure 1.9, it became clear that the ER in MRC-5V1 cells reaches almost up to the plasma membrane. Moreover, the distribution of the SERCA2A signal was reminiscent of the HA signal observed in cells expressing the MMP14 WT fusion protein. Therefore, 3HAMMP14 WT-EGFP-expressing cells were co-stained with anti-HA and anti-SERCA2A antibodies (see Supp. Fig. 1.10 for optimisation of the rabbit anti-HA antibody used for this co-staining). In cells expressing 3HA-EGFP as control, the HA signal was distributed diffusely throughout the cytoplasm (and the nucleus to a lesser extent) and did not seem to colocalise specifically with the SERCA2A signal (Fig. 2.9). In contrast, the HA signal in 3HA-MMP14 WT-EGFP-expressing cells was distributed almost identically to the SERCA2A signal. Together with the subcellular localisation of MMP14-EGFP shown in Figures 2.6 and 2.8, and the Western blot data in Figure 2.7, this data suggests that (exogenously expressed) MMP14 indeed undergoes serial cleavage, with its N-terminal SP (and attached 3HA tag) being removed upon incorporation into the ER membrane, and subsequent removal of the pro-domain in the Golgi apparatus as outlined in Fig. 2.1. This is further supported by the cytoplasmic localisation of MMP14 T17R, which fails to enter the ER membrane, does not traffic through the Golgi/trans-Golgi network, and as a result does not undergo any N-terminal cleavage. Moreover, these data provide the first evidence of MMP14 SP removal.

\section{MMP14 mutations impair pro-MMP2 activating cleavage}

Since MMP14 R111H seemed to be processed normally and localised to the cell membrane, its enzymatic functionality was subsequently assessed. As a key cellular role 


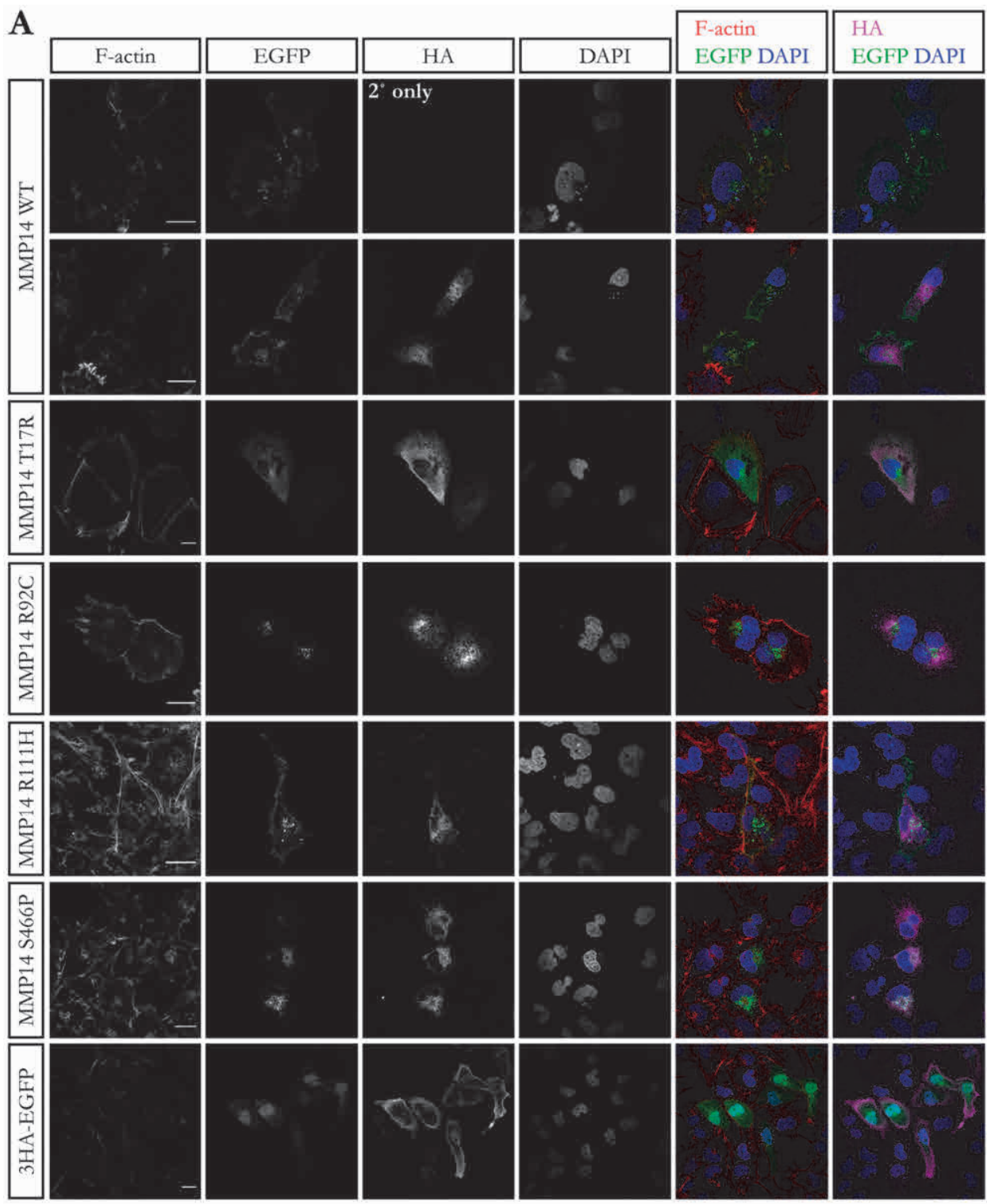

Figure 2.6 - R111H mutation does not affect subcellular localisation of exogenously expressed MMP14, whereas all other mutations disrupt its posttranslational cleavage and/or subcellular localisation. IF microscopy images of MRC-5V1 cells transiently expressing WT or mutant 3HA-MMP14EGFP or 3HA-EGFP fusion proteins, stained with a mouse anti-HA primary antibody (Ab16918) and/or Alexa 647-conjugated goat anti-mouse secondary antibody. A, subcellular localisation of the MMP14 WT fusion protein compared to single mutant MMP14 fusion proteins. In cells expressing 3HA-MMP14 WTEGFP, the EGFP signal localises to the cell surface and a perinuclear aggregate, whereas the signal intensity for the HA tag is highest perinuclearly and forms a gradient towards, but never reaches, the plasma membrane. Both signals show minimal colocalisation in the perinuclear region and do not colocalise at the cell surface. The subcellular localisation of both tags of 3HA-MMP14 R111H-EGFP is identical to those of 
B

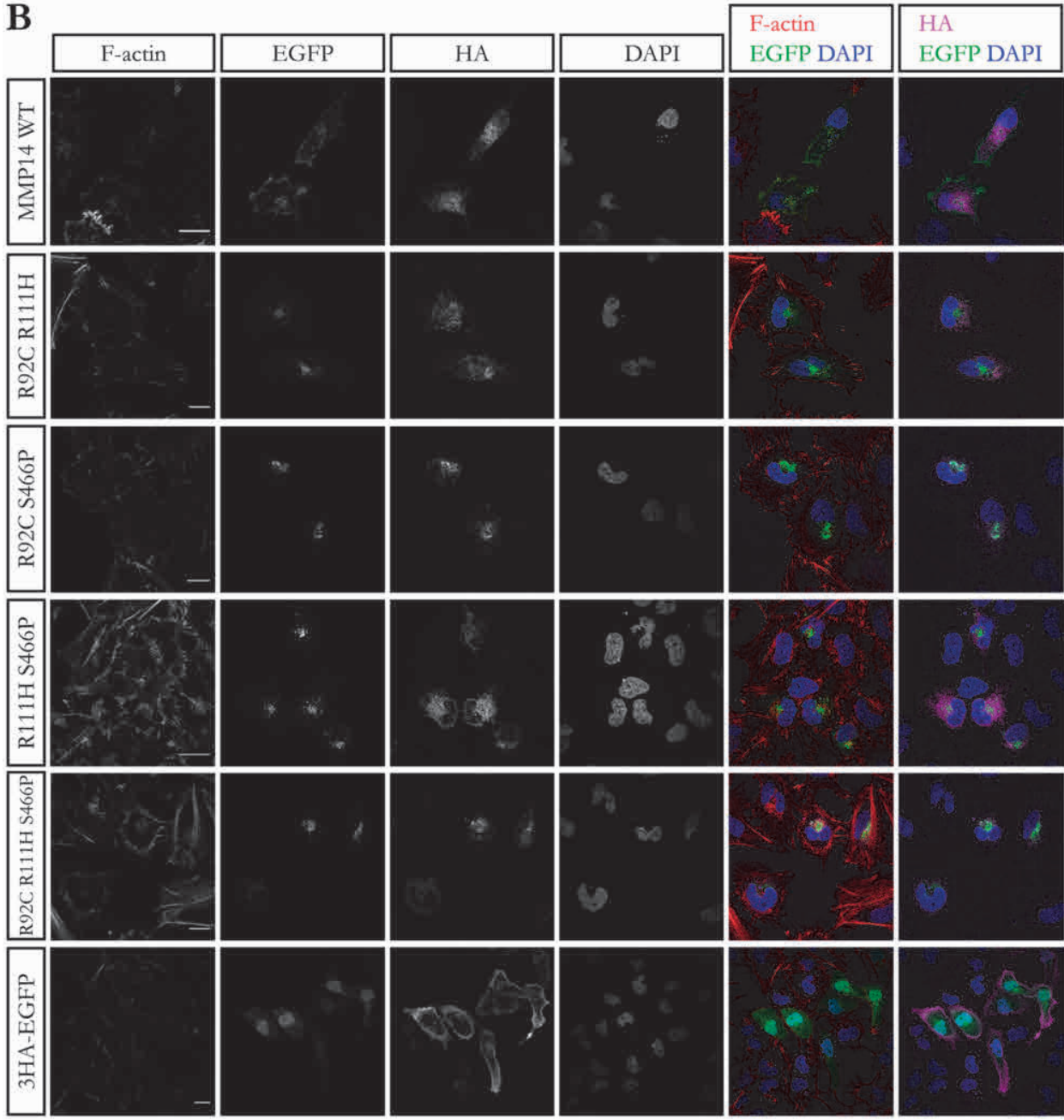

Figure 2.6 (continued) - the MMP14 WT fusion protein. The HA signal in cells expressing the MMP14 R92C and MMP14 S466P fusion proteins forms a gradient similar to cells expressing 3HA-MMP14 WTEGFP, whereas the EGFP signal is primarily located in a perinuclear aggregate that partially colocalises with the HA signal; hardly any EGFP signal can be detected at the cell surface. In cells expressing the MMP14 T17R fusion protein, both tags (partially) colocalise throughout the cytoplasm; no signal is detected at the cell surface. In contrast, the EGFP signal in cells expressing 3HA-EGFP is present in the entire cell, with the highest intensity in the nucleus, whereas the HA signal intensity is highest in the cell periphery. B, subcellular localisation of the MMP14 WT fusion protein compared to double and triple mutant MMP14 fusion proteins. The tags of all double and triple MMP14 mutants localise similarly to those of the MMP14 R92C fusion protein. Scale bars in A and B equal $20 \mu \mathrm{m}$.

of MMP14 is the activation of the gelatinase pro-MMP2 by proteolytic cleavage, the cleavage of pro-MMP2 by exogenously expressed W'T and mutant MMP14 fusion proteins were assessed [4]. MMP14 activates secreted pro-MMP2 at the cell surface, 


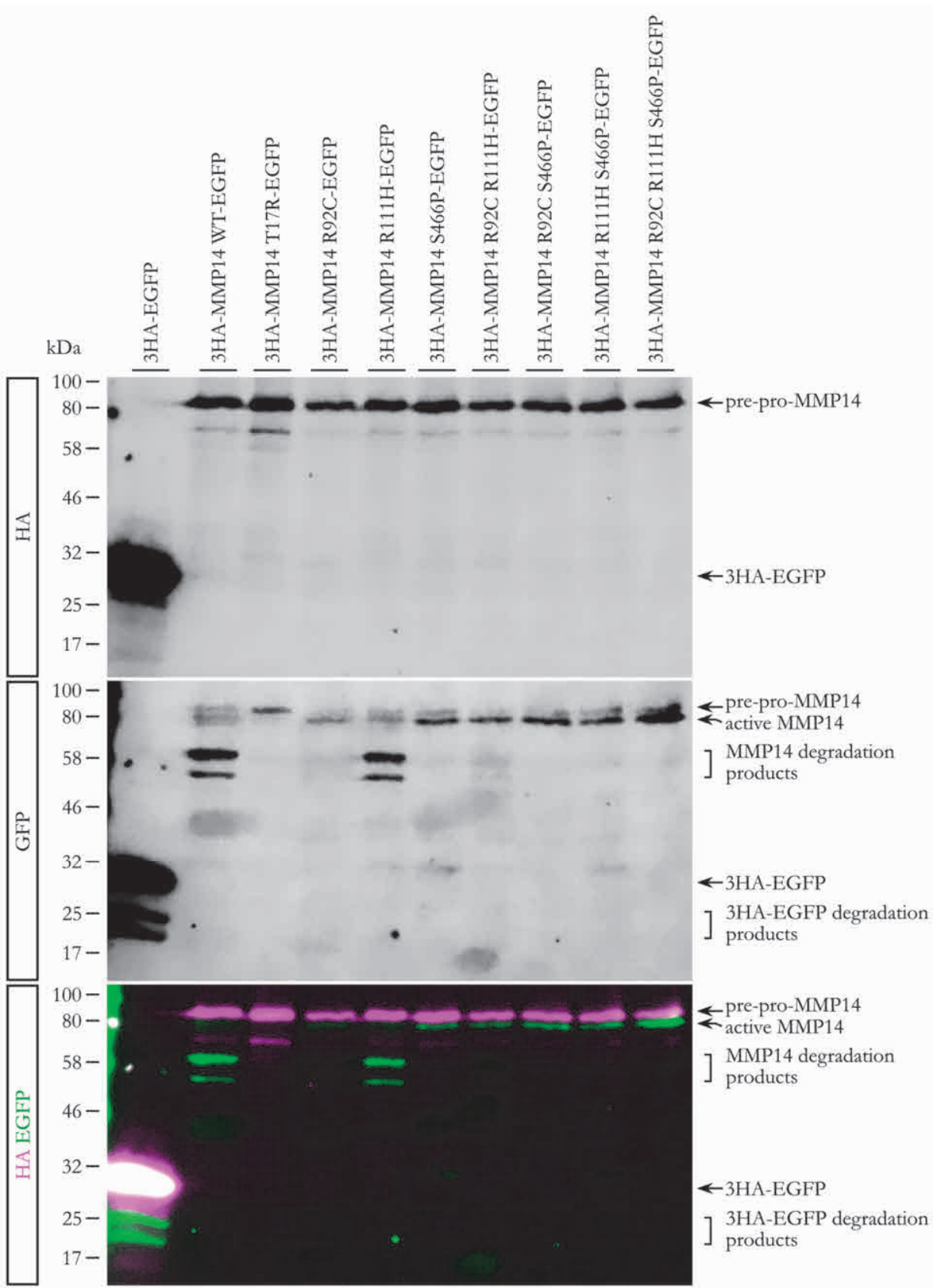

Figure 2.7 - Posttranslational processing of MMP14 is affected by all mutations except p.R111H. Immunoblot with anti-GFP and anti-HA antibodies on whole cell protein extracts of MRC-5V1 cells transiently expressing 3HA-EGFP, or WT or mutant 3HA-MMP14-EGFP fusion proteins. Anti-HA immunoblot (top panel) shows an intense band between 80 and $100 \mathrm{kDa}$ for cells expressing all MMP14 fusion proteins, that colocalises with the highest band detected by anti-GFP immunoblot (middle and bottom panels) and thus corresponds to the intact pro-protein. For cells expressing WT fusion protein, 
Figure 2.7 (continued) - the anti-GFP antibody shows three additional smaller bands: a single band around $80 \mathrm{kDa}$, corresponding to MMP14-EGFP (expected size: $82 \mathrm{kDa}$ ), and two bands around $58 \mathrm{kDa}$, most likely corresponding to inactive MMP14 degradation products. Bands for MMP14 R111H are identical to those of MMP14 WT, suggesting normal posttranslational processing of this mutant. The MMP14 T17R mutant only shows a single GFP band, overlapping with the HA band between 80 and 100 $\mathrm{kDa}$, suggesting it does not undergo any cleavage. For all other single, double and triple mutant fusion proteins, both the $96 \mathrm{kDa}$ and the $82 \mathrm{kDa}$ GFP bands can be seen, suggesting that in all the prodomain gets cleaved. However, further processing seems abnormal, given the absence of bands around $58 \mathrm{kDa}$.

and both pro-MMP2 and active MMP2 can, therefore, be detected in conditioned media by gelatin zymography. Under the conditions of this assay, both cleaved, active MMP2 and pro-MMP2 have gelatinolytic activity and can be distinguished by their size difference. The same holds for pro-MMP9 (and cleaved MMP9), which is an additional target of MMP14 [4, 6, 53]. First, it was determined that serum-free medium conditioned by 3HA-EGFP-expressing MRC-5V1 cells only contained the zymogen form of MMP2, as indicated by the presence of a single band between 50 and $75 \mathrm{kDa}$ corresponding to pro-MMP2 (Fig. 2.10A). This demonstrates that these cells secrete proMMP2, but that it does not get activated significantly, consistent with the very low levels of endogenous MMP14 in this cell type. In contrast, in media conditioned by cells expressing the MMP14 W'T fusion protein, two additional smaller bands between 50 and $75 \mathrm{kDa}$ could be detected, corresponding to active MMP2 (bottom band) and an intermediate form of MMP2 (middle band). This demonstrates that the exogenously expressed MMP14 WT fusion protein does not only localise normally but is also functionally active. Notably, in media of both 3HA-EGFP and 3HA-MMP14 WTEGFP-expressing cells, two additional bands could be detected between 75 and 100 $\mathrm{kDa}$ corresponding to pro-MMP9 (top band) and active MMP9 (lower band). This indicates that exogenous MMP14 expression does not alter activation of secreted proMMP9. Next, conditioned media of cells expressing mutant MMP14 fusion proteins was analysed for differences in pro-MMP2 activation. As shown in Figure 2.10A, both the MMP14 R111H and MMP14 S466 mutants retained partial activity to process proMMP2. Therefore, the p.R111H mutation is likely to retain some functionality with respect to activation of downstream MMP14 pathways (or targets). In contrast, the p.T17R and p.R92C mutations, as well as all combinations of mutations in double and triple MMP14 mutants (Fig. 2.10B), resulted in complete abrogation of pro-MMP2 processing.

\section{MMP14 mutations impair its in vitro gelatinase activity}

MMP14 has a key role in mediating invasive cell behaviour [5, 66]. Thus, the effect of the aforementioned MMP14 mutations on invasive extracellular matrix (ECM) degradation capacity was determined. As MMP14 acts as a gelatinase in vitro, Millipore's QCM $^{\text {TM }}$ Gelatin Invadopodia Assay system was combined with live cell imaging for this purpose. MRC-5V1 cells expressing 3HA-EGFP, or WT or mutant MMP14 

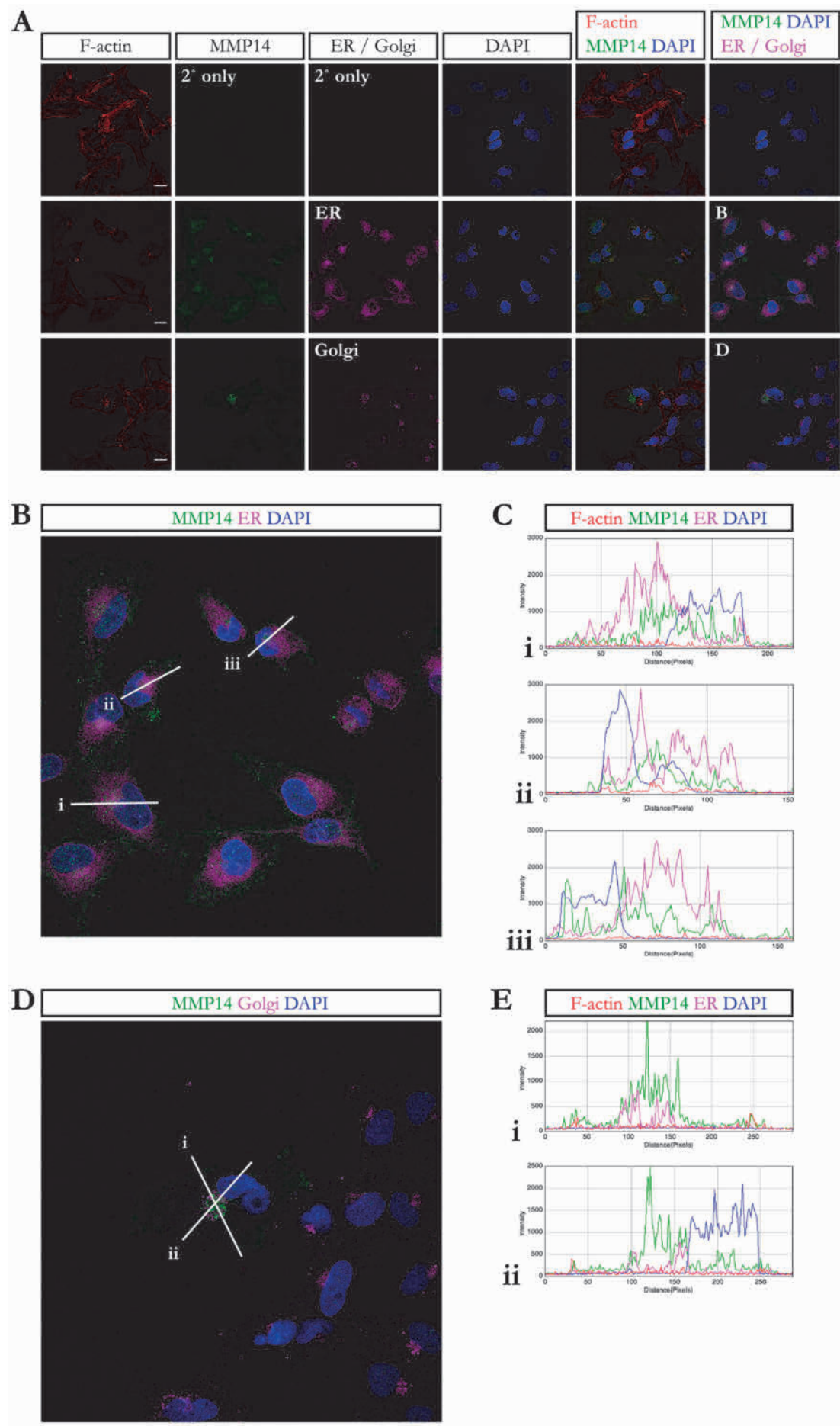

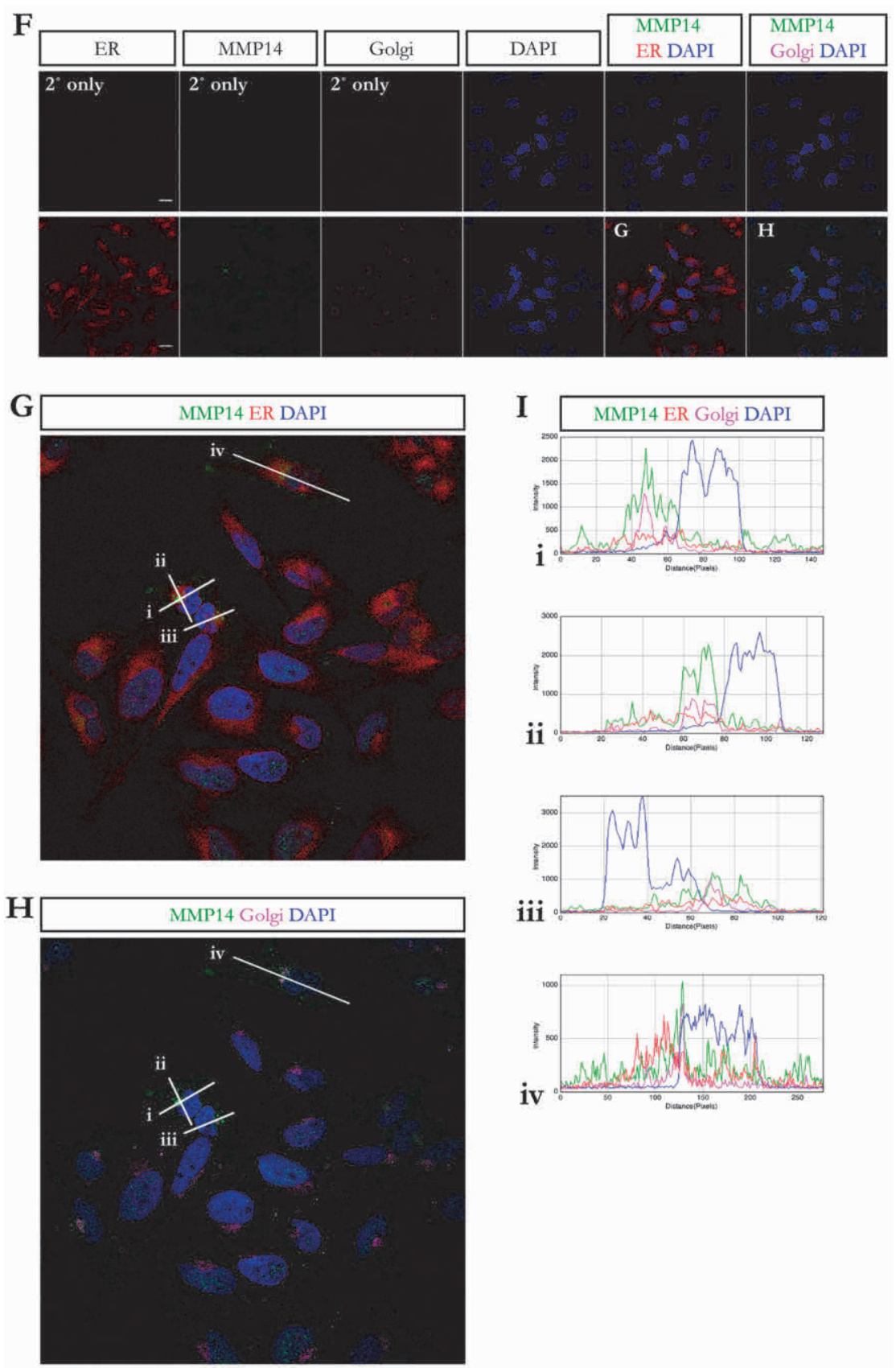

Figure 2.8 - Endogenous MMP14 forms a perinuclear aggregate that partially colocalises with the Golgi apparatus. IF microscopy images of MRC-5V1 cells stained with antibodies against endogenous MMP14, SERCA2A (used 1:1,000) and/or Golgin97 (used 1:1,000). Cells were seeded at a density of 75,000 cells per $\mathrm{mL}$ medium and fixed the next day. Isotype-specific antibodies were used for SERCA/Golgin97 co-staining. A-E, MMP14 forms a perinuclear aggregate (A-B) that only minimally 
Figure 2.8 (continued) - colocalises with the ER, which can be seen more clearly by the line plots in C showing signal intensity for the different channels along the lines in B. The perinuclear aggregate colocalises to a greater extent with the Golgi apparatus (D-E). F-I, colocalisation of MMP14 with the Golgi apparatus, and not with the ER, becomes more evident when cells are stained for MMP14 and both ER and Golgi markers (F-I). Scale bar equals $20 \mu \mathrm{m}$.

fusion proteins were hereto seeded on Cy3-labelled gelatin and imaged at different time points. Matrix digestion was readily visible by the presence of dark patches in the red fluorescent gelatin (Fig. 2.10C) as described before [67]. In line with our previous localisation and zymography data, expression of the MMP14 W'T fusion protein resulted in significantly more gelatin degradation than expression of 3HA-EGFP (Fig. 2.10D), further demonstrating the MMP14 WT fusion protein is functionally active. The p.R111H mutation resulted in a significant reduction, but not a complete loss, of gelatin degradation (see Supp. Table 1.1 for significance levels). Intriguingly, in this assay, the MMP14 T17R and MMP14 S466P mutants retained full activity when compared to the W'T protein, despite membrane localization and pro-MMP2 activation of these mutants is impaired. Moreover, the p.S466P mutation seemed to act as a gain-offunction mutation in this assay, as gelatin degradation by the MMP14 R111H S466P double mutant was not significantly different from that of MMP14 WT, whereas the p.R111H mutation when present alone significantly decreased gelatin degradation. The p.R92C mutation completely abolished gelatin degradation, also when present in combination with other mutations in double and triple mutant fusion proteins. The effects of the different MMP14 mutations on gelatin degradation at the cellular level cannot completely explain the similarities seen in phenotype on the organismal level. Where the phenotype of the Cartoon and Sabe mouse model are indistinguishable, cells expressing MMP14 R92C and MMP14 S466P behave drastically different in terms of ECM degradation. Therefore, we hypothesised that the p.S466P mutation impairs a different function of MMP14. As MMP14 has a role in stimulating cell migration that is independent of its proteolytic activity, which might contribute to the human and murine phenotype when affected, MMP14-dependent cell migration was subsequently assessed [47].

\section{The p.T17R, p.R92C and p.R111H mutations impair MMP14-dependent cell migration}

To analyse MMP14-dependent cell migration, cells expressing W'T or mutant MMP14 fusion proteins were subjected to a phagokinetic assay as previously described $[47,53]$. Although differences in ECM degradation were demonstrated with the Gelatin Invadopodia Assay, it was not possible to assess the ability of MMP14 to promote cellular migration. When the assay was repeated for a subset of fusion proteins, live confocal imaging demonstrated that cells expressing the WT or R111H mutant MMP14 fusion protein seemed to adhere strongly to the poly-L-lysine coated coverslip in areas where gelatin was degraded. When transfected cells did move, fragments of plasma 


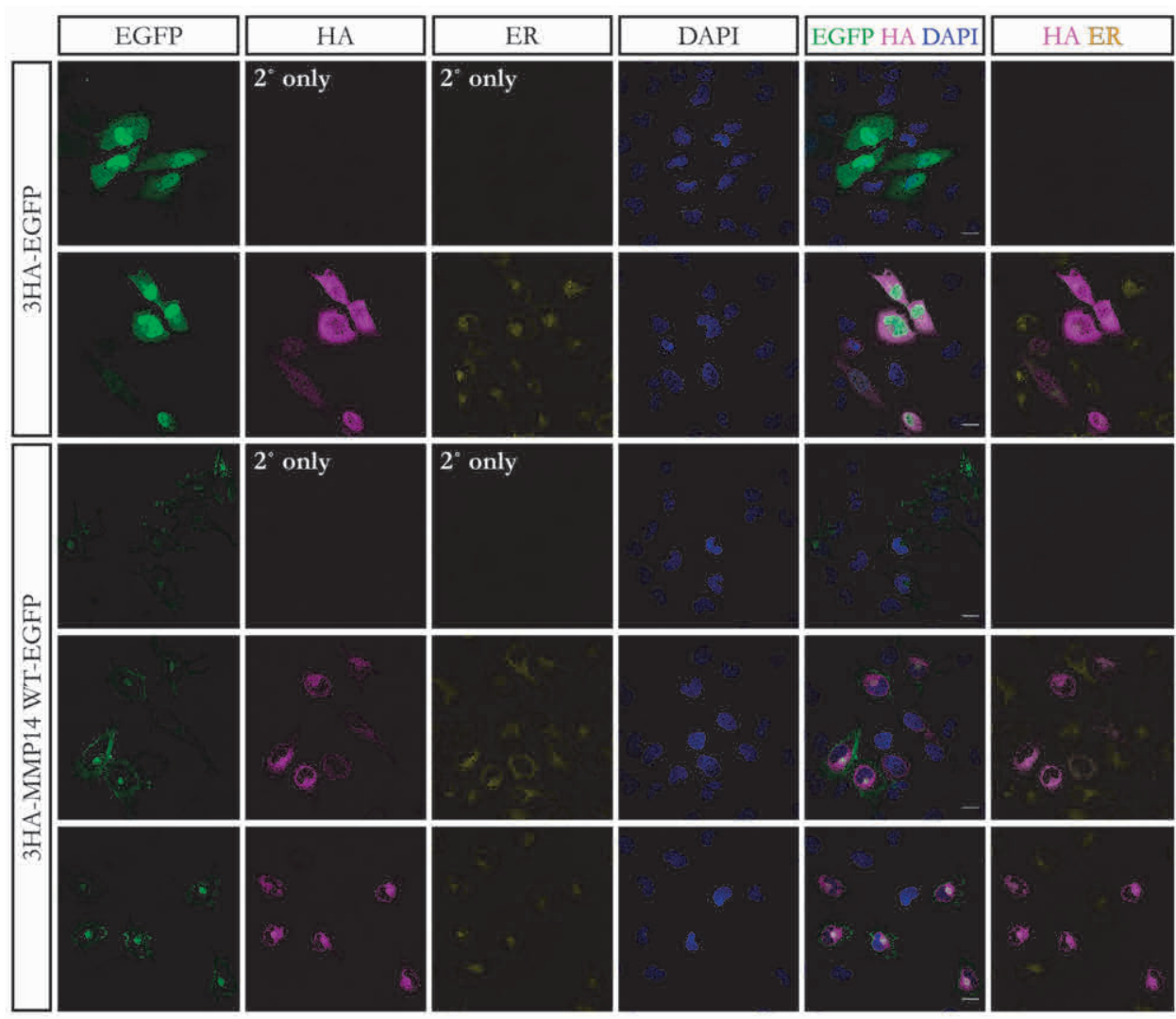

Figure 2.9 - The MMP14 signal peptide gets removed upon insertion into the ER membrane. IF microscopy images of MRC-5V1 cells transiently expressing 3HA-EGFP or 3HA-MMP14 WT-EGFP fusion proteins, incubated with rabbit anti-HA antibody (1:1,600 concentration) and mouse anti-SERCA2A antibody $(1: 40,000)$. The HA signal of the 3HA-EGFP fusion protein does not colocalise significantly with the ER. In contrast, the HA signal of the MMP14 WT fusion protein is almost identical to the SERCA2A signal. Note that the EGFP signal of the MMP14 fusion protein does not colocalise significantly with the ER staining, suggesting the SP and HA-tag get removed upon insertion of MMP14 into the ER membrane. Scale bar equals $20 \mu \mathrm{m}$.

membrane were left behind (shown in Fig. 2.10E by the trail of EGFP-positive foci), ultimately resulting in cell death. In contrast, cells expressing the 3HA-EGFP and MMP14 R92C fusion proteins, which do not degrade gelatin, migrated without EGFPpositive trail. Given the cells' strong adherence to the poly-L-lysine coating, fibronectin $(1 \mu \mathrm{g} / \mathrm{ml}$, without poly-L-lysine layer) was used as an alternative substrate on which the cells were seeded. As shown in Figure 2.11, cells expressing the MMP14 WT fusion protein had a significantly higher average migration rate $(4.60 \mu \mathrm{m} / \mathrm{h})$ compared to cells expressing 3HA-EGFP $(3.47 \mu \mathrm{m} / \mathrm{h}, \mathrm{p}=0.013)$. This demonstrates that the MMP14 WT fusion protein also behaves normally with respect to previously reported MMP14-stimulated cell migration. The p.T17R $(3.40 \mu \mathrm{m} / \mathrm{h}), \mathrm{p} . \mathrm{R} 92 \mathrm{C}(2.85 \mu \mathrm{m} / \mathrm{h})$ and 

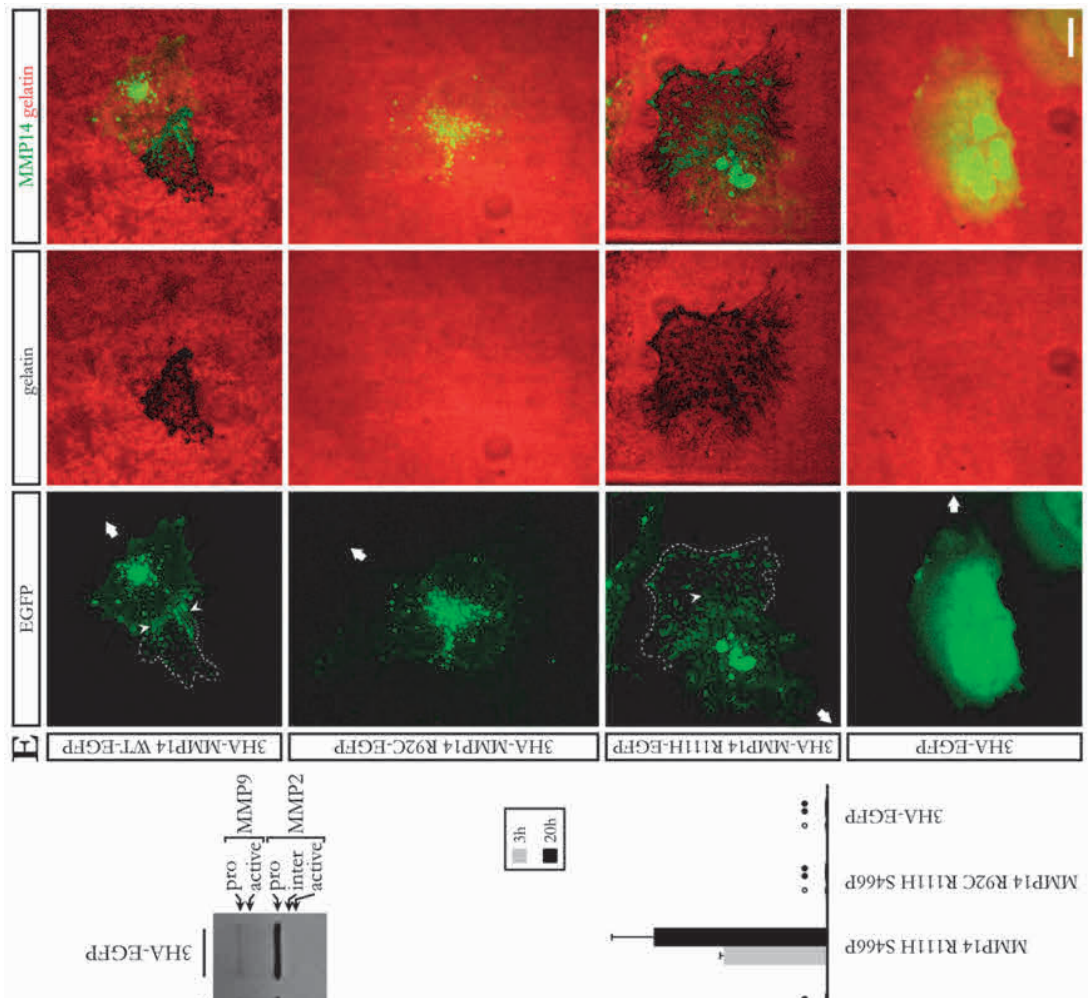

d99tS HนLบ כ264
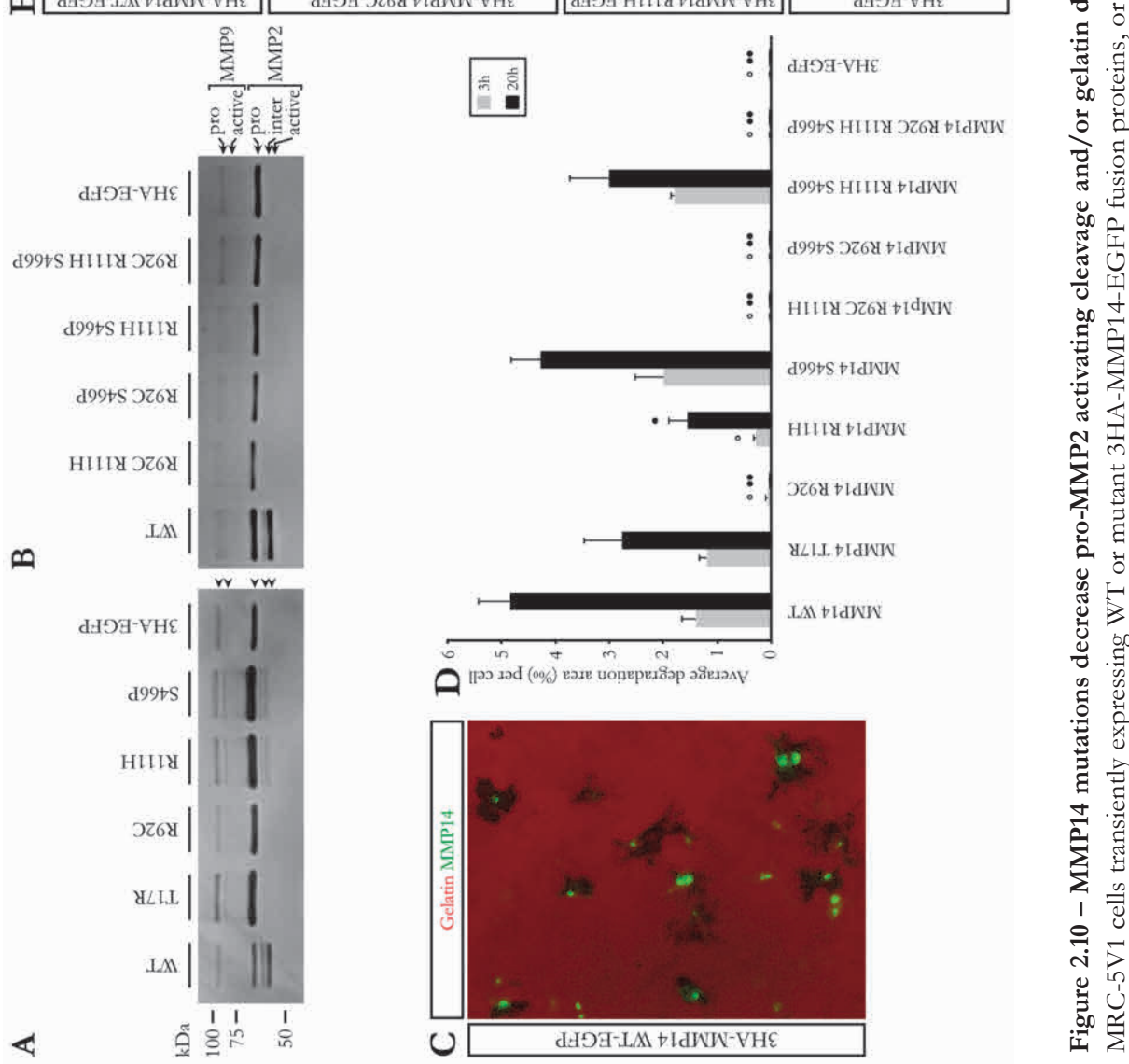
Figure 2.10 (continued) - expressing cells contained pro-MMP9 and active, cleaved MMP9, in addition to pro-MMP2. Media conditioned by cells expressing the MMP14 WT fusion protein additionally contained cleaved, active MMP2 and an intermediate form of MMP2. All analysed MMP14 mutations impair pro-MMP2 cleavage; it is completely abolished by the p.T17R and p.R92C mutations, whereas the p.R111H and p.S466P still allow some pro-MMP2 cleavage. C, IF microscopy image of MRC-5V1 cells, transiently expressing the MMP14 WT fusion protein, seeded on Cy3-labelled gelatin. Four hours post seeding, gelatin degradation becomes visible as dark patches. $\mathbf{D}$, quantification of the average Cy3-gelatin degradation surface area per green fluorescent cell at $4 \mathrm{~h}$ and $20 \mathrm{~h}$ post seeding for MRC-5V1 cells expressing WT or mutant MMP14 fusion proteins, or 3HA-EGFP (mean \pm SEM). Cells expressing the MMP14 W'T fusion protein digest significantly more gelatin than cells expressing the tags only. The p.T17R and p.S466P mutations do not significantly affect gelatin degradation. The p.S466P mutation seems to acts as a gain-of-function mutation in this assay, as gelatin degradation of MMP14 R111H S466P-expressing cells is not significantly different from MMP14 WT-expressing cells, whereas the p.R111H mutation alone significantly decreases gelatin degradation. The R92C mutation completely abolishes gelatin degradation, also when present in combination with other mutations. E, IF microscopy images of MRC-5V1 cells, transiently expressing the WT or mutant MMP14 fusion proteins or 3HA-EGFP, seeded on Cy3-labelled gelatin for qualitative analysis. Z-stacks were taken ever 15 minutes; maximum intensity projections $4 \mathrm{~h}$ post seeding are shown here. Both WT and R111H mutant MMP14 fusion protein expressing cells degrade gelatin and leave a trail of GFP-positive foci behind (direction of migration is indicated by the arrow, area with GFP foci is demarcated with dotted line). At the trailing edge of the cell, these foci seem still connected to the cell body by thin filaments (arrowheads). In contrast, cells expressing the 3HAEGFP and R92C mutant MMP14 fusion proteins, which do not degrade gelatin, migrate without GFPpositive trail. Scale bar in E equals $10 \mu \mathrm{m} .{ }^{*}, \mathrm{p}<0.05 ; * *, \mathrm{p}<0.01$.

p.R111H mutations $(2.65 \mu \mathrm{m} / \mathrm{h})$ were demonstrated to stimulate migration significantly less compared to MMP14 WT ( $p=0.011,0.0007$ and 0.0035, respectively), however without showing a clear correlation with the severity of the associated phenotypes. Interestingly, despite the plasma membrane localisation of MMP14 being impaired by the p.S466P mutation, motility of cells expressing the MMP14 S466P mutant fusion protein was not impinged compared to MMP14 WT-expressing cells $(4.56 \mu \mathrm{m} / \mathrm{h}, \mathrm{p}=$ $0.92)$.

\section{Exogenously expressed MMP14 fusion proteins that localise to the cell membrane form dome-shaped structures at the ventral cell surface}

When imaging MRC-5V1 cells expressing the MMP14 W'T fusion protein, a subset of cells showed ring-like MMP14-EGFP structures near the ventral surface of the cell, that is, the optical sections closest to the cover-slip on which the cells were growing. In Figure 2.12A, a single optical section of two 3HA-MMP14 WT-EGFP expressing cells is shown, demonstrating MMP14-EGFP rings. Multiple of such rings could typically be detected per cell, that varied in size (1 to $4.5 \mathrm{~mm}$ in diameter, Fig. 2.12A), had an overall round appearance, and were situated near the centre of the cell. These rings had a strong, yet dotted MMP14-EGFP signal, seemingly made up of several individual foci. The centre of these rings was relatively free of MMP14-EGFP signal, which is further demonstrated by the signal intensity plots shown in Figure 2.12B. Interestingly, 
the F-actin signal intensity only followed the same distribution in some (or at least only some part) of these rings (Fig. 2.12B, line plot iv). In orthogonal projection, these "rings" were shown to have a dome shape, with their base near the cover-slip surface (red line) and their apex pointing into the cytoplasm (Fig. 2.12C-E). This dome shape was present for $\mathrm{F}$-actin as well, but the signal intensity at the edge of the dome was not increased as was the case for MMP14-EGFP (compare GFP and F-actin signal in orthogonal projection in Fig. 2.12C). In cells expressing the MMP14 R111H mutant fusion protein, similar GFP structures could be identified (Fig. 2.12F) that were indistinguishable from the rings in MMP14 WT expressing cells with respect to their number, size, shape and subcellular localisation (Fig. 2.12F-K). However, it was noted that in MMP14 R111H-expressing cells, the F-actin signal followed the GFP signal distribution more closely in a larger number of structures (Fig. 2.12G, line plots i, iii, vi and ix). The question remained what the identity of these structures was, and whether lack of these structures in cells expressing mutant MMP14 fusion proteins that do not localise to the cell surface would help to explain the associated human and murine phenotype.

\section{MMP14-rich dome-shaped structures share characteristics with podosome rosettes}

We hypothesised that the structures shown in Figure 2.12 are podosomes. MMP14 plays a key role in podosome maturation, and localises to the podosome membrane [5]. Podosomes form at the ventral surface of the cell in $2 \mathrm{D}$ in vitro culture at the leading edge or underneath the nucleus $[5,68]$. Although individual podosomes are typically $0.5-2 \mu \mathrm{m}$ wide, they can cluster together into podosome rosettes that can eventually fuse together, forming structures similar in size to the rings observed in Figure 2.12 [5]. Furthermore, the podosome core is enriched in F-actin, as was the case in some (parts) of the structures shown in Figure 2.12. To analyse the possible podosome identity of these structures, 3HA-MMP14 WT-EGFP-expressing cells were subsequently stained for cortactin, another well-established podosome marker [69]. As shown in Figure 2.13A in cells expressing the MMP14 WT fusion protein, multiple MMP14EGFP rings could be observed in optical sections close to the ventral cell surface. Notably, in all three cells shown, the GFP rings were situated underneath the nucleus. From the overview of cells shown in Figure 2.13A, it became clear that the cortactin signal primarily localised to the nuclear region as well. When zooming in, it became clear that both F-actin (Fig. 2.13B), as well as cortactin (Fig. 2.13C) localised at least to some parts of the GFP rings. This supports the possibility of these structures indeed being podosome rosettes. To further assess their identity, these structures were subsequently compared to cells known to form podosomes as a positive control. 

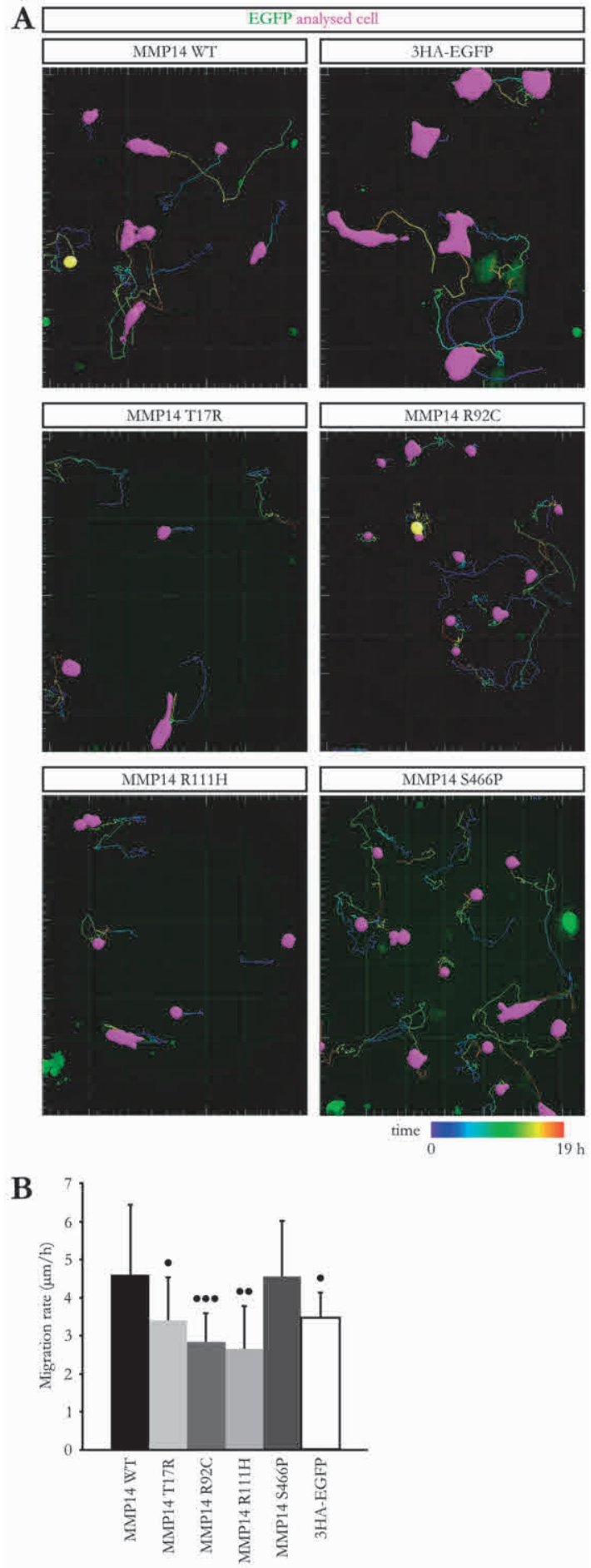

Figure 2.11 - MMP14 p.T17R, p.R92C, and p.R111H mutations all decrease MMP14-dependent cell motility. A, IF microscopy images of MRC-5V1 cells transiently expressing WT or mutant 3HA-MMP14-EGFP or 3HA-EGFP fusion proteins. Cells were seeded on fibronectin-coated glassbottom dishes and z-stacks were taken every 10 min from 2-21h post seeding; the last time point is shown here, with their migration path visualised by the rainbow trail. B, quantification of the average migration rate based on trails similar to the ones shown in A (mean \pm SEM). The MMP14 WTfusion protein significantly increases the average migration rate as compared to cells expressing 3HA-EGFP. The p.T17R, p.R92C and p.R111H mutations all decrease the average migration rate. In contrast, the p.S466P mutation does not significantly decrease the migration of cells. 


\section{MMP14-rich dome-shaped structures share features with Src-induced podosomes}

In order to compare the dome-shaped MMP14-EGFP structures shown in Figures 2.12 and 2.13 to podosomes, MRC-5V1 cells were transfected with expression vectors encoding either c-Src WT or c-Src 527. Src kinase has a central role in the initiation of podosome formation [70]. Its activity is (among other processes) regulated by phosphorylation of $\mathrm{Y}^{527}$, which reduces its kinase activity [71]. The c-Src 527 expression vector harbours a Y527F substitution. As a result, it cannot be phosphorylated, keeping the mutant Src kinase constitutively active [72]. Exogenous expression of c-Src 527 was therefore expected to induce podosome formation in cells, although overexpression of c-Src W'T might induce podosome formation as well. In untransfected MRC5V1 cells, endogenous Src was expressed at low levels and primarily localised to the plasma membrane, as supported by colocalisation with F-actin (Fig. 2.14A). No ringlike structures were observed near the ventral cell surface. As shown in Figure 2.14A, expression of exogenous c-Src WT altered the cell's morphology, inducing long cytoplasmic protrusions. Exogenous c-Src was expressed at higher levels, as indicated by the relatively high signal intensity as compared to endogenous Src, and localised to the cell surface. In addition, exogenous c-Src localised to ring-like structures (arrowhead in Fig. 2.14A, see also Fig. 2.14B-C) reminiscent in size and shape of the MMP14-EGFP structures shown above. Like the MMP14-EGFP structures, there were usually multiple c-Src rings, most evident in optical sections through the ventral part of the cell centre (shown here). Notably, at least part of the circumference of these rings was enriched in F-actin, as clearly demonstrated in Figure 2.14B (arrows). Orthogonal projection of the c-Src structures shown in Figure 2.14C furthermore showed they were dome-shaped, similar to the MMP14-EGFP structures (Fig. 2.14D-H). Similar rings could be observed in cells exogenously expressing c-Src 527 (Fig. 2.14I, arrows). However, c-Src 527 expression had a more dramatic effect on cell morphology, with about half of the transfected cells having long cytoplasmic protrusions, giving the cell a star-like appearance (Fig. 2.14A). Interestingly, c-Src 527 localised to bright foci in the distal edge of these cytoplasmic protrusions, which colocalised with F-actin (Fig. $2.14 \mathrm{~A}$, arrow). It is thought that these foci represent podosomes/podosome rosettes, which was further supported by the colocalisation of peripheral F-actin foci in these cells with endogenous cortactin (Fig. 2.14J). Staining c-Src 527 expressing MRC-5V1 cells with a custom-made antibody raised against SH3PXD2B revealed endogenous SH3PXD2B localises to the cell surface in these cells (Fig. 2.14J). However, it failed to demonstrate the presence of endogenous SH3PXD2B at the peripheral F-actin foci (Fig. 2.14J) [66]. This could, however, be demonstrated in c-Src 527 expressing cells co-stained against endogenous F-actin, cortactin and SH3PXD2B (Fig. 2.14K). In conclusion, exogenous expression of wild type c-Src, like the exogenous expression of MMP14, induces the formation of membrane structures with some, but not all, characteristics of podosome rosettes. 


\section{3-cis $\mathrm{RA}$ corrects the subcellular localisation of MMP14 S466P and MMP14 R111H S466P}

Retinoids are vitamin A derivates that are widely used to treat various skin problems $[73,74]$. Interestingly, they have been used to successfully treat different aspects of the MMP14-related phenotype. In our patients, a cumulative dose of orally administered 13-cis retinoic acid (RA) improved their acne [1]. Intraperitoneally administrated alltrans retinoic acid (ATRA), the active metabolite of 13-cis RA, has been reported to improve both bone and skin abnormalities in Mmp14 KO mice [58]. Having established the subcellular localisation and posttranslational cleavage of W'T and mutant MMP14, we hypothesised that both could be influenced by retinoid treatment. More specifically, we hypothesised that 13-cis RA could improve MMP14 activating cleavage and subsequent membrane localisation of mutant MMP14. Normal plasma concentrations of 13 -cis RA in patients being treated systemically range between 0.83 to 2.43 $\mathrm{ng} / \mathrm{mL}$ [75]. With a molecular weight of $300.44 \mathrm{~g} / \mathrm{mol}$, this corresponds to 2.76-8.09 nM. To test the effect of 13-cis RA treatment on the subcellular localisation of MMP14, MRC-5V1 cells transiently expressing the WT or mutant MMP14 fusion proteins were treated with 2-8 nM 13-cis RA, or were left untreated, in a trial experiment. Since 13-cis RA was dissolved in ethanol, treatment with $1.2 \%(v / v)$ ethanol served as vehicle control. Cells were treated for $4 \mathrm{~h}$ conform studies performed by others analysing the effect of retinoid treatment on the subcellular localisation of other proteins [76, 77]. As shown in Supplemental Figure 1.11, the subcellular distribution of both tags of the WT (A) and R111H (B) mutant MMP14 fusion protein was unaffected by either vehicle-only or 13-cis RA treatment. As seen before, in the majority of 3HA-MMP14 S466P-EGFP expressing cells, both tags localised to partially overlapping perinuclear aggregates, with only a minority $(<5 \%)$ of cells showing EGFP, but not HA, at the cell surface. Vehicle-only or 13-cis RA treatment did not affect the percentage of cells with seemingly normal processing and localisation of MMP14 S466P to the cell periphery. However, in cells treated with 13-cis RA, ring-like MMP14 S466P-EGFP structures could be observed, similar to those seen for WT and R111H mutant MMP14. These structures were not observed in MMP14 S466P-expressing cells receiving no or vehicle-only treatment.

To further study the effect of retinoid treatment on MMP14 processing and subcellular localisation, the experiment outlined above was repeated, with the addition of cells expressing the MMP14 R11H S466P fusion protein, and cells expressing GFP as a transfection control. Since there was no clear dose-dependent effect of retinoid treatment, cells were treated with $8 \mathrm{nM} 13$-cis RA or 9-cis RA, a vitamin A derivate that in contrast to 13-cis RA does not improve acne [78]. Treatment with 1.2\% $(v / v)$ ethanol served as vehicle control. As shown in Figure 2.15A, retinoid treatment did not affect the subcellular distribution of the HA and EGFP tags of the WT and R111H mutant MMP14 fusion proteins, thereby confirming the results of the trial experiment (Supp. Fig. 1.11). Again, only a minority $(<5 \%$ ) of ethanol-only treated cells expressing the 
A

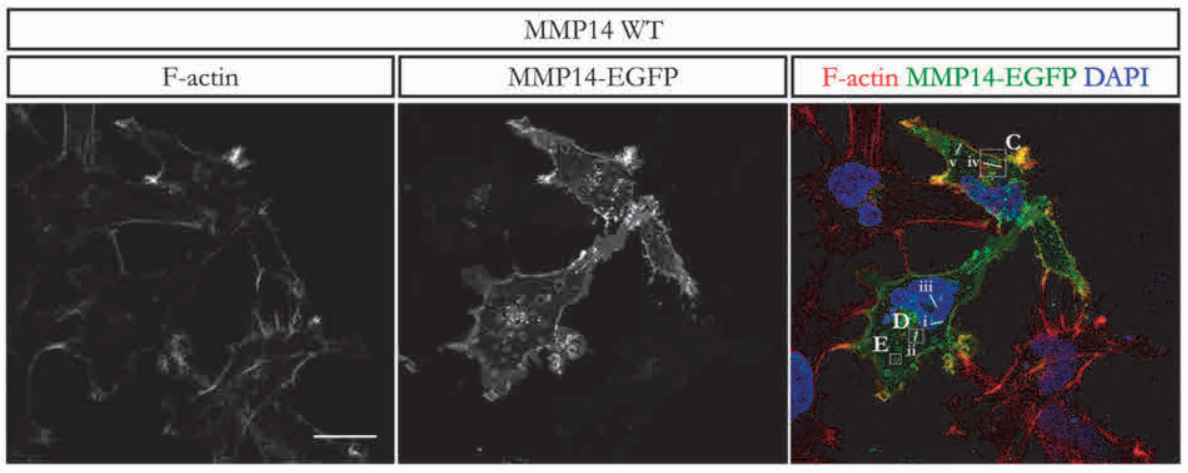

B
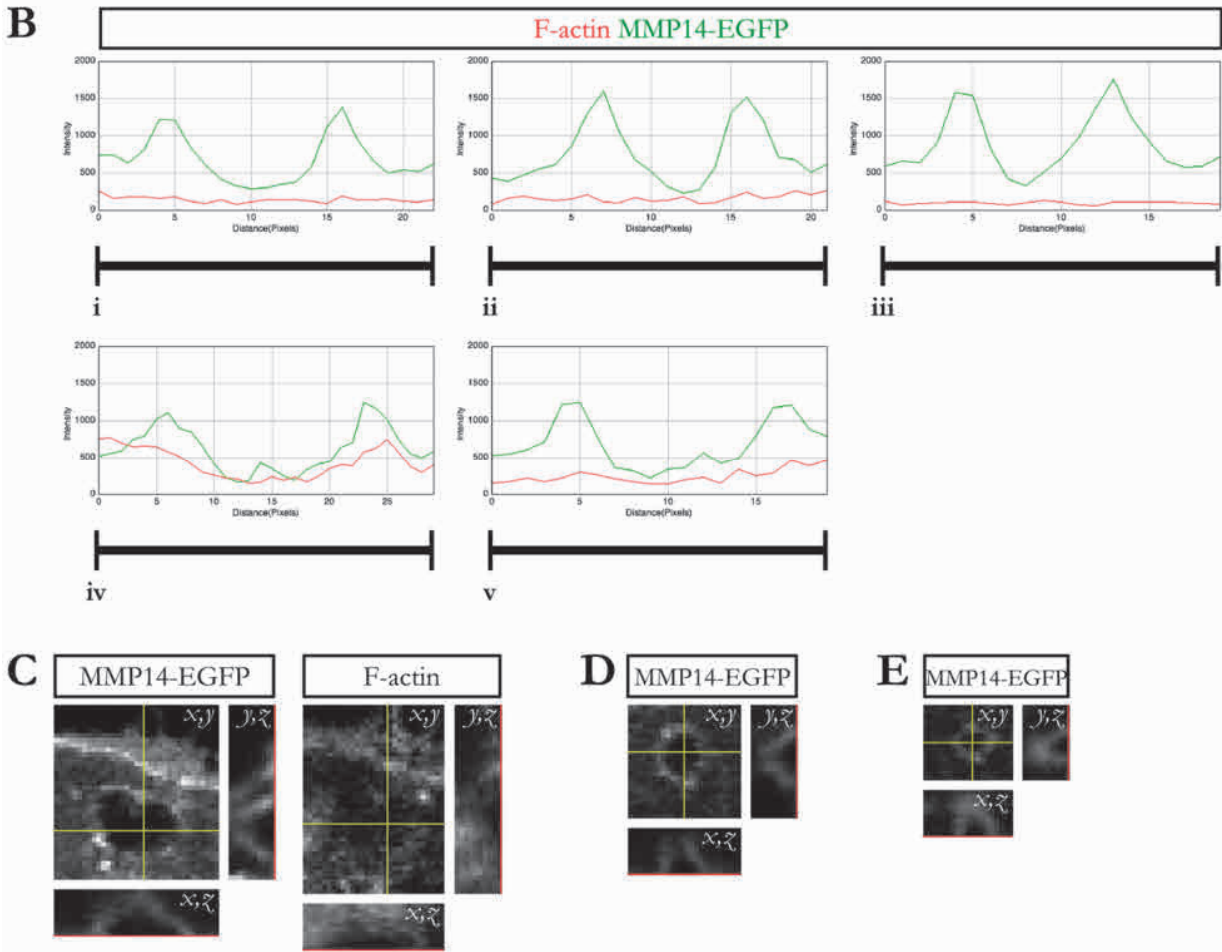

Figure 2.12 - MMP14 WT and MMP14 R111H form ring-like membrane structures near the central cell surface. A and F, IF microscopy images of MRC-5V1 cells transiently expressing 3HA-MMP14 WTEGFP (A) or 3HA-MMP14 R111H-EGFP (F) fusion proteins. Confocal z-stacks show that the EGFPsignal localises in rings, which is most evident in optical sections close to the coverslip surface on which the cells are grown (shown here). B and G, plots of MMP14-EGFP and F-actin signal intensity along the lines drawn in A and F further illustrate the ring-like distribution of MMP14-EGFP. Note that F-actin signal only follows the same distribution in some of these plots (iv in B, i-ii and ix in G). C-E and $\mathbf{H}-\mathbf{K}$, orthogonal projection demonstrates the structures shown in $\mathrm{A}$ and $\mathrm{F}$ are in fact dome-shaped, with their base near the cover-slip surface (red line). This dome-shaped appearance is more evident for MMP14-EGFP than for F-actin (C; compare to line plot iv in B). Scale bar equals $20 \mu \mathrm{m}$. 


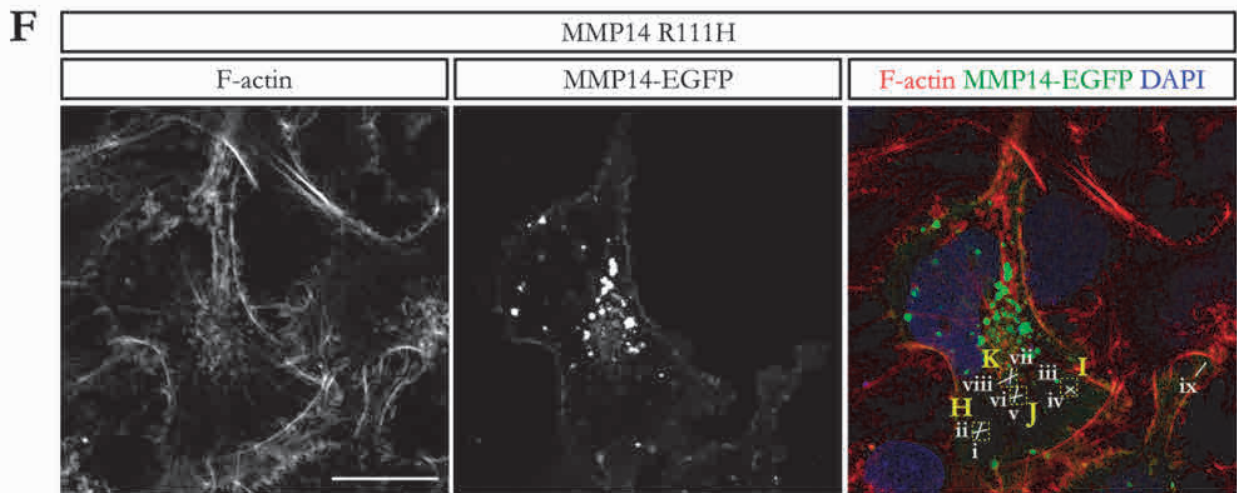

G
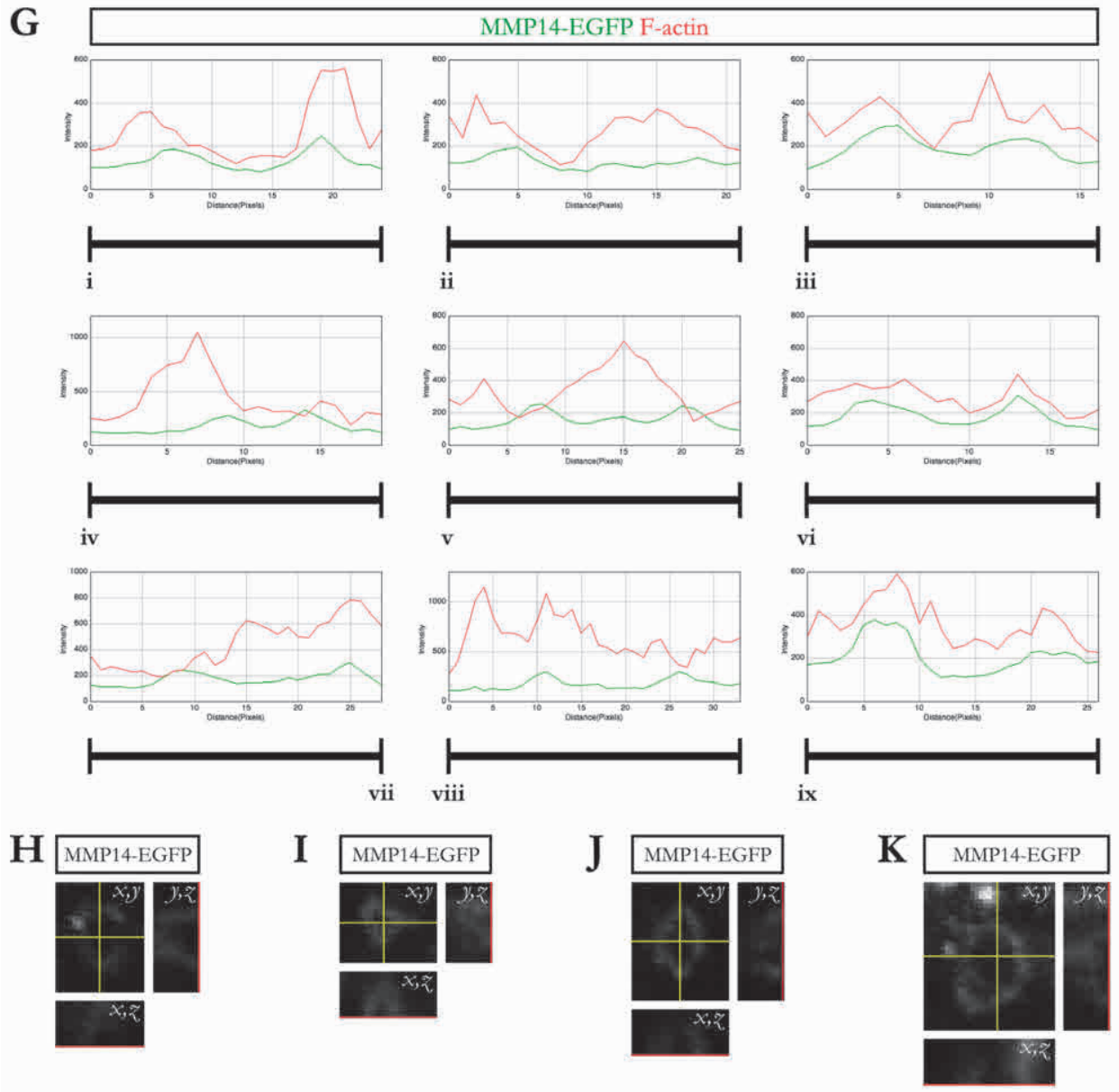

Figure 2.12 (continued). 


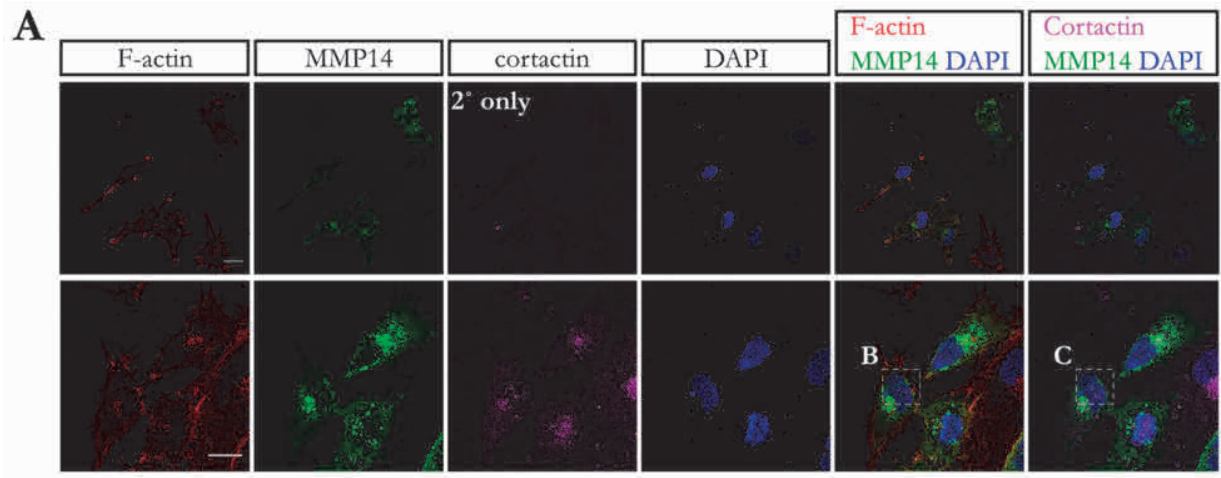

B
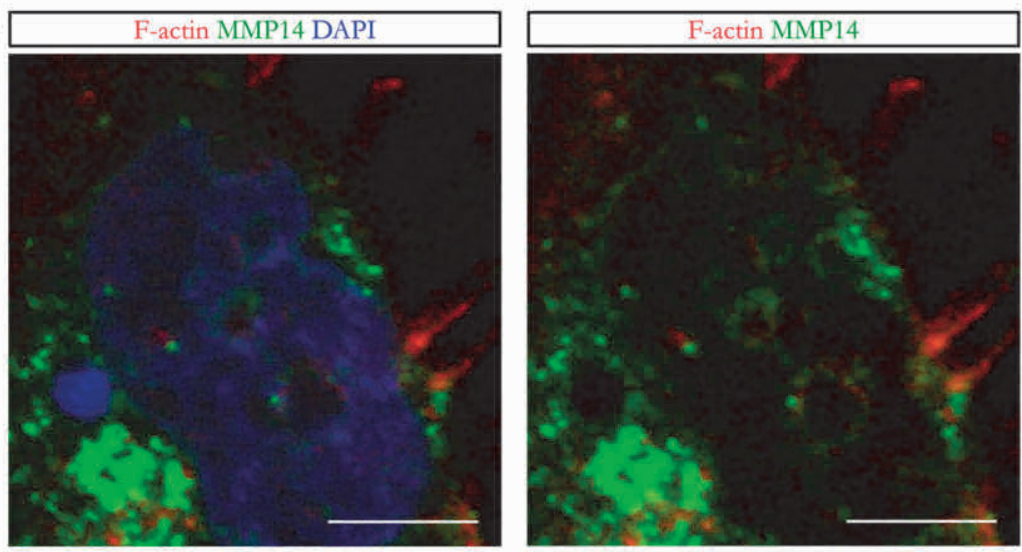

C
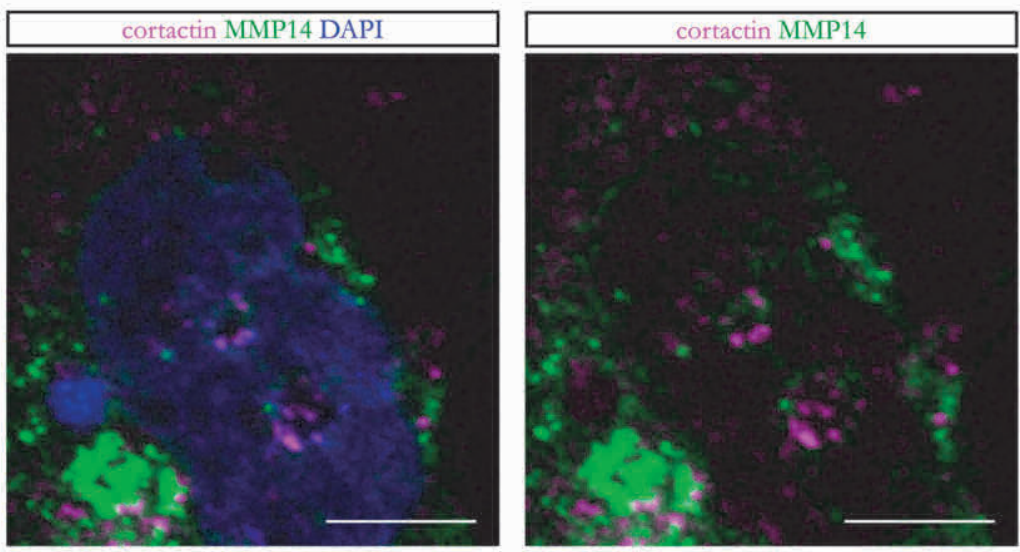

Figure 2.13 - MMP14 ring-like structures are enriched in cortactin. A, IF microscopy images of MRC-5V1 cells transiently expressing 3HA-MMP14 WT-EGFP and stained against the podosomal markers cortactin and F-actin. Merges are enlarged in B and C, either with (left) or without (right) DAPI signal shown. MMP14-EGFP signal forms ring-like structures, most evident in optical sections close to the coverslip surface on which the cells are grown (shown here). The centre of these structures is relatively free of MMP14-EGFP, F-actin, and DAPI signal. Both F-actin and cortactin colocalise to some extent with the MMP14-EGFP rings. Scale bar equals $20 \mu \mathrm{m}$. 
S466P mutant fusion protein showed localisation of the EGFP signal, but not HA, at the cell surface, suggesting normal processing and trafficking of the fusion protein. However, in contrast to the trial experiment, this percentage seemed to be increased by 13-cis RA treatment. As the HA staining pattern was unaltered, 13-cis RA treatment seemed to influence primarily trafficking, and not posttranslational cleavage, of MMP14 S466P. Ethanol-only treated cells expressing the MMP14 R111H S466P fusion protein showed severe impairment of MMP14-EGFP membrane localisation; only a very faint signal could be detected in a minority $(<5 \%)$ of transfected cells. This became more readily visible upon treatment with 13-cis RA, but not 9-cis RA (Fig. 2.15). Notably, in cells showing plasma membrane localisation of MMP14 S466P and MMP14 R111H S466P, faint ring-like structures could be detected in the centre of the cell's ventral surface. Retinoid treatment had no effect on the subcellular localisation of GFP in tag-only expressing cells. Interestingly, cells expressing the GFP control showed small circular areas free of GFP signal near the ventral surface, reminiscent of the MMP14-EGFP structures described above. Retrospectively, these circular GFPfree areas could also be observed in images of the 3HA-EGFP-expressing cells shown in Supplemental Figures 1.4 and 1.10. This would suggest that exogenously expressed MMP14 and c-Src do not necessarily induce these structures, but primarily localise to pre-existing structures, thereby making them more readily visible when fluorescently tagged or probed with an antibody.

As described above, 13-cis RA improved the trafficking of MMP14 mutants to the cell surface. To assess the effect of retinoid treatment on MMP14 processing, cell lysate of untransfected MRC-5V1 cells treated as described above or left untreated was subjected to immunoblotting with a custom-made anti-MMP14 antibody. Untreated MRC5V1 cells expressing the MMP14 W'T fusion protein served as positive control for the antibody. As shown in Figure 2.15C, the antibody recognised the different forms of both the exogenous MMP14 fusion protein and the endogenous MMP14; the size of the bands was similar to those seen before for endogenous (Fig. 2.4A) and exogenous MMP14 (Fig. 2.7). The size and the height of the different bands for endogenous MMP14 species were not altered by retinoid or vehicle-only treatment, suggesting retinoids do not alter MMP14 expression levels or posttranslational processing.

\section{Discussion}

We previously reported two brothers with a multisystem disorder encompassing skeletal anomalies, mitral valve insufficiency, facial dysmorphy, and severe acne, in which we identified a novel MMP14 missense mutation [1]. We used a novel in vitro model to assess the mutation's effect of on the protein level and compare it to previously reported human and murine MMP14 mutations. Our results confirmed our patients' mutation pathogenicity, and led to new insights into the effect of previously reported mutations on the protein level and normal MMP14 posttranslational processing. 
CHAPTER 2
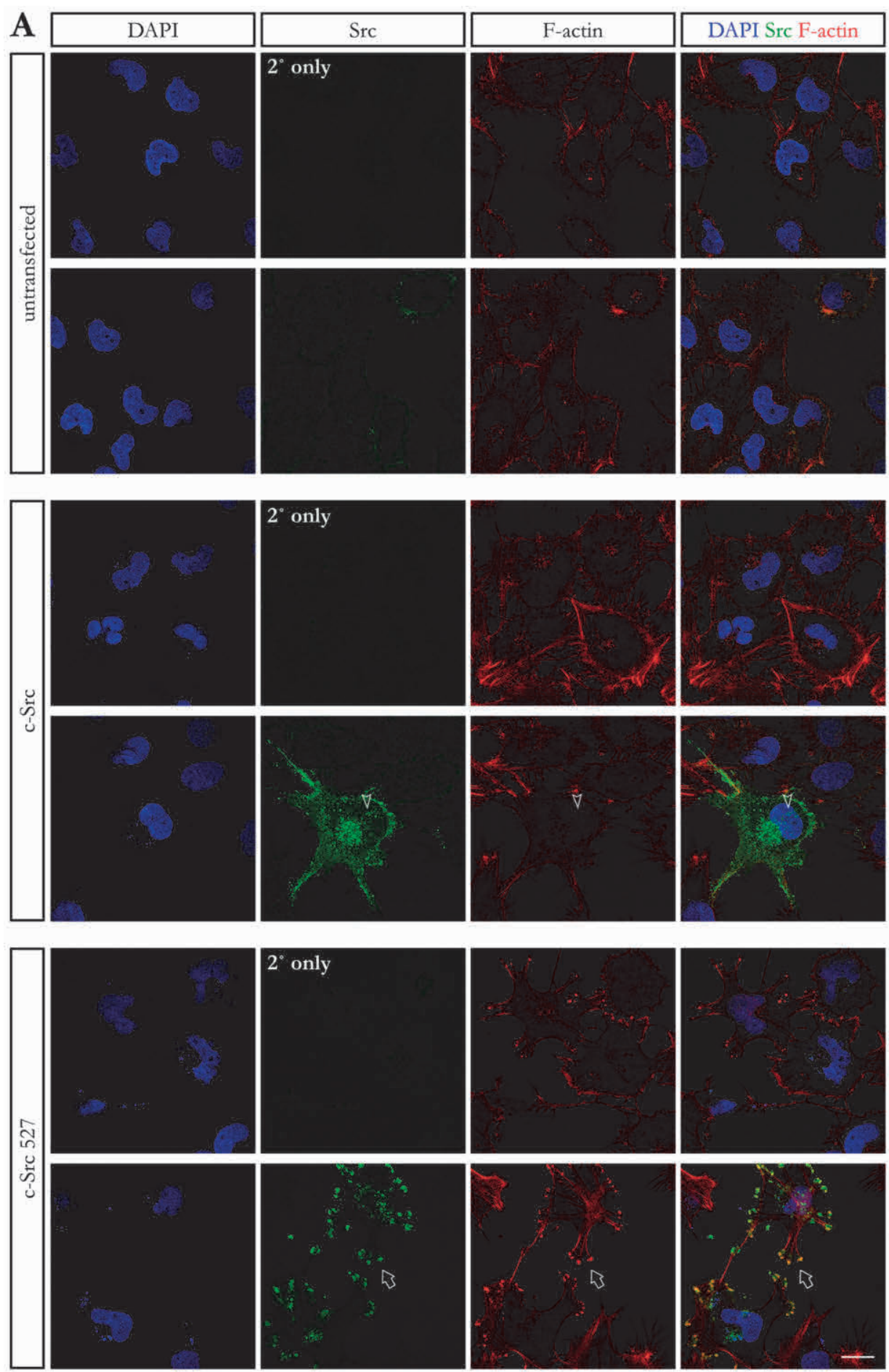

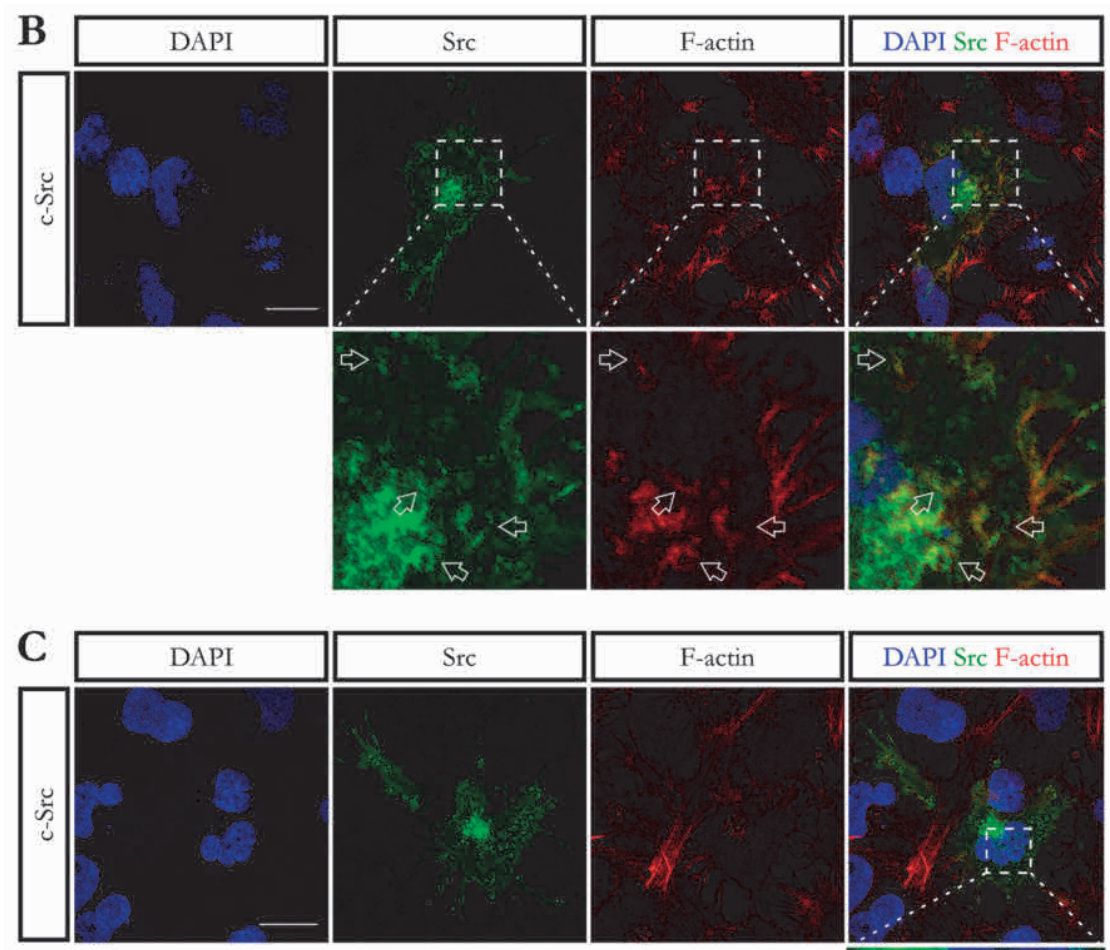

DAPI Src F-actin
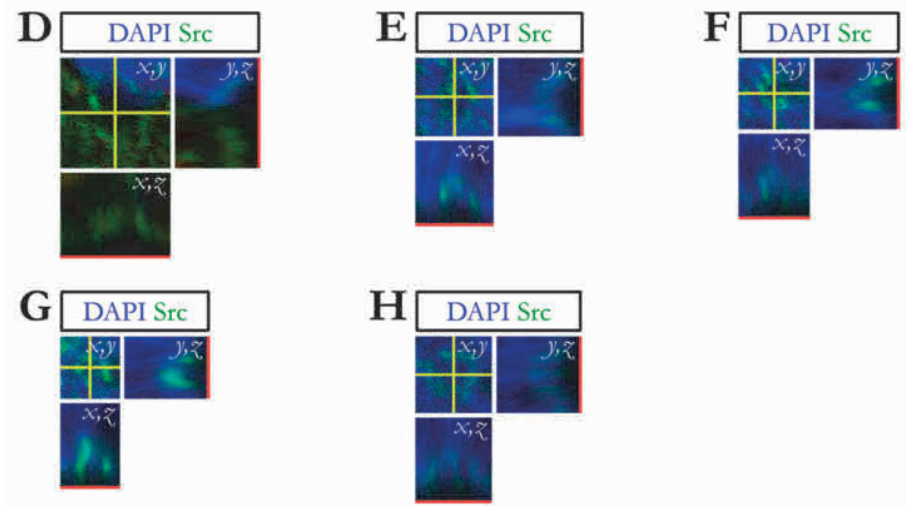

Figure 2.14 - c-Src induces ring-like structures in MRC-5V1 cells that differ from c-Src 527 induced podosome rosettes. A-K, IF microscopy images of MRC-5V1 cells transiently expressing exogenous c-Src WT or mutant c-Src 527, stained for the podosomal markers Src, F-actin, cortactin and/or SH3PXD2B. Cells were seeded at a density of 20,000 cell per mL of medium and left untransfected, or seeded at 50,000 

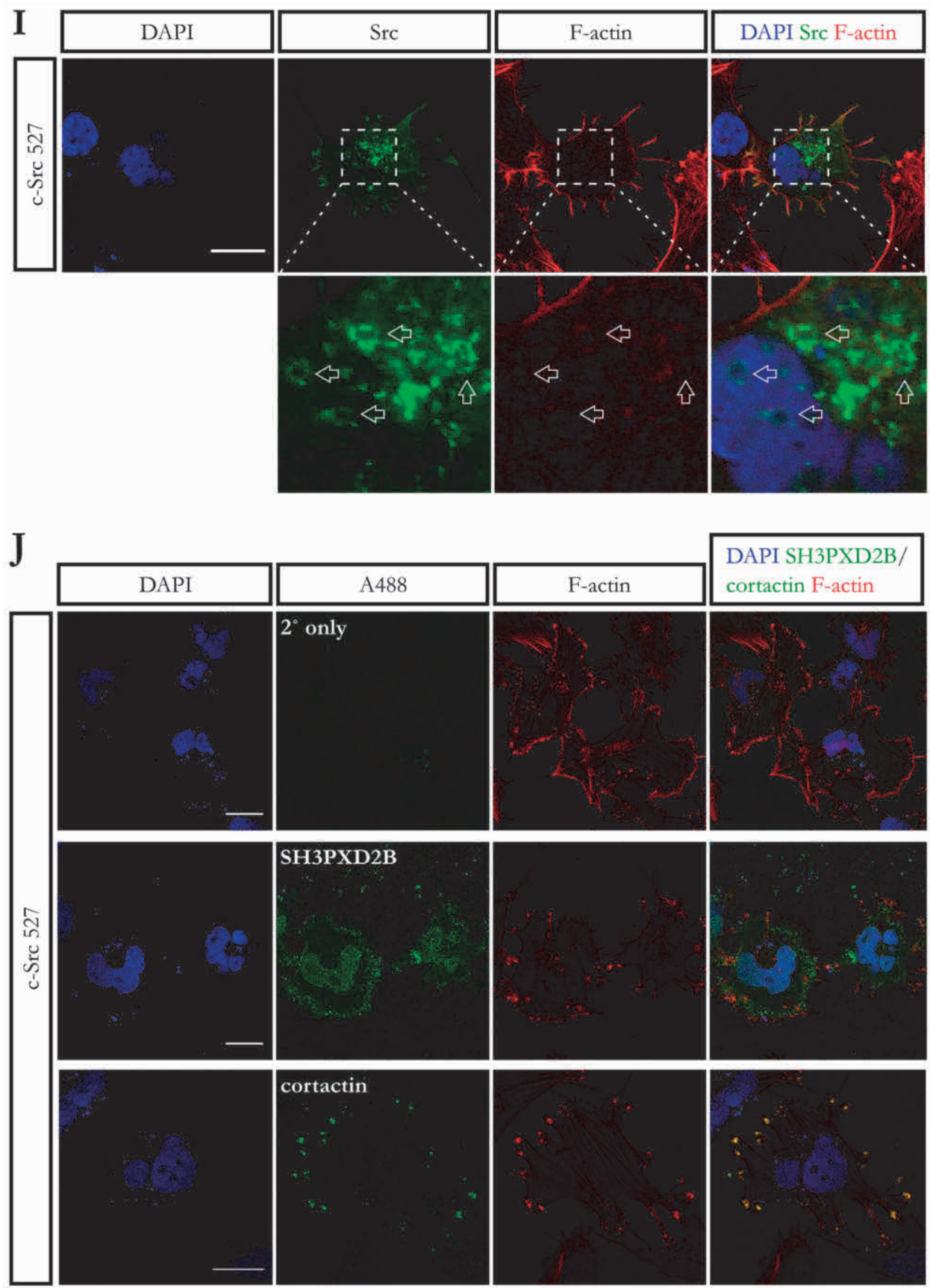

Figure 2.14 (continued) - cells per $\mathrm{mL}$ of medium and transfected $24 \mathrm{~h}$ later. All cells were fixed $72 \mathrm{~h}$ post seeding. A, endogenous Src in untransfected MRC-5V1 cells is expressed at low levels and primarily localises to the cell surface. Expression of exogenous c-Src changes the morphology of the cell, with long protrusions typically present as in the cell shown here. In addition, c-Src localises to ring-like structures 

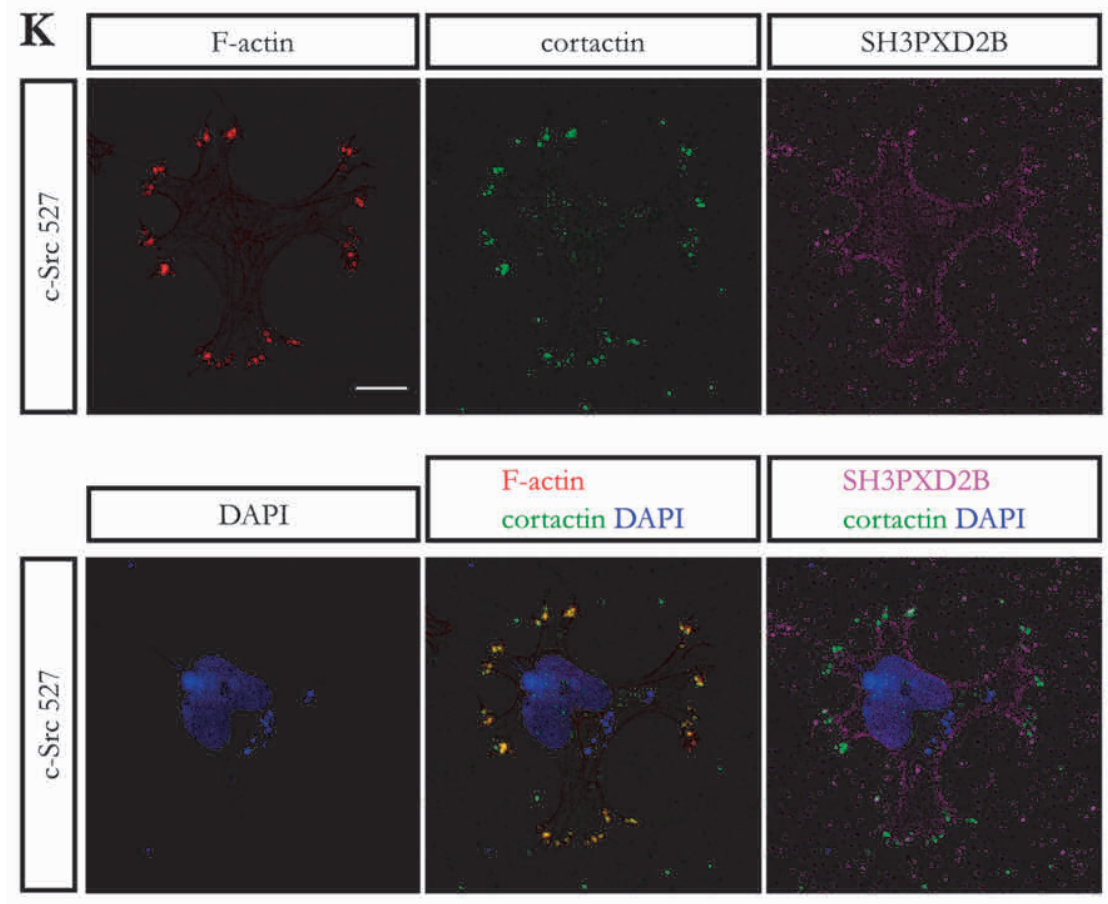

Figure 2.14 (continued) - (arrowhead) that are not enriched in F-actin, most evident in optical sections close to the coverslip surface on which the cells are grown (shown here). Expression of exogenous c-Src 527 induces a more dramatic starfish-like change of cell morphology. The distal edge of the cytoplasmic protrusions displays c-Src 527 foci (arrow) that colocalise with F-actin foci. B, C and I, exogenous c-Src and c-Src 527 localise to ring-like structures that partly colocalise with F-actin (B and I, arrows). D-H, orthogonal projection of the c-Src ring-like structures shown in $\mathrm{C}$ demonstrates their dome shape, with their base near the cover-slip surface (red line). Ring-like structures in the nuclear region are devoid of chromatin in their centre. J, staining c-Src 527-expressing cells for F-actin and either SH3PD2B or cortactin indicates that although SH3PXD2B localises to the cell surface in c-Src527 expressing cells, it does not clearly colocalise with the peripheral F-actin foci, in contrast to cortactin. K, staining c-Src 527 expressing cells for F-actin, cortactin, and SH3PXD2B shows even more clearly that SH3PXD2B does not colocalise with peripheral foci rich in F-actin and cortactin. Scale bars in A-J equal $20 \mu \mathrm{m}$; scale bar in $\mathrm{K}$ equals 10 $\mu \mathrm{m}$.

\section{A novel in vitro model to assess MMP14 subcellular localisation, processing and function}

By expressing an MMP14 fusion protein with N-terminal 3HA tag and C-terminal EGFP in MRC-5V1 fibroblasts, we established a novel in vitro model that enabled us to directly link posttranslational cleavage to subcellular localisation by IF microscopy and Western blot. We demonstrated that the subcellular localisation of exogenously expressed MMP14 WT-EGFP is identical to that of endogenous MMP14 and therefore unaffected by the tags. We furthermore showed by Western blot that the WT fusion protein is processed similarly to endogenous MMP14, both in our experiments as well as compared to previously published results [12, 13, 35, 60, 61]. Finally, we 
A
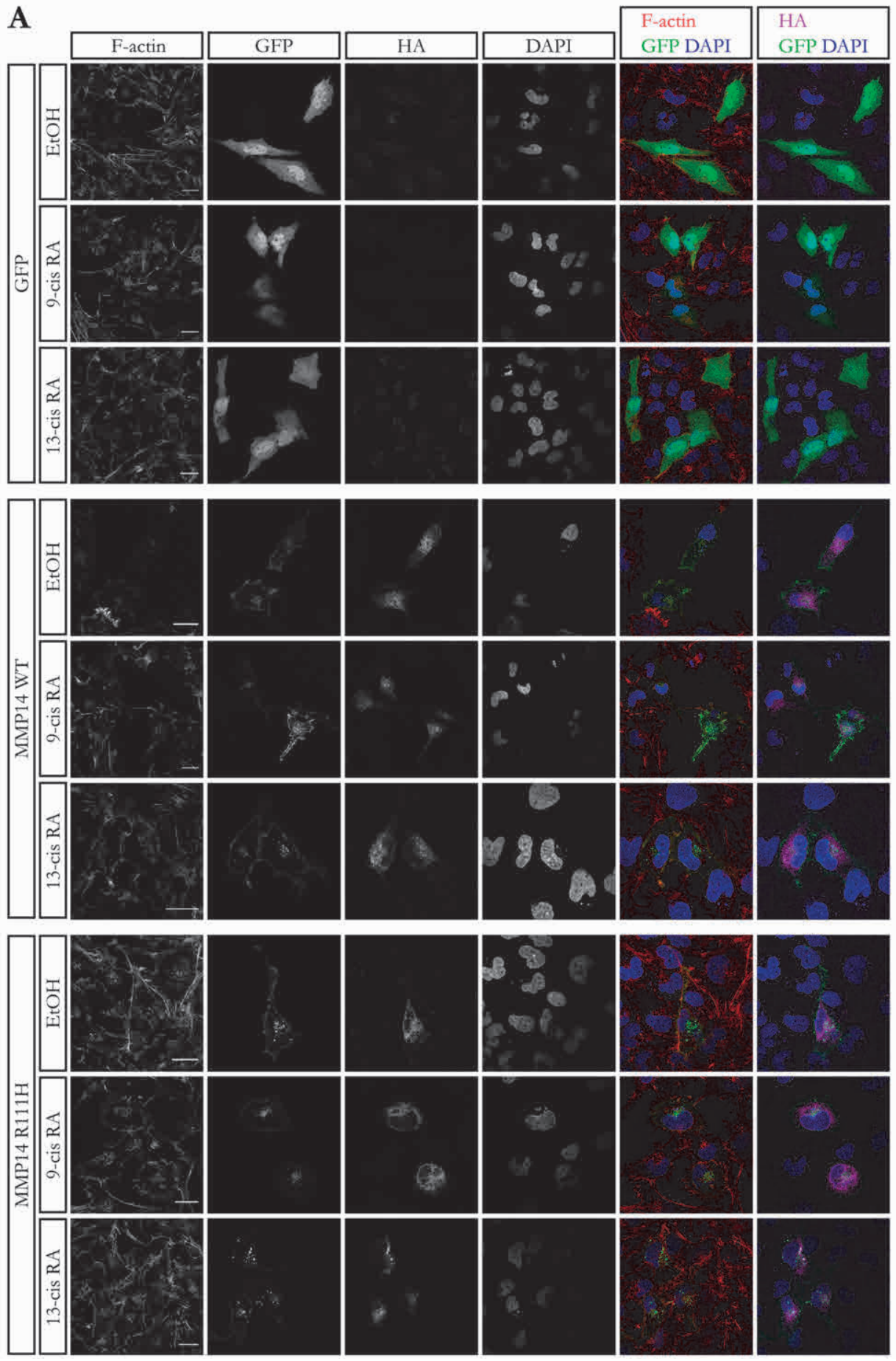

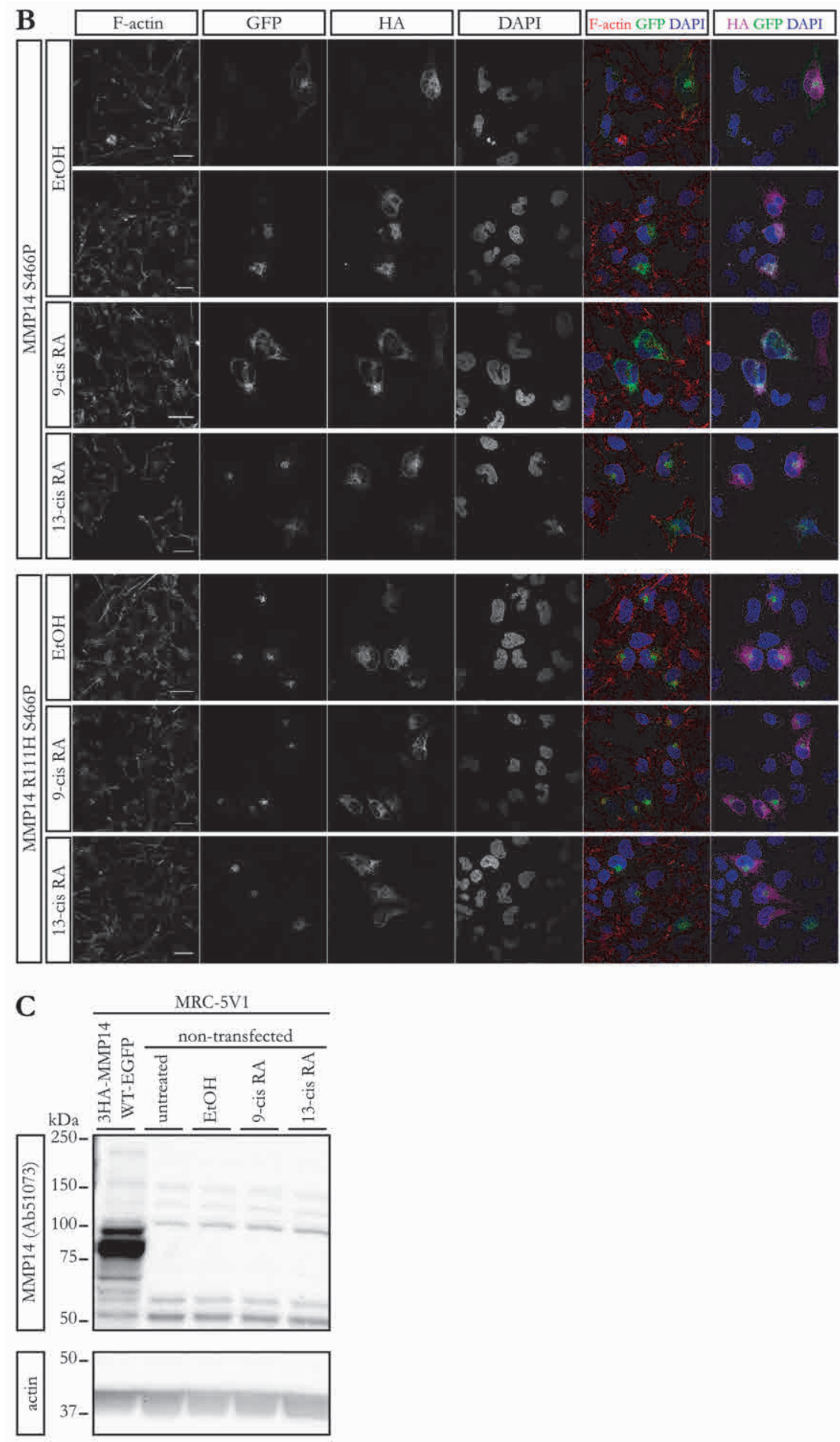
Figure 2.15 - Retinoids do not seem to affect posttranslational cleavage and subcellular localisation of MMP14, except for the S466P mutant. - A-B, IF microscopy images of MRC-5V1 cells transiently expressing WT or mutant 3HA-MMP14-EGFP fusion proteins or GFP as control, were treated with $8 \mathrm{nM} 9$-cis or 13-cis retinoic acid (RA) or vehicle $(1.2 \%(v / v)$ ethanol) only. Retinoid treatment does not seem to affect the subcellular distribution of the HA and EGFP tags of WT or R111H mutant MMP14 fusion protein. B, a small proportion $(<5 \%)$ of cells expressing the S466P mutant fusion protein show localisation of the EGFP signal, but not HA, at the cell surface, suggesting normal processing of the protein. This percentage seems to be increased by 13-cis RA treatment. Scale bars in A and B equal 20 $\mu \mathrm{m}$. C, immunoblot with anti-MMP14 antibody on protein extract of MRC-5V1 cells either transiently expressing 3HA-MMP14 WT-EGFP or untransfected and treated with retinoids, vehicle only, or left untreated. Bands for endogenous MMP14 between 50 and $75 \mathrm{kDa}$ are similar to those observed in Figure 2.4; bands over $100 \mathrm{kDa}$ for endogenous MMP14 likely correspond to multimers of active MMP14 and/or MMP14 degradation products, as reported before [39]. Expression levels and posttranslational processing of endogenous MMP14 in mIMCD-3 and MMP14 are not affected by the vehicle nor retinoid treatment.

demonstrated that the W'T fusion protein is functionally active with respect to enzymatic activity, and stimulation of cell migration in vitro. We were therefore confident to use this in vitro model to assess the effects of human and murine MMP14 missense mutations on the protein level.

\section{The N-terminus of MMP14 is sequentially cleaved in the biosynthetic-secretory pathway}

It has been demonstrated before that MMP14 is translated as a latent pre-pro-enzyme $[4,18]$. This also holds true for our WT fusion protein as evidenced by presence of both tags in the largest form detected by Western blot. MMP14 is a class I transmembrane protein that needs to be incorporated into the ER membrane for eventual plasma membrane localisation [4, 18, 23, 27]. Although previously assumed by others, we here show the first evidence supporting SP removal upon insertion of MMP14 into the ER membrane [11, 19-21]. Firstly, in silico analysis of the SP suggests its first 23 amino acids are likely to be removed by proteolytic cleavage. Secondly, only the largest form of the MMP14 WT fusion protein detected by Western blot contained the HA tag, while it was absent from all smaller, processed forms. Thirdly, we showed by IF microscopy that in cells expressing the WT fusion protein, the HA tag colocalised with the ER, whereas MMP14-EGFP did not. Others have previously demonstrated the pro-domain of MMP14 is sequentially cleaved in the exocytotic pathway prior to insertion into the plasma membrane $[12,33,34]$. We additionally demonstrated that MMP14-EGFP, like endogenous MMP14, forms a perinuclear aggregate that partially colocalises with the Golgi apparatus, where MMP14 prodomain cleavage by proprotein convertases (PCs) including furin is thought to take place [12, 37, 41]. Finally, we demonstrated that of MMP14-EGFP is present at the cell surface. 
The p.R111H mutation impairs MMP14 function, supporting its pathogenicity in WS

Consistent with in vitro our model, the p.R111H mutation did not affect SP removal. As the p.R111H mutation is present in the $\mathrm{R}^{108}-\mathrm{R}-\mathrm{Y}-\mathrm{R}-\downarrow_{-}-\mathrm{T}^{112} \mathrm{PC}$ recognition motif and mature MMP14 starts N-terminally with $\mathrm{T}^{112}$, we hypothesised this mutation impairs MMP14 activating cleavage and, possibly, subcellular localisation [12, 38, 39, 41, 42]. Indeed, our results demonstrated that the p.R111H mutation reduced catalytic activity and MMP14-stimulated migration, thereby supporting pathogenicity of this mutation. However, in contrast to our hypothesis, we found that 3HA-MMP14 R111H-EGFP is processed and trafficked seemingly identical to the MMP14 WT fusion protein (see Table 2.1 for an overview). Others have previously demonstrated that mutation of this PC recognition site impairs MMP14 processing and subsequent pro-MMP2 activation in vitro $[12,33,38,42]$. Only a single study showed that mutation of the PC motif had no adverse effects on MMP14 processing and function, which is likely a result of differences in cell types used [39]. In all of these studies, multiple residues of the PC motif were mutated, in contrast to our patients where only a single residue is altered. One possible explanation for the discrepancy between MMP14 $\mathrm{R} 111 \mathrm{H}$ seemingly normal processing and impaired activity could be abnormal prodomain cleavage that is not detected by Western blot analysis. Supporting this possibility is the observation by Golubkov et al. that MMP14 can be cleaved at other sites, including the $\mathrm{G}^{100}-\mathrm{A}-\mathrm{E}-\downarrow-\mathrm{I}^{103}$ motif by MMPs [34]. Although such forms have lost the cysteine switch domain, it is possible that their functional activity is reduced.

\section{The WS phenotype severity correlates with pro-MMP2 activation of mutant MMP14 in vitro}

Sequential cleavage in the secretory pathway is further supported by the total absence of posttranslational cleavage of the MMP14 T17R mutant fusion protein. Our observations suggest that the p.T17R mutation disrupts the SP, thereby preventing the protein from entering the ER and almost completely abolishing subsequent processing. In contrast to previously published, we did not observe pro-MMP14 T17R at the plasma membrane. This apparent discrepancy might be due to the greater sensitivity of surface biotinylation assay employed by Evans et al. as compared to our IF detection [6].

Table 2.1 - Overview of the effects of the studied mutations on pro-MMP14 processing, subcellular localization, pro-MMP2 activating cleavage, gelatin degradation and cell migration. Tick mark, unaltered; arrow, impaired; cross, severely impaired to absent.

\begin{tabular}{|c|c|c|c|c|c|}
\hline MMP14 & $\begin{array}{l}\text { Pro-MMP14 } \\
\text { processing }\end{array}$ & $\begin{array}{l}\text { MMP14 } \\
\text { localisation }\end{array}$ & $\begin{array}{l}\text { Pro-MMP2 } \\
\text { cleavage }\end{array}$ & $\begin{array}{l}\text { Gelatin } \\
\text { degradation }\end{array}$ & $\begin{array}{l}\text { Cell } \\
\text { migration }\end{array}$ \\
\hline WT & $\checkmark$ & $\checkmark$ & $\checkmark$ & $\checkmark$ & $\checkmark$ \\
\hline T17R & $x$ & X & $x$ & $\checkmark$ & $\downarrow$ \\
\hline R92C & $x$ & $x$ & $x$ & $x$ & $\downarrow$ \\
\hline R111H & $\checkmark$ & $\checkmark$ & $\downarrow$ & $\downarrow$ & $\downarrow$ \\
\hline S466P & $x$ & $\downarrow$ & $\downarrow$ & $\checkmark$ & $\checkmark$ \\
\hline
\end{tabular}


However, biotinylation demonstrated that only the pro-enzyme, and not the cleaved, active form of MMP14, was present at the cell membrane, supporting impaired processing. The lack of normal subcellular localisation and processing resulted as expected in a reduced pro-MMP2 cleavage, consistent with the findings of Evans et al., in addition to reduced MMP14-stimulated cell migration [6]. Notably, the p.T17R mutation did not significantly reduce in vitro gelatin degradation. One possible explanation for impaired pro-MMP2 activation, yet normal in vitro gelatin degradation, could be the dose of MMP14 needed at the cell surface for these functions in vitro. Less MMP14 present at the cell surface could impair homodimer formation, as it was shown previously that MMP14 homodimerisation is concentration dependent in E. coli [50]. It has been demonstrated that disturbed homodimer formation impairs pro-MMP2 activation, but not gelatin degradation $[48,50,52,79,80]$. As the phenotype of our patients is milder than that of the patients reported by Winchester et al., our observations suggest that MMP14's ability to activate pro-MMP2 may be the most important determinant of disease severity [7]. This is furthermore in line with the p.R92C mutation in the Sabe mouse, which phenotype is more severe.

\section{The N-terminal PC recognition motif plays an important role in pro-MMP14 activation}

Similar to the human p.R111H mutation, the p.R92C Sabe mutation is present at the cleavage site in the more $\mathrm{N}$-terminally located $\mathrm{R}^{89}$-R-P-R- $\downarrow-\mathrm{C}^{93}$ PC recognition motif [8]. Based on in silico analysis, it has previously been suggested that this site is inaccessible to furin $[12,33,40]$. In addition, it has been demonstrated that the effect of the p.R89A mutation in this motif is of limited significance to MMP14 processing and its capability to activate pro-MMP2 [12, 33, 38, 39]. Although we detected a form of MMP14 R92C-EGFP seemingly corresponding in size to the mature enzyme by Western blot, our functional data suggest that prodomain cleavage is aberrant, yet our Western analysis lacks the resolution to detect this. The devastating effect of this mutation on trafficking, additional processing and catalytic and migratory stimulating function of MMP14 (Table 2.1) strongly suggest that the p.R92C mutation impairs prodomain processing. This implies that the more $\mathrm{N}$-terminally located $\mathrm{PC}$ recognition site has an important role in pro-MMP14 activation. One possible explanation for the discrepancy between our results and previous studies could be that the p.R92C mutation is more damaging to the $\mathrm{PC}$ recognition motif than the previously studied p.R89A mutation. This is supported by the fact that in previous studies mutation of both PC recognition motifs acted synergistically and impaired MMP14 prodomain cleavage and pro-MMP12 activation to a greater extent than mutating either motif individually [12, 38]. The p.R92C mutation, however, disrupts MMP14 processing, trafficking and function to such extent that addition of any other mutation did not worsen the outcome of any of the assays we performed. 
The p.R92C mutation impairs MMP14 processing, subcellular localisation and functional activity to a greater extent than the p.R111H mutation, which could in part explain the more severe phenotype of the Sabe mice compared to our patients. Interestingly, MMP14 cleavage beyond the prodomain was only observed for the two MMP14 fusion proteins (WT and $\mathrm{R} 111 \mathrm{H}$ ) that were detected at the cell surface. Smaller MMP14 degradation products have previously been demonstrated to form multimers with intact, mature MMP14 at the cell surface [39, 62, 79, 81]. This suggests that additional MMP14 cleavages and cell surface localisation are linked, although the sequence of these two events remains unclear. Taken together, our findings suggest that cleavage of the $\mathrm{R}^{89}-\mathrm{R}-\mathrm{P}-\mathrm{R}-\downarrow-\mathrm{C}^{93} \mathrm{PC}$ recognition motif, in addition to the $\mathrm{R}^{108}-\mathrm{R}-\mathrm{Y}-\mathrm{R}-\downarrow_{-}-\mathrm{T}^{112}$ motif, plays an important role in the activation of MMP14.

\section{The hemopexin-like domain has an important role in MMP14 trafficking and functional activity}

As the Hx domain has previously been demonstrated essential for normal activating cleavage of the prodomain, trafficking to the plasma membrane and substrate binding, the p.S466P Cartoon mutation was expected to impair all of these aspects of MMP14 function $[10,11,32,45-52]$. Indeed, our data showed that the p.S466P mutation impaired MMP14 processing and trafficking to the cell surface similar to the p.R92C mutation (Table 2.1). In addition, there was some residual activity regarding to proMMP2 cleavage, which could be explained by the fact that in a small subset of cells $(<5 \%)$ MMP14 S466P-EGFP could be detected at the cell surface. However, gelatin degradation and MMP14-stimulated cell migration were not impaired by the p.S466P mutation. This was surprising, as the Sabe and Cartoon mice are phenotypically identical yet the corresponding mutant proteins behave dramatically different in these assays. As suggested for the T17R mutant, impaired pro-MMP2 activation, yet normal in vitro gelatin degradation could be explained by the amount of MMP14 present at the cell surface. In addition to a dose effect, the p.S466P mutation could directly impair MMP14 homodimer formation, which in turn has been shown to impair pro-MMP2 activation, but not gelatin degradation $[48,50,52,79,80]$. Although Tochowicz et al. have previously shown that blades II and III of the Hx domain are involved in physiological MMP14 homodimerisation, Zarrabi et al. demonstrated that exchange of blade IV (where $\mathrm{S}^{466}$ is located) with that of MMP1 abrogated dimerization [53, 80]. A third explanation could be that the p.S466P mutation alters the substrate specificity of the Hx domain, thereby enhancing gelatin degradation [11]. This putative gain-of-function would also explain how the combination of p.R111H and p.S466P mutations does not impair gelatin degradation, whereas the p.R111H mutation when present alone does. Notably, this remaining functionality is lost when the p.R92C mutation is present, stressing the disrupting effect of the Sabe mutation. A similar gain-of-function could explain the surprisingly normal motility of MMP14 S466P-expressing cells, as the Hx 
domain has been demonstrated to have a role in MMP14-enhanced motility independent of MMP14 catalytic activity [47, 82].

This, however, leaves us with the question of how the Sabe and Cartoon phenotype can be so similar, while the proteins differ in their in vitro activity. The answer to this question may lie in additional functions of MMP14 that were not assessed here. One particular function of relevance is cleavage of fibrillar collagen. It is known that for cleavage of intact collagen fibrils, MMP14 homodimerisation is crucial [48, 49]. If our abovementioned hypothesis is correct, both the Sabe and the Cartoon mutation should impair the collagenolytic activity of MMP14, either by reducing its trafficking to the cell surface (both mutations) or by disrupting Hx-dependent homodimerisation (p.S466P). The soft tissue fibrosis present in both mouse models supports this hypothesis [8 and our unpublished observation].

\section{Retinoid treatment stimulates trafficking of MMP14 S466P to podosome-like structures}

Finally, we demonstrated that MRC-5V1 cells form ventral structures sharing characteristics with podosome rosettes [5, 69]. We showed that the WT and R111H mutant MMP14 fusion proteins, which readily localise to the cell surface, could also be identified in these ventral structures. As 13-cis RA successfully treated acne in our patients, it was hypothesised that retinoid treatment could alter MMP14 processing and/or trafficking to the cell membrane [1]. Although we did not observe any effect on MMP14 processing by either 9-cis RA or 13-cis RA, the incubation period (4h) might have been too short. However, 13-cis RA partially corrected the subcellular localisation of the S466P and R111H S466P fusion proteins, which could be detected in ventral podosome-like rosettes upon retinoid treatment. This suggests that retinoids can stimulate trafficking of MMP14 to podosomes, which could (partially) explain their effect in acne.

\section{Conclusion and future perspectives}

In conclusion, we provide evidence that a mild manifestation of WS is caused by a novel homozygous p.R111H missense mutation in MMP14 that partially impairs the ability of the membrane-bound metalloproteinase to activate secreted pro-MMP2, digest gelatin and stimulate migration in vitro. The residual enzyme activity of MMP14 $\mathrm{R} 111 \mathrm{H}$ observed in our assays is likely to be responsible for the relatively mild disease phenotype evident in our patients. Our observations suggest that MMP14's catalytic activity, in particular, its ability to activate pro-MMP2, may be the most important determinant of disease severity in humans. However, further analysis is required for a full understanding of how different murine Mmp14 mutations lead to an identical phenotype, including assessing collagenolytic capacity and homodimerisation of the respective mutant proteins. Our assays furthermore provided additional insights in the pathogenesis of previously reported MMP14 mutations and normal MMP14 posttrans- 
lational processing. We presented the first evidence of MMP14 SP removal upon ER insertion. In addition, our data suggest that the $\mathrm{R}^{89}-\mathrm{R}-\mathrm{P}-\mathrm{R}-\downarrow-\mathrm{C}^{93}$ proprotein convertase recognition site plays a more important role in pro-MMP14 activation than previously thought. Future N-terminal protein sequencing could provide a definite answer to aberrant processing suggested by our data. Finally, we demonstrated that retinoid treatment influences the trafficking of MMP14, specifically to podosomes. The putative mechanism of retinoid treatment in acne will be assessed in Chapter 3 .

\section{Materials and methods}

\section{Cell culture}

MRC-5V1 immortalised human foetal lung fibroblasts (Prof. Alan Lehmann, University of Sussex, Brighton, UK) and HT-1080 fibrosarcoma cells (Dr. John Eykelenboom, University of Dundee, Dundee, UK) were grown in 2D culture in either high glucose Dulbecco's Modified Eagle Medium (DMEM, GE Healthcare Life Sciences, Pittsburgh, PA; SH3024.01) and mIMCD-3 immortalised murine kidney cells (ATCC; CRL-2123) were grown in low-glucose DMEM (GE; SH30021.01), both containing 10\% $(v / v)$ fetal bovine serum (FBS; GE; A15-101) and $100 \mathrm{U} / \mathrm{mL}$ penicillin / 100 $\mu \mathrm{g} / \mathrm{mL}$ streptomycin (ThermoFischer Scientific, Inc., Waltham, MA, USA; 15140122) at $37{ }^{\circ} \mathrm{C}$ in $100 \%$ humidity and $5 \% \mathrm{CO}_{2}$. Cells were kept growing in log phase and passaged when reaching 70-85\% confluence by detaching cells with $0.25 \%$ TrypsinEDTA (ThermoFischer Scientific, Inc.; 25200-056).

\section{Cloning and mutagenesis}

A series of expression vectors was created encoding 3HA and EGFP double-tagged versions of either WT or mutant human MMP14. The MMP14 W'T vector was generated by amplifying MMP14 (RefSeq NP_004986.1) from total cDNA obtained from MRC-5V1 cells (see Table 2.2 for primers used) using KOD high fidelity polymerase (Merck) and cloning the amplicon into the pJET3.1 vector (ThermoFisher Scientific, Inc.). MMP14 WT cDNA was cloned into the pQCXIB (w297-1, Addgene plasmid \#22800) backbone (a gift from Dr. Eric Campeau) with the addition of 3HA and EGFP tags in subsequent cloning steps. The WS p.R111H mutation was induced by site-directed mutagenesis (SDM) of Codon 111 of the MMP14 cDNA sequence in the WT vector from CGC into CAC using partially overlapping primers following the protocol of Werler et al. [83]. Likewise, the Cartoon p.S466P mutation was induced by SDM of Codon 466 from TCA into CCA. The WS p.T17R mutation was induced by SDM of Codon 17 from ACG into AGG with fully overlapping primers (see Supplementary Material, Table S1). Likewise, the Sabe p.R92C mutant vector was created by mutating Codon 92 from CGA into TGC with fully overlapping primers. The 3HA- 
EGFP control vector was generated by deletion of the MMP14 coding sequence from the WT vector by inverse-PCR with fully overlapping primers binding to $24 \mathrm{bp}$ of each linker sequence. The entire insert of mutant plasmids with correct band size was sequenced with primers \#1-6, control plasmid was sequenced with primers \#7-8 (AITbiotech, Singapore, Singapore); see Table 2.3. The wild type c-Src vector pcDNA3 cSrc, the mutant c-Src vector pcSrc527, and the control vector pMSCV-GFP were obtained via Addgene (Addgene; 42202, 17675 and 86537, respectively) [72, 84, 85].

\section{Retinoid treatment}

Seventy-two hours post seeding, cells were incubated with 0-8 nM 13-cis retinoic acid (RA) (Sigma-Aldrich, St. Louis, MO, USA; R3255) or $8 \mathrm{nM}$ 9-cis RA (Sigma; R4643) in 1.2\% $(v / v)$ ethanol (Merck, Darmstadt, Germany; 1.00983.2500) or left untreated for 4 hours at $37{ }^{\circ} \mathrm{C}$ in the dark, after which cells were fixed or harvested for IF or WB experiments, respectively.

\section{Immunofluorescence microscopy}

Cells were seeded on No. 1.5H microscope cover glasses (Paul Marienfeld GmbH \& Co. KG, Lauda-Königshofen, Germany; 0117580) in 12-well cell culture plates (Corning, Inc., Corning, NY, USA; 353043) at a density of 50,000 cells per well unless stated otherwise. Cells were transfected with $1.2 \mathrm{mg}$ vector DNA using jetPRIME®

Table 2.2 - Primers used for cloning and mutagenesis of pQCXIB 3HA-MMP14-EGFP vectors. Fw, forward; Rv, reverse; SDM, site-directed mutagenesis. The altered codon is underlined.

\section{MMP14WT cDNA \\ Fw 5'-TGAATTCCAATGTCTCCCGCCCCAAGA-3' \\ Rv 5'-AGATCTTGACCTTGTCCAGCAGGGAACGC-3'}

\section{SDM MMP14 T17R}

Fw 5'-CGTTGTCTCCTGCTCCCCCTGCTCAGGCTCGGCACCGCGCTCGCCTCCCTC-3';

Rv 5'-GAGGGAGGCGAGCGCGGTGCCGAGCCTGAGCAGGGGGAGCAGGAGACAACG-3'

\section{SDM MMP14 R92C}

Fw 5'-CCATGAGGCGCCCCTGCTGTGGTGTTCCAG-3'

Rv 5'- CTGGAACACCACAECAGGGGCGCCTCATGG-3'

\section{SDM MMP14 R111H}

Fw 5'-AATGTTCGAAGGAAGCACTACGCCATCCAGGGTCTCAAATGG-3'

Rv 5-ACCCTGGATGGCGTAGTGCTTCCTTCGAACATTGGCCTTGAT-3’

\section{SMD MMP14 S466P}

Fw 5'-GAGTCTCCCAGAGGGCCATTCATGGGCAGCGATGAAGTCT-3'

Rv 5'-CGCTGCCCATGAATGGCCCTCTGGGAGACTCAGGGATCCC-3'

\section{Generation of 3HA-EGFP vector}

Fw 5'-CTCGAGCTCAAGCT'TCGAAT'TCCAAAGATCCACCGGCCGGTAGCCACC-3'

Rv 5'-GGTGGCTACCGGCCGGTGGATCT'TTGGAATTCGAAGCTTGAGCTCGAG-3' 
(Polyplus transfection ${ }^{\circledR}$, VR SA, Illkirch, France; 114-15) 24h post-seeding and media refreshed $4 \mathrm{~h}$ post-transfection. Non-transfected cells were seeded $18 \mathrm{~h}$ prior to fixation. Cells were fixed with 4\% paraformaldehyde (PFA) in PBS 1x (Santa Cruz Biotechnology, Inc., Dallas, TX, USA; sc-281692), permeabilized with $0.2 \%$ Triton ${ }^{\circledR} \mathrm{X}$ 100 (Promega, Madison, WI, USA; H5141) in PBS 1x (GE Healthcare Life Sciences; SH30028.02) and blocked with Image-iT® FX Signal Enhancer (ThermoFisher Scientific, Inc.; R37107). Cells were incubated with primary antibodies (see Table 2.4 for antibody details and dilutions) overnight at $4{ }^{\circ} \mathrm{C}$, and with Alexa-fluorophore labeled secondary antibodies (1:200 unless stated otherwise) and phalloidin (1:100) (ThermoFisher Scientific, Inc.) for $1 \mathrm{~h}$ at RT, all dissolved in 3\% $(w / v)$ bovine serum albumin (BSA; MP Biomedicals, Santa Ana, CA, USA; 0219989880) in PBS 1x. Cells were mounted on microscope glasses (Biomedia, Singapore, Singapore; BMH.880102) using Vectashield antifade mounting medium containing 4',6-diamidino-2-phenylindole (Vector Laboratories, Burlingame, CA, USA; H-1200). Z-stacks of cells were recorded with an Olympus IX81 microscope with FV1000 scan head (Olympus Corporation, Tokyo, Japan). Images of randomly selected cells with average signal intensity were taken with a 63X 1.45 NA or 40X 1.30 NA oil immersion Plan Apochromat objective (Olympus), with fixed laser power and exposure time per experiment. The investigator taking and analyzing images was blinded for the transfection of the cells. Raw images were processed with Fiji software (U.S. National Institutes of Health, Bethesda, MD, USA; ImageJ version 2.0.0-rc-15/1.49k, adapted by IMU).

\section{Western blotting}

For endogenous MMP14 and analysis of double-tagged MMP14, cells were seeded at a density of 100,000 cells per well of a six-well plate. Cells were either transfected with pQCXIB vectors as described above or left non-transfected. Cells were harvested with trypsin-EDTA and whole cell lysate (WCL) was obtained using NP-40 lysis buffer (150 mM NaCl, 1\% NP-40, $250 \mathrm{mM}$ Tris $\mathrm{pH}$ 7.3) supplemented with protease and

Table 2.3 - Primers for sequencing of pQCXIB 3HA-MMP14-EGFP plasmids. The position of primers is given relative to the first base of the vector. Fw, forward; No, number; Or, orientation; Rv, reverse.

\begin{tabular}{lllll}
\hline No. & Or. & \multicolumn{2}{l}{ Sequence } & Position \\
\cline { 1 - 1 } & Fw & 5'-GAATTCCAATGTCTCCCGCCCCAAGACCCT-3' & c.6,468-6,597 \\
2 & Rv & 5'-AGTGGTGTGGCACTCTCCCA-3' & c.7,045-7,026 \\
3 & Fw & 5'-GAGGTGCCCTATGCCTACAT-3' & c.7,056-7,075 \\
4 & Fw & 5'-AACCGGGCTACCCCAAGTCA-3' & c.8,056-8,075 \\
5 & Fw & 5'-AGCGTTCCCTGCTGGACAAGGTCAAGATCC-3' & c.8,299-8,328 \\
6 & Rv & 5'-ACTTGTGGCCGTTTACGTC-3' & c.8,427-8,409 \\
7 & Fw & 5'-CATTGGAACGCTGCAGGAAT-3' & c.6,398-6,417 \\
8 & Rv & 5'-TAGGTCAGGGTGGTCACGAG-3' & c.6,799-6,780 \\
\hline
\end{tabular}


phosphatase inhibitors (Roche). Protein concentration was determined by Bradford protein assay (Sigma). An appropriate volume of Laemmli sample buffer was added to equal amounts of total protein lysate followed by boiling for $5 \mathrm{~min}$. Samples were subjected to SDS-PAGE before transfer to an Amersham Hybond ${ }^{\text {TM}}$-PVDF membrane (GE Healthcare Life Sciences, Buckinghamshire, UK). Membranes were blocked in 5\% $(w / v)$ milk (Marvel dried skimmed milk) in $0.1 \%(v / v)$ Tween-20 (Melford; P1362) in TBS 1x (Sigma; T5912-1L). After overnight incubation at $4{ }^{\circ} \mathrm{C}$ in primary antibody (Table 2.4) appropriately diluted in 0.5\% BSA in TBS block buffer, membranes were incubated with secondary IRDye ${ }^{\circledR}$ 680RD donkey anti-rabbit (LICOR Biosciences, Lincoln, Nebraska, USA; 926-68073) and Alexa fluor 680 donkey anti-mouse (ThermoFischer Scientific, Inc.; A10038) antibody appropriately diluted in block buffer. Blots were imaged on the Odyssey® Fc Imaging System (LI-COR).

To compare the processing of MMP14 upon retinoid treatment, cells were seeded at a density of 440,000 per $6 \mathrm{~cm}$ dish. Cells were harvested and WCL obtained by scraping in $4 \%(w / v)$ SDS in $\mathrm{dH}_{2} \mathrm{O}$. Protein concentration was determined by BCA kit (ThermoFischer Scientific, Inc.; 23225). Samples were subjected to SDS-PAGE before transfer to a PVDF membrane (ThermoFischer Scientific, Inc.; 88518). Membranes were blocked in $4 \%(w / v)$ milk/0.1\% $(v / v)$ Tween-20 in TBS $1 \mathrm{x}$. After overnight incubation at $4{ }^{\circ} \mathrm{C}$ in primary antibody (Table 2.4 ) appropriately diluted in $4 \%(w / v)$ milk in TBS block buffer, membranes were incubated with horseradish peroxidase (HRP)conjugated secondary antibodies (1:3,000 dilution; Thermo; 31450 and 31460) and Streptactin (1:10,000 dilution; Biorad; 161-0380) in 4\% $(w / v)$ milk in TBS block buffer.

Table 2.4 - Antibodies used. GFP, green fluorescent protein; HA, influenza hemagglutinin epitope (YPYDVPDYA); IF, indirect immunofluorescence microscopy; MMP14, matrix metalloproteinase 14; Ms, mouse; Rb, rabbit; WB, Western blot.

\begin{tabular}{|c|c|c|c|c|c|c|}
\hline \multirow[b]{2}{*}{ Raised against } & \multirow{2}{*}{$\begin{array}{l}\text { Raised } \\
\text { in }\end{array}$} & \multirow[b]{2}{*}{ Clonality } & \multirow[b]{2}{*}{ Manufacturer } & \multirow{2}{*}{$\begin{array}{l}\text { Product } \\
\text { code }\end{array}$} & \multicolumn{2}{|c|}{ Concentration } \\
\hline & & & & & IF & WB \\
\hline$\beta$-actin & Ms & Mono & Thermo & MA1-744 & - & $1: 4,000$ \\
\hline Cortactin & Ms & Mono & Merck & $05-180$ & $1: 200$ & - \\
\hline GFP & Ms & Duo & Roche & 11814460001 & & $1: 2,000$ \\
\hline Golgin-97 & Ms & Mono & Thermo & $\mathrm{A} 21270$ & $1: 1,000$ & - \\
\hline HA & Ms & Mono & Abcam & Ab16918 & $1: 200$ & - \\
\hline HA & $\mathrm{Rb}$ & Mono & Cell Signaling & 3724 & $1: 1,600$ & $1: 1,000$ \\
\hline KU-80 & $\mathrm{Rb}$ & Mono & Cell Signaling & 2180 & - & $1: 1,000$ \\
\hline MMP14 & $\mathrm{Rb}$ & Mono & Abcam & Ab51074 & $1: 100$ & $1: 1,000$ \\
\hline MMP14 AA 299-314 & $\mathrm{Rb}$ & Poly & Eurogentec & SY5336 & $1: 100$ & $1: 64,000$ \\
\hline MMP14 AA 218-233 & Ms & Mono & Merck & mab3328 & $1: 100$ & $1: 1,000$ \\
\hline SERCA2A & Ms & Mono & Abcam & Ab2861 & $1: 40,000$ & - \\
\hline SH3PXD2B (Hof1) & $\mathrm{Rb}$ & Poly & Millipore & & $1: 200$ & - \\
\hline Src & $\mathrm{Rb}$ & Poly & Abcam & Ab47405 & $1: 200$ & - \\
\hline Vimentin & $\mathrm{Rb}$ & Poly & Abcam & Ab45939 & $1: 50$ & - \\
\hline Vimentin & $\mathrm{Rb}$ & Mono & Abcam & Ab133260 & $1: 250$ & - \\
\hline
\end{tabular}


Protein bands were visualised by ECL Western Blotting substrate mix (Thermo; 32106) on BioRad Chemidoc ${ }^{\mathrm{TM}}$.

\section{Zymography}

Gelatin zymography was performed as previously described [86]. Briefly, MRC-5V1 cells were transfected with the aforementioned PQCXIB vectors. Culture medium was changed to FBS-free DMEM $24 \mathrm{~h}$ post-transfection to allow conditioning for $24 \mathrm{~h}$, after which conditioned medium was harvested and mixed with equal volumes of $2 \mathrm{x}$ sample buffer [0.125 M Tris-HCL, pH 6.8, 20\% (v/v) glycerol, 4\% (w/v) SDS, 0.005\% $(w / v)$ bromophenol blue (Sigma; B-6131)]. Samples were subjected to SDS-PAGE in $10 \%$ resolving gel containing $0.1 \%$ gelatin. After electrophoresis, proteins were renatured in freshly made 1x Zymogram Renaturing Buffer (Triton ${ }^{\circledR}$ X-100 2.5\% $(v / v)$ in $\mathrm{ddH}_{2} \mathrm{O}$ ) for $30 \mathrm{~min}$ at RT. The gel was subsequently incubated in 1x Zymogram Developing Buffer (0.01 M Tris-HCL pH 7.5 (Sigma; 10812846001), 1.25\% (v/v) Triton $\mathrm{X}-100,5 \mathrm{mM} \mathrm{CaCl}_{2}$ (Kanto Chemical Co., 07058-00)) for $30 \mathrm{~min}$ at RT to allow equilibration. Developing buffer was refreshed and the gel was incubated at $37{ }^{\circ} \mathrm{C}$ overnight. Finally, the gel was stained with Coomassie Blue R-250 (ThermoFischer Scientific, Inc.; 20278) $0.5 \%(w / v)$ for $30 \mathrm{~min}$, and subsequently washed in destaining solution (water:methanol:acetic acid at 5:4:1) for four times $15 \mathrm{~min}$. The gel was imaged with the Bio-Rad Image Lab system and the image analyzed in Fiji software.

\section{Gelatin digestion assay}

The QCMTM Gelatin Invadopodia Assay system (Merck Millipore, Billerica, MA, USA; ECM671) was used according to the manufacturer's protocol. In brief, a four-chamber glass bottom dish (Cellvis, Mountain View, CA, USA; D35C4-20-1.5-N) was coated with poly-L-Lysine, glutaraldehyde and Cy3-labeled gelatin in sequence. The coated dish was sterilized with $70 \%$ ethanol for $30 \mathrm{~min}$, followed by DMEM quenching for $30 \mathrm{~min}$ at RT. MRC-5V1 cells were transfected with the appropriate pQCXIB vectors $24 \mathrm{~h}$ prior to seeding onto the coated chamber dish. The ability of the transfected cells to digest the Cy3-labeled gelatin was determined at $3 \mathrm{~h}$ and $20 \mathrm{~h}$ post-seeding using an EVOS fluorescence microscope (Thermo; AMF4300). Average degradation per green fluorescent cell (degradation factor, d) was calculated by automated image analysis in Fiji and assessed for statistical difference by a two-sided student's T-test in Microsoft ${ }^{\circledR}$ Excel ${ }^{\circledR}$ (Microsoft Corporation, Redmond, WA, USA; version 14.6.6 (160626)). To quantify the number of transfected cells $(N)$ in a given field, a find-object macro was created in Fiji. The threshold was manually adjusted to cover the entire degradation area (A) that was measured. The degradation factor of each MMP14 mutant was defined as $d=A / N$. Three independent experiments $(N \geq 103)$ were carried out. For qualitative analysis, fluorescent cells were identified $2 \mathrm{~h}$ post-seeding and $\mathrm{z}$-stacks recorded ever 15 min for $5 \mathrm{~h}$ with a spinning disk (Yokogawa, Tokyo, Japan; CSU-22) 
confocal microscope (Nikon, Tokyo, Japan; Nikon Ti inverted) equipped with the Nikon Perfect Focus System. A maximum intensity projection was performed in MetaMorph 7.8.8.0 software (Molecular Devices, Sunnyvale, CA, USA) at the end of the experiment.

\section{Phagokinetic assay}

Four-chamber glass bottom dishes were coated with $1 \mu \mathrm{g} / \mathrm{ml}$ Rhodamine Fibronectin (Cytoskeleton, Denver, CO, USA; FNR01) according to the manufacturer's protocol. MRC-5V1 cells were transfected with the aforementioned $\mathrm{pQCXIB}$ vectors $24 \mathrm{~h}$ prior to seeding onto the coated chamber dish. Migration of the transfected cells was recorded by time-lapse imaging starting $2 \mathrm{~h}$ post-seeding with a spinning disk confocal microscope. A z-stack consisting of 16 steps with an interval of $1 \mu \mathrm{m}$ between successive steps of each field was acquired and a maximum intensity projection was performed in MetaMorph software. Time-lapse images were then subjected to customize cell-tracking analysis in Imaris Image Analysis Software 8.4.1 (Bitplane, Belfast, UK).

\section{Supplemental material}

Supplemental material is available in Addendum 1.

\section{Acknowledgements}

The authors acknowledge the invaluable assistance of Dr. Graham Wright, Dr. Jaron Liu, Mr. John Soon Yew Lim and Dr. Shuping Lin (Institute of Medical Biology, Microscopy Unit). This work was supported by the Skin Research Institute of Singapore (to M.A.M.v.S. and E.Y.T.), the Biomedical Research Council Singapore (to M.A.M.v.S.), the Agency for Science, Technology and Research (A*STAR Research Attachment Programme to I.J.H.M.d.V.), the Wellcome Trust (DGEM to M.A.M.v.S. and B.J.C.), Tenovus Scotland (T15/22 and T15/62 to M.A.M.v.S. and B.J.C.), the Victorian Government's Operational Infrastructure Support Program, and the Australian Government (NHMRC Senior Research Fellowship 110297 and NHMRC Program Grant 1054618 to M.B., NHMRC Career Development Fellowship GNT1032364 to P.J.L.).

\section{References}

1. Van Steensel MA, Ceulen RP, Delhaas T, de Die-Smulders C. Two Dutch brothers with Borrone dermato-cardio-skeletal syndrome. American Journal of Medical Genetics Part A. 2007;143a(11):1223-6.

2. Borrone C, Di Rocco M, Crovato F, Camera G, Gambini C. New multisystemic disorder involving heart valves, skin, bones, and joints in two brothers. American Journal of Medical Genetics. 1993;46(2):228-34. 
3. Wilson GR, Sunley J, Smith KR, Pope K, Bromhead CJ, Fitzpatrick E, et al. Mutations in SH3PXD2B cause Borrone dermato-cardio-skeletal syndrome. European Journal of Human Genetics. 2014;22(6):741-7.

4. Sato H, Takino T, Okada Y, Cao J, Shinagawa A, Yamamoto E, et al. A matrix metalloproteinase expressed on the surface of invasive tumour cells. Nature. 1994;370(6484):61-5.

5. Murphy DA, Courtneidge SA. The 'ins' and 'outs' of podosomes and invadopodia: characteristics, formation and function. Nature reviews Molecular Cell Biology. 2011;12(7):413-26.

6. Evans BR, Mosig RA, Lobl M, Martignetti CR, Camacho C, Grum-Tokars V, et al. Mutation of membrane type-1 metalloproteinase, MT1-MMP, causes the multicentric osteolysis and arthritis disease Winchester syndrome. American Journal of Human Genetics. 2012;91(3):572-6.

7. Winchester P, Grossman H, Lim WN, Danes BS. A new acid mucopolysaccharidosis with skeletal deformities simulating rheumatoid arthritis. The American Journal of Roentgenology, Radium Therapy, and Nuclear Medicine. 1969;106(1):121-8.

8. Curtain MM; Donahue LR. 2007. A possible new mutation to Mmp 14 MGI Direct Data Submission. MGI: J:127164. Updated Nov 2012. The Jackson Laboratory. URL: informatics.jax.org/downloads/Reference_texts/J127164.pdf.

9. Du X, Moresco EMY, Murray A, Beutler B. record for cartoon, updated Dec 12, 2013. MUTAGENETIX TM, B. Beutler and colleagues, center for the Genetics of Host Defense, UT Southwestern Medical Center, Dallas, TX. URL: mutagenetix. utsouthwestern.edu.

10. Massova I, Kotra LP, Fridman R, Mobashery S. Matrix metalloproteinases: structures, evolution, and diversification. FASEB Journal. 1998;12(12):1075-95.

11. Sternlicht MD, Werb Z. How matrix metalloproteinases regulate cell behavior. Annual Review of Cell and Developmental Biology. 2001;17:463-516.

12. Remacle AG, Rozanov DV, Fugere M, Day R, Strongin AY. Furin regulates the intracellular activation and the uptake rate of cell surface-associated MT1-MMP. Oncogene. 2006;25(41):5648-55.

13. Poincloux R, Lizarraga F, Chavrier P. Matrix invasion by tumour cells: a focus on MT1-MMP trafficking to invadopodia. Journal of Cell Science. 2009;122(Pt 17):3015-24.

14. Remacle AG, Rozanov DV, Baciu PC, Chekanov AV, Golubkov VS, Strongin AY. The transmembrane domain is essential for the microtubular trafficking of membrane type-1 matrix metalloproteinase (MT1-MMP). Journal of Cell Science. 2005;118(Pt 21):4975-84.

15. Williams BB, Cantrell VA, Mundell NA, Bennett AC, Quick RE, Jessen JR. VANGL2 regulates membrane trafficking of MMP14 to control cell polarity and migration. Journal of Cell Science. 2012;125(Pt 9):2141-7.

16. Pietraszek K, Chatron-Colliet A, Brezillon S, Perreau C, Jakubiak-Augustyn A, Krotkiewski H, et al. Lumican: a new inhibitor of matrix metalloproteinase-14 activity. FEBS letters. 2014;588(23):4319-24.

17. Koziol A, Martin-Alonso M, Clemente C, Gonzalo P, Arroyo AG. Site-specific cellular functions of MT1-MMP. European Journal of Cell Biology. 2012;91(11-12):889-95.

18. Takino T, Sato H, Yamamoto E, Seiki M. Cloning of a human gene potentially encoding a novel matrix metalloproteinase having a C-terminal transmembrane domain. Gene. 1995;155(2):293-8.

19. Maskos K. Crystal structures of MMPs in complex with physiological and pharmacological inhibitors. Biochimie. 2005;87(3-4):249-63.

20. Das S, Mandal M, Chakraborti T, Mandal A, Chakraborti S. Structure and evolutionary aspects of matrix metalloproteinases: a brief overview. Molecular and Cellular Biochemistry. 2003;253(1-2):3140 .

21. Saus J, Quinones S, Otani Y, Nagase H, Harris ED, Jr., Kurkinen M. The complete primary structure of human matrix metalloproteinase-3. Identity with stromelysin. The Journal of Biological Chemistry. 1988;263(14):6742-5.

22. Rehm A, Stern P, Ploegh HL, Tortorella D. Signal peptide cleavage of a type I membrane protein, HCMV US11, is dependent on its membrane anchor. The EMBO journal. 2001;20(7):1573-82. 
23. von Heijne G, Gavel Y. Topogenic signals in integral membrane proteins. European Journal of Biochemistry. 1988;174(4):671-8.

24. Kyte J, Doolittle RF. A simple method for displaying the hydropathic character of a protein. Journal of Molecular Biology. 1982;157(1):105-32.

25. Petersen TN, Brunak S, von Heijne G, Nielsen H. SignalP 4.0: discriminating signal peptides from transmembrane regions. Nature Methods. 2011;8(10):785-6.

26. von Heijne G. Patterns of amino acids near signal-sequence cleavage sites. European Journal of Biochemistry. 1983;133(1):17-21.

27. Blobel G, Dobberstein B. Transfer of proteins across membranes. I. Presence of proteolytically processed and unprocessed nascent immunoglobulin light chains on membrane-bound ribosomes of murine myeloma. The Journal of Cell Biology. 1975;67(3):835-51.

28. Sanchez-Lopez R, Nicholson R, Gesnel MC, Matrisian LM, Breathnach R. Structure-function relationships in the collagenase family member transin. The Journal of Biological Chemistry. 1988;263(24):11892-9.

29. Springman EB, Angleton EL, Birkedal-Hansen H, Van Wart HE. Multiple modes of activation of latent human fibroblast collagenase: evidence for the role of a Cys73 active-site zinc complex in latency and a "cysteine switch" mechanism for activation. Proceedings of the National Academy of Sciences of the United States of America. 1990;87(1):364-8.

30. Birkedal-Hansen H, Moore WG, Bodden MK, Windsor LJ, Birkedal-Hansen B, DeCarlo A, et al. Matrix metalloproteinases: a review. Critical Reviews in Oral Biology and Medicine. 1993;4(2):197250 .

31. Van Wart HE, Birkedal-Hansen H. The cysteine switch: a principle of regulation of metalloproteinase activity with potential applicability to the entire matrix metalloproteinase gene family. Proceedings of the National Academy of Sciences of the United States of America. 1990;87(14):5578-82.

32. Page-McCaw A, Ewald AJ, Werb Z. Matrix metalloproteinases and the regulation of tissue remodelling. Nature Reviews Molecular Cell Biology. 2007;8(3):221-33.

33. Golubkov VS, Chekanov AV, Shiryaev SA, Aleshin AE, Ratnikov BI, Gawlik K, et al. Proteolysis of the membrane type-1 matrix metalloproteinase prodomain: implications for a two-step proteolytic processing and activation. The Journal of Biological Chemistry. 2007;282(50):36283-91.

34. Golubkov VS, Cieplak P, Chekanov AV, Ratnikov BI, Aleshin AE, Golubkova NV, et al. Internal cleavages of the autoinhibitory prodomain are required for membrane type 1 matrix metalloproteinase activation, although furin cleavage alone generates inactive proteinase. The Journal of Biological Chemistry. 2010;285(36):27726-36.

35. Golubkov VS, Chernov AV, Strongin AY. Intradomain cleavage of inhibitory prodomain is essential to protumorigenic function of membrane type-1 matrix metalloproteinase (MT1-MMP) in vivo. The Journal of Biological Chemistry. 2011;286(39):34215-23.

36. Hosaka M, Nagahama M, Kim WS, Watanabe T, Hatsuzawa K, Ikemizu J, et al. Arg-X-Lys/Arg-Arg motif as a signal for precursor cleavage catalyzed by furin within the constitutive secretory pathway. The Journal of Biological Chemistry. 1991;266(19):12127-30.

37. Pei D, Weiss SJ. Furin-dependent intracellular activation of the human stromelysin-3 zymogen. Nature. 1995;375(6528):244-7.

38. Yana I, Weiss SJ. Regulation of membrane type-1 matrix metalloproteinase activation by proprotein convertases. Molecular Biology of the Cell. 2000;11(7):2387-401.

39. Rozanov DV, Deryugina EI, Ratnikov BI, Monosov EZ, Marchenko GN, Quigley JP, et al. Mutation analysis of membrane type-1 matrix metalloproteinase (MT1-MMP). The role of the cytoplasmic tail Cys(574), the active site Glu(240), and furin cleavage motifs in oligomerization, processing, and selfproteolysis of MT1-MMP expressed in breast carcinoma cells. The Journal of Biological Chemistry. 2001;276(28):25705-14. 
40. Seidah NG, Mayer G, Zaid A, Rousselet E, Nassoury N, Poirier S, et al. The activation and physiological functions of the proprotein convertases. The International Journal of Biochemistry \& Cell Biology. 2008;40(6-7):1111-25.

41. Sato H, Kinoshita T, Takino T, Nakayama K, Seiki M. Activation of a recombinant membrane type 1-matrix metalloproteinase (MT1-MMP) by furin and its interaction with tissue inhibitor of metalloproteinases (TIMP)-2. FEBS Letters. 1996;393(1):101-4.

42. Imai K, Ohuchi E, Aoki T, Nomura H, Fujii Y, Sato H, et al. Membrane-type matrix metalloproteinase 1 is a gelatinolytic enzyme and is secreted in a complex with tissue inhibitor of metalloproteinases 2. Cancer Research. 1996;56(12):2707-10.

43. Itoh Y, Seiki M. MT1-MMP: a potent modifier of pericellular microenvironment. Journal of Cellular Physiology. 2006;206(1):1-8.

44. Matrisian LM, Leroy P, Ruhlmann C, Gesnel MC, Breathnach R. Isolation of the oncogene and epidermal growth factor-induced transin gene: complex control in rat fibroblasts. Molecular and Cellular Biology. 1986;6(5):1679-86.

45. Mori H, Tomari T, Koshikawa N, Kajita M, Itoh Y, Sato H, et al. CD44 directs membrane-type 1 matrix metalloproteinase to lamellipodia by associating with its hemopexin-like domain. The EMBO Journal. 2002;21(15):3949-59.

46. Remacle AG, Golubkov VS, Shiryaev SA, Dahl R, Stebbins JL, Chernov AV, et al. Novel MT1-MMP small-molecule inhibitors based on insights into hemopexin domain function in tumor growth. Cancer Research. 2012;72(9):2339-49.

47. Cao J, Kozarekar P, Pavlaki M, Chiarelli C, Bahou WF, Zucker S. Distinct roles for the catalytic and hemopexin domains of membrane type 1-matrix metalloproteinase in substrate degradation and cell migration. The Journal of Biological Chemistry. 2004;279(14):14129-39.

48. Itoh Y, Ito N, Nagase H, Seiki M. The second dimer interface of MT1-MMP, the transmembrane domain, is essential for ProMMP-2 activation on the cell surface. The Journal of Biological Chemistry. 2008;283(19):13053-62.

49. Itoh Y, Ito N, Nagase H, Evans RD, Bird SA, Seiki M. Cell surface collagenolysis requires homodimerization of the membrane-bound collagenase MT1-MMP. Molecular Biology of the Cell. 2006;17(12):5390-9.

50. Itoh Y, Takamura A, Ito N, Maru Y, Sato H, Suenaga N, et al. Homophilic complex formation of MT1-MMP facilitates proMMP-2 activation on the cell surface and promotes tumor cell invasion. The EMBO Journal. 2001;20(17):4782-93.

51. McKleroy W, Lee TH, Atabai K. Always cleave up your mess: targeting collagen degradation to treat tissue fibrosis. American Journal of Physiology Lung Cellular and Molecular Physiology. 2013;304(11):L709-21.

52. Atkinson SJ, Roghi C, Murphy G. MT1-MMP hemopexin domain exchange with MT4-MMP blocks enzyme maturation and trafficking to the plasma membrane in MCF7 cells. The Biochemical Journal. 2006;398(1):15-22.

53. Zarrabi K, Dufour A, Li J, Kuscu C, Pulkoski-Gross A, Zhi J, et al. Inhibition of matrix metalloproteinase 14 (MMP-14)-mediated cancer cell migration. The Journal of Biological Chemistry. 2011;286(38):33167-77.

54. Sato T, del Carmen Ovejero M, Hou P, Heegaard AM, Kumegawa M, Foged NT, et al. Identification of the membrane-type matrix metalloproteinase MT1-MMP in osteoclasts. Journal of Cell Science. 1997;110 ( Pt 5):589-96.

55. Jacobs JP, Jones CM, Baille JP. Characteristics of a human diploid cell designated MRC-5. Nature. 1970;227(5254):168-70.

56. Huschtscha LI, Holliday R. Limited and unlimited growth of SV40-transformed cells from human diploid MRC-5 fibroblasts. Journal of Cell Science. 1983;63:77-99. 
57. Sidwell RU, Brueton LA, Grabczynska SA, Francis N, Staughton RC. Progressive multilayered banded skin in Winchester syndrome. Journal of the American Academy of Dermatology. 2004;50(2 Suppl):S53-6.

58. Gutierrez-Fernandez A, Soria-Valles C, Osorio FG, Gutierrez-Abril J, Garabaya C, Aguirre A, et al. Loss of MT1-MMP causes cell senescence and nuclear defects which can be reversed by retinoic acid. The EMBO Journal. 2015;34(14):1875-88.

59. Okada A, Bellocq JP, Rouyer N, Chenard MP, Rio MC, Chambon P, et al. Membrane-type matrix metalloproteinase (MT-MMP) gene is expressed in stromal cells of human colon, breast, and head and neck carcinomas. Proceedings of the National Academy of Sciences of the United States of America. 1995;92(7):2730-4.

60. Remacle AG, Shiryaev SA, Golubkov VS, Freskos JN, Brown MA, Karwa AS, et al. Non-destructive and selective imaging of the functionally active, pro-invasive membrane type-1 matrix metalloproteinase (MT1-MMP) enzyme in cancer cells. The Journal of Biological Chemistry. 2013;288(28):20568-80.

61. Bordoli MR, Yum J, Breitkopf SB, Thon JN, Italiano JE, Jr., Xiao J, et al. A secreted tyrosine kinase acts in the extracellular environment. Cell. 2014;158(5):1033-44.

62. Osenkowski P, Toth M, Fridman R. Processing, shedding, and endocytosis of membrane type 1matrix metalloproteinase (MT1-MMP). Journal of Cellular Physiology. 2004;200(1):2-10.

63. de Curtis I, Meldolesi J. Cell surface dynamics - how Rho GTPases orchestrate the interplay between the plasma membrane and the cortical cytoskeleton. Journal of Cell Science. 2012;125(Pt 19):4435-44.

64. Laurila P, Virtanen I, Lehto VP, Vartio T, Stenman S. Expression and distribution of vimentin and keratin filaments in heterokaryons of human fibroblasts and amnion epithelial cells. The Journal of Cell Biology. 1982;94(2):308-15.

65. Rauchman MI, Nigam SK, Delpire E, Gullans SR. An osmotically tolerant inner medullary collecting duct cell line from an SV40 transgenic mouse. The American Journal of Physiology. 1993;265(3 Pt 2):F416-24.

66. Buschman MD, Bromann PA, Cejudo-Martin P, Wen F, Pass I, Courtneidge SA. The novel adaptor protein Tks4 (SH3PXD2B) is required for functional podosome formation. Molecular Biology of the Cell. 2009;20(5):1302-11.

67. Williams KC, McNeilly RE, Coppolino MG. SNAP23, Syntaxin4, and vesicle-associated membrane protein 7 (VAMP7) mediate trafficking of membrane type 1-matrix metalloproteinase (MT1-MMP) during invadopodium formation and tumor cell invasion. Molecular Biology of the Cell. 2014;25(13):2061-70.

68. Linder S, Kopp P. Podosomes at a glance. Journal of Cell Science. 2005;118(Pt 10):2079-82.

69. Seano G, Chiaverina G, Gagliardi PA, di Blasio L, Puliafito A, Bouvard C, et al. Endothelial podosome rosettes regulate vascular branching in tumour angiogenesis. Nature Cell Biology. 2014;16(10):931-41, 1-8.

70. Hoshino D, Branch KM, Weaver AM. Signaling inputs to invadopodia and podosomes. Journal of Cell Science. 2013;126(Pt 14):2979-89.

71. Okada M, Nada S, Yamanashi Y, Yamamoto T, Nakagawa H. CSK: a protein-tyrosine kinase involved in regulation of stc family kinases. The Journal of Biological Chemistry. 1991;266(36):2424952.

72. Kmiecik TE, Shalloway D. Activation and suppression of pp60c-src transforming ability by mutation of its primary sites of tyrosine phosphorylation. Cell. 1987;49(1):65-73.

73. Dawson AL, Dellavalle RP. Acne vulgaris. BMJ. 2013;346:f2634.

74. Verfaille CJ, Borgers M, van Steensel MA. Retinoic acid metabolism blocking agents (RAMBAs): a new paradigm in the treatment of hyperkeratotic disorders. Journal der Deutschen Dermatologischen Gesellschaft. 2008;6(5):355-64.

75. Ganceviciene R, Zouboulis CC. Isotretinoin: state of the art treatment for acne vulgaris. Journal der Deutschen Dermatologischen Gesellschaft. 2010;8 Suppl 1:S47-59. 
76. Fernandez-Calotti P, Pastor-Anglada M. All-trans-retinoic acid promotes trafficking of human concentrative nucleoside transporter-3 (hCNT3) to the plasma membrane by a TGF-beta1-mediated mechanism. The Journal of Biological Chemistry. 2010;285(18):13589-98.

77. Kang JX, Bell J, Leaf A, Beard RL, Chandraratna RA. Retinoic acid alters the intracellular trafficking of the mannose-6-phosphate/insulin-like growth factor II receptor and lysosomal enzymes. Proceedings of the National Academy of Sciences of the United States of America. 1998;95(23):13687-91.

78. Ott F, Bollag W, Geiger JM. Oral 9-cis-retinoic acid versus 13-cis-retinoic acid in acne therapy. Dermatology. 1996;193(2):124-6.

79. Lehti K, Lohi J, Juntunen MM, Pei D, Keski-Oja J. Oligomerization through hemopexin and cytoplasmic domains regulates the activity and turnover of membrane-type 1 matrix metalloproteinase. The Journal of Biological Chemistry. 2002;277(10):8440-8.

80. Tochowicz A, Goettig P, Evans R, Visse R, Shitomi Y, Palmisano R, et al. The dimer interface of the membrane type 1 matrix metalloproteinase hemopexin domain: crystal structure and biological functions. The Journal of Biological Chemistry. 2011;286(9):7587-600.

81. Tobar N, Avalos MC, Mendez N, Smith PC, Bernabeu C, Quintanilla M, et al. Soluble MMP-14 produced by bone marrow-derived stromal cells sheds epithelial endoglin modulating the migratory properties of human breast cancer cells. Carcinogenesis. 2014;35(8):1770-9.

82. Dufour A, Sampson NS, Zucker S, Cao J. Role of the hemopexin domain of matrix metalloproteinases in cell migration. Journal of Cellular Physiology. 2008;217(3):643-51.

83. Werler PJH. A novel double strand break system in Schizosaccharomyces pombe, specific for single strand annealing. University of Sussex, 2005. URL: http://ethos.bl.uk/OrderDetails.do?uin= uk.bl.ethos.421429.

84. Luttrell LM, Ferguson SS, Daaka Y, Miller WE, Maudsley S, Della Rocca GJ, et al. Beta-arrestindependent formation of beta2 adrenergic receptor-Src protein kinase complexes. Science. 1999;283(5402):655-61.

85. Zhuo JM, Tseng HA, Desai M, Bucklin ME, Mohammed AI, Robinson NT, et al. Young adult born neurons enhance hippocampal dependent performance via influences on bilateral networks. eLife. 2016;5.

86. $\mathrm{Hu} \mathrm{X}$, and Beeton C. Detection of functional matrix metalloproteinases by zymography. Journal of Visualized Experiments. 2010;45:e2445. 



\section{CHAP'TER 3}

The role of MMP14 in de novo lumen formation and branching morphogenesis in acne pathogenesis 


\section{Abstract}

Frank-Ter Haar syndrome (FTHS) and Winchester syndrome (WS) are ultra-rare autosomal recessive skeletal dysplasias associated with severe acne responding to retinoid treatment. These disorders are caused by homozygous loss-of-function mutations in SH3PXD2B and MMP14, respectively. As the protein products of these genes directly cooperate in ECM remodelling, we hypothesized that defective dermal ECM remodelling might contribute to aberrant sebaceous gland morphogenesis resulting in cyst i.e. comedo formation, the hallmark of acne. By using two simple yet well-established in vitro 3D culture models of epithelial cells grown in ECM, we demonstrated an important role for MMP14 and SH3PXD2B in de novo lumen formation and branching morphogenesis. In addition, we demonstrated for the first time that therapeutic plasma concentrations of both 9-cis RA and 13-cis RA directly stimulate de novo lumen formation and branching morphogenesis of mIMCD-3 3D culture. Our findings suggest that MMP14, SH3PXD2B, and retinoids affect both processes by stimulating epithelial cell polarity, proliferation and/or ECM degradation. Finally, our retinoid experiments demonstrate the suitability of the spheroid in vitro model for drug treatment and support their use in drug screening. 


\section{Introduction}

Acne vulgaris is an extremely common skin condition, in some countries affecting almost $100 \%$ of adolescents [1]. It is characterised by the presence of comedones, which are cystic sebaceous glands (Fig. 3.1C-D), and inflammation around these structures [2]. From genetic and epidemiological studies, it is known that accelerated sebaceous gland growth, as a consequence of genetic predisposition, hormonal influences and/or a glycaemic diet, is an important precipitating factor [3, 4]. However, it does not emerge from these observations how or why such growth should become problematic and cause acne. Although Cutibacterium (formerly Propionibacterium) acnes has historically been associated with acne (as its name implies), there is no good evidence that $C$. acnes causes inflammation or is otherwise causally involved [1].

While mild acne resolves without long-term sequelae and is effectively addressed with topical drugs, moderate-to-severe disease can leave disfiguring scars and requires systemic therapy to prevent this [2]. However, treatment options are limited. Tetracyclines or macrolide antibiotics are often used for their anti-inflammatory properties but carry a risk of bacterial resistance [5]. Furthermore, they are less effective in severe acne, which requires intervention with 13-cis retinoic acid (RA) [6]. Although very effective, its exact mechanism of action in resolving acne is unknown. In addition, this drug is highly teratogenic and has dose-restricting side effects, limiting its usefulness. As there exists a profound lack of insight into acne pathogenesis, no novel drug classes are being developed for moderate-to-severe acne since $[6,7]$. Thus, there is a significant unmet medical need.

We may now have identified the missing link, providing a unique opportunity to better understand and treat acne. This link comes from studies of rare genetic syndromes that are associated with severe acne. The Frank-Ter Haar syndrome (FTHS, MIM 249420) is a rare autosomal recessive skeletal dysplasia that is characterized by premature osteoporosis, cardiac defects, craniofacial dysmorphism and severe acne [8-10]. It is caused by homozygous mutation or deletion of, SH3PXD2B at 5q25.1 (MIM 613293) [11-13]. We have previously reported two additional patients with clinical features strongly reminiscent of the FTHS patients reported by Borrone et al. (Fig. 3.1A-B), in which we recently identified a novel homozygous mutation in MMP14 at 14q11.2 (MIM 600754; see Chapter 1 and 2) [10, 14, 15]. Only a single loss-of-function mutation in MMP14 had been reported before in patients with Winchester syndrome (WS, MIM 277950) [16]. This is another ultra-rare skeletal dysplasia with clinical overlap with FTHS, including severe acne $[17,18]$. There is a compelling link between SH3PXD2B and MMP14. The protein products of these genes directly interact in the formation of podosomes, specialized membrane extensions that mediate extracellular matrix (ECM) degradation and invasion [19]. SH3PXD2B (SH3 and Phox-homology Domain Containing Protein 2B) is an adapter protein that recruits the membrane-bound MMP14 (matrix metalloproteinase 14) to the nascent podosome membrane, enabling the podo- 

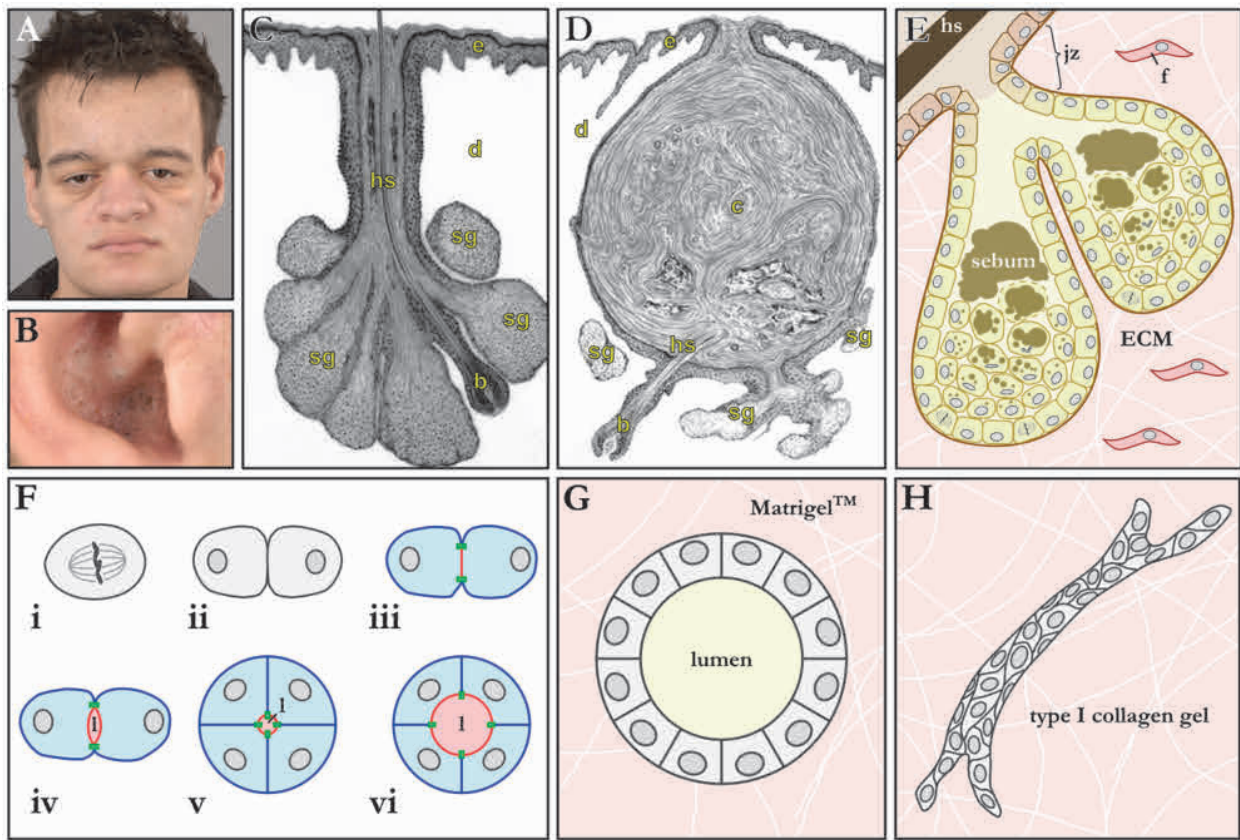

Figure 3.1 - A 3D in vitro model for aberrant sebaceous gland lumen formation and branching morphogenesis in Winchester syndrome. A-B, Winchester syndrome patient with severe acne, evidenced by numerous comedones in the concha of the right ear (B). C, sebaceous glands (sg) are situated in the dermis (d), are multilobulated and excrete sebum into the infundibulum around the hair shaft (hs). $\mathbf{D}$, in comedones (c), the infundibulum is expanded and filled with keratinous and sebaceous material, while the sebaceous gland itself appears hypotrophic [2]. E, the sebaceous gland is a multilobulated mini-organ, with a proliferative basal cell layer and more central cell layers of differentiating sebocytes undergoing various stages of lipid accumulation, cell volume increase, nuclear fragmentation, and cell lysis, resulting in holocrine secretin of sebum into a branched lumen [2]. F, Madin-Darby canine kidney (MDCK) cells form spheroids with a central lumen in 3D in vitro culture in Matrigel ${ }^{\mathrm{TM}}$ when exposed to serum by a process called "cord hollowing", following several well-defined steps. A nonpolarised cell (i) proliferates, generating two nonpolarised daughter cells (ii). Incorporation of tight junctions (green) between the two cells defines the apical (red) and basolateral (blue) membrane (iii). A lumen (l) is created between the apical/luminal membranes of both daughter cells by polarized exocytosis (iv). Subsequent cell divisions give rise to a spheroid, consisting of polarised cells in the periphery and a central lumen (v). By further apical secretion, the lumen and luminal membrane grow relatively compared to the surrounding cells, changing the morphology of the cells (vi) [31]. G-H, for murine inner medullary collecting duct (mIMCD-3) cells, luminogenesis follows a similar process as for MDCK cells, however, they behave differently in different extracellular matrices. In MatrigelTM, mIMCD-3 form spheroids with a lumen similar to MDCK cells (G). In type I collagen gel, mIMCD-3 cells form branched cell cords that later acquire a lumen $(\mathrm{H})$. B, hair bulb; e, epidermis; ECM, extracellular matrix; f, fibroblast; jz, junctional zone. A-B are adapted from Van Steensel et al. [14], C-D from Williams et al. [2], and F from Sigurbjornsdottir et al. [31], with permission.

some to locally digest the ECM [13, 19-21]. The identified SH3PXD2B and MMP14 mutations are loss-of-function mutations that result in reduced ECM degradation by cells expressing the mutant proteins $[11,12,16,20]$. As reported before for patients with FTHS and patients with a clinical diagnosis of WS, the facial skin of our patients 
was thickened due to dermal fibrosis, as evidenced by the dermal accumulation of collagen [10, 22 and unpublished data]. This demonstrates defective ECM remodelling and is thought to be linked to aberrant sebaceous gland growth resulting in comedo formation. This is further supported by the presence of large, open comedones in the postoperative hypertrophic scar in one of our patients [14].

The sebaceous gland is, in essence, a hollow multilobulated mini-organ situated in the collagen I-rich dermis. It consists of a proliferative basal cell layer and more central layers of differentiating sebocytes culminating in holocrine secretion of sebum into a branched lumen (Fig. 3.1E) [2]. Extracellular matrix remodelling is essential for normal morphogenesis of developing branching structures [23]. It has been shown that multiple human cell types, including fibroblasts and tumour cells of epithelial and mesenchyme origin, depend on the catalytic activity of MMP14 for the invasion of dense type I collagen matrices [24]. Furthermore, mouse mammary epithelial cells depend on the catalytic activity of MMP14 for branching morphogenesis in dense collagen matrix [25]. In addition, it has been shown that angiogenesis, a special form of branching morphogenesis, depends on the collagenolytic activity of MMP14 [26, 27]. Branching morphogenesis additionally requires planar cell polarity (PCP); abnormal PCP can cause cyst formation [28]. Intriguingly, membrane localisation of MMP14 is regulated by the PCP protein Van Gogh-like 2 (VANGL2) and is needed for normal PCP [29]. Thus, loss of SH3PXD2B or MMP14 could, therefore, disrupt ECM remodelling and/or PCP in growing sebaceous glands, resulting in cyst i.e. comedo formation. As the acne in our patients was successfully treated with 13-cis RA, we hypothesised that this drug could correct the putative defect in branching morphogenesis [14].

We aimed to assess the role of MMP14 and SH3PXD2B in, and the effects of retinoid treatment on, the morphogenesis of the developing sebaceous gland. In this pilot study, we used a well-established in vitro 3D epithelial cell culture model of murine inner medullary collecting duct (mIMCD-3) cells. When cultured in reconstituted basement membrane (Matrigel ${ }^{\mathrm{TM}}$ ), these cells form spheroids with a central lumen by a process called cord hollowing (Fig 3.1F-G) [30,32]. When cultured in a gel of type I collagen, these cells form branched cell cords that subsequently acquire a lumen by a similar process (Fig. 3.1H) [33]. Using mIMCD-3 cells in these two set-ups enabled us to study de novo lumen formation and branching morphogenesis separately, which is relevant as the sebaceous gland is a hollow, branched structure. We demonstrated an important role for MMP14 and SH3PXD2B in both de novo lumen formation and branching morphogenesis. Furthermore, we showed for the first time that therapeutic concentrations of both 9-cis and 13-cis RA directly stimulate both processes in in vitro mIMCD-3 3D culture. Our findings suggest that MMP14, SH3PXD2B, and retinoids affect both processes by stimulating epithelial cell polarity, proliferation and/or ECM degradation. As both 9-cis RA and 13-cis RA had similar effects on luminogenesis and branching morphogenesis, cell-type specific effects or additional modes of action are 
likely responsible for the effects of 13-cis RA on acne. Finally, our results further support the use of these in vitro models for drug screening affecting epithelial lumen formation and branching morphogenesis.

\section{Results}

\section{Lumen formation of mIMCD-3 cells in MatrigelIM is positively correlated with gel volume}

The role of MMP14 and SH3PXD2B in lumen formation was analysed by utilising an in vitro 3D culture model of mIMCD-3 cells in Matrigel ${ }^{\mathrm{TM}}$. First, normal luminogenesis by mIMCD-3 cells was established. For this, mIMCD-3 cells were seeded in MatrigelTM according to three different reported methods at comparable cell densities to determine the optimal culturing conditions. Cells were either seeded as single cell suspension in $10 \mu \mathrm{L}$ droplets of Matrigel TM ("standing droplets" method) that were incubated upside-down while the gel is setting, or seeded in $100 \mu \mathrm{L}$ Matrigel ${ }^{\mathrm{TM}}$ on top of a $15 \mu \mathrm{L}$ solidified layer of Matrigel ${ }^{\mathrm{TM}}$ ("sandwich" method), or seeded in $200 \mu \mathrm{L}$ Matrigel ${ }^{\text {TM }}$ ("thick gel") per well of a 8-well chambered cover glass [30, 34]. After the Matrigel $^{\mathrm{TM}}$ had set, $200 \mu \mathrm{L}$ of medium was added and cells were observed daily. Cells were fixed in situ (i.e. together with the gel) four days post seeding, when cells had formed round clusters (Fig. 3.2A). Staining the fixed cells for F-actin and chromatin (with fluorescently tagged phalloidin and DAPI, respectively) allowed for analysis of the cell cluster morphology by fluorescence microscopy. After four days, mIMCD-3 cells had formed round spheroids (Fig. 3.2B). However, there were differences in lumen formation between the three different culture methods. In standing droplets, only a minority $(\sim 10 \%)$ of cell clusters had a lumen. In sandwich gel, a lumen was more frequently ( $\sim 30 \%)$ observed in spheroids, whereas in thick gel, a slight majority (50-60\%) of spheroids had a lumen after four days of culture. Furthermore, various stages of lumen formation could be observed: cell clusters without a lumen, clusters with various small vesicles between the cells, and clusters with increasingly larger lumen were present side by side. In some spheroids, cell remnants with fragmented nuclei could be seen within or adjacent to the central lumen, suggesting apoptosis has a role in luminogenesis of mIMCD-3 cells. In general, average lumen size was positively correlated with Matrigel ${ }^{\mathrm{TM}}$ volume.

Keeping mIMCD-3 cells in log phase more strictly does not accelerate lumen formation in MatrigelTM

There was significant variation in spheroid size and lumen formation by mIMCD-3 cells between, but also within, the three tested 3D culturing methods. One possible explanation for the variation within a single method could be contact inhibition between cells in 2D culture prior to seeding in Matrigel ${ }^{\mathrm{TM}}$. Accordingly, some cells might take longer to recover from this contact inhibition, and the resulting cell clusters are 

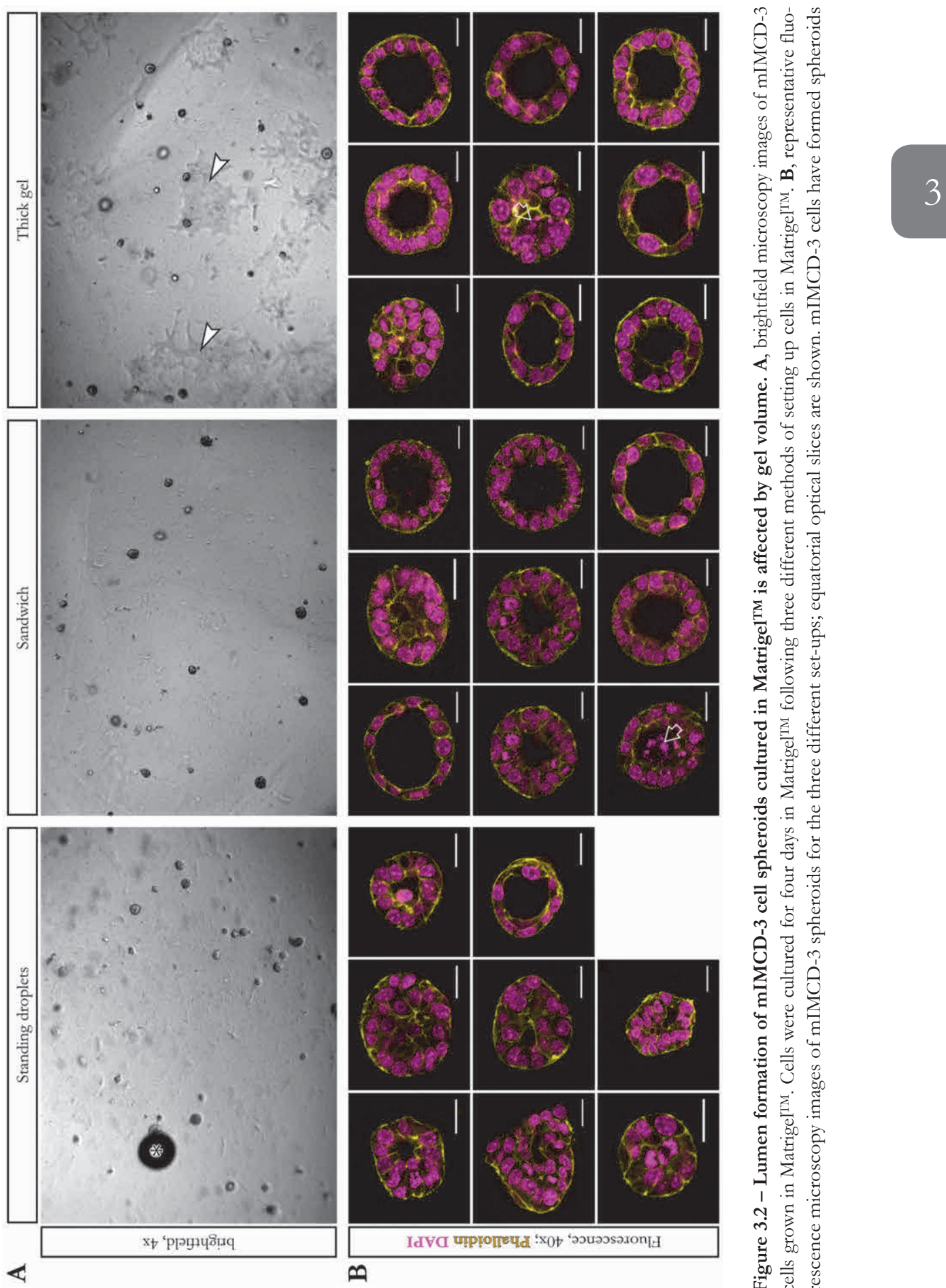

.

艺

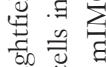

\&

ن.

ت

零

एँ

官芯

리

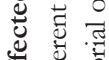

幽焉

至

ज्ञ

焉焉

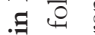

可焉

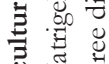

$0 \sum_{0} \pm$

-.

岂䓠

की

च 0

0 एँ

ค่ 己

总志

๘ँ ปे

을

త 0

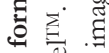

है

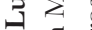

I.

กิ

Ð b

4

$\infty$

可司 
Figure 3.2 (continued) - after four days, but more spheroids have a distinct lumen and lumen size is on average larger in spheroids grown in the thick gel. Note however that in thick gel set-up, many cells are growing in 2D (A, arrowheads). In some spheroids, cell remnants with fragmented nuclei can be seen within or adjacent to the central lumen, suggesting apoptosis has a role in luminogenesis of mIMCD-3 cells. Asterisk indicates an air bubble. Scale bar equals $20 \mu \mathrm{m}$.

expected to grow slower and develop a lumen later than cells that were not or less affected by contact inhibition. Therefore, we hypothesised that keeping cells more strictly in log phase prior to seeding in Matrigel ${ }^{\mathrm{TM}}$ might enhance spheroid growth and lumen formation. To test this hypothesis, cells were kept at $85 \%$ or $40 \%$ confluence in 2D culture for five days prior to seeding in Matrigel ${ }^{\mathrm{TM}}$ standing droplets. Similar to the experiment shown in Figure 3.2, cells were fixed four days post seeding in MatrigelTM. However, there was significant variation in cell cluster size under both conditions. Notably, cells kept less confluently in 2D culture (Supp. Fig. 2.1C) formed less and smaller clusters in Matrigel ${ }^{\mathrm{TM}}$ than cells kept more confluently in $2 \mathrm{D}$ culture (Supp. Fig. 2.1A). As seen in Figure 3.2, under both conditions only a minority of cell clusters $(\sim 10 \%)$ had a lumen after four days of 3D culture (Supp. Fig. 2.1 B and D). Although lumen size varies within the two conditions, there was no overt difference in average lumen size between conditions. Taken together, our results suggest that contact inhibition in 2D culture does not impair lumen formation in subsequent $3 \mathrm{D}$ culture.

\section{Knockdown of Mmp14 or Sh3pxd2b disturbs mIMCD-3 spheroid architecture and luminogenesis}

Having established normal lumen formation by mIMCD-3 cells in MatrigelTM, the role of MMP14 and SH3PXD2B in luminogenesis was assessed next. As the mutations identified in FTHS and WS patients cause loss-of-function, this justified KD of these genes $[11,12,16,20]$. For KD of either Mmp14 or $S h 3 p x d 2 b$, mIMCD-3 cells were transfected with the TriFECTa ${ }^{\circledR}$ DsiRNA sets listed in Table 3.1 and seeded into MatrigelTM 24h later according to the "sandwich" method described above. Cells were fixed in situ seven days post seeding. Knockdown efficacy was assessed by qPCR, $24 \mathrm{~h}$ post transfection. As shown in Figure 3.3A, targeting $S h 3 p x d 2 b$ resulted in up to $40 \%$ $\mathrm{KD}$ of $\operatorname{Sh} 3 p x d 2 b$ mRNA expression (left panel). Albeit differences between the two primer sets used to assess $S h 3 p x d 2 b$ expression, it became clear that the level of Sh3pxd2b KD differs between DsiRNA sets, being highest for DsiRNA set 2 (35-40\% $\mathrm{KD})$ and lowest for set $3(6-30 \% \mathrm{KD})$. Knockdown of Mmp14 expression is more pronounced, reaching as high as $65 \%$ for Mmp14 DsiRNA set 2. Interestingly, KD of Sh3pxd2b resulted in a concurrent increase of Mmp14 mRNA levels (6-114\%, right panel). Conversely, KD of Mmp14 by DsiRNA sets 1 and 2 only led to a minor increase (2-17\%) in Sh3pxd2b expression and was absent for Mmp14 DsiRNA set 3.

After seven days of 3D culture in MatrigelTM ${ }^{\mathrm{TM}}$ mIMCD-3 cells had formed round cell clusters that varied in size, similar to previous experiments (Fig. 3.2 and Supp. Fig. 2.1). Transfection resulted in a substantial reduction in the number of spheroids 


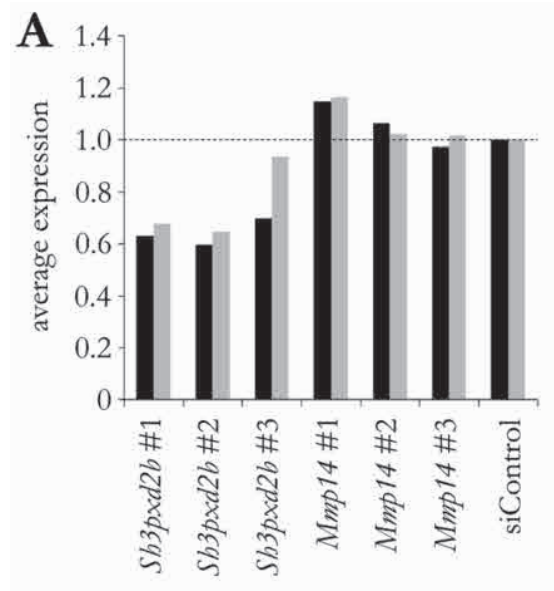

- Sh3pxd2b primer set 1

$\operatorname{Sb} 3 p x d 2 b$ primer set 2

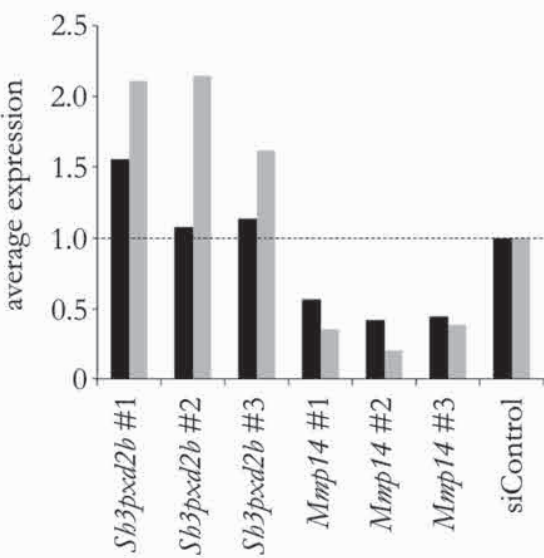

- Mmp14 primer set 1

Mmp14 primer set 2

Figure 3.3 - Knockdown of Mmp14, but not $S h 3 p x d 2 b$, disturbs spheroid architecture and lumen formation in mIMCD-3 cells cultured in Matrigel ${ }^{\mathrm{TM}}$. A, mean mRNA expression levels of $S h 3 p x d 2 b$ (left graph) and Mmp14 (right graph) 24h post transfection with the TriFECTa ${ }^{\circledR}$ DsiRNA sets listed on the $x$-axis (see Table 3.1 for details), analysed with two primer pairs (see Table 3.3), normalised for Hprt expression and denoted as the relative fold-difference to siControl. Transfection with Sh3p $x d 2 b$ DsiRNA resulted in max. $40 \% \mathrm{KD}$ of $S h 3 p x d 2 b$, with a concomitant upregulation of $M m p 14$ expression. Transfection with Mmp14 DsiRNA led to max. $65 \% \mathrm{KD}$ of Mmp14, with only a slight increase in $S h 3 p x d 2 b$ expression. B, brightfield microscopy images of mIMCD-3 spheroids in Matrigel ${ }^{\mathrm{TM}}$. Four regions per well are shown. Twenty-four hours post transfection, cells were seeded in Matrige ${ }^{\mathrm{TM}}$ and grown for seven days. Transfection reduced the average number of spheroids formed. C, equatorial optical sections of IF microscopy z-stacks of representative spheroids per treatment condition, stained for endogenous MMP14. Knockdown of Mmp14 and Sh3pxd2b disturbed normal lumen formation in mIMCD-3 spheroids. Cells transfected with siControl formed spheroids, most of which had a lumen after seven days. Transfection with $S h 3 p x d 2 b$ DsiRNA set 3 and Mmp14 set 1 had the most pronounced effect; in the majority of spheroids a lumen was absent and many spheroids had a disturbed morphology (see panel xii and xv for aberrantly shaped organoids). Transfection with $S b 3 p x d 2 b$ set 1 disturbs lumen formation in mIMCD-3 spheroids to a lesser extent. Scale bar equals $20 \mu \mathrm{m}$.

formed (Fig. 3.3B). From confocal immunofluorescence (IF) microscopy images it becomes clear that after seven days of 3D growth, the majority of spheroids formed by siControl-transfected cells had a distinct lumen (Fig. 3.3C). In contrast, although not quantified, transfection of cells with $S h 3 p x d 2 b$ DsiRNA disturbed normal lumen formation. For cells transfected with $S h 3 p x d 2 b$ DsiRNA set 1 , and to a lesser extent set 3, the fraction of organoids without a clear lumen seemed to be increased. Unfortunately, cells transfected with $S h 3 p x d 2 b$ DsiRNA set 2 were lost during the staining procedure and as a result were not imaged. Transfection of cells with Mmp14 DsiRNA sets 2 and 3 did not have an overt effect on luminogenesis; in the majority of spheroids visualised, a clear lumen could be detected, similar to the representative spheroid shown in 

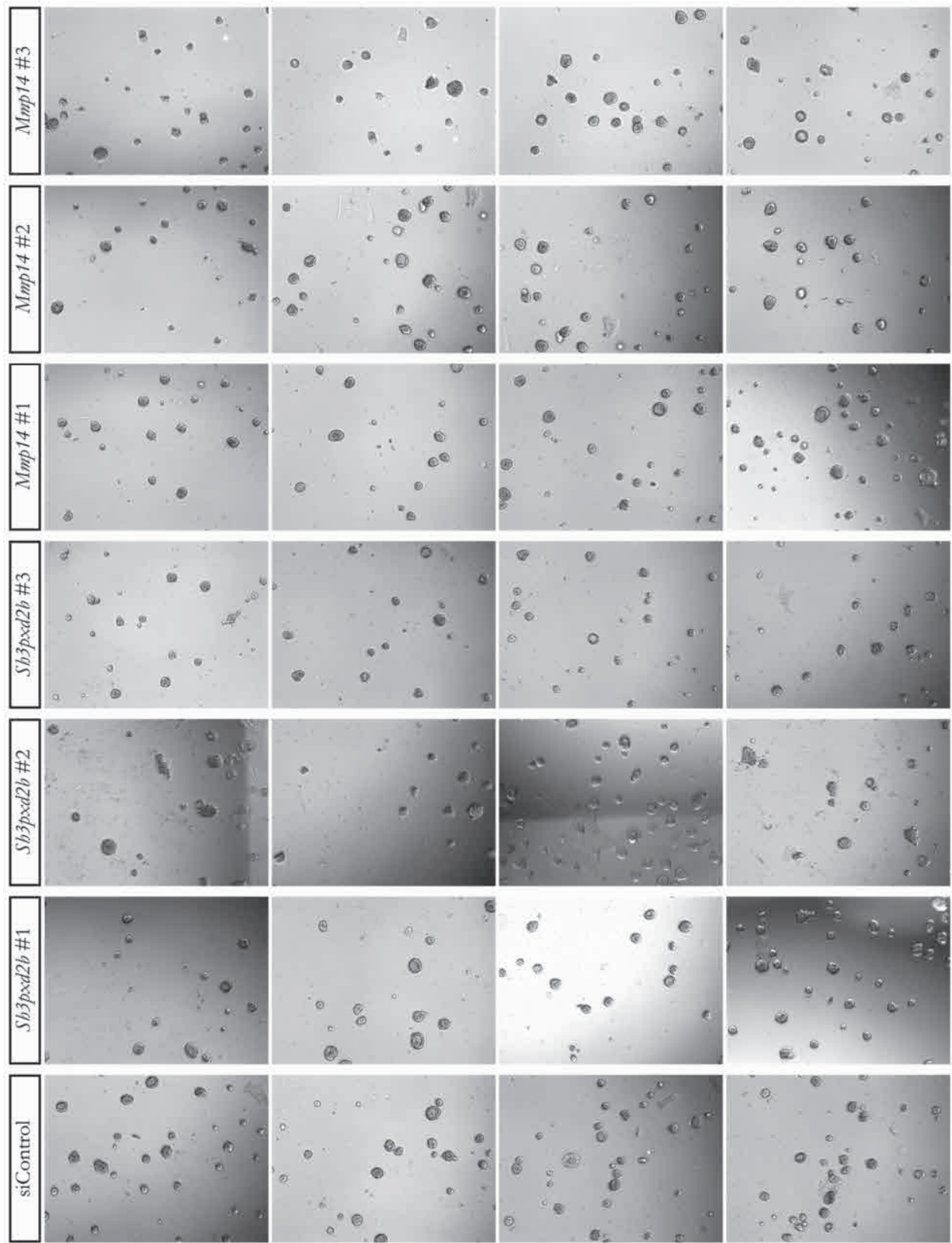

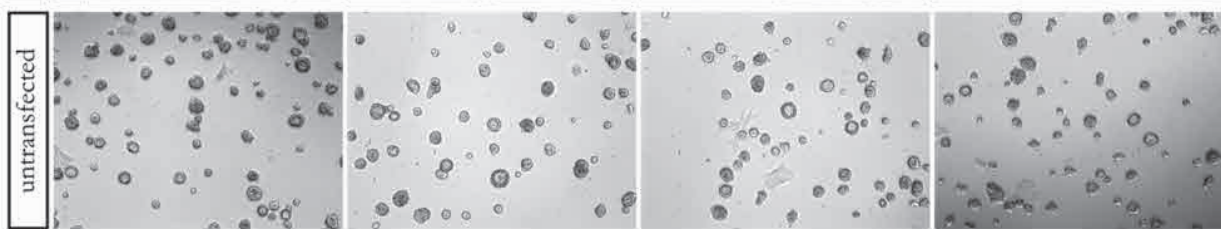



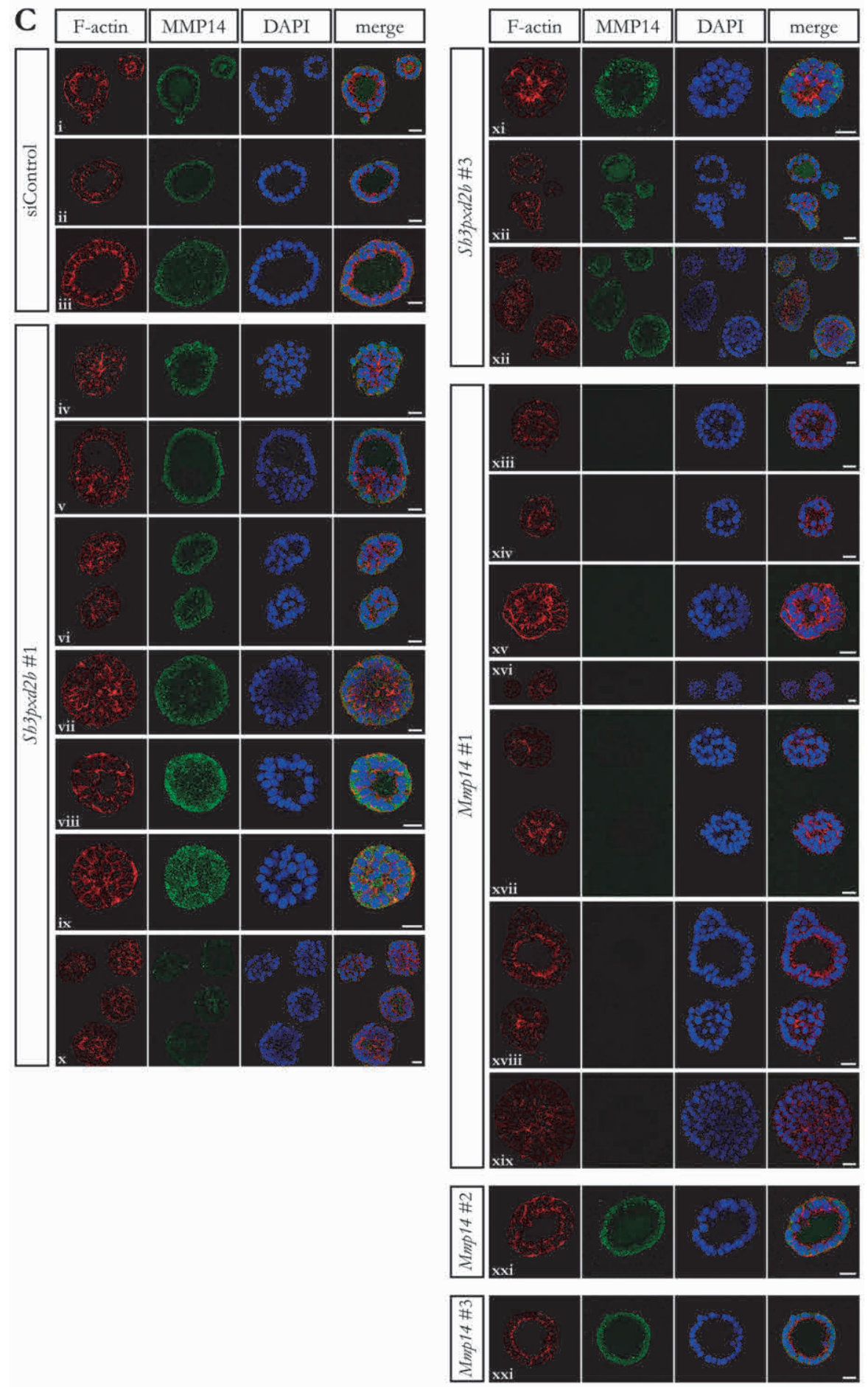
Figure 3.3C. Transfection with Mmp14 DsiRNA set 1, on the other hand, disturbed normal lumen formation, to a similar extent as $\operatorname{Sh} 3 p x d 2 b$ DsiRNA set 1 . Notably, no endogenous MMP14 could be detected in organoids of Mmp14 DsiRNA set 1 transfected cells. However, as qPCR showed the KD of Mmp14 was incomplete (Fig. 3.3A), it is unlikely that the organoids would not contain any MMP14 protein at seven days post transfection. As such, the lack of MMP14 signal in the IF image is more likely attributable to a technical error. Taken together, the results presented in Figure. 3.3 suggest KD of Mmp14 or $S h 3 p x d 2 b$ disturbs normal lumen formation in mIMCD-3 spheroids.

\section{Therapeutic plasma concentrations of retinoids stimulate mIMCD-3 spheroid size and luminogenesis}

After demonstrating a role for MMP14 and SH3PXD2B in lumen formation in an mIMCD-3 spheroid model, the effect of retinoids on lumen formation was analysed next. The standing droplet set-up was used to assess the effect of retinoids on lumen formation, expecting retinoid treatment to increase the number of spheroids with a

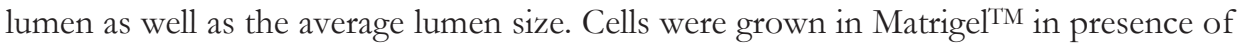
5-24 nM 9-cis retinoic acid (RA) or 13-cis RA. As discussed in Chapter 2, cells were treated before with 2-8 $\mathrm{nM}$ retinoids (corresponding to therapeutic plasma concentrations), to assess its effect on subcellular MMP14 localisation after $4 \mathrm{~h}$ of treatment. However, as spheroid formation is a relative slow process that requires cells to be cultured for multiple days, the range of retinoid doses was expanded. Retinoids were administered as a single dose to the culture medium immediately after the MatrigelTM had set. As the retinoids were dissolved in 100\% ethanol, treatment with 3.6\% $(v / v)$ ethanol served as vehicle control, corresponding to the volume added to cells treated with the highest retinoid dose $(24 \mathrm{nM})$. In addition, cells were left untreated to assess the effect of the vehicle on spheroid formation. After three days of growth in Matrigel $^{\mathrm{TM}}$, mIMCD-3 cells had formed small cell clusters (Fig. 3.4), and differences between organoids grown under different conditions were rather subtle. Treatment with therapeutic doses (5-8 nM) of 9-cis RA and 13-cis RA seemed to increase spheroid size compared to untreated cells (not quantified). At higher retinoid dose, the positive effect on spheroid size was absent; at $24 \mathrm{nM}$ RA treatment spheroids were less numerous and smaller compared to untreated control. This negative effect could, at least in part, be attributed to the relatively high concentration $(3.6 \%)$ of ethanol, as treatment with vehicle-only also negatively affected spheroid number and size. After six days, the effects of retinoid treatment on spheroid and lumen size became clearer. Treatment with 5-8 nM 9-cis RA or 13-cis RA increased the average spheroid and lumen diameter as compared to untreated spheroids (Fig. 3.5). At higher concentrations, the positive effect of retinoids on spheroid and lumen size was absent. Notably, treatment with $3.6 \%(v / v)$ ethanol with or without 24 nM RA decreased the average spheroid size. 

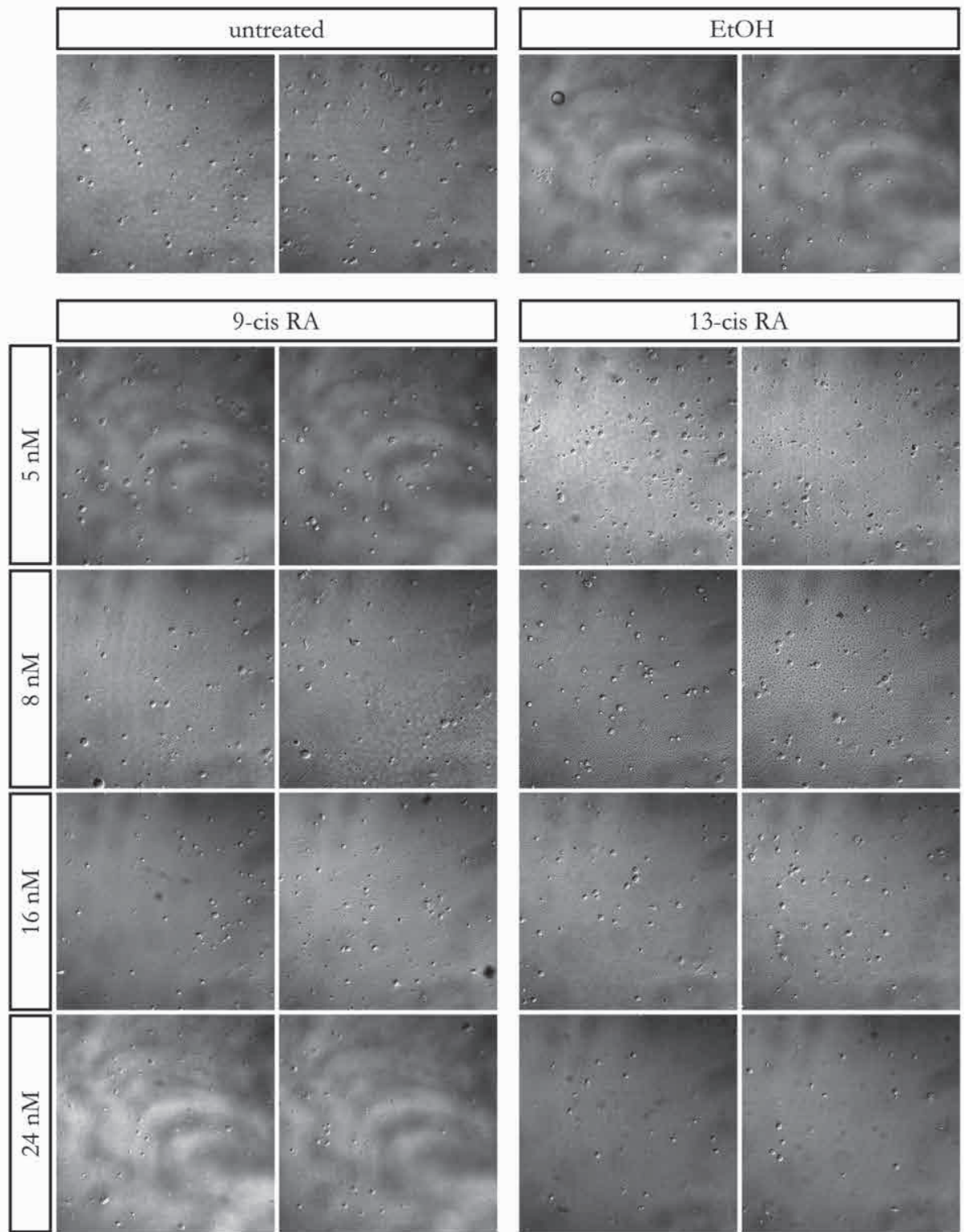

Figure 3.4 - Therapeutic doses of retinoids increase mIMCD-3 spheroid size after 3 days of 3D culture in Matrigel ${ }^{\mathrm{TM}}$. mIMCD-3 cells were seeded in $10 \mu \mathrm{L}$ droplets of Matrigel ${ }^{\mathrm{TM}}$ and cultured in media containing 5-24 nM 9-cis or 13-cis RA, vehicle (ethanol) only, or left untreated. A, brightfield overview showing small cell clusters. Treatment with 5-8 nM 9-cis RA or 13-cis RA increased the average spheroid size compared to untreated cells. At higher concentrations of retinoids (and vehicle), the positive effect on spheroid number and size was absent; at $24 \mathrm{nM}$ RA treatment there were fewer and smaller spheroids compared to untreated control. However, the highest concentration of vehicle only (3.6\% ethanol) seems to negatively affect spheroid number and size as well. 


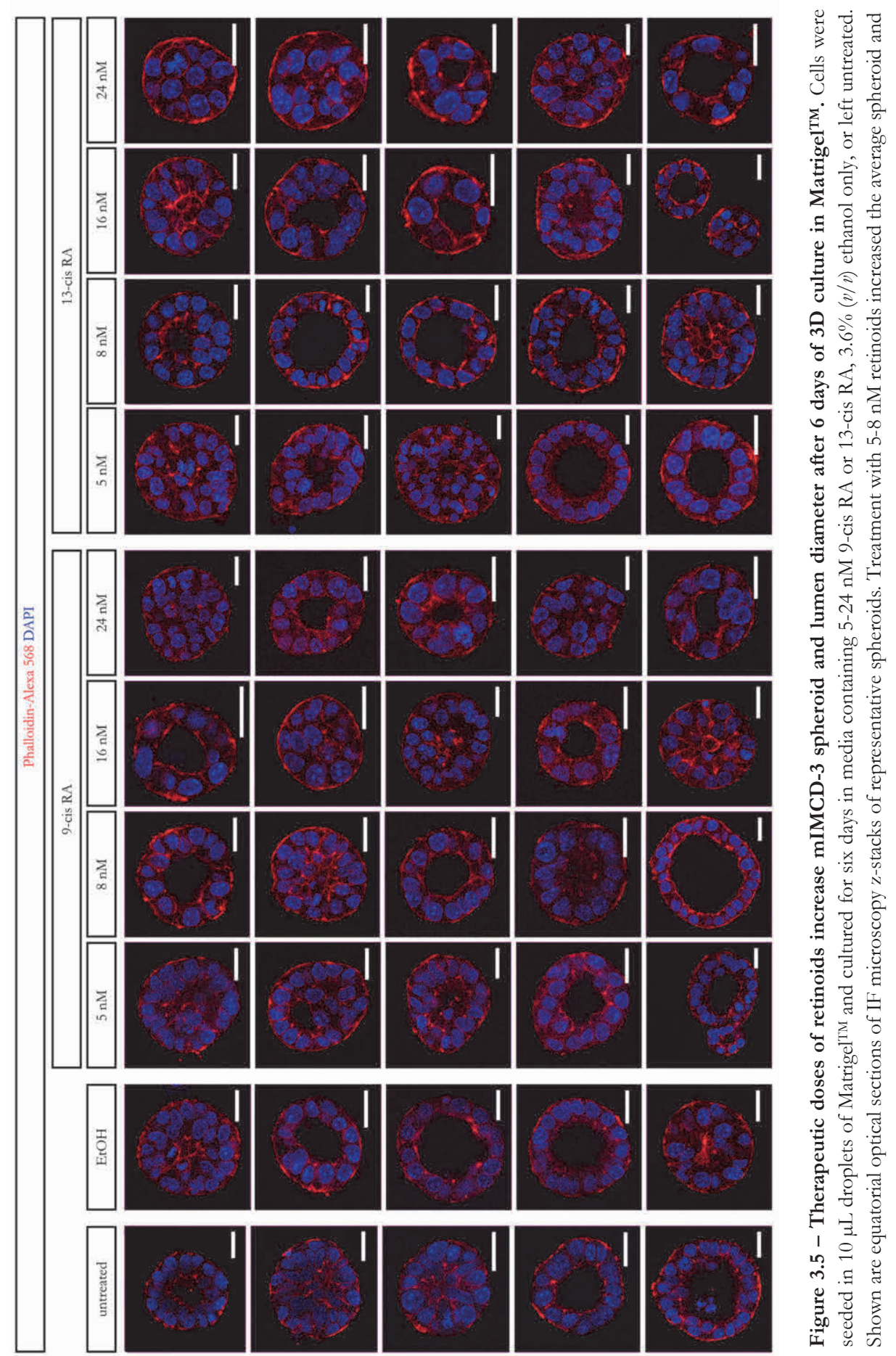


Figure 3.5 (continued) - lumen diameter as compared to untreated control. At higher concentrations, the positive effect of retinoids on spheroid and lumen size is absent. Scale bar equals $20 \mu \mathrm{m}$.

\section{mIMCD-3 cells form branched tubuli in 3D culture in type I collagen gel}

As sebaceous glands are not only a hollow but branched as well, tubulogenesis and branching morphogenesis were subsequently modelled by mIMCD-3 cells in type I collagen gel. Similar to the Matrigel ${ }^{\mathrm{TM}}$ experiments, normal tubulogenesis was assessed first. For this, mIMCD-3 cells were seeded as a single cell suspension in $10 \mu \mathrm{L}$ standing droplets type I collagen gel. Cells were grown for up to two weeks, as performed before by others [35]. After four days, the majority of cells had formed small cell clusters (Fig. 3.6, panel ii and iii), and to a lesser extent formed cell cords (panel i) with few branching points. After one week, longer cell cords had formed (panel iv) and more branching points were present (panel v). One week later, the cells had formed 2D sheets (panel vi, bottom part, and panel viii) and heavily branched cell cords (panel vi and vii). Even after two weeks, cell cords typically had a diameter of only 2-3 cells and were lacking a clear central lumen. Staining for MMP14 resulted in a rather diffuse signal distribution that only seemed to have a higher intensity at the cell surface in a minority of cells. One possible explanation is insufficient rinsing, thereby capturing an abundance of primary antibodies in the cells and the gel, as supported by the background staining in cell-free areas.

\section{Knockdown of Mmp14 or Sh3pxd2b disturbs tubulogenesis of mIMCD-3 cells in type I collagen gel}

After establishing normal tubulogenesis of mIMCD-3 cells in type I collagen gel, the role of MMP14 and SH3PXD2B in this process was assessed next. In parallel with the Matrigel $^{\mathrm{TM}}$ experiment shown in Figure 3.3B-C, part of the cells transfected with the TriFECTa ${ }^{\circledR}$ DsiRNA sets were seeded in type I collagen gel droplets $24 \mathrm{~h}$ post transfection. After seven days, untransfected mIMCD-3 cells have formed long, branched tubules (Fig. 3.7). As seen in Matrigel ${ }^{\mathrm{TM}}$ 3D culture, siControl transfection reduced the number and size of organoids formed in type I collagen gel. Knockdown of $S h 3 x d 2 b$ generally reduced tubule formation dramatically; the majority of cells formed small, star-like structures, although cells transfected with $S h 3 p x d 2 b$ DsiRNA set 2 still formed some tubules (second panel from top). Knockdown of $S h 3 p x d 2 b$ thus disturbed normal mIMCD-3 organogenesis in both Matrigel ${ }^{\mathrm{TM}}$ and type I collagen gel 3D culture systems. In contrast, transfection of mIMCD-3 cells with Mmp14 DsiRNA sets 1 and 2 had a milder effect on tubulogenesis, although the formed tubuli were generally shorter. Transfecting cells with Mmp14 DsiRNA set 3 had no overt effect on tubulogenesis. Knockdown of Mmp14 in mIMCD-3 cells disturbed both mIMCD-3 luminogenesis and tubulogenesis in the two respective 3D culture models, however, there were some discrepancies between the three DsiRNA sets used. Where KD of Mmp14 with DsiRNA set 1 disturbs both processes, set 2 only affected tubulogenesis. Although 

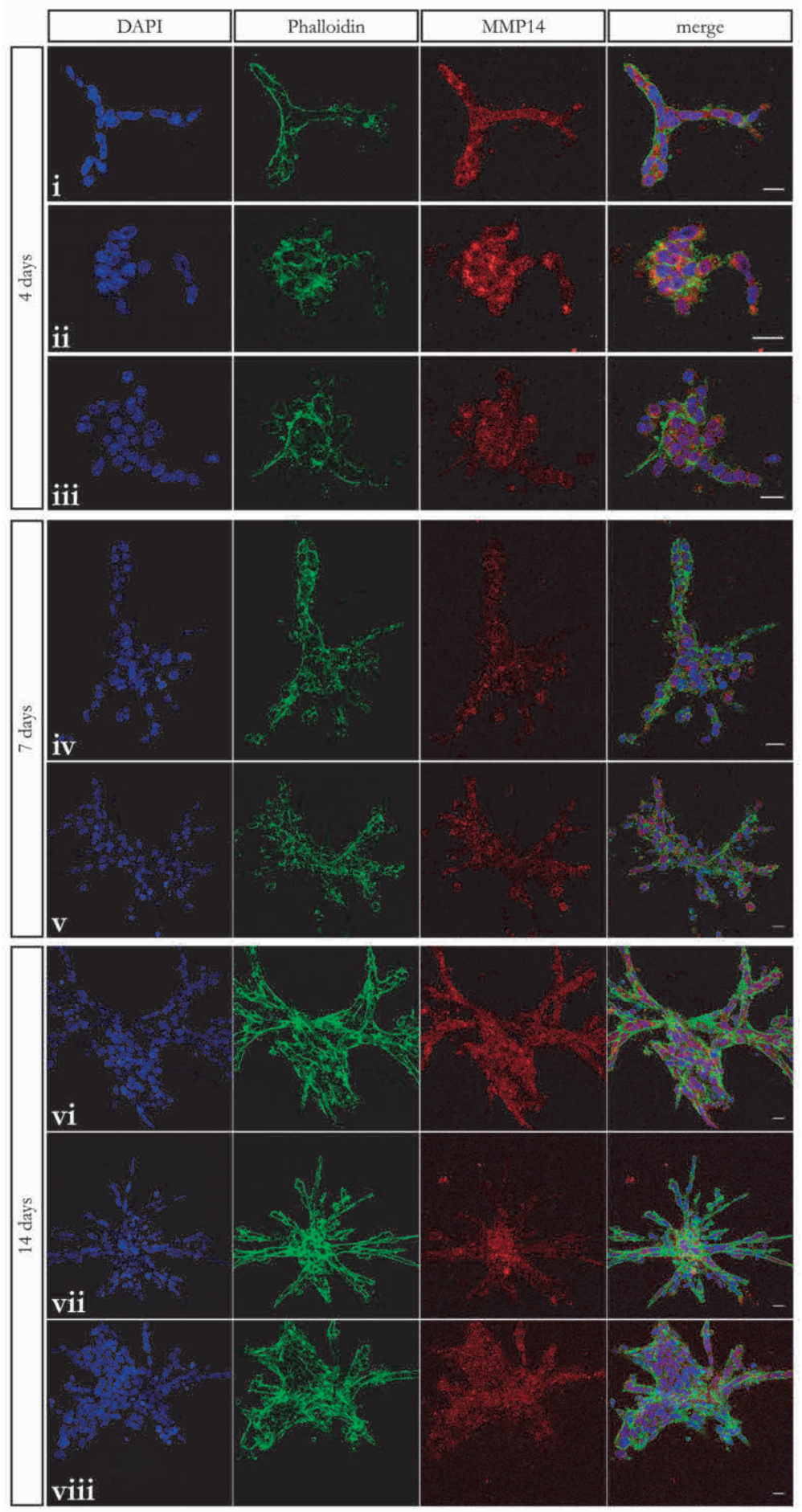
Figure 3.6 - Time course of mIMCD-3 cell tubulogenesis in type I collagen gel. mIMCD-3 cells started to grow in small cell clusters (ii and iii) and formed cell cords (i) within four days after seeding in type I collagen gel. After seven days, cell cords had elongated (iv) and started to branch out (v). After fourteen days, cells formed cellular sheets (vi, bottom, and viii) and heavily branched cell cords (vi and vii) without a clear lumen. Note that cells within the sheets formed actin stress fibers. Scale bars equal $20 \mu \mathrm{m}$.

Mmp14 was knocked-down as evidenced by qPCR, Mmp14 DsiRNA set 3 had no overt effect on lumen formation or tubulogenesis.

\section{Retinoid treatment stimulates tubulogenesis of mIMCD-3 cells in type I collagen gel}

The effect of retinoid treatment on tubulogenesis was assessed by growing mIMCD-3 cells in type I collagen gel in the presence or absence of 9-cis or 13-cis RA. In a first trial, cells were grown for three days, after which tubulogenesis was assessed by brightfield microscopy. As spheroid size and lumen formation were only stimulated by retinoid treatment dosed within the range of therapeutic plasma concentrations, cells were treated with 2-8 $\mathrm{nM} 9$-cis RA or 13-cis RA, or 1.2\% $(v / v)$ ethanol. After three days, the majority of untreated and vehicle-only treated mIMCD-3 cells had formed small cell clusters from which cell cords radiated; very few clear tubular structures could be observed (Supp. Fig. 2.2). Vehicle-only treatment did not affect tubulogenesis. Treatment of cells with increasing doses of 9-cis RA was positively correlated with the number of tubular structures that were formed. At all doses tested, 13-cis RA stimulated tubulogenesis of mIMCD-3 cells, which was reflected in both a larger amount of tubular structures as well as a longer average tubule length.

For five-day type I collagen 3D culture, cells were incubated with higher concentrations $(5-24 \mathrm{nM})$ of retinoids and an equivalent higher concentration $(3.6 \% v / \nu)$ of ethanol as a control. As shown in Figure 3.8, even after five days most untreated and vehicle-only treated mIMCD-3 cells formed cell clusters or sheets from which cell cords radiated; relatively few cells formed true tubular structures. Similar to three-day culture, there was no overt difference between untreated and vehicle-only treated cells. Increasing the retinoid concentration appeared to be positively correlated with the fraction of cells that formed tubular structures after five days, both for 9-cis RA and 13-cis RA.

\section{Discussion}

FTHS and WS are ultra-rare autosomal recessive skeletal dysplasias associated with severe acne responding to retinoid treatment $[8-10,14,17,18]$. These disorders are caused by homozygous loss- of-function mutations in SH3PXD2B and MMP14, respectively $[11,16]$. As the protein products of these genes directly cooperate in ECM remodelling, we hypothesized that defective dermal ECM remodelling might contribute to aberrant sebaceous gland morphogenesis resulting in cyst i.e. comedo for- 


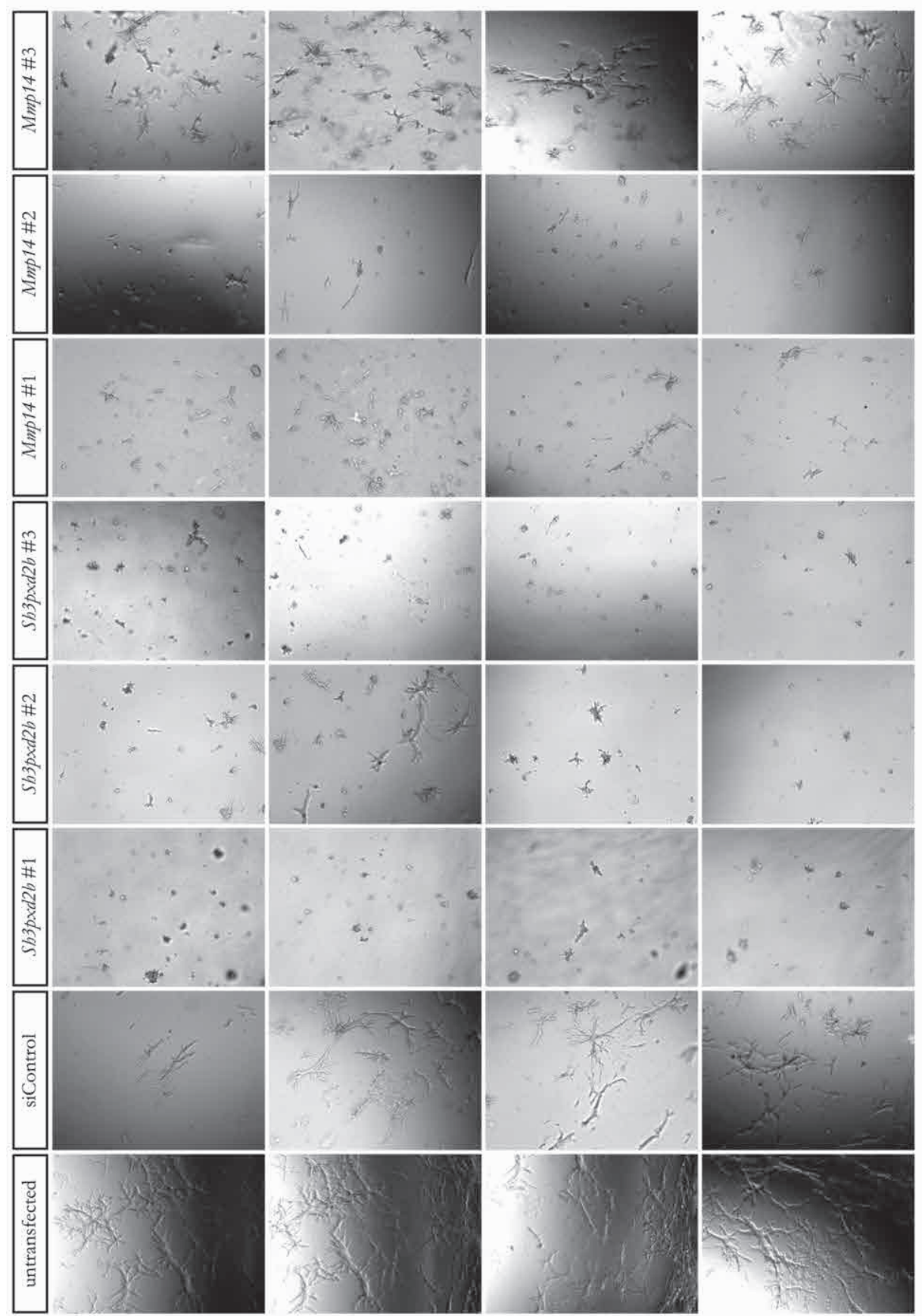

Figure 3.7 - Knockdown of $M m p 14$ or $S h 3 p x d 2 b$ disturbs tubulogenesis of mIMCD-3 cells cultured in type I collagen gel. After 7 days, untransfected mIMCD-3 cells form branching tubules. Transfection with siControl reduced the number and length of tubular structures to some extent. Although some tubules are formed by cells transfected with DsiRNA $\operatorname{Sh} 3 p x d 2 b$ set $2, \operatorname{Sh} 3 x d 2 b$ KD significantly inhibits 
Figure 3.7 (continued) - tubulogenesis. In contrast, transfection with DsiRNA Mmp14 set 1 and 2 has a milder effect on tubulogenesis, although the formed tubuli are generally shorter. DsiRNA Mmp14 set 3 does not affect tubulogenesis.

mation, the hallmark of acne $[2,20]$. By using two simple in vitro mIMCD-3 3D culture models, we confirmed an important role for Mmp14 and Sh3pxd2b in normal de novo lumen formation and branching morphogenesis [30, 32, 33]. In addition, we demonstrated for the first time that therapeutic plasma concentrations of 9-cis RA and 13-cis RA stimulate both processes in mIMCD-3 3D culture.

\section{In vitro mIMCD-3 3D culture models of lumen formation and branching morphogenesis}

The sebaceous gland is an epidermal adnex that, together with the hair follicle, invaginates the underlying dermis. In essence, it is a branched, hollow structure lined with epithelial cells and a central lumen filled with differentiating sebocytes and sebum [2, 36]. To study the role of MMP14 and SH3PXD2B in sebaceous gland branching morphogenesis, we used a simple in vitro 3D cell culture system to model the essential aspects of glandular epithelial organization [30, 37]. For de novo lumen formation, previously unpolarised epithelial cells need to sense their environment (ECM and neighbouring cells), establish apicobasal polarity that is coordinated with their neighbours, alter their shape, and finally create and expand a lumen between them [38-41]. To study these processes, we used the mIMCD-3 cell line. These are epithelial cells derived from the inner medullary collecting duct of mice transgenic for the early region (large T) of SV40 [32]. When cultured in ECM in vitro in presence of serum, these cells will rapidly polarise and form hollow 3D structures $[30,33]$. The advantage of this modular culture system is the possibility of growing these cells in different types of ECM [41]. When cultured in MatrigelTM, mIMCD-3 cells form spheroids with a central lumen lined by a single cell layer [30]. When cultured in collagen type I gel, these cells form branching tubuli [33]. These matrices are physiologically relevant. MatrigelTM is the protein extract of murine Engelbreth-Holm-Swarm (EHS) mouse sarcoma. It is rich in laminin, collagen type IV, nidogen/entactin and proteoglycans, and can thus serve as reconstituted basement membrane [37, 40]. In the skin, the epidermis including the sebaceous gland is separated from the dermis by the basement membrane. The reticular dermis, at the level of the sebaceous gland, is rich in collagen type I fibrils [36]. This modular system enabled us to study de novo lumen formation and branching morphogenesis of epithelial cells independently.

In Matrigel ${ }^{\mathrm{TM}}$, we demonstrated mIMCD-3 cells form spheroids within several days. Although lumen diameters were not quantified, the average lumen size observed and imaged of representative spheroids correlated positively with Matrigel ${ }^{\mathrm{TM}}$ volume. Spheroids formed asynchronously with various stages of lumen formation present at the same time, as reported before by others [30]. Keeping the cells more stringently in log-phase prior to seeding in MatrigelTM did not lead to more uniform spheroids, but 

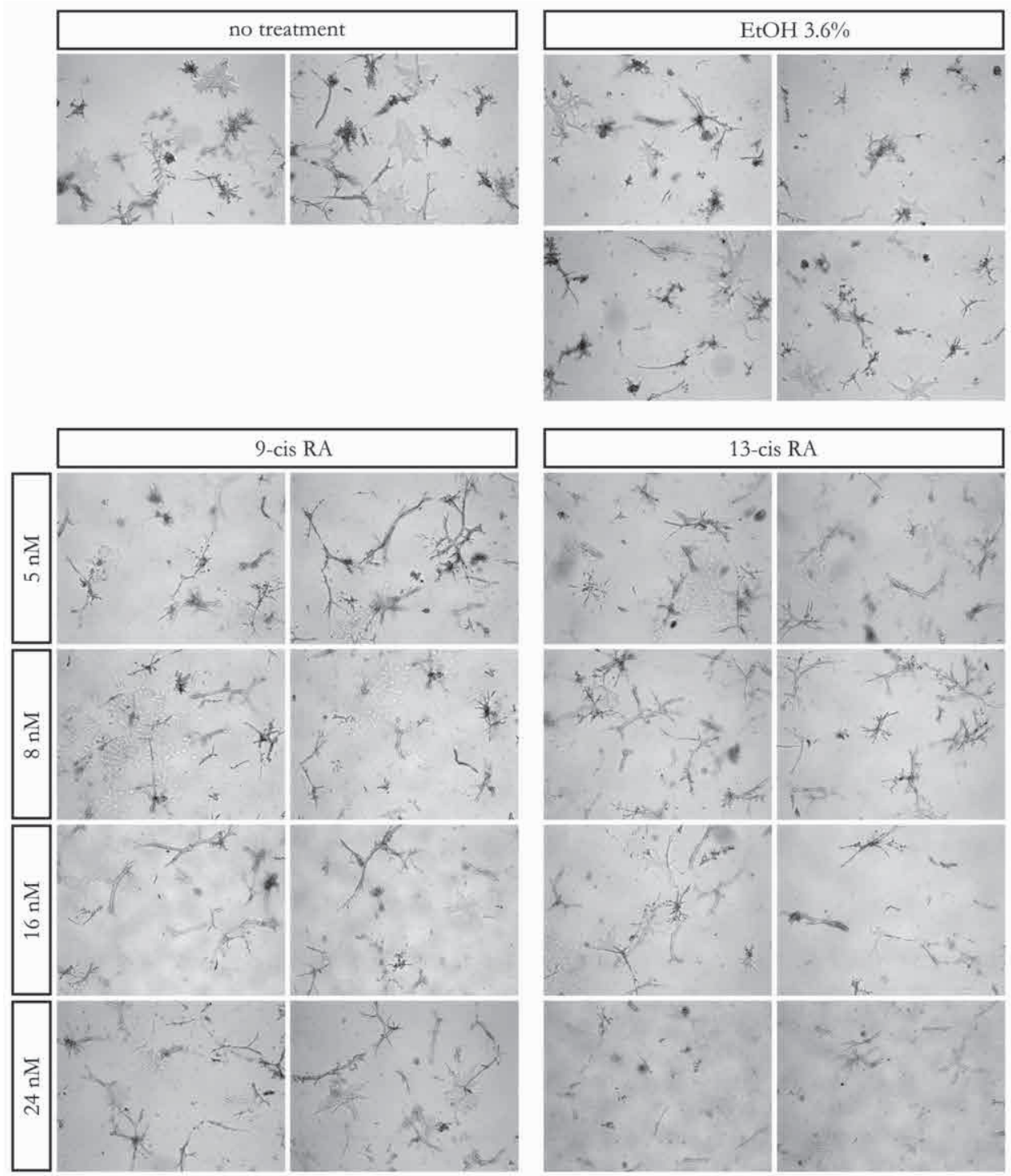

Figure 3.8 - Retinoids stimulate tubulogenesis of mIMCD-3 cells in type I collagen gel. Brightfield images of mIMCD-3 cells grown in type I collagen gel for five days in presence or absence of 5-24 nM 9cis RA or 13-cis RA, or vehicle $(3.6 \%(v / v)$ ethanol) only. After five days, most untreated and vehicle-only treated mIMCD-3 cells formed cell clusters or sheets from which cell cords radiated; relatively fewer cells formed true tubular structures. Treatment of cells with increasing doses of both 9-cis RA and 13-cis RA was positively correlated with the fraction of cells that form tubular structures after five days.

instead caused slower spheroids development. Frequent passaging might have caused cellular stress, resulting in slower cell growth and spheroid development. This is supported by our observation that the spheroids in general, and not only the lumen, were smaller for more frequently passaged cells. Alternatively, less frequently passaged cells 
could have been seeded in MatrigelTM as small clusters containing 2-3 cells instead of individual cells, thereby causing a growth advantage and resulting in larger spheroids over time. In addition, we observed fragmented nuclei in the lumen of some spheroids, suggestive of apoptosis. Similar to Madin-Darby canine kidney (MDCK) cells, mIMCD-3 cells were reported before to form a lumen by a process called cord hollowing, characterized by apical vesicular exocytosis of cytoplasmic vesicles [31, 39, 40]. Cavitation, i.e. luminal clearing by apoptosis, was previously reported not to be involved in mIMCD-3 luminogenesis [30]. In MDCK spheroids, cavitation occurs as a corrective mechanism when polarisation is slow [40]. Additional assays, including cleaved Caspase-9 staining, are required to assess whether these cells are indeed apoptotic.

In collagen type I gel, we observed mIMCD-3 cells forming cell clusters, radiating cell cords that grow in diameter and finally branched tubular structures over the course of several days as reported before [42, 43]. Piscione et al. previously demonstrated that after only $72 \mathrm{~h}$ growth in a collagen matrix, mIMCD-3 tubuli have a central lumen [43]. In contrast, we did not observe a central lumen by IF microscopy. It is likely that in this initial experiment, we only imaged tubule progenitors instead of structures that, in our subsequent experiments, were morphologically regarded as tubuli [44].

\section{Mmp14 and Sh3pxd2b are essential for epithelial lumen formation and branching morphogenesis}

As mIMCD-3 cells form organoids in 3D culture within days, the involvement of genes in luminogenesis and branching morphogenesis can be studied by siRNA knockdown (KD), and the phenotypic alterations assessed by microscopy when KD is still present $[30,39]$. By this approach, we found that MMP14 and SH3PXD2B play an important role in de novo lumen formation and tubulogenesis. Although not quantified, our results of Matrigel ${ }^{\mathrm{TM}} 3 \mathrm{D}$ culture suggested that KD of $\operatorname{Sh} 3 p x d 2 b$ and Mmp14 results in an increased fraction of spheroids without a clear lumen. We furthermore showed that in collagen type I gel $3 \mathrm{D}$ culture, $\operatorname{Sh} 3 p x d 2 b \mathrm{KD}$ resulted in a dramatic reduction of tubule formation, whereas $M m p 14 \mathrm{KD}$ had a less pronounced inhibitory effect of tubulogenesis. As the defects tubulogenesis were overtly visible, number and size of tubular structures were not quantified in this study. Although the used DsiRNA sets differed in the extent to which they affected luminogenesis and branching morphogenesis, and phenotypic rescue by overexpression was not performed, the fact that multiple DsiRNA sets per gene affected these two related processes makes explaining them as an off-target effect unlikely.

The question remains what process is exactly affected by the KD, resulting in the observed phenotypes. Three candidate processes are likely involved. The observed disturbed mIMCD-3 spheroid architecture and luminal filling upon KD of $5 h 3 p x d 2 b$ and Mmp14 are suggestive of impaired cellular polarity. This may be mediated through defective integrin signalling. Integrins are important in the cellular perception of the 
surrounding ECM and can thereby alter cellular behaviour [45]. It has previously been demonstrated that $\beta 1$-integrin has an important role in tissue polarization [38]. MDCK cells lacking $\beta 1$-integrin were shown to be incapable to initiate polarization, resulting in organoids lacking a lumen [31]. There is furthermore a compelling link between $\beta 1$ integrin and MMP14. Firstly, MMP14 can directly bind to $\beta 1$-integrin to activate Erk, increasing MAPK signalling, which facilitates branching morphogenesis in collagen matrices $[21,46]$. Secondly, shRNA KD of $\beta 1$-integrin has been demonstrated to decrease expression levels of $M m p 14$, and vice versa, in murine mammary epithelial cells. Expression levels of $\beta 1$-integrin are furthermore reduced in the mammary gland of Mmp14 KO mice [45]. Finally, KD of $\beta 1$-integrin was shown to block branching morphogenesis of mammary epithelial cells to the same extent as Mmp14 KD in $1 \mathrm{mg} / \mathrm{mL}$ type I collagen gel, the same concentration as used in our experiments [46].

In addition to aberrant polarity, there are indications that defective ECM degradation contributed to the observed phenotype in type I collagen gel. We demonstrated that KD of either Mmp14 or $S h 3 p x d 2 b$ reduced tubule length in collagen gel. The similar effect of $S h 3 p x d 2 b$ and Mmp14 KD on tubule formation could be explained by disturbed podosome formation, thereby decreasing focal collagen degradation. The type I collagen we used is prepared by acid extraction, keeping the $\mathrm{N}$ - and C-terminal telopeptides on the collagen monomers intact. These telopeptides contribute to crosslinking of the collagen fibrils, requiring proteolytic degradation for cells to move through the matrix [47]. Likewise, others previously demonstrated that murine ureteric bud (UB) cells require MMP-dependent ECM degradation to form similar branched structures [48]. This is further supported by defective renal branching morphogenesis in Mmp14 KO mice, which furthermore contained excessive amounts of ECM [49]. In addition, defective branching morphogenesis was shown to underlie the abnormal development of the submandibular gland in Mmp14 KO mice [50]. In both tissues, the defects in branching morphogenesis were shown to be independent of pro-MMP2 activation $[49,50]$.

Finally, knockdown of Mmp14 and possibly $S h 3 p x d 2 b$ could inhibit proliferation. This could explain the small mIMCD-3 structures that were observed in collagen gel. This is moreover likely to be a specific effect of $\mathrm{KD}$, as siControl transfected cells formed larger, branching tubular structures. In addition to the polarisation defects in the kidney of Mmp14 KO mice described above, an additional severe proliferative defect was reported. Furthermore, IMCD cells of these mice showed a low proliferative rate in vitro that could be increased by overexpressing MMP14 WT. Interestingly, overexpressing catalytic inactive MMP14 worsened the proliferative defect [49]. It was previously shown that overexpressing MMP14 stimulates the proliferative rate of MCF-7 human breast adenocarcinoma cells and UM-SCC-1 squamous cell carcinoma cells [51]. Similarly, overexpression of MMP14 resulted in a dramatic increase of MDCK cyst size grown in type I collagen gel, but not protease-resistant collagen gel or Mat- 


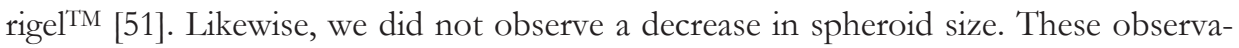
tions suggest the proliferative advantage depends specifically on the collagenolytic activity of MMP14. This is unlikely to result from the release of cell-derived factors into the matrix by MMP14, as it was previously demonstrated that co-culture of MDCK cells with or without overexpression of MMP14 only conferred the MMP14expressing cells with a growth advantage [51]. The proliferative advantage is furthermore unlikely attributable to the generation of collagen-derived growth enhancers by MMP14, as the incorporation of 1\% gelatin or $0.5 \%$ MMP14-generated collagen fragments was previously reported not to stimulate proliferation of UM-SCC-1 cells [51].

\section{Retinoids stimulate epithelial lumen formation and branching morphogenesis}

The in vitro 3D models outlined above can be used to directly observe the effects of drug treatment on luminogenesis and branching morphogenesis [30]. As acne was successfully treated in our patients by 13-cis RA, we assessed the effects of retinoid treatment on de novo lumen formation and branching morphogenesis was assessed. We demonstrated that retinoid treatment stimulated both spheroid size and lumen diameter in 3D culture of mIMCD-3 cells in MatrigelTM, in addition to an overt stimulatory effect on branching morphogenesis resulting in more and longer tubular structures in collagen type I gel. It has previously been demonstrated that that vitamin A and retinoids (synthetic vitamin A derivatives) are essential for normal branching morphogenesis of the murine lung, kidney and submandibular salivary gland [52-54]. In the kidney, all-trans retinoic acid (ATRA), the active isomer of both 9-cis RA and 13-cis RA, was shown to accelerate UB branching morphogenesis ex vivo $[52,55,56]$. However, this stimulatory effect on epithelial cells in lung and kidney was suggested to result from the paracrine release of the branching morphogen late-gestation lung protein 1 (Lgl1) by the surrounding mesenchymal cells. Both 9-cis RA and ATRA were able to induce Lg11 expression. Interestingly, IMCD cells were demonstrated to lack Lgr1 expression [52]. We here demonstrate that retinoids have a direct stimulatory effect on mIMCD-3 branching morphogenesis. The increase in tubular structure length and the increase in spheroid size could, at least in part, be explained by an increase in proliferative rate, as retinoids have been demonstrated to promote proliferation of basal keratinocytes by shortening the mitotic phase $[55,57]$. In addition, retinoids have been reported to increase the number of podosomes in chicken osteoclasts [58]. This could stimulate focal ECM degradation, thereby aiding tubulus formation.

Apart from stimulating proliferation and ECM degradation, tissue-specific effects are likely involved in the therapeutic effects of retinoids. Although 9-cis RA and 13-cis RA both stimulated de novo lumen formation and branching morphogenesis in our in vitro models, only 13-cis RA is effective in acne treatment [55]. In addition, previous studies have demonstrated that retinoids inhibit differentiation of hyperproliferative keratinocytes and sebocytes, and inhibit sebocyte differentiation and thereby sebum produc- 
tion $[55,57,59]$. These aspects were however not possible to assess with our currently used models.

\section{Conclusion and future perspectives}

In conclusion, we used two simple yet well-established in vitro models of epithelial cell 3D culture in ECM to demonstrate an important role for MMP14 and SH3PXD2B in de novo lumen formation and branching morphogenesis. In addition, we demonstrated for the first time that therapeutic plasma concentrations of both 9-cis RA and 13-cis RA directly stimulate luminogenesis and branching morphogenesis of mIMCD-3 cells. Our findings suggest that MMP14, SH3PXD2B, and retinoids affect both processes by stimulating epithelial cell polarity, proliferation and/or ECM degradation. Due to the observed variability in organoids per condition, further studies are needed to analyse larger numbers and quantify spheroid size, lumen diameter, and tubule length to confirm the observed effects of KD and retinoid treatment [30]. It would be interesting to assess whether the observed $\mathrm{KD}$ phenotypes can be rescued, either by cDNA overexpression or retinoid treatment. In addition, further studies are required to better understand the underlying mechanism of the observed phenotypes. In the current setup, additional staining for markers of polarisation including zonula occludens-1 (ZO-1) and $\beta$-catenin, and podosomal markers such as cortactin (see Chapter 1 ) could be used to assess the involvement of defective cell polarity and podosome formation, respectively. Although our findings support the involvement of MMP14 and SH3PXD2B in epithelial luminogenesis and branching morphogenesis, the experiments performed in this pilot study should be repeated with sebocytes to further assess whether similar processes could affect the sebaceous gland. Finally, our retinoid treatment experiments demonstrate the suitability of the spheroid system for drug treatment. As there is a significant unmet need in the treatment of acne, a sebocyte 3D model could potentially be used for drug screening [30, 37].

\section{Materials and methods}

\section{Cell culture}

mIMCD-3 immortalised murine inner medullary collecting duct cells (American Type Culture Collection, Manassas, Virginia, USA; CRL-2123) were grown in 2D culture in low-glucose Dulbecco's Modified Eagle Medium (DMEM, GE Healthcare Life Sciences, Pittsburgh, PA; SH30021.01) containing 10\% $(v / v)$ fetal bovine serum (FBS; GE; A15-101) and $100 \mathrm{U} / \mathrm{mL}$ penicillin / $100 \mu \mathrm{g} / \mathrm{mL}$ streptomycin (ThermoFischer Scientific, Inc., Waltham, MA, USA; 15140122) at $37{ }^{\circ} \mathrm{C}$ in $100 \%$ humidity and $5 \%$ $\mathrm{CO}_{2}$. Cells were kept growing in log phase and passaged when reaching $70-85 \%$ con- 
fluence by detaching cells with $0.25 \%$ trypsin-EDTA (ThermoFischer Scientific, Inc.; 25200-056).

\section{$3 D$ culture in MatrigelTM}

For gel droplets, $10 \mu \mathrm{L}$ single-cell suspension (100,000 cells/mL high-glucose DMEM (GE, SH3024.01)) was mixed with 10-15 $\mu \mathrm{L}$ Matrigel ${ }^{\mathrm{TM}}$ Basement membrane Matrix, growth factor reduced (Becton, Dickinson and Company, Franklin Lakes, NJ, USA; 354230) and $10 \mu \mathrm{L}$ droplets were placed on an 8-well Lab-Tek ${ }^{\mathrm{TM}}$ Chambered Coverglasses (ThermoFischer Scientific, Inc.; 15411). For "sandwich gel", each well was coated with $15 \mu \mathrm{L}$ MatrigelTM and solidified at $37{ }^{\circ} \mathrm{C}$. Next, $100 \mu \mathrm{L}$ cell suspension was mixed with $150 \mu \mathrm{L}$ MatrigelTM and $100 \mu \mathrm{L}$ of the mix was added per well. For "thick gel", $200 \mu \mathrm{L}$ cell suspension was mixed with $200 \mu \mathrm{L}$ Matrigel $^{\mathrm{TM}}$ and $200 \mu \mathrm{L}$ of the mix was added per well $[30,34]$. Matrigel ${ }^{\mathrm{TM}}$ was solidified at $37{ }^{\circ} \mathrm{C}$ for $60 \mathrm{~min}$ (droplets inverted to prevent cells from sinking to the coverglass surface), after which $200 \mu \mathrm{L}$ high-glucose DMEM was added per well. After 3-5 days, cells were fixed in 4\% $(w / v)$ PFA in PBS 1x (Santa Cruz Biotechnology, Inc., Dallas, TX, USA; sc-281692) or alternatively in $2 \%(w / v)$ PFA (Santa Cruz Biotechnology, Inc., Dallas, TX, USA; sc281692) / 0.5\% (w/v) gluteraldehyde (Sigma; G7651-10 mL) in PBS 1x.

\section{$3 D$ culture in type I collagen gel}

For gel droplets, $15.6 \mu \mathrm{L}$ dd $\mathrm{H}_{2} \mathrm{O}, 7.2 \mu \mathrm{L}$ PBS $10 \mathrm{x}$ and $1.2 \mu \mathrm{L} 1 \mathrm{M} \mathrm{NaOH}$ in $\mathrm{ddH}_{2} \mathrm{O}$ was mixed with $48 \mu \mathrm{L} 3 \mathrm{mg} / \mathrm{mL}$ type I collagen (ThermoFischer Scientific, Inc.; A10483-01) and $72 \mu \mathrm{L}$ cell suspension (100,000 cells/mL high-glucose DMEM). Single $10 \mu \mathrm{L}$ droplets of the mix ( $1 \mathrm{mg} / \mathrm{mL}$ type I collagen gel containing $\sim 500$ cells) were placed on No. 1.5H microscope cover glasses (Paul Marienfeld GmbH \& Co. KG, Lauda-Königshofen, Germany; 0117580) in 12-well cell culture plates. For "sandwich gel", $660 \mu \mathrm{L}$ high-glucose DMEM containing 15\% $(v / v)$ FBS and $10 \mu \mathrm{L} 1 \mathrm{M}$ $\mathrm{NaOH}$ was mixed with $333 \mu \mathrm{L} 3 \mathrm{mg} / \mathrm{mL}$ type I collagen. Each well of an 8 -well Chambered Coverglass was covered with $15 \mu \mathrm{L}$ of the mix and the collagen polymerised at $37{ }^{\circ} \mathrm{C}$. A $2 \mathrm{mg} / \mathrm{mL}$ type I collagen solution was made by mixing $780 \mu \mathrm{L}$ DMEM containing 30\% $(v / v) \mathrm{FBS}, 50 \mu \mathrm{L} 1 \mathrm{M} \mathrm{NaOH}$ and $1.67 \mathrm{~mL} \mathrm{3 \%}$ type I collagen, of which $100 \mu \mathrm{L}$ was mixed with $100 \mu \mathrm{L}$ cell suspension. Eighty $\mu \mathrm{L}$ of the final mix was added per well. The gels were incubated at $37{ }^{\circ} \mathrm{C}$ for the collagen to polymerise (droplets inverted for the first 5 min to prevent the sinking of cells), after which $1 \mathrm{~mL}$ (for 12-well plate) or $200 \mu \mathrm{L}$ (for 8-well chamber slide) DMEM (high glucose) was added. After 3-14 days, cells were fixed in 4\% (w/v) PFA in PBS $1 \mathrm{x}$ containing 5\% $(w / v)$ sucrose (Kanto Chemical Co., Tokyo, Japan; 37000-00) [42, 47]. 


\section{Brightfield and immunofluorescence microscopy}

After fixation, cells were incubated with $0.2 \mathrm{M}$ glycine (1 ${ }^{\text {st }}$ BASE, Singapore, Singa-

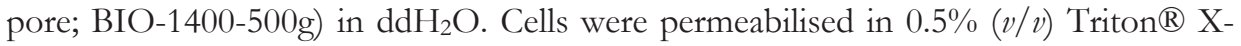
100 (Promega, Madison, WI, USA; H5141) in PBS 1x and blocked with Image-iT® FX Signal Enhancer (ThermoFischer Scientific, Inc.; R37107). Cells were incubated with a custom-made anti-MMP14 primary antibody (Eurogentec, Seraing, Belgium; SY53376) at 1:100 dilution overnight at $4{ }^{\circ} \mathrm{C}$ and Alexa-fluorophore labelled secondary antibodies and Alexa-fluorophore labelled phalloidin (ThermoFischer Scientific, Inc.; 1:200 and 1:100 dilution, respectively) for $1 \mathrm{~h}$ at RT, all dissolved in 3\% (w/v) bovine serum albumin (BSA; MP Biomedicals, Santa Ana, CA, USA; 0219989880) / 0.05\% Tween-20 (Promega; H5151) in PBS 1x. Gels were covered in $100 \mu \mathrm{L}$ Vectashield antifade mounting medium containing 4',6-diamidino-2-phenylindole (DAPI) (Vector Laboratories, Burlingame, CA, USA; H-1200). Gel droplets on cover slips were mounted on microscope slides with 15-18 mm wide, 0.6-0.8 $\mathrm{mm}$ deep cavity (Paul Marienfeld GmbH \& Co. KG, Lauda-Königshofen, Germany; 1320000) filled with 70 $\mu \mathrm{L}$ Vectashield with DAPI. Fluorescent z-stacks of organoids were recorded with an Olympus IX81 microscope with FluoView FV1000 scan head (Olympus Corporation, Tokyo, Japan). Images of randomly selected organoids that were entirely within focal reach were taken with a $63 \mathrm{X} 1.45 \mathrm{NA}$ or $40 \mathrm{X} 1.30 \mathrm{NA}$ oil immersion Plan Apochromat objective (Olympus Corporation, Tokyo, Japan), with fixed laser power and exposure time per experiment. Brightfield images of organoids in Matrigel ${ }^{\mathrm{TM}}$ were taken with an Olympus IX81, brightfield images of organoids in type I collagen gel were taken with a Zeiss Axiovert 200 M (Carl Zeiss EG, Oberkochen, Germany). Raw images were processed with Fiji software (U. S. National Institutes of Health, Bethesda, MD, USA; version 2.0.0-rc-15/1.49k, adapted by IMU).

Table 3.1 - Overview of the TriFECTA® DsiRNA duplex oligo-RNA sequences used.

\begin{tabular}{|c|c|c|c|}
\hline Gene & Set & Reference & Sequence \\
\hline \multirow[t]{3}{*}{ Mmp14 } & 1 & 121663819 & $\begin{array}{l}\text { oligo 1: 5'-GGGAAACAAAUACUGGAAGUUCAAC-3' } \\
\text { oligo 2: 3'-UUCCCUUUGUUUAUGACCUUCAAGUUG-5' }\end{array}$ \\
\hline & 2 & 121663816 & $\begin{array}{l}\text { oligo 1: 5'-AGUUUACCCUAAAGACUUUGAUAAG-3' } \\
\text { oligo 2: 3'-AGUCAAAUGGGAUUUCUGAAACUAUUC-5', }\end{array}$ \\
\hline & 3 & 121663813 & $\begin{array}{l}\text { oligo 1: 5'-CCAAGUGAUGGAUGGAUACCCAATG-3' } \\
\text { oligo 2: 3'-UUGGUUCACUACCUACCUAUGGGUUAC-5' }\end{array}$ \\
\hline \multirow[t]{3}{*}{$\int h 3 p x d 2 b$} & 1 & 121663833 & $\begin{array}{l}\text { oligo 1: 5'-GCAAAGAAAUGUUACAGUUCUGATT-3' } \\
\text { oligo 2: 3'-GUCGUUUCUUUACAAUGUCAAGACUAA-5', }\end{array}$ \\
\hline & 2 & 121663830 & $\begin{array}{l}\text { oligo 1: 5'-GGCUUAUUACAGCAAUAAACAAUTC-3' } \\
\text { oligo 2: 3'-GUCCGAAUAAUGUCGUUAUUUGUUAAG-5', }\end{array}$ \\
\hline & 3 & 121663827 & $\begin{array}{l}\text { oligo 1: 5'-GGACCACCUCAAUACAUAAUGGGAA-3' } \\
\text { oligo 2: 3'-UCCCUGGUGGAGUUAUGUAUUACCCUU-5', }\end{array}$ \\
\hline
\end{tabular}




\section{Retinoid treatment}

After cells were seeded in Matrigel ${ }^{\mathrm{TM}}$ or type I collagen gel, 5-24 nM 9-cis or 13-cis retinoic acid (Sigma; R3255-100MG) in 0.75-3.6\% ethanol, or 3.6\% $(v / v)$ ethanol (Merck; 1.00983.2500DE) was added to the medium, or left untreated.

siRNA knockdown of Mmp14 and Sh3pxd2b

For KD experiments, cells were seeded at a density of 100,000 per well of a 6-well plate and transfected $24 \mathrm{~h}$ post seeding with 100 pmol mouse TriFECTa ${ }^{\circledR}$ DsiRNA (Integrated DNA Technologies, Coralville, Iowa, USA) raised against Mmp14 (NM_008608) or Sh3pxd2b (NM_177364) as listed in Table 3.1, or siControl, with jetPRIME® (Polyplus-transfection ${ }^{\circledR}$ SA, Illkirch, France; 114-15), or were left untreated. Media was refreshed 5 h post transfection.

\section{qPCR analysis of siRNA knockdown}

Twenty-four hours post transfection, cells were incubated with Trizol (ThermoFischer Scientific, Inc.; 15596018), and total mRNA was chloroform/isopropanol precipitated and rinsed ice-cold 70\% $(v / v)$ ethanol (Merck; 1.00983.2500) in nuclease-free $\mathrm{dH}_{2} \mathrm{O}$

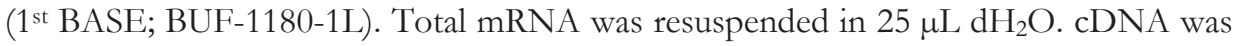
synthesised by iScript cDNA Synthesis Kit according to the manufacturer's protocol (Biorad; 1708898). qPCR was performed with iQ ${ }^{\mathrm{TM}}$ SYBR ${ }^{\circledR}$ Green Supermix (Biorad; $1708882 \mathrm{AP})$ in technical duplicates according to the manufacturer's protocol with a CFX96 Touch ${ }^{\text {TM }}$ Real-Time PCR Detection System (Biorad; 1855196). The primers used are summarised in Table 3.2. Expression of $M m p 14$ and $S h 3 p x d 2 b$ were normalised for Hprt expression and denoted as the relative fold-change to siControl.

Table 3.2 - Primers used for qPCR. Hprt, hypxanthine guanine phosphoribosyl transferase.

\begin{tabular}{|c|c|c|c|}
\hline Gene & \# & Sequence & $\operatorname{Tm}\left({ }^{\circ} \mathrm{C}\right)$ \\
\hline \multirow[t]{4}{*}{$\overline{M m p 14}$} & $\overline{1 F}$ & 5'-ACT'TCGTGT'TGCCTGATGAC-3' & $\overline{55.4}$ \\
\hline & $1 \mathrm{R}$ & 5'-CGAGAGGTAGT'TCTGGGTTG-3' & 54.7 \\
\hline & $2 \mathrm{~F}$ & 5'AGTGACAGGCAAGGCTGATTT-3' & 57.1 \\
\hline & $2 \mathrm{R}$ & 5'-AGGGGTGTAATTCTGAATGCAG-3' & 55.0 \\
\hline \multirow[t]{4}{*}{$\operatorname{Sh} 3 p x d 2 b$} & $1 \mathrm{~F}$ & 5'-CCATCTACCGGCGCTACAG-3' & 57.7 \\
\hline & $1 \mathrm{R}$ & 5'-GATCCTCTGCT'TGGGGTCT'T-3' & 56.7 \\
\hline & $2 \mathrm{~F}$ & 5'-CTATGGGCAGGGAGAAGGAG-3' & 56.8 \\
\hline & $2 \mathrm{R}$ & 5’-GGGGTCTTTGCCTCATCTTT-3’ & 55.1 \\
\hline \multirow[t]{2}{*}{ Hprt } & $\mathrm{F}$ & 5'-CTGGTGAAAAGGACCTCTCGAA-3' & 56.6 \\
\hline & $\mathrm{R}$ & 5'-CTGAAGTACTCATTATAGTCAAGGGCA-3' & 56.0 \\
\hline
\end{tabular}




\section{Supplemental material}

Supplemental material is available in Addendum 2.

\section{Acknowledgements}

The authors acknowledge the invaluable assistance Dr. Graham Wright and Dr. Shuping Lin (Institute of Medical Biology, Microscopy Unit), and Dr. Tijs Claessens (Maastricht University Medical Center+). This work was supported by the Skin Research Institute of Singapore (to M.A.M.v.S. and S.Y.Y.), the Biomedical Research Council Singapore (to M.A.M.v.S.), the Agency for Science, Technology and Research (A*STAR Research Attachment Programme to I.J.H.M.d.V.), the Wellcome Trust (DGEM to M.A.M.v.S. and B.J.C.), and Tenovus Scotland (T15/22 and T15/62 to M.A.M.v.S. and B.J.C.).

\section{References}

1. Bhate K, Williams HC. Epidemiology of acne vulgaris. The British Journal of Dermatology. 2013;168(3):474-85.

2. Williams HC, Dellavalle RP, Garner S. Acne vulgaris. Lancet (London, England). 2012;379(9813):361-72.

3. Chen W, Obermayer-Pietsch B, Hong JB, Melnik BC, Yamasaki O, Dessinioti C, et al. Acneassociated syndromes: models for better understanding of acne pathogenesis. Journal of the European Academy of Dermatology and Venereology. 2011;25(6):637-46.

4. Melnik BC, John SM, Schmitz G. Over-stimulation of insulin/IGF-1 signaling by western diet may promote diseases of civilization: lessons learnt from laron syndrome. Nutrition \& Metabolism. 2011;8:41.

5. Humphrey S. Antibiotic resistance in acne treatment. Skin Therapy Letter. 2012;17(9):1-3.

6. Dawson AL, Dellavalle RP. Acne vulgaris. BMJ. 2013;346:f2634.

7. Simpson RC, Grindlay DJ, Williams HC. What's new in acne? An analysis of systematic reviews and clinically significant trials published in 2010-11. Clinical and Experimental Dermatology. 2011;36(8):840-3; quiz 3-4.

8. Frank Y, Ziprkowski M, Romano A, Stein R, Katznelson MB, Cohen B, et al. Megalocornea associated with multiple skeletal anomalies: a new genetic syndrome? Journal de Genetique Humaine. 1973;21(2):67-72.

9. Ter Haar B, Hamel B, Hendriks J, de Jager J. Melnick-Needles syndrome: indication for an autosomal recessive form. American Journal of Medical Genetics. 1982;13(4):469-77.

10. Borrone C, Di Rocco M, Crovato F, Camera G, Gambini C. New multisystemic disorder involving heart valves, skin, bones, and joints in two brothers. American Journal of Medical Genetics. 1993;46(2):228-34.

11. Wilson GR, Sunley J, Smith KR, Pope K, Bromhead CJ, Fitzpatrick E, et al. Mutations in SH3PXD2B cause Borrone dermato-cardio-skeletal syndrome. European Journal of Human Genetics. 2014;22(6):741-7.

12. Iqbal Z, Cejudo-Martin P, de Brouwer A, van der Zwaag B, Ruiz-Lozano P, Scimia MC, et al. Disruption of the podosome adaptor protein TKS4 (SH3PXD2B) causes the skeletal dysplasia, eye, and cardiac abnormalities of Frank-Ter Haar Syndrome. American Journal of Human Genetics. 2010;86(2):254-61. 
13. Bendon CL, Fenwick AL, Hurst JA, Nurnberg G, Nurnberg P, Wall SA, et al. Frank-ter Haar syndrome associated with sagittal craniosynostosis and raised intracranial pressure. BMC Medical Genetics. 2012;13:104.

14. Van Steensel MA, Ceulen RP, Delhaas T, de Die-Smulders C. Two Dutch brothers with Borrone dermato-cardio-skeletal syndrome. American Journal of Medical Genetics Part A. 2007;143a(11):1223-6.

15. De Vos IJHM, Tao EY, Ong SLM, Goggi JL, Scerri T et al. Functional analysis of a hypomorphic allele shows that MMP14 catalytic activity is the prime determinant of the Winchester syndrome phenotype. Human Molecular Genetics. 2018; 27(16):2775-2788.

16. Evans BR, Mosig RA, Lobl M, Martignetti CR, Camacho C, Grum-Tokars V, et al. Mutation of membrane type-1 metalloproteinase, MT1-MMP, causes the multicentric osteolysis and arthritis disease Winchester syndrome. American Journal of Human Genetics. 2012;91(3):572-6.

17. Winchester P, Grossman H, Lim WN, Danes BS. A new acid mucopolysaccharidosis with skeletal deformities simulating rheumatoid arthritis. The American Journal of Roentgenology, Radium Therapy, and Nuclear Medicine. 1969;106(1):121-8.

18. Prapanpoch S, Jorgenson RJ, Langlais RP, Nummikoski PV. Winchester syndrome. A case report and literature review. Oral Surgery, Oral Medicine, and Oral Pathology. 1992;74(5):671-7.

19. Murphy DA, Courtneidge SA. The 'ins' and 'outs' of podosomes and invadopodia: characteristics, formation and function. Nature Reviews Molecular Cell Biology. 2011;12(7):413-26.

20. Buschman MD, Bromann PA, Cejudo-Martin P, Wen F, Pass I, Courtneidge SA. The novel adaptor protein Tks4 (SH3PXD2B) is required for functional podosome formation. Molecular Biology of the Cell. 2009;20(5):1302-11.

21. Poincloux R, Lizarraga F, Chavrier P. Matrix invasion by tumour cells: a focus on MT1-MMP trafficking to invadopodia. Journal of Cell Science. 2009;122(Pt 17):3015-24.

22. Sidwell RU, Brueton LA, Grabczynska SA, Francis N, Staughton RC. Progressive multilayered banded skin in Winchester syndrome. Journal of the American Academy of Dermatology. 2004;50(2 Suppl):S53-6.

23. Kim HY, Nelson CM. Extracellular matrix and cytoskeletal dynamics during branching morphogenesis. Organogenesis. 2012;8(2):56-64.

24. Sabeh F, Ota I, Holmbeck K, Birkedal-Hansen H, Soloway P, Balbin M, et al. Tumor cell traffic through the extracellular matrix is controlled by the membrane-anchored collagenase MT1-MMP. The Journal of Cell Biology. 2004;167(4):769-81.

25. Hotary K, Allen E, Punturieri A, Yana I, Weiss SJ. Regulation of cell invasion and morphogenesis in a three-dimensional type I collagen matrix by membrane-type matrix metalloproteinases 1,2 , and 3 . The Journal of Cell Biology. 2000;149(6):1309-23.

26. Chun TH, Sabeh F, Ota I, Murphy H, McDonagh KT, Holmbeck K, et al. MT1-MMP-dependent neovessel formation within the confines of the three-dimensional extracellular matrix. The Journal of Cell Biology. 2004;167(4):757-67.

27. Zhou Z, Apte SS, Soininen R, Cao R, Baaklini GY, Rauser RW, et al. Impaired endochondral ossification and angiogenesis in mice deficient in membrane-type matrix metalloproteinase I. Proceedings of the National Academy of Sciences of the United States of America. 2000;97(8):4052-7.

28. Happe H, de Heer E, Peters DJ. Polycystic kidney disease: the complexity of planar cell polarity and signaling during tissue regeneration and cyst formation. Biochimica et Biophysica Acta. 2011;1812(10):1249-55.

29. Williams BB, Cantrell VA, Mundell NA, Bennett AC, Quick RE, Jessen JR. VANGL2 regulates membrane trafficking of MMP14 to control cell polarity and migration. Journal of Cell Science. 2012;125(Pt 9):2141-7.

30. Giles RH, Ajzenberg H, Jackson PK. 3D spheroid model of mIMCD3 cells for studying ciliopathies and renal epithelial disorders. Nature Protocols. 2014;9(12):2725-31. 
31. Sigurbjornsdottir S, Mathew R, Leptin M. Molecular mechanisms of de novo lumen formation. Nature Reviews Molecular Cell Biology. 2014;15(10):665-76.

32. Rauchman MI, Nigam SK, Delpire E, Gullans SR. An osmotically tolerant inner medullary collecting duct cell line from an SV40 transgenic mouse. The American Journal of Physiology. 1993;265(3 Pt 2):F416-24.

33. Barros EJ, Santos OF, Matsumoto K, Nakamura T, Nigam SK. Differential tubulogenic and branching morphogenetic activities of growth factors: implications for epithelial tissue development. Proceedings of the National Academy of Sciences of the United States of America. 1995;92(10):4412-6.

34. Luijten MN, Basten SG, Claessens T, Vernooij M, Scott CL, Janssen R, et al. Birt-Hogg-Dube syndrome is a novel ciliopathy. Human Molecular Genetics. 2013;22(21):4383-97.

35. Li L, Black R, Ma Z, Yang Q, Wang A, Lin F. Use of mouse hematopoietic stem and progenitor cells to treat acute kidney injury. American journal of physiology Renal physiology. 2012;302(1):F9-f19.

36. Watt FM. Mammalian skin cell biology: at the interface between laboratory and clinic. Science. 2014;346(6212):937-40.

37. Debnath J, Brugge JS. Modelling glandular epithelial cancers in three-dimensional cultures. Nature Reviews Cancer. 2005;5(9):675-88.

38. Datta A, Bryant DM, Mostov KE. Molecular regulation of lumen morphogenesis. Current Biology. 2011;21(3):R126-36.

39. Lubarsky B, Krasnow MA. Tube morphogenesis: making and shaping biological tubes. Cell. 2003;112(1):19-28.

40. Marciano DK. A holey pursuit: lumen formation in the developing kidney. Pediatric Nephrology. 2017;32(1):7-20.

41. Andrew DJ, Ewald AJ. Morphogenesis of epithelial tubes: Insights into tube formation, elongation, and elaboration. Developmental Biology. 2010;341(1):34-55.

42. Leung-Hagesteijn C, Hu MC, Mahendra AS, Hartwig S, Klamut HJ, Rosenblum ND, et al. Integrinlinked kinase mediates bone morphogenetic protein 7-dependent renal epithelial cell morphogenesis. Molecular and Cellular Biology. 2005;25(9):3648-57.

43. Piscione TD, Yager TD, Gupta IR, Grinfeld B, Pei Y, Attisano L, et al. BMP-2 and OP-1 exert direct and opposite effects on renal branching morphogenesis. The American Journal of Physiology. 1997;273(6 Pt 2):F961-75.

44. Chen D, Chen Z, Zhang Y, Park C, Al-Omari A, Moeckel GW. Role of medullary progenitor cells in epithelial cell migration and proliferation. American Journal of Physiology Renal Physiology. 2014;307(1):F64-74.

45. Bissell MJ, Weaver VM, Lelievre SA, Wang F, Petersen OW, Schmeichel KL. Tissue structure, nuclear organization, and gene expression in normal and malignant breast. Cancer Research. 1999;59(7 Suppl):1757-63s; discussion 63s-64s.

46. Mori H, Lo AT, Inman JL, Alcaraz J, Ghajar CM, Mott JD, et al. Transmembrane/cytoplasmic, rather than catalytic, domains of Mmp14 signal to MAPK activation and mammary branching morphogenesis via binding to integrin beta1. Development. 2013;140(2):343-52.

47. Artym VV, Matsumoto KK. Imaging cells in three-dimensional collagen matrix. Current Protocols in Cell Biology. 2010 Sep;Chapter 10:Unit 10.18.1-20.

48. Pohl M, Sakurai H, Bush KT, Nigam SK. Matrix metalloproteinases and their inhibitors regulate in vitro ureteric bud branching morphogenesis. American Journal of Physiology Renal Physiology. 2000;279(5):F891-900.

49. Riggins KS, Mernaugh G, Su Y, Quaranta V, Koshikawa N, Seiki M, et al. MT1-MMP-mediated basement membrane remodeling modulates renal development. Experimental Cell Research. 2010;316(17):2993-3005.

50. Oblander SA, Zhou Z, Galvez BG, Starcher B, Shannon JM, Durbeej M, et al. Distinctive functions of membrane type 1 matrix-metalloprotease (MT1-MMP or MMP-14) in lung and submandibular 
gland development are independent of its role in pro-MMP-2 activation. Developmental Biology. 2005;277(1):255-69.

51. Hotary KB, Allen ED, Brooks PC, Datta NS, Long MW, Weiss SJ. Membrane type I matrix metalloproteinase usurps tumor growth control imposed by the three-dimensional extracellular matrix. Cell. 2003;114(1):33-45.

52. Quinlan J, Kaplan F, Sweezey N, Goodyer P. LGL1, a novel branching morphogen in developing kidney, is induced by retinoic acid. American Journal of Physiology Renal Physiology. 2007;293(4):F987-93.

53. Wright DM, Buenger DE, Abashev TM, Lindeman RP, Ding J, Sandell LL. Retinoic acid regulates embryonic development of mammalian submandibular salivary glands. Developmental Biology. 2015;407(1):57-67.

54. Takayama M, Miyatake K, Nishida E. Identification and characterization of retinoic acid-responsive genes in mouse kidney development. Genes to Cells. 2014;19(8):637-49.

55. Ganceviciene R, Zouboulis CC. Isotretinoin: state of the art treatment for acne vulgaris. Journal der Deutschen Dermatologischen Gesellschaft. 2010;8 Suppl 1:S47-59.

56. Verfaille CJ, Borgers M, van Steensel MA. Retinoic acid metabolism blocking agents (RAMBAs): a new paradigm in the treatment of hyperkeratotic disorders. Journal der Deutschen Dermatologischen Gesellschaft. 2008;6(5):355-64.

57. Melnik BC, Schmitz G, Zouboulis CC. Anti-acne agents attenuate FGFR2 signal transduction in acne. The Journal of Investigative Dermatology. 2009;129(8):1868-77.

58. O'Neill RP, Jones SJ, Boyde A, Taylor ML, Arnett TR. Effect of retinoic acid on the resorptive activity of chick osteoclasts in vitro. Bone. 1992;13(1):23-7.

59. Zouboulis CC. Isotretinoin revisited: pluripotent effects on human sebaceous gland cells. The Journal of Investigative Dermatology. 2006;126(10):2154-6. 



\section{CHAP'T'ER 4 \\ A zebrafish model for Winchester syndrome}

Adapted from:

Ivo J.H.M. de Vos, Evelyn Yaqiong Tao, Sheena Li Ming Ong, Julian L. Goggi, Thomas Scerri, Gabrielle R. Wilson, Chernis Guai Mun Low, Arnette Shi Wei Wong, Dominic Grussu, Alexander P.A. Stegmann, Michel van Geel, Renske Janssen, David J. Amor, Melanie Bahlo, Norris R. Dunn, Thomas J. Carney, Paul J. Lockhart, Barry J. Coull and Maurice A.M. van Steensel. Functional analysis of a hypomorphic allele shows that MMP14 catalytic activity is the prime determinant of the Winchester syndrome phenotype. Human Molecular Genetics 2018; 27(16):2775-2788

Ivo J.H.M. de Vos, Sheena Li Ming Ong, John Lim, Julian Goggi, Chee Bing Ong, Barry J. Coull, Norris R. Dunn, Thomas J. Carney and Maurice A.M. van Steensel. A zebrafish model for Winchester syndrome. Mechanisms of Development. 2017;145:S28 


\section{Abstract}

Winchester syndrome is an ultra-rare, autosomal recessive skeletal dysplasia caused by deleterious mutations in the gene encoding MMP14, a membrane-bound metalloproteinase. So far, only two human MMP14 missense mutations have been reported; another two were identified in mouse models. Although the resulting phenotype varies in severity, humans, and mice share stunted growth, craniofacial malformations and drastically reduced bone mineral density leading to pervasive deformities including kyphosis. The reasons for the craniofacial malformations and the osteopenia are insufficiently understood, and treatment, therefore, is a hit-and-miss affair. Here, we report a novel mmp14a/b knockout zebrafish model that recapitulates essential aspects of the Winchester syndrome phenotype including craniofacial malformations, kyphosis, short-stature, and reduced bone density. In our fish model, we found that cranial neural crest induction, delamination, migration and homing to the pharyngeal arches, as well as early steps in skeletogenesis, were unaffected by mmp14a/ $b$ knockout. Instead, skeletal remodelling turned out to be abnormal, showing similarities with previously reported Mmp14 knockout mice and owing to defective collagen remodelling. The fish will be a valuable tool for developing novel therapeutic approaches to this devastating disorder, as well as reduced bone density in general. 


\section{Introduction}

Winchester syndrome (WS, MIM \#277950) is an extremely rare autosomal recessive skeletal dysplasia, caused by mutations in the gene encoding membrane-bound metalloproteinase 14 (MMP14) [1, 2]. To date, four missense mutations in conserved regions are known in patients and mouse models, all affecting protein function. In both humans and mice, these mutations lead to short stature, progressive osteopenia, thoracic kyphosis and craniofacial malformations including (Fig. 4.1A) [1-5]. Interestingly, the majority of the affected craniofacial skeletal elements is of neural crest (NC) origin (Fig. 4.1B) [6]. The NC comprises a population of multipotent stem cells that is induced at the lateral borders of the neural plate during embryogenesis. These cells delaminate from the closing neural tube and subsequently migrate extensively to many parts of the developing body, where they differentiate and give rise to various derivatives. For cranial NC cells, this includes major parts of the skull $[6,7]$. It seems therefore likely that the cranial NC is involved in the MMP14-related craniofacial phenotype, however, it is unknown which aspect of NC development would be affected. MMP14 has an important role in invasive cell motility by locally degrading structural components of the extracellular matrix (ECM) [8-13]. As NC cells migrate extensively, we hypothesized that loss of MMP14 function could lead to craniofacial malformations (at least in part) due to impaired cranial NC cell migration. In addition, it is unclear how the loss of MMP14 function causes a general reduction in bone density. These questions are best addressed in an in vivo model.

A well-established model organism for vertebrate development is the zebrafish (Danio rerio), a bony fish that has been extensively used for genetic studies [19]. Bone has only been introduced once during evolution and the characteristics of skeletal tissues are
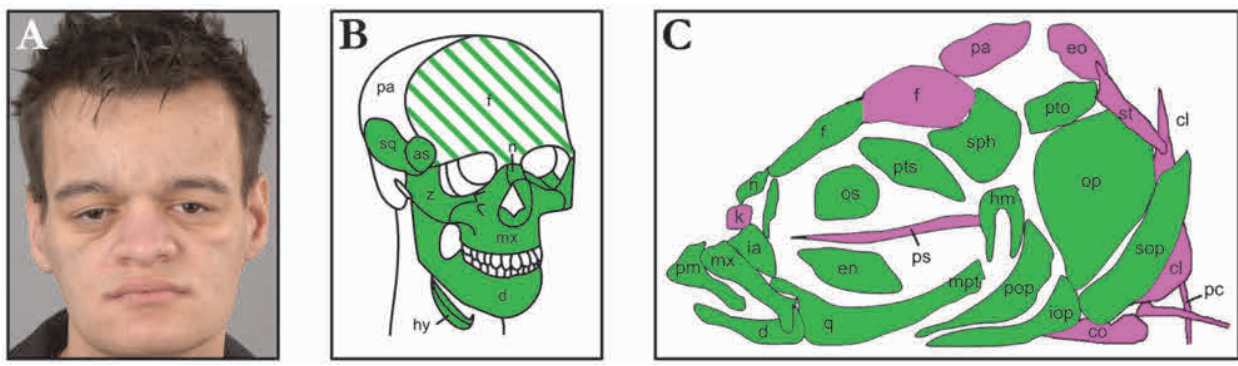

Figure 4.1 - Craniofacial skeletal elements affected in Winchester syndrome are of neural crest origin. A, facial appearance of a Winchester syndrome patient, with a high and broad forehead, hypertelorism, and a prominent lower jaw. B, the affected craniofacial skeletal elements are of neural crest (NC) origin (green). The frontal bone (f) is of mixed NC and mesodermal origin. $\mathbf{C}$, in zebrafish, as in humans, the majority of craniofacial skeletal elements are of neural crest origin (green). Note that the anterior part of the frontal bone is of neural crest origin, whereas the posterior part is of mesodermal origin (magenta). As, alisphenoid; sq, squamosal; hy, hyoid; z, zygomatic bone. See Table 4.9 for additional abbreviations of skeletal elements. A is reproduced from Van Steensel et al., B is adapted from Santagati and Rijli, and C is reproduced from Kague et al., all with permission [3, 6, 14]. 


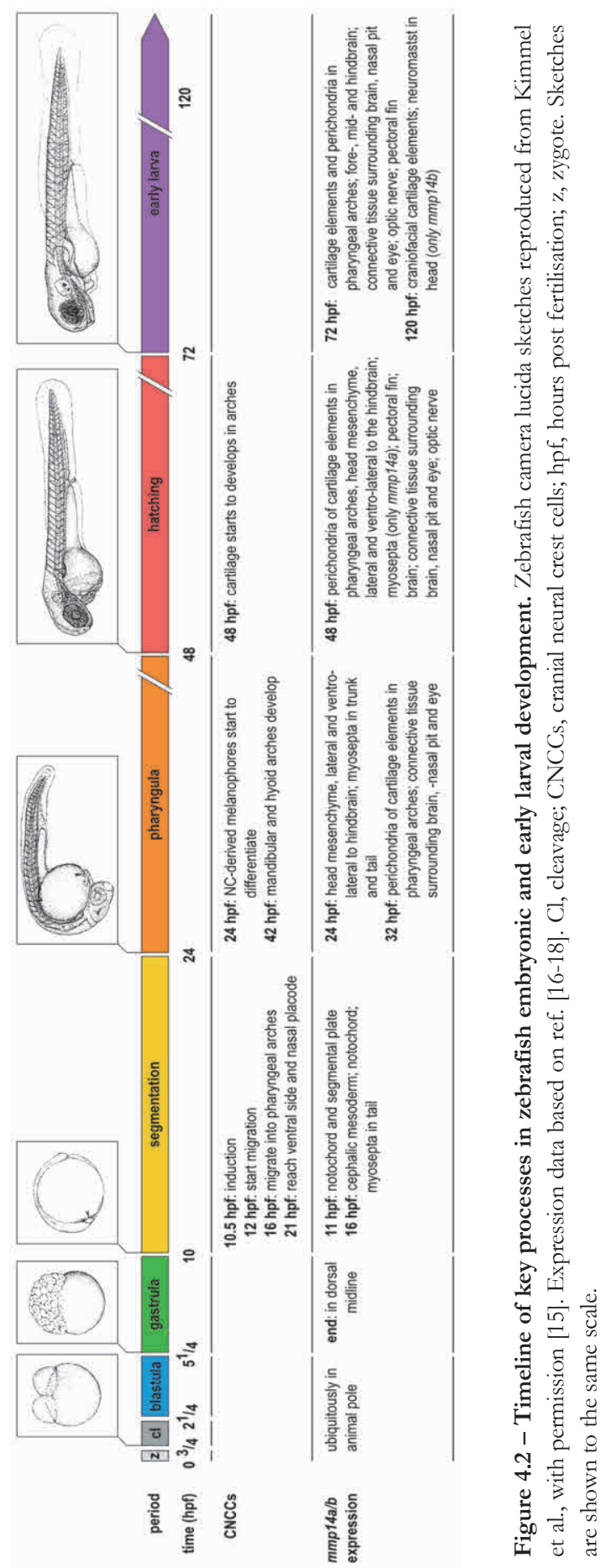


well conserved among vertebrates [19, 20]. Zebrafish form all major types of skeletal cells and tissues found in higher vertebrates including humans [20-22]. Furthermore, bone formation and remodelling is similar in zebrafish and humans, and the pathways regulating these processes are well conserved [19, 20, 22, 23]. Although the zebrafish skull is more complex, it contains similar skeletal elements as higher vertebrates, which furthermore develop in similar manner [21,22]. As in humans, the cranial NC contributes to major parts of the zebrafish skull (Fig. 4.1C), giving rise to chondroblasts, chondrocytes, and osteoblasts of many craniofacial skeletal elements [14, 24]. As such, the zebrafish is a good model organism for human bone disorders, especially those affecting the craniofacial skeletal elements such as WS.

The use of zebrafish has important advantages over other model organisms such as mice. Zebrafish are relatively cheap to house, easy to handle and readily produce large clutches of offspring, improving the statistical power of experiments [19, 21, 25]. Furthermore, zebrafish embryos develop externally, making them readily available for use in experiments $[19,26]$. In addition, the zebrafish embryonic development proceeds rapidly. By $24 \mathrm{~h}$ post fertilization (hpf), the embryo has a recognizable head and tail and contains rudiments of primary organs (Fig. 4.2) [15]. The NC cells are induced, and delaminate and migrate during the first day. The NC-derived melanophores differentiate only after $24 \mathrm{hpf}$; as a result, the zebrafish embryo is completely transparent until the majority of NC migration is completed [15]. A transgenic background expressing a fluorescent signal under sox10 promoter, a marker of NC cells, can therefore be used to visualise NC cells in vivo $[14,27,28]$. During the second day, the pharyngeal arches develop and on the third day, cartilage starts to develop in the chondrocranium, the jaws, and the branchial arches, and the first bone (cleithrum) appears. Embryos hatch sporadically from the chorion, mostly during the third day. [15]. After $72 \mathrm{hpf}$, the larvae start to swim actively. During the larval period (up to 30 days post fertilization, dpf), cartilage development continues and ossification of the skeletal elements proceeds in an anterior-to-posterior sequence as part of the larval-to-juvenile metamorphosis [26, 29]. All of the aforementioned characteristics make the zebrafish highly suitable to study both NC migration and craniofacial bone development.

Zebrafish express 25 matrix metalloproteinases. However, their set of Mmps is less complex compared to humans. Firstly, there are no orthologs of many human MMPs $(1,3,7,8,10,12,26$ and 27). Secondly, zebrafish express duplicates of many Mmps due to genomic duplication during their evolution [33]. As such, zebrafish express two paralogs of Mmp14: Mmp14a and Mmp14b [32-34]. Both paralogs are well conserved, with an amino acid sequence that is $>80 \%$ identical to human MMP14 (Fig. 4.3A), and a similar domain structure (Fig. 4.3B) [32]. Importantly, similar to the expression pattern of MMP14 in mice, both Mmp14a and Mmp14b are expressed in the zebrafish head mesenchyme during embryonic and larval development, (Fig. 4.2) [16-18, 35, 36]. As we have demonstrated that all four MMP14 missense mutations known in humans 
A

MMP14
MSPAPRPPRC

MMP14

Mmp14a

Mmp14b

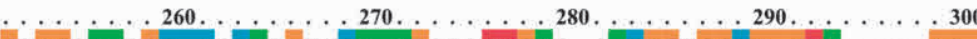
SDPSAIMAPF YQWMDTENFV LPDDDRRGIQ QLYGGESGFP TKM- ... . PP GDPSAIMAPF YQWMDTENFV LPEDDRRGIQ QIYGAGSEDK PQP...... NDPSAIMAPF YQWMETDEFV LPDDDRKGIQ KLYGPGSGGH PRPPV- - SP

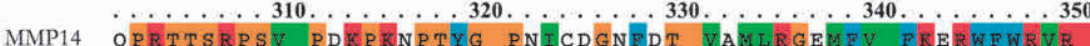
Mmp14a -...PAPRPP TRTPDRPSFG PDICEGHFDT IAFLRGEMFV FKEKWFWRVR MmP14b ETPHHTPYPT PYRPGGPSYG PNICEGHEDT IGIFRGEMFV FKGKWFWRVR MMP14 NNQVMDGYPM PIGQFWRGLP ASINTAYER DGKFVFFKGD KHWVFDEA Mmp14a DGKPQQGYPM PIGHFWKGLP PSINAAYERN DGKFVFFKGD KYWVFNEAKM Mmp14b NNQVMENYPM PIGHFWRGLP TDINAAYERE DGKFVFFRGD RHWVFTESNL

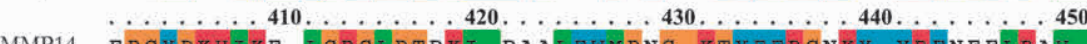
MMP14 EPGYPKHIKE LGRGLPTDKI DAALFWMPG KTYFFRGNKY YRFNEELRAV Mmp14a EEGYPKTFKE LGTGLPRDKL DAAIFYTPTG NTYFFRGTRY YRFNEESRSV MmP14b EPGYPKVLGE LGTGVPKDKL DAALLYTPTG YTYFFRGNKY YRYNEDTHSV

MMP14 DSEYPXNIKV WEGIPESPRG SFMGSDEVFT YFYKGNXYWR FNNQKIXVEP Mmp14a DSDYPRDIGV WQGVPDNVKG AFMSEDGANA YFYKANKYWK FNNQQLKVEP MmP14b DPDYPKPISK WQGVPDNIKA AFMSRDQGYT YFYKANKYWK FNNQLLKVEP

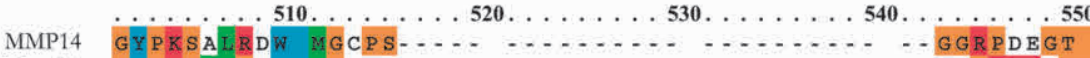

Mmp14a GRPKSVLTNW MGCEA... ............. . ERPKRRAG

Mmp14b GYPKSALKDW MGCPNEDSNT GGGGSDRDE RERERERER REREARERE

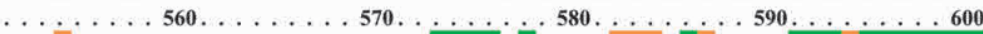

MMP14 EEET-............. - EVIIIEVDE EGGGAVSAAA VVLPVLLLLL

Mmp14a TDEE...................... VIIEVDG EGGAMGGAAA IVIPLFLIAC

Mmp14b REQDRTNEVD RTEERGKRE TEVLIIEVED APSSRgGAAA VVVPLMLLVC

MMP14

Mmp14a

Mmp14b

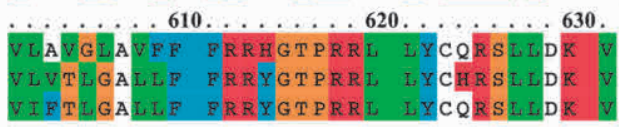

\section{B}

\begin{tabular}{|c|c|c|c|c|c|c|c|c|c|}
\hline MMP14 N SP & Pro & Catalytic & $\mathrm{Hi}$ & $\mathrm{Hx} 1$ & $\mathrm{H} \times 2$ & $\mathrm{H} \times 3$ & $\mathrm{Hx} 4$ & \begin{tabular}{|l|l|}
$\mathrm{T}$ & $\mathrm{I}$ \\
\end{tabular} & ] $\mathrm{C}$ \\
\hline Mmp14a N SP & Pro & Catalytic & $\mathrm{Hi}$ & $\mathrm{Hx} 1$ & $\mathrm{H} \times 2$ & $\mathrm{H} \times 3$ & $\mathrm{Hx} 4$ & \begin{tabular}{l|l|}
$\mathrm{T}$ & $\mathrm{I}$ \\
\end{tabular} & C \\
\hline Mmp14b N SP & Pro & Catalytic & $\mathrm{Hi}$ & $\mathrm{Hx} 1$ & $\mathrm{Hx} 2$ & $\mathrm{H} \times 3$ & $\mathrm{Hx} 4$ & St & \begin{tabular}{|l|l}
$T$ \\
\end{tabular} \\
\hline
\end{tabular}


Figure 4.3 - MMP14 is well conserved from zebrafish to human. A, alignment of human MMP14 (NP_004986.1), zebrafish Mmp14a (NP_919397.1) and zebrafish Mmp14b (NP_919395.1) amino acid sequence using the PRALINE online server, displayed in ClustalX colour scheme [30, 31]. Overall homology between MMP14 and Mmp14a/b is $>80 \%$. B, Mmp14a and Mmp14b have a similar domain structure to MMP14. Mmp14a is similar in length (574 amino acids, AA) to MMP14 (582 AA). Note the insertion in Mmp14b shown in A, resulting in a larger stalk region (St) shown in B (drawn to the same scale) and an overall larger protein (621 AA) [32]. The red dot indicates the location of catalytic zinc-ion binding residues. C, carboxyl-terminus; Hi, hinge region; Hx, hemopexin-like domain; I, cytoplasmic tail; N, aminoterminus; SP, signal peptide; T, transmembrane domain.

and mice cause loss-of-function (see Chapter 2), a knockout (KO) approach would be justified to study the role of Mmp14a/b in cranial NC migration and bone development. Previously, transient knockdown (KD) of mmp14a/ $b$ by antisense Morpholino oligonucleotides (MOs) injection of embryos during 1-4 cell stage was shown to cause gastrulation defects and to disrupt craniofacial cartilage development [16, 32]. However, it is known that MOs can cause off-target changes in gene expression, which could confound phenotypic analysis $[37,38]$. In a recent study, Kok et al. demonstrated that $\sim 80 \%$ of the analysed previously published morphant phenotypes were not recapitulated in mutant embryos [37]. One well-known off-target effect of MOs is the induction of p53-dependent apoptosis $[37,38]$. Indeed, a later study demonstrated that gastrulation defects were absent and only mild craniofacial defects occurred upon simultaneous MO KD of mmp14a and $p 53$ [17]. Although genome editing can also induce off-target mutations, these can be segregated away from the mutation of interest by outcrossing, which is impossible for MO [38]. Consequently, it has been advocated to only use MOs that result in the same phenotype as the corresponding genetic mutant $[37,38]$. As no mutant mmp14a/ $b$ fish existed, we performed targeted mutagenesis of mmp14a/ $b$ by CRISPR/Cas9 approach, aiming to knock both genes out. This furthermore enabled us to study later stages in bone remodelling, which is not possible by transient KD by MO.

The CRISPR (Clustered Regularly Interspaced Short Palindromic Repeats)/Cas (CRISPR-associated) systems were originally identified as a form of adaptive immunity of eubacteria and archaea against viral and plasmid DNA [39-41]. The type II system of $S$. pyogenes has been adapted for use as a fast and accurate genome-editing tool that has been used successfully in zebrafish in recent years [40-44]. This system only requires two components: Cas9 protein and guide RNA (gRNA). Cas9 is an endonuclease that cleaves double-stranded (ds) DNA [40-42]. Figure 4.4 demonstrates the general overview of genome editing by CRISPR/Cas9 in zebrafish. In vitro generated Cas9 mRNA and gRNA are microinjected into the yolk cell of the zygote. After entering the animal cell, Cas9 mRNA is translated into Cas9 protein and enters the nucleus together with the gRNA [42]. The gRNA guides Cas9 to the desired locus in the genomic DNA for site-specific editing. For this, the gRNA contains a Cas9 binding site and a variable 20-nucleotide (nt) protospacer sequence complementary to the target site [41, 43-45]. 
i

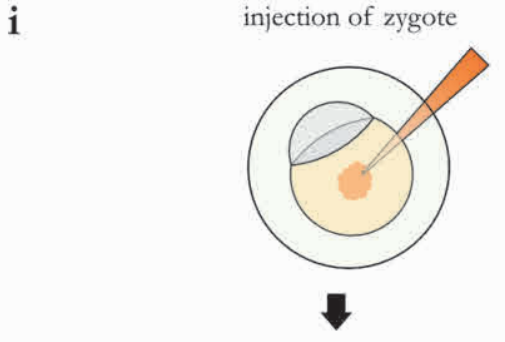

ii

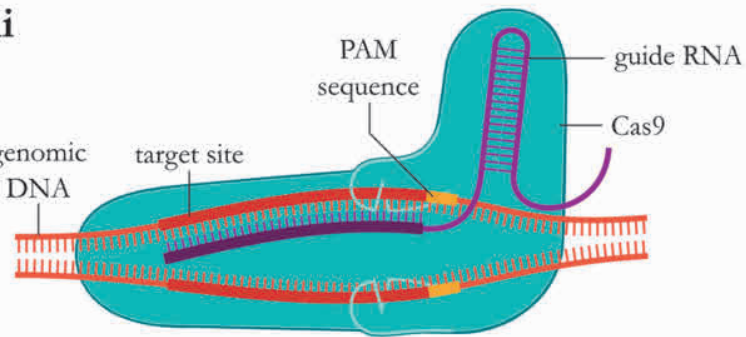

iii

double-stranded break

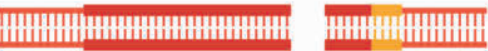

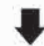

iv repair by NHEJ generates random in/del

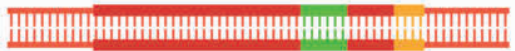

73 months

$\mathbf{v}$ $\mathrm{F}_{0}$

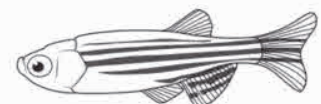

mosaic mutant (founder)

73 months

vi

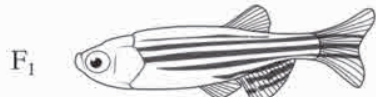

heterozygous mutant

73 months

vii

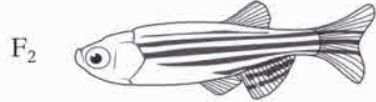

homozygous mutant
Figure 4.4 - Overview of the CRISPR approach to generate mutant fish. The yolk cell of zebrafish zygotes is injected with gRNA and Cas? RNA. Phenol red is added for easier estimation of droplet size and visualisation of successful injection (i). Both RNAs enter the animal cell, where Cas 9 RNA is translated into Cas9 protein. gRNA and Cas9 enter the nucleus, where the gRNA guides Cas9 to the target site. Here, Cas9 generates a double-stranded DNA break (ii, iii) that, in absence of a template for homology-directed repair, is repaired by nonhomologous end-joining, creating random insertions and/or deletions (iv). At the reproductive age of three months, injected fish are genotyped to select for fish in which genomic targeting has occurred. These fish are subsequently intercrossed, and part of their F1 offspring genotyped to identify F0 founders in which the germline was targeted (v). F1 fishes heterozygous for a favourable frameshift mutation leading to an immediate stop codon at/near the target site are selected (vi) and intercrossed to generate F2 homozygous mutants (vii). The entire procedure from injection until homozygous mutants takes at least nine months, and every additional crossing needed will add three months. Adapted from Charpentier et al., with permission. [42].

Here, Cas9 generates a blunt double-stranded DNA break [37, 41, 42]. In absence of template DNA, the break is repaired by error-prone non-homologous end joining (NHEJ), generating small insertions or deletions (in/dels) [42, 43]. When targeting coding sequence, this can result in a frameshift, inducing a premature termination 
codon [37]. As different mutations can occur in different cells even within the same embryo, the injected embryos are typically mosaic mutants. In a proportion of the injected embryos, the germline will be targeted (F0 founders) [37]. Further crosses lead to F1 heterozygous and F2 homozygous mutants. As zebrafish reach sexual maturity typically around $90 \mathrm{dpf}$, generating homozygous mutant adults takes at least 9 months $[26,29,44]$. When two genes need to be knocked out, as is the case for mmp14a/b, each additional crossing will add another 3 months to the timeline.

Here, we demonstrate a novel zebrafish model of WS. Similar to human MMP14 and murine Mmp14 mutants, mmp14a/ $b$ KO fish have a short stature, kyphosis, and craniofacial dysmorphology. In addition, the bone density of mmp14a/b KO fish is reduced, albeit limited to the skull. Similar to the mutant mice, mmp14a/b KO fish have a shortened lifespan and do not reproduce. Knockout of mmp14a and mmp14b did not affect cranial NC migration, although the possibility of maternal transcript rescuing the phenotype cannot be ruled out. In contrast to earlier MO studies, craniofacial cartilage developed normally in mmp14a/ $b \mathrm{KO}$ fish. Initial mineralisation proceeded normally as well, and only subtle craniofacial abnormalities became apparent at juvenile age. Notably, both NC- and mesoderm-derived bones are affected, which is furthermore not limited to a single ossification type. Affected bones show disorganisation of cartilage content with a relative lack of cortical bone. In conclusion, our mmp14a/b KO fish recapitulates the skull bone defects seen in WS well. This new model can not only aid to shed light on the underlying pathogenesis, but is also be a valuable tool for developing novel treatments for decreased bone density in general.

\section{Results}

\section{Genome editing of mmp14a and mmp14b in zebrafish by CRISPR/Cas9 approach}

To knock out mmp14a and mmp14b in D. rerio by CRISPR/Cas9, gRNA and Cas9 RNA were generated. gRNA targeting either mmp14a or mmp14b was generated by in vitro T7 transcription of dsDNA fragments encoding all necessary elements of functional gRNA, including the protospacer and the Cas9-binding scaffold sequence (Fig. 4.5C) [46]. The protospacer sequence determines the binding site in the genomic DNA (gDNA) of the gRNA and is, therefore, the only variable part of the DNA oligonucleotide. As shown in Figure 4.5C, the protospacer (in blue) is directly adjacent to the 3' end of the T7 promoter (in red). For better compatibility with T7 transcription, the optimal protospacer starts with GG on its 5 ' end $[43,46]$. In the gDNA, the protospacer needs to bind to a target site directly adjacent to an NGG protospacer adjacent motif (PAM) sequence on its 3' end (see Fig. 4.4) [41, 45]. Possible gRNA target sequences that meet these criteria were identified with $\mathrm{ZiFiT}$ Targeter $[47,48]$. The ideal target site is located in an exon $5^{\prime}$ of regions predicted to be important for protein function so that upon inducing a frameshift mutation, the majority of the functional 
A $\operatorname{chr} 7$
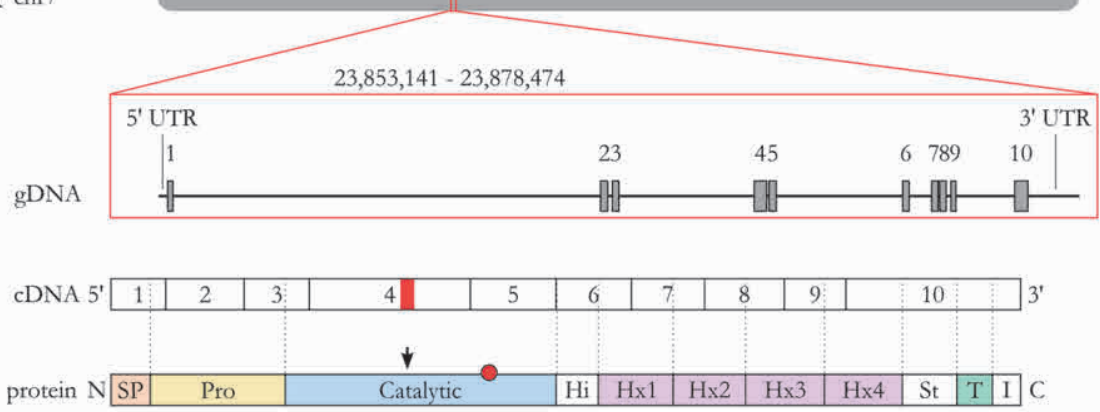

B chr2

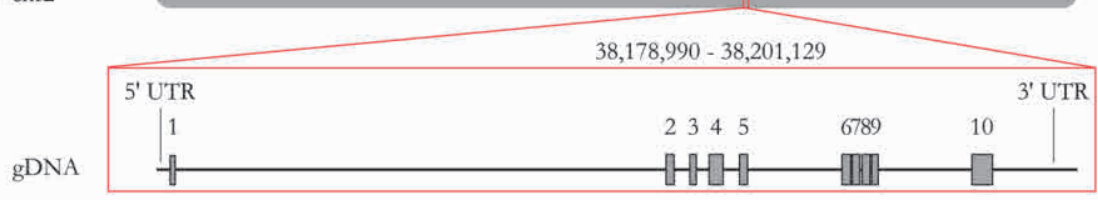

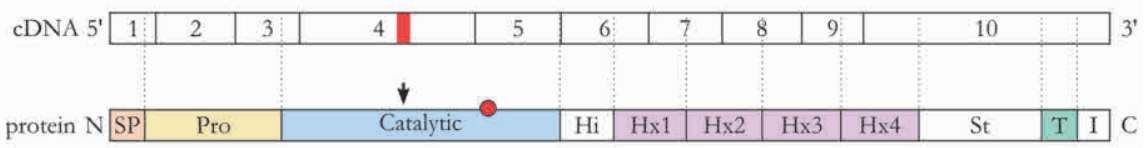

C 5'- CATTATGGTGAAAGTTGGAACCTCTTACGTGCCGATCAAGTCAAAAGCCTCCGGTCGGAGGCTTT TGACTTTCTGCTATGGAGGTCAGGTATGATTTAAATGGTCAGTATTGAGCCTCAGGAAACAGCTA TGACATCAAGCTGACTAGATAATCTAGCTGATCGTGGACCGATCATACGTATAATGCCGTAAGAT CACGGGTCGCAGCACAGCTCGCGGTCCAGTAGTGATCGACACTGCTCGATCCGCTCGCACCGCTA GCTAATACGACTCACTATAGGAGACAGCACCCCATTTGAGTTTTAGAGCTAGAAATAGCAAGTTA AAATAAGGCTAGTCCGTTATCAACTTGAAAAAGTGGCACCGAGTCGGTGCTTTT - -3'

D

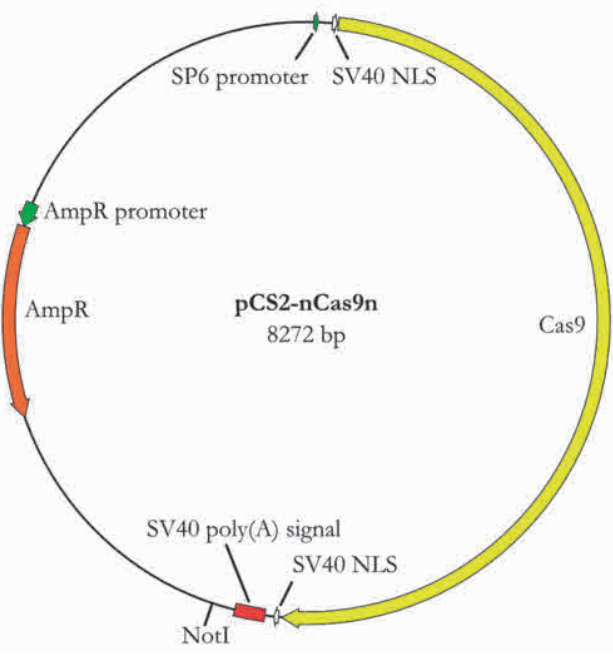


Figure 4.5 - Knockout of mmp14a and mmp14b by CRISPR/Cas9. A-B, schematic overview of the localisation of mmp14a on chromosome 7 (A) and mmp14b on chromosome 2 (B), gDNA with exons (grey boxes), cDNA with CRISPR target site in exon 4 (red bar) and the protein domains. The red dot indicates the catalytic zinc-ion binding residues. Abbreviations as in Figure 4.3. C, gBlock ${ }^{\circledR}$ oligonucleotide sequence. Forward and reverse primer binding sites are underlined at the 5' and 3' end, respectively. The T7 promoter is shown in red, the target sequence (shown here for mmp14a exon 4) in blue. D, simplified vector map of the pCS2 nls-zCas9-nls vector, with Cas9 coding sequence under SP6 promoter [44]. AmpR, ampicillin resistance cassette; SV40 NLS, SV40 virus nuclear localisation signal.

domains of the resulting protein product are lost [44]. On the other hand, the target sequence needs to be located far enough from the original start ATG to minimize the risk of an alternative start codon being used to generate a functional protein product, thereby circumventing the effect of any induced mutation [37, 44]. Taking all these requirements into consideration, target sequences in exon 4 of mmp14a and mmp14b (Fig. 4.5A-B, red bar) seemed optimal. Blasting the selected target sequences against the $D$. rerio genome by CRISPRscan predicted only a single binding site for each [49]. The resulting dsDNA fragments were in vitro transcribed into RNA (Supp. Fig. 3.1AB). mRNA encoding zebrafish codon-optimised Cas? with $\mathrm{N}$ - and C-terminal NLS was generated by in vitro SP6 transcription of the NotI-linearised pCS2 nls-zCas9-nls vector (Fig. 4.5D and Supp. Fig. 3.1C-E) [40].

\section{Successful targeting of mmp14a and mmp14b in zebrafish}

Twenty-four hours after injecting $D$. rerio zygotes with Cas 9 RNA and gRNA targeting mmp14a or mmp14b, part of embryos was lysed to test for successful targeting. Primers were hereto designed (Table 4.12) and optimised (Supp. Fig. 3.2) for PCR amplification of a 200-500 bp region around the respective target site using the gDNA of single-embryo lysate as template. In uninjected embryos, Sanger sequencing of the amplicon resulted in single peaks in the region around the target site, corresponding to the W'T sequence (shown for mmp14a in Fig. 4.6A). Mosaic targeting of injected embryos was evidenced by the presence of double and triple peaks in the chromatogram starting in the 3' region of the target site (Fig. 4.6B). Injected F0 clutch mates of embryos with demonstrated targeting were grown until fertile age, intercrossed and part of their F1 offspring genotyped at $24 \mathrm{hpf}$. Identification of F1 heterozygous mutants (shown for mmp14a in Fig. 4.6C) implicates at least one of the F0 parents is a founder with germline targeting, which were subsequently outcrossed into the $\operatorname{Tg}(\operatorname{sox} 10:: \operatorname{eg} f \mathrm{p})$ transgenic background. At $24 \mathrm{hpf}$, their green fluorescent offspring was selected and a subset genotyped as described above. F1 fish were fin-clip genotyped at two months of age. The identified frameshift mutations are listed in Figure 4.6D and E. Most of these mutations occurred at the 3' end of the target site, as reported before [43, 45]. Some mutations caused a termination codon at the target site, which is predicted to result in loss of the majority of the functional domains including the residues binding the catalytic zinc ion (red dot in Fig. 4.5A-B). As such, fish with a 2 bp deletion in 
A

mmp14a exon 4

CRISPR target site

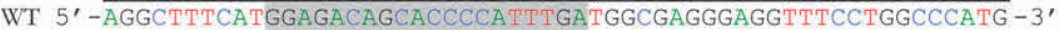

B

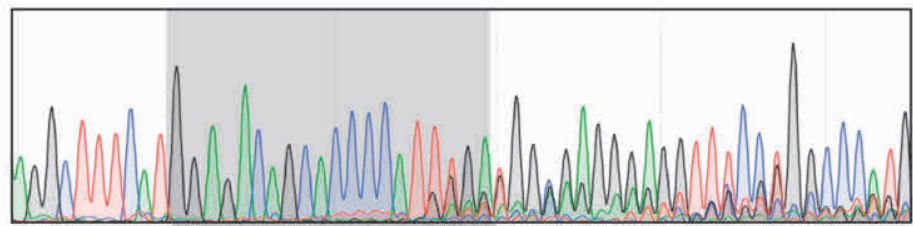

WT 5' -AGGCTTTCATGGAGACAGCACCCCATTTGATGGCGAGGGAGGTTTCCTGGCCCATG-3'

-1 bp $5^{\prime}$-AGGCTTTCATGGAGACAGCACCCCATTGATGGCGAGGGAGGTTTCCTGGCCCATGC-3'

-2 bp $5^{\prime}$-AGGCTTTCATGGAGACAGCACCCCATGATGGCGAGGGAGGTTTCCTGGCCCATGCG $-3^{\prime}$
? $5^{\prime}-$
$A A$
TT C GGG CATTTG $-3^{\prime}$

C

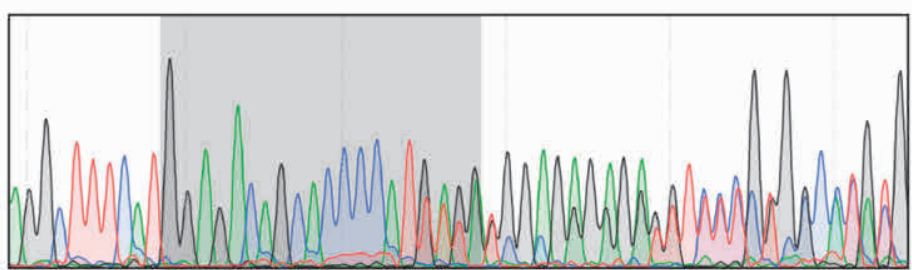

W'T 5' - AGGCTTTCATGGAGACAGCACCCCATTTGATGGCGAGGGAGGTTTCCTGGCCCATG - 3 ' -2 bp $5^{\prime}$-AGGCTTTCATGGAGACAGCACCCCATGATGGCGAGGGAGGTTTCCTGGCCCATGCG - $3^{\prime}$

D

\section{WT}

$-1 \mathrm{bp}$

-2 bp

$-4 \mathrm{bp}$

$-5 \mathrm{bp}$

$-10 \mathrm{bp}$

$+1 \mathrm{bp}$

+2 bp

+11 bp

$-2,+4$ bp

$-2,+7$ bp

\section{mmp14a exon 4}

CRISPR target site

5' - GGAGACAGCACCCCATTT GATGGCGAGGGAGGTTTCC-3'

5' - GGAGACAGCACCCCATT-GATGGCGAGGGAGGTTTCC-3'

5' - GGAGACAGCACCCCAT --GATGGCGAGGGAGGTTTCC - 3 '

5' -GGAGACAGCACCCCATT-----GGCGAGGGAGGTTTCC -3'

5' - GGAGACAGCACCCCAT------GGCGAGGGAGGTTTCC-3'

5' - GGAGACAGCA--------TGGCGAGGGAGGTTTCC-3'

5' - GGAGACAGCACCCCATGTTGATGGCGAGGGAGGTTTC-3'

5' - GGAGACAGCACCCCATTGATGATGGCGAGGGAGGTTT -3'

5' - GGAGACAGCACCCCATT GTTACAAACCATGATGGCGA -3'

5' - GGAGACAGCACCCCAAATATGATGGCGAGGGAGGTTT -3'

5' - GGAGACAGCACCCCAGAACTACTTGATGGCGAGGGAG-3'

\begin{tabular}{ccc}
$\Delta$ AA to stop & & $\#$ \\
\cline { 3 - 3 } & & 76 \\
$190-459$ & & 23 \\
190 & 34 \\
$190-458$ & 6 \\
$190-211$ & 9 \\
$188-456$ & 7 \\
190 & 4 \\
$190-460$ & 4 \\
$190-460$ & 2 \\
$190-460$ & 8 \\
$190-461$ & 2
\end{tabular}

$\mathbf{E}$

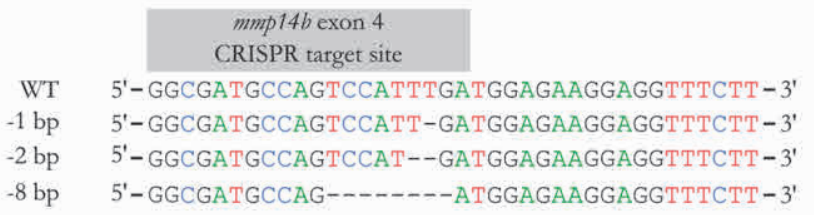

$\begin{array}{ccc}\frac{\Delta \text { AA to stop }}{} & \frac{\#}{61} \\ 185-201 & 26 \\ 184 & 37 \\ 183-205 & 11\end{array}$

Figure 4.6 - Results of mmp14a and mmp14b genome targeting. A-C, chromatography results of direct Sanger sequencing around the mmp14a CRISPR target site of a single WT embryo showing single 
Figure 4.6 (continued) - peaks (A), a representative F0 embryo injected with mmp14a gRNA and Cas9 RNA showing double and triple peaks starting at the target site (B), and a representative F1 embryo heterozygous for a 2 bp deletion in mmp14a (C), all analysed at 24 hpf. D and E, overview of the identified mutations in mmp14a (D) and $m m p 14 b$ (E) and their effect on any putative protein product (number of altered amino acids up to the premature termination codon) in analysed 2-month-old F1 heterozygous nontransgenic mutants.

exon 4 of mmp14a were selected for further crossings. As shown in Figure 4.6E, all the identified mmp14b mutations induced a termination codon at or relatively close to the target site. Therefore, F1 fish heterozygous for a 2 bp deletion in mmp14a were crossed with F0 mmp14b founders. F2 offspring heterozygous for an mmp14a 2 bp deletion (p.F190X) and an mmp14b 8 bp deletion (p.S183Rfs23X) were identified, hereafter referred to as $m m p 14 a^{+/ \Delta}$; mmp14 $14 b^{+/ \Delta}$ fish.

\section{mmp14a $a^{\Delta / \Delta}$; mmp $14 b^{\Delta / \Delta}$ fish have a distinct phenotype}

The F2 mmp14 $a^{+/ \Delta}$; mmp $14 b^{+/ \Delta}$ fish were subsequently intercrossed. As mmp14a and mmp14b are located on different chromosomes (Fig. 4.5A-B), the mutant alleles of both genes segregate independently. Therefore, all possible combinations of WT and mutant alleles were expected in the F3 offspring of mmp $14 a^{+/ \Delta}$; mmp $14 b^{+/ \Delta}$ fish. Two clutches of F3 offspring were generated and observed regularly, starting at every 6 hours during the first week and once daily afterward until one month of age. In the first 6 days of life, $7 \%$ off the embryos/larvae died; another $12 \%$ died over the next 10 days. Embryos and early larvae were inspected for morphology of the head, eyes, yolk ball and yolk extension, and pigmentation pattern. At $6 \mathrm{dpf}$, larvae were inspected for presence and inflation of the swim bladder [26]. Up till one-month post fertilisation, no over phenotype could be observed. At three months of age, a total of 111 adult F3 fish were fin-clip genotyped (Table 4.1). All nine possible genotypes were identified, close to the expected Mendelian ratio. Notably, only two mmp14a $a^{\Delta / \Delta} ; m m p 14 b^{\Delta / \Delta}$ double homozygous mutants were identified. The majority of genotypes developed

Table 4.1 - Relative frequency of different genotypes in F3 offspring of F2 $\mathrm{mmp} 14 \mathrm{a}^{+/ \Delta}$; $m m p 14 \mathrm{~b}^{+/ \Delta}$ parents. $n$, number of fish.

\begin{tabular}{|c|c|c|}
\hline Genotype & Actual \% (n) & Expected \% \\
\hline mmp14a $a^{+/+} ;$mmp14b & $6.31(7)$ & 6.25 \\
\hline$m m p 14 a^{+/ \Delta} ; m m p 14 b^{+/+}$ & $17.12(19)$ & 12.50 \\
\hline mmp14a $a^{+/+} ;$mmp14b & $6.31(7)$ & 12.50 \\
\hline mmp14a $a^{\Delta / \Delta} ; m m p 14 b^{+/+}$ & $6.31(7)$ & 6.25 \\
\hline 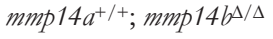 & $4.50(5)$ & 6.25 \\
\hline$m m p 14 a^{+/ \Delta} ; \operatorname{mmp} 14 b^{+/ \Delta}$ & $31.53(35)$ & 25.00 \\
\hline$m m p 14 a^{\Delta / \Delta} ; m m p 14 b^{+/ \Delta}$ & $13.51(15)$ & 12.50 \\
\hline$m m p 14 a^{+/ \Delta} ; m m p 14 b^{\Delta / \Delta}$ & $12.61(14)$ & 12.50 \\
\hline$m m p 14 a^{\Delta / \Delta} ; m m p 14 b^{\Delta / \Delta}$ & $1.80(2)$ & 6.25 \\
\hline
\end{tabular}



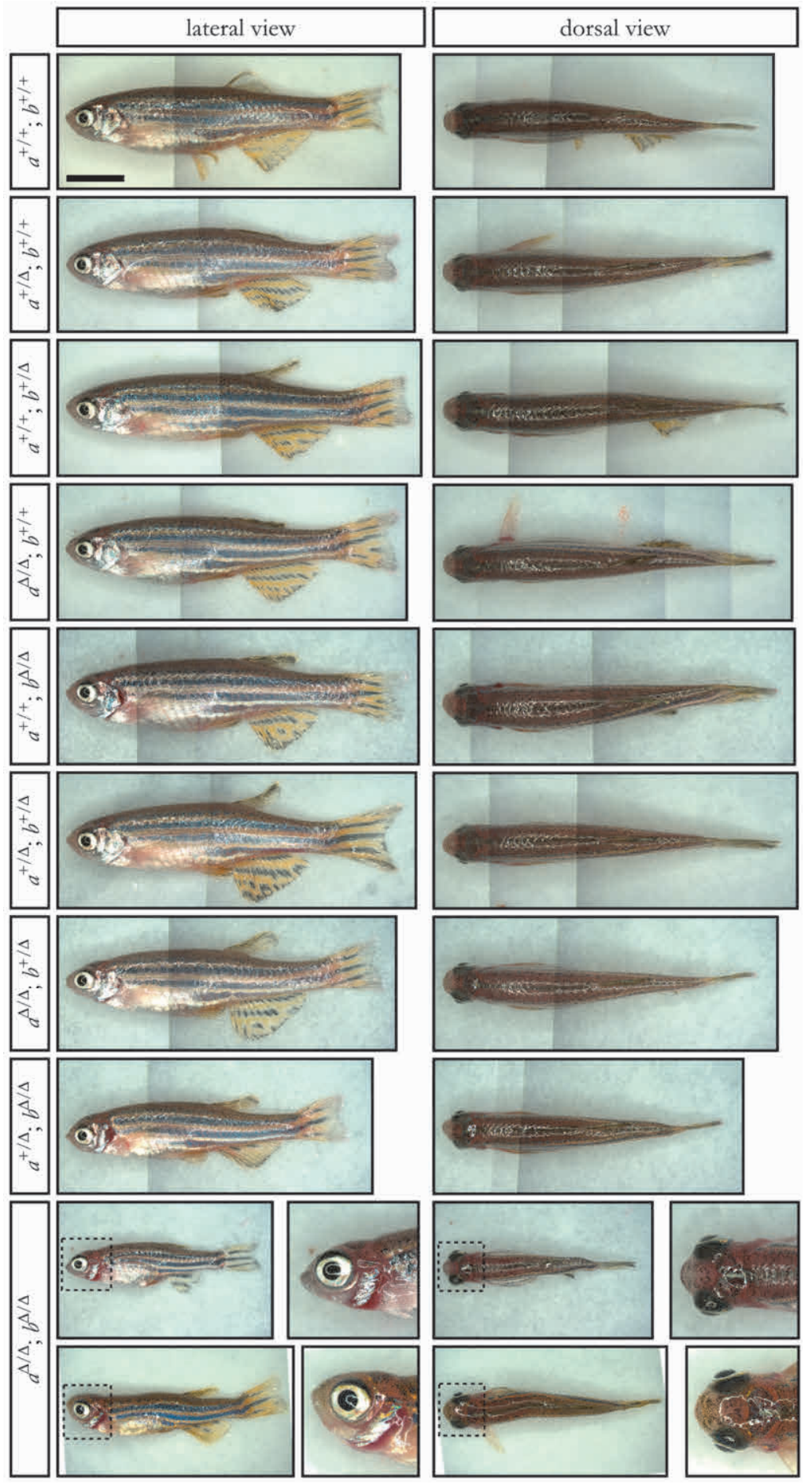
Figure 4.7 - Genomic targeting of mmp14a and mmp14b affects growth and craniofacial morphology. Gross anatomy images of representative 3-month-old offspring of F2 parents heterozygous for a $2 \mathrm{bp}$ deletion in exon 4 of mmp14a and an 8 bp deletion in exon 4 of mmp14b. The majority of fish developed normally. Fish with genotype $m m p 14 a^{+/ \Delta} ; m m p 14 b^{\Delta / \Delta}$ had a shorter length and a relatively short head. Fish with genotype $m m p 14 a^{\Delta / \Delta}$; mmp $14 b^{\Delta / \Delta}$ were shorter than their clutch mates, had kyphosis, a short, dorsally tilted head, a short operculum that exposed the gills, and protruding eyes. Note that these fish were fin-clip genotyped 1-2 weeks prior to imaging, which is reflected in the regrown tail fin that is not fully pigmented yet. All fish sharing the same scale bar were resized identically; scale bar equals $5 \mathrm{~mm}$.

normally and were indistinguishable from mmp14 $a^{+/+}$; mmp $14 b^{+/+}$fish at 3 months of age (Fig. 4.7). Fish with genotype $m m p 14 a^{+/ \Delta} ; m m p 14 b^{\Delta / \Delta}$ had a shorter total body length and a relatively short head. In contrast, the two mmp $14 a^{\Delta / \Delta} ; m m p 14 b^{\Delta / \Delta}$ fish, belonging to different clutches, looked strikingly different from their clutch mates: they were shorter, had kyphosis, and had a relatively short and dorsally tilted head and a short operculum that exposed the underlying gills. Inspecting the fish in dorsal view demonstrated the relative short head and revealed exophthalmos in the double homozygous mutant fish. Interestingly, these $m m p 14 a^{\Delta / \Delta} ; m m p 14 b^{\Delta / \Delta}$ fish shared many features with the mutant Mmp14 mouse models: both have a short stature, small head, short snout and relatively large, protruding eyes compared to their WT siblings. To verify the observed phenotype was indeed caused by mmp14a/b KO, the effect of the selected mutations at the mRNA and protein level was assessed. In addition, larger numbers of double homozygous fish were generated to assess the reproducibility of the observed phenotype.

\section{Mutant mmp14a/b $m \mathrm{RN} A$ undergoes nonsense-mediated decay}

To analyse the effect of the selected mmp14a/b mutations at the mRNA level, mmp14a/b mRNA levels were assessed in 1-5 dpf embryos/larvae of F3 mmp14a $a^{\Delta / \Delta}$;

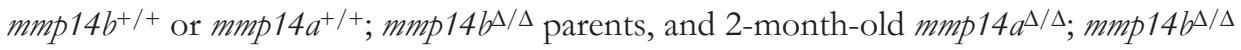
juveniles, and compared to those in age-matched WT fish. The expression levels of mutant mmp14a and mmp14b were very low compared to the WT alleles $(21-51 \%$ of W'T mRNA levels at $1 \mathrm{dpf}$ for mmp14a, 13-58\% for mmp14b; Fig. $4.8 \mathrm{~A}-\mathrm{B})$. In addition, the increase in expression of WT alleles in the first 5 days is absent for mutant alleles. These results are suggestive of nonsense-mediated mRNA decay (NMD), a process that degrades faulty mRNAs with premature termination codons. This process is especially likely to be responsible for mRNA degradation if the premature termination codon is present more than 50 nucleotides upstream of the (original) exon junction $[50,51]$. Following this rule-of-thumb, NMD was expected for the mmp14a mutation, but seemed less likely for the mmp14b mutation, as it induced a termination codon within 50 nucleotides of the downstream exon junction. Interestingly, while the mutant mRNA levels are very low, the expression of the non-mutated paralog is upregulated compared to its expression in WT larvae (30-135\% increase). In accordance with 

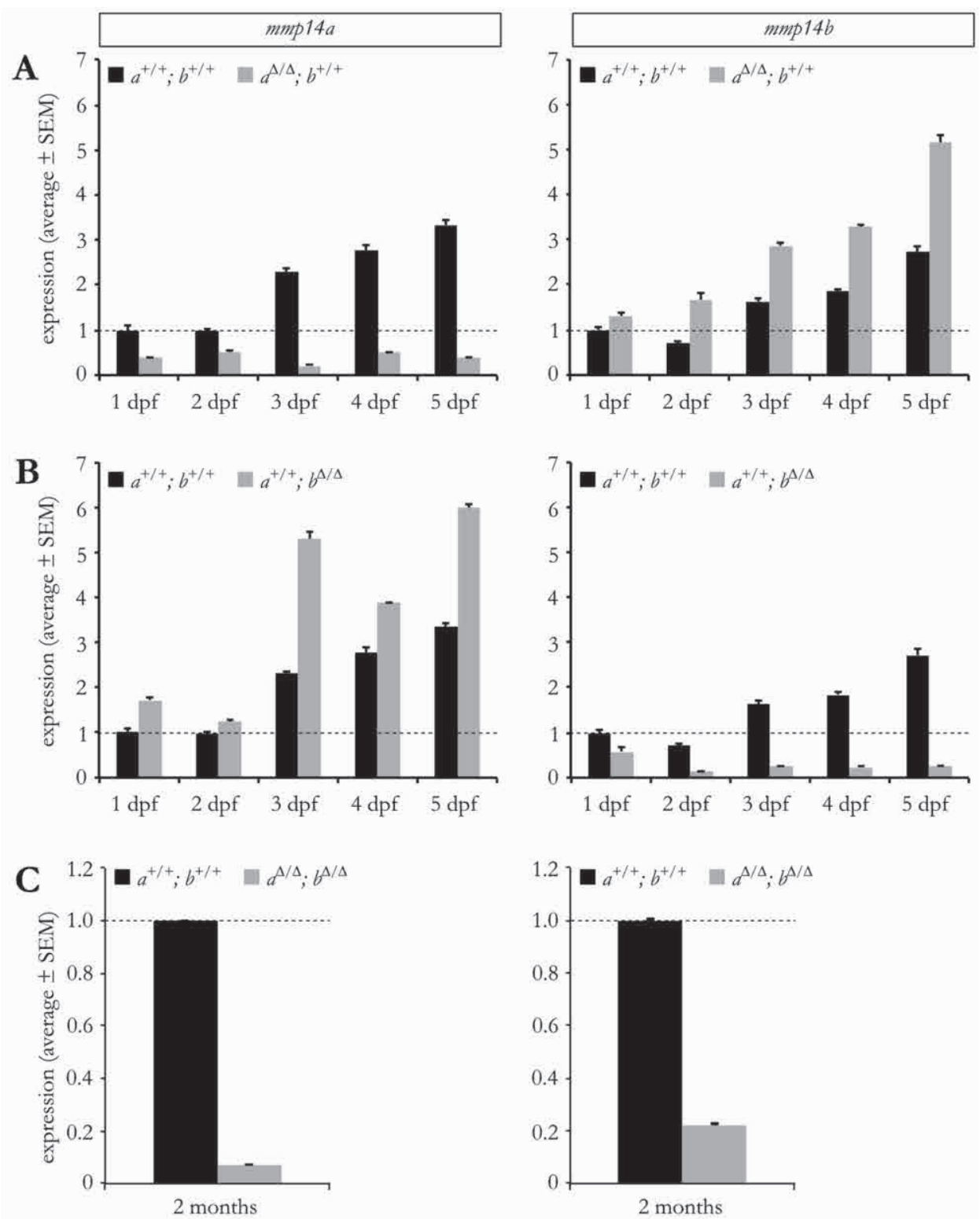

Figure 4.8 - Mutant mmp14a and mmp14b mRNA undergo nonsense-mediated decay. A-B, mRNA expression levels for mmp14a (left) and mmp14b (right) of 1-5 dpf offspring of $m m p 14 a^{\Delta / \Delta}$;

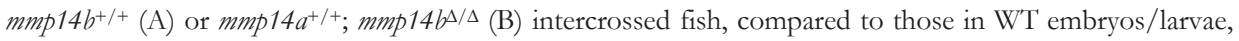
normalised for $\beta$-actin expression and denoted relative to the expression level in $1 \mathrm{dpf}$ WT embryos (dotted line). Values are average \pm SEM of technical triplicates. mRNA levels of mutant mmp14a and mmp14b are very low, suggestive of nonsense-mediated mRNA decay. Note that the expression of the WT paralog in mutant fish is upregulated. C, mRNA expression levels of mmp14a (left) and mmp14b (right) of 2-month-old

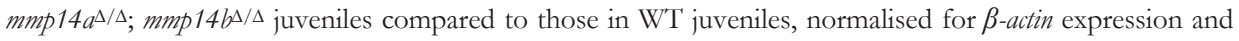
denoted relative to expression in WT juveniles (dotted line). 
our findings in embryos/larvae, the levels of mutant mmp14a and mmp14b mRNA are very low (7\% for mmp14a, 22\% for mmp14b) in 2-month-old double homozygous juveniles (Fig. 4.8C) as compared to W'T fish.

To assess the contribution of maternal transcripts, the experiment shown in Figure 4.8A-B was repeated with offspring of either F3 mmp14a $a^{+/ \Delta} ; m m p 14 b^{\Delta / \Delta}$ or F3 $m m p 14 a^{\Delta / \Delta} ; m m p 14 b^{+/ \Delta}$ parents and WT larvae. Similarly to the previous experiment, the expression levels of the mmp14 paralog for which the parents were homozygous mutant were very low $(9-30 \%$ for $m m p 14 a, 22-23 \%$ for mmp14b) in their larval offspring (Supp. Fig. 3.3). Interestingly, in the offspring of mmp14a $a^{+/ \Delta}$; mmp $14 b^{\Delta / \Delta}$ parents, the expression level of mmp14a did not differ from that in WT larvae. It is im-

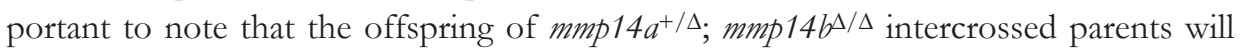

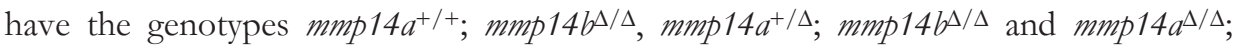
$m m p 14 b^{\Delta / \Delta}$, that are theoretically present at a 1:2:1 ratio. The observed 'normalisation' of the mmp14a mRNA levels could, therefore, result from NMD of mutant mmp14a mRNA and concurrent upregulation of W' mmp14a mRNA. In the offspring of $m m p 14 a^{\Delta / \Delta} ; m m p 14 b^{+/ \Delta}$ parents, however, mmp14b expression was upregulated to a similar extent (12-156\%) as observed in mmp14a $a^{\Delta / \Delta}$; mmp $14 b^{+/+}$larvae, except for a higher expression (150\% increase) at $1 \mathrm{dpf}$. However, as the exact ratio of the genotypes are unknown due to pooling RNA of 20 randomly selected larvae per time point, it is impossible to draw any definite conclusions. Furthermore, it is impossible to make any definitive statements about the contribution of any maternal transcripts. Taken together, our qPCR results suggest that the selected mutations indeed knocked out mmp14a and mmp14b.

\section{The selected mmp14a/ $b$ mutations have a deleterious effect on any putative protein product}

After demonstrating the effect of the mmp14a and mmp14b mutation at the mRNA level, the effect of these mutations at the protein level was assessed. Both the $2 \mathrm{bp}$ deletion in mmp14a and 8 bp deletion in mmp14b are frameshift mutations, that are predicted to induce a premature termination codon within exon 4 of the respective gene (Fig. 4.6). In case any mRNA would escape degradation, any putative protein product is expected to lack its $\mathrm{C}$-terminal half. To test this hypothesis, protein lysate of

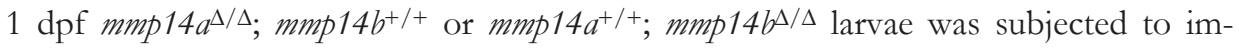
munoblotting with antibodies reportedly raised against zebrafish Mmp14a or Mmp14b, respectively. However, the antibodies turned out to cross-react with both Mmp14 paralogs in addition to other zebrafish proteins and were therefore not useful for testing our hypothesis (data not shown). As an alternative approach, four vectors were created encoding either WT or mutant Mmp14a or Mmp14b, all with a C- terminal Hemagglutinin (HA) tag (Fig. 4.9A). Based on the predicted effect of these mutations on the protein level, the HA sequence should not be presented in any resulting mutant protein. Primers were designed to amplify mmp14a or mmp14b coding sequence 
A

\begin{tabular}{|c|c|c|c|}
\hline kozak & mmp $14 a / b$ cDNA without stop codon & HA & stop \\
\hline
\end{tabular}
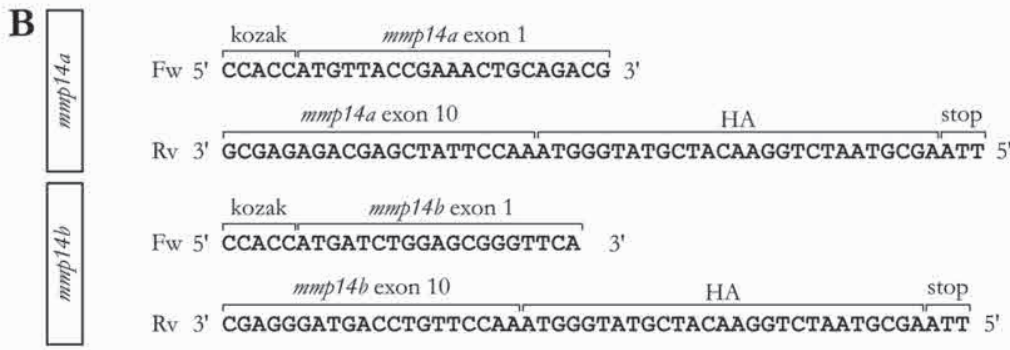

C
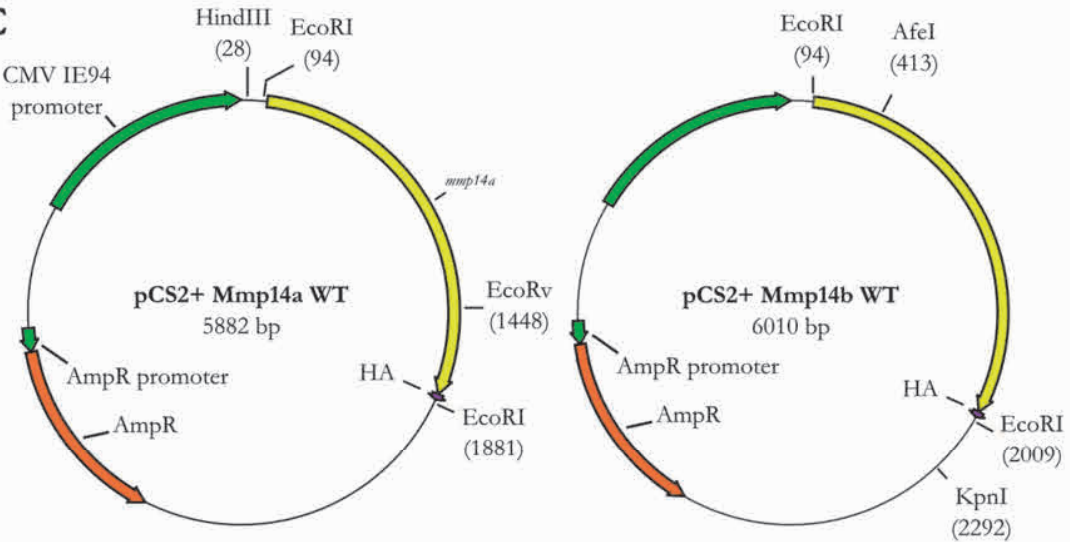

D

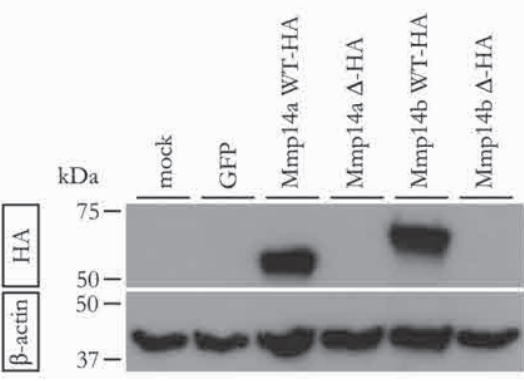

Figure 4.9 - Mutant mmp14a and mmp14b mRNA does not form full-length Mmp14. A, to assess the effect of the selected mmp14a/b frameshift mutations on any putative protein product, plasmid inserts were designed encoding a zebrafish optimised kozak sequence, WT or mutant mmp14a/b cDNA (without stop codon) and a hemagglutinin (HA) tag sequence. B, to generate these four inserts, forward ( $\mathrm{Fw}$ ) primers containing the kozak sequence and reverse $(\mathrm{Rv})$ primers containing the HA coding sequence and a stop codon were used to PCR amplify WT or mutant mmp14a/ $b$ coding sequence from cDNA of 2-monthold WT or mmp14a $a^{\Delta / \Delta} ; m m p 14 b^{\Delta / \Delta}$ juveniles, respectively. C, the resulting amplicons were cloned into the pCS2+ backbone; only the two WT expression vectors are shown here. $\mathbf{D}$, the four vectors were transfected into MRC-5V1 cells and whole cell protein extract analysed by immunoblot against the HA-tag. 
Figure 4.9 (continued) - A strong band is visible at the expected height for cells expressing the WT Mmp14a-HA (60 kDa) and Mmp14b-HA (71 kDa) fusion protein. No bands are seen in cells transfected with the mutant vectors, demonstrating the disrupting effect of the frameshift mutations.

while adding a 3' kozak sequence and a 5' HA tag encoding sequence (Fig.4.9B and Table 4.15). cDNA of a single 2-month-old mmp14a $a^{\Delta / \Delta} ; m m p 14 b^{\Delta / \Delta}$ or $m m p 14 a^{+/+}$; mmp $14 b^{+/+}$juvenile served as template. The resulting amplicons were inserted into the pCS2+ vector (Fig. 4.9C; see Supp. Fig. 3.4 for details on the cloning strategy). Initial$\mathrm{ly}$, the four vectors in vitro transcribed and the mRNA injected into W'T zygotes. As immunoblotting $24 \mathrm{hpf}$ protein extract against the HA tag resulted in nonspecific bands, the vectors were transiently expressed in MRC-5V1 human fibroblasts as an alternative approach (see Chapter 2 for details on MRC-5V1 cells). As shown in Figure 4.9D, subjecting the whole cell protein extract to anti-HA immunoblotting resulted in strong bands at the expected height for Mmp14a WT-HA (60 kDa) and Mmp14b WT-HA $(71 \mathrm{kDa})$. Importantly, no bands were seen in the lysate of cells transfected with the mutant vectors. Although it is impossible to distinguish between mRNA decay, a premature stop codon or an altered protein sequence caused by frameshift, the results shown in Figure 4.9D demonstrate the disrupting effect of the mutations on the resulting protein product.

\section{CRISPR-induced mutations in mmp14a and mmp14b are not circumvented by alternative splicing}

Although it was demonstrated that mutant mmp14a and mmp14b mRNA was downregulated, and any surviving full-length mRNA was shown not to result in a full-length Mmp14 protein, it could be argued that the mutations in exon 4 could be circumvented by alternative splicing, thereby forming a (shorter) functional protein [37]. Alternative splicing could not be assessed in the in vitro model presented in Figure 4.9, as the vectors do not include any intronic sequences. As shown in Supplemental Figure 3.4, the primers shown in Figure 4.9B generated multiple smaller amplicons in addition to the full-length mmp14a and mmp14b cDNA. To assess whether this result was dependent on the primers used, new primers to amplify exons 1-10 of mmp14a and mmp14b cDNA were designed (Table 4.14) and optimised (Supp. Fig. 3.5A-B). As shown in supplemental Figure 3.5C, the new mmp14a primers generated only two amplicons from WT cDNA template; one of a length between 1-1.5 kb corresponding to the fulllength mRNA (expected size of 1,326 bp) and one smaller fragment of 500 bp. When mutant cDNA was used as template, the mmp14a primers only amplified a single $\sim 80$ bp fragment that is too short to encode any protein product of significance. For both WT and mutant cDNA, the new mmp14b primers amplified two fragments, one around $1.5 \mathrm{~kb}$ corresponding to the full-length mmp14b mRNA (expected size of 1,458 bp) and a smaller fragment just under $400 \mathrm{bp}$. Both amplicons were subsequently analysed by direct Sanger sequencing, confirming the larger fragment indeed corresponds to full-length mmp14b mRNA, containing an 8 bp deletion in the mutant sample. The 


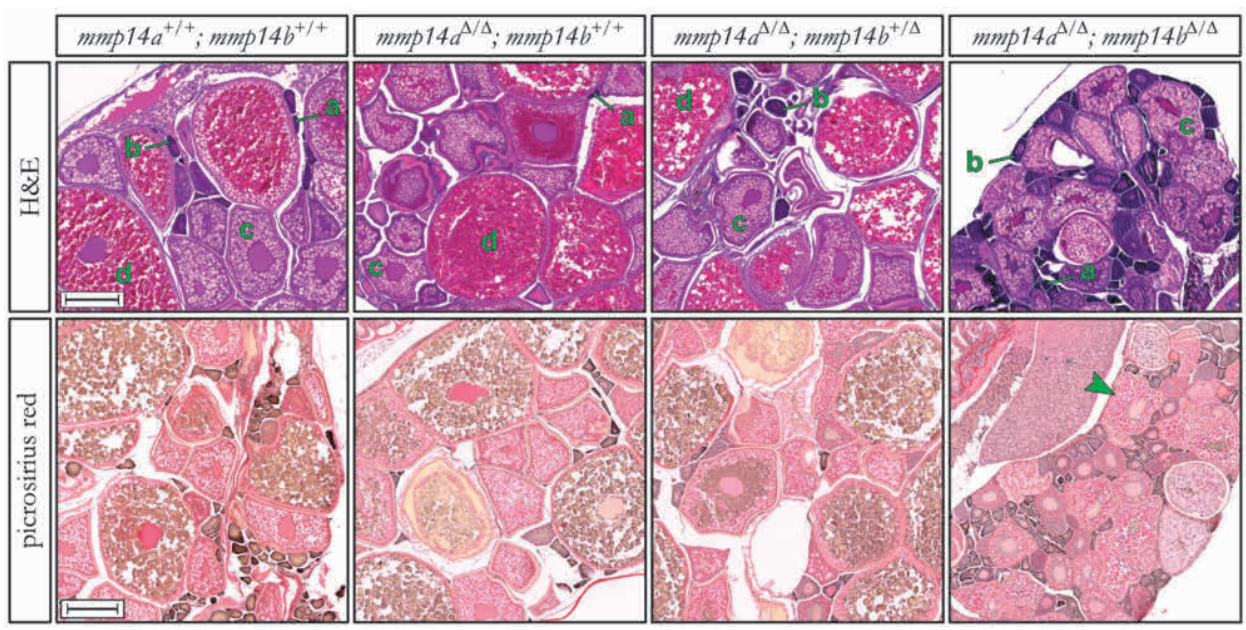

Figure 4.10 - mmp14a/b KO impairs follicular maturation in the ovary. Ovaria on midsagittal sec-

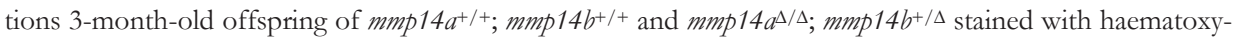
lin and eosin (top panel) and picrosirius red (bottom panel) demonstrating the relative lack of final stages in follicular maturation, which does not improve at age 3.5 months (not shown here). Note the intense collagen staining in vitellogenic follicles of mmp14a/ $b$ KO fish (arrowhead), not present in the other genotypes. a, oogonium; b, pre-vitellogenic follicle; c, vitellogenic follicle; d, pre-ovulatory follicle. Scale bars equal 200 $\mu \mathrm{m}$.

$\sim 400$ bp amplicon corresponded to the first four exons of mmp14b mRNA, and ended before the CRISPR target site. These results suggest no alternative splicing of any significance takes place in mmp14a $a^{\Delta / \Delta} ; m p 14 b^{\Delta / \Delta}$ fish that would circumvent the mutations and lead to a functional protein product. Together with the qPCR and Western blot results, this strongly suggests mmp14a and mmp14b were knocked out.

\section{mmp14a/b KO fish do not reproduce}

To verify the phenotype of mmp14a/b KO fish as shown in Figure 4.7 was reproducible, more mmp14a/b KO fish needed to be generated. As the two mmp14a/b KO fish in the initial two F3 clutches did not produce any offspring, F3 mmp14 $a^{\Delta / \Delta} ; m m p 14 b^{+/ \Delta}$ fish were intercrossed to generate additional mmp14a $a^{\Delta / \Delta} ; m m p 14 b^{\Delta / \Delta}$ fish. These F4 mmp14a/b KO fish were used for the Western blot experiments described above. Multiple attempts to intercross mmp14a/b KO fish failed to produce any offspring; normal courtship and chasing behaviour was absent and no eggs were laid. Attempts to outcross phenotypically female F4 mmp14a/ $b$ KO fish with size-matched adult WT males failed as well. Sectioning confirmed that the analysed mmp14a/b KO fish were indeed female, as evidenced by the presence of ovaria. In ovaria of mmp14a/ $b \mathrm{KO}$ fish, oogonia and early-stage follicles were relatively abundant, while pre-ovulatory follicles were relatively scarce. In addition, an intense picrosirius red staining indicated a relatively high collagen content of vitellogenic follicles (Fig. 4.10, arrowhead) in mmp14a/b KO 
ovaria as compared to their clutch mates' and WT fish ovaria. In D. rerio, body size rather than age correlates best with reproductive maturity, for which typically a standard length of $\sim 23 \mathrm{~mm}$ (SL, distance from the anteriormost tip of the snout, excluding the lower jaw, to the caudal peduncle) needs to be reached [25]. As shown in Figure 4.7, the short body length of mmp14a/ $b \mathrm{KO}$ fish might explain their sexual immaturity at the age of 3 months. Alternatively, Mmp14a and Mmp14b might have a direct role in follicular development. In the rat ovarium, expression of Mmp14 and Mmp2 are spatiotemporally controlled, as previously demonstrated by RNA in situ hybridisation [52]. Both MMP14 and MMP2 (which is activated by MMP14) are collagenases, and the altered collagen content of vitellogenic follicles in mmp14a/b KO fish supports a direct effect of mmp14a/b KO on follicle development. Although all nine sectioned mmp14a/b KO fish turned out to be female, this does not automatically imply a role of Mmp14a/b in sex determination. Although sex determination in zebrafish is incompletely understood, it is known that environmental factors play an important role. Rearing density is a well-documented factor influencing sex determination, with a lower density favouring female development [25]. As fish were reared at a relative low density (max. 20 fish per $3 \mathrm{~L}$ ), and even lower after sorting fish according to genotype per clutch (see below), this might have skewed the male/female ratio. For further experiments, mmp14a/b KO fish were therefore generated by intercrossing either mmp $14 a^{\Delta / \Delta}$; mmp $14 b^{+/ \Delta}$ or mmp14a $a^{+/ \Delta}$; mmp14b $b^{\Delta / \Delta}$ fish.

\section{mmp14a/b KO fish have stunted growth, craniofacial malformations, and byperkyphosis}

To confirm the phenotype of mmp14a/b KO fish, and to assess when the phenotype arises, F3 mmp14a $a^{\Delta / \Delta}$; mmp14 $b^{+/ \Delta}$ fish were intercrossed and their offspring imaged between 2.5 weeks to 3 months of age. Offspring of uninjected $m m p 14 a^{+/+}$; mmp $14 b^{+/+}$ fish served as control. As shown in Figure 4.11, at the age of 17 days, there were no overt differences in physical appearance between the different genotypes. The F4 mmp14a/ $b$ KO fish started to look phenotypically different from their clutch mates

Table 4.2 - Number of individuals imaged and measured per genotype and time point. Dpf, days post fertilization.

\begin{tabular}{|c|c|c|c|c|c|c|c|}
\hline & Genotype & $17 \mathrm{dpf}$ & $34 \mathrm{dpf}$ & $43 \mathrm{dpf}$ & $53 \mathrm{dpf}$ & $73 \mathrm{dpf}$ & $90 \mathrm{dpf}$ \\
\hline WT intercrossed & $m m p 14 a^{+/+} ; m m p 14 b^{+/+}$ & 12 & 28 & 41 & 43 & 47 & 75 \\
\hline$m m p 14 a^{\Delta / \Delta} ; m m p 14 b^{+/ \Delta}$ & mmp14a $a^{\Delta / \Delta} ; m m p 14 b^{+/+}$ & 5 & 10 & 14 & 4 & 15 & 11 \\
\hline \multirow[t]{2}{*}{ intercrossed } & $m m p 14 a^{\Delta / \Delta} ; m m p 14 b^{+/ \Delta}$ & 3 & 20 & 27 & 10 & 24 & 15 \\
\hline & $m m p 14 a^{\Delta / \Delta} ; m m p 14 b^{\Delta / \Delta}$ & 4 & 13 & 13 & 8 & 17 & 21 \\
\hline \multirow{3}{*}{$\begin{array}{l}m m p 14 a^{+/ \Delta} ; m m p 14 b^{\Delta / \Delta} \\
\text { intercrossed }\end{array}$} & $m m p 14 a^{+/+} ; \operatorname{mmp} 14 b^{\Delta / \Delta}$ & - & - & 9 & - & - & 17 \\
\hline & $m m p 14 a^{+/ \Delta} ; m m p 14 b^{\Delta / \Delta}$ & - & - & 19 & - & - & 29 \\
\hline & $m m p 14 a^{\Delta / \Delta} ; m m p 14 b^{\Delta / \Delta}$ & - & - & 13 & - & - & 13 \\
\hline
\end{tabular}



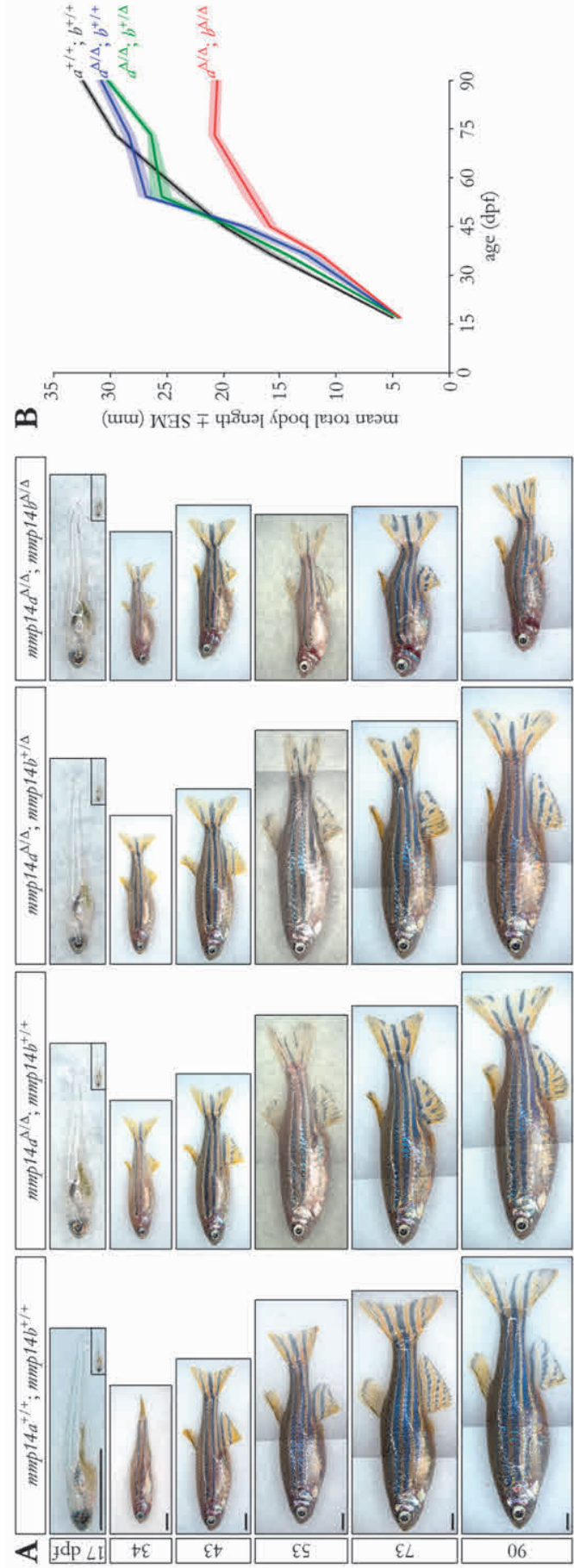

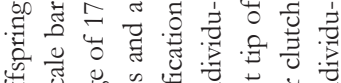

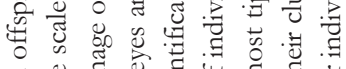
च छ 员

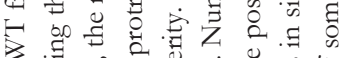

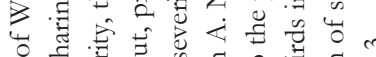

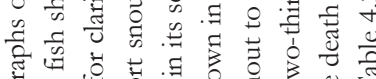

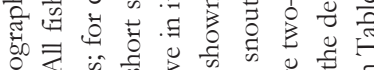
ये की

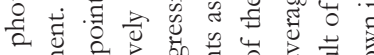

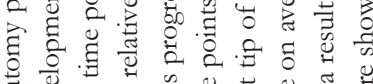
ปै

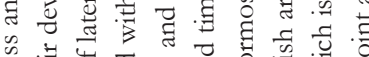

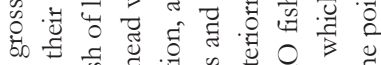

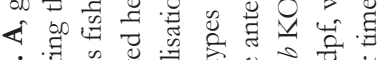

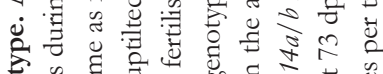

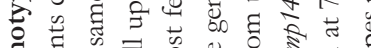

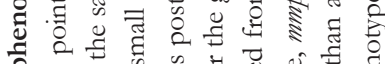

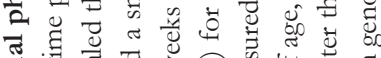
可 च चु 氜 गु

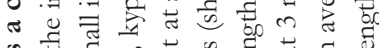

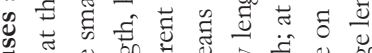
ซु

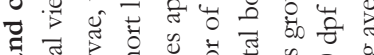

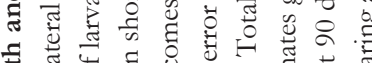

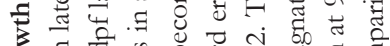

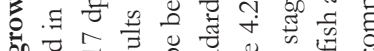
कo

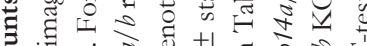
का वी ช

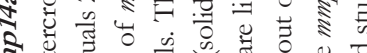

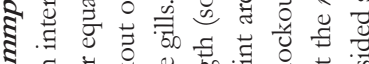

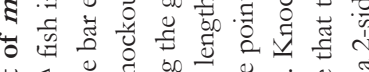

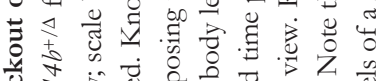

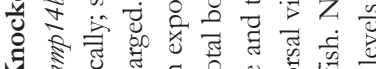

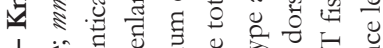
$=\dot{\Delta}$.

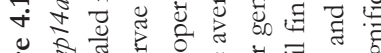

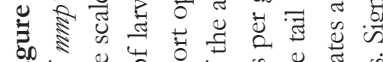

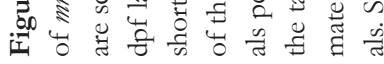


from about one month of age, and could be identified by their appearance from $43 \mathrm{dpf}$ 4.2. The average total body length was compared between genotypes per time point by a 2-sided student T-test; the resulting significance levels are summarised in Table 4.3. onwards. Over time, their phenotype gradually worsened and encompassed short body length, a short and dorsally tilted head with relatively short snout, relatively large, protruding eyes, a short operculum exposing the gills, and kyphosis. This phenotype was identical to that observed in the F3 mmp14a/b KO fish (Fig. 4.7). Total body length quantification confirmed that mmp14a/b KO fish were on average significantly shorter than their clutch mates and age-matched WT fish. As shown in Figure 4.11B, the growth of mmp14a/b KO fish stagnated relatively early during their development, reaching a total body length at $90 \mathrm{dpf}$ of about two-thirds of their clutch mates'. The number of fish measured per genotype and time point is summarised in Table 4.3.

\section{The zebrafish mmp14a/b KO phenotype is independent of the parental genotype}

To assess whether the parental genotype influences the phenotype of mmp14a/b KO fish, F3 mmp14a $a^{+/ \Delta} ; m m p 14 b^{\Delta / \Delta}$ fish were intercrossed and their offspring compared to

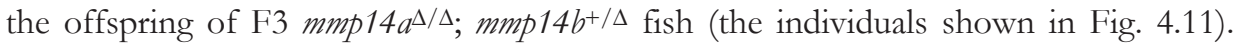
However, intercrossing $m m p 14 a^{+/ \Delta} ; m m p 14 b^{\Delta / \Delta}$ fish repeatedly resulted in either no

Table 4.3 - Levels of significance of two-sided student T-test on average total body length between different genotypes at different ages. Genotype colours as in Figure 4.12.

\begin{tabular}{|c|c|c|c|c|c|c|}
\hline & $17 \mathrm{dpf}$ & $33 \mathrm{dpf}$ & $43 \mathrm{dpf}$ & $53 \mathrm{dpf}$ & $73 \mathrm{dpf}$ & $90 \mathrm{dpf}$ \\
\hline$a^{+/+} ; b^{+/+}$vs. $a^{\Delta / \Delta} ; b^{+/+}$ & 0.0010 & 0.011 & 0.11 & 0.051 & 0.091 & 0.011 \\
\hline$a^{+/+} ; b^{+/+}$vs. $a^{+/+} ; b^{\Delta / \Delta}$ & - & - & 0.61 & - & - & 0.028 \\
\hline$a^{+/+} ; b^{+/+}$vs. $a^{\Delta / \Delta} ; b^{+/ \Delta}$ & 0.0091 & 0.11 & 0.52 & 0.073 & $<0.0001$ & 0.0003 \\
\hline$a^{+/+} ; b^{+/+}$vs. $a^{+/ \Delta} ; b^{\Delta / \Delta}$ & - & - & 0.31 & - & - & $<0.0001$ \\
\hline$a^{+/+} ; b^{+/+}$vs. $a^{\Delta / \Delta} ; b^{\Delta / \Delta}$ & 0.0004 & $<0.0001$ & 0.0001 & $<0.0001$ & $<0.0001$ & $<0.0001$ \\
\hline$a^{+/+} ; b^{+/+}$vs. $a^{\Delta / \Delta} ; b^{\Delta / \Delta}$ & - & - & 0.0043 & - & - & $<0.0001$ \\
\hline$a^{\Delta / \Delta} ; b^{+/+}$vs. $a^{+/+} ; b^{\Delta / \Delta}$ & - & - & 0.39 & - & - & 0.82 \\
\hline$a^{\Delta / \Delta} ; b^{+/+}$vs. $a^{\Delta / \Delta} ; b^{+/ \Delta}$ & 0.85 & 0.040 & 0.26 & 0.40 & 0.0069 & 0.35 \\
\hline$a^{\Delta / \Delta} ; b^{+/+}$vs. $a^{+/ \Delta} ; b^{\Delta / \Delta}$ & - & - & 0.38 & - & - & 0.0003 \\
\hline$a^{\Delta / \Delta} ; b^{+/+}$vs. $a^{\Delta / \Delta} ; b^{\Delta / \Delta}$ & 0.29 & 0.17 & 0.037 & $<0.0001$ & $<0.0001$ & $<0.0001$ \\
\hline$a^{\Delta / \Delta} ; b^{+/+}$vs. $a^{\Delta / \Delta} ; b^{\Delta / \Delta}$ & - & - & 0.25 & - & - & $<0.0001$ \\
\hline$a^{+/+} ; b^{\Delta / \Delta}$ vs. $a^{+/ \Delta} ; b^{\Delta / \Delta}$ & - & - & 0.76 & - & - & $<0.0001$ \\
\hline$a^{+/+} ; b^{\Delta / \Delta}$ vs. $a^{\Delta / \Delta} ; b^{+/ \Delta}$ & - & - & 0.94 & - & - & 0.23 \\
\hline$a^{+/+} ; b^{\Delta / \Delta}$ vs. $a^{\Delta / \Delta} ; b^{\Delta / \Delta}$ & - & - & 0.015 & - & - & $<0.0001$ \\
\hline$a^{+/+} ; b^{\Delta / \Delta}$ vs. $a^{\Delta / \Delta} ; b^{\Delta / \Delta}$ & - & - & 0.0012 & - & - & $<0.0001$ \\
\hline$a^{\Delta / \Delta} ; b^{+/ \Delta}$ vs. $a^{+/ \Delta} ; b^{\Delta / \Delta}$ & - & - & 0.67 & - & - & 0.0019 \\
\hline$a^{\Delta / \Delta} ; b^{+/ \Delta}$ vs. $a^{\Delta / \Delta} ; b^{\Delta / \Delta}$ & 0.29 & $<0.0001$ & 0.0004 & $<0.0001$ & $<0.0001$ & $<0.0001$ \\
\hline$a^{\Delta / \Delta} ; b^{+/ \Delta}$ vs. $a^{\Delta / \Delta} ; b^{\Delta / \Delta}$ & - & - & 0.012 & - & - & $<0.0001$ \\
\hline$a^{+/ \Delta} ; b^{\Delta / \Delta}$ vs. $a^{\Delta / \Delta} ; b^{\Delta / \Delta}$ & - & - & 0.0068 & - & - & $<0.0001$ \\
\hline$a^{+/ \Delta} ; b^{\Delta / \Delta}$ vs. $a^{\Delta / \Delta} ; b^{\Delta / \Delta}$ & - & - & 0.0001 & - & - & $<0.0001$ \\
\hline$a^{\Delta / \Delta} ; b^{\Delta / \Delta}$ vs. $a^{\Delta / \Delta} ; b^{\Delta / \Delta}$ & - & - & 0.18 & - & - & $<0.0001$ \\
\hline
\end{tabular}



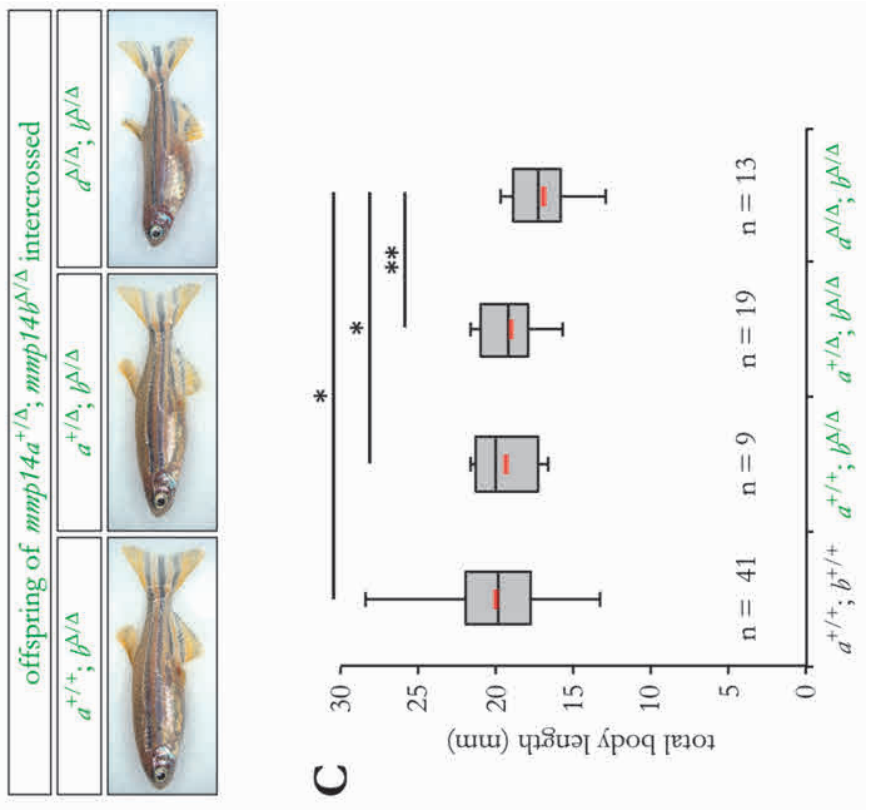

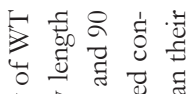

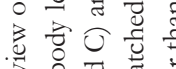

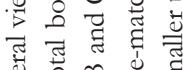

䒕

छ

ते है

क्ष०

南至

ह

ต

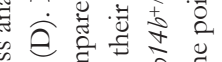

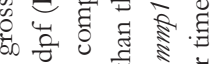

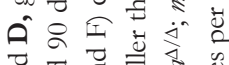

䒕 吉

$4 \lesssim U$ क ह

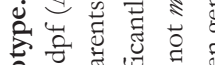

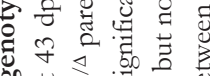

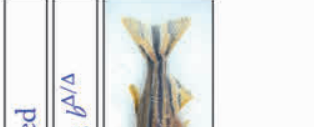

00 त क बी

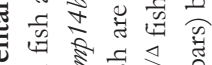
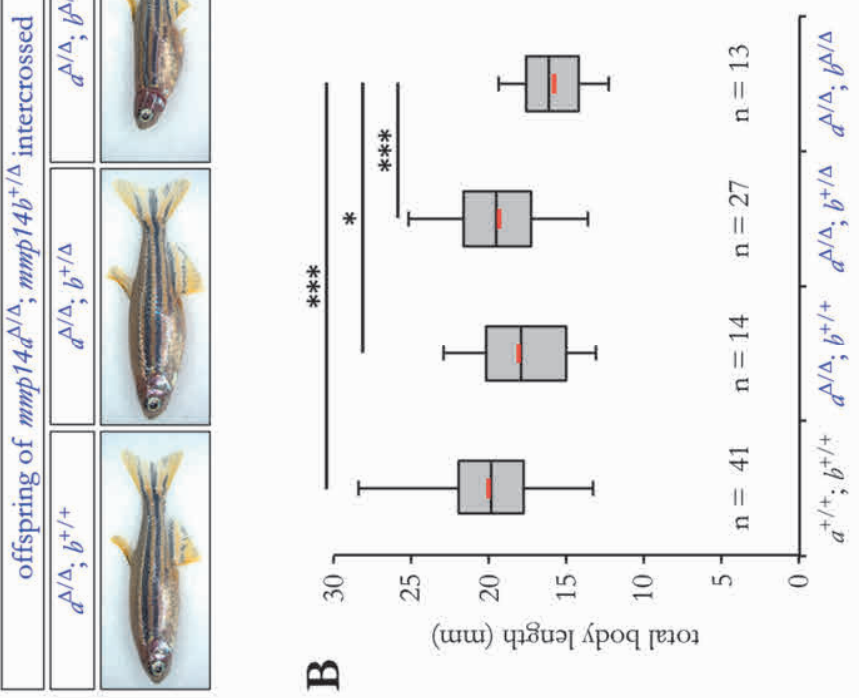

च च

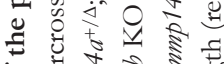

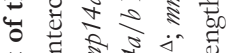

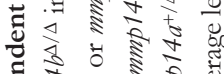

व 0 ह

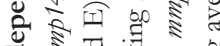

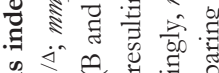

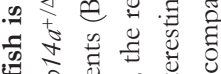

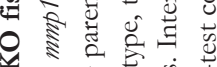

0 ปे

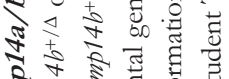

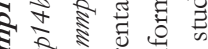

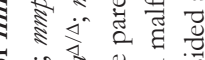

पैं

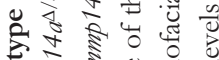

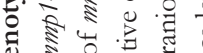
¿

\&.

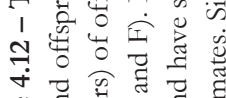

व चै तै च

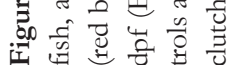



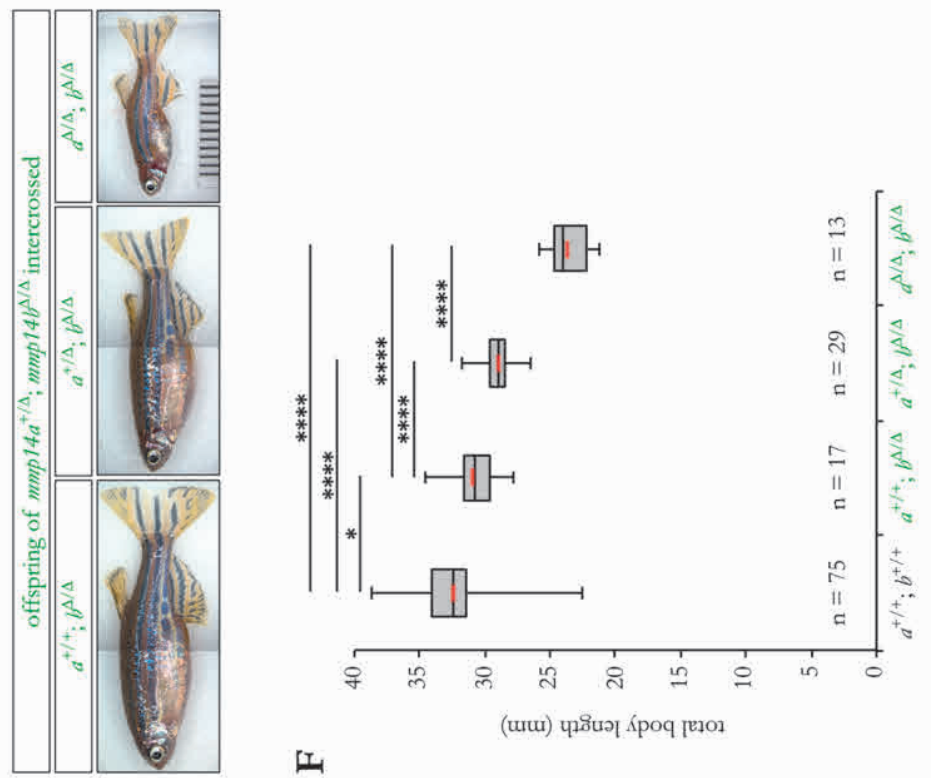

है
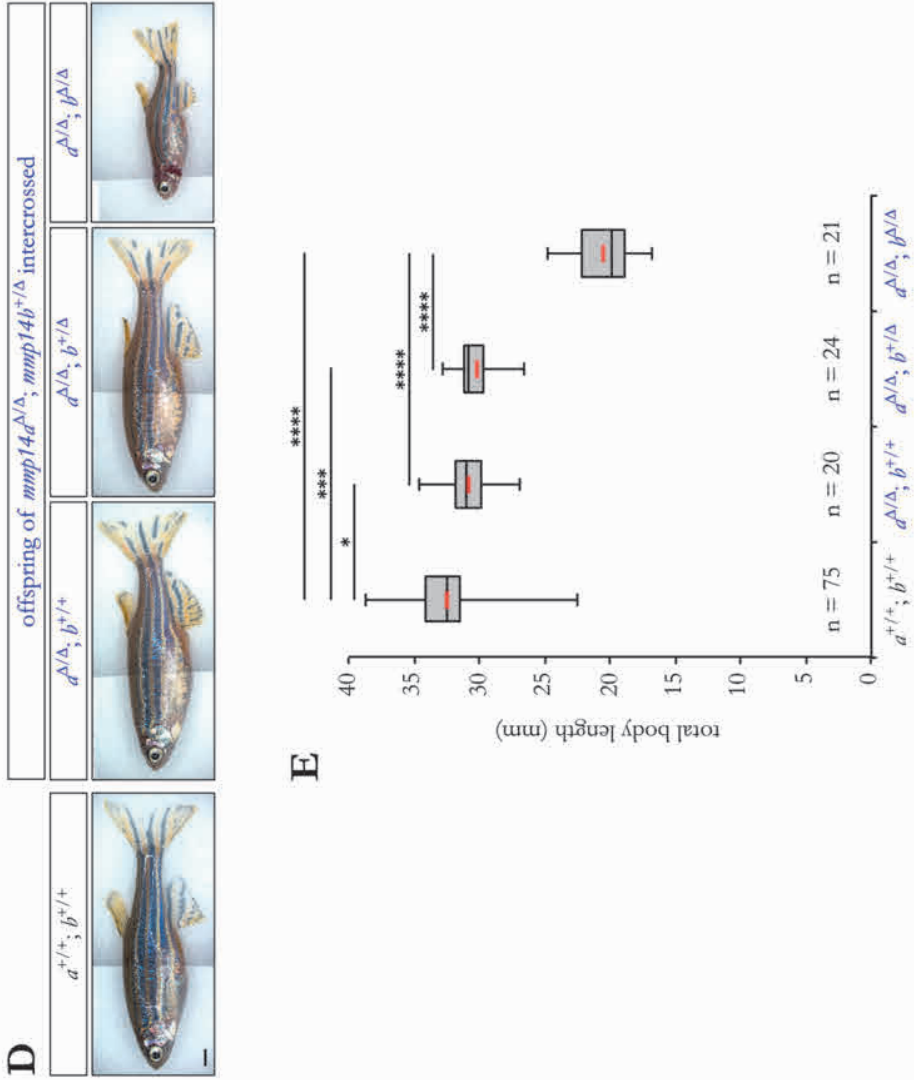

II

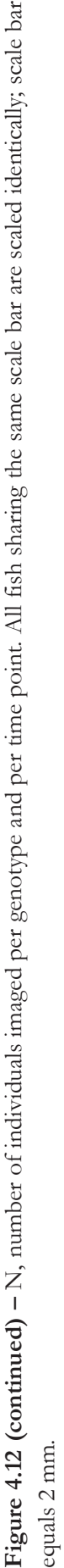


offspring or relatively small clutches (30-80 eggs). Notably, ovaria of 3-month-old $m m p 14 a^{+/ \Delta} ; m m p 14 b^{\Delta / \Delta}$ females were phenotypically normal including presence of late stages of follicular development (not shown). In contrast, the majority of $m m p 14 a^{\Delta / \Delta}$; $m m p 14 b^{+/ \Delta}$ couples set up for crossing repeatedly produced large clutches (up to $\sim 300$ eggs). It is noteworthy that the number of eggs laid seems to be the limiting factor, as the majority of eggs laid was fertilised. This strengthens the hypothesis that Mmp14a/b have a role in ovarian follicle development. At $43 \mathrm{dpf}$, the mmp14a/b KO fish resulting from both crossings were indistinguishable in phenotype and body size (15.8 vs. $17.0 \mathrm{~mm}$; $\mathrm{p}=0.18$; see Fig. 4.12A). In both crossings, the mmp14a/ $b \mathrm{KO}$ fish were significantly smaller than their clutch mates and age-matched WT fish (see Fig. 4.12B-C; see Table 4.3 for significance levels). At $90 \mathrm{dpf}$, mmp14a/b KO offspring from both crossings had the typical phenotype encompassing all the features described above (Fig. 4.12D). Although the offspring of F3 mmp14a $a^{\Delta / \Delta} ; m p 14 b^{+/ \Delta}$ fish (the individuals shown before in Fig. 4.11). Although the double homozygous $\mathrm{KO}$ fish of both crossings were significantly smaller than their clutch mates and WT fish, mmp $14 a / b \mathrm{KO}$ fish of $m m p 14 a^{+/ \Delta}$; mmp $14 b^{\Delta / \Delta}$ parents had a slight yet significant larger

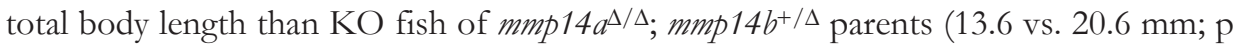
$<0.0001$; Fig. 4.12E-F). Interestingly, the F4 mmp14 $a^{+/ \Delta}$; mmp $14 b^{\Delta / \Delta}$ fish were found to be on average significantly smaller than their mmp14 $a^{+/+} ; m m p 14 b^{\Delta / \Delta}$ clutch mates (29.0 vs. $30.9 \mathrm{~mm}, \mathrm{p}<0.0001)$, whereas there was no significant difference in total body length between $m m p 14 a^{\Delta / \Delta}$; mmp $14 b^{+/+}$and $m m p 14 a^{\Delta / \Delta} ; m m p 14 b^{+/ \Delta}$ fish at $90 \mathrm{dpf}$ (30.8 vs. $30.2 \mathrm{~mm}$; $=0.35$ ). Although this confirms the shorter length of mmp $14 a^{+/ \Delta}$; $m m p 14 b^{\Delta / \Delta}$ fish in the F3 generation, the relative shortness of the head as present as shown in Figure 4.7 was not observed in the F3 generation. In addition, part of the F2 and F3 mmp14 $a^{+/ \Delta}$; mmp $14 b^{\Delta / \Delta}$ fish showed dorsal tilting of the head, similar as seen in mmp14a/b KO fish (Fig. 4.12D). Scoring the number of fish with dorsally tilted head per genotype demonstrated that this feature becomes more evident over time in $m m p 14 a / b \mathrm{KO}$ fish, and is less penetrant in mmp14a $a^{+/ \Delta}$; mmp14 $b^{\Delta / \Delta}$ fish (see Tables 4.4 and 4.5). Notably, there was no statistical difference in the penetrance of this feature

Table 4.4 - Number of individuals per genotype with an uptilted head. n, number of individuals with an uptilted head; N, total number of assessed individuals. Genotypes are coloured as in Figure 4.12.

\begin{tabular}{|c|c|c|c|c|}
\hline & \multicolumn{2}{|c|}{$43 \mathrm{dpf}$} & \multicolumn{2}{|c|}{$90 \mathrm{dpf}$} \\
\hline & $\%$ & $\mathrm{n} / \mathbf{N}$ & $\%$ & $\mathrm{n} / \mathrm{N}$ \\
\hline mmp14a $a^{+/+} ;$mmp14b & 0 & $0 / 39$ & 0 & $0 / 75$ \\
\hline mmp14a $a^{\Delta / \Delta} ; \operatorname{mmp} 14 b^{+/+}$ & 0 & $0 / 14$ & 0 & $0 / 20$ \\
\hline$m m p 14 a^{\Delta / \Delta} ; m m p 14 b^{+/ \Delta}$ & 0 & $0 / 27$ & 0 & $0 / 24$ \\
\hline$m m p 14 a^{\Delta / \Delta} ; m m p 14 b^{\Delta / \Delta}$ & 46 & $6 / 13$ & 86 & $18 / 21$ \\
\hline mmp14a $a^{+/+} ;$mmp14b $1 / \Delta$ & 0 & $0 / 9$ & 0 & $0 / 17$ \\
\hline mmp14a $a^{+/ \Delta} ; m m p 14 b^{\Delta / \Delta}$ & 16 & $3 / 19$ & 14 & $4 / 29$ \\
\hline$m m p 14 a^{\Delta / \Delta} ; m m p 14 b^{\Delta / \Delta}$ & 69 & $9 / 13$ & 77 & $10 / 13$ \\
\hline
\end{tabular}


between mmp14a/b KO offspring of the two crossings $(86$ vs. $77 \%$ at $90 \mathrm{dpf}, \mathrm{p}=$ 0.653). In conclusion, the phenotype of mmp14a/ $b \mathrm{KO}$ fish is independent of the parental genotype. Additionally, $m m p 14 a^{+/ \Delta} ; m m p 14 b^{\Delta / \Delta}$ display a partial phenotype with larger inter-individual variability.

\section{mmp14a/b KO fish have a shortened life-span}

In the growth curve shown in Figure 4.11B, the mmp14a/b KO fish at $90 \mathrm{dpf}$ were on average shorter than at $73 \mathrm{dpf}$, due to the death of several larger individuals. The mmp14a/b KO fish had a shorter life-span compared to their clutch mates and to WT fish. Up till one-month post fertilisation, the different genotypes in the offspring of $m m p 14 a^{\Delta / \Delta} ; m m p 14 b^{+/ \Delta}$ parents followed a Mendelian ratio. At later time points, the percentage of mmp14a/ $b \mathrm{KO}$ fish decreased progressively. When genotyping offspring of $m m p 14 a^{\Delta / \Delta}$; mmp $14 b^{+/ \Delta}$ or mmp14a $a^{+/ \Delta}$; mmp14 $4^{\Delta / \Delta}$ parents between 2 and 3 months of age, only 73 out of a total of $492(14.8 \%)$ fish turned out to be double homozygous $\mathrm{KO}$. This implies that at least $41 \%$ of $m m p 14 a / b \mathrm{KO}$ fish died before three months of age. The majority of $\mathrm{KO}$ fish that survived past $71 \mathrm{dpf}$ died within the next 7 months, as shown in the Kaplan-Meyer survival curve in Supplemental Figure 3.6. In contrast, there was no significant death of the other genotypes between the first week and the first three months. Afterwards only a handful of WT fish and the clutch mates of mmp14a/b KO fish died, usually due to illness not related to the CRISPR-induced mutation (confirmed by pathology evaluation, data not shown). Interestingly, short stature and a shorter life-span have also been reported for patients and mouse models with MMP14 mutations (see Chapter 1 and 2). However, the cause of death differs between patients and mice. Where patients usually die of (untreated) heart failure, the Mmp14 mutant mice are not successfully weaned and die of wasting [1, 4, 5]. It has been suggested by Zhou et al. that the facial abnormalities present in the Mmp14 mu-

Table 4.5 - Levels of significance of Fisher Exact test comparing the number of individuals with uptilted head per genotype. Genotypes are coloured as in Figure 4.12.

\begin{tabular}{|c|c|c|}
\hline & $43 \mathrm{dpf}$ & $90 \mathrm{dpf}$ \\
\hline 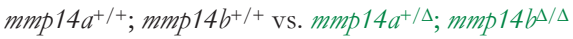 & 0.031 & 0.000 \\
\hline$m m p 14 a^{+/+} ; m m p 14 b^{+/+}$vs. $m m p 14 a^{\Delta / \Delta} ; m m p 14 b^{\Delta / \Delta}$ & 0.000 & 0.000 \\
\hline$m m p 14 a^{+/+} ; m m p 14 b^{+/+}$vs. $m m p 14 a^{\Delta / \Delta} ; m m p 14 b^{\Delta / \Delta}$ & 0.000 & 0.000 \\
\hline$m m p 14 a^{\Delta / \Delta} ; m m p 14 b^{+/+}$vs. mmp14a $a^{\Delta / \Delta} ; m m p 14 b^{+/ \Delta}$ & 0.530 & 0.281 \\
\hline$m m p 14 a^{\Delta / \Delta} ; m m p 14 b^{+/+}$vs. $m m p 14 a^{\Delta / \Delta} ; m m p 14 b^{\Delta / \Delta}$ & 0.006 & 0.000 \\
\hline$m m p 14 a^{\Delta / \Delta} ; m m p 14 b^{+/ \Delta}$ vs. mmp14a $a^{\Delta / \Delta} ; m m p 14 b^{\Delta / \Delta}$ & 0.000 & 0.000 \\
\hline$m m p 14 a^{+/+} ; m m p 14 b^{\Delta / \Delta}$ vs. mmp14a $a^{\Delta / \Delta} ; m m p 14 b^{\Delta / \Delta}$ & 0.002 & 0.000 \\
\hline$m m p 14 a^{+/+} ; m m p 14 b^{\Delta / \Delta}$ vs. mmp14a $a^{+/ \Delta} ; m m p 14 b^{\Delta / \Delta}$ & 0.004 & 0.000 \\
\hline$m m p 14 a^{\Delta / \Delta} ; m m p 14 b^{+/ \Delta}$ vs. mmp14a $a^{+/ \Delta} ; m m p 14 b \Delta / \Delta$ & 0.064 & 0.117 \\
\hline$m m p 14 a^{\Delta / \Delta} ; m m p 14 b^{+/ \Delta}$ vs. mmp14a $a^{\Delta / \Delta} ; m m p 14 b^{\Delta / \Delta}$ & 0.109 & 0.000 \\
\hline$m m p 14 a^{\Delta / \Delta} ; m m p 14 b^{\Delta / \Delta}$ vs. $m m p 14 a^{\Delta / \Delta} ; m m p 14 b^{\Delta / \Delta}$ & 0.428 & 0.653 \\
\hline
\end{tabular}




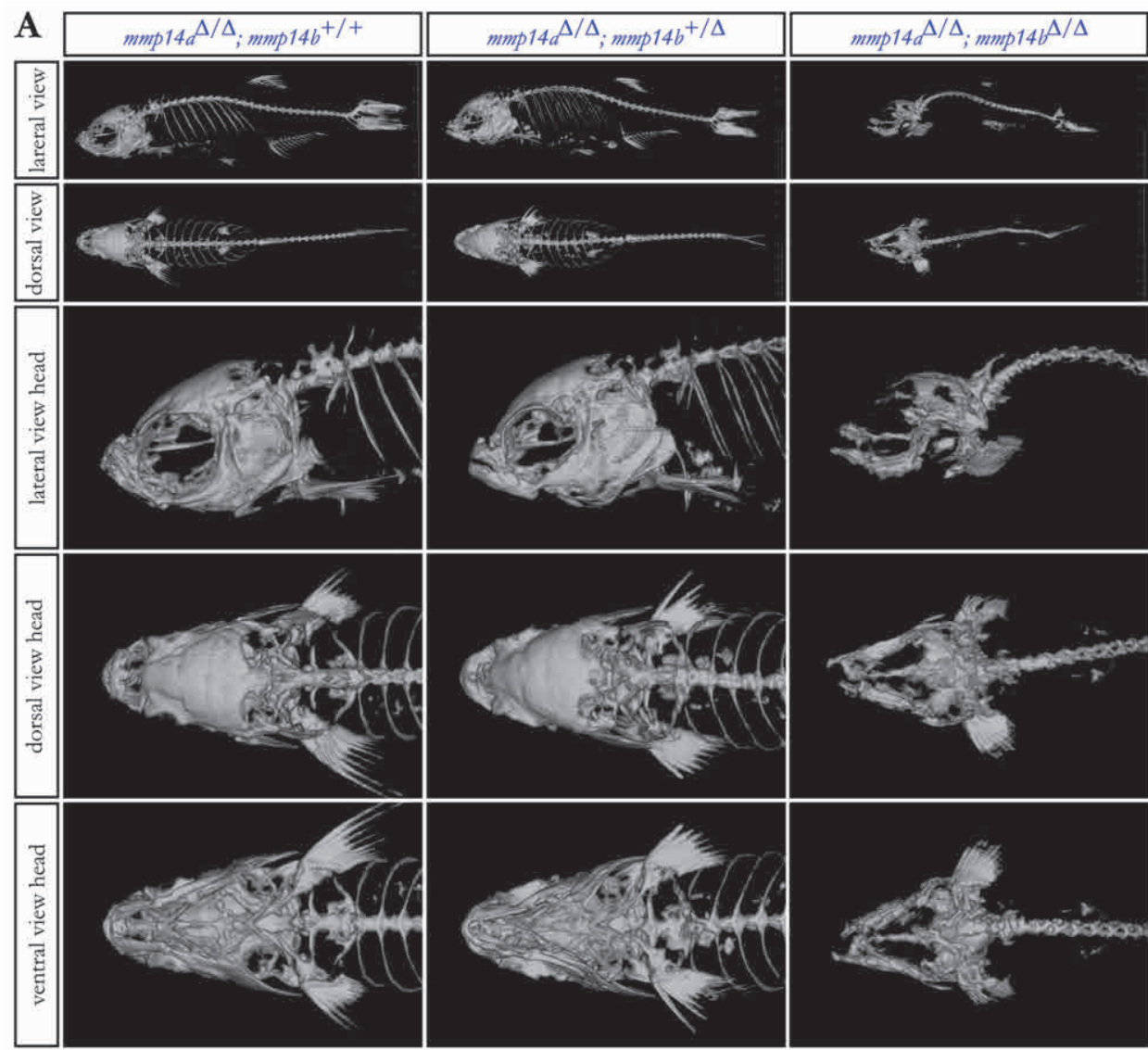

Figure 4.13 - Skull bone density is reduced in $m m p 14 a^{\Delta / \Delta} ; m m p 14 b^{\Delta / \Delta}$ and $m m p 14 a^{+/ \Delta} ; m m p 14 b^{\Delta / \Delta}$ fish. A, 3D renders of the skeleton of offspring of 3 -month-old $m m p 14 a^{\Delta / \Delta} ; m m p 14 b^{+/ \Delta}$ parents, imaged by microcomputed tomography. Skull bones of mmp14a/b KO fish have significantly reduced density (see B) and hence appear as incomplete in 3D reconstruction, as do the ribs. Note the exaggerated Weberian/prehemal kyphosis in mmp14a/b KO fish. B-C, bone density measurement of 3-month-old WT fish and

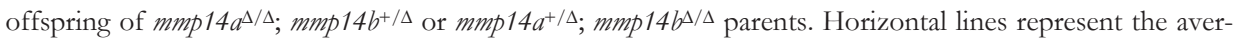
age \pm standard error. The total bone density (average of the entire skeleton) of mmp $14 a / b \mathrm{KO}$ fish is significantly lower than its clutch mates' (see Table 4.6), which is completely attributable to a reduced skull bone density. C, $m m p 14 a^{+/ \Delta} ; m m p 14 b^{\Delta / \Delta}$ fish have significantly reduced bone density, not only of their skull bones, but also of their vertebrae. Note the increase in the spread of measurements for $m m p 14 a^{+/ \Delta}$; $m m p 14 b^{\Delta / \Delta}$ fish. Genotypes are coloured as in Figure 4.12.

tant mice might contribute to poor feeding and malnutrition [53]. Although feeding problems could contribute to the death of mmp14a/b KO fish, there is evidence (as shown in Fig. 4.15 and discussed below) suggesting a different cause of death. 

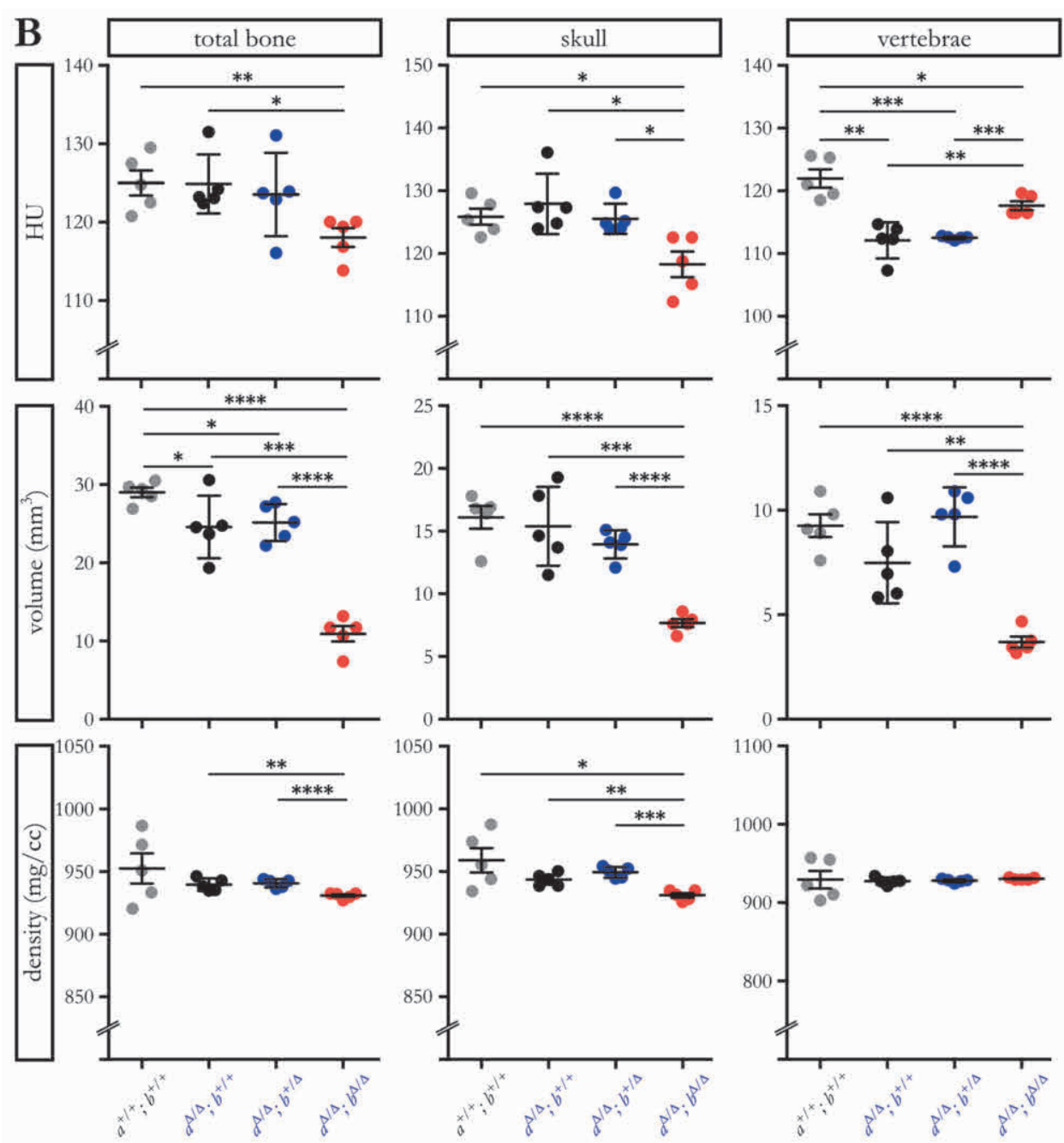

Figure 4.13 (continued).

\section{Knockout of mmp14a/ $b$ causes a reduced skull bone density in zebrafish}

As mmp14a/b KO fish have a short stature, craniofacial malformations and kyphosis, their skeleton was subsequently analysed by microcomputed tomography (micro-CT). Apart from visualising the entire skeleton, this technique allows for the measurement of the bone density as well. A total of five fish per genotype (WT fish and the offspring of both F3 mmp14a $a^{\Delta / \Delta}$; mmp $14 b^{+/ \Delta}$ and F3 mmp14 $a^{+/ \Delta}$; mmp14b $b^{\Delta / \Delta}$ fish intercrossed) were analysed. As development in $D$. rerio correlates with their body size more than their age, it was expected that larger fish would have a higher bone density. The difference in average total body length between mmp14a/b KO fish and their clutch mates increased over time and may confound the bone density results. However, the 

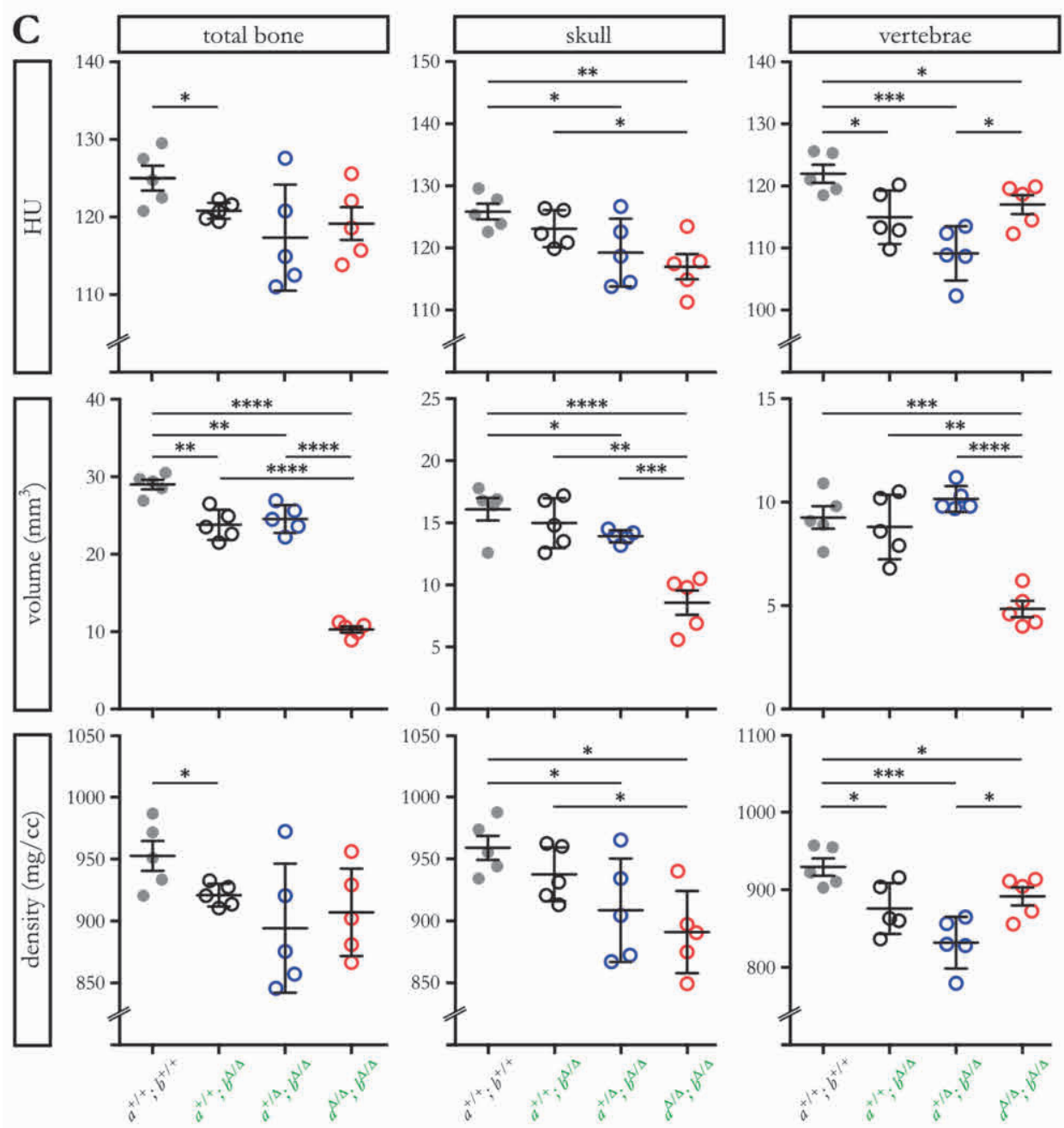

Figure 4.13 (continued).

first trial with 4.5-month-old fish revealed that the bone density in the smaller mmp $14 a / b \mathrm{KO}$ fish is a limiting factor. Although size differences between the genotypes are smaller at juvenile age, imaging mmp14a/b KO juveniles would not yield reproducible density measurements. Therefore, fish were imaged at $90 \mathrm{dpf}$. For a fairer comparison, fish of average total body length were selected with exception of mmp14a/b KO fish, where only the largest individuals were imaged. Micro-CT scanning at $90 \mathrm{dpf}$ revealed mmp14a/b KO fish had a significantly reduced average bone density compared to their clutch mates and W'T fish. For mmp14a/b KO fish with $m m p 14 a^{\Delta / \Delta}$; mmp $14 b^{+/ \Delta}$ parents, this difference in bone density was completely attributable to the reduced density of skull bones, while the density of the vertebral col- 
umn was normal (Fig. 4.13B; see Table 4.6 for levels of significance). Similar results have been reported for the Mmp14 mutant Sabe mouse before [4]. The low skull bone density is reflected in the 3D reconstructions of the skeleton shown in Figure 4.13A, where the skull bones of mmp14a/ $b \mathrm{KO}$ fish had such low density they appeared to be partially missing. The bone density of $m m p 14 a^{\Delta / \Delta}$; mmp14b $1 /+$ and $m m p 14 a^{\Delta / \Delta}$; mmp $14 b^{+/ \Delta}$ was not significantly different from that of age-matched WT fish. Although there was no significant difference in the total bone density between mmp14a/b KO fish of the two crossings 930.9 vs. $908.0 \mathrm{mg} / \mathrm{cc}, \mathrm{p}=0.20$ ), the average bone density measurement of the skull and the vertebral column separately demonstrated a lower average density in double homozygous offspring of mmp $14 a^{+/ \Delta} ; m m p 14 b^{\Delta / \Delta}$ parents (931.1 vs. $891.5 \mathrm{mg} / \mathrm{cc}$ for skull, $\mathrm{p}=0033$; 930.5 vs. 891.5 for vertebrae, $\mathrm{p}=0.0099$ ). One explanation could be differences in volume of the skull relative to the vertebral column in individual fish, thereby contributing to a different extent to the bone density of the total skeleton. This is supported by the notion that there are two mmp $14 a / b \mathrm{KO}$ fish with a skull volume that was almost half of that of other mmp14a/b KO fish, although their vertebral column volume was comparable (Fig. 4.13C). This, in addition to

Table 4.6 - Significance levels of two-sided student T-test on microcomputed tomography measurements between genotypes. Soc, supraoccipital bone; sn2, supraneural of the second Weberian vertebra. Genotypes are coloured as in Figure 4.12.

\begin{tabular}{|c|c|c|c|c|c|}
\hline & \multicolumn{3}{|c|}{ Bone density } & \multicolumn{2}{|c|}{ Angles } \\
\hline & total & skull & $\overline{\text { vertebrae }}$ & kyphosis & soc-sn2 \\
\hline$a^{+/+} ; b^{+/+}$vs. $a^{\Delta / \Delta} ; b^{+/+}$ & 0.32 & 0.17 & 0.87 & 0.60 & 0.84 \\
\hline$a^{+/+} ; b^{+/+}$vs. $a^{+/+} ; b^{\Delta / \Delta}$ & 0.038 & 0.18 & 0.020 & 0.58 & 0.94 \\
\hline$a^{+/+} ; b^{+/+}$vs. $a^{\Delta / \Delta} ; b^{+/ \Delta}$ & 0.36 & 0.36 & 0.90 & 0.16 & 0.15 \\
\hline$a^{+/+} ; b^{+/+}$vs. $a^{+/ \Delta} ; b^{\Delta / \Delta}$ & 0.057 & 0.043 & 0.0008 & 0.38 & 0.015 \\
\hline$a^{+/+} ; b^{+/+}$vs. $a^{\Delta / \Delta} ; b^{\Delta / \Delta}$ & 0.11 & 0.022 & 0.92 & 0.0002 & 0.0001 \\
\hline$a^{+/+} ; b^{+/+}$vs. $a^{\Delta / \Delta} ; b^{\Delta / \Delta}$ & 0.058 & 0.0057 & 0.047 & 0.0011 & $<0.0001$ \\
\hline$a^{\Delta / \Delta} ; b^{+/+}$vs. $a^{+/+} ; b^{\Delta / \Delta}$ & 0.0040 & 0.58 & 0.0084 & 0.91 & 0.75 \\
\hline$a^{\Delta / \Delta} ; b^{+/+}$vs. $a^{\Delta / \Delta} ; b^{+/ \Delta}$ & 0.68 & 0.12 & 0.82 & 0.51 & 0.30 \\
\hline$a^{\Delta / \Delta} ; b^{+/+}$vs. $a^{+/ \Delta} ; b^{\Delta / \Delta}$ & 0.089 & 0.095 & 0.0002 & 0.33 & 0.030 \\
\hline$a^{\Delta / \Delta} ; b^{+/+}$vs. $a^{\Delta / \Delta} ; b^{\Delta / \Delta}$ & 0.0080 & 0.0020 & 0.19 & 0.0004 & 0.0003 \\
\hline$a^{\Delta / \Delta} ; b^{+/+}$vs. $a^{\Delta / \Delta} ; b^{\Delta / \Delta}$ & 0.089 & 0.0091 & 0.016 & 0.0029 & $<0.0001$ \\
\hline$a^{+/+} ; b^{\Delta / \Delta}$ vs. $a^{+/ \Delta} ; b^{\Delta / \Delta}$ & 0.29 & 0.20 & 0.068 & 0.30 & 0.0022 \\
\hline$a^{+/+} ; b^{\Delta / \Delta}$ vs. $a^{\Delta / \Delta} ; b^{+/ \Delta}$ & 0.0019 & 0.061 & 0.0077 & 0.38 & 0.055 \\
\hline$a^{+/+} ; b^{\Delta / \Delta}$ vs. $a^{\Delta / \Delta} ; b^{\Delta / \Delta}$ & 0.46 & 0.034 & 0.43 & 0.0016 & $<0.0001$ \\
\hline$a^{+/+} ; b^{\Delta / \Delta}$ vs. $a^{\Delta / \Delta} ; b^{\Delta / \Delta}$ & 0.047 & 0.52 & 0.0060 & 0.0002 & $<0.0001$ \\
\hline$a^{\Delta / \Delta} ; b^{+/ \Delta}$ vs. $a^{+/ \Delta} ; b^{\Delta / \Delta}$ & 0.082 & 0.061 & 0.0002 & 0.11 & 0.16 \\
\hline$a^{\Delta / \Delta} ; b^{+/ \Delta}$ vs. $a^{\Delta / \Delta} ; b^{\Delta / \Delta}$ & 0.0007 & 0.0001 & 0.059 & 0.0002 & 0.0003 \\
\hline$a^{\Delta / \Delta} ; b^{+/ \Delta}$ vs. $a^{\Delta / \Delta} ; b^{\Delta / \Delta}$ & 0.079 & 0.0055 & 0.014 & 0.0012 & $<0.0001$ \\
\hline$a^{+/ \Delta} ; b^{\Delta / \Delta}$ vs. $a^{\Delta / \Delta} ; b^{\Delta / \Delta}$ & 0.64 & 0.49 & 0.013 & 0.0068 & $<0.0001$ \\
\hline$a^{+/ \Delta} ; b^{\Delta / \Delta}$ vs. $a^{\Delta / \Delta} ; b^{\Delta / \Delta}$ & 0.15 & 0.27 & 0.0002 & 0.0008 & 0.0008 \\
\hline$a^{\Delta / \Delta} ; b^{\Delta / \Delta}$ vs. $a^{\Delta / \Delta} ; b^{\Delta / \Delta}$ & 0.20 & 0.033 & 0.0099 & 0.12 & 0.26 \\
\hline
\end{tabular}



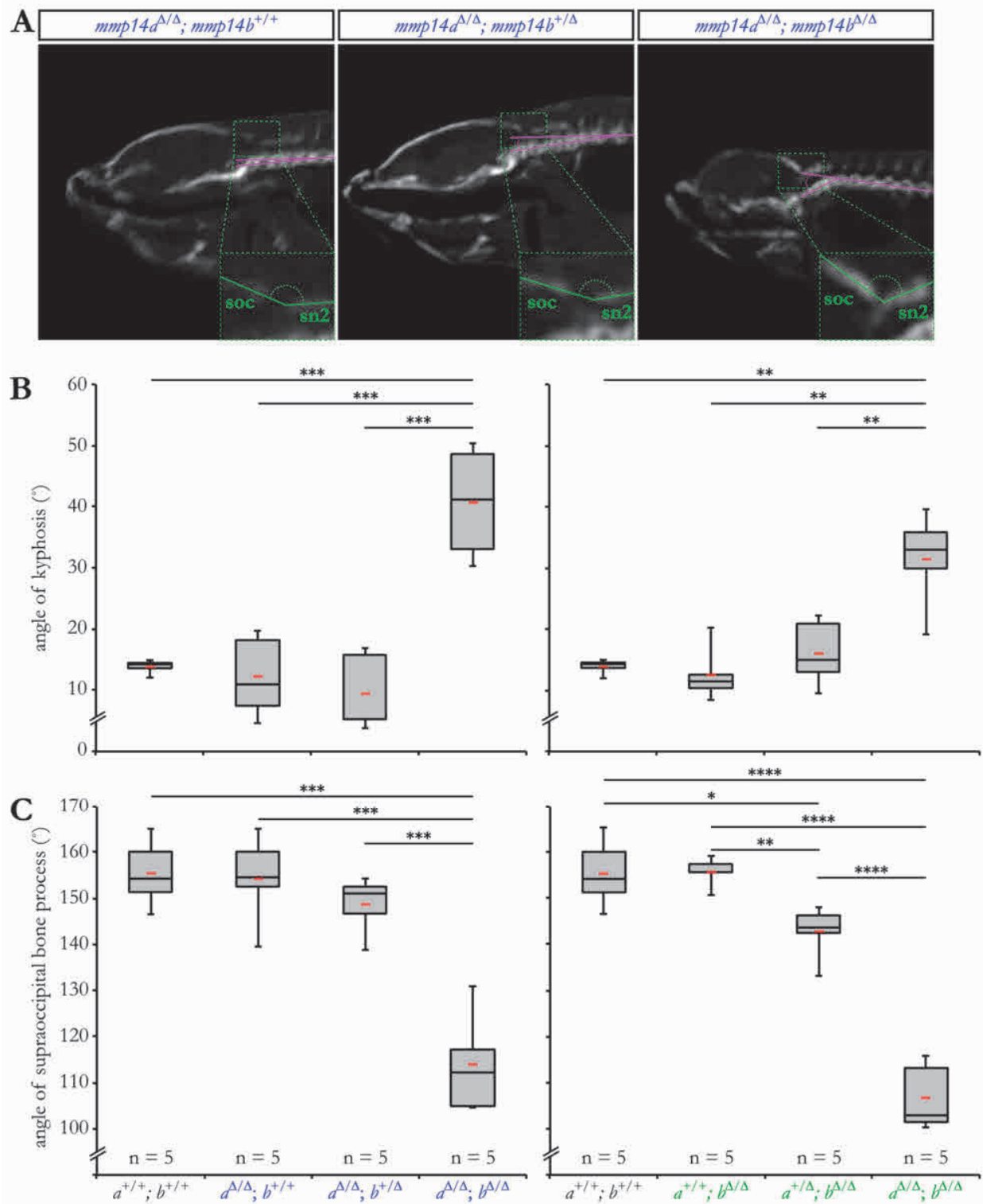

Figure $4.14-m m p 14 a / b$ KO fish have significantly increased Weberian-prehemal kyphosis and a sharper supraoccipital-supraneural 2 angle. A, Midsagittal virtual microcomputed tomography sections of 3-month-old offspring of mmp14 $a^{\Delta / \Delta}$; mmp $14 b^{+/ \Delta}$ parents, with the angle of kyphosis (magenta) and the angle between the supraoccipital bone (soc) and the supraneural of the second Weberian vertebra (sn2; green) indicated. B, The average soc-sn2 angle (red bars) is significantly sharper in mmp14a/b KO fish, irrespective of the crossing. $\mathbf{C}$, the average angle of kyphosis (red lines) is increased in mmp14a/b KO fish, as well as in $m m p 14 a^{+/ \Delta}$; $m m p 14 b^{\Delta / \Delta}$, albeit to a lesser degree. See Table 4.6 for levels of significance. Genotypes are coloured as in Figure 4.12. 
differences in density between the skull bones and the vertebral column within a single fish, was likely to cause the large spread in total bone density in mmp14a/ $b \mathrm{KO}$ fish of

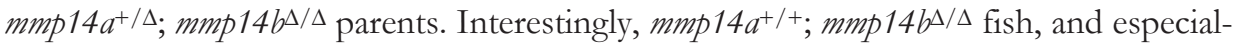
ly $m m p 14 a^{+/ \Delta}$; $m m p 14 b^{\Delta / \Delta}$ fish, had a lower average density of the skull and vertebral column (Fig. 4.13C). However, average total bone density was only significantly reduced for mmp $14 a^{+/+}$; mmp14b $b^{\Delta / \Delta}$ fish compared to age-matched WT fish. This was likely due to the considerable inter-individual spread in skull bone density seen for $m m p 14 a^{+/ \Delta} ; m m p 14 b^{\Delta / \Delta}$ fish, which suggests reduced bone density is a variable feature of this genotype, similar to the dorsal tilting of the head described above. Levels of significance for the 2-sided student T-tests performed on the data of Figure 4.13B-C are summarised in Table 4.6.

\section{Knockout of mmp14a/b causes byperkyphosis and cranial vault abnormalities in zebrafish}

Hyperkyphosis is part of both the human and murine MMP14-related phenotype. Gross anatomy images of mmp14a/b KO fish at $90 \mathrm{dpf}$ suggested the presence of hyperkyphosis. The skeletal 3D renders shown in Figure 4.13A clearly confirmed an increased Weberian-prehemal hyperkyphosis and dorsal tilting of the skull in $90 \mathrm{dpf}$ mmp14a/b KO fish. Differences in Weberian-prehemal kyphosis between the different genotypes were quantified subsequently. For this, the angle between a line connecting the centra of the Weberian vertebrae and a line connecting the centra of the prehemal vertebrae was measured on midsagittal digital sections (magenta lines in Fig. 4.14A). As expected, the angle of Weberian-prehemal kyphosis was significantly increased in mmp $14 a / b$ KO fish compared to their clutch mates and age-matched WT fish. In addition, there was no significant difference between the average angle of kyphosis of

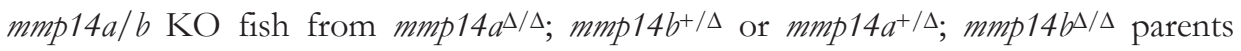
(40.7 vs. $31.4^{\circ}, \mathrm{p}=0.12$ ). On the digital midsagittal micro-CT sections, it appeared that the dorsal tilting of the head was accompanied by morphological differences of the posterior cranial vault. The posterior roof of the skull is formed by the supraoccipital process (soc), which forms a fibrous connection with the supraneural of the second Weberian vertebra $(\operatorname{sn} 2)[54,55]$. In mmp14a/b KO fish, the angle between these two bones was significantly sharper $\left(107-113^{\circ}\right)$, independent of the parental genotype. Notably, the soc-sn2 angle was sharper, yet to a lesser extent, in $m m p 14 a^{+/ \Delta}$; $m m p 14 b^{\Delta / \Delta}$ fish $\left(143^{\circ}\right)$ compared to WT fish $(p=0.015)$. Levels of significance for 2 sided student $\mathrm{T}$-tests performed on the measured angles are summarised in Table 4.6.

\section{mmp14a/b KO fish have an abnormal bone architecture}

After demonstrating mmp14a/b KO fish have a reduced bone density, the bone histology was subsequently analysed on midsagittal sections of 3-month-old WT fish and the offspring of F3 mmp14a $a^{\Delta / \Delta}$; mmp $14 b^{+/ \Delta}$ parents. Haematoxylin and eosin (H\&E) staining revealed an abnormal architecture of craniofacial bones and Weberian vertebrae. 

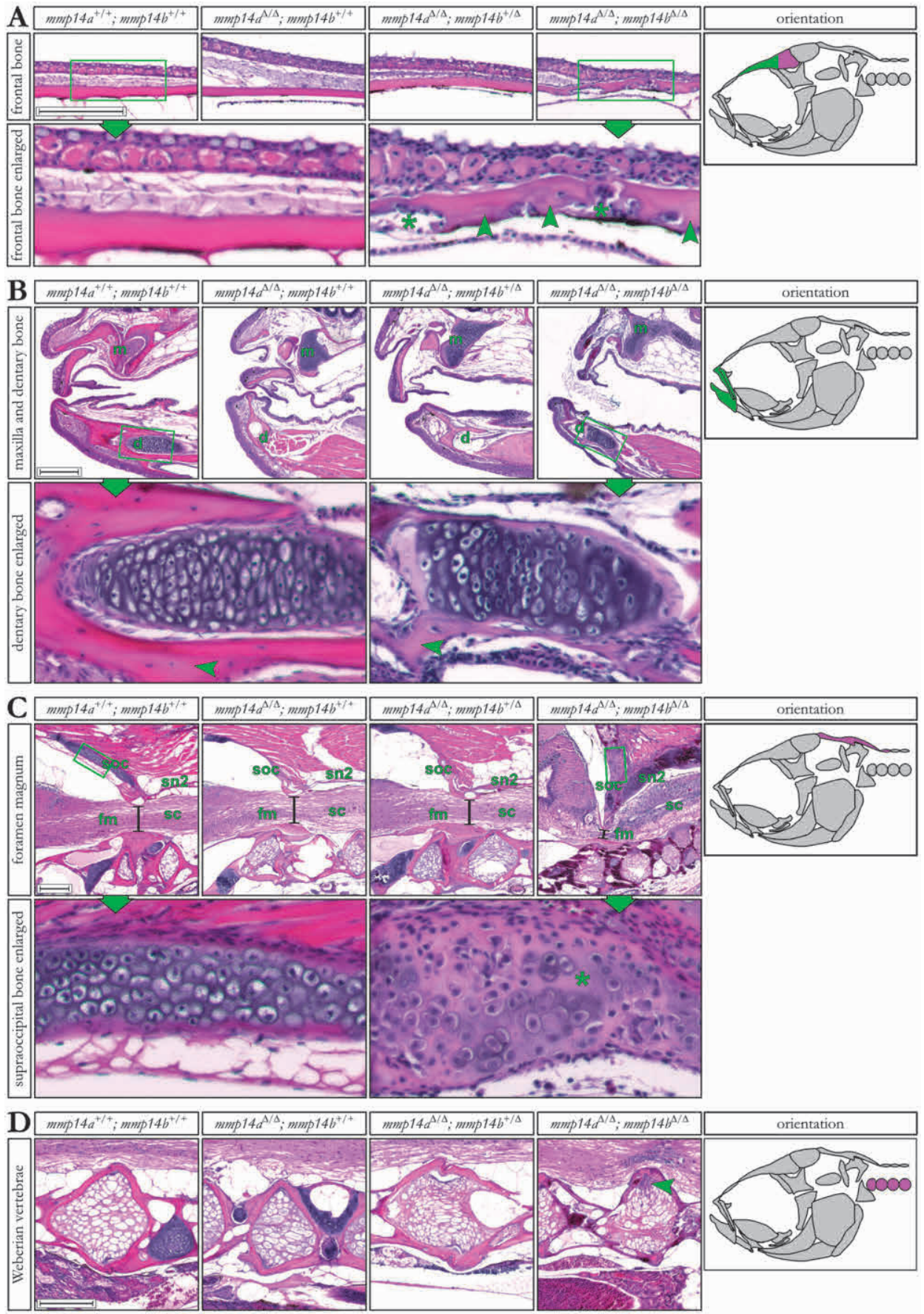

Figure 4.15 - Knockout of mmp14a/b affects ossification both types of ossification of bones of neural crest and mesodermal origin. A-D, haematoxylin and eosin stained midsagittal sections of 3month-old WT fish and offspring of mmp14a $a^{\Delta / \Delta}$; mmp $14 b^{+/ \Delta}$ parents. The cartoons on the right highlight 
Figure 4.15 (continued) - the bones shown on the sections in green (neural crest origin) or magenta (mesodermal origin). A, the frontal bone of WT fish has a smooth surface and even thickness on sagittal sections. In contrast, the frontal bone in $m m p 14 a / b \mathrm{KO}$ fish has a threadbare appearance (stars) with a heavily undulated surface and uneven eosin staining (note the lighter parts highlighted by arrowheads). B, bones forming both the upper (maxilla, $\mathrm{m}$ ) and lower (dentary, d) jaw of mmp14a/ $b$ KO fish have a relative lack of bone and (as a result) relative abundance of cartilage. The chondrocytes (enlarged for dentary) appear less organised and vary in size. $\mathbf{C}$, the suppraoccipital bone (soc) and the supraneural of the second Weberian vertebra (sn2) form a much sharper angle in mmp14a/b KO fish. Both soc and sn2 contain little bone but an abundance of cartilage. In the soc, the cartilage contains disorganised chondrocytes and areas that lack cells (star). Note the relative lack of haematoxylin staining, indicating the lower proteoglycan content of the cartilage. The soc and sn 2 are ventrally extended, and compress the spinal cord (sc) at the foramen $(\mathrm{fm})$ against the Weberian vertebrae (compare the diameter of the foramen magnum indicated by the black line). D, the Weberian vertebral bodies of mmp14a/b KO fish have a disorganised aspect and contain cells in the superior part of the vertebral bodies (arrowhead). Scale bars equal $200 \mu \mathrm{m}$.

As shown in Figure 4.15A, the frontal bones (and parietal bones, not shown) of mmp14a/b KO fish were irregularly thickened and had a variably undulated surface. Furthermore, the eosin staining of the frontal (and parietal) bones of mmp14a/b KO fish was uneven, with parts being less intensely stained (arrowheads). The frontal (and parietal) bone contained cell clusters (stars), resulting in a threadbare appearance on sagittal sections. These cells differed in staining pattern and morphology from chondrocytes (see below) and were suggested to be osteoclasts, locally digesting the bone matrix. Similar to the cells observed in the frontal and parietal bone, zebrafish osteoclasts can be multinucleated [23]. Furthermore, osteoclastic resorption of the outer bone plate of calvarial bones has previously been demonstrated in Mmp14 KO mice $[56,57]$. In contrast, the frontal (and parietal) bones of their clutch mates and WT fish had a smooth surface and even thickness and eosin intensity on sagittal sections. Importantly, both the coronal (between the frontal and the parietal bones) and the lambdoid suture (between the parietal and supraoocipital bones) were open in all genotypes at the age of three months as reported before [58]. On midsagittal sections of mmp14a/b KO fish, skeletal elements of the upper (maxillary bone) and lower (dentary bone) jaw appeared to contain a relatively small amount of bone matrix, whereas cartilage was relatively abundant (Fig. 4.15B). The chondrocytes in the jawbones appeared disorganised and varied considerably in size. The abnormalities in bone architecture of both the cranial vault and upper and lower jaw could account for the low skull bone density as measured by micro-CT. Although in both regions some of the affected bones (including the frontal bone, maxilla and dentary bone, see Table 4.9) are of NC origin, bones of mesodermal origin (parietal bone) were affected as well. In addition, the mesoderm-derived soc and sn 2 of $m m p 14 a / b \mathrm{KO}$ fish are also affected. Midsagittal sections confirmed the much sharper angle between the latter two bones in mmp14a/ $b$ KO fish compared to their clutch mates and WT fish (Fig 4.15C). Furthermore, both structures contained only a minimal amount of bone matrix, while cartilage was relatively abundant. The cartilage in the soc contained disorganised chondrocytes and cell- 
free areas (star). The ECM in these cell-free areas was relatively devoid of proteoglycans, indicated by the minimal haematoxylin staining as a result of the loss of basophilia [57]. Proteoglycan loss has previously been reported in the murine parietal cartilage when this structure is removed during intramembranous ossification of the overlying parietal bone. However, apoptotic bodies, another hallmark of cartilage removal, were absent in the mmp14a/b KO soc [57]. Importantly, the soc and sn2 of mmp14a/b KO fish were ventrally extended, thereby narrowing the foramen magnum and spinal canal, compressing the spinal cord focally against the first Weberian vertebra. This was even further exaggerated by the dorsal tilting of the head. In contrast, in their clutch mates and WT fish, there was only minimal indentation of the spinal cord at the foramen magnum. As described above, mmp14a/b KO died earlier than their clutch mates. Multiple mmp14a/b KO fish were observed swimming inadequately 1-2 days before dying with a laterally bent tail, making tumbling movements at the water surface. After euthanizing such fish by hypothermic shock, the tail straightened, suggesting the tail bending was caused by muscle spasm. Upon dissection, the swim bladder was normal in shape and size, intact and inflated (not shown). Therefore, we hypothesise that the focal spinal cord impingement causes muscle spasm, thereby severely limiting normal movement and feeding behaviour, eventually causing death. In mmp14a/ $b \mathrm{KO}$ fish, the mesoderm-derived Weberian vertebral bodies had a disorganised aspect and contained clusters of multinucleated cells in their dorsal aspect (Fig 4.15D, arrowhead), not observed in their clutch mates or WT fish. In addition, the amount of vertebral bone matrix was relatively small.

During imaging of mmp14a/b KO fish at $90 \mathrm{dpf}$, it was noted that some individuals were unable to adduct their pectoral fins. On gross anatomical inspection in ventral view, the pectoral muscles seemed enlarged (not shown). Horizontal sections through the pectoral girdle demonstrated bilaterally deformed and thickened scapulae, with hypertrophy of subscapular muscle bundles relative to other muscle groups of the pectoral girdle (not shown). It is of note that (apart from the cleithrum) the NC is not reported to contribute to the skeletal elements of the pectoral girdle in D. rerio.

In addition to the musculoskeletal abnormalities mentioned above, diffuse hypoplasia of skeletal and cardiac muscles was observed on midsagittal sections of double homozygous mutants. In contrast to human patients, there were no valvular or outflow tract abnormalities in mmp14a/b KO fish. Apart from the smaller size of visceral organs as part of generalised smaller stature, the organ architecture was well differentiated and organised. No significant histopathological changes were observed in brain, sensory organs, pituitary gland, gill lamellae and pseudobranch, pharyngeal pad and oesophageal sacs, teeth, intestine, kidney, pancreas and spleen, assessed according to Menke et al. [59]. At the age of 3.5 months, mmp14a/b KO fish showed less cytoplasmic glycogen vacuoles in the liver as compared to their clutch mates. The gall bladder was relatively large, although no pathological changes were identified on the sections (not 
shown). There were no significant findings in the sections of mmp14a $a^{\Delta / \Delta} ; m m p 14 b^{+/+}$ and $m m p 14 a^{\Delta / \Delta} ; m m p 14 b^{+/ \Delta}$ fish compared to age-matched WT fish.

Taken together, the data presented in Figure 4.15 suggest Mmp14a and Mmp14b have a crucial role in normal bone modelling and/or remodelling, which is not restricted to bones of $\mathrm{NC}$ origin.

\section{mmp14a/b KO fish have abnormal collagen content in affected bones}

As Mmp14a and Mmp14b are collagenases, the collagen content of the affected bones in the fish shown in Figure 4.15 was subsequently assessed by picrosirius red (PSR) staining of additional sagittal sections. Similar to the H\&E staining shown in Figure 4,17A, the frontal bone of W'T fish, mmp14a $a^{\Delta / \Delta}$; mmp14 $b^{+/+}$and mmp14a $a^{\Delta / \Delta}$; mmp $14 b^{+/ \Delta}$ fish was homogenously stained by PSR (Fig. 4.16A). In contrast, the frontal bone in mmp14a/b KO fish appeared threadbare with a normointense core and hypointense periphery (arrowheads). The staining intensity was lowest in the cell-rich lacunae (stars). The maxillary bone and soc of WT fish, mmp $14 a^{\Delta / \Delta} ; m m p 14 b^{+/+}$and $m m p 14 a^{\Delta / \Delta}$; $m m p 14 b^{+/ \Delta}$ fish were homogenously stained, with a hyperintense peripheral layer (Fig. 4.16B). In mmp14a/b KO fish, both bones were overall less intensely stained; the periphery was not hyperintense and appeared threadbare (arrowheads). Similar to the frontal bone, these peripheral hypointense regions contained cells that differed in morphology from the chondrocytes that were present more towards the centre of these skeletal elements. Taken together, the affected bones in mmp14a/b KO fish clearly had an altered collagen content. To assess whether the collagen fibrils differed in their orientation between bones in mmp14a/b KO fish, their clutch mates and WT fish, the sections shown in Figure 4.16A-C were subsequently imaged by polarised differential interference contrast (DIC) microscopy. In addition to stain collagen, PSR enhances birefringence of fibrillar collagen and can thereby be used to assess collagen fibrillogenesis [60]. Birefringence was assessed in mmp14a/b KO fish in bones with abnormal ossification as shown in Figures 4.15 and 4.16A-C, and compared to their clutch mates and WT fish. As demonstrated in Figure 4.16D-F, no overt changes in birefringence between the different genotypes could be observed. This suggests that the initial collagen fibrillogenesis is unaffected by mmp14a/b KO.

\section{mmp14a/b KO fish have an increased density of epidermal mucous cells}

When analysing the midsagittal H\&E stained sections as shown in Figure 4.15, it was noted that the aspect of the skin of mmp14a/b KO fish was different. Although mmp14a/b KO fish had dermal scales that were covered by an intact epidermis consisting of all reported cell layers, there seemed to be a relative abundance of mucous cells. These mucous secreting cells are located in the middle cell layer of the zebrafish epidermis, covering the entire surface of the fish with their secrete. This mucous layer contributes to the barrier function of the skin, reduces shear stress between the epi- 

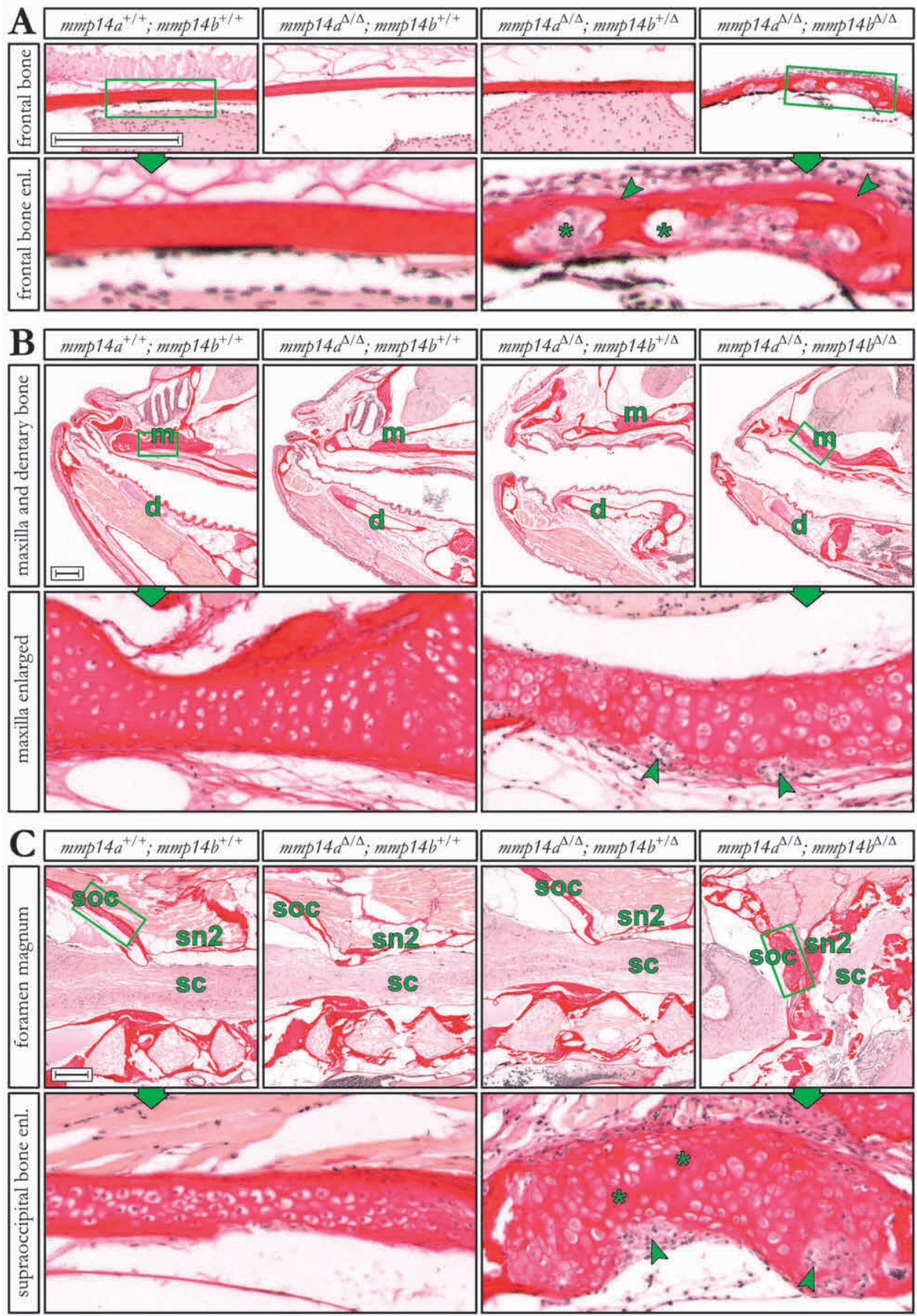

Figure 4.16 - Affected bones in mmp14a/b KO have altered collagen content. A-C, picrosirius red stained midsagittal sections of 3-month-old WT fish and offspring of mmp14a $a^{\Delta / \Delta} ; m m p 14 b^{+/ \Delta}$ parents. A, the frontal bone of WT fish is smooth and homogenously stained by picrosirius red. In contrast, the frontal 

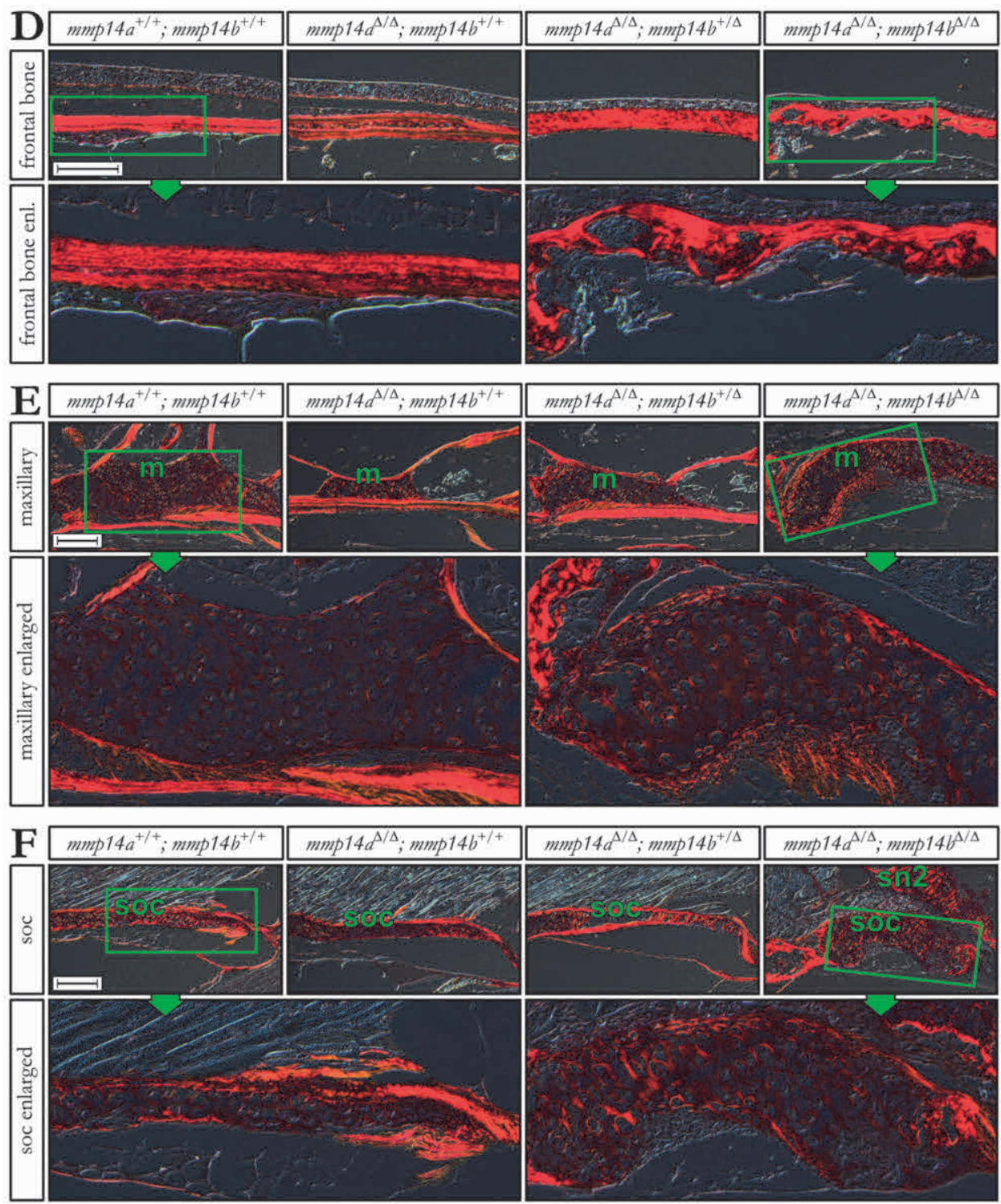

Figure 4.16 (continued) - bone in mmp14a/b KO fish appears threadbare (stars) with hyperintense and hypointense parts (arrowheads). B-C, the maxillary bone ( $\mathrm{m}$ ) and supraoccipital bone (soc) in WT fish are homogenously stained, with a hyperintense peripheral layer. In mmp14a/b KO fish, both bones are overall less intensely stained; the periphery is not hyperintense and appears threadbare (arrowheads). C, cell-free areas in the suppraoccipital bone (soc) are intensely stained by picrosirius red (stars). D-F, representative polarised DIC microscopy images of bones shown in A-C. Birefringence is similar in mmp $14 a / b \mathrm{KO}$ compared to their clutch mates and WT fish, indicating the organisation of collagen fibrils is unaltered by mmp14a/b KO. See legend of Figure 4.15 for additional abbreviations. Scale bars equal $200 \mu \mathrm{m}$. 

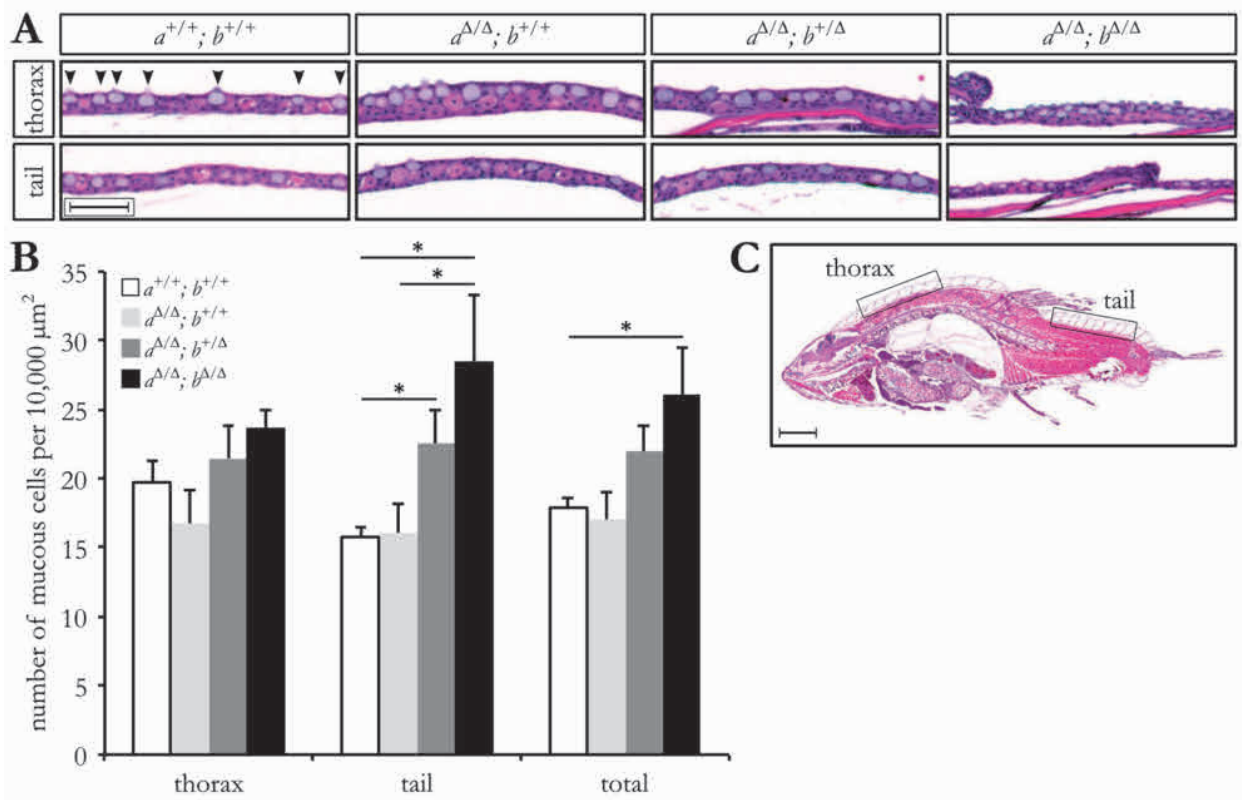

Figure $4.17-m m p 14 a / b$ KO fish have an increased density of epidermal mucous cells. A, representative images of scales in haematoxylin and eosin stained midsagittal sections of $90 \mathrm{dpf}$ WT fish and offspring of $m m p 14 a^{\Delta / \Delta}$; mmp $14 b^{+/ \Delta}$ parents of dorsal thoracic (top row) and tail (bottom row) epidermis, in which mucous cells (arrowheads) can readily be observed. Scale bar equals $50 \mu \mathrm{m}$. B, quantification of the number of mucous cells per $10,000 \mu \mathrm{m}^{3}$ (close to the cross-sectional area of the epidermis covering an average WT scale), demonstrating the density of mucous cells is significantly increased in mmp $14 a / b \mathrm{KO}$ fish, which is completely attributable to an increased density in the tail skin. Values are shown as average \pm SEM. Note that all cell types in mmp14a/b KO skin are smaller compared to their clutch mates and WT skin. C, midsagittal section of a WT fish, indicating where images of scales were taken and quantified. Scale bar equals $2 \mathrm{~mm}$.

dermis and the surrounding water, contains an array of antimicrobial factors [61, 62]. There is an interesting link between mucous cells and human sebocytes. Both cell types die by holocrine secretion and share the expression of certain markers including Lrig1 [63 and unpublished, personal communication from Dr. T.J. Carney]. In addition, WS patients have severe acne, characterized by the presence of comedones, which are cystic sebaceous glands (see Chapter 3). Mucous cells can readily be identified on H\&E stained sections by their round, lilac mucous core (Fig. 4.17A). To assess differences in mucous cell number, these cells were counted in five fields of view per region (thorax and tail, see Fig. 4.17C) per fish on midsagittal H\&E stained sections of 3-month-old fish at $10 \mathrm{X}$ magnification, for a total of five individuals per genotype. As mmp14a/b KO fish were smaller than their clutch mates and wild type fish, their scales were smaller as well. To correct for differences in scale size, the mucous cell density was expressed as the number of counted mucous cells per cross-sectional scale area. Although the mucous cells in mmp14a/b KO skin looked smaller compared to the skin 
of their clutch mates and WT fish (Fig. 4.17A), their density was increased (26 vs. 17 per $10,000 \mu \mathrm{m}^{2}$, Fig. 4.17B) compared to W'T fish. This increase was only statistically significant for scales of the dorsal tail (28 vs. 16 per $10,000 \mu \mathrm{m}^{2}$ ), but not the dorsal thorax ( 24 vs. 20 per 10,000 $\mathrm{m}^{2}$ ). The latter is likely attributable to the relatively large inter-individual spread in mucous cell density and the relatively small number of fish that were analysed per genotype. It is notable that also in $m m p 14 a^{\Delta / \Delta} ; m m p 14 b^{+/ \Delta}$ fish, the density of mucous cells was significantly increased in tail skin (23 vs. 16 per 10,000 $\left.\mu \mathrm{m}^{2}\right)$. These data suggest mmp14a and/or mmp14b play a role in mucous cell development, which is altered upon $\mathrm{KO}$ of $m m p 14 a / b$.

\section{Live imaging of cranial neural crest cells in zebrafish embryos and larvae with a custom-built light sheet microscope}

Having demonstrated craniofacial bone abnormalities in mmp14a/b KO fish, subsequent experiments aimed to elucidate when these abnormalities arise. In zebrafish, several major processes are involved in the formation of the craniofacial skeletal elements. As shown in Figure 4.1C, the majority of the craniofacial skeletal elements are of NC origin. The NC cells are induced at the dorsolateral aspect of the neural keel and later the (closing) neural tube and, after delamination, migrate extensively throughout the embryo [15, 64-67]. After reaching their final destination, NC cells subsequently differentiate and give rise to a vast array of cell types and tissues. In zebrafish, the cranial NC cells contribute to the larval craniofacial cartilage elements, which at a later stage are remodelled into the adult skeletal elements [14]. Given the role of MMP14 in invasive cell motility, disturbed cranial NC cell migration in mmp14a/b KO fish seemed a plausible explanation for the bone phenotype in these fish. To visualise NC cells in zebrafish embryos/larvae, the $\operatorname{Tg}(\operatorname{sox} 10::$ eg $p$ ) background was utilised. As Sox10 is a key regulator of NC cell development in mammals as well as in zebrafish, all NC cells in this background are labelled with Egfp [28]. However, imaging the green fluorescent NC cells in situ while three-dimensionally migrating in a region of interest over $200 \mu \mathrm{m}$ in each direction would be challenging using regular line scanning confocal fluorescence microscopy. To overcome the limitations in scanning speed and sample size, single plane illumination fluorescence microscopy was used. For this, a custom-built dual-sided light sheet microscope, under development in our institute, was optimised for usage with live zebrafish embryos and larvae. The basic set-up of this microscope is shown in Figure 4.18B. A thin sheet of excitation light illuminates the plane at which emitted fluorescence is detected, perpendicular to the light sheet, thereby greatly reducing out-of-focus light and achieving a higher signal-to-noise ratio [68]. Focussing a light sheet from both sides onto the sample guaranteed an even illumination, even in thick samples. Together with a long working distance 20X water lens, optical z-stacks up to $300 \mu \mathrm{m}$ in thickness could be imaged at a speed of 50-200 ns per optical section. With this set-up, the system had an optical 

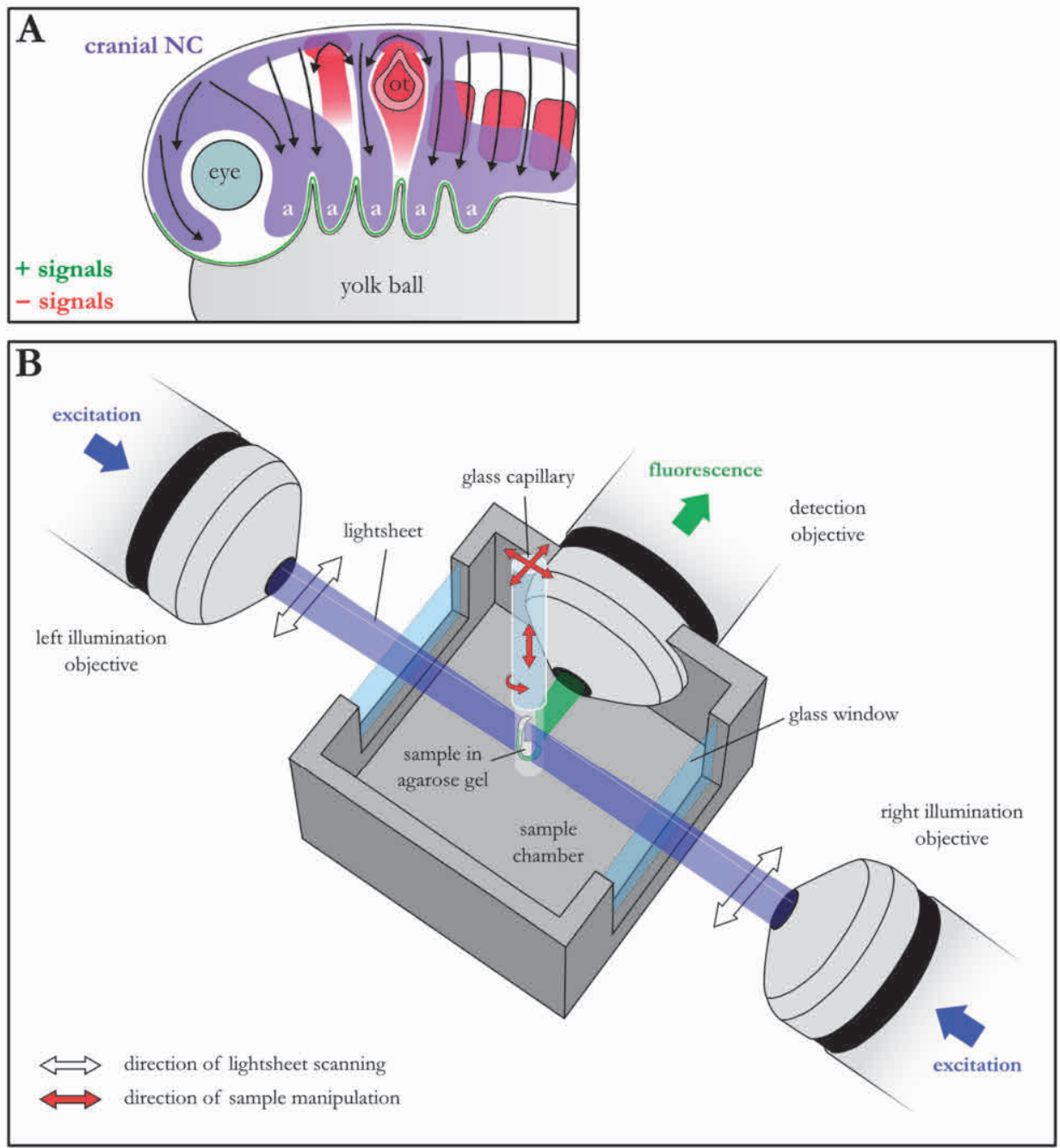

Figure 4.18 - Live in vivo imaging of cranial neural crest cell migration in zebrafish embryos with a custom-built dual-sided light sheet fluorescence microscope. A, in zebrafish, cranial neural crest (NC, purple) cells are induced around 10.5 hpf dorsolaterally in the embryo. By $12 \mathrm{hpf}$ the cranial NC cells delaminate and start migrating, first as a single wave that shortly after splits into distinct streams (black arrows): one anterior of the eye, and three into the developing branchial arches (a). Their migratory path is determined by positive (green) and negative (red) signals from the surrounding mesoderm. By $24 \mathrm{hpf}$, the cranial NC cells have completed the majority of their migration. B, schematic overview of single-plane illumination microscopy. The sample, i.e. a dechorionated $\operatorname{Tg}(\operatorname{sox} 10:$ : eg $p$ p $)$ zebrafish embryo, is embedded in a column of low-melting point agarose, which is kept in place in a water-filled sample chamber by a glass capillary attached to a sample holder (not shown for clarity). An excitation light sheet is focussed on the sample from two sides; the light that the sample emits is focused on a camera by a long-working distance water lens. Ot, otic vesicle. A is adapted from Mayor et al [64], with permission; B is adapted from OpenSPIM Wiki; the original is available at http://openspim.org/File:SPIM_logo.jpg\#filelinks. 
resolution of $300 \mathrm{~nm}$ in the $x, y$ plane and $10 \mu \mathrm{m}$ in $z$-direction, sufficient to distinguish individual cells. In addition, the high speed with which z-stacks could be imaged cause relative low fluorophore bleaching and phototoxicity, thereby improving the viability of the embryos and allowing for longer observation periods [68].

In the first series of trial experiments, $\mathrm{NC}$ cells were imaged at different time points in $\operatorname{Tg}(\operatorname{sox} 10:: \mathrm{g} g \mathrm{p})$ embryos WT for both mmp14a and mmp14b. In zebrafish, cranial NC cells are reportedly induced around $10.5 \mathrm{hpf}$. Around $12 \mathrm{hpf}$, the cranial NC cells start migrating (Fig 4.18A) [65]. The first NC cells reach the pharyngeal arches around 17 hpf, and by 24 hpf the majority of cranial NC migration is completed [15, 24, 64-67, 69]. In accordance with the literature, in $16 \mathrm{hpf}$ embryos the cranial NC cells had initiated their migration but had not yet reached the pharyngeal arches (Supp. Fig. 3.7A). At $28 \mathrm{hpf}$, cranial NC cells had completed the majority of their migration and had reached the pharyngeal arches (Supp. Fig. 3.7B). For imaging at later stages, embryos and larvae were grown in presence of $200 \mu \mathrm{M}$ 1-phenyl 2-thiourea (PTU) from $24 \mathrm{hpf}$ onwards to inhibit melanophore formation [70-73]. At $3 \mathrm{dpf}$, cranial NC cells were observed to contribute to the developing larval craniofacial cartilage elements (Supp. Fig. 3.7C), which are larger and more distinct at $6 \mathrm{dpf}$ (Supp. Fig. 3.7D). Importantly, at all imaged developmental stages, individual cranial NC cells could be visualised in almost the entire head. Having optimised live in vivo imaging of cranial NC cells, the effect of $m m p 14 a / b \mathrm{KO}$ on their migration was assessed next.

\section{Knockout of mmp14a/b does not affect cranial NC migration in zebrafish}

To analyse the effect of mmp $14 a / b \mathrm{KO}$ on cranial NC cell migration, F2 mmp14at/ $m m p 14 b^{+/ \Delta}$ fish were outcrossed into the $\operatorname{Tg}($ sox10::egfp $)$ background. Their F3 offspring was selected for green fluorescence at $24 \mathrm{hpf}$, and fin-clip genotyped for

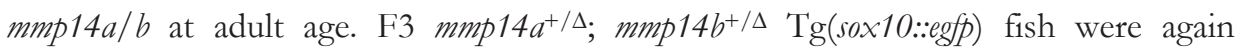
crossed with F2 mmp14a $a^{+/ \Delta}$; mmp $14 b^{+/ \Delta}$ fish to generate all possible combinations of mmp14a/b genotypes in the transgenic background for subsequent crossing. At 26-29 hpf, a single z-stack was recorded of the head of the selected embryos. At this time point, the majority of NC migration has been completed. Differences between genotypes in cranial NC migration should, therefore, be reflected in the relative position of $\mathrm{NC}$ cells at this time point. In addition, the chosen interval is small enough to make meaningful comparisons between embryos. By this approach, multiple embryos per clutch could be imaged. Accordingly, F5 green fluorescent embryos of three clutches

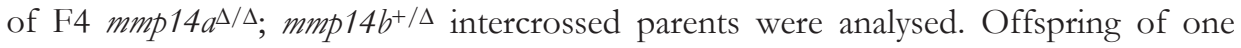

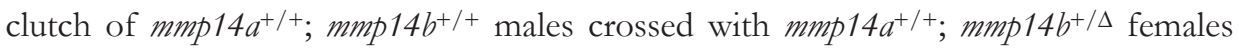
served as control. As melanophore formation only starts around this time and because PTU treatment can potentially disrupt normal craniofacial development, embryos were not treated with PTU during this experiment [70, 75]. As shown in Figure 4.19, no overt differences in $\mathrm{NC}$ cell localisation were observed between the different geno- 

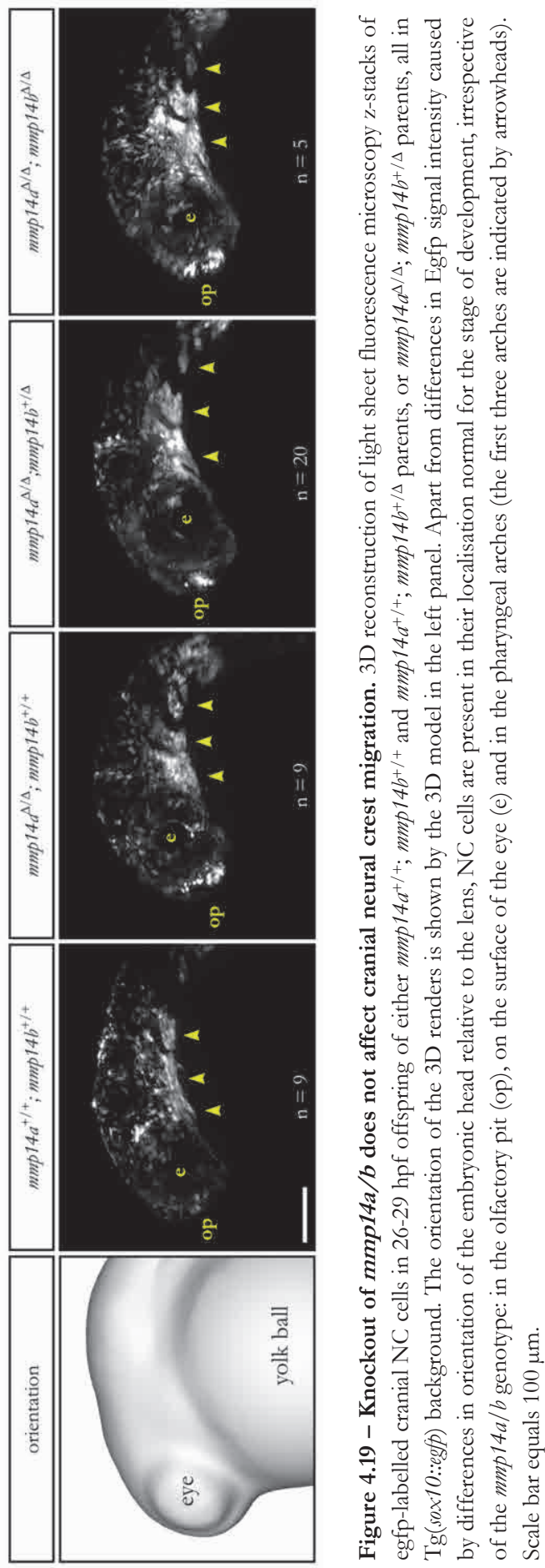
types. In mmp14a/b KO embryos, as in their clutch mates and WT cousins, NC cells were present conform the developmental stage of the embryos: in the pharyngeal arches (arrowheads), in the olfactory pit, and on the surface of the eye. There were, however, some differences in relative signal intensity in these locations between, but also within (not shown) the different genotypes. This is likely a result of differences in orientation of the embryo relative to the detection objective when imaging. Nevertheless, these data suggest that cranial NC migration up to $26 \mathrm{hpf}$ proceeded normally and was unaffected my mmp14a/b KO. One would be inclined to conclude that Mmp14a and Mmp14b, therefore, do not play a crucial role in cranial NC migration. However, due to the manner of crossing, a possible contribution of mmp14b maternal transcripts "rescuing" a putative impaired NC migration cannot be ruled out. To further assess whether the NC distribution and function is normal in mmp14a/b KO larvae, the larval craniofacial cartilage elements were subsequently analysed.

\section{Knockout of mmp14a/b does not affect larval craniofacial cartilage formation in zebrafish}

Having demonstrated a normal distribution of cranial NC cells in 26-29 hpf embryos, the larval craniofacial cartilage elements of $\mathrm{NC}$ origin were assessed next. For this, 5 dpf F5 offspring of F4 mmp14 ${ }^{\Delta / \Delta}$; mmp $14 b^{+/ \Delta}$ parents were stained with alcian blue; W'T larvae served as control. Alcian blue is a basic blue dye that binds to acidic mucopolysaccharides such as glycosaminoglycans present in cartilage [76]. Qualitative analysis did not identify overt differences between the genotypes. In mmp14a/b KO larvae, all elements were present and had normal size, shape and staining intensity (Fig. 4.20). Formation of larval craniofacial cartilage elements, therefore, does not seem to depend on Mmp14a/b, although the possible contribution of maternal transcripts cannot be entirely ruled out even at this age. After showing the larval craniofacial cartilage is unaffected in mmp14a/b KO fish, mineralization was assessed next.

Knockout of mmp14a/b does not delay mineralisation during D. rerio larval and juvenile development In zebrafish, mineralisation of the skeleton initiates in the craniofacial elements around $5 \mathrm{dpf}$ [77]. Around $4 \mathrm{~mm}$ total length ( $\sim 7 \mathrm{dpf})$, mineralisation of the axial skeleton, formed by the vertebral column and the median fins, starts in perichordal centra [26, 77-79]. Similar to the majority of the craniofacial bones (see Table 4.9), the vertebrae form by membranous ossification. Ossification of the vertebral column can be divided into two parts. Anteriorly, unsegmented ossification gives rise to the baso-occipital articulary process (bop). Posteriorly, metameric mineralisation forms the vertebrae. The latter process starts at the third and fourth vertebral body; subsequent ossification centra are added bidirectionally [78]. At $5.5 \mathrm{~mm}$ total length, preural 1 and ural 1 ossify, after which ossification proceeds bidirectionally in the tail fin [78]. As ossification of the vertebral column proceeds following a fixed pattern and forms separate ossification centra, the number of centra that have calcified at any given time point can be 

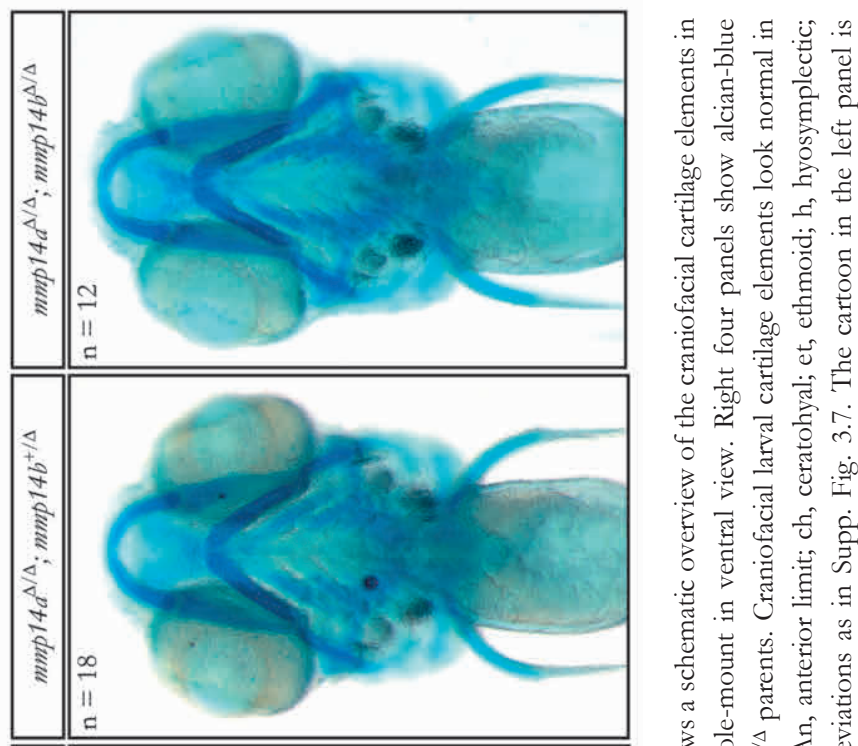

पु 苞苟

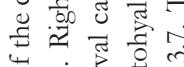

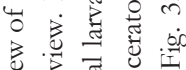

至焉 语

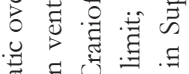

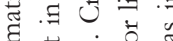

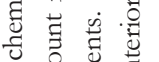

๘

光匹

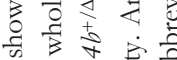

च वे है

苛

芯氙方

E

ơ

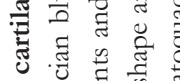

तี $\frac{\pi}{0}$ है
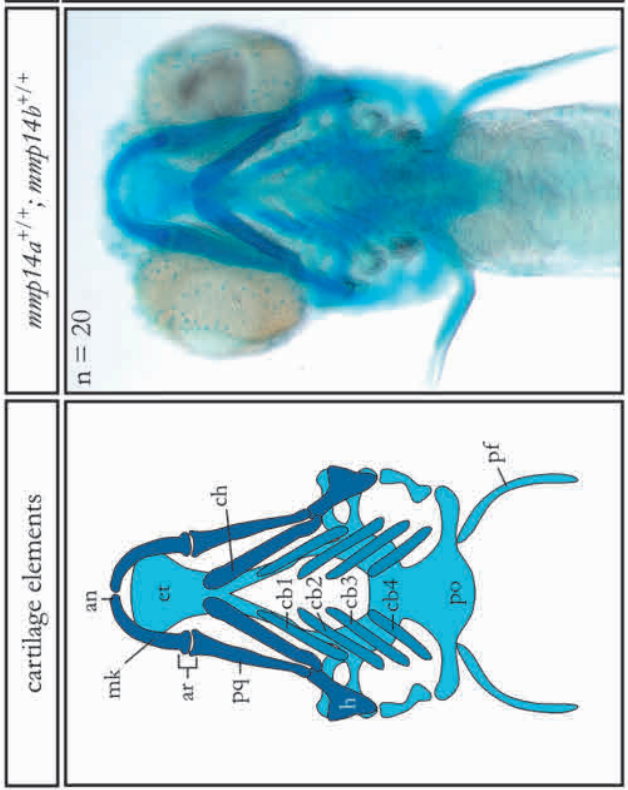

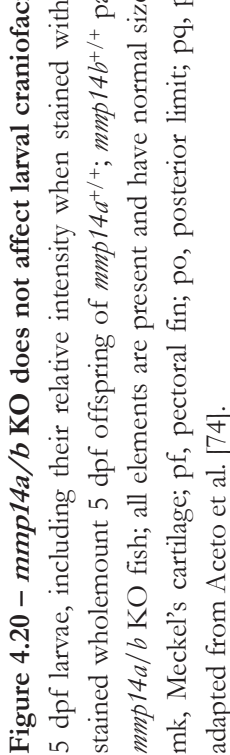



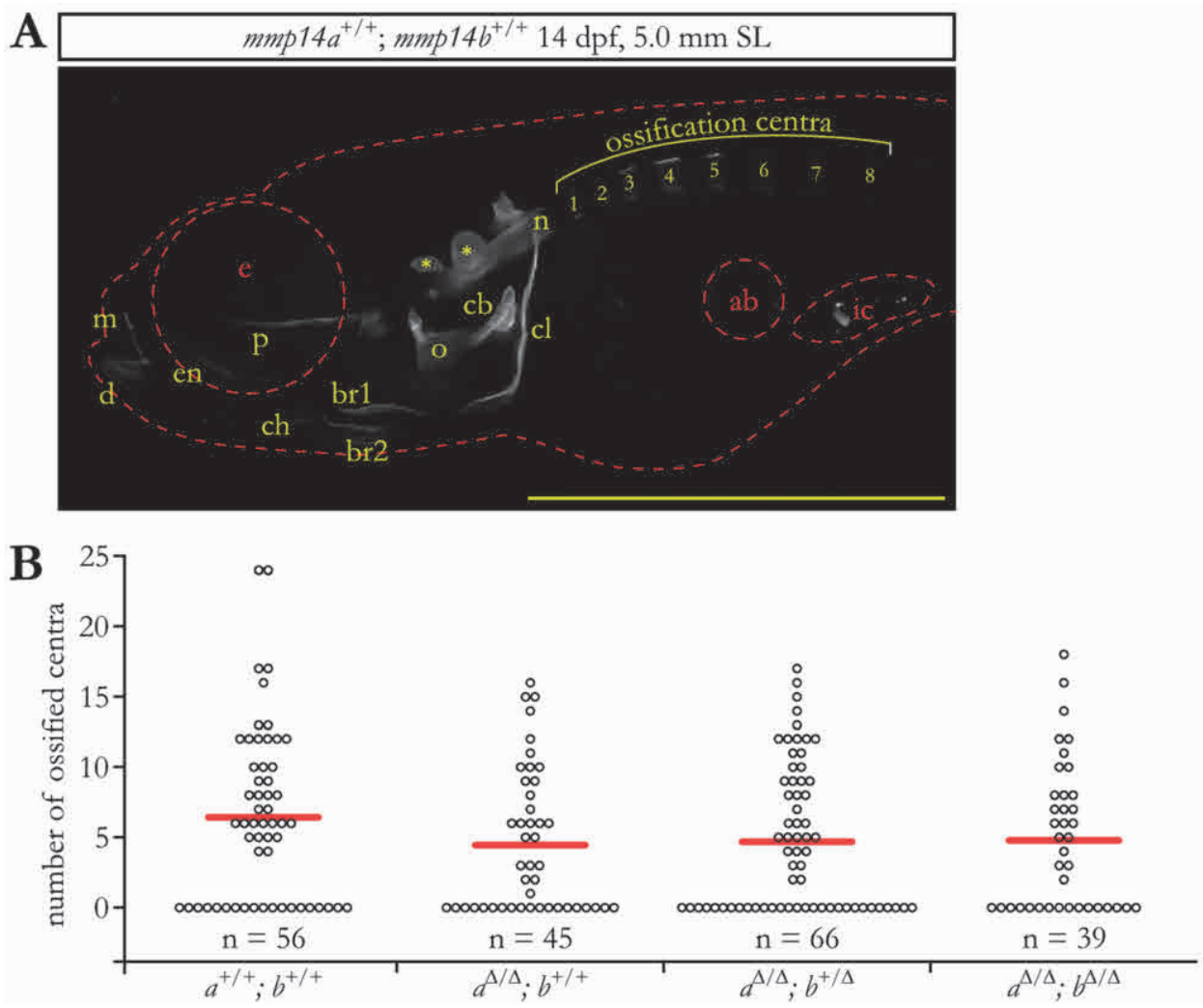

Figure $4.21-m m p 14 a / b$ KO does not delay mineralisation of the vertebral column. A, fluorescence microscopy image of a whole mount $14 \mathrm{dpf}$ WT larva $(5 \mathrm{~mm}$ standard length, SL) stained for calcified bone with alizarin red. Lateral view, anterior to the left. At two weeks of age, multiple craniofacial skeletal elements have started ossifying. In addition, the vertebral ossification centra start to calcify around the notochord in a rostrocaudal wave, which has progressed until the eighth vertebra for the larva shown here. B, quantification of the number of calcified vertebral ossification centra at $14 \mathrm{dpf}$ in WT larvae and offspring of $m m p 14 a^{\Delta / \Delta} ; m m p 14 b^{+/ \Delta}$ parents. Red lines represent average value per genotype. There is no significant difference in the average number of calcified vertebral ossification centra between the different genotypes. Note the large proportion of larvae that have not yet started ossification of the vertebral column; this proportion does not significantly differ between the different genotypes. Ab, air bubble; br, branchiostegal ray, cl, cleithrum; e, eye; en, entopterygoid; ic, intestinal content; o, opercle; p, parasphenoid. Additional abbreviations as in Figure 4.20. Stars indicate the otholiths. Scale bar equals $1 \mathrm{~mm}$.

counted as measure for (membranous) ossification rate. For this, $14 \mathrm{dpf}$ offspring of $m m p 14 a^{\Delta / \Delta} ; m m p 14 b^{+/ \Delta}$ parents and $14 \mathrm{dpf}$ wild type larvae were stained with alizarin red (AR), a calcium-binding dye. Although AR stained skeletal elements can be visualised with brightfield microscopy, a higher sensitivity and contrast can be achieved by using fluorescence microscopy [76]. As shown in Figure 4.21A, many calcified craniofacial skeletal elements, conform the larvae's age, could be identified by this method [74]. In addition, vertebral ossification centra could be visualised and scored. In all imaged 14 dpf larvae, ossification had started in the head. Although there was no sig- 
nificant difference in the average number of calcified vertebral centra between the genotypes, there were considerable inter-individual differences (Fig. 4.21B). For all analysed genotypes, in many larvae did ossification did not proceed to the vertebrae yet (Fig. 4.21B and Table 4.7). However, the number of fish in which vertebral calcification had not yet started did not differ significantly between the genotypes (Table 4.8). As the SL did not differ in these clutches at $21 \mathrm{dpf}$ (see below) and differences in length between the genotypes have been shown to increase over time, SL was not measured in the imaged $14 \mathrm{dpf}$ larvae. At $21 \mathrm{dpf}$, the experiment was repeated. At this time point, ossification of craniofacial skeletal elements had proceeded, although no overt differences in ossification or bone shape could be observed between the different genotypes (Fig. 4.22A). In the vertebral column, ossification had proceeded further caudally and ossification of the tail fin had initiated. To assess differences in ossification rate, the number of calcified elements in both the vertebral column and the tail fin was scored (see Fig. 4.22C for an overview) and corrected for the SL to achieve an "ossification score". The measured SL was corrected for the effects of fixation $(+0.29$ $\mathrm{mm}$ ) [29]. Apart from differences between juveniles with the same genotype, there were no statistically significant differences in SL between the assessed genotypes at 21 dpf (on average 5.5-5.8 mm; Fig. 4.22D and Table 4.8). In addition, there were no significant differences in ossification score between the different genotypes (9.07-9.59; Fig. 4.22E and Table 4.8). Normal ossification in mmp14a/b KO 14 dpf larvae and 21 dpf juveniles is consistent with the mutant Mmp14 mouse models, where initial ossification is normal, suggesting this process is not Mmp14-dependent [56].

\section{At 30 dpf, mmp14a/b KO develop aberrantly shaped calvarian bones}

Having demonstrated that membranous ossification is not delayed in mmp14a/b KO fish in the first three weeks of life, juveniles were stained with alizarin red at $30 \mathrm{dpf}$. This revealed the first skeletal differences between the genotypes, just before the gross anatomical features become apparent. At $30 \mathrm{dpf}$, there were significant differences in SL (Fig. 4.23A and Table 4.8). For a fair comparison, only juveniles with similar SL $(10.0-10.3 \mathrm{~mm})$ were compared in Figure 4.23B-H. The entire skeleton is calcified in

Table 4.7 - Quantification of vertebral mineralization in 14 dpf larvae. "no 0", excluding larvae without vertebral calcification.

\begin{tabular}{|c|c|c|c|c|}
\hline Genotype & $\begin{array}{c}\text { Average } \\
\text { calcified } \\
\text { vertebrae, } \\
\text { all larvae }\end{array}$ & $\begin{array}{c}\text { Average } \\
\text { calcified } \\
\text { vertebrae, } \\
\text { "no 0" }\end{array}$ & $\begin{array}{c}\text { Larvae } \\
\text { without } \\
\text { calcified } \\
\text { vertebrae }\end{array}$ & $\begin{array}{c}\text { Total } \\
\text { number } \\
\text { of } \\
\text { larvae }\end{array}$ \\
\hline$\overline{m m p 14 a^{+/+} ; m m p 14 b^{+/+}}$ & 6.4 & 9.7 & 19 & 56 \\
\hline$m m p 14 a^{\Delta / \Delta} ; m m p 14 b^{+/+}$ & 4.4 & 7.7 & 19 & 45 \\
\hline$m m p 14 a^{\Delta / \Delta} ; m m p 14 b^{+/ \Delta}$ & 4.7 & 8.4 & 29 & 66 \\
\hline$m m p 14 a^{\Delta / \Delta} ; m m p 14 b^{\Delta / \Delta}$ & 4.8 & 8.2 & 16 & 39 \\
\hline
\end{tabular}


$30 \mathrm{dpf}$ mmp14a/b KO juveniles (Fig. 4.23B and $\mathrm{H}$ ). However, the skull of the mmp14a/ $b$ KO fish looked smaller and its shape differed from that of its clutch mates and age-matched WT juveniles. When zooming in, subtle differences could be detected in the shape of the frontal, parietal and soc bones of mmp14a/b KO (Fig. 4.23D, F and $G$, green and magenta arrows, respectively). At $21 \mathrm{dpf}$, there were no differences in the soc between the different genotypes. The frontal and parietal bones, however, were not yet calcified in the 21-dpf juveniles and could therefore not be assessed.

\section{Discussion}

Winchester syndrome is an ultra-rare skeletal dysplasia that is caused by homozygous missense mutations in MMP14 [1, 2]. The mechanism underlying the associated reduced bone density and craniofacial malformations is incompletely understood. In the present study, we generated a novel mmp14a/b KO zebrafish model to gain insights in the pathogenesis of WS. Our zebrafish model recapitulated important aspects of WS and previous Mmp14 mutant and $\mathrm{KO}$ mouse models. As we outcrossed our fish at least once when generating $\operatorname{mmp} 14 a / b$ double mutants, the selected mutations were demonstrated to be deleterious on mRNA and protein level, and the phenotype of $m m p 14 a^{\Delta / \Delta} ; m m p 14 b^{\Delta / \Delta}$ mutant fish was reproducible and independent of the parental genotype, we are convinced this phenotype is a specific effect of mmp14a/b KO. Similar to the patients and mice, our fish demonstrated a gradually worsening phenotype that includes stunted growth, craniofacial anomalies involving skeletal elements of both the viscerocranium and neurocranium, hyperkyphosis, a reduced bone density and shortened lifespan $[1,3-5,53,56,81]$. Similar to the mouse models, our mutant fish had a relatively short head, exophthalmos and the inability to produce offspring [4, $5,56]$. Additional abnormalities in our fish, not present in WS patients or Mmp14

Table 4.8 - Levels of significance for Mann-Whitney-U test comparing the average number of calcified vertebrae per genotype at $14 \mathrm{dpf}$, SL at 21 and $30 \mathrm{dpf}$, and ossification scores at $21 \mathrm{dpf}$, and for Chi-square test comparing the number of larvae without calcified vertebrae per genotype. "no 0", excluding larvae without vertebral calcification. No vert, fraction of larvae without vertebral ossification; Oss. score, ossification score as defined in Figure 4.22.

\begin{tabular}{|c|c|c|c|c|c|c|}
\hline \multirow[b]{3}{*}{ Comparison } & \multicolumn{3}{|c|}{$14 \mathrm{dpf}$} & & & \multirow{3}{*}{$\frac{30 \mathrm{dpf}}{\mathrm{SL}}$} \\
\hline & \multicolumn{3}{|c|}{$\begin{array}{l}\text { Average number of } \\
\text { calcified vertebrae }\end{array}$} & \multicolumn{2}{|c|}{$21 \mathrm{dpf}$} & \\
\hline & All & "no 0" & No vert. & SL & Oss. score & \\
\hline$a^{+/+} ; b^{+/+}$vs. $a^{\Delta / \Delta} ; b^{+/+}$ & 0.136 & 0.131 & 0.392 & 0.11 & 0.10 & 0.0001 \\
\hline$a^{+/+} ; b^{+/+}$vs. $a^{\Delta / \Delta} ; b^{+/ \Delta}$ & 0.132 & 0.285 & 0.259 & 0.19 & 0.12 & $<0.0001$ \\
\hline$a^{+/+} ; b^{+/+}$vs. $a^{\Delta / \Delta} ; b^{\Delta / \Delta}$ & 0.244 & 0.268 & 0.481 & 0.81 & 0.99 & $<0.0001$ \\
\hline$a^{\Delta / \Delta} ; b^{+/+}$vs. $a^{\Delta / \Delta} ; b^{+/ \Delta}$ & 0.913 & 0.547 & 0.858 & 0.67 & 0.31 & 0.45 \\
\hline$a^{\Delta / \Delta} ; b^{+/+}$vs. $a^{\Delta / \Delta} ; b^{\Delta / \Delta}$ & 0.762 & 0.658 & 0.912 & 0.088 & 0.15 & $<0.0001$ \\
\hline$a^{\Delta / \Delta} ; b^{+/ \Delta}$ vs. $a^{\Delta / \Delta} ; b^{\Delta / \Delta}$ & 0.876 & 0.813 & 0.771 & 0.16 & 0.11 & 0.003 \\
\hline
\end{tabular}



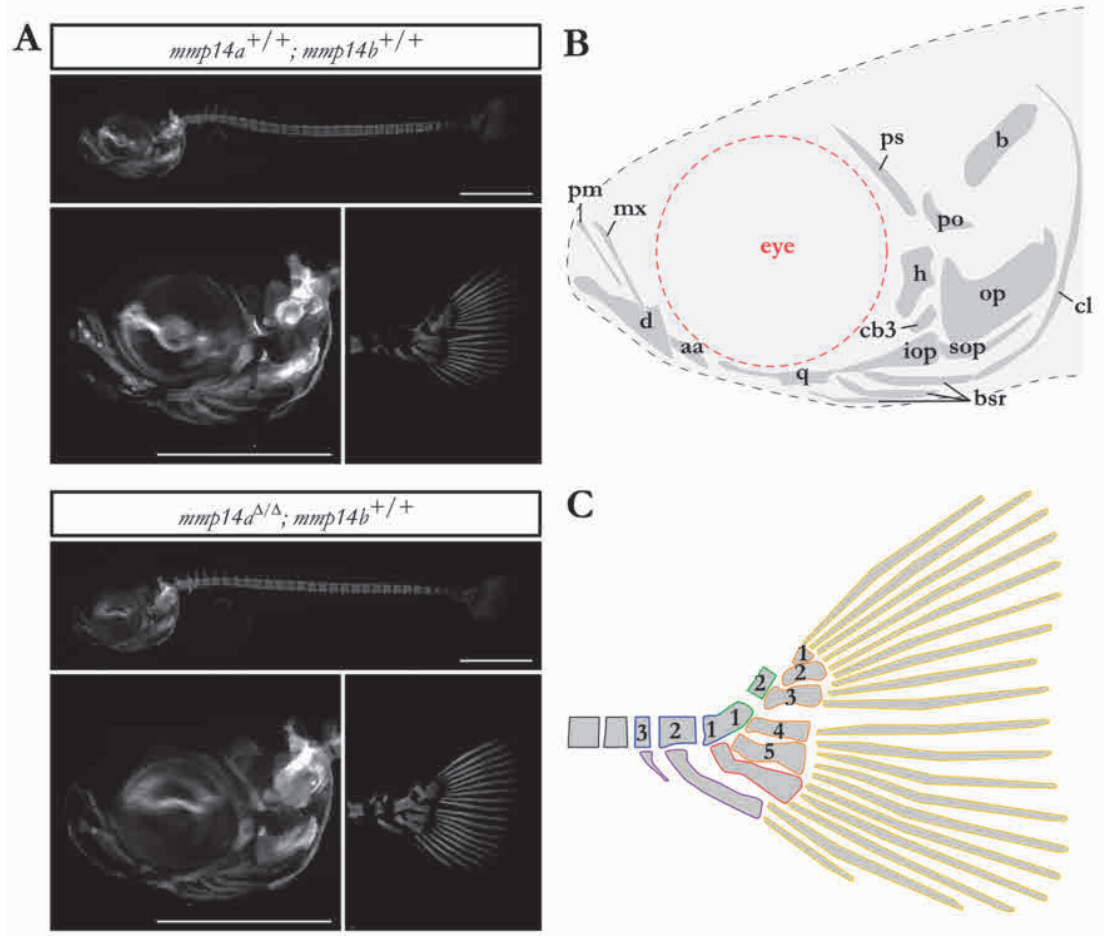

C
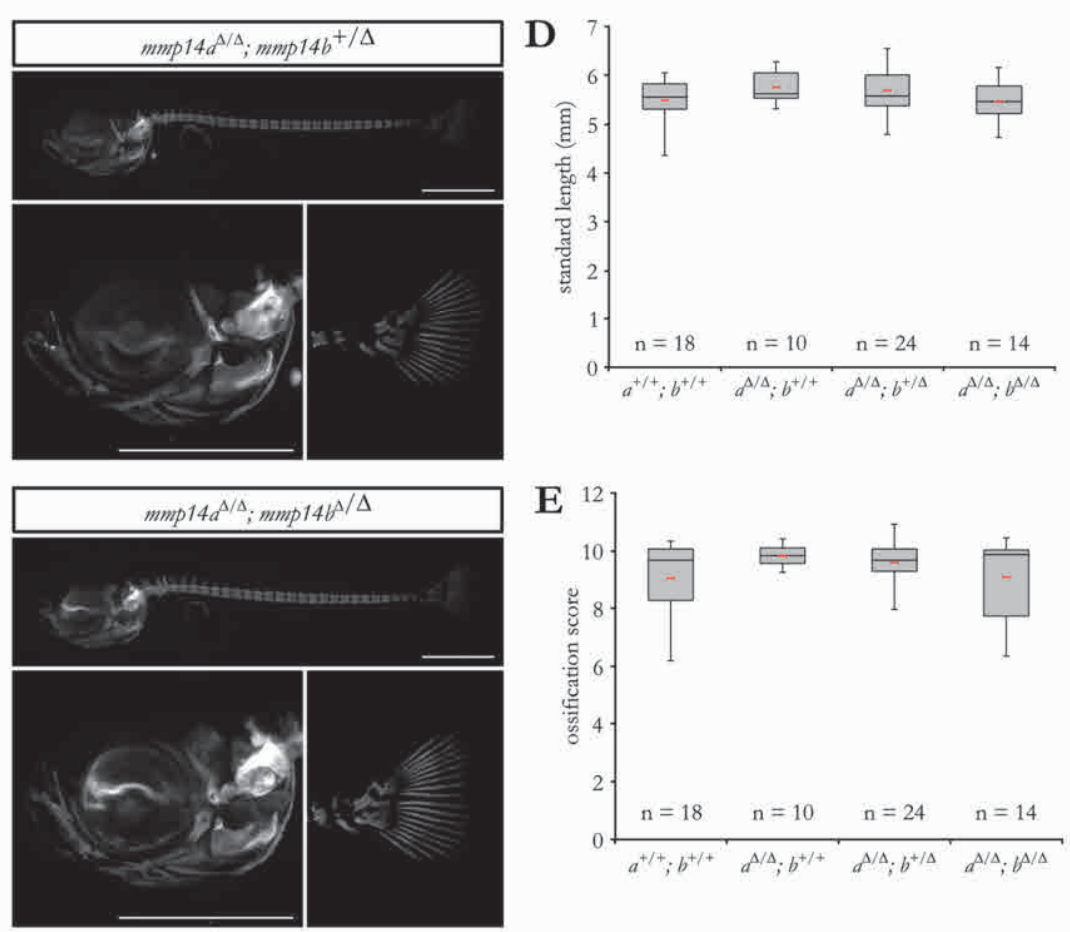

E

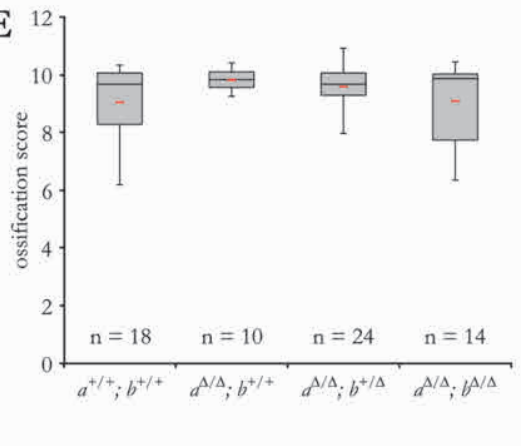


Figure 4.22 - Knockout of mmp14a/b does not delay mineralisation of the vertebral column and tail fin. A, fluorescence microscopy images of representative whole mount 21-dpf (5.7 mm SL) WT larvae or offspring of $m m p 14 a^{\Delta / \Delta}$; mmp14 $b^{+/ \Delta}$ parents, stained for calcified bone with alizarin red. Lateral view, anterior to the left. No obvious differences in overall skeletal structure or craniofacial skeletal elements can be detected. B, schematic overview of the craniofacial skeletal elements that can be identified in the images shown in A. C, schematic overview of the tail fin skeletal elements that can be identified the images shown in A. D, quantification of the SL of fish as shown in A. There are no statistically significant differences in average SL (red lines) between the genotypes analysed. E, quantification of the number of calcified vertebrae and tail fin elements as shown in C (black, up to 28 ossified centra; blue, 0-3 preural centra; green, 0-2 ural centra, cave preural and ural 1 are fused; purple, 0-2 hemal arches; red, parhypural; orange, 0-5 hypurals, yellow, 0-19 subnumeral rays). Ossification score is the sum of ossified elements, divided by the standard length (in $\mathrm{mm}$ ). There are no statistically significant differences in the average number of ossified elements (red bars) between the genotypes analysed. See Table 4.9 for abbreviations of craniofacial skeletal elements. Scale bar equals $1 \mathrm{~mm}$.

mouse models, included dorsal tilting of the skull accompanied by foramen magnum stenosis. Joint destruction reported in the mouse models was absent in our fish. In our model, we found that cranial NC migration was unaffected. Instead, skeletal remodelling turned out to be abnormal, showing similarities with previously reported Mmp14

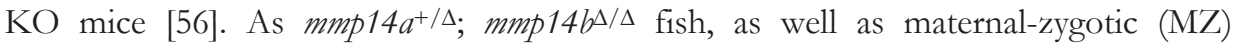
$m m p 14 b^{\Delta / \Delta}$ fish showed a partial phenotype, whereas mmp14a $a^{\Delta / \Delta} ; m m p 14 b^{+/ \Delta}$ and $\mathrm{MZ}$

Table 4.9 - Abbreviation, neural crest contribution and ossification type of craniofacial skeletal elements in zebrafish. Data from Cubbage and Mabee, and Kague et al. [14, 80]. Abb, abbreviation; C, cartilage replacement; D, dermal; NCO, neural crest origin; OT, ossification type.

\begin{tabular}{|c|c|c|c|c|c|c|c|}
\hline Abb. & Full name & $\mathrm{NCO}$ & OT & Abb. & Full name & NCO & OT \\
\hline aa & anguloarticular & yes & $\mathrm{D}$ & op & operculum & yes & $\mathrm{D}$ \\
\hline $\mathrm{b} / \mathrm{boc}$ & basioccipital & no & $\mathrm{C}$ & os & orbitosphenoid & yes & $\mathrm{C}$ \\
\hline bsr & branchiostegal ray & yes & $\mathrm{D}$ & $\mathrm{p}$ & palatine & yes & $\mathrm{C}$ \\
\hline $\mathrm{cb}$ & ceratobranchial & yes & $\mathrm{C}$ & $\mathrm{pa}$ & parietal & no & $\mathrm{D}$ \\
\hline $\mathrm{cl}$ & cleithrum & yes & $\mathrm{D}$ & $\mathrm{pc}$ & postcleithrum & no & $\mathrm{D}$ \\
\hline $\mathrm{CO}$ & coracoid & no & $\mathrm{C}$ & $\mathrm{pm}$ & premaxilla & yes & $\mathrm{D}$ \\
\hline d & dentary & yes & $\mathrm{D}$ & po & prootic & yes & $\mathrm{C}$ \\
\hline e & ethmoid & yes & $\mathrm{C}$ & pop & preopercle & yes & $\mathrm{D}$ \\
\hline en & entopterygoid & yes & $\mathrm{D}$ & ps & parasphenoid & no & $\mathrm{D}$ \\
\hline eo & epioccipital & no & $\mathrm{C}$ & pto & pterotic & yes & $\mathrm{C}$ \\
\hline eoc & exoccipital & no & $\mathrm{C}$ & pts & pterosphenoid & yes & $\mathrm{C}$ \\
\hline $\mathrm{f}$ & frontal & $\operatorname{mix}$ & $\mathrm{D}$ & $\mathrm{q}$ & quadrate & yes & $\mathrm{C}$ \\
\hline $\mathrm{h} / \mathrm{hm}$ & hyomandibula & yes & $\mathrm{C}$ & $\mathrm{ra}$ & retroarticular & yes & $\mathrm{C}$ \\
\hline k & kinethmoid & no & $\mathrm{C}$ & sop & supraorbital & yes & $\mathrm{D}$ \\
\hline iop & interopercle & yes & $\mathrm{D}$ & sph & sphenotic & no & $\mathrm{C}$ \\
\hline le & lateral ethmoid & yes & $\mathrm{C}$ & st & subopercle & yes & $\mathrm{D}$ \\
\hline $\mathrm{mpt}$ & metapterygoid & yes & $\mathrm{C}$ & st & sphenotic & yes & $\mathrm{C}$ \\
\hline $\mathrm{mx}$ & maxilla & yes & $\mathrm{D}$ & st & supratemporal & no & $\mathrm{D}$ \\
\hline $\mathrm{n}$ & nasal & yes & $\mathrm{D}$ & sy & symplectic & yes & $\mathrm{C}$ \\
\hline
\end{tabular}



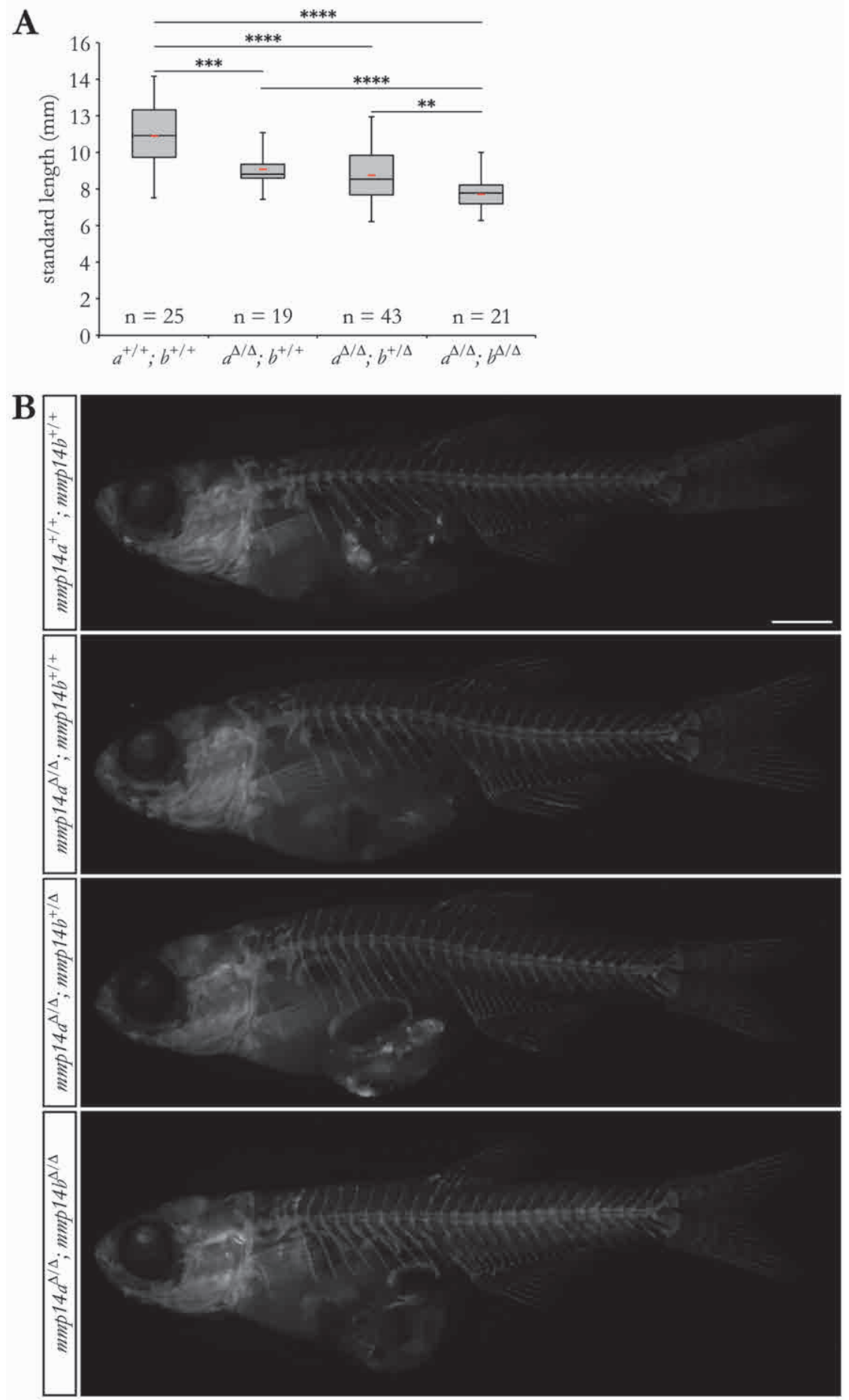

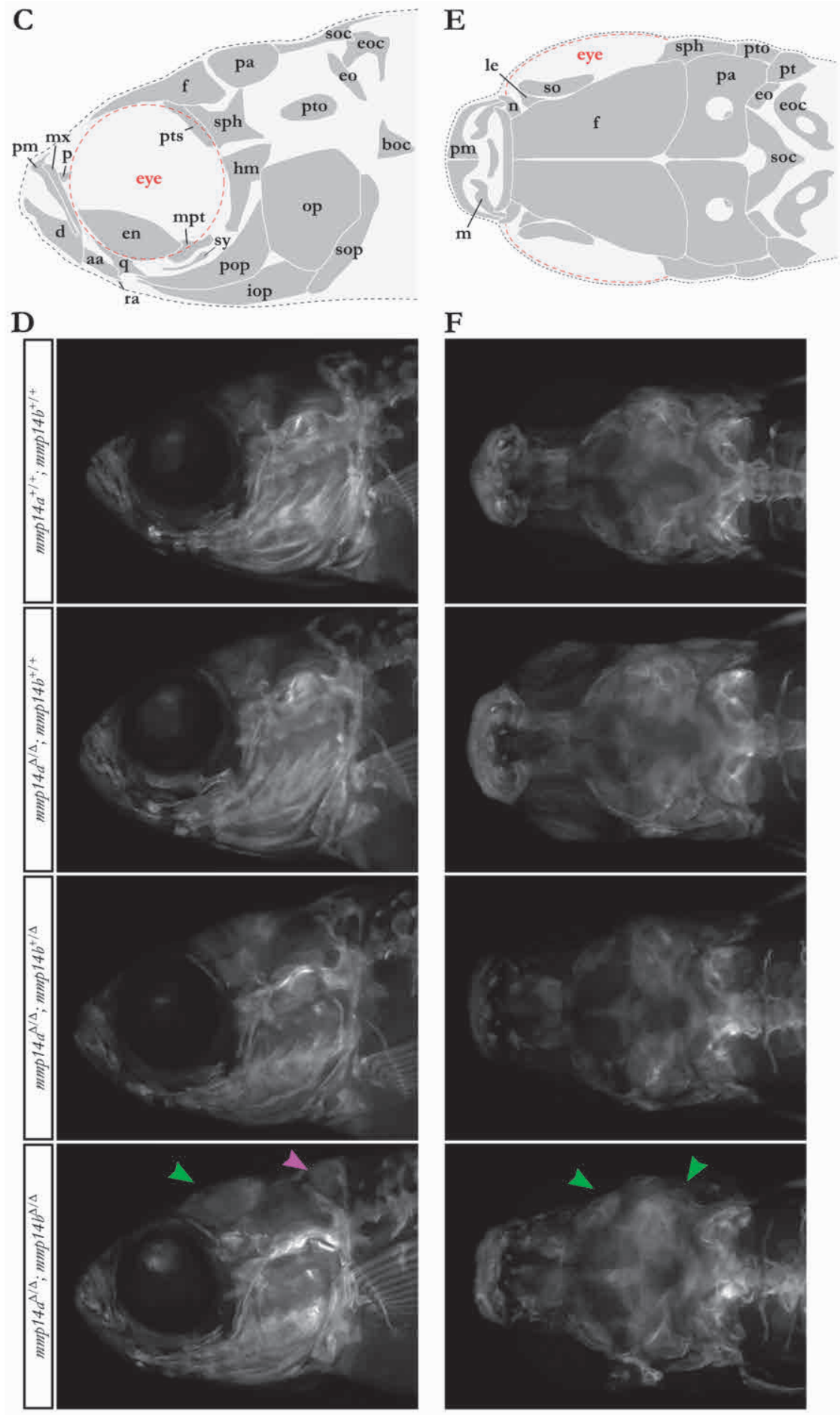

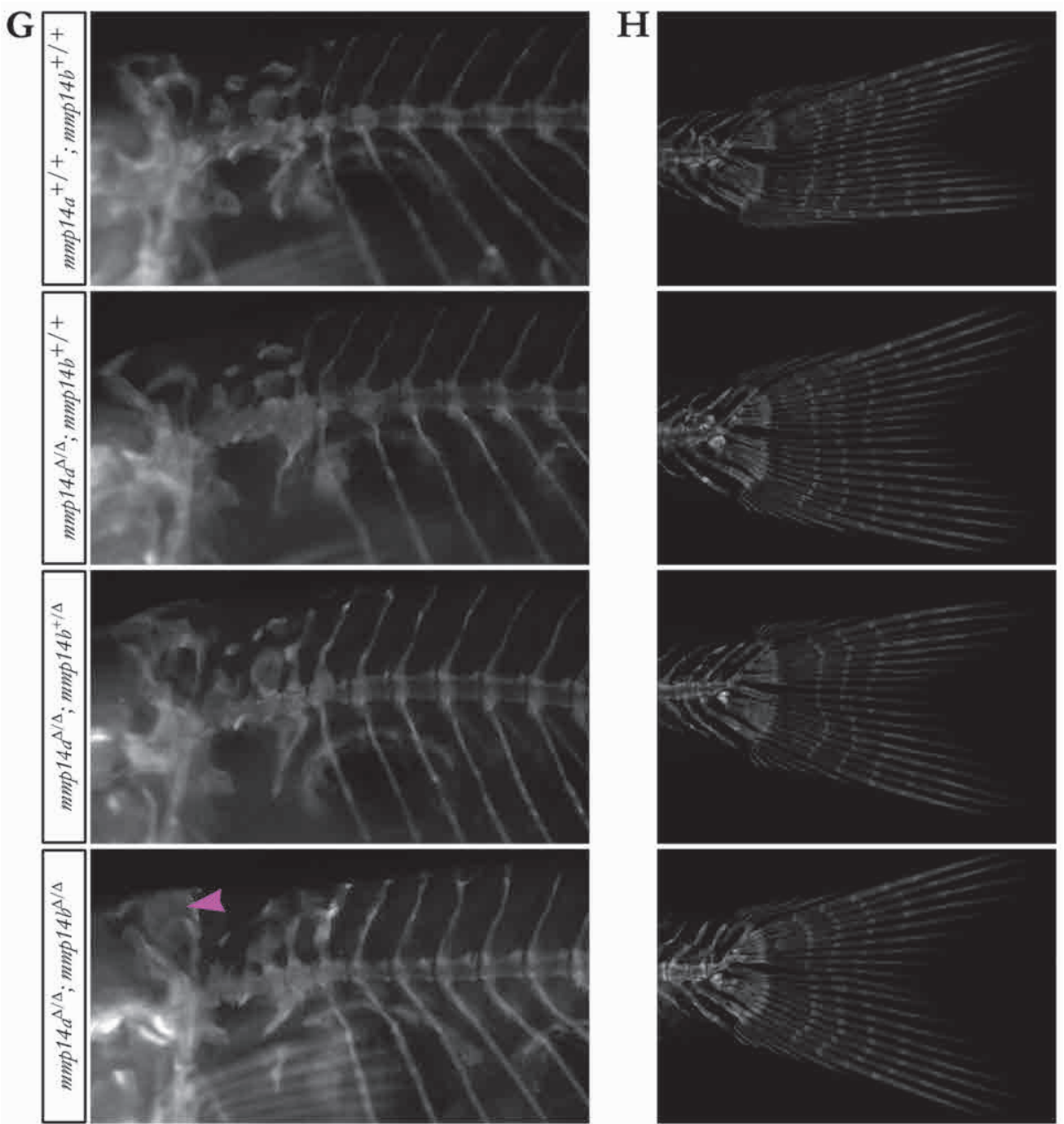

Figure 4.23 - At $30 \mathrm{dpf}, \mathrm{mmp} 14 \mathrm{a} / \mathrm{b} \mathrm{KO}$ frontal, parietal and supraoccipital bone shape is aberrant. A, quantification of SL of 30-dpf WT juveniles and offspring of $m m p 14 a^{\Delta / \Delta} ; m m p 14 b^{+/ \Delta}$ parents imaged in B-H. Average SL (red lines) is significantly different between the analysed genotypes, with WT juveniles being larger and mmp14a/b KO fish being shorter on average. B, whole mount fluorescence microscopy images of representative $30 \mathrm{dpf}$ juveniles (10.0-10.3 mm SL), stained for calcified bone with alizarin red. Lateral view, anterior to the left. $\mathbf{C}$ and $\mathbf{E}$, schematic overview of the craniofacial skeletal elements that can be identified in the images shown in $\mathrm{D}$ and $\mathrm{F}$ in lateral and dorsal view, respectively. $\mathbf{D}, \mathbf{F}$ and $\mathbf{G}$, enlarged images of the skull and Weberian vertebrae of fish shown in B. In mmp14a/b KO fish, the shape of the frontal and parietal bone (green arrowhead) and the supraoccipital bone (magenta arrowhead) are slightly different to those in the other genotypes. $\mathbf{H}$, enlarged images of the tail fin of fish shown in B. No differences in tail anatomy or ossification can be observed. See Table 4.9 for abbreviations. Scale bar equals 1 $\mathrm{mm}$.

mmp14a $a^{\Delta / \Delta}$ fish did not (data not shown), it seems that Mmp14b has a more crucial role in skeletal remodelling. 


\section{Mmp14a/ b may not be crucial for cranial neural crest migration}

The NC is the prime embryonic structure contributing to the development of the face $[7,64,82]$. As a result, genetic disorders that affect NC development often manifest with craniofacial dysmorphology $[82,83]$. Similarly, patients and mice with homozygous MMP14 loss-of-function mutations had craniofacial malformations affecting skeletal structures of $\mathrm{NC}$ origin $[1,3-5,56]$. After delamination, NC cells migrate extensively; for both processes, remodelling of the ECM is essential [64, 67, 83-85]. As MMP14 has an important role in invasive cell motility and NC cells have been demonstrated to express mmp14 in Xenopus laevis, a role for Mmp14a/b was implied in cranial NC cell migration during zebrafish embryogenesis [8-13, 83]. This is further supported by the observation that MMP-inhibitors can reduce chicken NC motility rate in vivo and in vitro [86]. In addition, inhibition of MMP2, which is activated by MMP14, has been demonstrated to block NC delamination in the chicken embryo [66, 87]. Furthermore, $\mathrm{MO} \mathrm{KD}$ of thes5, encoding an adaptor protein that together with Mmp14 is involved in the formation of podosomes (specialised membrane structures involved in invasive cell motility, see Chapter 1), was shown to decrease the number of migrating truncal NC cells in zebrafish [84]. However, in our zebrafish model, we found that mmp14a/b KO did not affect cranial NC induction, delamination, migration or homing to the pharyngeal arches. In addition, the NC-derived larval cartilage elements appeared qualitatively normal. In contrast, others have previously demonstrated that mmp14a/b KD by MO impaired convergence and extension (C\&E) cell movements and dramatically disrupted larval craniofacial cartilage organisation in zebrafish [16, 32]. Although these studies did not specifically analyse the NC, the migrational defect and cartilage abnormalities suggest that NC migration was likely impaired. An important difference between the used approaches to $\mathrm{KD}$ or $\mathrm{KO}$ mmp14 is the timing and site of action [88]. Where we crossed fish homozygous/heterozygous for genomic mutations in mmp14a/b, respectively, previous studies used MOs to inhibit Mmp14 translation at the mRNA level. Although both mutant and morphant embryos still contain maternally derived proteins, it cannot be excluded that our mmp14a/b KO fish additionally contain maternal transcripts of WT mmp14b [32, 89]. MOs, on the other hand, are injected into the individual embryos to be analysed and can target the maternal transcripts as well [38]. It is, therefore, possible that complete lack of Mmp14a/b results in NC migrational defects, which is masked in our model by the presence of maternally derived W'T Mmp14b throughout embryonic development. However, the fact that Coyle et al. and Zhang et al. reported craniofacial abnormalities in larvae injected with MOs targeting either mmp14a or mmp14b, whereas its homolog remained unaffected, makes this hypothesis less plausible [16, 32]. Alternatively, the lack of an early phenotype in our mutant fish could be explained by genomic compensation, although the overt phenotype at later age would suggest such compensation would be time and/or tissue specific [90]. 


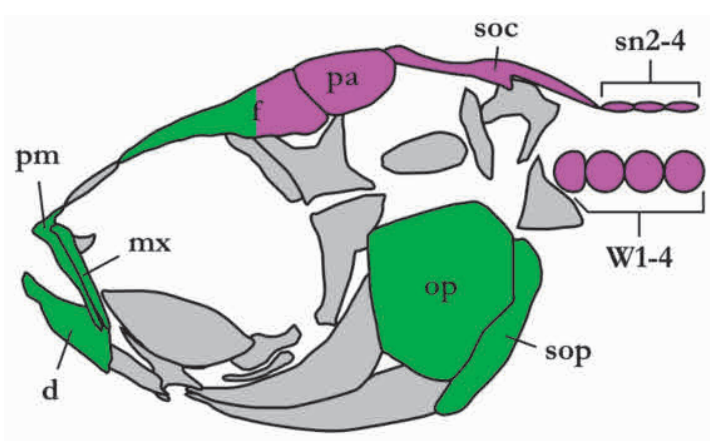

Figure 4.24 - Schematic overview of skeletal elements with aberrant ossification in mmp14a/b KO fish. Elements highlighted in green (NC-derived) or magenta (mesoderm-derived) are demonstrated to have aberrant morphology and/or architecture. Grey elements are normal or could not be assessed by the used mode of imaging/sectioning. W, Weberian vertebrae.

An alternative explanation for the discrepancy between the mutant and morphant phenotype could be a non-specific effect of the used MOs. Although some MOs efficiently mimic mutant phenotypes, it has been demonstrated that MOs can induce false-positive features due to off-target changes in gene expression [37, 38, 89, 90]. Coyle et al. showed that their mmp14a morphant phenotype can be partially rescued by injecting embryos with mRNA encoding W'T, but not catalytically inactive, Mmp14a, suggesting the phenotype is a specific result of mmp14a KD. The authors additionally reported that $p 53 \mathrm{KD}$ by $\mathrm{MO}$ had no effect on the mmp14 morphant phenotype, however, the used MO doses are unclear [16]. In contrast, Janssens et al. later clearly demonstrated that p53-dependent apoptosis contributed significantly to the mmp14a MO-induced phenotype [17]. In addition, Boer et al. have demonstrated that MOs can induce p53-independent NC cell death [89]. The observation that in our mutant fish besides multiple NC-derived structures (frontal bone, opercle, subopercle, maxilla, premaxilla, dentary) also multiple mesoderm-derived skeletal elements (parietal bone, soc, sn2, Weberian vertebral bodies, see Fig. 4.24) are affected, whereas other NCderived structures (caudal fin lepidotrichae) and cell types (melanocytes) are not, argues against a NC-specific problem $[14,91]$. The progressive skeletal phenotype present in WS patients, previous mouse models, and our mutant zebrafish is more suggestive of a more general problem in bone remodelling that affects the mutants later during development $[1,3-5,53,56]$. For a definite answer, MZ mmp14a/b mutant fish lacking maternally derived protein and mRNA should be generated. Although a direct role for Mmp14 in follicular development is likely, ovarian transplantation was successfully used to overcome the breeding inability of Mmp mutant Sabe mice [4]. As our $m m p 14 a / b \mathrm{KO}$ fish were unable to generate offspring, transplantation of mmp14a/b $\mathrm{KO}$ ovaria into WT females and subsequent crossing with mmp $14 a^{\Delta / \Delta} ; m m p 14 b^{+/ \Delta}$ males would be the only way to generate MZ mmp14a/b KOs. Furthermore, any additional features caused by injection of previously published MOs into these MZ mutant embryos would be confirmed as off-target. 


\section{Mmp14a/b play an important role in perichondral and intramembranous ossification in zebrafish}

After the larval craniofacial cartilage elements are formed, they are replaced during metamorphosis by the more permanent mature skeleton [29]. We found that the timing and rate of mineralization are unaffected in mmp14a/b KO larvae. Instead, we demonstrated that later aspects of skeletal remodelling and bone formation are disrupted, which became evident for the first time around $30 \mathrm{dpf}$ and seemed progressive in nature given the worsening gross phenotype of mutant fish.

In fish, like in mammals, bone can be formed by two different processes: intramembranous ossification and endochondral ossification [20]. The majority of the bones that had an abnormal architecture in our mutant fish, except for the soc, are formed by intramembranous ossification (see Table 4.9) [14, 20, 23, 58, 78-80]. Intramembranous bones, in fish often referred to as dermal bones, develop directly from mesenchymal condensation [78]. This occurs entirely or partially in association with cartilage, but not within the cartilage itself [92]. During endochondral ossification, bone forms within a cartilage precursor [78]. In fish, true endochondral ossification is rare, and the common mechanism by which cartilage is replaced by bone is perichondral ossification (together referred to as "cartilage replacement" in Table 4.9) [14, 20]. During perichondral ossification, bone is laid down by cells at the surface of the cartilage that previously formed the perichondrium. In contrast to mammals, the cartilage precursor can remain present inside the bone [20]. In our mutant fish, we observed a relatively small amount of bone matrix in all affected bones, irrespective of their ossification type. The maxilla, dentary bone and soc contained a relative abundance of cartilage with irregular hyperthrophic chondrocytes and, in the soc, loss of peptidoglycans and cell-free collagen-rich areas. The calvariae and maxilla additionally contained lacunae filled with multinucleated cells, seemingly resorbing the bone matrix locally. Finally, we observed a distorted shape and morphology of frontal and parietal bone, soc and Weberian vertebrae. Similar to our fish, both types of ossification were affected in previously reported Mmp14 $\mathrm{KO}$ mice [56]. In these mice, bone formation, in general, is reduced. Cartilage templates for endochondral ossifying bones, as well as those associated with intramembranous ossifying elements, are not timely and properly replaced or removed, respectively (see below). The intramembranously ossifying parietal bone is underdeveloped and undergoes osteoclastic resorption $[53,56]$. Endochondrally ossifying bones showed disturbed formation and retarded ossification of secondary ossification centra [56]. Apart from species-related differences in bone morphology and ossification mechanisms, the bone abnormalities of our mmp14a/b KO fish seem, at least to a certain extent, comparable with those seen in Mmp14 KO mice.

\section{Possible mechanism of disrupted bone remodelling upon loss of Mmp14}

The question remains how the loss of MMP14 results in aberrant bone remodelling. Studies in mice have indicated a crucial role for MMP14 in endochondral and non- 
endochondral cartilage dissolution $[53,56]$. In these two processes, MMP14 has been demonstrated to be essential for remodelling of unmineralised collagen matrix [93]. In murine endochondral ossifying bones, the maturation, hypertrophy, and calcification of pre- and early postnatal metaphyseal growth plates were shown to proceed normally. However, postnatal development of the secondary ossification centra turned out to be delayed $[53,56]$. During this process, epiphyseal chondrocytes normally switch from expressing collagen type II to type X, hypertrophy, and promote mineralisation of the ECM [57, 92]. In addition, they release vascular endothelial growth factor (VEGF) to stimulate ingrowth of vascular canals [92]. At this point, the peripheral ECM is not yet mineralised and proteolytic cleavage of the collagen matrix is needed for vessel ingrowth. In contrast, the central matrix is mineralised and is subsequently resorbed by osteoclasts, and the chondrocytes undergo apoptosis [92, 94]. Bone is subsequently deposited around the cartilage core by newly differentiated osteoblasts [57]. In Mmp14 KO mice, vascular invasion of the epiphyseal cartilage is defective [53, $56,94]$. Instead of a well-organised secondary ossification centre, a mass of hypertrophic chondrocytes surrounded by a chondroid matrix is retained that eventually ossifies via an alternative mechanism from the perichondrium $[53,56]$. The disturbed endochondral ossification contributes to the impaired growth of the Mmp14 KO mice [56]. It has been suggested that the failure of epiphyseal vessel ingrowth is part of a more general defect in postnatal angiogenesis. Although supported by impaired corneal FGF2-stimulated angiogenesis in Mmp14 KO mice, additional vascular abnormalities are absent [53].

Non-endochondral dissolution of cartilages associated with intramembranous ossifying skeletal elements also depends on the collagenolytic action of MMP14 [56]. During this process, first identified in the murine parietal cartilage, the chondrocytes stop synthesizing peptidoglycans and type II collagen. In contrast with endochondral ossification, the chondrocytes neither hypertrophy nor produce type X collagen, but instead start expressing MMP14 and eventually undergo apoptosis [57, 92, 53]. In absence of MMP14, the peptidoglycan content is lost, but the collagenous matrix persists and is enriched in type I collagen by chondrocytes. The chondrocytes eventually undergo apoptosis, but at a later time point than normal and in a random distribution, creating an acellular fibrotic tissue described as "ghost cartilage" [53, 56]. In Mmp14 KO mice, this is thought to contribute to growth arrest and cranial dysmorphism [57, 92].

The non-endochondral cartilage dissolution described above was additionally found to be impaired in bone-soft tissue interfaces [92]. Concurrently, excessive osteoclastic resorption of adjacent mineralized bone was observed [56, 53, 92]. Importantly, MMP14 is not needed for cathepsin-dependent dissolution of mineralized collagen matrix by osteoclasts [93]. It was thought that the impaired soft tissue remodelling disturbs the balanced development of different skeletal compartments. The osteoclastic resorption was hypothesised by Holmbeck et al. to be a compensating mechanism 
to reduce stress on (peri-) skeletal tissues and enable continued growth up to some extent $[56,92]$.

In addition to its role in cartilage dissolution, additional functions for MMP14 in bone formation have been demonstrated that likely contribute to the MMP14 mutant phenotype. MMP14 was shown to stimulate the osteogenic potential of murine osteoprogenitor cells and bone marrow stem cells $[56,93]$. Tang et al. demonstrated that catalytic remodelling of the ECM by MMP14 allows murine skeletal stem cells to change their shape, thereby stimulating commitment to the osteogenic lineage over the chondrogenic and adipogenic lineage in 3D in vitro culture [95]. Accordingly, mice with skeletal stem cell-specific Mmp14 KO have thickened cartilage, delayed membranous ossification and general osteopenia. Their appearance furthermore shares similarities with the Mmp14 KO mice, including short length, a short snout and dome-shaped skull [95]. In addition, MMP14 was shown to protect FGFR2 against the sheddase activity of ADAM9 (a disintegrin and metalloprotease domain 9). In absence of MMP14, FGFR2 shedding by ADAM9 abrogated FGF-dependent proliferation of osteoblasts [96]. It is unknown whether a general defect in FGF-dependent proliferation could as well account for the defect in angiogenesis reported by Zhou et al. [53]. Although reduced pro-MMP2 activation has previously been implied in Mmp14 KO, its importance remains unclear as MMP2 stimulates both the growth and differentiation of both osteoblasts and osteoclasts [53, 97]. However, MMP14 additionally functions as a sheddase for receptor activator of NF- $\mathrm{KB}$ ligand (RANKL) expressed on the plasma membrane of osteoblasts. In the absence of MMP14, binding of RANKL to its receptor RANK on osteoclast precursors is increased, resulting in increased osteoclast differentiation, activation, and survival [98]. Finally, increased cell senescence was demonstrated in multiple tissues of Mmp14 KO mice, which may contribute to the bone phenotype when present in osteoblasts or their progenitors [81]. Further studies are needed to assess whether these processes also underlie the skeletal abnormalities observed in our mmp14a/b KO fish.

\section{Conclusion and future perspectives}

We generated a novel zebrafish model for Winchester syndrome, recapitulating essential aspects of the skeletal phenotype. Similar to previous Mmp14 KO mouse models, early steps in skeletogenesis are unaffected. Instead, perichondral and intramembranous ossification are disturbed in our fish. Our results moreover suggest Mmp14b is more crucial in these processes than its homolog Mmp14a. Bones of both $\mathrm{NC}$ and mesodermal origin were affected in our mutant fish yet cranial NC migration was unaffected. However, with our current model, we cannot exclude a possible role for Mmp14a/b in NC migration, masked in our assay by maternal transcripts. Generating MZ mmp14a/b KO fish is required for a definite answer. Additional assays could shed light on the exact processes underlying the observed skeletal phenotype. Sections at 
juvenile age could help to assess the sequence of events in aberrant bone remodelling. Staining for tartrate resistant acid phosphatase (TRAP) could assess to what extent osteoclastic one resorption plays a role in the bone anomalies. Transgenic lines could be used to assess the differentiation of osteoblasts and osteoclasts as well. Moreover, our observations highlight a central role for impaired collagen remodelling in pathophysiology underlying the WS bone phenotype, a potential target for any novel pharmacotherapy. We believe that our mmp14a/b KO fish could be used to develop and test such treatments, which might also be able to address osteoporosis and other conditions with decreased bone density. In contrast to existing mouse models, our model can be used for high-throughput drug screening. In addition, it would be worth exploring whether our fish could be used to model, and develop novel therapies for, postinflammatory dermal fibrosis seen in our patients. Finally, our fish could serve as a novel model for follicular maturation as well as epidermal mucous cell differentiation and/or function.

\section{Materials and methods}

\section{Zebrafish husbandry}

Danio rerio larvae of the $\mathrm{AB}$ strain were grown in $\mathrm{E} 3$ medium $(0.1 \mathrm{mM} \mathrm{NaCl}, 3.4 \mu \mathrm{M}$ $\left.\mathrm{HCl}, 6.6 \mu \mathrm{M} \mathrm{CaCl}_{2}, 6.6 \mu \mathrm{M} \mathrm{MgSO}_{4}, \mathrm{pH} 7.4\right)$ at $28.5{ }^{\circ} \mathrm{C}$ at a rearing density of $80-100$ individuals per liter until $14 \mathrm{dpf}$, after which larvae were transferred to a closed water system at a density of 13-16 per liter. Feeding schedule was fixed according to the age of the fish; light/dark $(14 \mathrm{~h} / 10 \mathrm{~h})$ circadian rhythm remained fixed from $5 \mathrm{dpf}$ onwards. From 4 weeks of age onwards, fish were kept at $27.5{ }^{\circ} \mathrm{C}$. Transgenic fish $\operatorname{Tg}(\operatorname{sox} 10:: \mathrm{eg} p)$ were a generated before by T.J. Carney [28]. All zebrafish experiments were conducted under IACUC licence 140924.

\section{Genomic editing of mmp14a and mmp14b in zebrafish by CRISPR/Cas?}

Optimal CRISPR target sites in mmp14a (NP_919397.1) and mmp14b (NP_919395.1) were identified by ZiFiT Targeter Version 4.2 (Zinc Finger Consortium, URL: zifit.partners.org/ZiFiT, date last accessed May 2014; see Table 4.10), ordered as gBlocks ${ }^{\circledR}$ Gene Fragments (Integrated DNA Technologies, Coralville, Iowa, USA), PCR amplified (see Table 4.11 for primers used) and transcribed into gRNA with the

Table 4.10 - Overview of identified CRISPR target sites for knockout of $m m p 14 a / b$ in $D$. rerio. Fw, forward; Rv, reverse.

\begin{tabular}{|c|c|c|c|}
\hline Gene & Exon & Orientation & Sequence \\
\hline$m m p 14 a$ & 4 & $\overline{\mathrm{FW}}$ & 5'-GGAGACAGCACCCCATT'TGA-3' \\
\hline$m m p 14 b$ & 4 & $\mathrm{FW}_{\mathrm{W}}$ & 5'-GGCGATGCCAGTCCATT'TGA-3' \\
\hline
\end{tabular}


InvitrogenTM MEGAshortscript T7 kit (ThermoFischer Scientific, Inc., Waltham, MA, USA; AM1354) according to the manufacturer's protocol [46-48]. Cas9 mRNA was generated by transcribing the NotI-linearised pCS2 nls-z Cas9-nls vector (Addgene, Cambridge, MA, USA; 47929) with the Invitrogen ${ }^{\text {TM }}$ mMESSAGE mMACHINETM SP6 Transcription Kit (ThermoFischer Scientific, Inc.; AM1340) according to the manufacturer's protocol [40]. Danio rerio zygotes were micro-injected in the yolk cell with $2.5 \mathrm{~nL} \mathrm{1x}$ Danieau's solution (58 mM NaCl, $0.7 \mathrm{mM} \mathrm{KCl,} 0.4 \mathrm{mM} \mathrm{MgSO} 4,0.6$ $\mathrm{mM} \mathrm{Ca}\left(\mathrm{NO}_{3}\right.$ )2, $5.0 \mathrm{mM}$ HEPES; $\mathrm{pH}$ 7.6)/1x PhenolRed (Sigma; P0290) in $\mathrm{dH}_{2} \mathrm{O}$ containing $0.375 \mathrm{ng}$ Cas9 RNA and $0.375 \mathrm{ng}$ mmp14a gRNA or $1 \mathrm{ng}$ mmp14b gRNA as previously described [99]. Genomic editing was assessed by lysis of $24 \mathrm{hpf}$ embryos or juvenile/adult fin-clips (in $10 \mathrm{mM}$ Tris, $50 \mathrm{mM} \mathrm{KCl,} \mathrm{0.3 \%}(v / v)$ Tween-20, 0.3\% $(v / v)$ $\mathrm{NP}-40$, and $0.488 \mathrm{mg} / \mathrm{mL}$ proteinase $\mathrm{K}$ in $\mathrm{dH}_{2} \mathrm{O}, \mathrm{pH}$ 8.3) and direct Sanger sequencing of a 200-500 bp gDNA amplicon encompassing the target site (primers for short genomic PCR are listed in Table 4.12) using BigDye Terminators version 3.1 (ThermoFischer Scientific, Inc.; 4337455) and a 3730XL sequencer (ThermoFischer Scientific, Inc.). Chromatography reads were analysed for frameshift mutations with Poly Peak Parser web tool (Yost lab, Salt Lake City, UT, USA) and checked manually in SnapGene version 3.3.4 (Clontech Laboratories, Mountain View, CA, USA) [100]. Three-month-old F0 mosaic mutants were intercrossed and their F1 offspring genotyped at 2 months of age to identify fish with heterozygous frameshift mutations in $m m p 14 a$ or mmp14b. F1 mmp14a $a^{+/ \Delta}$ fish were outcrossed with F0 mmp14b founders to generate F2 mmp14a $a^{+/ \Delta}$; mmp14 $b^{+/ \Delta}$ fish, which were intercrossed to generate all possible mmp14a/b genotypes in the F3 generation. For NC cell imaging, F2 mmp14a $a^{+/ \Delta}$; $m m p 14 b^{+/ \Delta}$ fish were outcrossed into the $\operatorname{Tg}(\operatorname{sox} 10::$ egf $)$ background. F3 mmp14a $a^{+/ \Delta}$; $m m p 14 b^{+/ \Delta} \operatorname{Tg}(\operatorname{sox} 10:: e g p p)$ fish were outcrossed with F2 mmp14a $a^{+/ \Delta} ; m m p 14 b^{+/ \Delta}$ fish to generate all possible mmp14a/b genotypes in $\operatorname{Tg}($ sox10::eg $p$ ) background.

\section{Verification of mmp14a/b KO at the $m \mathrm{RN} A$ level}

Twenty 1-5 dpf embryos/larvae per genotype were pooled per time point, or 2-monthold juveniles were kept separately, homogenised in Trizol (ThermoFischer Scientific, Inc.; 15596018), and total mRNA was chloroform/isopropanol precipitated and rinsed with ice-cold $75 \%(v / v)$ ethanol in diethyl pyrocarbonate-treated water (Sigma; 159220). mRNA was resuspended in RNase-free water, DNase treated (ThermoFischer Scientific, Inc.; AM2238) and cleaned-up with the RNeasy Mini Kit (Qiagen; 74106).

Table 4.11 - Overview of primers used for PCR amplification of the gRNA template. Fw, forward; Rv, reverse; Tm, melting temperature.

\begin{tabular}{|c|c|c|}
\hline Name & $\operatorname{Tm}\left({ }^{\circ} \mathrm{C}\right)$ & Sequence \\
\hline$\overline{\text { gBlock Fw }}$ & $\overline{49.6}$ & 5'-CATTATGGTGAAAGTTGGAAC-3' \\
\hline gBlock Rv & 62.6 & 5'AAAAGCACCGACTCGGTGCCAC-3' \\
\hline
\end{tabular}


cDNA was synthesised with the High-Capacity cDNA Reverse Transcription Kit (ThermoFischer Scientific, Inc.; 4368813), all according to the manufacturers' protocols. Primers listed in Table 4.13 were tested at different concentrations (1.95-500 nM) on cDNA of $24 \mathrm{hpf}$ W'T embryos in technical triplicate with SYBR ${ }^{\mathrm{TM}}$ Select Master Mix according to the manufacturer's protocol. Generation of a single amplicon was verified by direct Sanger sequencing. Expression of mmp14a/b was normalised to $\beta$ beta-actin (and expressed relative to the expression level in WT embryos at $1 \mathrm{dpf}$ for larvae or 2-month-old WT for juveniles). Two-month-old cDNA was amplified (primers listed in Table 4.14), run on a 1.5\% (w/v) agarose in TAE $1 \mathrm{x}$ gel, isolated bands purified with the Wizard® SV Gel \& PCR Clean-Up System (Promega, Madison, WI, USA; A9281) and sent for sequencing with the respective forward primer (AITbiotech, Singapore, Singapore).

\section{Cloning of pCS2+ mmp14a/b-HA plasmids}

Four expression vectors encoding either WT or mutant Mmp14a or Mmp14b with a C-terminal Haemagglutinin (HA) tag were generated. cDNA was obtained from 2month-old W'T and mmp14a $a^{\Delta / \Delta} ; m m p 14 b^{\Delta / \Delta}$ juveniles, respectively, and W'T respectively mutant mmp14a/b coding sequence PCR amplified with the primers listed in Table 4.15. The forward primers started with zebrafish-optimised kozak sequence 5'CCACC-3', the reverse primer ended with the HA coding sequence. The amplicon was

Table 4.12 - Primers used for genotyping. Fw, forward; Rv, reverse; Tm, melting temperature.

\begin{tabular}{|c|c|c|c|}
\hline Name & Sequence and binding site & Prod. & $\operatorname{Tm}\left({ }^{\circ} \mathrm{C}\right)$ \\
\hline mmp14a exon 4 Fw1 & $\begin{array}{l}\text { 5'-GCCACACATGAGGCCATCAA-3' } \\
\text { binds } 144 \text { bp upstream of target site }\end{array}$ & $307 \mathrm{bp}$ & 61.0 \\
\hline mmp14a exon 4 Rv1 & $\begin{array}{l}\text { 5'-ACCCTAGACACAATTCTAGATCATCT-3' } \\
\text { binds } 118 \text { bp downstream of target site }\end{array}$ & & 58.9 \\
\hline mmp14a exon 4 Fw2 & $\begin{array}{l}\text { 5'-CCTGAAATGCCCACTCAGACC-3' } \\
\text { binds } 235 \text { bp upstream of target site }\end{array}$ & 513 bp & 60.4 \\
\hline mmp14a exon 4 Rv2 & $\begin{array}{l}\text { 5'-GCTCCAAACCAAGTGCATGACC-3' } \\
\text { binds } 258 \text { bp downstream of target site }\end{array}$ & & 60.4 \\
\hline$m m p 14 b$ exon $4 \mathrm{Fw}_{\mathrm{w}} 1$ & $\begin{array}{l}\text { 5'-AAGTGTGGGAAAGCGTTACG-3' } \\
\text { binds } 113 \text { bp upstream of target site }\end{array}$ & 223 bp & 58.8 \\
\hline mmp14b exon 4 Rv1 & $\begin{array}{l}\text { 5'-ATAGTCCAAGGCTCAGCTGC-3' } \\
\text { binds } 90 \text { bp downstream of target site }\end{array}$ & & 59.8 \\
\hline mmp14b exon 4 Fw2 & $\begin{array}{l}\text { 5'-CGCAGCATACAGAACTACACGC-3' } \\
\text { binds } 183 \text { bp upstream of target site }\end{array}$ & $326 \mathrm{bp}$ & 60.4 \\
\hline mmp14b exon $4 \mathrm{Rv} 2$ & $\begin{array}{l}\text { 5'-GAGTGT'TTTCGCTGACATGCGG-3' } \\
\text { binds } 123 \text { bp downstream of target site }\end{array}$ & & 60.4 \\
\hline
\end{tabular}


gel-extracted and cloned into the pCR2.1 TOPO TA vector (ThermoFischer Scientific, Inc.; 450641) according to the manufacturer's protocol. The kozak-mmp14a/b-HA insert was subsequently cloned into the pCS2+ backbone.

\section{Cell culture}

MRC-5V1 immortalised human fetal lung fibroblasts were provided by Prof. Alan Lehmann (University of Sussex, Brighton, UK). Cells were grown in 2D culture in high-glucose Dulbecco's Modified Eagle Medium (DMEM, GE Healthcare Life Sciences, Pittsburgh, PA; SH3024.01) containing 10\% ( $/ / v)$ fetal bovine serum (FBS; GE Healthcare Life Sciences, Pittsburgh, PA; A15-101) and $100 \mathrm{U} / \mathrm{mL}$ penicillin / 100 $\mu \mathrm{g} / \mathrm{mL}$ streptomycin (ThermoFischer Scientific, Inc.; 15140122) at $37{ }^{\circ} \mathrm{C}$ in $100 \%$ humidity and $5 \% \mathrm{CO}_{2}$. Cells were kept growing in log phase and passaged when reaching $70-85 \%$ confluence by detaching cells using $0.25 \%$ trypsin-EDTA (ThermoFischer Scientific, Inc.; 25200-056).

\section{Western blotting}

For analysis of the effect of mmp14a/b frameshift mutations on putative protein products, 400,000 cells were seeded in $6 \mathrm{~cm}$ dishes. Cells were transfected with $24 \mathrm{~h}$ postseeding with $8 \mu \mathrm{g}$ pCS2+ vector using Invitrogen ${ }^{\mathrm{TM}}$ Lipofectamine 3000 (ThermoFischer Scientific, Inc.; L3000008) according to the manufacturer's protocol, or left non-transfected. Media was refreshed $6 \mathrm{~h}$ post transfection. Cells were harvested $24 \mathrm{~h}$ post-transfection using trypsin-EDTA and whole cell protein extract obtained using Triton ${ }^{\circledR}$ X-100 lysis buffer (50 mM Tris- $\mathrm{HCl} \mathrm{pH} 7.5,150 \mathrm{mM} \mathrm{NaCl}, 1 \%(v / v) \mathrm{mM} \beta$ glycerophosphate) supplemented with protease inhibitors (Roche) and phosphatase inhibitors $\left(10 \mathrm{mM} \mathrm{NaF}\right.$ and $\left.1 \mathrm{mM} \mathrm{Na} \mathrm{VO}_{4}\right)$. Protein concentration was determined by Bradford protein assay (Biorad; 500-0006). Samples were mixed with Laemmli sample buffer and boiled for $5 \mathrm{~min}$. Samples were subjected to SDS-PAGE before transfer to a PVDF membrane (Merck; ISEQ00010). Membranes were blocked in 5\% (w/v) milk (Biorad; 170-6404) in 0.1\% $(v / v)$ Tween-20 in TBS $1 \mathrm{x}$ and incubated with rabbit antiHA (Cell Signaling Technology Inc., Danvers, MA, USA; 3724) or rabbit anti- $\beta$-actin

Table 4.13 - Primers used for qPCR. Fw, forward; Rv, reverse; Tm, melting temperature.

\begin{tabular}{|c|c|c|c|c|}
\hline Name & Sequence & $\begin{array}{l}\text { Binds to } \\
\text { exon }\end{array}$ & Product & $\operatorname{Tm}\left({ }^{\circ} \mathrm{C}\right)$ \\
\hline mmp14a F1331 & 5'CAAGGAAAAGTGGTTCTGGCGA-3' & $6-7$ & $186 \mathrm{bp}$ & 58.6 \\
\hline$m m p 14 a \mathrm{R} 1516$ & 5'СCTTCCTCCATTT'TAGCCTCGT'TG-3' & 8 & & 60.5 \\
\hline$m m p 14 b \mathrm{~F} 1265$ & 5'-ACTCCATACСССАСАCCGTA-3' & 6 & $130 \mathrm{bp}$ & 58.4 \\
\hline$m m p 14 b \mathrm{R} 1394$ & 5'-CACGCCAGAACCACT'TACCCT'TA-3' & $6-7$ & & 60.5 \\
\hline$\beta$-actin & 5’-CGAGCAGGAGATGGGAACC-3' & $1-2$ & $100 \mathrm{bp}$ & 60.4 \\
\hline$\beta$-actin & 5'CAACGGAAACGCTCAT'TGC-3' & $2-3$ & & 56.1 \\
\hline
\end{tabular}


(Cell Signaling; 4967) primary antibodies at 1:1,000 respectively 1:4,000 in blocking buffer. Membranes were incubated with horseradish peroxidase-conjugated goat antirabbit secondary antibody $(1: 3,000)$ and Streptactin $(1: 10,000)$ in $4 \%(w / v)$ milk in $0.1 \%(v / v)$ Tween-20 in TBS 1x. Protein bands were visualised by ECL Western Blotting substrate mix (ThermoFischer Scientific, Inc.; 32106) on the BioRad Chemidoc ${ }^{\mathrm{TM}}$ and gel images edited in Image Lab ${ }^{\text {TM }} 5.1$ build 8 software (Biorad).

\section{Gross anatomy of larval, juvenile and adult zebrafish}

Fish were sedated with in $200 \mu \mathrm{g} / \mathrm{mL}$ ethyl 3-aminobenzoate methanesulfonate (Tricaine/MS222; Sigma; A5040) in system water buffered to $\mathrm{pH}$ 7.0-7.5 with $\mathrm{Na}$ $\mathrm{HCO}_{3}$ and imaged on moist filter paper with a MZ16 FA fluorescence stereomicroscope (Leica, Wetzlar, Germany) at 1.4 MPixel resolution at 7.11x and 14x magnification [101]. Images were stitched together in Pixelmator 3.5 Canyon software (Pixelmator Team, Vilnius, Lithuania). Total body length was measured with Fiji software (U. S. National Institutes of Health, Bethesda, MD, USA; version 2.0.0-rc-15/1.49k, adapted by IMU).

\section{Microcomputed tomography}

Three month-old fish were euthanised by hypothermic shock, fixed in 4\% PFA and dehydrated through graded water into ethanol. Average sized fish were fixed per geno-

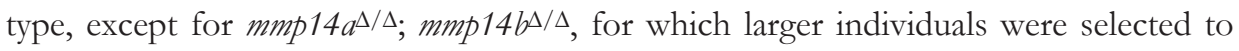
better match the size of the other genotypes. Microcomputed tomography images were acquired using an Inveon CT (Siemens AG, Berlin, Germany) at $55 \mathrm{kVp} / 110$ $\mathrm{mA}$. The exposure time per projection was $2,500 \mathrm{~ms}$, and a binning factor of 2 was used, resulting in a reconstructed pixel size of $35 \mu \mathrm{m}$. Planar images were acquired from 181 projections over $360^{\circ}$ of rotation in step-and-shoot mode. The images were reconstructed using a Feldkamp cone-beam algorithm. Three-dimensional renders of the skeleton were made with AMIRA software (FEI, Mérignac Cedex, France) with constant window settings. Images were exported as TIFF files and extracorporeal skeletal elements of previously imaged fish were manually removed with Pixelmator software. Raw data was viewed with AMIDE-bin 1.0.5 software (Andreas Loening), individual virtual sections exported as TIFF files and angles measured in Fiji software.

Table 4.14 - Primers for sequencing mmp14 cDNA. Fw, forward; Rv, reverse; Tm, melting temperature.

\begin{tabular}{|c|c|c|c|}
\hline Name & Sequence & Binds exon & $\operatorname{Tm}\left({ }^{\circ} \mathrm{C}\right)$ \\
\hline$m m p 14 a \mathrm{~F}_{\mathrm{w}} 1$ & 5'-CAAAGAAGTGAGACCAGAGGCATG-3' & $1-2$ & 60.5 \\
\hline mmp14a Rv2 & 5'TAGAAATAGGCGTTGGCTCCATCC-3' & $9-10$ & 60.5 \\
\hline$m m p 14 b$ Fw1 & 5'-AAGATATGAAACCTGAGGCATGGC-3' & $1-2$ & 58.8 \\
\hline$m m p 14 b \mathrm{Rv} 2$ & 5'-TATTGGAGTCTTCGTTAGGGCAGC-3' & 10 & 60.5 \\
\hline
\end{tabular}




\section{Histology}

Three-month-old fish were euthanized and fixed as described above. After trimming, the fish were placed into cassettes and processed with Sakura VIP Tissue Processor. Fish were dehydrated through graded ethanol into xylene (Chemtech Trading) before paraffin (Leica; 39601006) infiltration. After processing, tissues were embedded into paraffin blocks and sectioned midsagittally with a rotary microtome into $5 \mu \mathrm{m}$ thick sections. The slides with sections were dried and placed into an incubator $\left(60^{\circ} \mathrm{C}, 15\right.$ min). The sections were deparaffinised and rehydrated through graded ethanol into water. Rehydrated sections were subjected to hematoxylin solution (Richard-Allan Scientific ${ }^{\text {TM}}$; 7231), Bluing (Richard-Allan Scientific ${ }^{\text {TM}}$; 7301), Clarifier (Richard-Allan Scientific ${ }^{\mathrm{TM}}$; 7442), and eosin-phloxine B Solution (AMPL). A separate batch of $5 \mu \mathrm{m}$ thick sections of all the samples was stained with Weigert's hematoxylin, followed by picrosirius red $0.1 \%(w / v)$ (AMPL) staining and rinsing with two changes of acidified $(0.5 \%)$ water. Stained sections were dehydrated through graded ethanol into xylene and a cover-slip added. Slides were imaged with an automated slide scanner equipped with Zeiss AxioImager Z.2 body, MetaSystems stage, Zeiss Plan-Neofluar 20x/0.5 Ph2 lens, SSCOPED TL light source, and CoolCube 1 camera with 1.4 Mpixel resolution controlled by Metafer4 software. Images were viewed with VSViewer V2.1.103 software (MetaSystems GmbH, Altlussheim, Germany). Mucous cell number per crosssectional scale surface was quantified with Fiji software.

\section{Light sheet fluorescence microscopy of cranial neural crest cells}

Dechorionated green fluorescent $\operatorname{Tg}(\operatorname{sox} 10:: \mathrm{eg} / \mathrm{p})$ embryos/larvae were stained with 500 nM MitoTracker ${ }^{\circledR}$ Red CMXRos (Invitrogen; M7512), $2.5 \mu \mathrm{M}$ DRAQ5 (Abcam; ab108410) or left unstained. Larvae imaged after 48 hpf were grown in E3 medium containing $200 \mu \mathrm{M}$ N-phenylthiourea ( $w / \nu)$ (Sigma; P7629) from 24 hpf onwards [71]. Embryos/larvae were sedated with $200 \mu \mathrm{g} / \mathrm{mL}$ Tricaine, mounted in 2\% $(w / v)$ lowmelting point agarose $/ 200 \mu \mathrm{g} / \mathrm{mL}$ Tricaine in E3 medium in a glass capillary (Brand, Wertheim, Germany; 701904) in the sample chamber of a custom built dual-sided

Table 4.15 - Primers used to generate WT and mutant Mmp14a/b-HA-encoding plasmids. Fw, forward; HA, hemagglutinin; Rv, reverse; Tm, melting temperature.

\begin{tabular}{|c|c|c|}
\hline Name & Sequence & $\operatorname{Tm}\left({ }^{\circ} \mathrm{C}\right)$ \\
\hline mmp14a Fw Kozak & 5'CCACCATGTTACCGAAACTGCAGACG-3' & 56 \\
\hline mmp14a Rv HA-Stop & $\begin{array}{l}\text { 5'-TTAAGCGTAATCTGGAACATCGTATGGGTAAACCT'TA } \\
\text { 'TCGAGCAGAGAGCG-3' }\end{array}$ & 58 \\
\hline mmp14b Fw Kozak & 5'CCACCATGATCTGGAGCGGGT'TCA-3' & 56 \\
\hline mmp14b Rv HA-Stop & $\begin{array}{l}\text { 5'-TTAAGCGTAATCTGGAACATCGTATGGGTAAACCT'TG } \\
\text { TCCAGTAGGGAGC-3' }\end{array}$ & 58 \\
\hline$M 13 F$ & 5’-GTAAAACGACGGCCAGT-3’ & 53 \\
\hline M13R & 5'-GGAAACAGCTATGACCATG-3' & 53 \\
\hline
\end{tabular}


scanning light sheet microscope (IMB microscopy unit) containing $200 \mu \mathrm{g} / \mathrm{mL}$ Tricaine in the water as previously described [102]. The system was equipped with 488 $\mathrm{nm}$ and $561 \mathrm{~nm}$ lasers (Coherent OBIS), guided via a beam splitter cube and two optical fibers onto Galvanometer mirrors. The collimated beam on the Galvanometer mirrors and the back focal plane of the objective (Leica air NPLAN 10x NA 0.25) formed a conjugated plane. A virtual light sheet was formed by scanning the Gaussian beam with the Galvanometer mirror using an analogue signal from an Arduino controller with DAC chip. A beam expander controlled the thickness of the light sheet. A Z-stack was recorded either a single time point or every $7 \mathrm{~min}$. The thinnest full width at half maximum of the Gaussian beam was $4.9 \mu \mathrm{m}$. Embryos/larvae were imaged in lateral view unless this was impossible due to their rotation in the agarose column. The raw data was exported and 2D projections of 3D renders were made with Imaris Image Analysis Software 8.4.1 (Bitplane, Belfast, UK). After imaging, gDNA was obtained by lysing the embryos/larvae and genotyped for mmp14a/b by direct PCR/Sanger sequencing as described above.

\section{Whole mount cartilage and calcified bone staining}

Larvae were stained for cartilage and calcified bone according to a protocol adapted from Walker et al. [76]. Larvae were euthanised by an overdose of Tricaine, fixed in $4 \%$ PFA and dehydrated in 50\% $(v / v)$ ethanol. Larvae were stained with either $0.02 \%$ $(w / v)$ Alcian blue 8 GX (Sigma; A5268)/60 mM MgCl 2 or $40 \mu \mathrm{g} / \mathrm{mL}$ 3,4-dihydroxy9,10-dioxo-2-anthracenesulfonic acid sodium salt (alizarin red, Sigma; A5533) in 70\% $(v / v)$ ethanol for $14 \mathrm{~h}$ at RT unless stated otherwise. Larvae were rehydrated in $50 \%$ ethanol $(v / v)$. Thirty dpf alizarin red stained larvae were de-stained with $1 \%(w / v)$ $\mathrm{KOH}$ for $45 \mathrm{~min}$ at RT. All larvae were bleached with $1.5 \%(v / v) \mathrm{H}_{2} \mathrm{O}_{2} / 1 \%(w / v)$ $\mathrm{KOH}$ for $20 \mathrm{~min}$ at RT. Larvae were cleared by going through successive stages (20$50 \%, v / v)$ of glycerol. Five and $14 \mathrm{dpf}$ larvae were mounted individually in 50\% glycerol $(v / v)$ between a microscope slide and a cover-slip, separated by modelling clay to prevent squishing of the sample. Fourteen dpf alizarin red stained larvae and picrosirius red stained sections were imaged with a Zeiss AxioImager M2 upright fluorescence microscope with X-Cite ${ }^{\circledR}$ 120Q (120 W) light source (Excelitas Technologies Corp., Waltham, MA, USA), dsRed filter, DIC polariser and Zeiss Plan-Neofluar 5X / 0.16 NA, 10X / 0.3 NA and 20X / 0.5 NA lenses. The system was operated with AxioVision version 4.8.2 SP3 software (Zeiss) and images were taken with an AxioCam HRc camera (Zeiss) with 1.4 Mpixel resolution. Alcian Blue stained larvae and 21 and $30 \mathrm{dpf}$ alizarin red stained larvae were imaged with a Leica MZ16FA fluorescence stereomicroscope with Leica CLS150 (for brightfield) and MZ16FA (for fluorescence) light sources, dsRed filter and Planapo 1.0x lens (Leica; 10447157). The system was operated with Leica Application Suite software version 2.5.0 R1 (Build 975) and images were taken with a Leica DFC 300 FX R2 camera with 1.4 Mpixel resolution. 


\section{Statistics}

Standard length, total body length, bone volume and density, mucous cell count and $21 \mathrm{dpf}$ ossification score were assessed for statistically significant differences between genotypes by two-sided Student's T-test (College of Saint Benedict \& Saint John's University, Collegeville, Minnesota, USA; URL: www.physics.csbsju.edu/stats/ttest_bulk_form.html). The number of fish with head overextension was assessed by Fisher Exact test in SPSS software version 22 (IBM, Armonk, NY, USA). Vertebral calcification at $14 \mathrm{dpf}$ was assessed for significant differences between genotypes both by chi-squared test and Mann-Whitney-U test in SPSS software.

\section{Supplemental material}

Supplemental material is available at in Addendum 3.

\section{Acknowledgements}

The authors acknowledge the invaluable assistance of Mr. John Soon Yew Lim and Dr. Sohail Ahmed, (Institute of Medical Biology, Microscopy Unit), Dr. Monique L.H. Luijten (Institute of Molecular and Cell Biology), and Irma van de Beek (IMB). This work was supported by the Skin Research Institute of Singapore (to M.A.M.v.S.), the Biomedical Research Council Singapore (to M.A.M.v.S.), the Agency for Science, Technology and Research (A*STAR Research Attachment Programme to I.J.H.M.d.V.), the Wellcome Trust (DGEM to M.A.M.v.S. and B.J.C.), and Tenovus Scotland (T15/22 and T15/62 to M.A.M.v.S. and B.J.C.).

\section{References}

1. Winchester P, Grossman H, Lim WN, Danes BS. A new acid mucopolysaccharidosis with skeletal deformities simulating rheumatoid arthritis. The American Journal of Roentgenology, Radium Therapy, and Nuclear Medicine. 1969;106(1):121-8.

2. Evans BR, Mosig RA, Lobl M, Martignetti CR, Camacho C, Grum-Tokars V, et al. Mutation of membrane type-1 metalloproteinase, MT1-MMP, causes the multicentric osteolysis and arthritis disease Winchester syndrome. American Journal of Human Genetics. 2012;91(3):572-6.

3. Van Steensel MA, Ceulen RP, Delhaas T, de Die-Smulders C. Two Dutch brothers with Borrone dermato-cardio-skeletal syndrome. American Journal of Medical Genetics Part A. 2007;143a(11):1223-6.

4. Curtain MM; Donahue LR. 2007. A possible new mutation to Mmp 14 MGI Direct Data Submission. MGI: J:127164. Updated Nov 2012. The Jackson Laboratory. URL: informatics.jax.org/downloads/Reference_texts/J127164.pdf.

5. Du X, Moresco EMY, Murray A, Beutler B. record for cartoon, updated Dec 12, 2013. MUTAGENETIX TM, B. Beutler and colleagues, center for the Genetics of Host Defense, UT Southwestern Medical Center, Dallas, TX. URL: mutagenetix. utsouthwestern.edu.

6. Santagati F, Rijli FM. Cranial neural crest and the building of the vertebrate head. Nature reviews Neuroscience. 2003;4(10):806-18. 
7. Schoenwolf GC. Larsen's Human Embryology. Philadelphia,PA:Churchill Livingstone/Elsevier; 2009.

8. d'Ortho MP, Will H, Atkinson S, Butler G, Messent A, Gavrilovic J, et al. Membrane-type matrix metalloproteinases 1 and 2 exhibit broad-spectrum proteolytic capacities comparable to many matrix metalloproteinases. European Journal of Biochemistry. 1997;250(3):751-7.

9. Ohuchi E, Imai K, Fujii Y, Sato H, Seiki M, Okada Y. Membrane type 1 matrix metalloproteinase digests interstitial collagens and other extracellular matrix macromolecules. The Journal of Biological Chemistry. 1997;272(4):2446-51.

10. Nakahara H, Howard L, Thompson EW, Sato H, Seiki M, Yeh Y, et al. Transmembrane/cytoplasmic domain-mediated membrane type 1-matrix metalloprotease docking to invadopodia is required for cell invasion. Proceedings of the National Academy of Sciences of the United States of America. 1997;94(15):7959-64.

11. Buschman MD, Bromann PA, Cejudo-Martin P, Wen F, Pass I, Courtneidge SA. The novel adaptor protein Tks4 (SH3PXD2B) is required for functional podosome formation. Molecular Biology of the Cell. 2009;20(5):1302-11.

12. Gawden-Bone C, Zhou Z, King E, Prescott A, Watts C, Lucocq J. Dendritic cell podosomes are protrusive and invade the extracellular matrix using metalloproteinase MMP-14. Journal of Cell Science. 2010;123(Pt 9):1427-37.

13. Sabeh F, Ota I, Holmbeck K, Birkedal-Hansen H, Soloway P, Balbin M, et al. Tumor cell traffic through the extracellular matrix is controlled by the membrane-anchored collagenase MT1-MMP. The Journal of Cell Biology. 2004;167(4):769-81.

14. Kague E, Gallagher M, Burke S, Parsons M, Franz-Odendaal T, Fisher S. Skeletogenic fate of zebrafish cranial and trunk neural crest. PloS One. 2012;7(11):e47394.

15. Kimmel CB, Ballard WW, Kimmel SR, Ullmann B, Schilling TF. Stages of embryonic development of the zebrafish. Developmental Dynamics. 1995;203(3):253-310.

16. Coyle RC, Latimer A, Jessen JR. Membrane-type 1 matrix metalloproteinase regulates cell migration during zebrafish gastrulation: evidence for an interaction with non-canonical Wnt signaling. Experimental Cell Research. 2008;314(10):2150-62.

17. Janssens E, Gaublomme D, De Groef L, Darras VM, Arckens L, Delorme N, et al. Matrix metalloproteinase 14 in the zebrafish: an eye on retinal and retinotectal development. PloS One. 2013;8(1):e52915.

18. Thisse, B., Pflumio, S., Fürthauer, M., Loppin, B., Heyer, V., Degrave, A., Woehl, R., Lux, A., Steffan, T., Charbonnier, X.Q. and Thisse, C. (2001) Expression of the zebrafish genome during embryogenesis (NIH R01 RR15402). ZFIN Direct Data Submission. URL: http://zfin.org.

19. Mackay EW, Apschner A, Schulte-Merker S. A bone to pick with zebrafish. BoneKEy Reports. 2013;2:445.

20. Apschner A, Schulte-Merker S, Witten PE. Not all bones are created equal - using zebrafish and other teleost species in osteogenesis research. Methods in Cell Biology. 2011;105:239-55.

21. Yelick PC, Schilling TF. Molecular dissection of craniofacial development using zebrafish. Critical Reviews in Oral Biology and Medicine. 2002;13(4):308-22.

22. Hammond CL, Schulte-Merker S. Two populations of endochondral osteoblasts with differential sensitivity to Hedgehog signalling. Development. 2009;136(23):3991-4000.

23. Witten PE, Huysseune A. A comparative view on mechanisms and functions of skeletal remodelling in teleost fish, with special emphasis on osteoclasts and their function. Biological Reviews of the Cambridge Philosophical Society. 2009;84(2):315-46.

24. Schilling TF, Kimmel CB. Segment and cell type lineage restrictions during pharyngeal arch development in the zebrafish embryo. Development. 1994;120(3):483-94.

25. Harper C, Lawrence C. The Laboratory Zebrafish: Crc Press; 2016.

26. Schilling TF. The morphology of larval and adult zebrafish. Zebrafish. 2002;261:59-94. 
27. Kwak J, Park OK, Jung YJ, Hwang BJ, Kwon SH, Kee Y. Live image profiling of neural crest lineages in zebrafish transgenic lines. Molecules and Cells. 2013;35(3):255-60.

28. Carney TJ, Dutton KA, Greenhill E, Delfino-Machin M, Dufourcq P, Blader P, et al. A direct role for Sox10 in specification of neural crest-derived sensory neurons. Development. 2006;133(23):4619-30.

29. Parichy DM, Elizondo MR, Mills MG, Gordon TN, Engeszer RE. Normal table of postembryonic zebrafish development: staging by externally visible anatomy of the living fish. Developmental Dynamics. 2009;238(12):2975-3015.

30. Simossis VA, Heringa J. The PRALINE online server: optimising progressive multiple alignment on the web. Computational Biology and Chemistry. 2003;27(4-5):511-9.

31. Thompson JD, Gibson TJ, Plewniak F, Jeanmougin F, Higgins DG. The CLUSTAL_X windows interface: flexible strategies for multiple sequence alignment aided by quality analysis tools. Nucleic Acids Research. 1997;25(24):4876-82.

32. Zhang J, Bai S, Zhang X, Nagase H, Sarras MP, Jr. The expression of novel membrane-type matrix metalloproteinase isoforms is required for normal development of zebrafish embryos. Matrix Biology. 2003;22(3):279-93.

33. Wyatt RA, Keow JY, Harris ND, Hache CA, Li DH, Crawford BD. The zebrafish embryo: a powerful model system for investigating matrix remodeling. Zebrafish. 2009;6(4):347-54.

34. Taylor JS, Braasch I, Frickey T, Meyer A, Van de Peer Y. Genome duplication, a trait shared by 22000 species of ray-finned fish. Genome Research. 2003;13(3):382-90.

35. Kinoh H, Sato H, Tsunezuka Y, Takino T, Kawashima A, Okada Y, et al. MT-MMP, the cell surface activator of proMMP-2 (pro-gelatinase A), is expressed with its substrate in mouse tissue during embryogenesis. Journal of Cell Science. 1996;109 ( Pt 5):953-9.

36. Apte SS, Fukai N, Beier DR, Olsen BR. The matrix metalloproteinase-14 (MMP-14) gene is structurally distinct from other MMP genes and is co-expressed with the TIMP-2 gene during mouse embryogenesis. The Journal of Biological Chemistry. 1997;272(41):25511-7.

37. Kok FO, Shin M, Ni CW, Gupta A, Grosse AS, van Impel A, et al. Reverse genetic screening reveals poor correlation between morpholino-induced and mutant phenotypes in zebrafish. Developmental Cell. 2015;32(1):97-108.

38. Schulte-Merker S, Stainier DY. Out with the old, in with the new: reassessing morpholino knockdowns in light of genome editing technology. Development. 2014;141(16):3103-4.

39. Horvath P, Barrangou R. CRISPR/Cas, the immune system of bacteria and archaea. Science. 2010;327(5962):167-70.

40. Jao LE, Wente SR, Chen W. Efficient multiplex biallelic zebrafish genome editing using a CRISPR nuclease system. Proceedings of the National Academy of Sciences of the United States of America. 2013;110(34):13904-9.

41. Jinek M, East A, Cheng A, Lin S, Ma E, Doudna J. RNA-programmed genome editing in human cells. eLife. 2013;2:e00471.

42. Charpentier E, Doudna JA. Biotechnology: Rewriting a genome. Nature. 2013;495(7439):50-1.

43. Hwang WY, Fu Y, Reyon D, Maeder ML, Tsai SQ, Sander JD, et al. Efficient genome editing in zebrafish using a CRISPR-Cas system. Nature Biotechnology. 2013;31(3):227-9.

44. Talbot JC, Amacher SL. A streamlined CRISPR pipeline to reliably generate zebrafish frameshifting alleles. Zebrafish. 2014;11(6):583-5.

45. Mali P, Yang L, Esvelt KM, Aach J, Guell M, DiCarlo JE, et al. RNA-guided human genome engineering via Cas9. Science. 2013;339(6121):823-6.

46. Bassett AR, Tibbit C, Ponting CP, Liu JL. Highly efficient targeted mutagenesis of Drosophila with the CRISPR/Cas9 system. Cell Reports. 2013;4(1):220-8.

47. Sander JD, Zaback P, Joung JK, Voytas DF, Dobbs D. Zinc Finger Targeter (ZiFiT): an engineered zinc finger/target site design tool. Nucleic Acids Research. 2007;35(Web Server issue):W599-605.

48. Sander JD, Maeder ML, Reyon D, Voytas DF, Joung JK, Dobbs D. ZiFiT (Zinc Finger Targeter): an updated zinc finger engineering tool. Nucleic Acids Research. 2010;38(Web Server issue):W462-8. 


\section{CHAPTER 4}

49. Moreno-Mateos MA, Vejnar CE, Beaudoin JD, Fernandez JP, Mis EK, Khokha MK, et al. CRISPRscan: designing highly efficient sgRNAs for CRISPR-Cas9 targeting in vivo. Nature Methods. 2015;12(10):982-8.

50. Kurosaki T, Maquat LE. Nonsense-mediated mRNA decay in humans at a glance. Journal of Cell Science. 2016;129(3):461-7.

51. Popp MW, Maquat LE. Leveraging Rules of Nonsense-Mediated mRNA Decay for Genome Engineering and Personalized Medicine. Cell. 2016;165(6):1319-22.

52. Liu K, Wahlberg P, Ny T. Coordinated and cell-specific regulation of membrane type matrix metalloproteinase 1 (MT1-MMP) and its substrate matrix metalloproteinase 2 (MMP-2) by physiological signals during follicular development and ovulation. Endocrinology. 1998;139(11):4735-8.

53. Zhou Z, Apte SS, Soininen R, Cao R, Baaklini GY, Rauser RW, et al. Impaired endochondral ossification and angiogenesis in mice deficient in membrane-type matrix metalloproteinase I. Proceedings of the National Academy of Sciences of the United States of America. 2000;97(8):4052-7.

54. Grande T, Young B. The ontogeny and homology of the Weberian apparatus in the zebrafish Danio rerio (Ostariophysi: Cypriniformes). Zoological Journal of the Linnean Society. 2004;140(2):241-54.

55. Higgs DM, Rollo AK, Souza MJ, Popper AN. Development of form and function in peripheral auditory structures of the zebrafish (Danio rerio). The Journal of the Acoustical Society of America. 2003;113(2):1145-54.

56. Holmbeck K, Bianco P, Caterina J, Yamada S, Kromer M, Kuznetsov SA, et al. MT1-MMP-deficient mice develop dwarfism, osteopenia, arthritis, and connective tissue disease due to inadequate collagen turnover. Cell. 1999;99(1):81-92.

57. Holmbeck K, Bianco P, Chrysovergis K, Yamada S, Birkedal-Hansen H. MT1-MMP-dependent, apoptotic remodeling of unmineralized cartilage: a critical process in skeletal growth. The Journal of Cell Biology. 2003;163(3):661-71.

58. Topczewska JM, Shoela RA, Tomaszewski JP, Mirmira RB, Gosain AK. The Morphogenesis of Cranial Sutures in Zebrafish. PloS One. 2016;11(11):e0165775.

59. Menke AL, Spitsbergen JM, Wolterbeek AP, Woutersen RA. Normal anatomy and histology of the adult zebrafish. Toxicologic Pathology. 2011;39(5):759-75.

60. Borges LF, Gutierrez PS, Marana HR, Taboga SR. Picrosirius-polarization staining method as an efficient histopathological tool for collagenolysis detection in vesical prolapse lesions. Micron. 2007;38(6):580-3.

61. Rakers S, Gebert M, Uppalapati S, Meyer W, Maderson P, Sell AF, et al. 'Fish matters': the relevance of fish skin biology to investigative dermatology. Experimental Dermatology. 2010;19(4):313-24.

62. Cline A, Feldman SR. Zebrafish for modeling skin disorders. Dermatology online journal. 2016;22(8).

63. Henrikson RC, Matoltsy AG. The fine structure of teleost epidermis. II. Mucous cells. Journal of Ultrastructure Research. 1967;21(3):213-21.

64. Mayor R, Theveneau E. The neural crest. Development (Cambridge, England). 2013;140(11):2247-51.

65. Klymkowsky MW, Rossi CC, Artinger KB. Mechanisms driving neural crest induction and migration in the zebrafish and Xenopus laevis. Cell Adhesion \& Migration. 2010;4(4):595-608.

66. Theveneau E, Mayor R. Neural crest delamination and migration: from epithelium-to-mesenchyme transition to collective cell migration. Developmental Biology. 2012;366(1):34-54.

67. Halloran MC, Berndt JD. Current progress in neural crest cell motility and migration and future prospects for the zebrafish model system. Developmental Dynamics. 2003;228(3):497-513.

68. Stelzer EH. Light-sheet fluorescence microscopy for quantitative biology. Nature Methods. 2015;12(1):23-6.

69. Kimmel CB, Miller CT, Keynes RJ. Neural crest patterning and the evolution of the jaw. Journal of Anatomy. 2001;199(Pt 1-2):105-20.

70. Karlsson J, von Hofsten J, Olsson PE. Generating transparent zebrafish: a refined method to improve detection of gene expression during embryonic development. Marine Biotechnology. 2001;3(6):522-7. 
71. Li Z, Ptak D, Zhang L, Walls EK, Zhong W, Leung YF. Phenylthiourea specifically reduces zebrafish eye size. PloS One. 2012;7(6):e40132.

72. Wu SY, Wang HM, Wen YS, Liu W, Li PH, Chiu CC, et al. 4-(Phenylsulfanyl)butan-2-One Suppresses Melanin Synthesis and Melanosome Maturation In Vitro and In Vivo. International Journal of Molecular Sciences. 2015;16(9):20240-57.

73. Grush J, Noakes DL, Moccia RD. The efficacy of clove oil as an anesthetic for the zebrafish, Danio rerio (Hamilton). Zebrafish. 2004;1(1):46-53.

74. Aceto J, Nourizadeh-Lillabadi R, Bradamante S, Maier JA, Alestrom P, van Loon JJ, et al. Effects of microgravity simulation on zebrafish transcriptomes and bone physiology-exposure starting at 5 days post fertilization. NPJ Microgravity. 2016;2:16010.

75. Bohnsack BL, Gallina D, Kahana A. Phenothiourea sensitizes zebrafish cranial neural crest and extraocular muscle development to changes in retinoic acid and IGF signaling. PloS One. 2011;6(8):e22991.

76. Walker MB, Kimmel CB. A two-color acid-free cartilage and bone stain for zebrafish larvae. Biotechnic \& Histochemistry. 2007;82(1):23-8.

77. Du SJ, Frenkel V, Kindschi G, Zohar Y. Visualizing normal and defective bone development in zebrafish embryos using the fluorescent chromophore calcein. Developmental Biology. 2001;238(2):239-46.

78. Bird NC, Mabee PM. Developmental morphology of the axial skeleton of the zebrafish, Danio rerio (Ostariophysi: Cyprinidae). Developmental Dynamics. 2003;228(3):337-57.

79. Bensimon-Brito A, Cardeira J, Cancela ML, Huysseune A, Witten PE. Distinct patterns of notochord mineralization in zebrafish coincide with the localization of Osteocalcin isoform 1 during early vertebral centra formation. BMC Developmental Biology. 2012;12:28.

80. Cubbage CC, Mabee PM. Development of the cranium and paired fins in the zebrafish Danio rerio (Ostariophysi, Cyprinidae). Journal of Morphology. 1996;229(2):121-60.

81. Gutierrez-Fernandez A, Soria-Valles C, Osorio FG, Gutierrez-Abril J, Garabaya C, Aguirre A, et al. Loss of MT1-MMP causes cell senescence and nuclear defects which can be reversed by retinoic acid. The EMBO Journal. 2015;34(14):1875-88.

82. Cordero DR, Brugmann S, Chu Y, Bajpai R, Jame M, Helms JA. Cranial neural crest cells on the move: their roles in craniofacial development. American journal of medical genetics Part A. 2011;155a(2):270-9.

83. Christian L, Bahudhanapati H, Wei S. Extracellular metalloproteinases in neural crest development and craniofacial morphogenesis. Critical Reviews in Biochemistry and Molecular Biology. 2013;48(6):544-60.

84. Murphy DA, Diaz B, Bromann PA, Tsai JH, Kawakami Y, Maurer J, et al. A Src-Tks5 pathway is required for neural crest cell migration during embryonic development. PloS One. 2011;6(7):e22499.

85. Erickson CA, Perris R. The role of cell-cell and cell-matrix interactions in the morphogenesis of the neural crest. Developmental Biology. 1993;159(1):60-74.

86. Cai DH, Brauer PR. Synthetic matrix metalloproteinase inhibitor decreases early cardiac neural crest migration in chicken embryos. Developmental Dynamics. 2002;224(4):441-9.

87. Duong TD, Erickson CA. MMP-2 plays an essential role in producing epithelial-mesenchymal transformations in the avian embryo. Developmental Dynamics. 2004;229(1):42-53.

88. Morcos PA, Vincent AC, Moulton JD. Gene Editing Versus Morphants. Zebrafish. 2015;12(5):319.

89. Boer EF, Jette CA, Stewart RA. Neural Crest Migration and Survival Are Susceptible to MorpholinoInduced Artifacts. PloS One. 2016;11(12):e0167278.

90. Rossi A, Kontarakis Z, Gerri C, Nolte H, Holper S, Kruger M, et al. Genetic compensation induced by deleterious mutations but not gene knockdowns. Nature. 2015;524(7564):230-3.

91. Nieto MA. The early steps of neural crest development. Mechanisms of Development. 2001;105(12):27-35. 


\section{CHAPTER 4}

92. Holmbeck K, Szabova L. Aspects of extracellular matrix remodeling in development and disease. Birth defects research Part C, Embryo Today: Reviews. 2006;78(1):11-23.

93. Szabova L, Yamada SS, Wimer H, Chrysovergis K, Ingvarsen S, Behrendt N, et al. MT1-MMP and type II collagen specify skeletal stem cells and their bone and cartilage progeny. Journal of bone and mineral research : the official journal of the American Society for Bone and Mineral Research. 2009;24(11):1905-16.

94. Blumer MJ, Longato S, Fritsch H. Localization of tartrate-resistant acid phosphatase (TRAP), membrane type-1 matrix metalloproteinases (MT1-MMP) and macrophages during early endochondral bone formation. Journal of Anatomy. 2008;213(4):431-41.

95. Tang Y, Rowe RG, Botvinick EL, Kurup A, Putnam AJ, Seiki M, et al. MT1-MMP-dependent control of skeletal stem cell commitment via a beta1-integrin/YAP/TAZ signaling axis. Developmental Cell. 2013;25(4):402-16.

96. Chan KM, Wong HL, Jin G, Liu B, Cao R, Cao Y, et al. MT1-MMP inactivates ADAM9 to regulate FGFR2 signaling and calvarial osteogenesis. Developmental Cell. 2012;22(6):1176-90.

97. Mosig RA, Dowling O, DiFeo A, Ramirez MC, Parker IC, Abe E, et al. Loss of MMP-2 disrupts skeletal and craniofacial development and results in decreased bone mineralization, joint erosion and defects in osteoblast and osteoclast growth. Human Molecular Genetics. 2007;16(9):1113-23.

98. Hikita A, Yana I, Wakeyama H, Nakamura M, Kadono Y, Oshima Y, et al. Negative regulation of osteoclastogenesis by ectodomain shedding of receptor activator of NF-kappaB ligand. The Journal of Biological Chemistry. 2006;281(48):36846-55.

99. Rosen JN, Sweeney MF, Mably JD. Microinjection of zebrafish embryos to analyze gene function. Journal of Visualized Experiments. 2009(25).

100. Hill JT, Demarest BL, Bisgrove BW, Su YC, Smith M, Yost HJ. Poly peak parser: Method and software for identification of unknown indels using Sanger sequencing of polymerase chain reaction products. Developmental Dynamics. 2014;243(12):1632-6.

101. Carter KM, Woodley CM, Brown RS. A review of tricaine methanesulfonate for anesthesia of fish. Reviews in Fish Biology and Fisheries. 2011;21(1):51-9.

102. Weber M, Mickoleit M, Huisken J. Multilayer mounting for long-term light sheet microscopy of zebrafish. Journal of Visualized Experiments. 2014(84):e51119. 
This chapter is embargoed at request

\section{CHAP'TER 5}

\section{General discussion}

Adapted from:

Ivo J.H.M. de Vos, Arnette Shi Wei Wong, Tim J.M. Welting, Barry J. Coull and Maurice A.M. van Steensel. Multicentric osteolytic syndromes represent a phenotypic spectrum defined by defective collagen remodelling. In revision for American Journal of Medical Genetics Part A 
CHAP'T'ER 6

Addendum 



\section{Addendum 1}

A

N-region

H-region

C-region

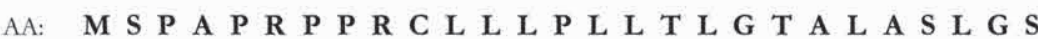

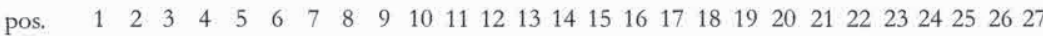
$\begin{array}{lllll}-5 & -4 & -3 & -2 & -1 \\ +1\end{array}$

B
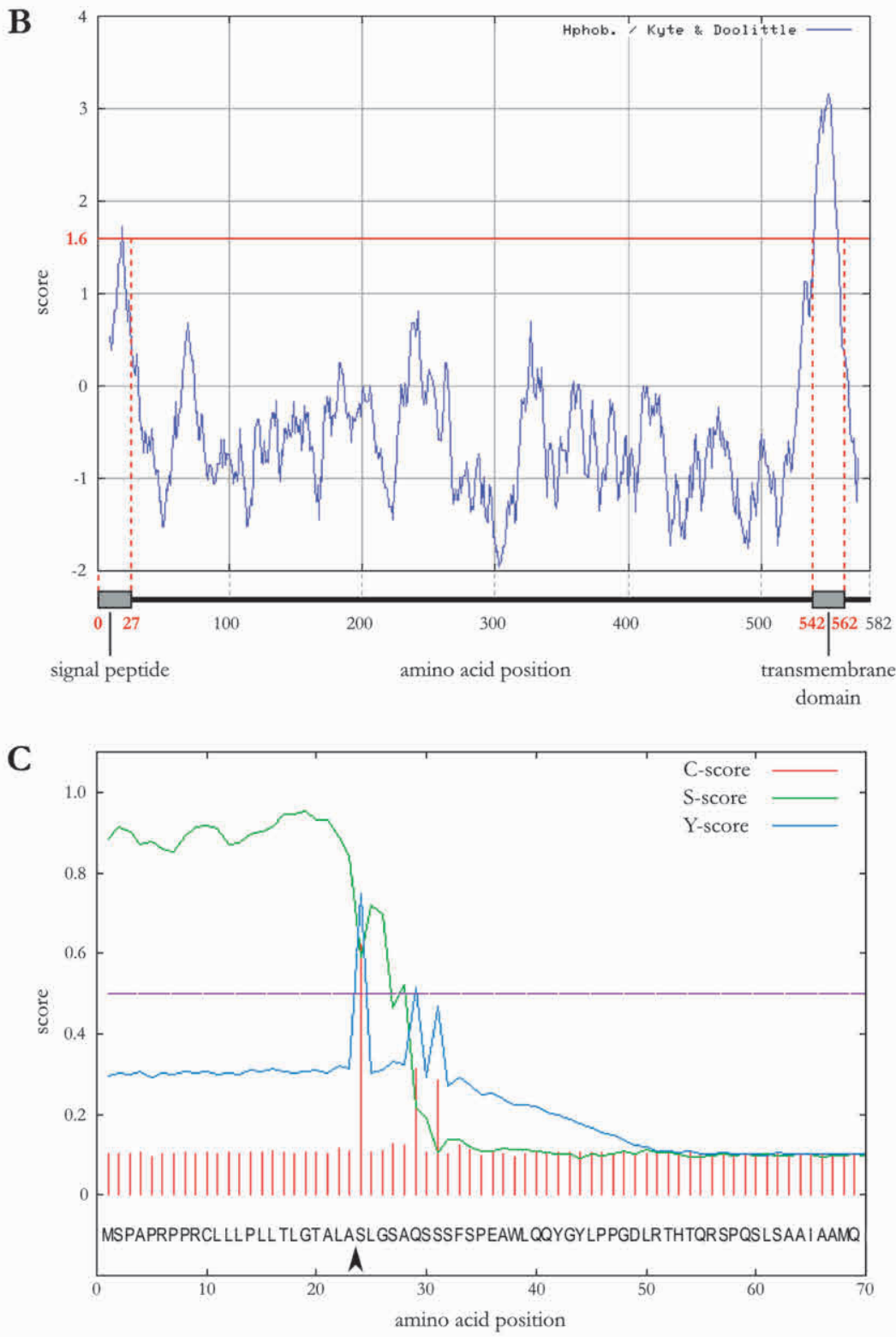
Supplemental Figure 1.1 - In silico analysis of the MMP14 signal peptide. A, MMP14 SP sequence has all the characteristics of traditional SPs: an N-region with positively charged Arg residues (position 6 and 9) flanking six hydrophobic Leu residues (position 11-18) in the H-region, and a C-region containing polar residues [1, 2]. B, Kyte-Doolittle hydropathy plot, showing the mean hydropathy of 19 amino acid segments, predicts membrane-spanning sequences (hydropathy $>1.6$ ) in the MMP14 N-terminus (SP) and C-terminus (transmembrane domain) [3]. C, the SP is predicted to be cleaved between positions 23 and 24 by SignalP 4.0 software (arrowhead in A and C), based on the raw cleavage (C-) score (max. at position 24), SP (S-) score (max. at pos. 19), combined cleavage site (Y-) score (calculated from C-score and S-score slope, max. at pos. 24), and discrimination (D-) score (weighted average of mean S and max. Y scores, > 0.450 as cut-off for pos. 1-23) [4]. This is in perfect agreement with consensus for SP cleavage sites, which includes small neutral residues at positions -1 and -3 from the cleavage site (Ala at positions 23 and 21), Gly and Pro at positions -4 and -5 from the cleavage site (Gly at position 19), and hydrophobic residues that are dominant from position -6 onwards from the cleavage site (Leu at position 11-18) [5].
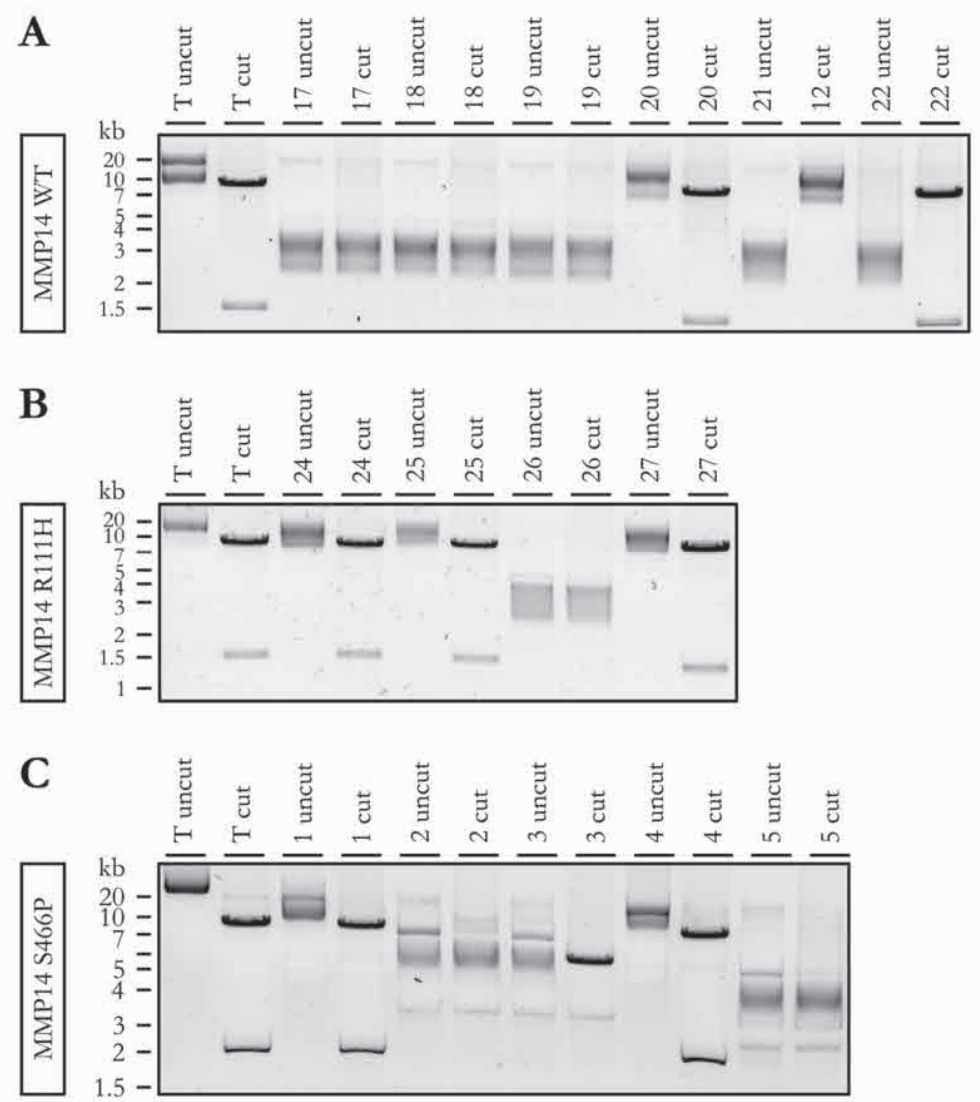

Supplemental Figure 1.2 - Site-directed mutagenesis of pQCXIB 3HA-MMP14-EGFP vectors. DNA gel electrophoresis results of template vectors (T) and SDM reaction products (numbered) of pQCXIB 3HA-MMP14-EGFP (A), pQCXIB 3HA-MMP14 R111H-EGFP (B), pQCXIB 3HA-MPP14 S466P-EGFP (C) or pQCXIB 3HA-MMP14 R111H S466P-EGFP (D) to induce the p.R92C mutation, or SDM of PQCXIB 3HA-MMP14-EGFP to induce the p.T17R mutation (E), digested with BamHI or undigested after SDM. SDM products yielding restriction fragments of the correct length $(8,130$ and 1,438 

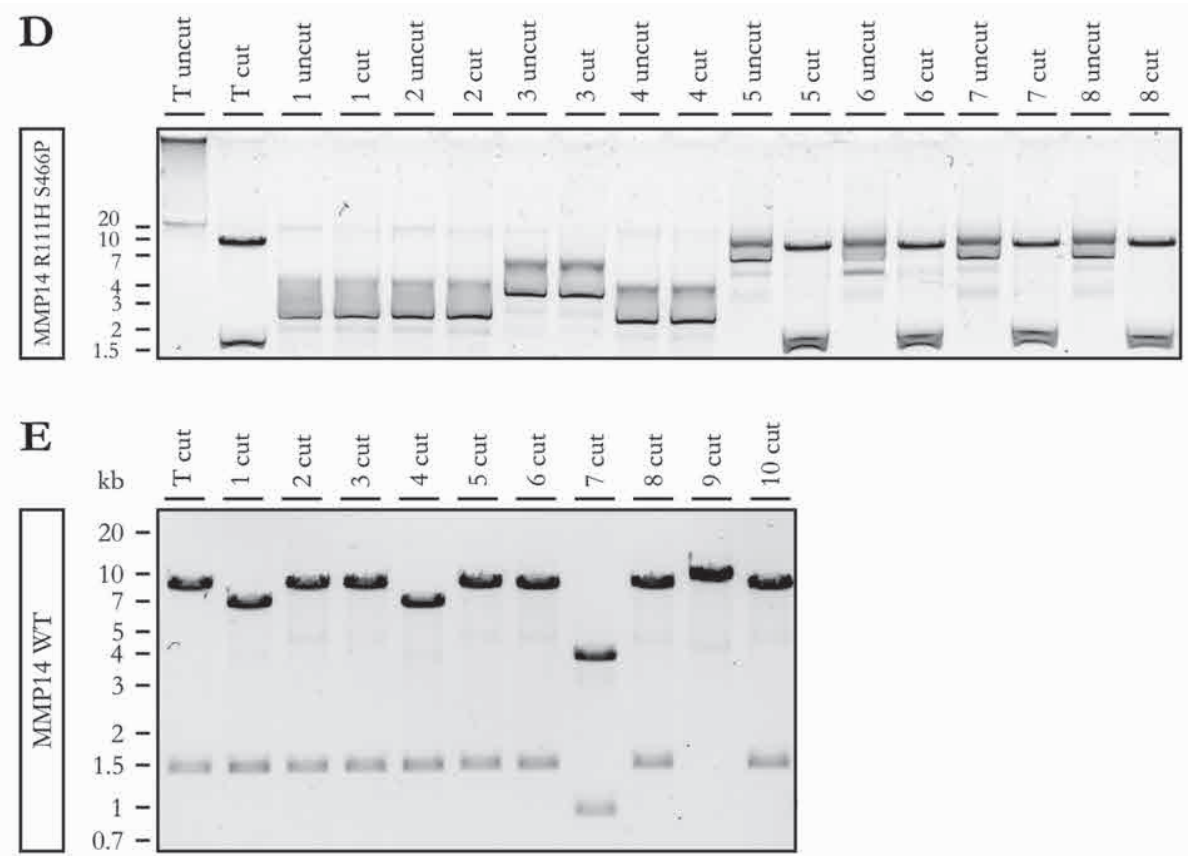

Supplemental Figure 1.2 (continued) - bp) were subsequently analysed by Sanger sequencing of the entire insert. Samples 20 (A), 24 (B), 1 (C), 8 (D) and 2 (E) were used for the experiments in Chapter 2.

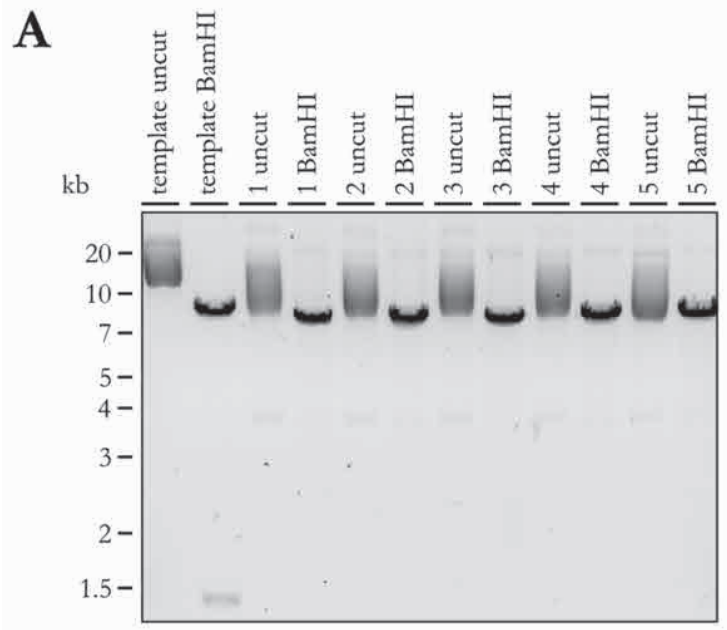

Supplemental Figure 1.3 - Generation of the pQCXIB 3HA-EGFP vector by inverse-PCR. A, DNA gel electrophoresis result of PQCXIB 3HA-MMP14 WT-EGFP from single bacterial colonies after inverse-PCR to remove MMP14 coding sequence, digested with BamHI or undigested. Digestion of template vector results in two fragments $(8,130$ and $1,438 \mathrm{bp})$, whereas the PCR product yields a single band (expected size 7,822 bp) for samples 1-5. B, sequencing result of sample 1 with reverse (Rv) primer 8 compared to the theoretical sequence of the PQCXIB 3HA-EGFP vector, displayed in ClustalW2 format. MMP14 coding sequence is absent; 3HA and EGFP coding sequences are fused by the two linker sequenc- 
B 3HA-EGFP Rv

3HA-EGFP Rv 3HA-BGFP Rv

3HA-BGFP Rv

3HA-EGFP RV

3HA-EGFP Rv

3HA-BGFP RV

3HA-EGFP RV

3HA-EGFP Rv

3HA-EGFP Rv

3HA-EGFP Rv

3HA-EGFP

Rv

3HA-EGFP Rv

3HA-EGFP Rv

3HA-EGFP Rv

3HA-EGFP Rv

3HA-EGFP Rv

CMV enhancer

TTACATAACTTACGGTAAATGGCCCCCTGGCTGACCGCCCAACGACCCCCCCCATTGA 300 TTACATAACTTACGGTAAATGGCCCGCCTGGCTGACCGCCCAACGACCCCCGCCCATTGA 292

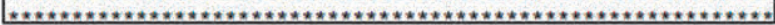
CMV enhancer CFTCAATANTGACGTATGTTCCCATAGTAACGCCAATAGGGACTTTCCATTGACGTCAAT 360 CGTCAATAATGACGTATGTTCCCATAGTAACGCCAATAGGGACTTTCCATTGACGTCAAM 352 .

CMV enhancer

GGGTGGAGTATTWACGTTAAACTGCCCACTTGGCAGTACATCAAGTGTATCATATGCCAA 420 GGGTGGAGTATTTACGGTAAACTGCCCACTTGGCAGTACATCAAGTGTATCATATGCCAA 412

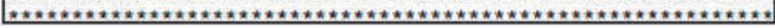
CMV enhancer

GTACGCCCCCTATT GACGTCAATGACGGTAAATGGCCCGCCTGGCATTATGCCCAGTACA 480 GTACGCCCCCTATTGACGTCAATGACGGTAAATGGCCCGCCTGGCATTATGCCCAGTACA 472

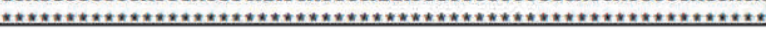

CMV enhancer

TGACCTTATGGGACTTTCCTACTTGGCAGTACATCTACGTATTAGTCATCGCTATTACCA 540 TGACCTTATGGGACTTTCCTACTTGGCAGTACATCTACGTATTAGTCATCGCTATTACCA 532

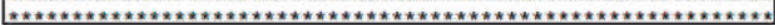

CMV enhancer CMV promoter CMV promoter TQETGATGCGGTTTTGGCAGTACATCAATGGGCGTOA ATAGCGGTTTGACTCACGGGGAT 600 TCETGATGCGGTTTTGGCAGTACATCAATGGGCGTC CATAGCGGTTTCACTCACGGGGAT CMV promoter

TTCCAAGTCTCCACCCCATTGACGTCAATGGGAGTTTGTTTTGGCACCAAAATCAACGGG 660 TTCCAAGTCTCCACCCCATTGACGTCAATGGGAGTTTGTTTTGGCACCAAAATCAACGGC 652

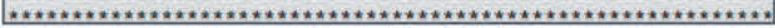
CMV promoter

ACTTTCCAAAATGTCGTAACAACTCCGCCCCATTGACGCAAATGGGCGTTAGGCTGTAC 720 ACTTTCCAAAATGTCGTAACAACTCCGCCCCATTGACGCAAATGGGCGTAGGCGTGTAO 712

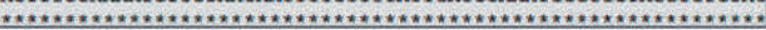
CMV promoter

GGTGGGAGGTCTATATAAGCAGAGCTCGTTTAGTGAACCGTCAGATCGCCTGGAGACGCC 780 GGTGGGAGGTCTATATAAGCAGAGCICGTTTAGTGAACCGTCAGATCGCCTGGAGACGCC 772 *****************************************************************

ATCCACGCTGTTTTGACCTCCATAGAAGACACCGGGACCGATCCAGCCTCCGCGGCCGGG 840 ATCCACGCTGTTTTGACCTCCATAGAAGACACCGGACCGATCCAGCCTCCGCGGCCGG 832 $* * * * * * * * * * * * * * * * * * * * * * * * * * * * * * * * * * * * * * * * * * * * * * * * * * * * * * * * * * * * * * * * * * *$

AACGGTGCATTGGAACG-DTGCAGGAATTGATCCGCGGCCGCACCGGTGCCACCATGG 897 AACGGTGCATTGGAACQ GAC TTGCAGGAATTGATCCGCGGCCGCACCGGTGCCACCATGG 892 *****************0.07*********************************** $3 \mathrm{HA}$

CGAGATACCCATACGATGTCCCTGACTATGCGGGCTATCCCTATGACGTCCCGGACTATG 957 CGAGATACCCATACGATGTCCCTGACTATGCGGGCTATCCCTATGACGTCCCGGACTATG 952 ******************************************************************) 3HA linker 1 CAGGATCCTACCCATATGACGTTCCAGATTACGCTPCAAGAGATCTCGAGCTCAAGCTTC 1017 CAGGATCCTACCCATATGACGTTCCAGATTACGCTRCAAGAGATCTCGAGCTCAAGCTTC 1012 С******************************************************************* linker 1 linker 2 EGFP

GAATT CCAAAGATCCACCGGCCGGTCGCCACOATGGTGAGCAAGGGCAGGAGCTGTTCA 1077 GAAT CCAAAGATCCACCGGCCGGTCGCCACCATGGTGAGCAAGGGCGAGGAGCTGTTCA 1072 等, EGFP

CCGGGGTGGTGCCCATCCTGGTCGAGCTGGACGGCGACGTAAACGGCCACAAGTTCAGCG 1137 CCGGGGTGGTGCCCATCCTGGTCGAGCTGGACGGCGACGTAAACGGCCACAAGTTCAGCG 1132 ******************************************************************* EGFP

TGTCCGGCGAGGGCGAGGGCGATGCCACCTACGGCAAGCTGACCCTGAAGTTCATCTGCA 1197 TGTCCGGCGAGGGCGAGGGCGATGCCACCTACGGCAAGCTGACCCTGAAGT-CATCTGCA 1191 ***************************************************************** EGFP

EGFP
CCACCGGCAAGCTGCCCGTGCCTGGCCACCCTCGTGACCACCCTGACCTA 1249

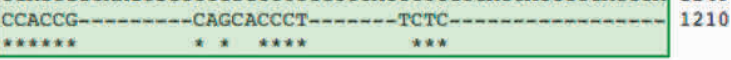

Supplemental Figure 1.3 (continued) - es. The A>G mutation in CMV promoter and the 3 bp insertion between CMV promoter and the 3HA coding sequence are also present in the pQCXIB 3HA-MMP14 WT-EGFP vector. Sample 1 was maxiprepped and used in the experiments in Chapter 2. 
A
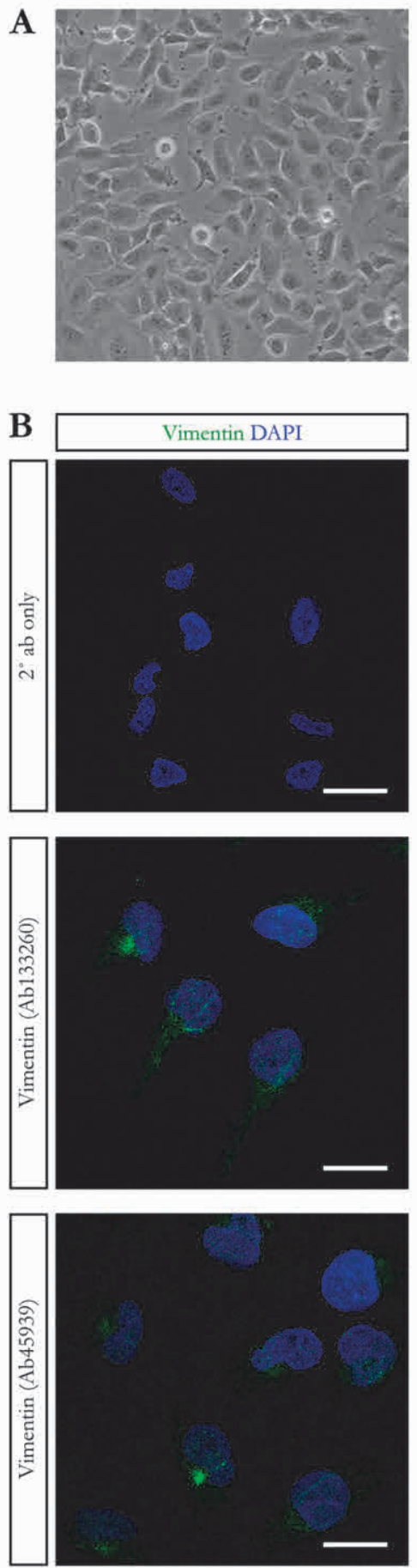

Supplemental Figure 1.4 - Morphology of MRC-5V1 cells. A, Brightfield image of MRC-5V1 cells at $10 \mathrm{x}$ magnification. As opposed to the primary MRC5 cells, MRC-5V1 cells are less uniform in cell size and shape, and have an epithelial to fibroblast-like morphology that is more epithelial as cells grow more confluent, as described before [6]. B, maximum intensity projections of IF microscopy z-stacks at 60X magnification of MRC$5 \mathrm{~V} 1$ cells stained with anti-Vimentin primary antibodies and/or Alexa 488-conjugated goat antirabbit secondary antibody, revealing a filamentous Vimentin staining pattern, characteristic for fibroblasts. Scale bars equal $20 \mu \mathrm{m}$. 


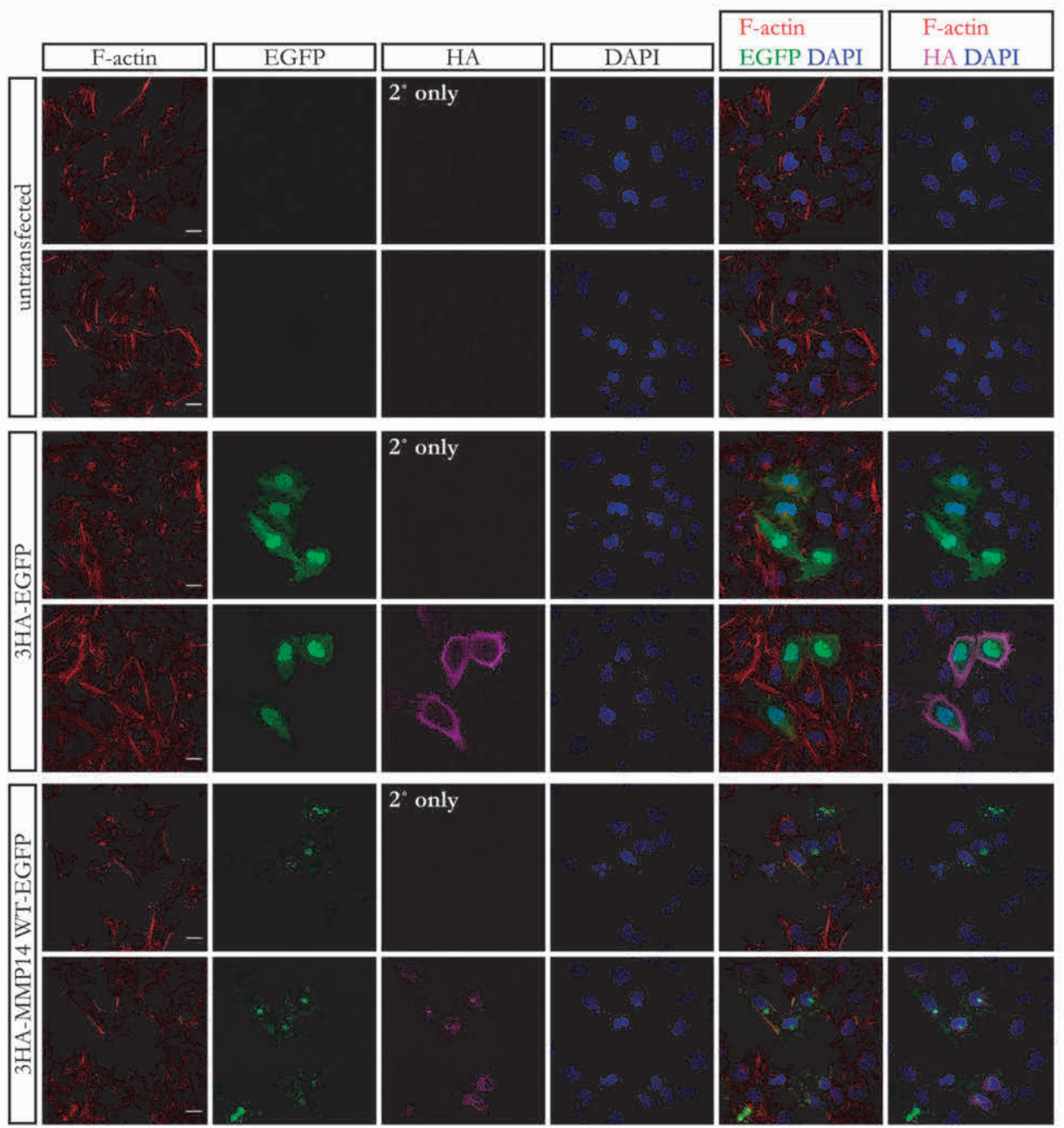

Supplemental Figure 1.5 - Testing the mouse anti-HA antibody for indirect IF microscopy. IF microscopy images of MRC-5V1 cells transfected with pQCXIB 3HA-MMP14 WT-EGFP or pQCXIB 3 HA-EGFP (seeded at 50,000 cells per mL of medium) or left untransfected (seeded at 20,000 cells per mL of medium). Cells were fixed $72 \mathrm{~h}$ post seeding, and stained with mouse anti-HA antibody (ab16918) and/or Alexa 647-conjugated goat-anti-mouse secondary antibody. The subcellular localisation of the EGFP signal in MRC-5V1 cells transiently expressing 3HA-MMP14 WT-EGFP is similar to that of endogenous MMP14 in MRC-5V1 cells, that is, at the cell surface and in a perinuclear aggregate. The HA signal in 3HA-MMP14 WT-EGFP-expressing cells is highest in the region of the perinuclear EGFP aggregate, and forms a gradient towards but not reaching the plasma membrane. The EGFP signal in MRC-5V1 cells transiently expressing 3HA-EGFP is present in the entire cell, with seemingly highest intensity in the nucleus. The HA signal in 3HA-EGFP-expressing MRC-5V1 cells is distributed differently, with a higher signal intensity in the cell periphery but a relatively lower intensity in the nucleus. Scale bars equal $20 \mu \mathrm{m}$. 


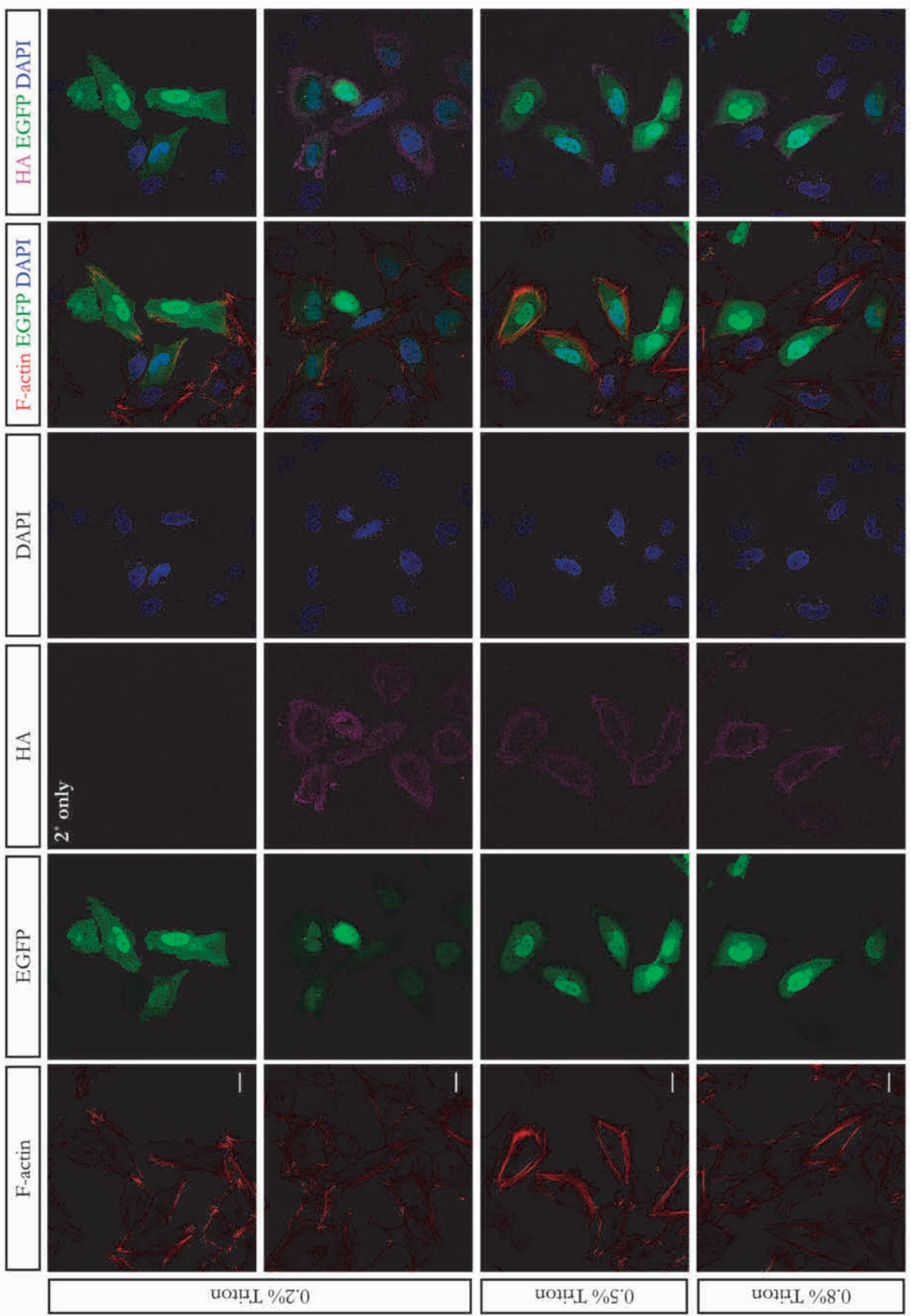

Supplemental Figure 1.6 - Permeabilisation optimisation for indirect IF microscopy. IF microscopy images of MRC-5V1 cells transiently expressing 3HA-EGFP, permeabilised with $0.2-0.8 \%(v / v)$ Triton X100 and stained with mouse anti-HA antibody (ab16918) and/or Alexa 647-conjugated goat-anti-mouse secondary antibody. Increasing the Triton X-100 concentration did not result in an increase in nuclear HA signal intensity. Scale bar equals $20 \mu \mathrm{m}$. 


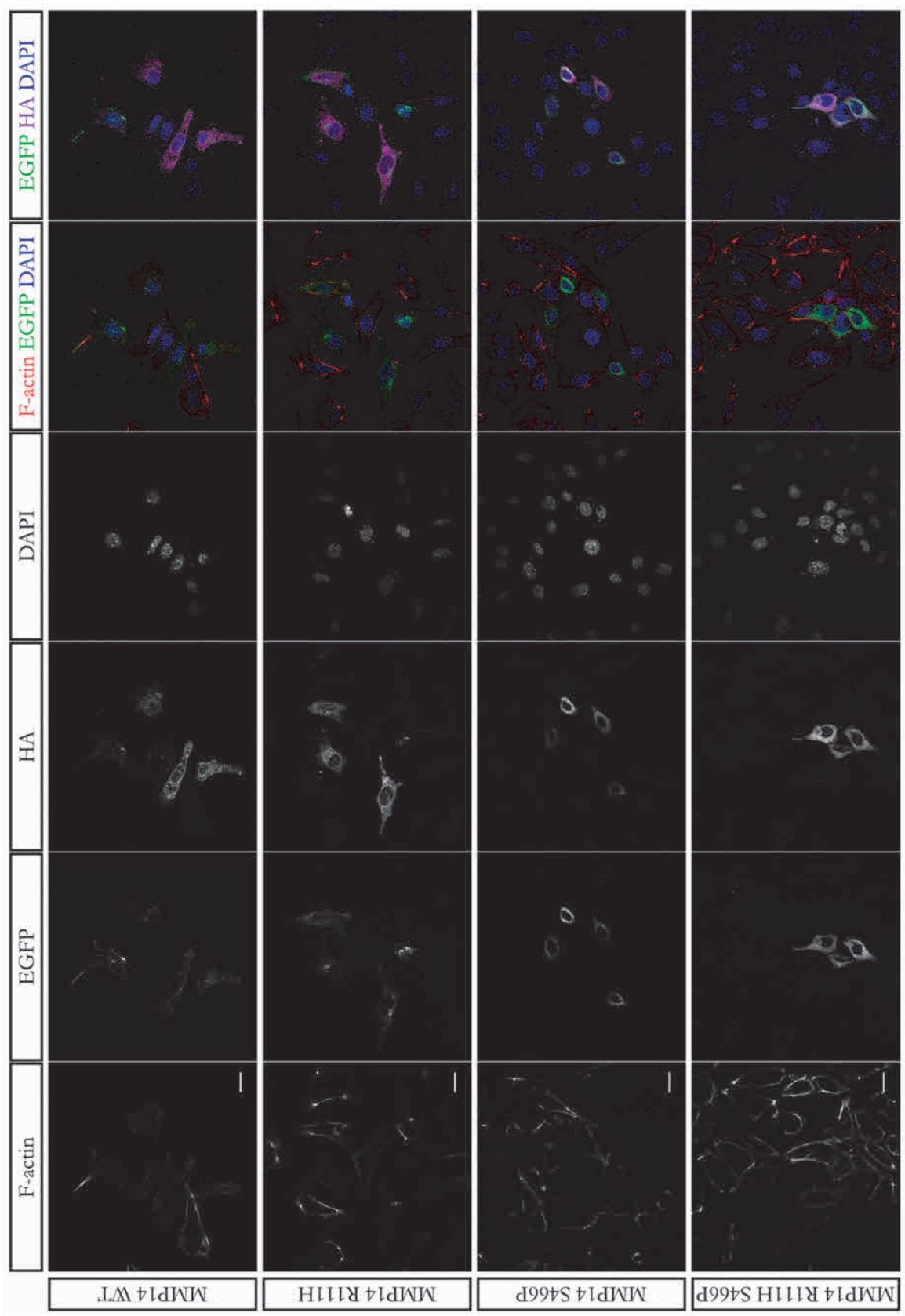

Supplemental Figure 1.7 - Exogenous 3HA-MMP14-EGFP fusion proteins localise similarly in mIMCD-3 cells and MRC-5V1 cells. IF microscopy images of mIMCD-3 cells transiently expressing W'T or mutant 3HA-MMP14-EGFP fusion proteins demonstrate that their subcellular localisation is similar to that in MRC-5V1 cells (Fig. 2.7). Scale bars equal $20 \mu \mathrm{m}$. 


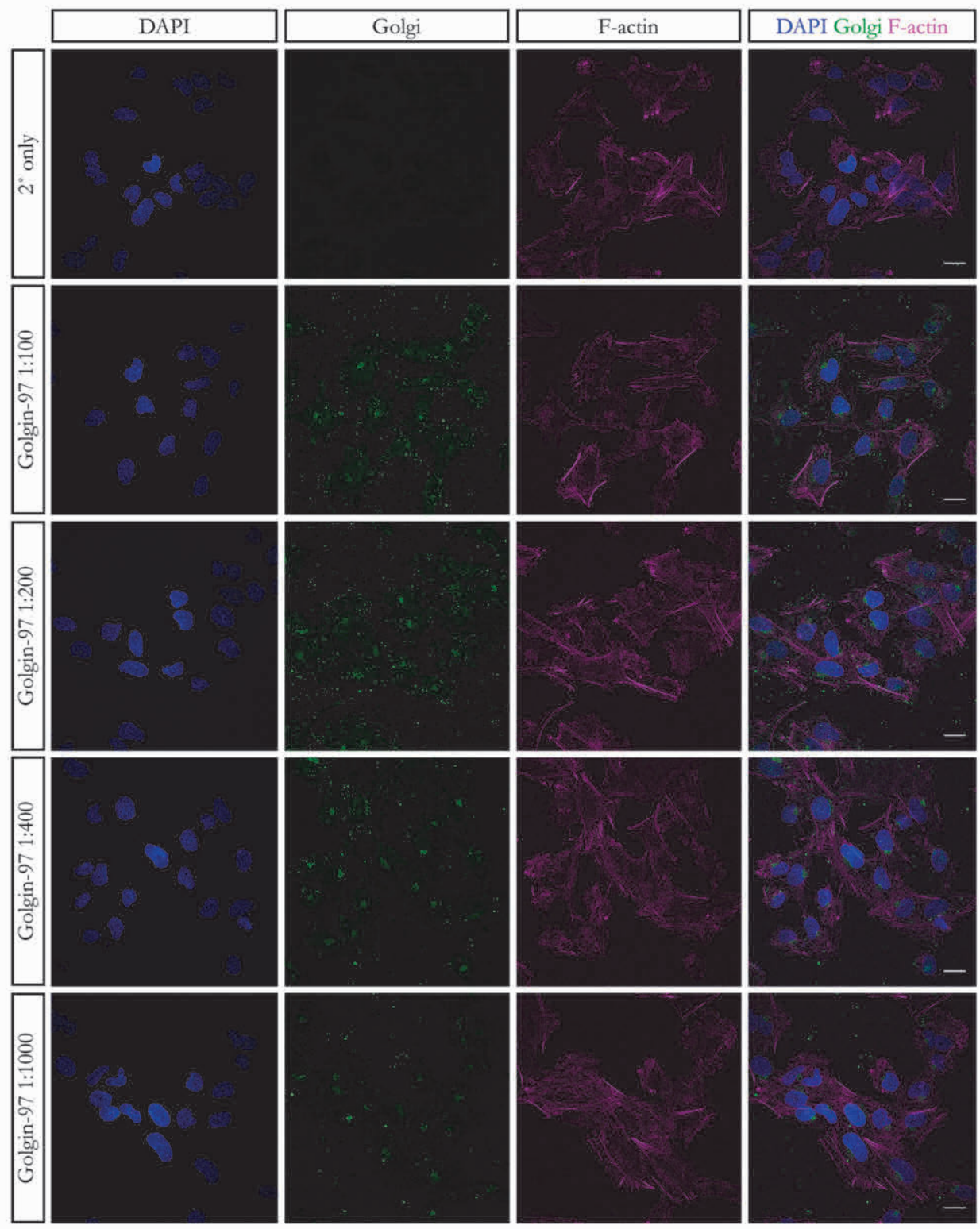

Supplemental Figure 1.8 - Testing the mouse anti-Golgin97 antibody for indirect IF microscopy. IF microscopy images of MRC-5V1 cells incubated with mouse anti-Golgin97 primary antibody at concentrations 1:100-1,000 and/or Alexa 488-conjugated goat-anti-mouse secondary antibody. Within the tested concentration range, using the anti-Golgin97 antibody at 1:1,000 yields a specific staining in the perinuclear region without the nonspecific cytoplasmic and extracellular speckles present at higher concentrations. Scale bars equal $20 \mu \mathrm{m}$. 
A
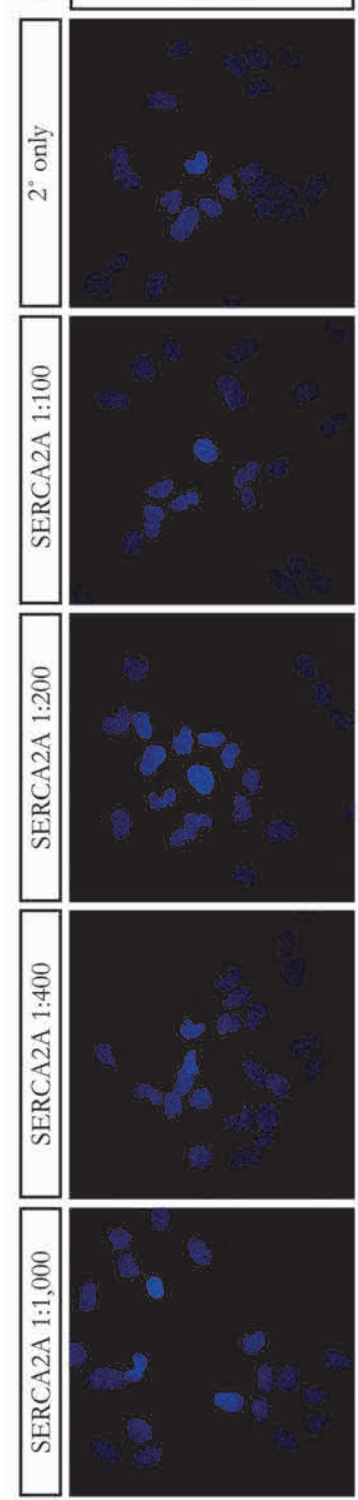
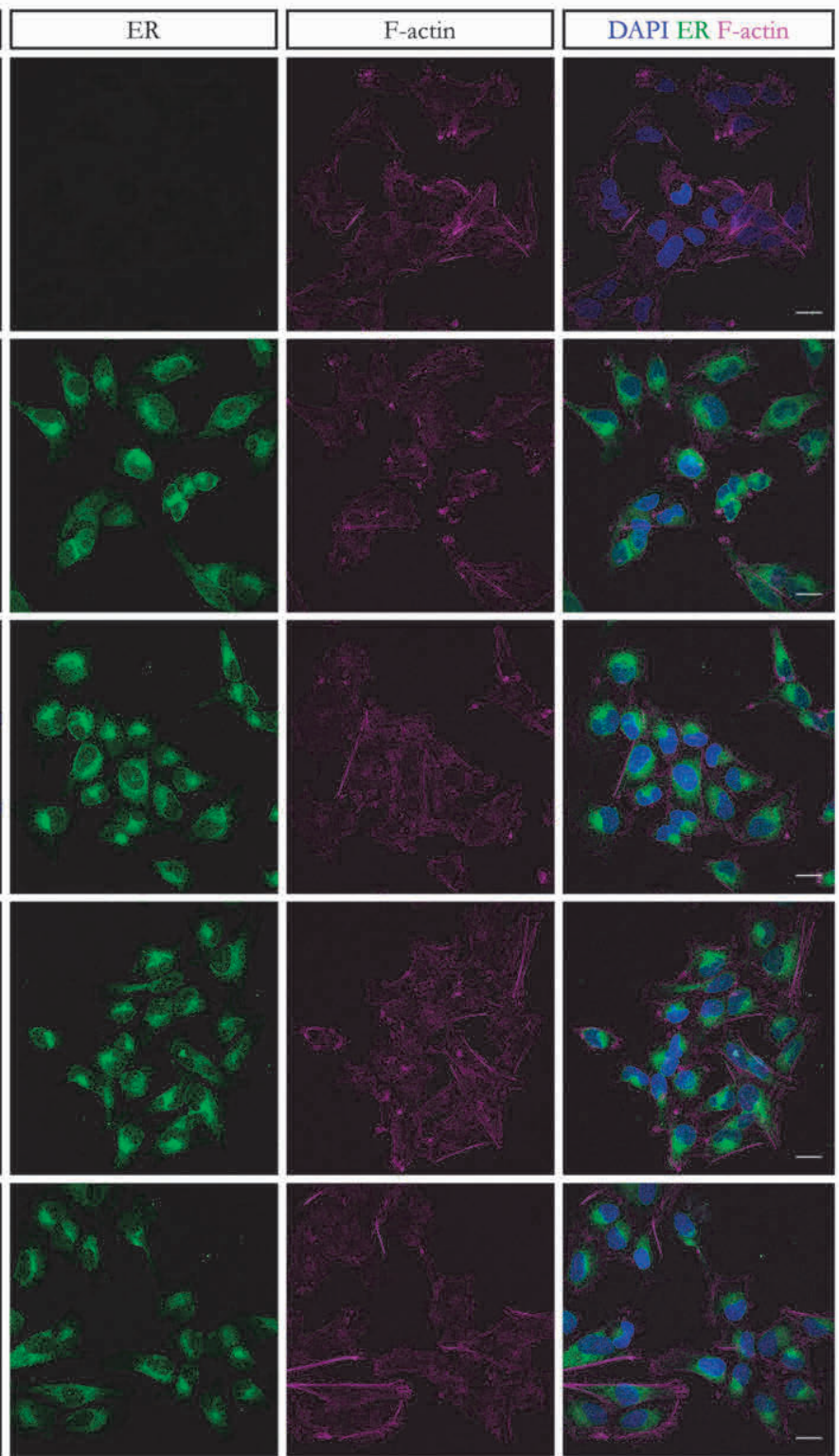

Supplemental Figure 1.9 - Testing the mouse anti-SERCA2A antibody for indirect IF microscopy. A-C, IF microscopy images of MRC-5V1 cells incubated with mouse anti-SERCA2A primary antibody at concentrations 1:100-1,000 (A), 1:1,000-10,000 (B), or 1:10,000-100,000 (C), and/or Alexa 488-conjugated goat-anti-mouse secondary antibody. Within the tested concentration range, using the anti-SERCA2A antibody at concentrations 1:40,000-100,000 yields a specific perinuclear staining. Scale bars equal $20 \mu \mathrm{m}$. 


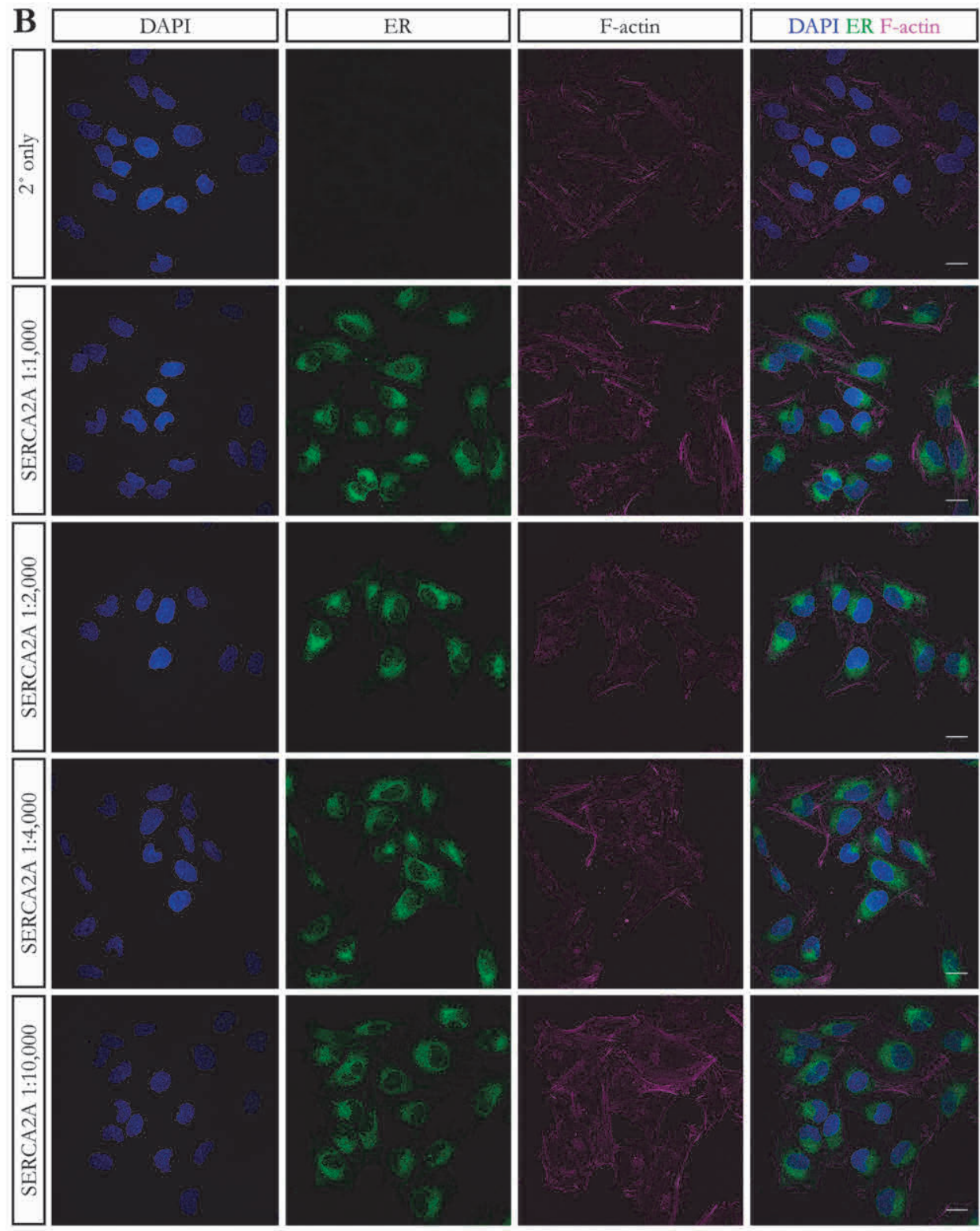

Supplemental Figure 1.9 (continued). 


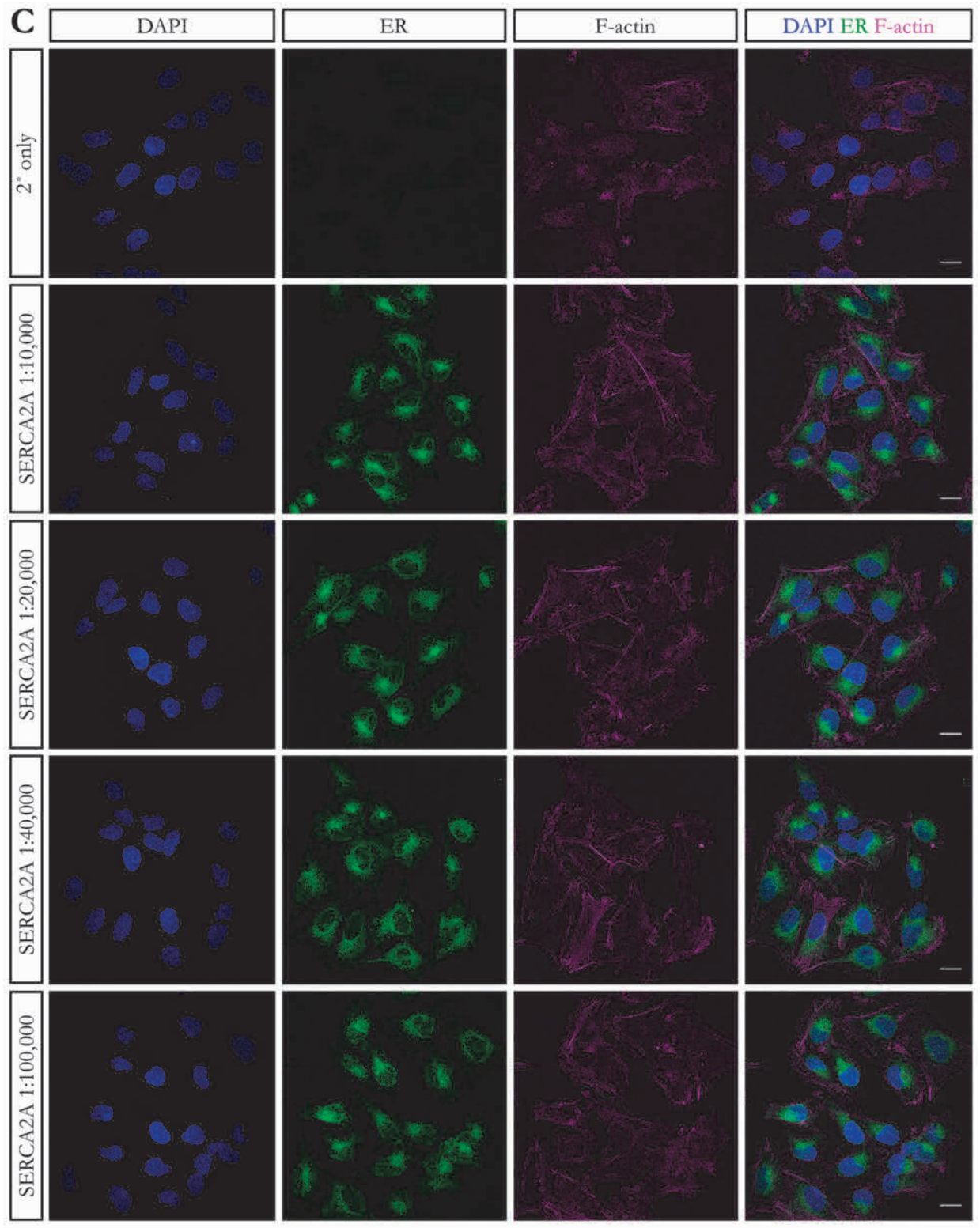

Supplemental Figure 1.9 (continued). 


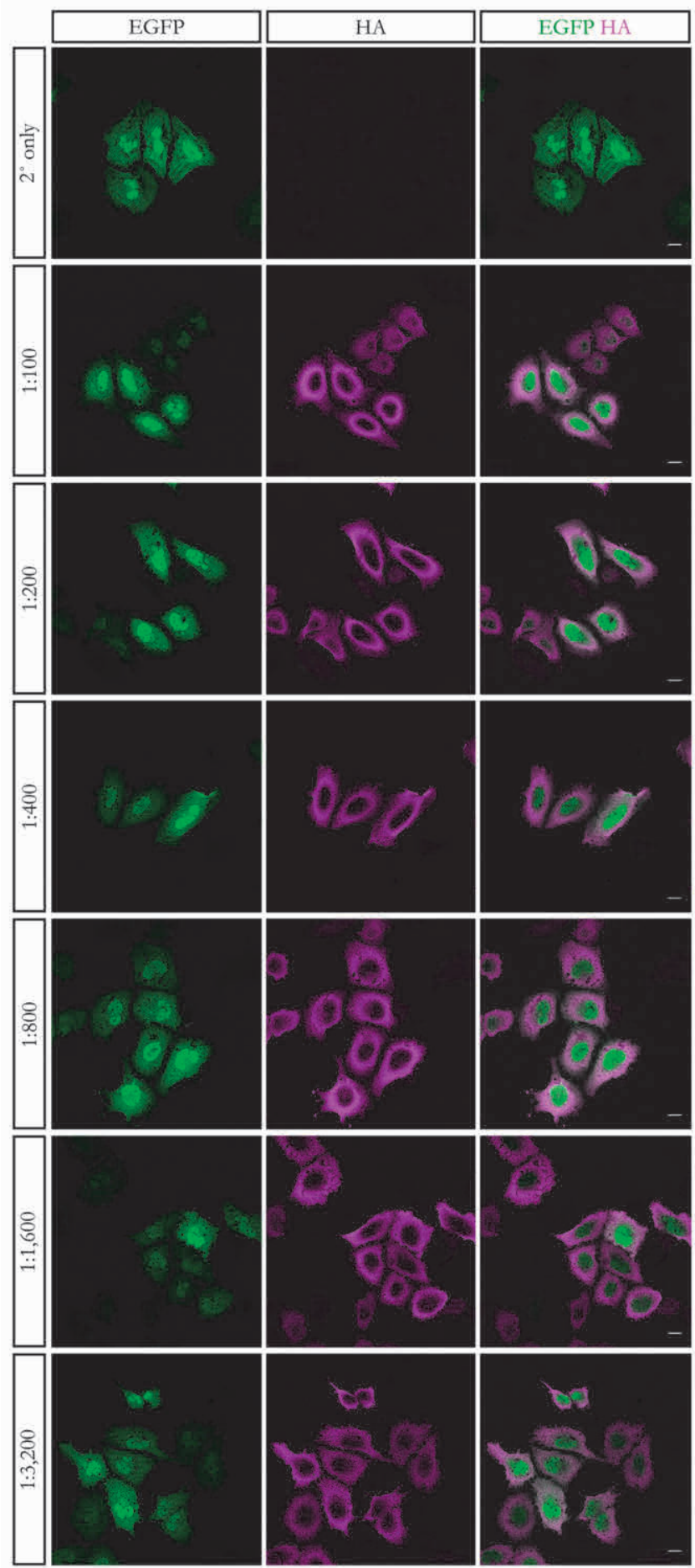

Supplemental Figure 1.10 - Testing the rabbit anti-HA antibody for indirect IF microscopy. IF microscopy images of MRC-5V1 transiently expressing 3HA-EGFP, incubated with a rabbit anti-HA primary antibody (Cell signalling 3724) at concentrations 1:100-3,200 and/or Alexa 568conjugated goat anti-rabbit secondary antibody. Within the tested concentration range, using the anti-HA antibody up to concentrations as low as 1:1,600 still yields a strong staining, which diminishes with further dilution of the antibody. Scale bars equal $20 \mu \mathrm{m}$. 
Supplemental Table 1.1 - Average degraded gelatin area (promille per cell) for cells expressing different fusion proteins, compared to 3HA-MMP14 WT-EGFP expressing cells by two-sided Student's T-test. H, hour post seeding.

\begin{tabular}{|c|c|c|c|c|}
\hline \multirow[b]{2}{*}{ Fusion protein } & \multicolumn{2}{|c|}{$3 \mathrm{~h}$} & \multicolumn{2}{|c|}{$21 \mathrm{~h}$} \\
\hline & average & p-value & average & p-value \\
\hline MMP14 WT & 0.139 & - & 0.485 & - \\
\hline MMP14 T17R & 0.119 & 0.590 & 0.276 & 0.137 \\
\hline MMP14 R92C & 0.005 & 0.013 & $6.80 \mathrm{E}-4$ & 0.002 \\
\hline MMP14 R111H & 0.027 & 0.023 & 0.154 & 0.016 \\
\hline MMP14 S466P & 0.199 & 0.453 & 0.426 & 0.585 \\
\hline MMP14 R92C R111H & 6.67E-05 & 0.011 & $5.00 \mathrm{E}-5$ & 0.002 \\
\hline MMP14 R92C S466P & $1.33 \mathrm{E}-4$ & 0.011 & $1.00 \mathrm{E}-4$ & 0.002 \\
\hline MMP14 R111H S466P & 0.179 & 0.269 & 0.299 & 0.181 \\
\hline MMP14 R92C R111H S466P & 3.33E-05 & 0.011 & $1.87 \mathrm{E}-4$ & 0.002 \\
\hline 3HA-EGFP & $6.21 \mathrm{E}-4$ & 0.011 & $3.48 \mathrm{E}-05$ & 0.002 \\
\hline
\end{tabular}



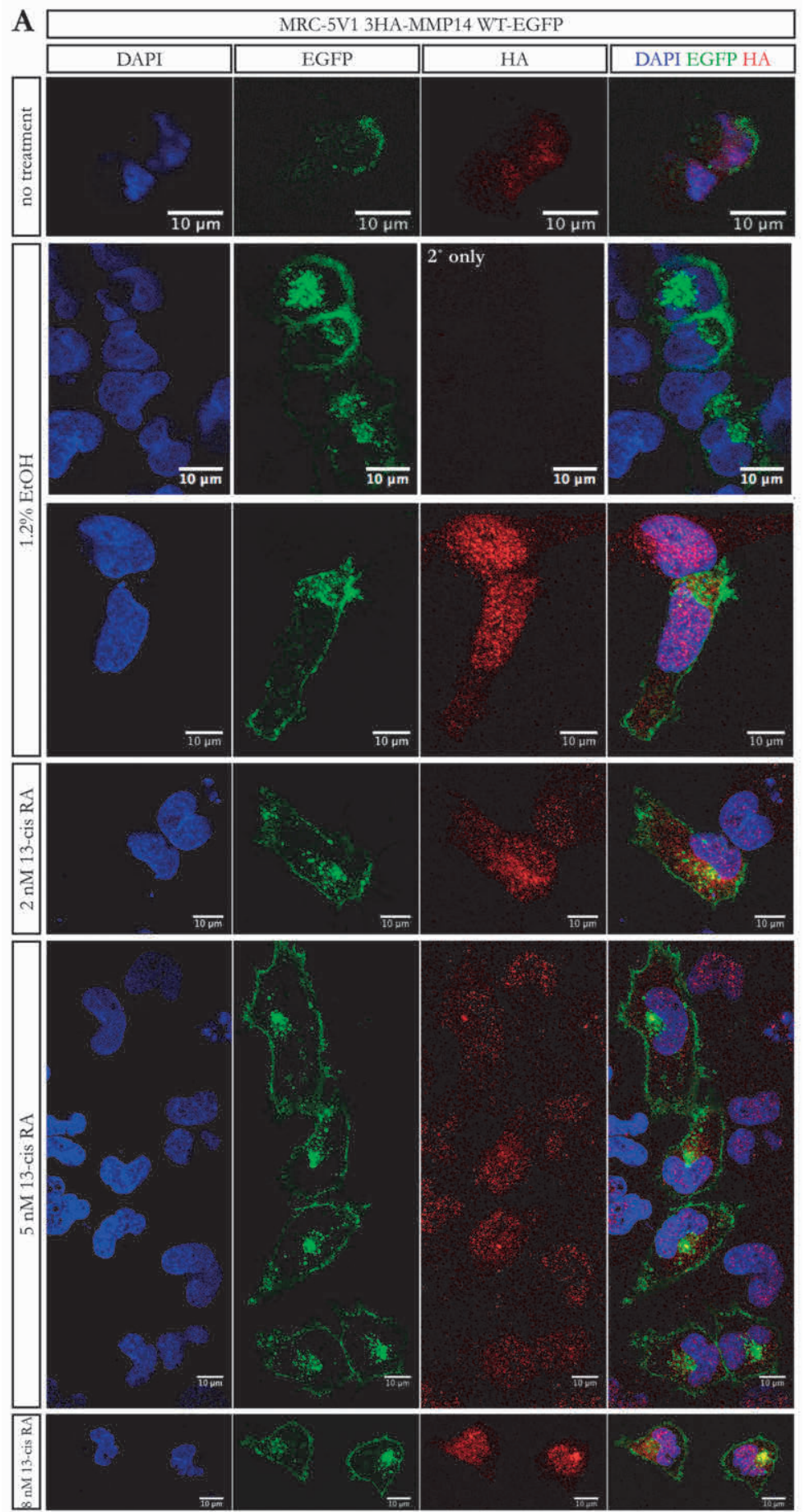

Supplemental Figure 1.11 - Retinoids do not alter subcellular distribution of exogenous MMP14. 


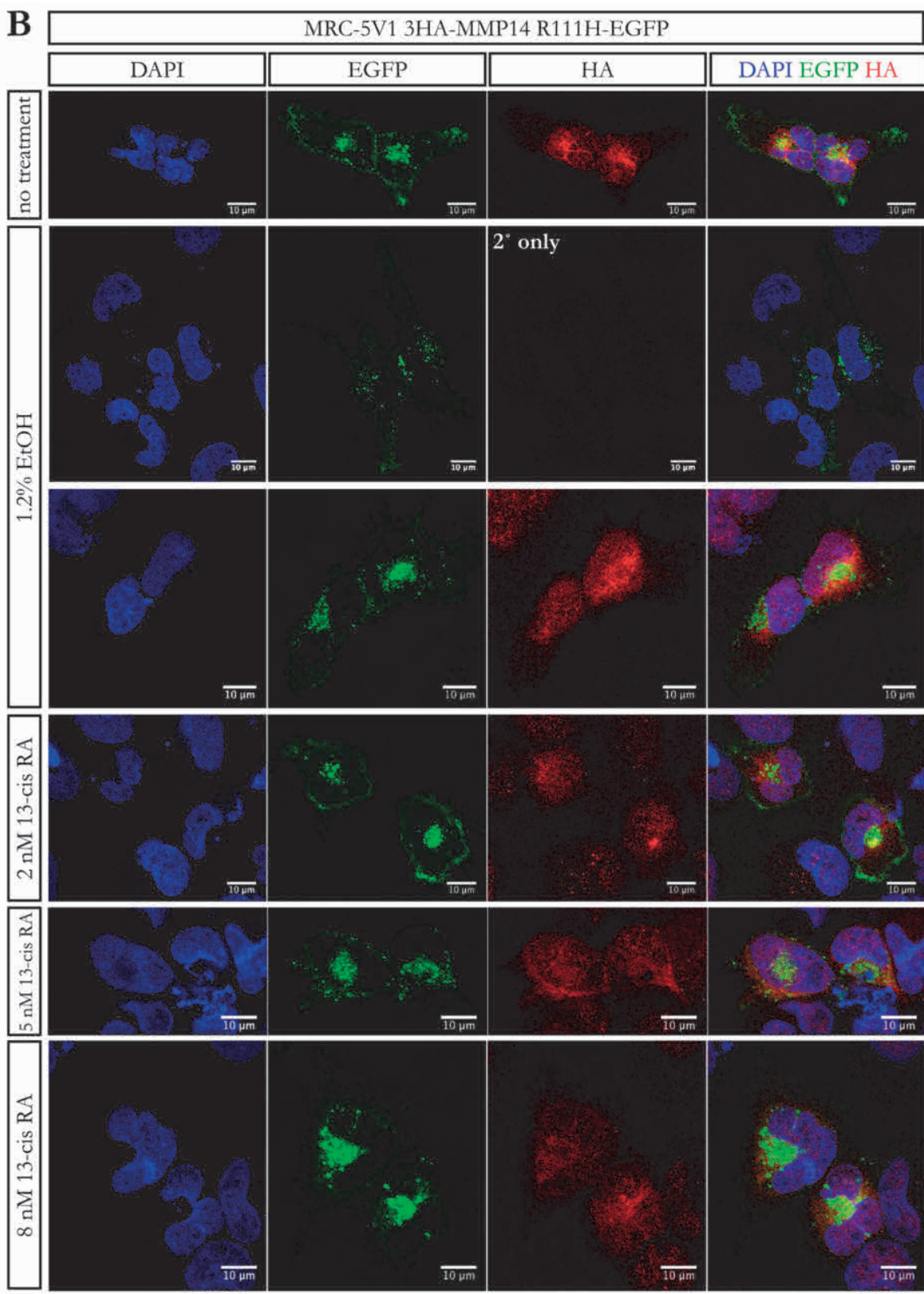

Supplemental Figure 1.11 (continued) - IF microscopy images of MRC-5V1 cells transiently expressing WT or mutant 3HA-MMP14-EGFP fusion proteins, treated with 2-8 nM 13-cis retinoic acid (RA) or vehicle $(1.2 \%(v / v)$ ethanol) only, or left untreated. A-C, subcellular localisation of the fusion proteins' tags in untreated cells is similar to previous experiments (Fig. 2.6) and unaltered by vehicle treatment. A-B, 13cis RA did not affect the subcellular distribution of the tags of the WT and R111H mutant fusion proteins. C, $<5 \%$ of cells expressing the S466P mutant fusion protein show localisation of the EGFP signal, but not $\mathrm{HA}$, at the cell surface. This percentage is unaffected by 13-cis RA treatment. Scale bars equal $10 \mu \mathrm{m}$. 


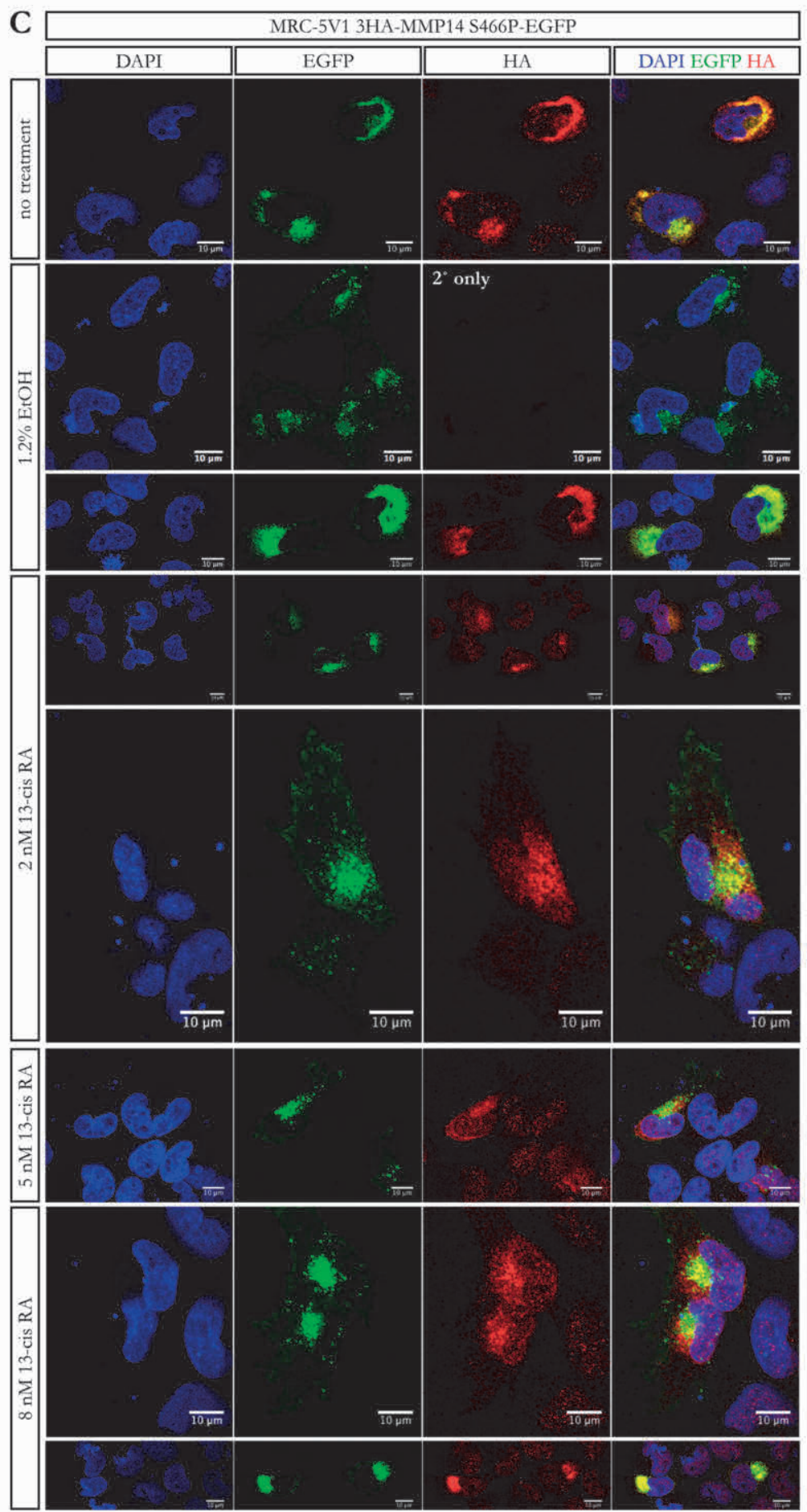

Supplemental Figure 1.11 (continued). 



\section{Addendum 2}

A

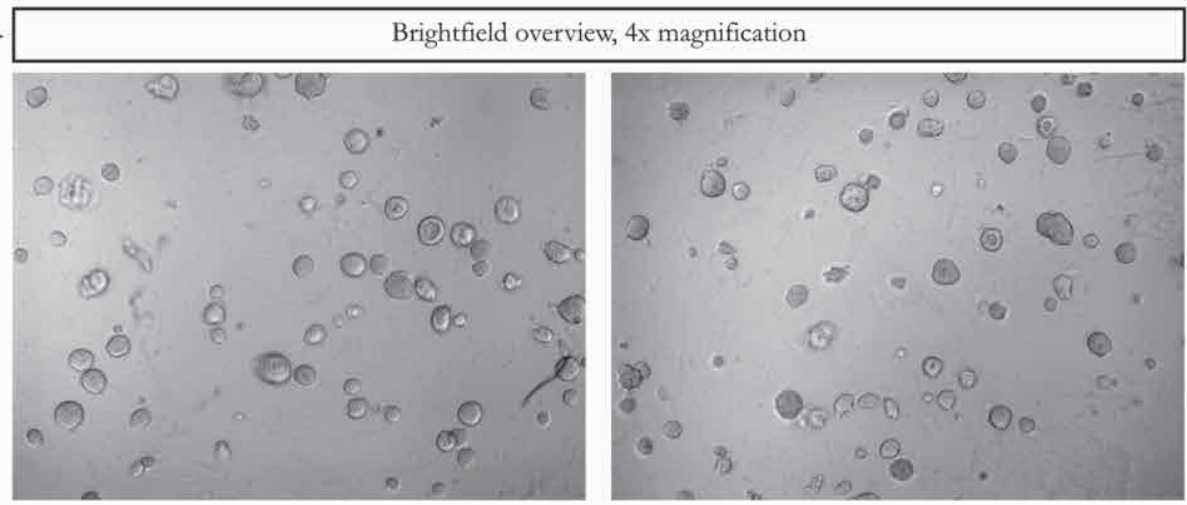

B
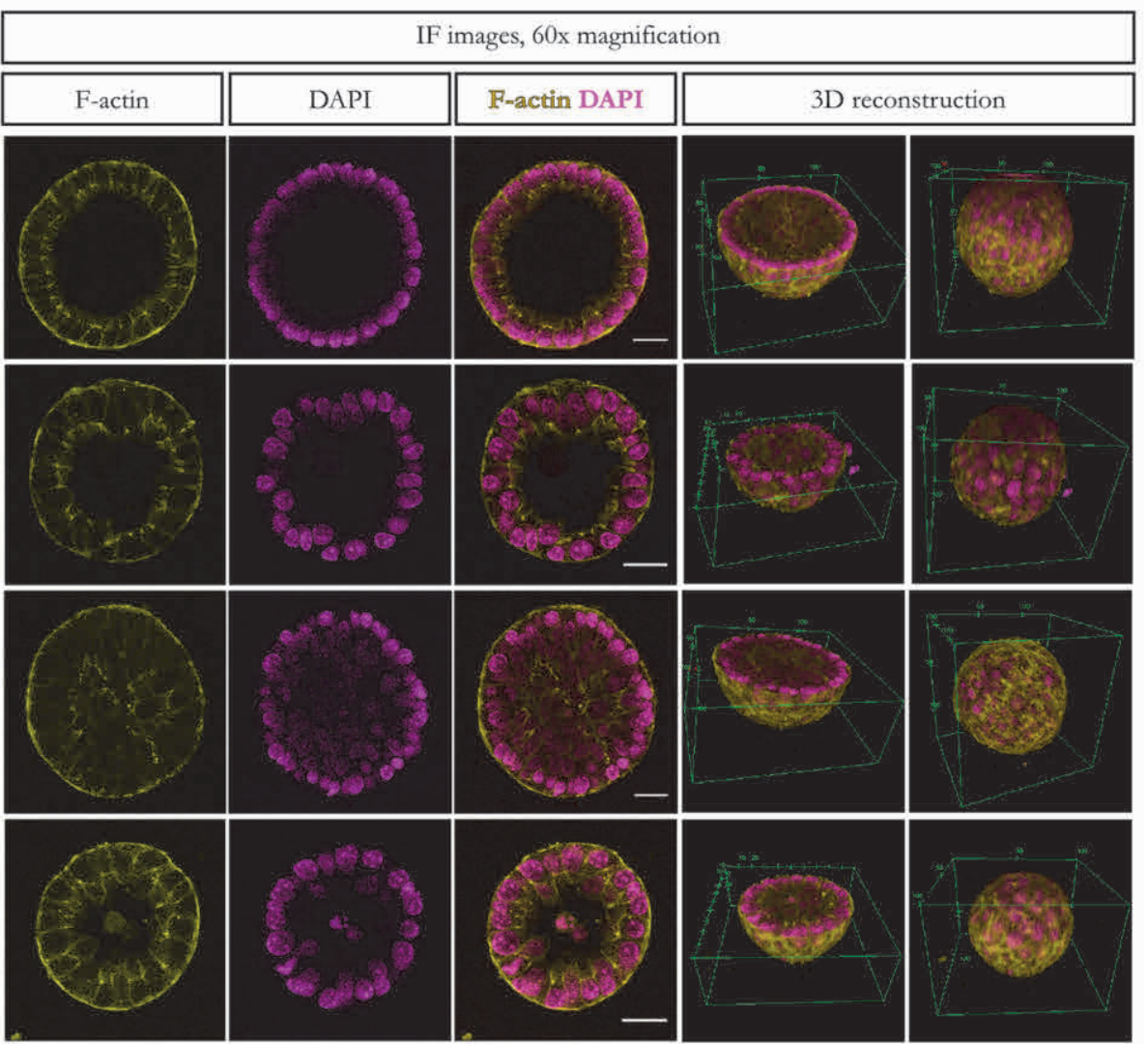

Supplemental Figure 2.1 - Lumen formation of mIMCD-3 spheroids does not accelerate by growing cells less confluent in $2 \mathrm{D}$ culture prior to seeding in Matrigel ${ }^{\mathrm{TM}}$. Brightfield (A and C) and IF (B and D) microscopy images of mIMCD-3 spheroids in Matrigel. In B and D, equatorial optical slices of the imaged spheroids are shown. Cells were kept at $85 \%$ (A- B) or 40\% (C-D) confluence in $2 \mathrm{D}$ culture prior 

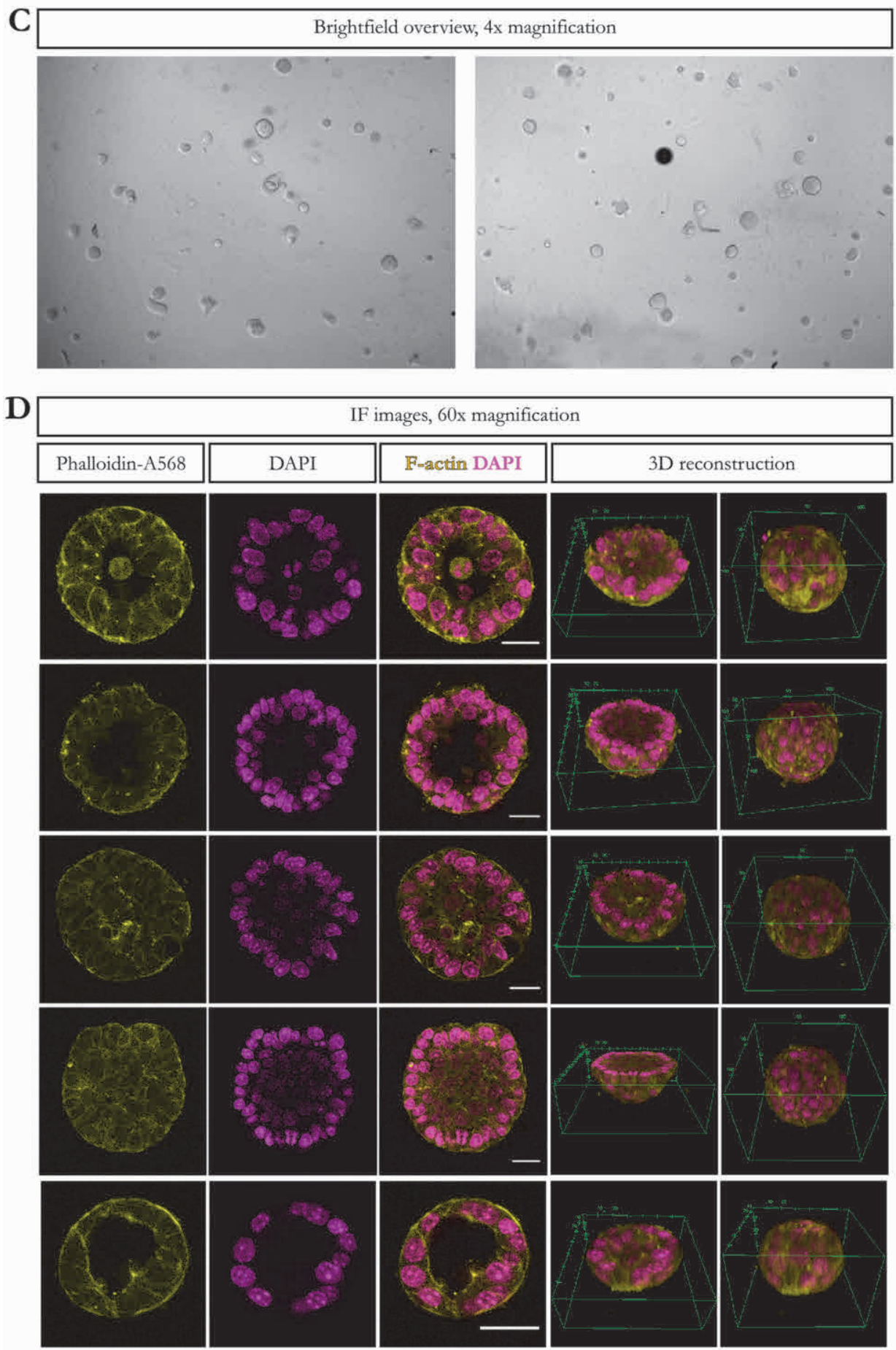

Supplemental Figure 2.1 (continued) - to seeding in Matrigel ${ }^{\mathrm{TM}}$. Cells that were kept less confluent in 
Supplemental Figure 2.1 (continued) - 2D culture formed less and smaller clusters than more confluent cells. With both culture methods, only about $10 \%$ cell clusters has a lumen after four days. Scale bars equal $20 \mu \mathrm{m}$.
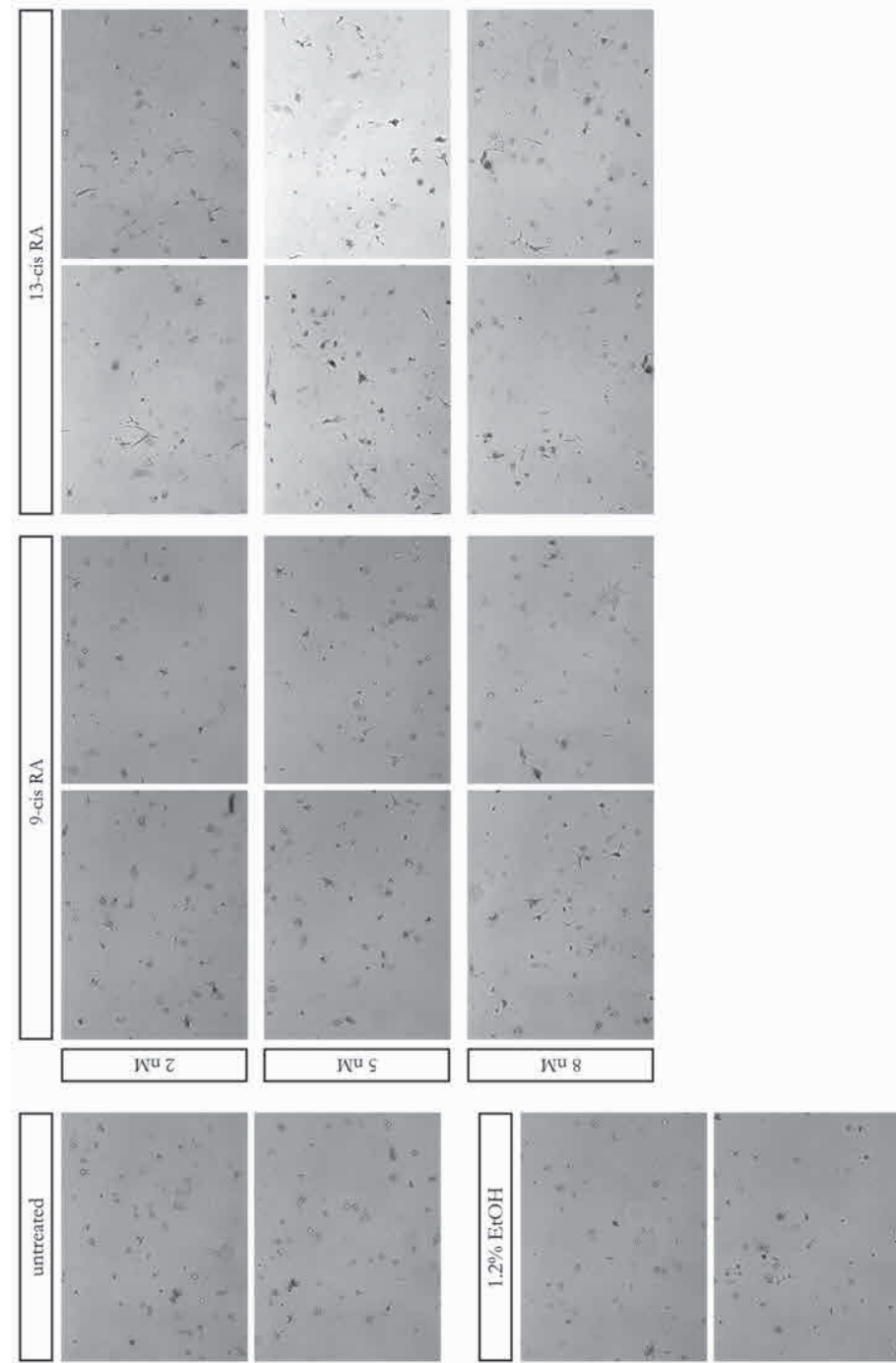

Supplemental Figure 2.2 - Retinoids stimulate tubulogenesis of mIMCD-3 cells in collagen I gel. Brightfield images of mIMCD-3 cells grown in type I collagen gel for three days in presence of 2-8 nM 9cis RA or 13-cis RA, vehicle $(1.2 \%(v / v)$ ethanol) only, or left untreated. After three days, the majority of untreated and vehicle-only treated mIMCD-3 cells form small cell clusters from which cell cords radiate; very few tubular structures can be observed. Treatment of cells with increasing doses of 9-cis RA is positively correlated with the number of tubular structures that are formed after three days. At all doses tested, 13-cis RA stimulates tubulogenesis of mIMCD-3 cells, which is reflected in both a larger amount of tubular structures as well as a longer average tubule length. 



\section{Addendum 3}

A

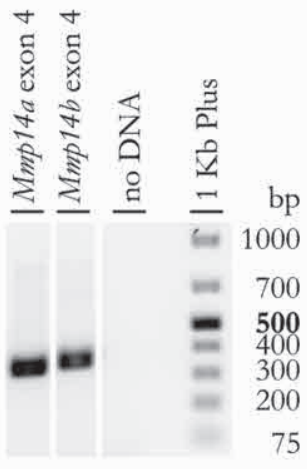

B

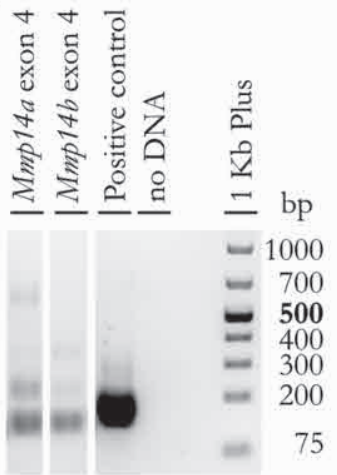

C

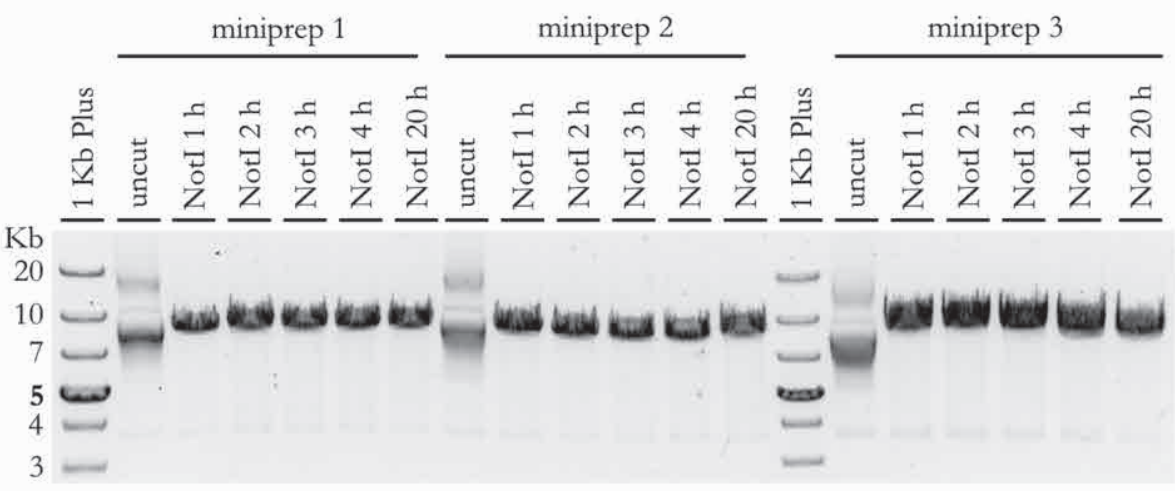

D

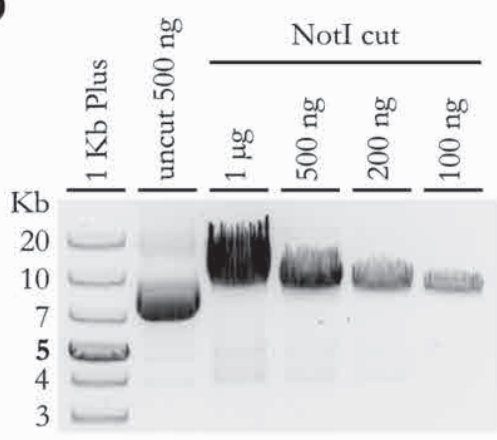

E

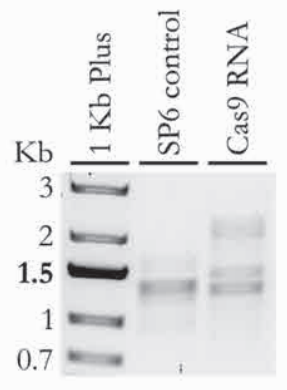

Supplemental Figure 3.1 - Generating mmp14a/b gRNA and Cas9 RNA for genome editing. A, DNA gel electrophoresis result of PCR-amplified gBlock ${ }^{\circledR}$ DNA. B, DNA gel electrophoresis result of T7 transcribed mmp14a/b gRNA, compared to positive (from T7 kit) and negative control. 3-4 bands can be detected in every lane, corresponding to the different RNA conformations, with the smallest band being 
Supplemental Figure 3.1 (continued) - the most intense. Several lanes were removed from the gel images, as these contained gBlocks ${ }^{\circledR}$ DNA respectively RNA to target genes not relevant to this project. C, DNA gel electrophoresis result of miniprepped pCS2 nls-zCas9-nls plasmid DNA, digested with NotI for a different duration or undigested, to find the optimal incubation time for complete plasmid linearisation. Digestion seems complete even after $1 \mathrm{~h}$ incubation; bacteria of culture 1 (corresponding to miniprep 1) were subsequently grown in larger volume and DNA isolated by maxiprep. D, DNA gel electrophoresis result of different amounts of maxiprepped pCS2-nCas9n plasmid DNA (from culture 1), digested with NotI for $2.2 \mathrm{~h}$ or undigested, to rule out any additional bands that were previously not visible due to overloading. When loading as little as $100 \mathrm{ng}$ of linearized Cas9 plasmid, only a single band can be detected; the plasmid is completely linearised. E, DNA gel electrophoresis result of SP6 RNA transcript of linearised Cas9 plasmid, compared to positive control (from the used kit). Similar to gRNA (B), four bands (two < $1.5 \mathrm{~Kb}$, two $>2 \mathrm{~Kb}$ ) can be observed, with the smallest band being the most intense.

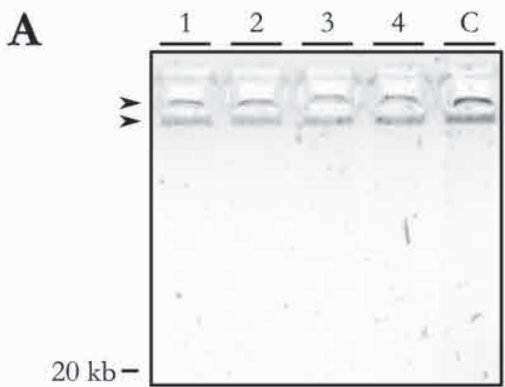

B

\begin{tabular}{|c|c|c|c|c|c|c|c|c|c|c|c|c|c|}
\hline \multirow[b]{2}{*}{ lysate $(\mu \mathrm{L})$} & \multicolumn{6}{|c|}{ lysate 1} & \multicolumn{6}{|c|}{ lysate C } & \multirow{2}{*}{$\begin{array}{l}0 \\
1\end{array}$} \\
\hline & 1 & 1 & 3.3 & 3.3 & 1 & 1 & 1 & 1 & 3.3 & 3.3 & 1 & 1 & \\
\hline $\mathrm{MgCl}_{2}(\mathrm{mM})$ & 1 & 2 & 1 & 2 & 1 & 1 & 1 & 2 & 1 & 2 & 1 & 1 & 1 \\
\hline$F / R$ & $+1+$ & $+1+$ & $+1+$ & $+1+$ & $+1-$ & $-1+$ & $+1+$ & $+1+$ & $+1+$ & $+1+$ & $+1-$ & $-1+$ & $+1+$ \\
\hline $\begin{array}{r}\text { bp } 500- \\
400- \\
300- \\
200- \\
75-\end{array}$ & $=$ & $=$ & 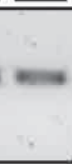 & 5 & & & $=$ & $=$ & $=$ & $m$ & & 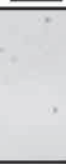 & \\
\hline
\end{tabular}

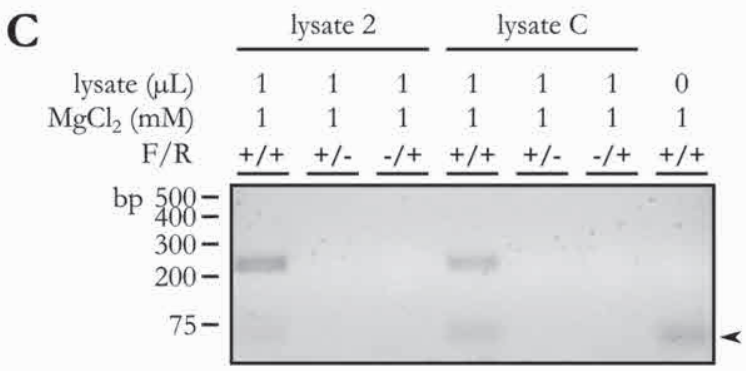

Supplemental Figure 3.2 - Optimisation of mmp14a/genotyping. A, DNA gel electrophoresis result of the unpurified lysate of single $24 \mathrm{hpf}$ WT zebrafish embryos (1-4) and purified gDNA of a single adult WT zebrafish (C). gDNA was present in and just below the wells (arrowheads). Lysates 1, 2 and C were used in B and C. B-C, DNA gel electrophoresis result of optimisation of PCR amplification of region 
Supplemental Figure 3.2 (continued) - around CRISPR site in exon 4 of mmp14a (B) and mmp14b (C). Different amounts of lysate as template and $\mathrm{MgCl}_{2}$ concentrations were tested and compared to negative controls (absence of either forward (F) or reverse (R) primer or template DNA). A single amplicon band at the expected height (307 bp for mmp14a, $223 \mathrm{bp}$ for mmp14b) can be seen in all reactions with both primers and gDNA template present. Note the presence of a primer dimer (arrowhead) for the mmp14b primer set that becomes more clearly visible in absence of template gDNA. D-E, result of direct Sanger sequencing with forward primer of the mmp14a exon 4 (D) and mmp14b exon 4 (E) amplicon, compared to reference sequence shown (mmp14a, NP_919397.1; mmp14b, NP_913395.1) as ClustalW2 output. The CRISPR target site is highlighted by the red box. Note the SNP in mmp14b exon 4 (arrow). F-G, halfway the project, new primers were designed to amplify larger regions the target site in exon 4 of mmp14a (F) and mmp14b(G). Both primer sets were tested in the absence and presence of $1 \mu \mathrm{L}$ purified gDNA of an adult WT zebrafish; only a single concentration of $\mathrm{MgCl}_{2}(2 \mathrm{mM})$ was used. A single amplicon band at the expected height (513 bp for mmp14a, $326 \mathrm{bp}$ for mmp14b) can be seen in all reactions with both primers and gDNA template present. 
D

14 a4 GCCACACATGAGGCCATCAAGAAGGCATTCAGAGTTTGGGAGGCCGTGACGCCACTCAAG seq1 CGGGTA-_-_-_-- AACTTGTGAGCCTGTCGCGCTGCT---G

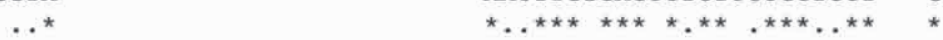

$14 a 4$ TTTCGTGAAATCCCATACAGTCAGATCAATGGCAAGGTGGAGAAGTTTGCAGACATCATG seq1 CTCCGCG-AATCCCATACCGTCAGATCAATGGCAAGGTGGAGAAGTTTGCAGACATCATG

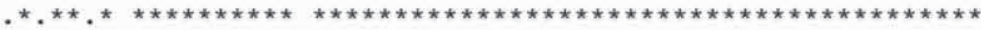

14 a4 CTTTTCTTTGCGGAAGGCTTTCATGGAGACAGCACCCCATTTGATGGCGAGGGAGGTTTC seq1 CTTTTCTTTGCGGAAGGCTTTCAIGGAGACAGCACCCCATTTGATGGCGAGGGAGGTTTC

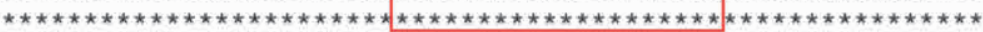

14 a4 CTGGCCCATGCGTACTTCCCTGGTCACGGCATCGGAGGAGACACACATTTTGATGAAGCA seq1 CTGGCCCATGCGTACTTCCCTGGTCACGGCATCGGAGGAGACACACATTTTGATGAAGCA

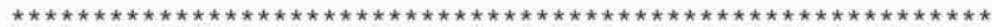

14 a4 GAGCCTTGGACAACAGGCAACGTTGACAAGGGAGgtgaggaagatgatctagaattgtgt seq1 GAGCCTTGGACAACAGGCAACGTTGACAAGGGAGGTGAGGAAGATGATCTAGAATTGTGT

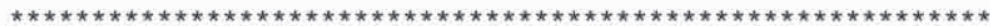

$14 \mathrm{a} 4$ ctagggt

seq 1 CTAGGGTAATCTACCGCCCGATCGTTCCGTTCGGGGGAGGTGTCAGGAATCAGGACACAC

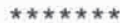

$\mathbf{E}$

14b4 AAGTGTGGGAAAGCGTTACGCCTTTGCGATTCAGAGAGATCTCTTATAGTGACATCAGGG seq2 ----GGGGGGCTTCGGCGCTTGGC---- TCACGGCTCGGGA $\star * \star \ldots . . . * * \star * *$ ** $\quad * \ldots{ }^{*}{ }^{*}{ }^{* *} . * *$.

$14 \mathrm{~b} 4$ ACAAGGTTGTAGACTTTGCAGACATCATGCTCT-TCTTCGCTGATGGCTTTCATGGCGAT seq2 CTGG--------CGCAGACATCATGCTCTCTCTTCGCTGATGGCTTTCAT GGCGAT

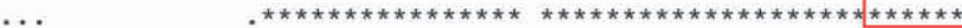

$14 \mathrm{~b} 4$ GCCAGTCCATTTGATGGAGAAGGAGGTTTCTTGGCCCATGCTTACTTCCCAGGGATGGC seq2 GCCAGTCCATTTGATGGAGAAGGAGGTTTCTTGGCCCATGCTTACTTCCCAGGGAATGGC

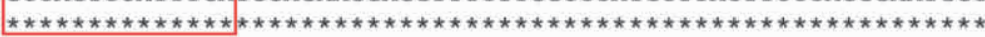

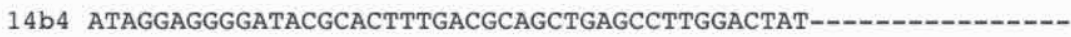
seq2 ATAGGAGGGGATACGCACTTTGACGCAGCTGAGCCTTGGACTAATGACCCCCTGCATGAC

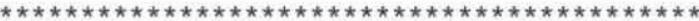
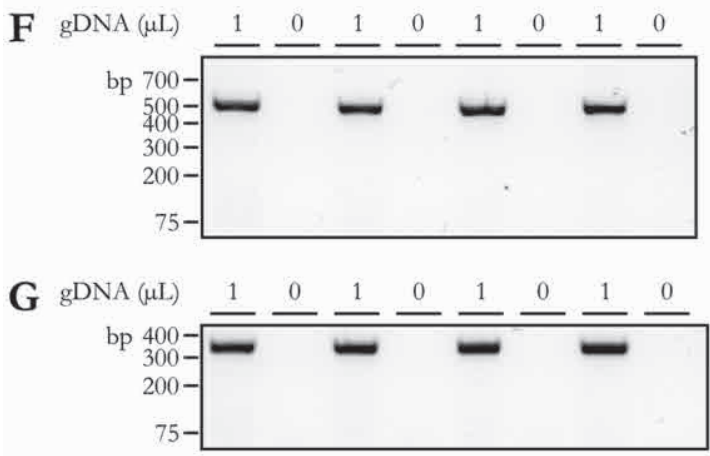

Supplemental Figure 3.2 (continued). 

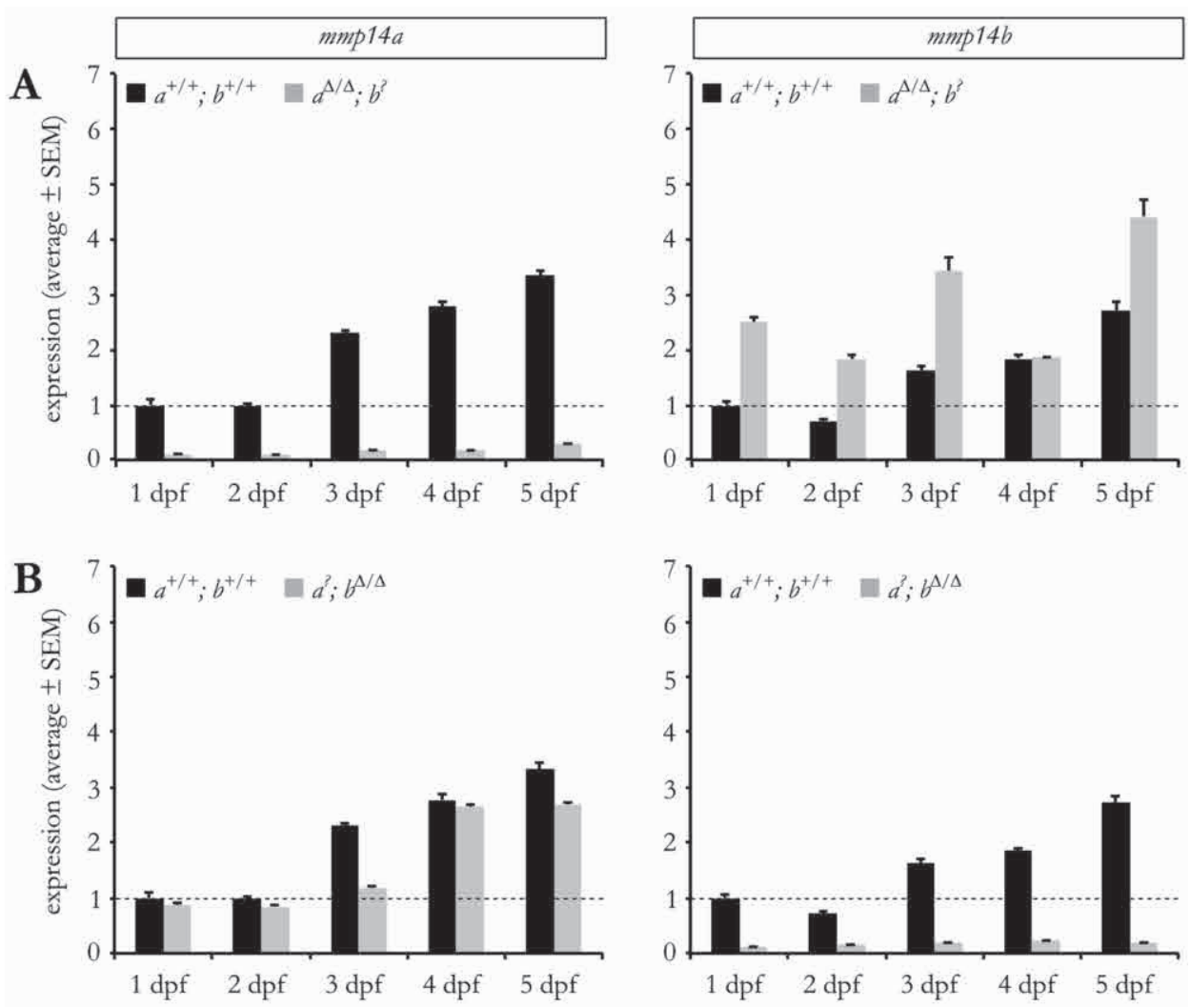

Supplemental Figure 3.3 - Analysis of WT mmp14a/b mRNA during early larval development of offspring of $m m p 14 a^{+/ \Delta}$; mmp14b $14 / \Delta$ or $m m p 14 a^{\Delta / \Delta}$; mmp $14 b^{+/ \Delta}$ parents. A-B, mRNA expression levels for mmp14a (left) and mmp14b (right) of 1-5 dpf offspring of $m m p 14 a^{\Delta / \Delta} ; m m p 14 b^{+/ \Delta}$ (A) or $m m p 14 a^{+/ \Delta} ; m m p 14 b^{\Delta / \Delta}$ (B) intercrossed (grey bars) compared to WT larvae (black bars), normalised for $\beta$ actin expression and denoted relative to $1 \mathrm{dpf}$ WT embryos (dotted line). mRNA levels of mutant mmp14a and mmp14b are very low, suggestive of nonsense-mediated mRNA decay. Note the upregulation of

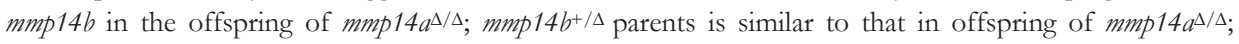
mmp14b ${ }^{+/+}$parents (Fig. 4.9). 

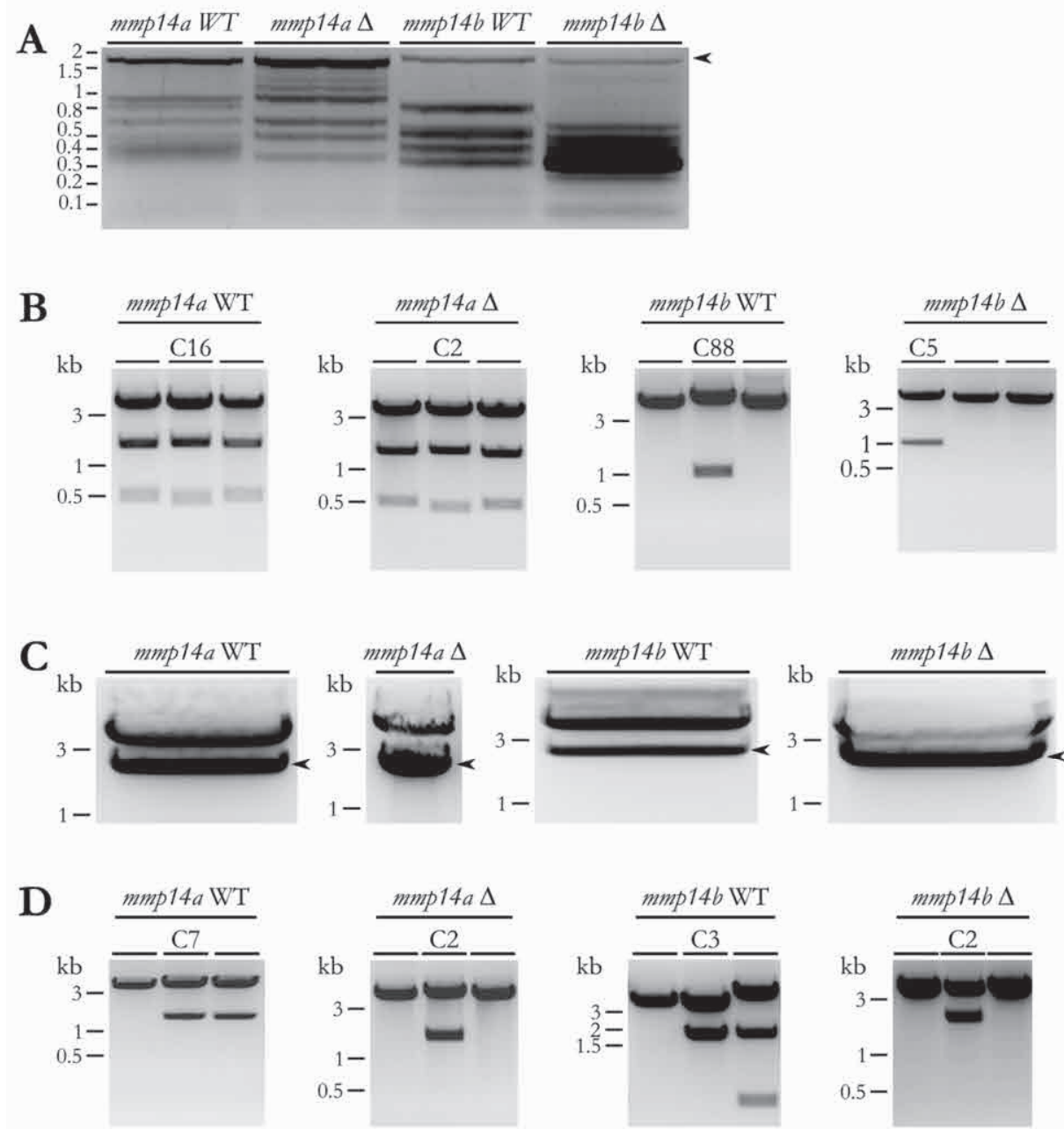

Supplemental Figure 3.4 - Generating WT and mutant Mmp14a/b-HA expression plasmids. A, DNA gel electrophoresis results of PCR amplified mmp14a/b coding sequences from WT and mutant cDNA template. The largest amplicon per lane corresponding to the full-length cDNA of $m m p 14 a(1754$ bp for WT) and mmp14b (1895 bp for WT) with kozak sequence and HA-tag coding sequence (arrowhead) was excised, column purified and cloned into the pCR2.1 TOPO TA vector. B, DNA gel electrophoresis result of miniprepped pCR2.1 TOPO TA vectors with WT or mutant mmp14a-HA insert digested with EcoRV and HindIII, or WT or mutant mmp14b-HA insert digested with HindIII. Plasmids with correct restriction fragment size (3,808 bp, 1,401 bp and 445 bp bands for WT mmp14a; 4,964 bp and 831 bp for W'T mmp14b) were sent for Sanger sequencing. C, DNA gel electrophoresis result of EcoRI linearised plasmids with correct sequence insert sequence (C16, C2, C88 and C5). The insert (lower band, arrowhead) was cut out, column purified and ligated into EcoRI-linearised pCS2+ backbone. D, DNA gel electrophoresis result of WT/mutant pCS2+ Mmp14/b-HA plasmids, digested with EcoRV and HindIII (mmp14a) or KpnI and AfeI (mmp14b). Plasmids with correct restriction fragments (4,443 bp and 1,406 bp for WT mmp14a; 4,144 bp and 1,846 bp for W'T mmp14b) were sent for sequencing. Plasmids with correct sequence were used for further experiments. 

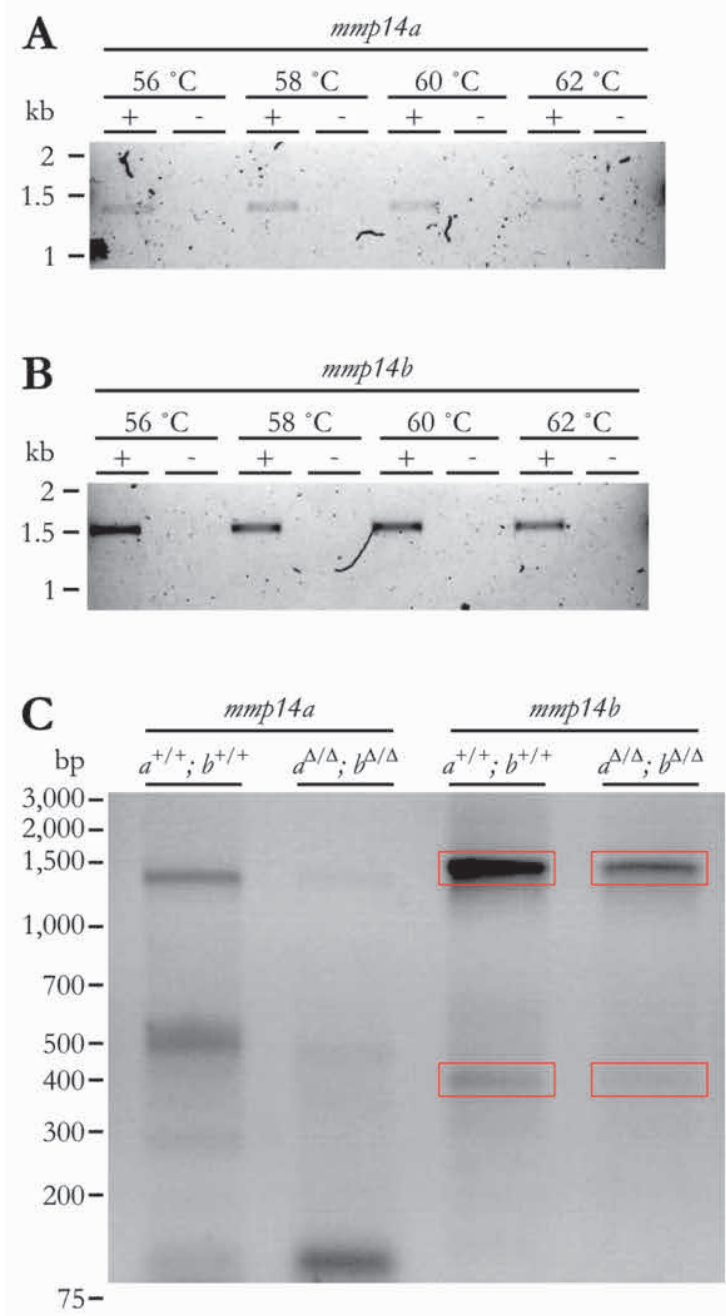

Supplemental Figure 3.5 - Alternative splicing of mmp $14 b$ mRNA does not encode a catalytically active form of Mmp14b. A-B, DNA gel electrophoresis result of optimisation of primer pairs for amplification (and subsequent sequencing) of mmp14a (A) and mmp14b (B) cDNA. Both primer pairs were tested in the presence and absence of purified gDNA of an adult WT zebrafish at different annealing temperatures; only a single concentration of $\mathrm{MgCl}_{2}(2 \mathrm{mM})$ was used. A single amplicon band at the expected height of $1326 \mathrm{bp}$ (A) and $1458 \mathrm{bp}$ (B) for full-length mmp14a respectively mmp14b cDNA can be seen in all reactions with gDNA template present. C, DNA gel electrophoresis result of amplification products of WT and $m m p 14 a^{\Delta / \Delta} ; m m p 14 b^{\Delta / \Delta}$ cDNA. In WT fish, there are two mmp14a and mmp14b cDNA amplicons. Both mmp14a bands can hardly be detected in mutant fish, whereas the mmp14b bands can be clearly observed. The mmp14b cDNA amplicons of both WT and mutant fish (red boxes) were excised, column purified and sent for direct Sanger sequencing with the forward primer. The top band corresponds to the full-length mmp14b cDNA sequence, which in mutant fish contains the expected 8 bp deletion in exon 4 . The smaller amplicon corresponds to exons 1-4, ends upstream of the CRISPR target site in both WT and mutant fish (chromatography reads not shown). 


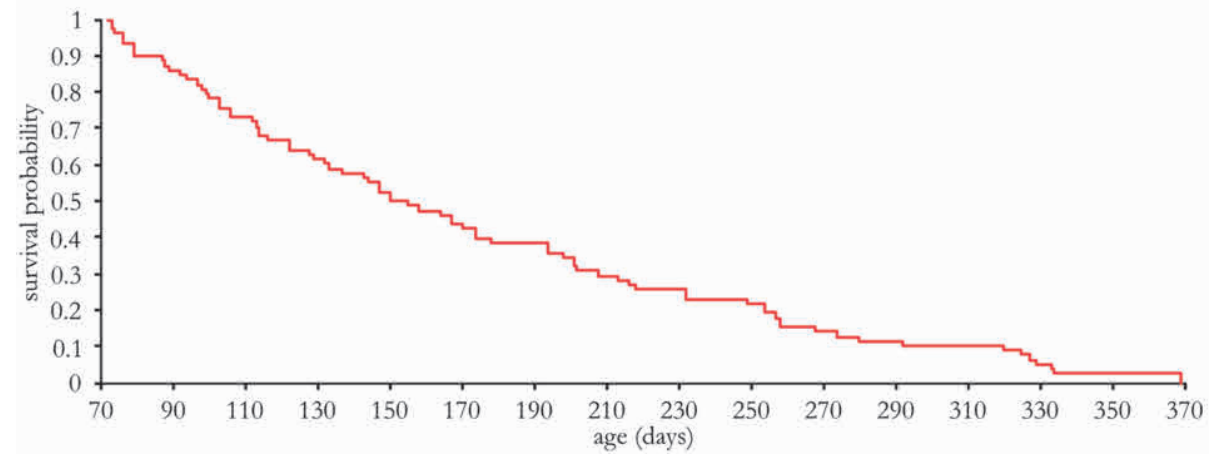

Supplemental 3.6 - Kaplan-Meier survival curve of mmp14a/b KO fish. Survival curve for 79 mmp14a/b KO fish that lived longer than $72 \mathrm{dpf}$.

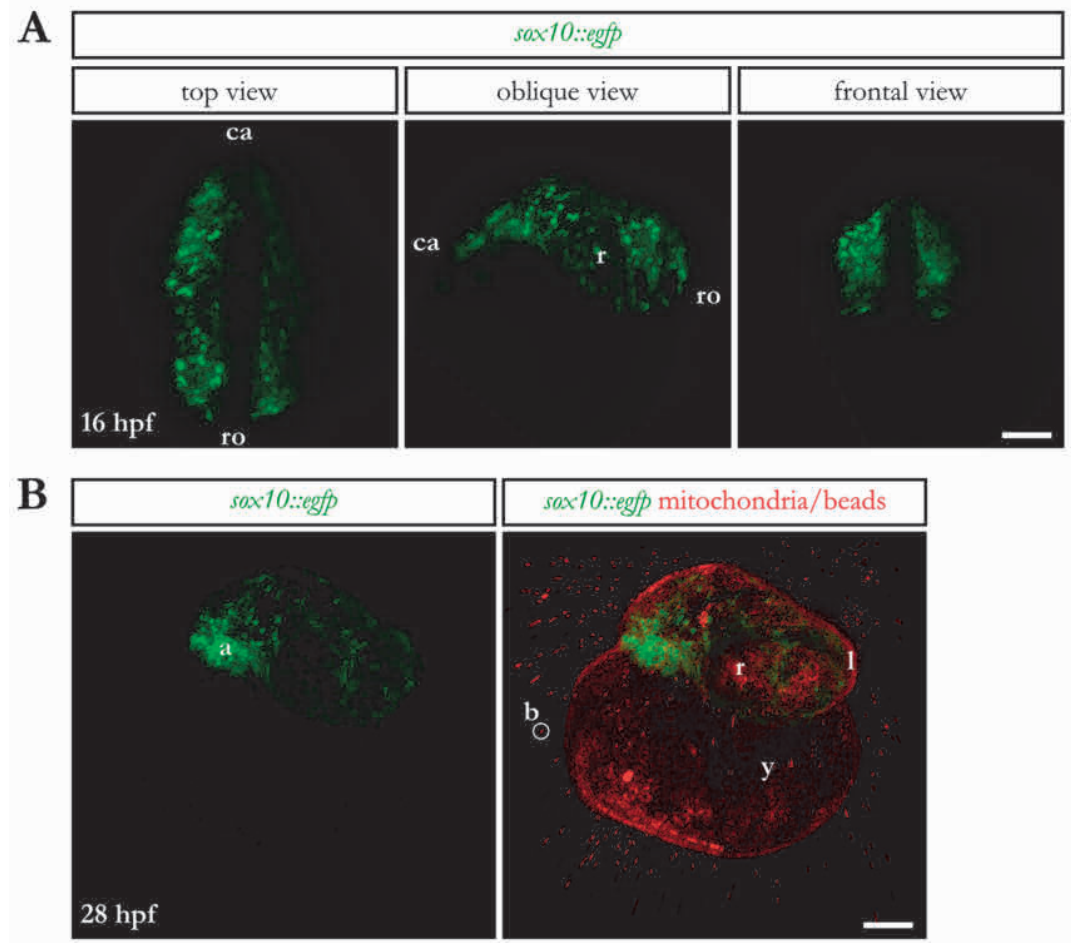

Supplemental Figure 3.7 - Live imaging of neural crest cells in zebrafish embryos/larvae. 3D reconstruction of light sheet fluorescence microscopy z-stacks of cranial NC cells in $\operatorname{Tg}(\operatorname{sox} 10::$ eg $f$ ) embryos/larvae. A, at $16 \mathrm{hpf}$, migrating cranial NC cells have not yet reached the pharyngeal arches. B, at $28 \mathrm{hpf}$, cranial NC cells can be observed in the pharyngeal arches (a) as a condensation on the ventrolateral surface of the head, close to the yolk ball (y). Note that $30 \mathrm{~min}$ incubation with MitoTracker ${ }^{\circledR}$ Red CMXRos stained the outermost layer of the embryo and the yolk ball. Red fluorescent beads were embedded in the agarose column for stitching of 3D stacks imaged from different angles. C, at $78 \mathrm{hpf}$, cranial NC cells contribute to the developing craniofacial cartilage structures, as well as the olfactory pit (op; ventral view). Overnight incubation with MitoTracker ${ }^{\circledR}$ Red CMXRos resulted in bright staining of the outline of the 
Addendum
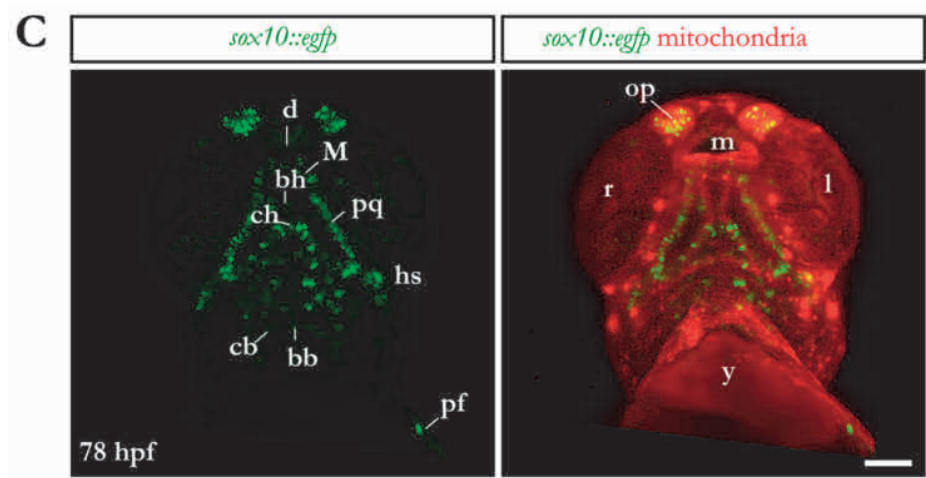

D

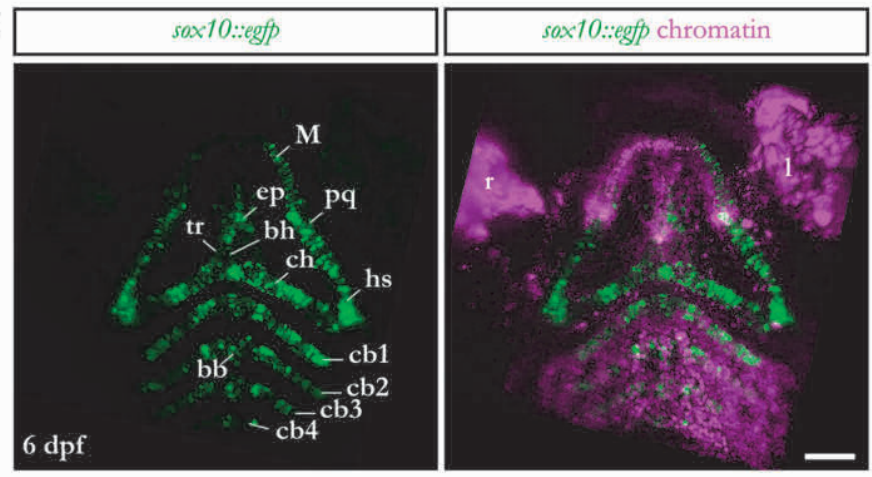

Supplemental Figure 3.7 (continued) - larva, but failed to penetrate beyond the epidermis. D, at $6 \mathrm{dpf}$, the fluorescent cranial NC cells clearly demarcated craniofacial cartilage structures (ventral view). DRAQ5TM penetrated very well and labelled nuclei throughout the larval head. b, bead embedded in agarose column; bb, basibranchial; bh, basihyal; ca, caudal; cb, ceratobranchial; ch, ceratohyal; d, diencephalon; ep, ethmoid plate; hs, hyosymplectic; l, left eye; m, mouth; Meckel's cartilage; pf, pectoral fin; pq, palatoquadrate; r, right eye ro, rostral; tr, trabeculae. Scale bars equal $100 \mu \mathrm{m}$.

\section{References}

1. Evans BR, Mosig RA, Lobl M, Martignetti CR, Camacho C, Grum-Tokars V, et al. Mutation of membrane type-1 metalloproteinase, MT1-MMP, causes the multicentric osteolysis and arthritis disease Winchester syndrome. American journal of human genetics. 2012;91(3):572-6.

2. von Heijne G, Gavel Y. Topogenic signals in integral membrane proteins. European Journal of Biochemistry. 1988;174(4):671-8.

3. Kyte J, Doolittle RF. A simple method for displaying the hydropathic character of a protein. Journal of Molecular Biology. 1982;157(1):105-32.

4. Petersen TN, Brunak S, von Heijne G, Nielsen H. SignalP 4.0: discriminating signal peptides from transmembrane regions. Nature Methods. 2011;8(10):785-6.

5. von Heijne G. Patterns of amino acids near signal-sequence cleavage sites. European Journal of Biochemistry. 1983;133(1):17-21.

6. Huschtscha LI, Holliday R. Limited and unlimited growth of SV40-transformed cells from human diploid MRC-5 fibroblasts. Journal of Cell Science. 1983;63:77-99. 



\section{CHAP'TER 7}

Valorisation 



\section{The relevance of a diagnosis}

In this thesis, we identified a novel homozygous mutation in MMP14 in two patients with a multisystem disorder (see Chapter 1) and subsequently confirmed its pathogenicity at the cellular level (see Chapter 2). This enabled us to provide the patients with a final diagnosis: Winchester syndrome (WS). Although this diagnosis does not alter the therapy that the patients are currently receiving, it is nevertheless important for several reasons. Firstly, it becomes possible to provide the patients with adequate information about their disorder and the underlying cause. This not only helps the patients in understanding of, but may also improve coping with their symptoms [1, 2]. Secondly, a diagnosis can focus additional examinations based on tissues and organ systems known to be involved in the particular disorder. Third, as WS is an autosomal recessive disorder, confirming the molecular diagnosis provides the opportunity to offer more precise genetic counselling to the patients (and partners), informing them about inheritance and the potential risk to their future offspring [1].

\section{The value of investigating rare genetic disorders}

WS is an ultra-rare autosomal recessive disorder; the molecular diagnosis has only been confirmed in four patients worldwide [3-5, see Chapter 1]. The clinically overlapping and genetically related skeletal dysplasias Frank-Ter Haar syndrome (FTHS) and multicentric osteolysis, nodulosis, and arthropathy (MONA) only add a limited number of patients [6-9]. Spending significant amounts of time, effort and taxpayer's money on investigating these rare disorders may, therefore, seem only to benefit a handful of affected patients. However, the power of studying rare monogenetic disorders lays not so much in the number of patients with that exact diagnosis, but rather in the unique opportunity the direct genotype-phenotype correlation offers to study more common pathological processes from a unique and yet unexplored angle. This approach has previously been demonstrated successful and has contributed to major advanced in various fields. For instance, many genes now known to regulate osteoblast and osteoclast differentiation and function, including Runx2, Msx2, Sox9, FGFR1-3, and cathepsin K, were first identified by studying monogenetic disorders [10]. Moreover, studying rare monogenetic disorders can lead to the identification of novel therapeutic targets. By this, studying rare genetic disorders can be beneficial to patients suffering from more prevalent disorders [5].

\section{Winchester syndrome as a model for acne and reduced bone density}

WS is a skeletal dysplasia characterised by a progressive generalised reduction of bone density, thoracic kyphosis, craniofacial dysmorphology and severe nodulocystic acne with dermal fibrosis and excessive scar formation [3, 4]. We are therefore convinced that WS can accurately model reduced bone density and acne (scarring). As mentioned 
above, there is significant clinical overlap between WS, FTHS, and MONA [6-9]. The protein products of the involved genes MMP14, SH3PXD2B, and MMP2, respectively, cooperate in extracellular matrix (ECM) remodelling and invasive cell motility (see Chapter 1) [9, 11-15]. As such, this led to innovative lines of research studying the pathogenesis of acne and reduced bone density from a novel angle.

\section{Acne affects a significant part of the population and novel therapeutic options are needed}

Acne vulgaris is the most common skin disorder in the world and one of the most common ailments overall. In developing countries, it affects almost 100\% of adolescents [16]. Although usually mild and self-limiting, it is a chronic disease that is typically present for many years. In addition, acne is moderate-to-severe in $15-20 \%$ of patients and often persists into adulthood, with an estimated prevalence of $12 \%$ in adult females [17]. Apart from physical symptoms that are present while acne is fulminant, including soreness, itching, and pain, moderate-to-severe acne can leave disfiguring scars $[16,18]$. Furthermore, acne can have a large impact on the patient's quality of life and is often associated with significant psychosocial morbidity such as selfconsciousness and anxiety in social interaction, even when the acne itself is comparatively mild [18]. This psychological burden can be explained by the involvement of directly visible skin and occurance during puberty, an age that is crucial for building confidence and self-esteem, while popular culture furthermore emphasises the importance of flawless skin [18]. The psychosocial comorbidity moreover has been demonstrated to have a negative impact on school and work performance. In the US alone, it was estimated that annually over 3 billion dollars are lost to the costs of treatment and reduced productivity [16].

Although mild acne can be effectively addressed with topical drugs, moderate-tosevere disease requires systemic therapy to prevent excessive scar formation [18]. However, current treatment options are limited and their use restricted by their side effects $[19,20]$. No novel drug classes are being developed for moderate-to-severe acne since there is a profound lack of insight into acne pathogenesis [21, 22]. As such, there is a significant unmet medical need.

\section{In vitro $3 D$-culture for development of novel anti-acne therapeutics}

As stressed above, novel anti-acne therapeutics need to be developed. Acne is characterised by the presence of comedones, which are cystic sebaceous glands, accompanied by inflammation around these structures [18]. The sebaceous gland is in essence a hollow, branched mini-organ. For normal morphogenesis of branching structures, ECM remodelling is essential [23]. Our WS patients demonstrated defective ECM remodelling and moreover, have a loss-of-function mutation in MMP14 (see Chapter 
2), a central player in pericellular ECM degradation [24]. This urged us to explore the possible involvement of disturbed lumen formation, as a consequence of impaired ECM remodelling, in the pathogenesis of acne. In a pilot study, we used a simple, wellestablished in vitro 3D cell culture model to confirm a crucial role of MMP14 and SH3PXD2B in lumen formation by epithelial cells [25]. Moreover, we demonstrated that therapeutic plasma concentrations of 13-cis RA have a stimulatory effect on lumen formation (see Chapter 3). The latter further highlighted the suitability of this in vitro model for assessing the effects of drug treatment. These promising results led to two subsequent studies. Firstly, as part of a multi-million dollar industry collaboration with a major international cosmetics producer, the in vitro model used in our pilot study was further developed for use with sebocytes. As this was successful, this novel sebocyte 3D model was subsequently used to assess the effects of existing (e.g. 13-cis RA) and novel drugs on organoid formation and sebum production. Secondly, collaboration was started with the University of Dundee Drug Discovery Unit. This latter project highlighted the potential of this in vitro model: upscaling with semi-automated analysis for drug screening, as reported before [25].

\section{The zebrafish is a valuable model organism for skin research}

The aforementioned in vitro 3D cell culture model offers the possibility to study lumen formation and the effect of prospective therapeutic compounds thereon. However, other aspects of acne, such as scarring, are hard - if not impossible - to model in vitro. To study these aspects, ultimately an organismal model is needed. Although MMP14 mutant and knockout (KO) mice have been previously generated, mice are unsuitable for drug screening for a variety of reasons, such as high cost and the consequent inability to analyse large numbers of animals. A more suitable model organism that does not have these limitations is the zebrafish $[26,27]$. Besides its use in genetic studies, the zebrafish is a powerful model organism for skin research. Adult zebrafish have dermal scales that are covered by a non-keratinizing epidermis. In addition, zebrafish do not have sebaceous glands, but instead harbour mucous glands [28, 29]. Apart from these adaptations to aquatic life, the architecture of zebrafish skin is remarkably similar to that of mammals. Similar to humans, zebrafish epidermis consists of multiple cell layers that moreover correspond to their human counterparts including the stratum basale, spinosum and granulosum [28, 29]. The epidermis is separated from the underlying dermis, containing the scales, by a basement membrane zone. Another attractive feature of zebrafish is their rapid external embryonic development; zebrafish form a fully functional multi-layered skin in only six days [29]. During this process, zebrafish are optically transparent and hence suitable for live in vivo microscopy experiments. Moreover, wound healing in zebrafish skin follows a similar basic sequence of events compared to mammalian skin [29, 30]. The causative gene of WS is conserved between human and zebrafish and in this thesis, we presented the first mmp14a/b KO zebrafish 
model (see Chapter 4). We furthermore demonstrated that the phenotype of our fish recapitulates key aspects of the human pathology, suggestive of defective collagen remodelling as the underlying central problem. Therefore, we believe our fish can serve as a useful in vivo model for cutaneous tissue remodelling and scarring and designed a novel project to be carried out by the A*STAR Skin Research Institute of Singapore in collaboration with the NTU Lee Kong Chian School of Medicine. Within this project, which will commence in September 2018, collagen remodelling will be assessed during cutaneous wound repair in our mutant fish. Subsequently, this will be used as readout for the effect of different commercially available and newly generated therapeutics. As such, this model may lead to the development of novel strategies targeting acne scarring.

\section{Reduced bone density is an increasing problem}

The skeletal dysplasias, including the multicentric osteolyses WS, FTHS, and MONA, are a heterogeneous group of over 450 inheritable disorders characterised by a generalised abnormal growth and development of bone and cartilage [31, 32]. Although the individual dysplasias are rare, a recent study conducted in South America analysing over 1.5 million births demonstrated that collectively the prevalence of skeletal dysplasia is 3.2 per 10,000 births [32]. A large number of skeletal dysplasias, including WS, present with reduced bone density [33]. In the general population, osteoporosis (defined by bone mineral density more than 2.5 standard deviations below the average of a young adult) is an increasing worldwide problem [34]. In the Netherlands, the prevalence of osteoporosis has been estimated to encompass 431,400 people in 2015, the majority of which are postmenopausal women [35]. In this subgroup, about $40 \%$ is affected by osteoporosis [36]. A reduction in bone density increases the risk of fractures: patients with osteoporosis have a lifetime risk for fractures of $40 \%$, primarily of the spine, hip and wrist $[34,36]$. These fractures often result in chronic pain and/or loss of mobility and autonomy, and can have a drastic effect on the patient's quality of life $[34,36]$. In addition, fractures of the hip and spine are associated with a significant mortality as a result of complications due to hospitalisation [36]. In 2010, the annual health care costs of osteoporosis-related fractures in the Netherlands amounted to nearly 200 million euros [34]. As the prevalence of osteoporosis increases with age and the Dutch population is ageing, the number of osteoporosis-related fractures and accompanying costs are expected to increase in the future $[35,36]$. Although present therapies are efficient, most have side effects limiting their long-term adherence [36]. Therefore, development of novel therapeutics is needed. 


\section{Zebrafish are invaluable in bone research}

For the development of drugs targeting bone density, an organismal model is needed. The zebrafish is a well-established model organism for vertebrate development. Zebrafish form all major types of skeletal cells and tissues found in higher vertebrates including humans [37-39]. Furthermore, bone formation and remodelling is similar in zebrafish and humans, and the pathways regulating these processes are well conserved [37, 39-41]. As such, the zebrafish is a good model organism for human bone disorders. Our zebrafish model of WS had an overt skeletal phenotype that included decreased bone density. Therefore, we believe our WS zebrafish model can be used for testing novel therapeutic strategies aimed at improving skeletal development and increasing bone density. As part of the planned project mentioned above, our mutant fish will serve as a model to test the effect on bone development and bone density of existing therapeutics currently not used for this purpose. This collaboration will enable the development of novel therapeutic strategies addressing two common conditions. Due to its focus on tissue remodelling and invasion, there is considerable potential for spin-off into other common pathological conditions such as scarring and cancer. In addition, the workflow developed in the program can be applied to other (skin) disorders.

\section{Conclusion}

In conclusion, studying the molecular basis of rare disorders such as Winchester syndrome is important for two main reasons. Firstly, providing the affected patients with a definite diagnosis is important to improve coping with their symptoms, focussing clinical follow-up examinations and offering genetic counselling. Secondly, rare disorders can provide novel insights into more common pathologies. As such, the models used and developed in this thesis have been or will be subsequently used in academic and industry collaborations to develop novel therapeutic strategies for two common disorders: acne vulgaris and reduced bone density.

\section{References}

1. Schrander-Stumpel CTRM, De Nijs Bik H. In: Schrander-Stumpel CTRM, Curfs LMG, Van Ree JW. Klinische genetica: Bohn Stafleu van Loghum; 2005.

2. Veening EP, Gans ROB, Kuks JBM. Medische consultvoering: Bohn Stafleu van Loghum; 2009.

3. Winchester P, Grossman H, Lim WN, Danes BS. A new acid mucopolysaccharidosis with skeletal deformities simulating rheumatoid arthritis. The American Journal of Roentgenology, Radium Therapy, and Nuclear Medicine. 1969;106(1):121-8.

4. Van Steensel MA, Ceulen RP, Delhaas T, de Die-Smulders C. Two Dutch brothers with Borrone dermato-cardio-skeletal syndrome. American Journal of Medical Genetics Part A. 2007;143a(11):1223-6. 
5. Evans BR, Mosig RA, Lobl M, Martignetti CR, Camacho C, Grum-Tokars V, et al. Mutation of membrane type-1 metalloproteinase, MT1-MMP, causes the multicentric osteolysis and arthritis disease Winchester syndrome. American Journal of Human Genetics. 2012;91(3):572-6.

6. Frank Y, Ziprkowski M, Romano A, Stein R, Katznelson MB, Cohen B, et al. Megalocornea associated with multiple skeletal anomalies: a new genetic syndrome? Journal de Genetique Humaine. 1973;21(2):67-72.

7. Ter Haar B, Hamel B, Hendriks J, de Jager J. Melnick-Needles syndrome: indication for an autosomal recessive form. American Journal of Medical Genetics. 1982;13(4):469-77.

8. Maas SM, Kayserili H, Lam J, Apak MY, Hennekam RC. Further delineation of Frank-ter Haar syndrome. American Journal of Medical Genetics Part A. 2004;131(2):127-33.

9. Zankl A, Pachman L, Poznanski A, Bonafe L, Wang F, Shusterman Y, et al. Torg syndrome is caused by inactivating mutations in MMP2 and is allelic to NAO and Winchester syndrome. Journal of Bone and Mineral Research. 2007;22(2):329-33.

10. Mosig RA, Dowling O, DiFeo A, Ramirez MC, Parker IC, Abe E, et al. Loss of MMP-2 disrupts skeletal and craniofacial development and results in decreased bone mineralization, joint erosion and defects in osteoblast and osteoclast growth. Human Molecular Genetics. 2007;16(9):1113-23.

11. Martignetti JA, Aqeel AA, Sewairi WA, Boumah CE, Kambouris M, Mayouf SA, et al. Mutation of the matrix metalloproteinase 2 gene (MMP2) causes a multicentric osteolysis and arthritis syndrome. Nature Genetics. 2001;28(3):261-5.

12. Iqbal Z, Cejudo-Martin P, de Brouwer A, van der Zwaag B, Ruiz-Lozano P, Scimia MC, et al. Disruption of the podosome adaptor protein TKS4 (SH3PXD2B) causes the skeletal dysplasia, eye, and cardiac abnormalities of Frank-Ter Haar Syndrome. American Journal of Human Genetics. 2010;86(2):254-61.

13. Wilson GR, Sunley J, Smith KR, Pope K, Bromhead CJ, Fitzpatrick E, et al. Mutations in SH3PXD2B cause Borrone dermato-cardio-skeletal syndrome. European Journal of Human Genetics. 2014;22(6):741-7.

14. Murphy DA, Courtneidge SA. The 'ins' and 'outs' of podosomes and invadopodia: characteristics, formation and function. Nature Reviews Molecular Cell Biology. 2011;12(7):413-26.

15. Buschman MD, Bromann PA, Cejudo-Martin P, Wen F, Pass I, Courtneidge SA. The novel adaptor protein Tks4 (SH3PXD2B) is required for functional podosome formation. Molecular Biology of the Cell. 2009;20(5):1302-11.

16. Bhate K, Williams HC. Epidemiology of acne vulgaris. The British Journal of Dermatology. 2013;168(3):474-85.

17. Goulden V, Stables GI, Cunliffe WJ. Prevalence of facial acne in adults. Journal of the American Academy of Dermatology. 1999;41(4):577-80.

18. Williams HC, Dellavalle RP, Garner S. Acne vulgaris. Lancet. 2012;379(9813):361-72.

19. Humphrey S. Antibiotic resistance in acne treatment. Skin Therapy Letter. 2012;17(9):1-3.

20. Tan J, Boyal S, Desai K, Knezevic S. Oral Isotretinoin: New Developments Relevant to Clinical Practice. Dermatologic Clinics. 2016;34(2):175-84.

21. Dawson AL, Dellavalle RP. Acne vulgaris. BMJ. 2013;346:f2634.

22. Simpson RC, Grindlay DJ, Williams HC. What's new in acne? An analysis of systematic reviews and clinically significant trials published in 2010-11. Clinical and Experimental Dermatology. 2011;36(8):840-3; quiz 3-4.

23. Kim HY, Nelson CM. Extracellular matrix and cytoskeletal dynamics during branching morphogenesis. Organogenesis. 2012;8(2):56-64.

24. Sabeh F, Ota I, Holmbeck K, Birkedal-Hansen H, Soloway P, Balbin M, et al. Tumor cell traffic through the extracellular matrix is controlled by the membrane-anchored collagenase MT1-MMP. The Journal of Cell Biology. 2004;167(4):769-81.

25. Giles RH, Ajzenberg H, Jackson PK. 3D spheroid model of mIMCD3 cells for studying ciliopathies and renal epithelial disorders. Nature Protocols. 2014;9(12):2725-31. 
26. Mackay EW, Apschner A, Schulte-Merker S. A bone to pick with zebrafish. BoneKEy Reports. 2013;2:445.

27. Yelick PC, Schilling TF. Molecular dissection of craniofacial development using zebrafish. Critical Reviews in Oral Biology and Medicine. 2002;13(4):308-22.

28. Rakers S, Gebert M, Uppalapati S, Meyer W, Maderson P, Sell AF, et al. 'Fish matters': the relevance of fish skin biology to investigative dermatology. Experimental Dermatology. 2010;19(4):313-24.

29. Cline A, Feldman SR. Zebrafish for modeling skin disorders. Dermatology Online Journal. 2016;22(8).

30. Richardson R, Slanchev K, Kraus C, Knyphausen P, Eming S, Hammerschmidt M. Adult zebrafish as a model system for cutaneous wound-healing research. The Journal of Investigative Dermatology. 2013;133(6):1655-65.

31. Krakow D, Rimoin DL. The skeletal dysplasias. Genetics in Medicine. 2010;12(6):327-41.

32. Barbosa-Buck CO, Orioli IM, da Graca Dutra M, Lopez-Camelo J, Castilla EE, Cavalcanti DP. Clinical epidemiology of skeletal dysplasias in South America. American Journal of Medical Genetics Part A. 2012;158a(5):1038-45.

33. Krakow D. Skeletal dysplasias. Clinics in Perinatology. 2015;42(2):301-19, viii.

34. Lotters FJ, van den Bergh JP, de Vries F, Rutten-van Molken MP. Current and Future Incidence and Costs of Osteoporosis-Related Fractures in The Netherlands: Combining Claims Data with BMD Measurements. Calcified Tissue International. 2016;98(3):235-43.

35. NIVEL Zorgregistraties eerse lijn, NIVEL Zorgregistraties. URL: https://www.volksgezondheid enzorg.info/onderwerp/osteoporose/cijfers-context/huidige-situatie\#bronverantwoording.

36. Rachner TD, Khosla S, Hofbauer LC. Osteoporosis: now and the future. Lancet. 2011;377(9773):1276-87.

37. Apschner A, Schulte-Merker S, Witten PE. Not all bones are created equal - using zebrafish and other teleost species in osteogenesis research. Methods in Cell Biology. 2011;105:239-55.

38. Yelick PC, Schilling TF. Molecular dissection of craniofacial development using zebrafish. Critical Reviews in Oral Biology and Medicine. 2002;13(4):308-22.

39. Hammond CL, Schulte-Merker S. Two populations of endochondral osteoblasts with differential sensitivity to Hedgehog signalling. Development. 2009;136(23):3991-4000.

40. Mackay EW, Apschner A, Schulte-Merker S. A bone to pick with zebrafish. BoneKEy Reports. 2013;2:445.

41. Witten PE, Huysseune A. A comparative view on mechanisms and functions of skeletal remodelling in teleost fish, with special emphasis on osteoclasts and their function. Biological Reviews of the Cambridge Philosophical Society. 2009;84(2):315-46. 



\section{CHAP'TER 8}

\section{Summary}

Samenvatting

Acknowledgements / Dankwoord

Curriculum vitae 



\section{Summary}

In 2007, we reported two brothers with a multisystem disorder encompassing progressive mitral valve insufficiency, osteopenia, thoracic kyphosis, craniofacial dysmorphism, dermal fibrosis, and severe nodulocystic acne [1]. In these patients, we identified a novel homozygous MMP14 c.332G > A missense mutation. This gene encodes matrix metalloproteinase 14, a membrane-bound endopeptidase that primarily cleaves structural components of the extracellular matrix (ECM), which is expressed in various tissues including bone and skin [2-5]. Multiple lines of evidence suggested that this mutation could be pathogenic. As shown in Chapter 1, in silico analysis predicted that the resulting p.R111H substitution would be damaging. Secondly, a homozygous MMP14 p.T17R mutation has previously been reported in patients with a clinical diagnosis of Winchester syndrome (WS), a constellation of features similar to our patients' phenotype [6, 7]. Thirdly, the mouse models Sabe and Cartoon with homozygous MMP14 p.R92C and p.S466P missense mutation, respectively, share many skeletal features with our patients $[8,9]$. Fourthly, there is significant phenotypical overlap between our patients and individuals with Frank-Ter Haar syndrome (FTHS) and multicentric osteolysis, nodulosis, and arthropathy (MONA), which are caused by homozygous loss-of-function mutations in SH3PXD2B respectively MMP2 [10-16]. Importantly, the protein products of these three genes directly cooperate in the formation of podosomes, which are specialised membrane protrusions involved in ECM remodelling and invasive cell motility [17-20]. Together, this evidence led us to diagnose our patients with Wichester syndrome, pending confirmation of the p.R111H mutation's pathogenicity. In this thesis, we aimed to elucidate how mutation of MMP14 results in the WS phenotype.

In Chapter 2, we first assessed how our novel p.R111H mutation, and previously reported human and murine mutations, affect MMP14. MMP14 is synthesised as a latent pre-proenzyme, whose activity is controlled by different processes, including proteolytic activation of the zymogen and exposure at the cell surface [2, 3, 21-24]. To reach the plasma membrane, the full-length zymogen contains an amino-terminal signal peptide (SP) for its insertion into the endoplasmatic reticulum (ER) membrane during translation $[2,3]$. Next to the SP lies a prodomain that keeps the adjacent catalytic domain inactive [2, 3, 21, 25-27]. After ER insertion, pro-MMP14 is activated by sequential cleavage of its $\mathrm{N}$-terminus by MMPs and proprotein convertases (PCs), and is subsequently trafficked to the plasma membrane [22, 28-32]. The abovementioned mutations are all present at sites that are thought to be crucial for normal activation and/or trafficking of MMP14: the SP (p.T17R), PC recognition motifs (p.R92C and p.R111H), and the hemopexin-like (Hx) domain (p.S466P) [2, 3, 7-9, 28-30, 33-35]. To assess the effect of these mutations on MMP14 processing, trafficking, and activity, we developed a novel in vitro model consisting of fibroblasts expressing either wild type 
(W'T) or mutant MMP14 with two different tags at their amino respectively carboxyl terminus. As expected, the full-length WT fusion protein was sequentially processed along the biosynthetic-exocytotic pathway and finally localised at the cell surface as a functionally active enzyme. We provided the first direct evidence for MMP14 SP removal in the ER in vitro. The p.T17R mutation impaired ER insertion and subsequent processing and trafficking of MMP14. In contrast, MMP14 R111H was processed and trafficked seemingly normally. However, the p.R111H mutation partially impaired MMP14's catalytic activity and its stimulatory effect on cell migration in vitro, suggesting $\mathrm{R}^{111}$ is a crucial residue for pro-MMP14 activation. It is possible that the impaired activity is caused by a subtle aberration in pro-domain cleavage that we were unable to detect by Western blot. Compared to the patients originally reported by Winchester et al., our patients had a relatively mild phenotype, correlating best with mutant MMP14's ability to activate pro-MMP2 in vitro [6]. Our results thus confirmed the pathogenicity of the novel hypomorphic MMP14 allele, causing a mitigated form of WS. Compared to WS patients, the Mmp14 mutant mice have a more severe phenotype. In line with this, the p.R92C mutation dramatically impaired MMP14's intracellular trafficking and activity in vitro. This implies a more important role for the $\mathrm{R}^{89}-\mathrm{R}-\mathrm{P}-\mathrm{R}-\mathrm{C}^{93} \mathrm{PC}$ recognition motif in MMP14 activation and subsequent trafficking than previously thought $[23,31,36]$. Although the Sabe and Cartoon phenotype are virtually indistinguishable and the respective mutations impaired MMP14's trafficking and pro-MMP2 cleavage, MMP14 S466P retained its ability to digest gelatin and stimulate cell motility [8, 9]. A possible explanation for this discrepancy is that in contrast to MMP14 R92C, some MMP14 S466P still makes it to the plasma membrane, leaving its functions that do not require homodimerisation intact [37-40]. Homodimerisation might be impaired by a dose effect, or by a direct effect of the p.S466P mutation on the Hx domain [41]. In addition, the p.S466P mutation might alter MMP14's substrate specificity, enhancing gelatin digestion, but not pro-MMP2 cleavage. Taken together, we demonstrated that these four mutations each impair MMP14 functional activity in a unique manner, resulting in a similar clinical phenotype. We subsequently used $3 \mathrm{D}$ in vitro and in vivo models to assess how the loss of MMP14 function resulted in specific aspects of the WS phenotype.

One aspect of the WS and FTHS phenotype of our particular interest is acne $[1,6,10$, $11,42]$. Although it is well known that acne is characterised by cystic sebaceous glands i.e. comedones, it is unknown why such cystic changes occur. The sebaceous gland is, in essence, a hollow, branched epithelial structure that is separated from the type I collagen-rich dermis by a basement membrane [43]. Morphogenesis of such structures generally relies on de novo lumen formation and branching morphogenesis [44]. Planar cell polarity (PCP) and ECM remodelling play a crucial role in these two processes and when disturbed, can result in cyst formation [45-48]. Interestingly, multiple cell types 
depend on MMP14's catalytic activity for branching morphogenesis, and on cell surface localisation of MMP14 for normal PCP [49-52]. Therefore, we hypothesised that disrupted luminogenesis and branching morphogenesis of the sebaceous gland due to impaired function of MMP14 or, given their direct functional link outlined above, SH3PXD2B, could underly comedogenesis. As the acne in our patients was successfully treated with 13-is retinoic acid, we hypothesised that this drug could correct the putative defective branching morphogenesis [1]. In Chapter 3, we conducted a pilot study to assess the effects of Mmp14 or $S h 3 p x d 2 b$ knockdown (KD) and retinoid treatment on luminogenesis and branching morphogenesis of epithelial cells in vitro $[53,54]$. We demonstrated that KD of either of these genes impaired lumen formation in reconstituted basement membrane (Matrigel ${ }^{\mathrm{TM}}$ ) and reduced branching morphogenesis in type I collagen gel, whereas retinoid treatment directly stimulated both processes. The disturbed luminogenesis upon KD of Mmp14 or $S h 3 p x d 2 b$ is suggestive of impaired PCP [52]. In addition, the observed shortened tubule length in crosslinked type I collagen matrix suggests that KD of Mmp14 or $S h 3 p x d 2 b$ impairs ECM degradation $[55,56]$. Finally, the reduced organoid size in type I collagen gel upon KD could additionally be caused by a reduction in cell proliferation. The latter two mechanisms are likely linked, as IMCD cells isolated from Mmp14 KO mice were previously reported to have a proliferative defect that depended on MMP14's collagenolytic activity [57]. The fact that KD of either $M m p 14$ or $S h 3 p x d 2 b$ caused a similar effect in vitro suggests that a shared pathway is affected. Given its role in ECM remodelling, podosome function is the most likely candidate. Retinoids could act through stimulating podosome formation, as we demonstrated that 13-cis retinoic acid stimulated trafficking of MMP14 S466P to podosomes in vitro. This could explain the therapeutic effect of 13 -cis retinoic acid in acne treatment, although additional tissue-specific effects are likely involved $[58,59]$. Future studies should repeat our experiments with sebocytes to assess whether these processes could affect the sebaceous gland. Such sebocyte 3D model could potentially be used in screening for anti-acne therapeutics.

Apart from acne, the WS phenotype is characterised by craniofacial dysmorphism and generalised osteopenia $[1,6]$. The majority of the affected craniofacial skeletal elements are of neural crest $(\mathrm{NC})$ origin, the primary embryonic structure contributing to the development of the face [60-62]. After their induction during embryogenesis, NC cells delaminate and migrate extensively, for which ECM remodelling is essential [60, 61, 63-65]. As MMP14 is involved in ECM remodelling and invasive cell motility, we hypothesised that impaired cranial NC migration underlies the WS craniofacial phenotype $[4,5,21,66]$. To test this hypothesis, and to study the underlying cause of osteopenia in WS, we decided to use a zebrafish model. Zebrafish form the same skeletal tissues as humans, which furthermore develop in a similar manner. This especially holds true for the zebrafish skull bones, the majority of which is of NC origin [67-70]. 
Zebrafish have two well-conserved MMP14 paralogs, Mmp14a and Mmp14b, which are expressed in the head mesenchyme during development [71-76]. The rapid external development and optical transparency of zebrafish embryos furthermore enable live in vivo imaging of NC cells [77-79]. In Chapter 4, we used the CRISPR/Cas9 approach to successfully knock out (KO) both mmp14a and mmp14b in zebrafish. Our mmp14a/b $\mathrm{KO}$ fish recapitulated essential aspects of the WS phenotype, including stunted growth, gradually worsening craniofacial anomalies, hyperkyphosis, osteopenia (albeit limited to the skull), and a shortened lifespan [1, 6]. In contrast to our hypothesis, NC induction, delamination, pharyngeal arch invasion, and differentiation into larval craniofacial cartilage elements were unaffected by mmp14a/b KO. Previously described craniofacial defects in mmp14a of mmp14b morphants are likely off-target effects of the used Morpholino oligonucleotides [74, 75, 80]. In our mmp14a/b KO larvae, skeletal mineralisation during larval-to-juvenile metamorphosis was unaffected [81]. Adult mutant fish showed impaired endochondral/perichondral and intramembranous ossifying bones of both NC and mesodermal origin, further arguing against an NCspecific problem $[68,82]$. Affected bones generally contained relatively little amounts of bone matrix with altered collagen content, clusters of multinucleated cells, and a relatively volumous, disorganised cartilage core. In mice, loss of MMP14 impairs timely cartilage removal during both ossification types and is accompanied by excessive absorption of mineralised bone matrix, resulting in similar bone abnormalities as those observed in our mmp14a/b KO fish [51, 83]. Although it was previously demonstrated in vitro that MMP14 stimulated osteoblast differentiation and inhibited osteoclast differentiation and activation, additional assays should shed light on the exact processes and cell types involved in the observed skeletal phenotype in the mmp14a/ $b$ KO fish [84-86]. In addition, it is still unknown to what extent impaired podosome function is involved in the resulting human, murine and zebrafish phenotype. The generation of various combinations of mmp $14 a / b \mathrm{KO}, \operatorname{sh} 3 p x d 2 b \mathrm{KO}$ and mmp2 KO zebrafish might shed light on how their protein products interact, at least in fish, in bone remodelling. Finally, the newly generated mmp $14 a / b \mathrm{KO}$ fish could be a suitable model for development of novel therapeutics addressing not only the WS bone phenotype, but also low bone density in general. 


\section{Samenvatting}

In 2007 beschreven wij twee broers met een multisysteemaandoening, bestaande uit progressieve mitraalklepinsufficiëntie, osteopenie, thoracale hyperkyfose, craniofaciale dysmorfie, dermale fibrose, en ernstig nodulocysteus acne [1]. Recent toonden wij aan dat deze patiënten homozygoot zijn voor een niet eerder gerapporteerde c.332G>A missense mutatie in MMP14. Dit gen codeert voor matrix metalloproteinase 14, een membraangebonden endopeptidase dat vooral structurele componenten van de extracellulaire matrix (ECM) hydrolyseert en tot expressie komt in diverse weefsels, waaronder de huid en bot [2-5]. Het is om meerdere redenen aannemelijk dat deze mutatie pathogeen is. Zoals getoond in Hoofdstuk 1, voorspelde in silico analyse dat de resulterende p.R111H substitutie schadelijk is. Eerder werd een homozygote MMP14 p.T17R mutatie geïdentificeerd in twee patiënten met Winchester syndroom (WS), die qua fenotype sterk lijken op onze patiënten $[6,7]$. Ten derde hebben de muismodellen Sabe en Cartoon, met respectievelijk homozygote MMP14 p.R92C en p.S466P mutatie, skeletafwijkingen vergelijkbaar met die van onze patiënten $[8,9]$. Ten vierde is er een sterke gelijkenis tussen het fenotype van onze patiënten en dat van mensen met FrankTer Haar syndroom (FTHS) dan wel multicentrische osteolyse, nodulose, en arthropathie (MONA). FTHS en MONA worden veroorzaakt door homozygote mutaties in respectievelijk SH3PXD2B en MMP2 [10-16]. Deze twee genen coderen voor eiwitten die direct samenwerken met MMP14 in de vorming van podosomen, gespecialiseerde membraanuitstulpingen die betrokken zijn bij ECM remodellering en invasieve celmotiliteit [17-20]. Om deze vier redenen hebben wij onze patiënten gediagnosticeerd met WS, hoewel de pathogeniciteit van de MMP14 p.R111H mutatie nog bevestogd moest worden. In dit proefschrift probeerden wij te ontrafelen hoe mutatie van MMP14 leidt tot het WS phenotype.

In Hoofdstuk 2 hebben wij onderzocht of onze nieuw ontdekte p.R111H mutatie daadwerkelijk pathogen is, en hoe deze en eerder gerapporteerde mutaties MMP14 beïnvloeden. MMP14 wordt gesynthetiseerd als een latent pre-proenzym, waarvan de activiteit gereguleerd wordt door verschillende processen waaronder proteolytische activatie van het zymogeen en blootstelling op het celoppervlak [2, 3, 21-24]. Om het plasmamembraan te bereiken bevat pro-MMP14 een signaalpeptide (SP) aan zijn aminoterminus voor insertie in het endoplasmatisch reticulum (ER) membraan tijdens translatie [2, 3]. Naast het SP ligt een prodomein, dat het nabij gelegen katalytisch domein inactief houdt [2, 3, 21, 25-27]. Na ER insertie wordt pro-MMP14 geactiveerd door sequentiële hydrolyse van zijn aminoterminus door MMPs en proproteine convertasen (PCs) en wordt actief MMP14 vervolgens naar het plasmamembraan getransporteerd [22, 28-32]. Al de bovengenoemde mutaties zijn aanwezig op plekken die cruciaal worden geacht voor activatie en/of transport van MMP14: het SP (p.T17R), de PC herkenningsmotieven (p.R92C en p.R111H), en het hemopexine (Hx) domein 
(p.S466P) [2, 3, 7-9, 28-30, 33-35]. Wij hebben het effect van deze mutaties op hydrolyse, transport en activiteit van MMP14 onderzocht aan de hand van een nieuw ontwikkeld in vitro model. Dit model bestond uit fibroblasten die wild-type (WT) dan wel mutant MMP14 met twee verschillende labels aan respectievelijk hun amino- en carboxylterminus tot expressie brachten. Zoals verwacht, werd het W'T fusie-eiwit sequentieel gehydrolyseerd in de biosynthetische-exocytaire route en bevond zich uiteindelijk op het celoppervlak als functioneel actief enzym. Dit leverde tevens het eerste directe bewijs voor verwijdering van het SP van MMP14 in het ER in vitro. De p.T17R mutatie verstoorde ER insertie en daaropvolgende hydrolyse en transport van MMP14. Daarentegen verliep de hydrolyse en het transport van MMP14 R111H schijnbaar normaal. De p.R111H mutatie verstoorde de katalytische activiteit en het stimulerende effect op celmigratie van MMP14 in vitro echter deels, wat suggereert dat $\mathrm{R}^{111}$ een cruciaal residu is voor MMP14 activatie. Het is mogelijk dat de verminderde activiteit veroorzaakt wordt door een subtiele afwijking in prodomeinhydrolyse, die wij niet konden detectered door middel van Western blot. Vergeleken met de door Winchester et al. beschreven patiënten, hadden onze patiënten een relatief mild fenotype; dit correleerde het beste met de mate van pro-MMP2 activatie door mutant MMP14 in vitro [6]. Onze resultaten bevestigden de pathogeniciteit van een nieuw, hypomorf MMP14 allel, dat een milde vorm van WS veroorzaakt. Vergeleken met de WS patiënten, hebben de $M m p 14$ mutante muizen een ernstiger fenotype. In overeenstemming hiermee verstoorde de p.R92C mutatie het intracellulair transport en de activiteit van MMP14 in vitro dramatisch. Dit impliceert dat het $\mathrm{R}^{89}-\mathrm{R}-\mathrm{P}-\mathrm{R}-\mathrm{C}^{93} \mathrm{PC}$ herkenningsmotief een belangrijkere rol speelt in deze processen dan eerder werd aangenomen [23, 31, 36]. Hoewel het Sabe en Cartoon phenotype nagenoeg identiek zijn aan elkaar en de respectievelijke mutaties de pro-MMP2 activatie door MMP14 ernstig verstoorden, behield MMP14 S466P zijn vermogen tot hydrolyse van gelatine en stimulatie van celmotiliteit in vitro $[8,9]$. Een mogelijke verklaring voor deze discrepantie is dat in tegenstelling tot MMP14 R92C, enig MMP14 S466P het celmembraan bereikt, en daarmee functies die onafhankelijk zijn van homodimerisatie gespaard blijven [37-40]. Homodimerisatie kan verstoord zijn door een dosis-effect, of door een direct effect van de p.S466P mutatie op het Hx domein [41]. Daarnaast zou de p.S466P mutatie de substraatspecificiteit van MMP14 kunnen veranderen, wat de proteolyse van gelatine, maar niet van pro-MMP2, vergemakkelijkt. Samengevat hebben wij aangetoond dat de bovengenoemde vier mutaties de functionele activiteit van MMP14 elk op een unieke manier verstoren, hetgeen restulteert in een vergelijkbaar klinisch fenotype.

Eén aspect van het WS en FTHS fenotype dat onze belangstelling heeft is acne [1, 6 , 10, 11, 42]. Hoewel het alom bekend is dat acne gekenmerkt wordt door cysteuze talgklieren oftewel comedonen, is het onbekend waarom dergelijke cysteuze veranderingen optreden. De talgklier is in principe een holle, vertakte epitheliale structuur die 
gescheiden is van de type I collageen-rijke dermis door een basaal membraan [43]. Vorming van dergelijke structuren hangt doorgaans af van de novo lumenformatie en vertakkende morfogenese [44]. Planaire celpolariteit (PCP) en ECM remodellering spelen een cruciale rol in deze twee processen en kunnen, indien verstoord, leiden tot cystevorming [45-48]. Opvallend genoeg zijn meerdere celtypen afhankelijk van de katalytische activiteit van MMP14 voor vertakkende morfogenese, en tevens van celmembraanlocalisatie van MMP14 voor PCP [49-52]. Daarom hypothetiseerden wij dat verstoorde luminogenese en vertakkende morfogenese van de talgklier door verminderde functie van MMP14 of SH3PXD2B (gezien hun hierboven beschreven directe interactive) ten grondslag kan liggen aan comedovorming. Aangezien acne in onze patiënten succesvol werd behandeld met 13-cis retinoïnezuur, hypothetiseerden wij dat dit medicament het veronderstelde defect in vertakkende morfogenese zou kunnen corrigeren [1]. In Hoofdstuk 3 beschrijven wij een pilotstudie die het effect van Mmp14 of $S h 3 p x d 2 b$ knockdown (KD) dan wel retinoïden op lumenvorming en vertakkende morfogenese van epitheelcellen in vitro onderzoekt $[53,54]$. We toonden aan dat $\mathrm{KD}$ van deze genen lumenvorming in gereconstrueerd basaal membraan (Matrigel ${ }^{\mathrm{TM}}$ ) verstoorde en vertakkende morfogenese in type I collageengel verminderde, terwijl retinoïden beide processen direct stimuleerden. Verstoorde lumenvorming door KD van Mmp14 of $S h 3 p x d 2 b$ impliceert dat PCP afwijkend is [52]. Verder suggereert de verkorte tubuluslengte in gecrosslinkte type I collageenmatrix dat KD van Mmp14 of Sh3pxd2b de ECM degradatie verstoort $[55,56]$. Tenslotte kan de KD de celdeling inhiberen, wat de kleinere organoïden in type I collageengel kan verklaren. De laatste twee mechanismen zijn waarschijnlijk gekoppeld, aangezien eerder is aangetoond dat IMCD cellen geïsoleerd uit Mmp14 KO muizen in vitro een proliferatief defect hadden door verlies van de collagenolytische activiteit van MMP14 [57]. Het feit dat KD van Mmp14 of Sh3pxd2b eenzelfde effect veroorzaakt in vitro suggereert dat een gemeenschappelijke functionele pathway aangedaan is. Vanwege haar rol in ECM remodellering, is podosoomfunctie de meest waarschijnlijke kandidaat. Retinoïden zouden hun effect kunnen uitoefenen door podosoomvorming te stimuleren, aangezien we in Hoofdstuk 2 hebben aangetoond dat 13-cis retinoïnezuur het transport van MMP14 S466P naar podosomen in vitro stimuleerde. Dit kan het therapeutisch effect van 13-cis retinoïnezuur in de behandeling van acne verklaren, hoewel aanvullende weefselspecifieke effecten waarschijnlijk ook een rol spelen [58, 59]. Toekomstig onderzoek dat onze experimenten met sebocyten herhaalt, zal moeten uitwijzen of deze processen een rol kunnen spelen in de morfogenese van talgklieren. Een dergelijk 3D sebocytmodel kan mogelijk gebruikt worden in screening naar anti-acne farmaca.

Naast acne wordt het WS fenotype gekenmerkt door craniofaciale dysmorfie en gegeneraliseerde osteopenie [1, 6]. De meeste aangedane craniofaciale skeletelementen zijn afgeleid van de van neurale lijst (NL), de voornaamste embryonale structuur die bij- 
draagt aan de ontwikkeling van het gelaat [60-62]. Na hun inductie, delamineren NL cellen en migreren ze uitvoerig, waarvoor ECM remodellering essentieel is [60, 61, 6365]. Aangezien MMP14 betrokken is bij ECM remodellering en invasieve celmotiliteit, hypothetiseerden wij dat verstoorde NL migratie ten grondslag ligt aan de craniofaciale dysmorfie van WS [4, 5, 21, 66]. Om deze hypothese te testen en de onderliggende oorzaak van osteopenie bij WS te bestuderen, besloten wij een zebravismodel te ontwikkelen. Zebravissen vormen dezelfde skeletweefsels als mensen, die zich bovendien op een vergelijkbare wijze ontwikkelen. Dit geldt met name voor de schedelbeenderen van de zebravis, waarvan de meerderheid is afgeleid van de NL [67-70]. Zebravissen hebben twee goed geconserveerde MMP14 paralogen, Mmp14a en Mmp14b, die tijdens de ontwikkeling in het mesenchym van de kop tot expressie komen [71-76]. De snelle uitwendige ontwikkeling en optische transparantie van het zebravisembryo maken bovendien live in vivo beeldvorming mogelijk [77-79]. In Hoofdstuk 4 hebben wij de CRISPR/Cas9 techniek gebruikt voor knockout (KO) van zowel mmp14a als mmp14b in zebravissen. Onze mmp14a/b KO vissen toonden essentiële aspecten van het WS fenotype, inclusief verminderde groei, geleidelijk verergerende craniofaciale afwijkingen, hyperkyfose, osteopenie (hoewel beperkt tot de schedel), en een verkorte levensduur [1, 6]. In tegenstelling tot onze hypothese, waren NL inductie, delaminatie, farynxbooginvasie, en differentiatie tot craniofaciaal kraakbeen onaangetast door mmp14a/b KO. Zoals eerder gesuggereerd, zijn voorheen beschreven craniofaciale defecten in mmp14a en mmp14b morfanten waarschijnlijk aspecifieke neveneffecten van de gebruikte Morfolino oligonucleotiden [74, 75, 80]. In onze mmp14a/b KO larven was de skeletmineralisatie tijdens metamorfose onaangetast [81]. Volwassen mutanten hadden een verstoorde enchondrale/perichondrale en intramembraneuze ossificatie van zowel NL- als mesoderm-afgeleide beenderen, wat verder tegen een NL-specifiek problem pleit $[68,82]$. Aangedane botten bevatten in het algemeen relatief weinig botmatrix met een afwijkende collageeninhoud en clusters multinucleaire cellen, en een relatief volumineuze, ongeorganiseerde kraakbeenkern. In muizen verstoort verlies van MMP14 de tijdige verwijdering van kraakbeen tijdens beide vormen van ossificatie, wat gepaard gaat met overmatige resorptie van gemineraliseerde botmatrix. Dit resulteert in vergelijkbare botafwijkingen als aanwezig in onze mmp14a/b KO vissen [51, 83]. Hoewel in vitro is aangetoond dat MMP14 de osteoblastdifferentiatie stimuleert en osteoclastdifferentiatie en -activatie inhibeert, zal aanvullend onderzoek uit moeten wijzen welke processen en celtypes betrokken zijn in het skeletfenotype van onze mmp14a/b KO vissen [84-86]. Verder is het nog onbekend in welke mate afwijkende podosoomfunctie betrokken is in het WS fenotype. Analyse van zebravissen met verschillende combinaties van mmp14a/b, sh3pxd2b en/of mmp2 KO kan helpen om de samenwerking van de respectievelijke eiwitproducten in botremodellering te begrijpen. Tenslotte kan de mmp14a/b KO vis gebruikt worden voor de ontwikkeling van medicatie voor botafwijkingen in WS alsook osteopenie in het algemeen. 


\section{References}

1. Van Steensel MA, Ceulen RP, Delhaas T, de Die-Smulders C. Two Dutch brothers with Borrone dermato-cardio-skeletal syndrome. American Journal of Medical Genetics Part A. 2007;143a(11):1223-6.

2. Sato H, Takino T, Okada Y, Cao J, Shinagawa A, Yamamoto E, et al. A matrix metalloproteinase expressed on the surface of invasive tumour cells. Nature. 1994;370(6484):61-5.

3. Takino T, Sato H, Yamamoto E, Seiki M. Cloning of a human gene potentially encoding a novel matrix metalloproteinase having a C-terminal transmembrane domain. Gene. 1995;155(2):293-8.

4. Ohuchi E, Imai K, Fujii Y, Sato H, Seiki M, Okada Y. Membrane type 1 matrix metalloproteinase digests interstitial collagens and other extracellular matrix macromolecules. The Journal of Biological Chemistry. 1997;272(4):2446-51.

5. d'Ortho MP, Will H, Atkinson S, Butler G, Messent A, Gavrilovic J, et al. Membrane-type matrix metalloproteinases 1 and 2 exhibit broad-spectrum proteolytic capacities comparable to many matrix metalloproteinases. European Journal of Biochemistry. 1997;250(3):751-7.

6. Winchester P, Grossman H, Lim WN, Danes BS. A new acid mucopolysaccharidosis with skeletal deformities simulating rheumatoid arthritis. The American Journal of Roentgenology, Radium Therapy, and Nuclear Medicine. 1969;106(1):121-8.

7. Evans BR, Mosig RA, Lobl M, Martignetti CR, Camacho C, Grum-Tokars V, et al. Mutation of membrane type-1 metalloproteinase, MT1-MMP, causes the multicentric osteolysis and arthritis disease Winchester syndrome. American Journal of Human Genetics. 2012;91(3):572-6.

8. Curtain MM; Donahue LR. 2007. A possible new mutation to Mmp 14 MGI Direct Data Submission. MGI: J:127164. Updated Nov 2012. The Jackson Laboratory. URL: informatics.jax.org/downloads/Reference_texts/J127164.pdf.

9. Du X, Moresco EMY, Murray A, Beutler B. record for cartoon, updated Dec 12, 2013. MUTAGENETIX TM, B. Beutler and colleagues, center for the Genetics of Host Defense, UT Southwestern Medical Center, Dallas, TX. URL: mutagenetix. utsouthwestern.edu.

10. Frank Y, Ziprkowski M, Romano A, Stein R, Katznelson MB, Cohen B, et al. Megalocornea associated with multiple skeletal anomalies: a new genetic syndrome? Journal de Genetique Humaine. 1973;21(2):67-72.

11. Ter Haar B, Hamel B, Hendriks J, de Jager J. Melnick-Needles syndrome: indication for an autosomal recessive form. American Journal of Medical Genetics. 1982;13(4):469-77.

12. Maas SM, Kayserili H, Lam J, Apak MY, Hennekam RC. Further delineation of Frank-ter Haar syndrome. American Journal of Medical Genetics Part A. 2004;131(2):127-33.

13. Iqbal Z, Cejudo-Martin P, de Brouwer A, van der Zwaag B, Ruiz-Lozano P, Scimia MC, et al. Disruption of the podosome adaptor protein TKS4 (SH3PXD2B) causes the skeletal dysplasia, eye, and cardiac abnormalities of Frank-Ter Haar Syndrome. American Journal of Human Genetics. 2010;86(2):254-61.

14. Martignetti JA, Aqeel AA, Sewairi WA, Boumah CE, Kambouris M, Mayouf SA, et al. Mutation of the matrix metalloproteinase 2 gene (MMP2) causes a multicentric osteolysis and arthritis syndrome. Nature Genetics. 2001;28(3):261-5.

15. Zankl A, Bonafe L, Calcaterra V, Di Rocco M, Superti-Furga A. Winchester syndrome caused by a homozygous mutation affecting the active site of matrix metalloproteinase 2. Clinical Genetics. 2005;67(3):261-6.

16. Zankl A, Pachman L, Poznanski A, Bonafe L, Wang F, Shusterman Y, et al. Torg syndrome is caused by inactivating mutations in MMP2 and is allelic to NAO and Winchester syndrome. Journal of Bone and Mineral Research. 2007;22(2):329-33.

17. Nakahara H, Howard L, Thompson EW, Sato H, Seiki M, Yeh Y, et al. Transmembrane/cytoplasmic domain-mediated membrane type 1-matrix metalloprotease docking to invadopodia is required for 
cell invasion. Proceedings of the National Academy of Sciences of the United States of America. 1997;94(15):7959-64.

18. Buschman MD, Bromann PA, Cejudo-Martin P, Wen F, Pass I, Courtneidge SA. The novel adaptor protein Tks4 (SH3PXD2B) is required for functional podosome formation. Molecular Biology of the Cell. 2009;20(5):1302-11.

19. Murphy DA, Courtneidge SA. The 'ins' and 'outs' of podosomes and invadopodia: characteristics, formation and function. Nature Reviews Molecular Cell Biology. 2011;12(7):413-26.

20. Gawden-Bone C, Zhou Z, King E, Prescott A, Watts C, Lucocq J. Dendritic cell podosomes are protrusive and invade the extracellular matrix using metalloproteinase MMP-14. Journal of Cell Science. 2010;123(Pt 9):1427-37.

21. Sternlicht MD, Werb Z. How matrix metalloproteinases regulate cell behavior. Annual Review of Cell and Developmental Biology. 2001;17:463-516.

22. Remacle AG, Rozanov DV, Baciu PC, Chekanov AV, Golubkov VS, Strongin AY. The transmembrane domain is essential for the microtubular trafficking of membrane type-1 matrix metalloproteinase (MT1-MMP). Journal of Cell Science. 2005;118(Pt 21):4975-84.

23. Remacle AG, Rozanov DV, Fugere M, Day R, Strongin AY. Furin regulates the intracellular activation and the uptake rate of cell surface-associated MT1-MMP. Oncogene. 2006;25(41):5648-55.

24. Koziol A, Martin-Alonso M, Clemente C, Gonzalo P, Arroyo AG. Site-specific cellular functions of MT1-MMP. European Journal of Cell Biology. 2012;91(11-12):889-95.

25. Springman EB, Angleton EL, Birkedal-Hansen H, Van Wart HE. Multiple modes of activation of latent human fibroblast collagenase: evidence for the role of a Cys73 active-site zinc complex in latency and a "cysteine switch" mechanism for activation. Proceedings of the National Academy of Sciences of the United States of America. 1990;87(1):364-8.

26. Van Wart HE, Birkedal-Hansen H. The cysteine switch: a principle of regulation of metalloproteinase activity with potential applicability to the entire matrix metalloproteinase gene family. Proceedings of the National Academy of Sciences of the United States of America. 1990;87(14):5578-82.

27. Page-McCaw A, Ewald AJ, Werb Z. Matrix metalloproteinases and the regulation of tissue remodelling. Nature Reviews Molecular Cell Biology. 2007;8(3):221-33.

28. Massova I, Kotra LP, Fridman R, Mobashery S. Matrix metalloproteinases: structures, evolution, and diversification. FASEB Journal. 1998;12(12):1075-95.

29. Yana I, Weiss SJ. Regulation of membrane type-1 matrix metalloproteinase activation by proprotein convertases. Molecular Biology of the Cell. 2000;11(7):2387-401.

30. Rozanov DV, Deryugina EI, Ratnikov BI, Monosov EZ, Marchenko GN, Quigley JP, et al. Mutation analysis of membrane type-1 matrix metalloproteinase (MT1-MMP). The role of the cytoplasmic tail Cys(574), the active site Glu(240), and furin cleavage motifs in oligomerization, processing, and selfproteolysis of MT1-MMP expressed in breast carcinoma cells. The Journal of Biological Chemistry. 2001;276(28):25705-14.

31. Golubkov VS, Chekanov AV, Shiryaev SA, Aleshin AE, Ratnikov BI, Gawlik K, et al. Proteolysis of the membrane type-1 matrix metalloproteinase prodomain: implications for a two-step proteolytic processing and activation. The Journal of Biological Chemistry. 2007;282(50):36283-91.

32. Golubkov VS, Cieplak P, Chekanov AV, Ratnikov BI, Aleshin AE, Golubkova NV, et al. Internal cleavages of the autoinhibitory prodomain are required for membrane type 1 matrix metalloproteinase activation, although furin cleavage alone generates inactive proteinase. The Journal of Biological Chemistry. 2010;285(36):27726-36.

33. Mori H, Tomari T, Koshikawa N, Kajita M, Itoh Y, Sato H, et al. CD44 directs membrane-type 1 matrix metalloproteinase to lamellipodia by associating with its hemopexin-like domain. The EMBO Journal. 2002;21(15):3949-59.

34. Atkinson SJ, Roghi C, Murphy G. MT1-MMP hemopexin domain exchange with MT4-MMP blocks enzyme maturation and trafficking to the plasma membrane in MCF7 cells. The Biochemical Journal. 2006;398(1):15-22. 
35. Lehti K, Lohi J, Juntunen MM, Pei D, Keski-Oja J. Oligomerization through hemopexin and cytoplasmic domains regulates the activity and turnover of membrane-type 1 matrix metalloproteinase. The Journal of Biological Chemistry. 2002;277(10):8440-8.

36. Seidah NG, Mayer G, Zaid A, Rousselet E, Nassoury N, Poirier S, et al. The activation and physiological functions of the proprotein convertases. The International Journal of Biochemistry \& Cell Biology. 2008;40(6-7):1111-25.

37. Itoh Y, Takamura A, Ito N, Maru Y, Sato H, Suenaga N, et al. Homophilic complex formation of MT1-MMP facilitates proMMP-2 activation on the cell surface and promotes tumor cell invasion. The EMBO Journal. 2001;20(17):4782-93.

38. Itoh Y, Ito N, Nagase H, Seiki M. The second dimer interface of MT1-MMP, the transmembrane domain, is essential for ProMMP-2 activation on the cell surface. The Journal of Biological Chemistry. 2008;283(19):13053-62.

39. Cao J, Kozarekar P, Pavlaki M, Chiarelli C, Bahou WF, Zucker S. Distinct roles for the catalytic and hemopexin domains of membrane type 1-matrix metalloproteinase in substrate degradation and cell migration. The Journal of Biological Chemistry. 2004;279(14):14129-39.

40. Dufour A, Sampson NS, Zucker S, Cao J. Role of the hemopexin domain of matrix metalloproteinases in cell migration. Journal of Cellular Physiology. 2008;217(3):643-51.

41. Zarrabi K, Dufour A, Li J, Kuscu C, Pulkoski-Gross A, Zhi J, et al. Inhibition of matrix metalloproteinase 14 (MMP-14)-mediated cancer cell migration. The Journal of Biological Chemistry. 2011;286(38):33167-77.

42. Borrone C, Di Rocco M, Crovato F, Camera G, Gambini C. New multisystemic disorder involving heart valves, skin, bones, and joints in two brothers. American Journal of Medical Genetics. 1993;46(2):228-34.

43. Williams BB, Cantrell VA, Mundell NA, Bennett AC, Quick RE, Jessen JR. VANGL2 regulates membrane trafficking of MMP14 to control cell polarity and migration. Journal of Cell Science. 2012;125(Pt 9):2141-7.

44. Kim HY, Nelson CM. Extracellular matrix and cytoskeletal dynamics during branching morphogenesis. Organogenesis. 2012;8(2):56-64.

45. Datta A, Bryant DM, Mostov KE. Molecular regulation of lumen morphogenesis. Current Biology. 2011;21(3):R126-36.

46. Lubarsky B, Krasnow MA. Tube morphogenesis: making and shaping biological tubes. Cell. 2003;112(1):19-28.

47. Andrew DJ, Ewald AJ. Morphogenesis of epithelial tubes: Insights into tube formation, elongation, and elaboration. Developmental Biology. 2010;341(1):34-55.

48. Marciano DK. A holey pursuit: lumen formation in the developing kidney. Pediatric Nephrology. 2017;32(1):7-20.

49. Hotary K, Allen E, Punturieri A, Yana I, Weiss SJ. Regulation of cell invasion and morphogenesis in a three-dimensional type I collagen matrix by membrane-type matrix metalloproteinases 1,2 , and 3 . The Journal of Cell Biology. 2000;149(6):1309-23.

50. Chun TH, Sabeh F, Ota I, Murphy H, McDonagh KT, Holmbeck K, et al. MT1-MMP-dependent neovessel formation within the confines of the three-dimensional extracellular matrix. The Journal of Cell Biology. 2004;167(4):757-67.

51. Zhou Z, Apte SS, Soininen R, Cao R, Baaklini GY, Rauser RW, et al. Impaired endochondral ossification and angiogenesis in mice deficient in membrane-type matrix metalloproteinase I. Proceedings of the National Academy of Sciences of the United States of America. 2000;97(8):4052-7.

52. Happe H, de Heer E, Peters DJ. Polycystic kidney disease: the complexity of planar cell polarity and signaling during tissue regeneration and cyst formation. Biochimica et Biophysica Acta. 2011;1812(10):1249-55.

53. Debnath J, Brugge JS. Modelling glandular epithelial cancers in three-dimensional cultures. Nature Reviews Cancer. 2005;5(9):675-88. 
54. Giles RH, Ajzenberg H, Jackson PK. 3D spheroid model of mIMCD3 cells for studying ciliopathies and renal epithelial disorders. Nature Protocols. 2014;9(12):2725-31.

55. Artym VV, Matsumoto KK. Imaging cells in three-dimensional collagen matrix. Current Protocols in Cell Biology. 2010 Sep;Chapter 10:Unit 10.18.1-20.

56. Pohl M, Sakurai H, Bush KT, Nigam SK. Matrix metalloproteinases and their inhibitors regulate in vitro ureteric bud branching morphogenesis. American Journal of Physiology Renal Physiology. 2000;279(5):F891-900.

57. Riggins KS, Mernaugh G, Su Y, Quaranta V, Koshikawa N, Seiki M, et al. MT1-MMP-mediated basement membrane remodeling modulates renal development. Experimental Cell Research. 2010;316(17):2993-3005.

58. Ganceviciene R, Zouboulis CC. Isotretinoin: state of the art treatment for acne vulgaris. Journal der Deutschen Dermatologischen Gesellschaft. 2010;8 Suppl 1:S47-59.

59. Zouboulis CC. Isotretinoin revisited: pluripotent effects on human sebaceous gland cells. The Journal of Investigative Dermatology. 2006;126(10):2154-6.

60. Santagati F, Rijli FM. Cranial neural crest and the building of the vertebrate head. Nature Reviews Neuroscience. 2003;4(10):806-18.

61. Mayor R, Theveneau E. The neural crest. Development (Cambridge, England). 2013;140(11):2247-51.

62. Cordero DR, Brugmann S, Chu Y, Bajpai R, Jame M, Helms JA. Cranial neural crest cells on the move: their roles in craniofacial development. American journal of medical genetics Part A. 2011;155a(2):270-9.

63. Halloran MC, Berndt JD. Current progress in neural crest cell motility and migration and future prospects for the zebrafish model system. Developmental Dynamics. 2003;228(3):497-513.

64. Christian L, Bahudhanapati H, Wei S. Extracellular metalloproteinases in neural crest development and craniofacial morphogenesis. Critical Reviews in Biochemistry and Molecular Biology. 2013;48(6):544-60.

65. Erickson CA, Perris R. The role of cell-cell and cell-matrix interactions in the morphogenesis of the neural crest. Developmental Biology. 1993;159(1):60-74.

66. Sabeh F, Ota I, Holmbeck K, Birkedal-Hansen H, Soloway P, Balbin M, et al. Tumor cell traffic through the extracellular matrix is controlled by the membrane-anchored collagenase MT1-MMP. The Journal of Cell Biology. 2004;167(4):769-81.

67. Yelick PC, Schilling TF. Molecular dissection of craniofacial development using zebrafish. Critical Reviews in Oral Biology and Medicine. 2002;13(4):308-22.

68. Kague E, Gallagher M, Burke S, Parsons M, Franz-Odendaal T, Fisher S. Skeletogenic fate of zebrafish cranial and trunk neural crest. PloS One. 2012;7(11):e47394.

69. Schilling TF, Kimmel CB. Segment and cell type lineage restrictions during pharyngeal arch development in the zebrafish embryo. Development. 1994;120(3):483-94.

70. Witten PE, Huysseune A. A comparative view on mechanisms and functions of skeletal remodelling in teleost fish, with special emphasis on osteoclasts and their function. Biological Reviews of the Cambridge Philosophical Society. 2009;84(2):315-46.

71. Zhang J, Bai S, Zhang X, Nagase H, Sarras MP, Jr. The expression of novel membrane-type matrix metalloproteinase isoforms is required for normal development of zebrafish embryos. Matrix Biology. 2003;22(3):279-93.

72. Wyatt RA, Keow JY, Harris ND, Hache CA, Li DH, Crawford BD. The zebrafish embryo: a powerful model system for investigating matrix remodeling. Zebrafish. 2009;6(4):347-54.

73. Taylor JS, Braasch I, Frickey T, Meyer A, Van de Peer Y. Genome duplication, a trait shared by 22000 species of ray-finned fish. Genome Research. 2003;13(3):382-90.

74. Coyle RC, Latimer A, Jessen JR. Membrane-type 1 matrix metalloproteinase regulates cell migration during zebrafish gastrulation: evidence for an interaction with non-canonical Wnt signaling. Experimental Cell Research. 2008;314(10):2150-62. 
75. Janssens E, Gaublomme D, De Groef L, Darras VM, Arckens L, Delorme N, et al. Matrix metalloproteinase 14 in the zebrafish: an eye on retinal and retinotectal development. PloS One. 2013;8(1):e52915.

76. Thisse, B., Pflumio, S., Fürthauer, M., Loppin, B., Heyer, V., Degrave, A., Woehl, R., Lux, A., Steffan, T., Charbonnier, X.Q. and Thisse, C. (2001) Expression of the zebrafish genome during embryogenesis (NIH R01 RR15402). ZFIN Direct Data Submission. URL: http://zfin.org.

77. Kimmel CB, Ballard WW, Kimmel SR, Ullmann B, Schilling TF. Stages of embryonic development of the zebrafish. Developmental Dynamics. 1995;203(3):253-310.

78. Schilling TF. The morphology of larval and adult zebrafish. Zebrafish. 2002;261:59-94.

79. Mackay EW, Apschner A, Schulte-Merker S. A bone to pick with zebrafish. BoneKEy Reports. 2013;2:445.

80. Boer EF, Jette CA, Stewart RA. Neural Crest Migration and Survival Are Susceptible to MorpholinoInduced Artifacts. PloS One. 2016;11(12):e0167278.

81. Parichy DM, Elizondo MR, Mills MG, Gordon TN, Engeszer RE. Normal table of postembryonic zebrafish development: staging by externally visible anatomy of the living fish. Developmental Dynamics. 2009;238(12):2975-3015.

82. Nieto MA. The early steps of neural crest development. Mechanisms of Development. 2001;105(12):27-35.

83. Holmbeck K, Bianco P, Caterina J, Yamada S, Kromer M, Kuznetsov SA, et al. MT1-MMP-deficient mice develop dwarfism, osteopenia, arthritis, and connective tissue disease due to inadequate collagen turnover. Cell. 1999;99(1):81-92.

84. Tang Y, Rowe RG, Botvinick EL, Kurup A, Putnam AJ, Seiki M, et al. MT1-MMP-dependent control of skeletal stem cell commitment via a beta1-integrin/YAP/TAZ signaling axis. Developmental Cell. 2013;25(4):402-16.

85. Chan KM, Wong HL, Jin G, Liu B, Cao R, Cao Y, et al. MT1-MMP inactivates ADAM9 to regulate FGFR2 signaling and calvarial osteogenesis. Developmental Cell. 2012;22(6):1176-90.

86. Hikita A, Yana I, Wakeyama H, Nakamura M, Kadono Y, Oshima Y, et al. Negative regulation of osteoclastogenesis by ectodomain shedding of receptor activator of NF-kappaB ligand. The Journal of Biological Chemistry. 2006;281(48):36846-55. 



\section{Acknowledgements / Dankwoord}

Lieve ouders, bedankt voor de onvoorwaardelijke liefde en steun die jullie mij gegeven hebben, niet alleen tijdens mijn promotie, maar ook al die jaren daarvóór. Jullie hebben mij het vertrouwen gegeven om aan de andere kant van de wereld mijn opleiding voort te zetten, al moet het zwaar voor jullie zijn geweest mij uitendelijk te zien vertrekken en ik bovendien langer wegbleef dan vooraf voorzien. Ik heb jullie zeker ook gemist, en was blij dat ik vooral tijdens de eerste maanden de cultuurshock via Skype met jullie kon delen. Het was dan ook fijn om mij eens per jaar lekker thuis te laten verwennen, om daarna weer een jaar flink aan te poten. Pap, ik vond het leuk dat je me kwam opzoeken en ik je mijn leven in Singapore kon laten zien - en dat je vervolgens al mijn Daiso-souvenirs mee naar Nederland wilde zeulen!

My dearest Sheena, although I could try to be funny by saying I wrote this thesis not thanks to, but despite you (and your K-pop blaring through our apartment), not only might it turn out cringy, but I also genuinely owe a lot to you. You taught me essential zebrafish techniques, helped me with the necessary controls, taught me to effectively multitask in the lab and by that not only sped up my work but also helped me restore my work-life balance. And most of all: you (and your family) made me truly feel at home in Singapore. Thank you for sticking with me, even when during thesis writing everything else came in second place... I am very grateful for your support throughout the second half of my $\mathrm{PhD}$, and hope to return the favour in the future - you are a gifted scientist worthy of a $\mathrm{PhD}$ yourself.

Beste Maurice, na jouw college over acne en de diagnostiek van genodermatosen tijdens mijn co-schap Dermatologie wist ik het zeker: dat wil ik óók! Ik was dan ook blij dat ik mijn wetenschapsstage in je laboratorium in Maastricht mocht volgen en hoopte vurig dat ik er een promotieonderzoek uit kon slepen. Toen het gerucht ging dat je naar Singapore vertrok, grapten je PhD studenten er al over dat ik - na de Kootstra Talent Fellowship nét te zijn misgelopen - maar moest vragen of je daar geen baan voor me had. Toch was ik overrompeld toen je me daadwerkelijk benaderde om mijn promotie te starten in Singapore. Ik ben je erg dankbaar dat je me deze unieke kans hebt geboden en het vertrouwen had om me, enkel met supervisie op afstand, in je nieuwe lab te laten werken. Je enthousiasme voor dermatologie en wetenschap straalde op onze groep af en heeft me telkens weer gemotiveerd, ook wanneer de experimenten eens tegenzaten... Waar andere groepen blij waren dat hun PI eindelijk eens met vakantie ging, vonden wij het juist leuk als je weer voor een maand naar Singapore kwam - en vonden we het net zo jammer als je weer vertrok. Ik ben blij dat je uiteindelijk full-time naar Singapore kwam, ik hier mijn promotie mocht afmaken, en we daarna onze samenwerking hebben voortgezet binnen SRIS. 
Dear Xinhong, I want to thank you for teaching me to be critical in science, especially about my own work. You challenged me to think independently about every aspect of my project and experimental design instead of 'blindly' following other people, and to question the obtained results. Although your (many) questions during my first lab meeting presentation(s) were rather tough to answer, they helped me to distinguish main and side issues and to take a more focused approach to my project. In addition, your contribution to my IMB seminars was invaluable (I still carry a copy of "Dr X's Rules for Clear Scientific Communication" around).

Dear Barry, I want to thank you for all the time and effort you put into my project. Even though it was not your primary focus, every week you managed to find the time to attend our lab meeting via Skype - not only to give feedback on my results and project progress, but also to provide practical tips on how to optimally carry out the planned experiments. Finally, I appreciate your feedback on the text and figures, which significantly improved the quality of my thesis and our papers.

Dear Tom, thank you for 'adopting' me into your group, teaching me the basics of zebrafish research and allowing me to use your space in the lab and fish facility. Although my schedule did not allow me to attend your group's lab meeting more frequently, the feedback I got from presenting there contributed significantly to the overall narrative of our MMP14 paper and this thesis, and helped me troubleshooting the fish experiments.

Dear Ray, thank you for your supervision and allowing Sheena to spend some of her time assisting me with the final bits of my project. Even though our collaboration was relatively brief, I really appreciate your effort in making me feel part of your team.

Dear Prof. Lane, thank you for providing me the opportunity of carrying out my entire $\mathrm{PhD}$ project in such a well-equipped institute, in addition to gaining significant working experience in Asia.

Beste prof. dr. Steijlen, ik waardeer het zeer dat u, ondanks uw drukke agenda, elk jaar tijdens mijn vakantie in Nederland de tijd nam voor een persoonlijke update van mijn PhD project. Bedankt dat u mij de gelegenheid bood om tijdens de wetenschappelijke vergadering aan mijn Maastrichtse collega's te tonen wat ik aan de andere kant van de aardbol uitvoerde.

Beste Michel, dit proefschrift was er niet geweest zonder jouw bevestiging van de MMP14 mutatie in onze patiënten. Bedankt dat je ook tijdens de laatste fase van mij 
promotietraject betrokken bleef en ons hebt geholpen met de publicatie van onze nieuw ontdekte mutatie.

Dear colleagues of the Acne/MvS/LXH/SHARE/HAPZ/ASGP lab, it was a joy to work together with you at IMB A*STAR, and later SRIS (you really love abbreviations, don't you?).

Niva, you were my first colleague in IMB. Thank you for being my "buddy", guiding me around during my first few weeks in Singapore - and telling me all the important things HR assumes "everybody knows" (getting a phone number, bank account, business cards, and bringing me to a GP when I thought I had Dengue fever). Even though our first few experiments did not always work out, we had a lot of fun setting up the lab.

Shi Yun, you were the first post-doc to supervise me (and boss me around). Thank you for your help in setting up the 3D cultures, and helping prioritising our work whenever Maurice and Barry came up with another 10 new hypotheses for us to test during our weekly Skype meeting. Although it was sad that both you and Niva left our lab prematurely, we thankfully found new hires to strengthen our team.

Evelyn, your pragmatic approach and "lets do this!" attitude were very inspiring. I liked how every Monday we would sit together, plan that week's experiments, and discuss whatever either of us was uncertain about. Of course, I cannot forget to mention your hospitality, hosting countless lab gatherings at your penthouse where we would cook together and play Avalon until we were just able to catch the last train home! Thank you for your contributions to the in vitro functional assays and, even after you had already left IMB, our MMP14 paper.

Arnette, you arrived just at the right moment, when I could use a hand in handling and genotyping - all the fish lines I had been generating for months. Thank you for your general support in the lab, handling all our complex orders and taking care of the interns when I was too busy writing this thesis.

Finally, I would like to thank all other current and former lab members (Sophia, Khek Chian, Shang Wei, Alvin, Selwyn, Carol, Pei Wen, Jing Shun, Faith, Keith, Rosanna, Melvin, Daniel, Yun, Jun Ren, Yun Pei, Richard, and Kenneth) for making it such an inspiring and fun environment to work in. I appreciate all the feedback I got throughout the years, which helped to improve both my technical skills and my data presentation. Furthermore, I enjoyed the countless times we went for lunch at the Tanglin Halt Hawker Center, playing musical chairs to "chope" a table and defying the grumpy cleaners together...

Beste Monique, mijn buddy in IMCB! Ik kan me nog goed herinneren dat je naar Singapore kwam om je promotieonderzoek te presenteren. Ik had al allerlei plannen gemaakt om je rond te leiden - tot ik griep kreeg en de helft niet doorging! Een paar 
maanden later was het eindelijk zover en kwam je (voorgoed?) naar Singapore. Eindelijk iemand die me wegwijs kon maken in het zebravisonderzoek. Behalve je praktische tips en duidelijke adviezen over hoe complexe problemen op een gestructureerde manier aan te pakken, heb ik ook mentaal veel steun aan je gehad. Bedankt voor jouw luisterend oor waaraan ik al de obstakels van mijn promotieonderzoek kwijt kon tijdens onze wekelijkse lunch bij Raj (bollen!) of de Thai. Het was een verademing om te zien dat jij je over dezelfde dingen verwonderde en aan dezelfde dingen ergerde in Singapore (het lag dus niet aan míj) - en we nagegoeg dezelfde fases doorliepen van aanpassing aan een nieuwe cultuur. Bedankt dat je als paranimf bij mijn promotie kon zijn!

Beste Irma, het was fijn om eindelijk niet meer de enige Nederlandse promovendus binnen IMB te zijn. Bedankt voor je hulp bij het fotograferen van mijn visjes en het delen van je SPSS-skills. Fijn dat je mijn paranimf wilde zijn!

Dear Oliver and Peh Fern, thank you for your general support during my first few months in IMB. Even though your team is relatively small, you are still the lab to go to whenever I need "rare" consumables - you really have everything!

Dear interns in our lab, thank you for your interest in our group's research. Your curiosity has provided me with many basic questions I would have otherwise overlooked to ask - and have definitely contributed to my overall knowledge and final thesis. Dear Chernis, thank you for your contribution to my project - especially considering your background was completely different and the speed with which you picked up both biology and built a set of technical skills.

Beste Stefan, jij was de eerste student die ik mocht begeleiden - een leuke en leerzame ervaring voor ons beiden. Frappant dat we ons in Singapore leerden kennen, terwijl we jarenlang 'thuis' hemelsbreed $5 \mathrm{~km}$ van elkaar woonden!

Dear PhD students, ROs and RFs in IMB and IMCB, thank you for all the discussions, borrowed samples, and not the least our gatherings to unwind after yet another busy week! Dear Abraha, Josie, Kat, Sowjanya, Shi Young, Ritika, Simone and Serene, thank you for all the advices that got my zebrafish work up to par. Dear Wen Chiy, Paul, Federica and Navin, thank you for making the tissue culture rooms one of my favourite places to work.

Dear Graham, thank you for providing such a great microscopy suite that enabled most of my research. Dear Shuping, Sarah, and Jaron, thank you for training me to use the various microscopes. Dear John, your ImageJ course is invaluable, as are your custom plug-ins that made my job so much easier. Thank you for your support in 
using the lightsheet microscope - even when it meant we both had to stay until midnight!

Dear Agus and aunties of the IMCB zebrafish facility, thank you for taking care of my fishes throughout the years - and telling me the little tricks in zebrafish mating and husbandry nobody else told me.

I would like to thank all collaborators for their contribution to this thesis. Dear Dominic, thank you for your help on the Western blots for our MMP14 paper. Even though we have never met in person, I highly appreciate your prompt action whenever it was needed.

Beste Tijs, bedankt voor al je voorbereidend werk in ons nieuwe lab in Singapore - en natuurlijk voor al je praktische tips tijdens mijn stage in Maastricht en de eerste maanden in Singapore - ik heb er veel aan gehad!

Beste Renske, bedankt voor al je werk aan het MMP14-project voordat we het in Singapore voortzetten.

Beste secretaresses van de afdeling Dermatologie, elke keer wanneer mijn visum afliep begon een race tegen de klok om tijdig alle formulieren bij het Ministry of Manpower in Singapore te krijgen. Bedankt dat het toch elke keer weer lukte!

Dear Tate, it was great having you as my housemate when I just arrived in Singapore. It made a huge difference to me to have at least one person to share experiences with when everything was still new and strange...

Tenslotte wil ik graag de leden van de beoordelingscommissie bedanken voor de tijd en moeite die $\mathrm{u}$ in het lezen en beoordelen van dit proefschrift heeft gestoken. 


\section{Curriculum vitae}

Ivo Johannes Hendrikus Marie de Vos was born in Maastricht on July 18 ${ }^{\text {th }}$ 1985. After receiving his secondary education degree (pre-university stream) from the Stella Maris College Meerssen in 2003, he enrolled in Molecular Life Sciences at the transnational University of Limburg, spending time in both the Netherlands (UM) and Belgium (Hasselt University). In 2006, he obtained his bachelors degree with first class honours and started studying Medicine in Maastricht. In 2008, he worked as Education and Research Assistant at the UM Department of Pharmacology and Toxicology. Under the supervision of Dr. Blankestijn, he investigated the role of Wnt/Fz-signalling in wound healing following myocardial infarction. In 2010 he obtained his second bachelors degree and won the Top 3\% Student Award. After an elective clinical clerkship in Dermatology, he dedicated his final year of the Medicine master programme to rare genetic disorders with special attention to genodermatoses, first at the MUMC+ Department of Clinical Genetics under the supervision of Prof. Stumpel, and later at the Experimental Dermatology Laboratory under the supervision of Prof. Van Steensel and Dr. Coull. Here, he studied the physiological function of folliculin, involved in Birt-Hogg-Dubé syndrome. After graduating in 2013, he undertook a volunteer research internship in the same lab. In 2014 he started his PhD project on MMP14related pathology in Prof. Van Steensel's newly founded Acne Lab at the A*STAR Institute of Medical Biology in Singapore.

Ivo Johannes Hendrikus Marie de Vos werd geboren op 18 juli 1985 te Maastricht. Na het behalen van zijn VWO-diploma aan het Stella Maris College Meerssen in 2003, ging hij Moleculaire Levenswetenschappen studeren aan de transnationale Universiteit Limburg, waarbij hij de opleiding deels in Nederland (UM) en deels in België (Universiteit Hasselt) volgde. In 2006 behaalde hij zijn bachelordiploma cum laude aan de UM, en startte vervolgens zijn studie Geneeskunde in Maastricht. In 2008 werkte hij als Onderwijs- en Onderzoeksassistent bij de afdeling Farmacologie en Toxicologie van de UM. Onder supervisie van dr. Blankestijn onderzocht hij de rol van Wnt/Fzsignalering in wondgenezing na een myocardinfarct. In 2010 behaalde hij zijn tweede bachelordiploma en won de Top 3\% Student Award. Na een keuzestage Dermatologie wijdde hij het laatste jaar van zijn master Geneeskunde aan zeldzame genetische aandoeningen, met speciale aandacht voor genodermatosen; eerst binnen de afdeling Klinische Genetica van het MUMC+ onder begeleiding van prof. dr. Stumpel, en later in het Laboratorium voor Experimentele Dermatologie onder begeleiding van prof. dr. Van Steensel en dr. Coull. Hier onderzocht hij de fysiologische rol van folliculine, betrokken bij Birt-Hogg-Dubé syndroom. Na het behalen van zijn artsdiploma in 2013, volgde hij een vrijwillige stage in hetzelfde lab. In 2014 startte hij zijn promotieonderzoek naar MMP14-gerelateerde pathologie in het nieuw geopende Acne Lab van prof. dr. Van Steensel in het A*STAR Institute of Medical Biology in Singapore. 


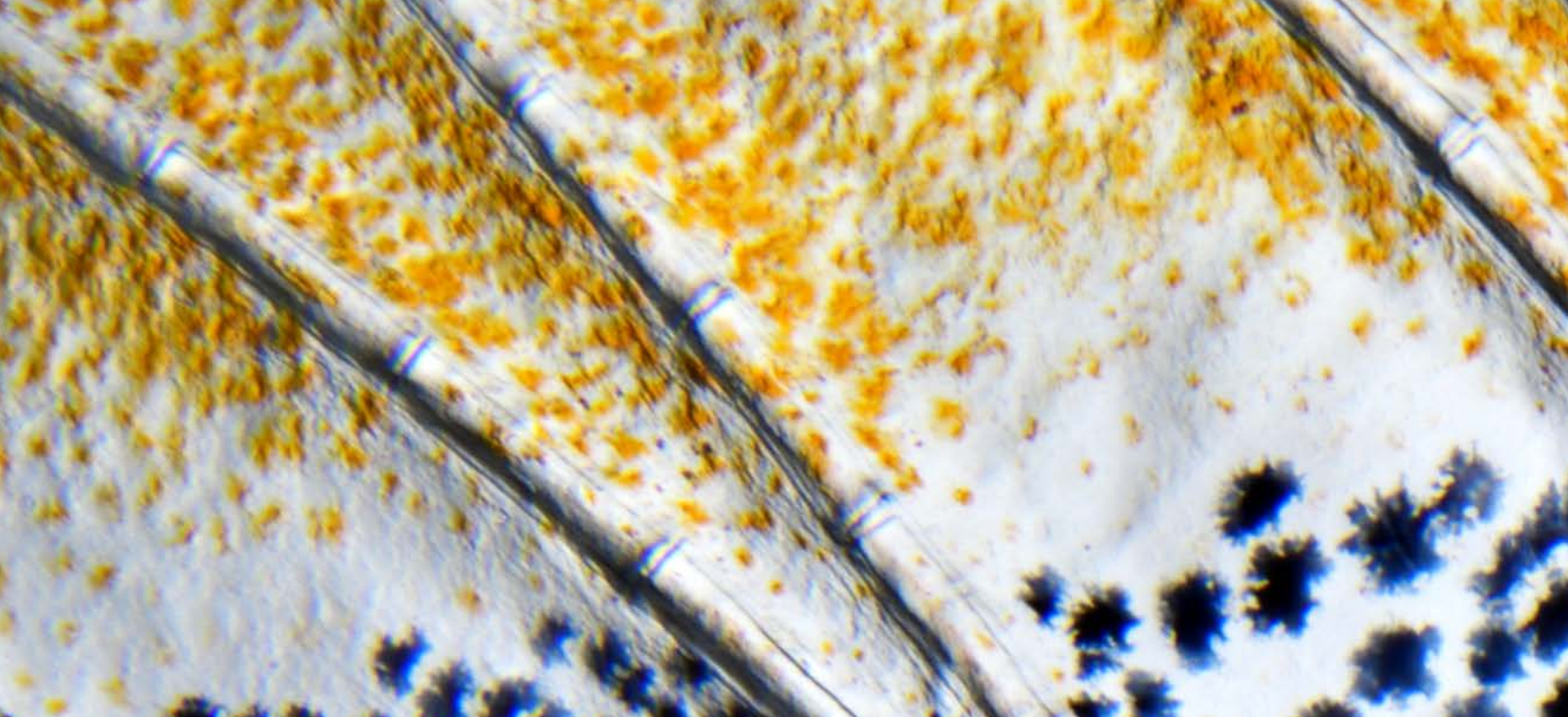

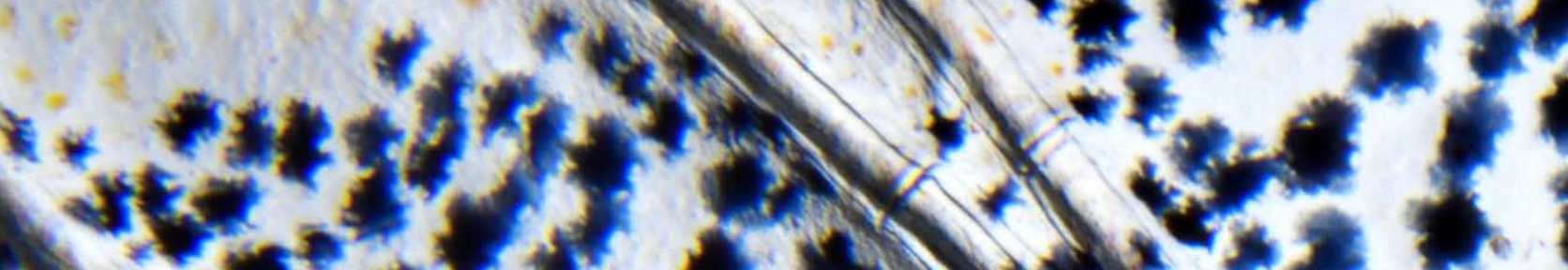

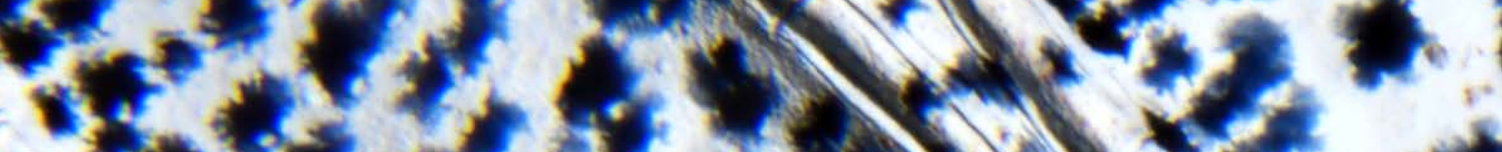
(5)

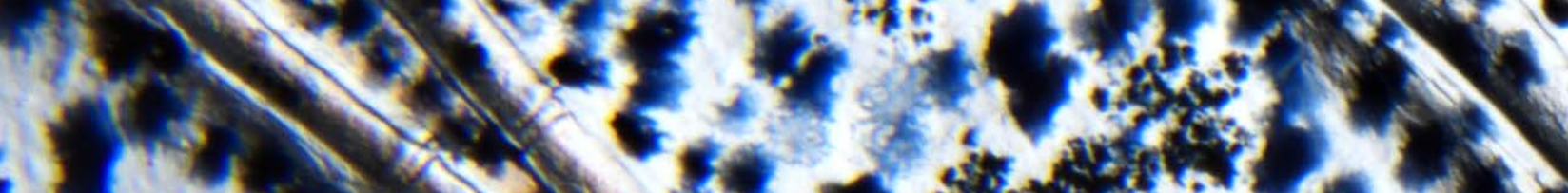

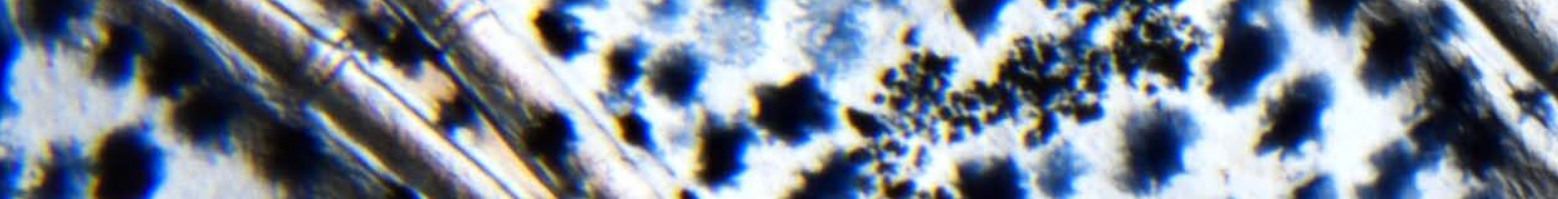
$x^{4}+x^{2}+x^{2}+2 x^{2}$

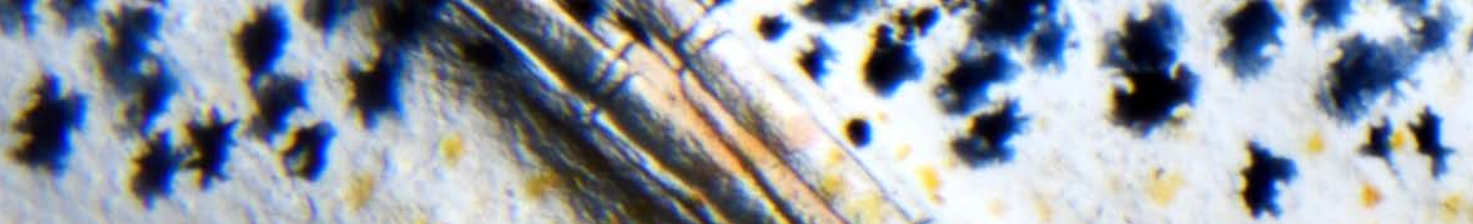

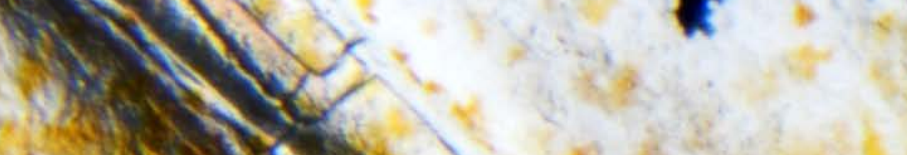

M. 